\title{
Influence of Seabed Response on Fatigue Performance of Steel Catenary Risers in Touchdown Zone
}

\author{
by
}

\section{Hodjat Shiri}

This thesis is presented for the degree of

Doctor of Philosophy

at

The University of Western Australia

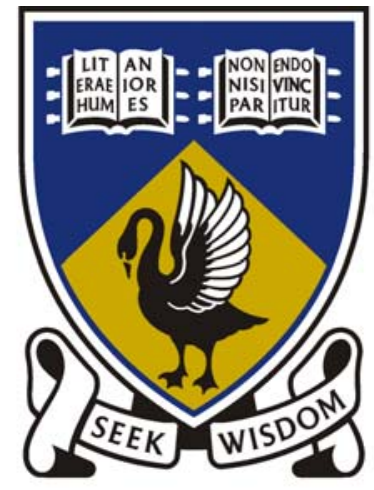

Centre for Offshore Foundation Systems

School of Civil and Resource Engineering

February 2010 

In the name of the most compassionate

"To Yoosof-e-Zahra, Aale-e-Taha and Khatam-e-Sofara (SAA) who never stopped their heartfelt supports to me and my family" 



\section{DECLARATION}

I hereby declare that, except where specific reference is made in the text to the work of others, the contents of this thesis are original and have not been submitted to any other university.

\section{Hodjat Shiri}

February 2010 


\section{ABSTRACT}

The influence of the riser-seabed interaction on fatigue performance of steel catenary risers (SCRs) is now widely accepted. Due to uncertainties associated with the complex nature of the riser-seabed interaction, existing analysis software and design recommendations have mostly been limited to consideration of a linear elastic seabed response, which is a significant over-simplification from a geotechnical point of view. Indeed, observations from ROV surveys have shown that trenches several diameters deep can develop in the touchdown zone of the SCR. Sophisticated non-linear hysteretic seabed models have recently been introduced, which are able to simulate the reduction in secant stiffness with increasing displacement of the riser, the gradual embedment of the riser into the seabed, and even trench development under cyclic perturbations of the floating vessel supporting the SCR.

The current dissertation has focused on the effect of the seabed model in estimating fatigue damage in the touchdown zone of SCRs. The thesis starts with a review of analytical approaches for modelling the profile and stress distributions within risers. A generic Spar system, with a particular riser geometry and wave scatter diagram based on conditions in the Gulf of Mexico, were adopted to evaluate how the fatigue damage was affected by the seabed stiffness, initially for elastic response of the seabed. Finite element analyses were undertaken using the software package ABAQUS. All analyses were carried out as two-dimensional, quasi-static analyses, with the main focus being to explore the relative effect of different seabed responses rather than on assessment of the absolute fatigue damage. The applicability of analytical solutions for the SCR system was explored, combining catenary equations with a boundary layer method in order to estimate the shear force distribution and hence fatigue damage under the action of lifetime wave loading, comparing the results with those from the finite element analyses.

A hysteretic non-linear seabed model was then implemented within ABAQUS, in order to examine the effect of a non-linear seabed model on the calculated fatigue damage. The effects of different numbers of waves, and the ordering of wave packages of different amplitude, were explored in order to arrive at an appropriate strategy for conducting fatigue analysis with a non-linear seabed response. The investigations showed that the fatigue damage in a non-linear hysteretic seabed is independent of the number of representing wave cycles and the hierarchy of individual sea states, if the system experiences the most severe sea state in the beginning of the analysis. Therefore, the applicability of Miner's rule for superposition of individual damages was proved for non-linear seabed with particular considerations. Parametric studies were undertaken to evaluate how different aspects of the seabed model, such as the shear strength profile, the degree of suction resistance mobilised during uplift of the riser and the maximum unloading stiffness, affected the fatigue damage. 
The non-linear model was then used to examine the effect of gradual embedment of the riser, initially for moderate penetration into the seabed but then using modified parameters in the non-linear seabed model, to simulate the development of deep trenches. The effect of the trench depth on the stress distribution along the riser was studied, and consequently how the trench depth affected the fatigue performance in the touchdown zone.

The results show that in both linear elastic and non-linear hysteretic seabeds, the ultimate fatigue damage increases with increasing seabed stiffness and the peak damage point is moved slightly towards the anchor end of the riser. The gradual embedment of the riser into the non-linear hysteretic seabed under cyclic vessel excitation also increases the fatigue damage, slightly moving the peak damage point towards the vessel end of the SCR. The pre-trenching studies show that the development of deep trenches has significant effect on fatigue damage, considerably increasing the peak damage point with extreme relocation towards the vessel end of the SCR. The comparison of the shear force distribution and the ultimate fatigue damage for various trench depths shows the peak shear force in mean vessel position and the peak fatigue damage are relocated in opposite directions as the trench depth is increased. This suggests that the peak shear force in the vessel mean position is not a direct indicator of maximum fatigue damage. The fatigue damage is mostly driven by riser fluctuations from moderate waves, with a considerable number of cycles, at the trench edge towards the vessel end of the SCR. 


\section{ACKNOWLEDGEMENTS}

I would like to express my sincere gratitude to my supervisor, Prof. Mark Randolph, whose excellent guidance, professional suggestions and patience made completion of this thesis possible. His continual illustrative comments and enthusiasm through the difficult situations and the give-up moments is a sign of his eminency in worldwide academic community.

I am also deeply grateful for Prof. Mark Cassidy for his valuable suggestions and supports particularly in numerical simulations and modelling techniques; and also Prof. Martin Fahey for his excellent advice on thesis writing.

I would like to give my profound appreciation to Dr. Mehrdad Kimiaei, for his genuine and invaluable suggestions and sharing his extensive industrial experience in numerical simulations and particularly in fatigue calculations.

My special thanks go to Dr. Dong Wong and Dr. Tian Yinghui for their professional suggestions and supports on programming the subroutines for user-defined elements of ABAQUS and their excellent ideas and comments for code improvement and trouble shooting.

In addition, I acknowledge the financial support provided by the University Postgraduate Award (U.P.A.), the WA:ERA PhD Scholarship and the COFS Ad-Hoc Scholarship. I would like to give many thanks to the COFS and CRE staff for their friendly support over the duration of this research and the numerous visitors to COFS who became good friends over the years.

I am also extremely appreciative to my friends in industry round the world for their generous help keeping me in the loop during my $\mathrm{PhD}$ research, providing me opportunities to participate in various mooring analyses of floating structures and design of offshore foundations as well.

I would like also to thank the special breed of my fellow PhD students and all my UWA and WA friends for their companionship and creative discussions and for all of those who have helped me in various ways during my stay, many thanks!

Finally, my boundless and sincere thanks to my wife and children for their love and the sacrifices they have endured and never complained about deficiencies; to my parents and my wife's parents for their continual spiritual supports to ensure a better life for their loving children.

\section{Hodjat Shiri}

February 2010 


\section{TABLE OF CONTENTS}

CHAPTER 1. Introduction .....................................................................................18

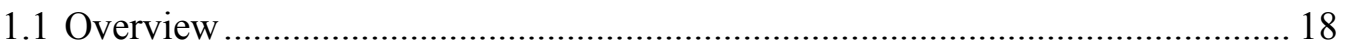

1.2 SCR fatigue performance.............................................................................. 19

1.3 riser-seabed interaction and fatigue damage in TDZ ....................................... 20

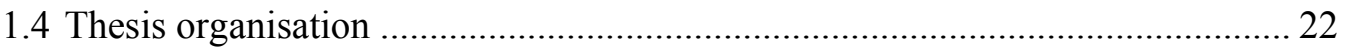

CHAPTER 2. Literature review ................................................................................24

2.1 Overview of steel catenary risers ………………………………………...... 24

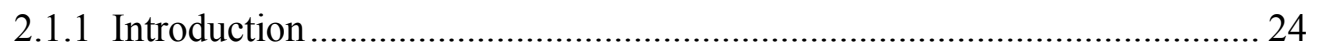

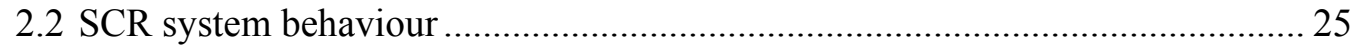

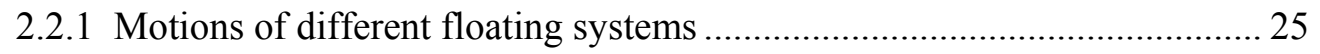

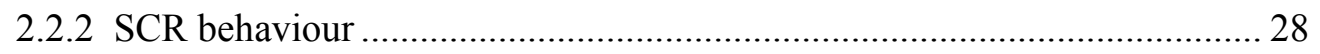

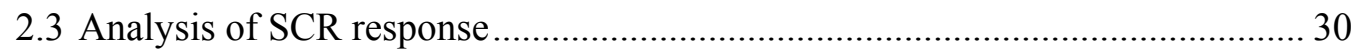

2.3.1 SCR static configuration in linear seabed …………….................................. 30

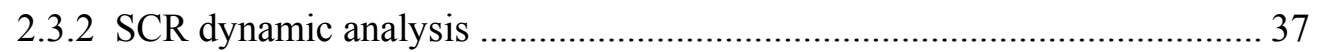

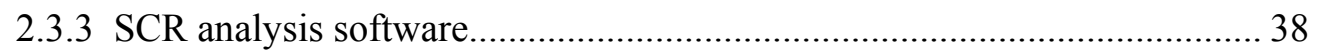

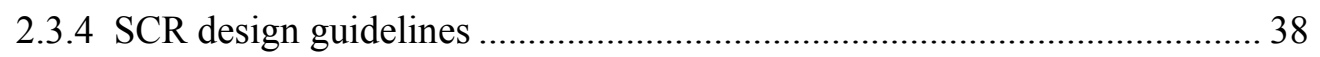

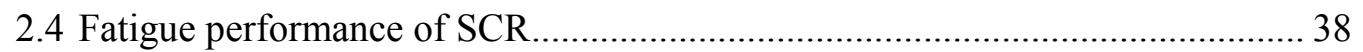

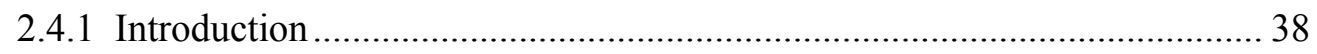

2.4.2 Fatigue performance of SCR in touchdown zone ..................................... 40

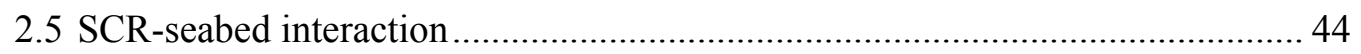

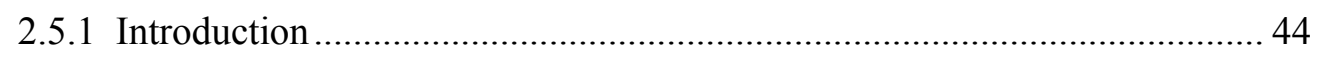

2.5.2 Deepwater seabed sediments characterization ........................................... 44

2.5.3 Classical static penetration of a cylinder into soil........................................ 46

2.5.4 Seabed response to system dynamics in TDZ .......................................... 52

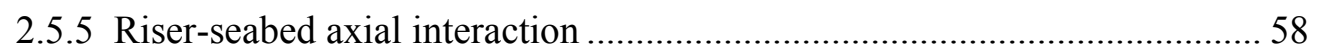

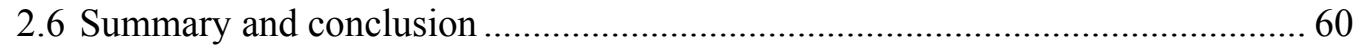

CHAPTER 3. Closed form seabed interaction models .............................................61

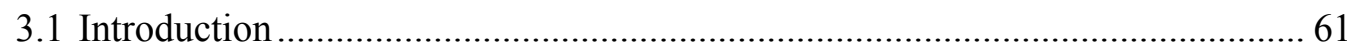

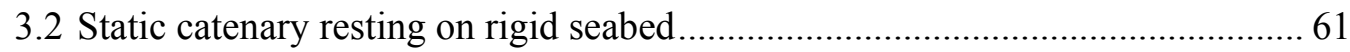

3.2.1 Catenary equations for vessel's near and far offset .................................... 64

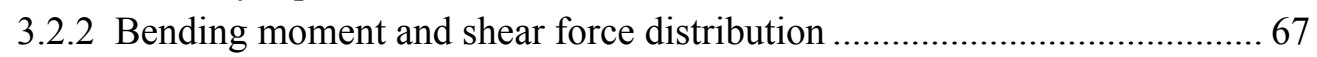

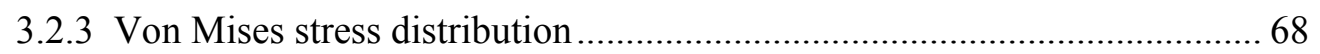

3.3 Static catenary resting on elastic seabed .......................................................... 71

3.3.1 Hetenyi's solutions for beam on Winkler elastic foundation....................... 71

3.3.2 Tensionless beam on Winkler elastic foundation........................................ 73

3.3.3 Tension beam on Winkler elastic foundation.............................................. 77

3.3.4 Boundary layer method for seabed interaction modelling ............................ 84

3.3.5 Fatigue damage prediction at TDZ by boundary-layer method .................. 90

3.4 Summary and conclusions........................................................................... 94 
CHAPTER 4. Fatigue performance of SCRs on linear elastic seabed .....................96

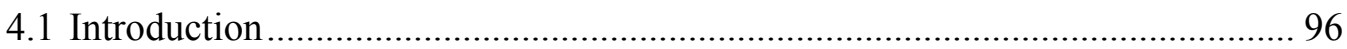

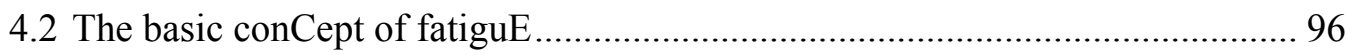

4.3 Fatigue damage of steel catenary risers …..................................................... 99

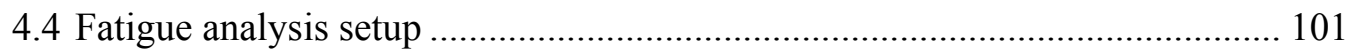

4.4.1 ABAQUS finite element analysis program........................................ 101

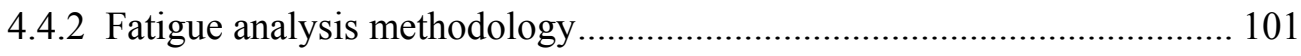

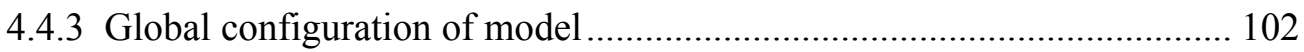

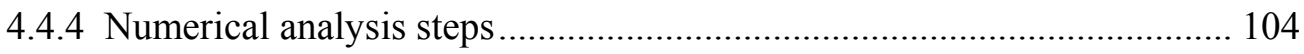

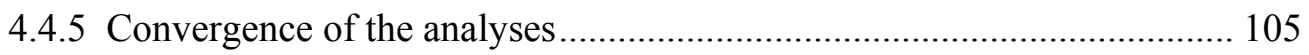

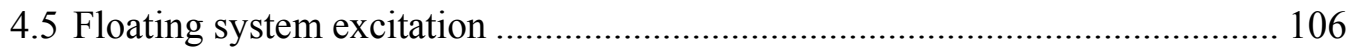

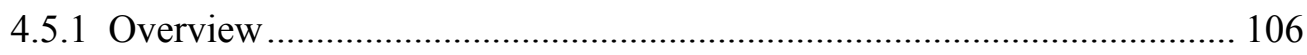

4.5.2 Selection of Spar vessel as floating system .......................................... 106

4.5.3 Environmental data / wave scatter diagram .......................................... 110

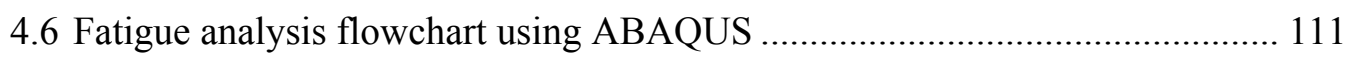

4.6.1 The global flowchart of analysis setup ................................................ 111

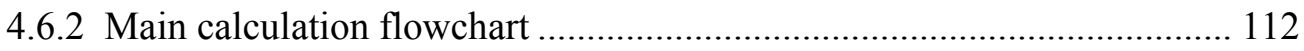

4.6.3 Vessel excitation internal flowchart........................................................ 114

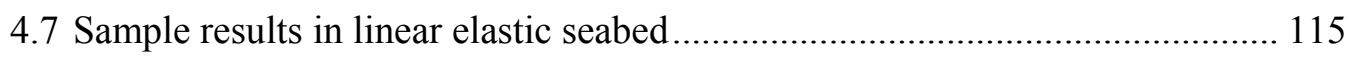

4.7.1 Fatigue damage distribution morphology ............................................ 118

4.7.2 Influence of wave orders and wave number of cycles ............................ 119

4.7.3 Influence of seabed stiffness on fatigue ................................................. 120

4.8 Fatigue damage prediction by closed form solutions.................................... 125

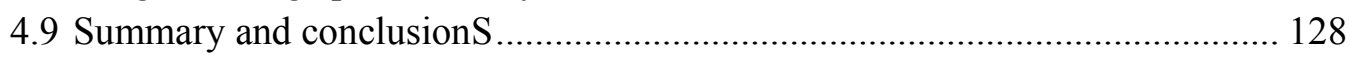

CHAPTER 5. Non-linear SCR-soil interaction theory ......................................... 130

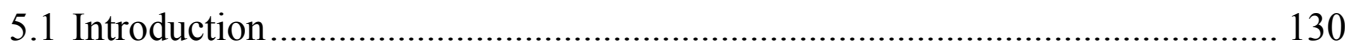

5.2 Principles of non-linear soil models........................................................... 130

5.3 Randolph and Quiggin's non-linear hysteretic soil model............................. 132

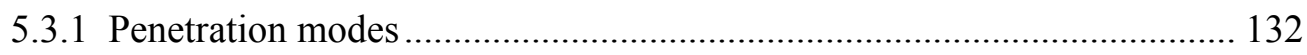

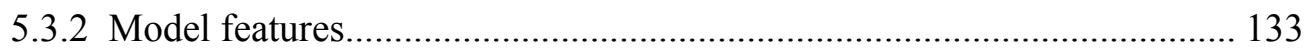

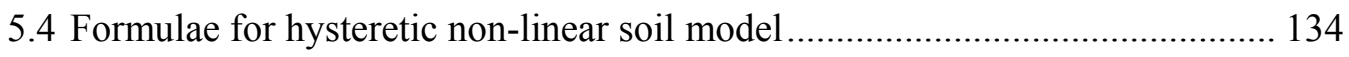

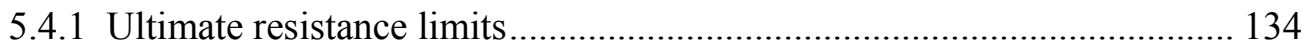

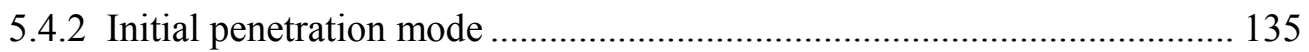

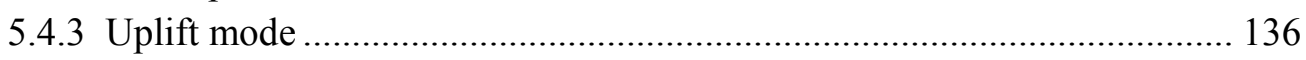

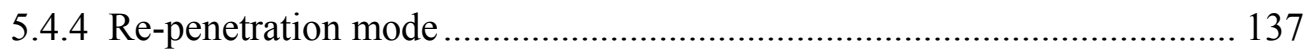

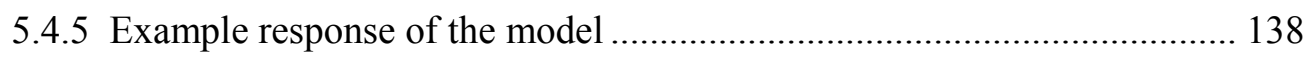

5.4.6 Typical values for model parameters ................................................... 140

5.5 Non-linear soil model implemented in abaqus ........................................... 144

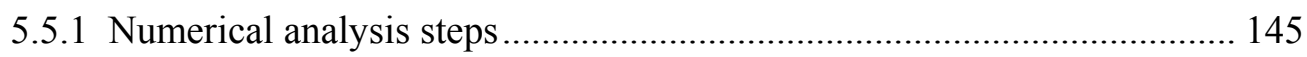

5.5.2 Load-displacement response in quasi-static analysis............................. 145

5.5.3 Gradual embedment and stress distribution.......................................... 149

5.5.4 Comparison with linear elastic seabed in an in-place static analysis........ 154 
5.5.5 Deep trenching by hysteretic non-linear seabed model .......................... 157

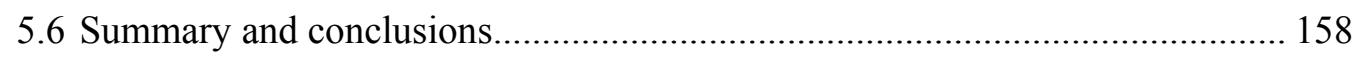

CHAPTER 6. Fatigue performance of SCR on hysteretic non-linear seabed....... 160

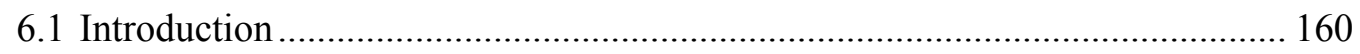

6.2 Fatigue damage at TDZ under single wave................................................ 161

6.3 Von mises stress range variation by wave hierarchy ..................................... 165

6.3.1 Two single waves with different orders .................................................. 165

6.3.2 Full package of waves with regular order ............................................... 169

6.3.3 Full package of waves with various random orders.............................. 171

6.3.4 Full package of waves with various random orders and most severe

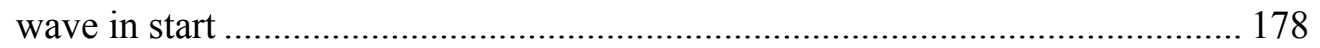

6.4 Influence of suction force on fatigue damage ................................................ 183

6.5 Influence of seabed stiffness on fatigue damage........................................... 189

6.6 Fatigue calculation procedure for plastic seabed ........................................... 193

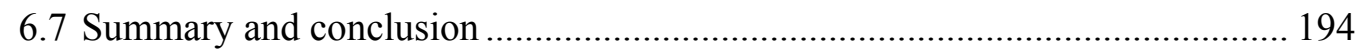

CHAPTER 7. Ultimate trench insertion and influence on fatigue performance .. 196

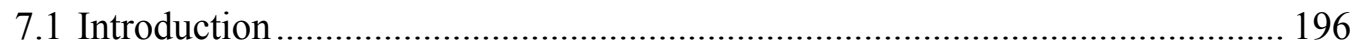

7.2 The morphology of SCR trench profile …..................................................... 196

7.2.1 SCR trench profile observed by ROV surveys ....................................... 196

7.2.2 Mathematical approximation of SCR trench............................................ 198

7.3 Artificial trench insertion and fatigue performance.......................................... 204

7.3.1 Ultimate quadratic exponential trench insertion in elastic seabed ........... 204

7.3.2 Ultimate trench insertion by hysteretic non-linear seabed model ............. 212

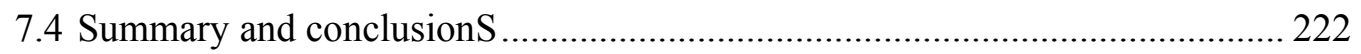

CHAPTER 8. Summary and conclusions ...................................................................... 224

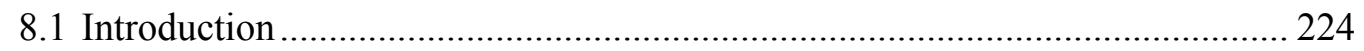

8.2 Linear elastic seabed and SCR fatigue performance...................................... 225

8.3 Non-linear hysteretic riser-seabed interaction .............................................. 226

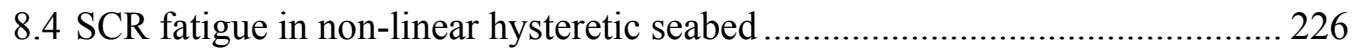

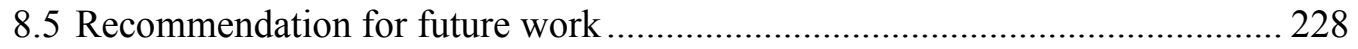

\section{APPENDICES}

Appendix A: ABAQUS UEL\& DISP Subroutines

Appendix B: Parametric study of stress and fatigue

Appendix C: Parametric Study of model individual nodes 


\section{TABLE OF FIGURES}

Figure 1-1: Schematic layout of SCR attached to a Spar ( Spar by (Marinetalk 2000)) 18

Figure 1-2: Trench formation in touchdown zone for an SCR connected to a Spar....... 20

Figure 2-1: Schematic side view of SCR connected to floating system

Figure 2-2: The relative hull response of various floating systems to environment (FloaTec 2006)..... 26

Figure 2-3: Sample surge RAO of various floating system (Murray 2007) ................... 27

Figure 2-4: Sample heave RAO of various floating system (Murray 2007).................. 27

Figure 2-5: Sample pitch RAO of various floating systems (Murray 2007) .................. 27

Figure 2-6: SCR/Spar TDP global dynamic movement (Theti and Moros 2001) .......... 29

Figure 2-7: Schematic horizontal movement of TDP relative to vessel offset ............... 30

Figure 2-8: Static configuration of SCR connected to a Spar ....................................... 31

Figure 2-9: Maximum shear force in touchdown zone (Randolph and White 2008b) 36

Figure 2-10: Variation of first order fatigue damage with seabed stiffness (Theti and Moros 2001)

Figure 2-11: Penetration and extraction data for cyclic penetration test (Low et al. 2008)

Figure 2-12: Plasticity solutions and empirical approaches for pipe embedment (Cathie et al. 2005) 48

Figure 2-13: Collapse load for vertical pipe embedment (a) rough pipe (b) smooth pipe (Merifield et al. 2008)

Figure 2-14: Comparison of penetration resistance curves (Randolph and White 2008b)

Figure 2-15: Non-dimensional bearing capacity factor, load-controlled tests (Dunlap et al. 1990)

Figure 2-16: Banana-shaped cyclic vertical pipe-soil interaction and vertical resistance (Hodder et al. 2008).

Figure 2-17: Penetration-resistance curves in large amplitude tests (Aubeny et al. 2005; Murff et al. 1989)

Figure 2-18: Accumulation of pipe embedment during lay effect model test (Cheuk and White 2008) 57

Figure 3-1: Generic SCR overall configuration on rigid seabed 62

Figure 3-2 : Generic configuration of SCR connected to a Spar... 63

Figure 3-3: Vessel offsets 65

Figure 3-4: Non-linear regression of the left hand side of equation (3-20) 67 
Figure 3-5: SCR catenary shape based on mathematical equations.............................. 70

Figure 3-6: Bending moment distribution along SCR based on catenary equations ...... 70

Figure 3-7: Shear force distribution along SCR based on catenary equations................ 71

Figure 3-8: Von Mises stress along SCR based on catenary equations ......................... 71

Figure 3-9: Static catenary combination with beam on Winkler elastic foundation....... 72

Figure 3-10 : Section A-A, Tension beam on Winkler elastic foundation (Hetenyi

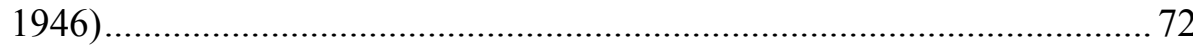

Figure 3-11: Tensionless beam on Winkler foundation under point load...................... 73

Figure 3-12: Tensionless beam on Winkler foundation under concentrated moment .... 73

Figure 3-13: SCR profile in TDZ for tensionless beam on Winkler foundation ............. 75

Figure 3-14: SCR shear force in TDZ for tensionless beam on Winkler foundation ..... 76

Figure 3-15: SCR bending moment in TDZ for tensionless beam on Winkler

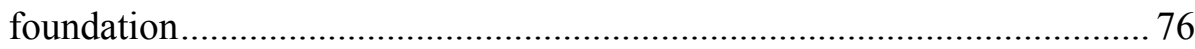

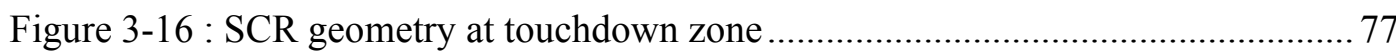

Figure 3-17: Tension beam on Winkler foundation under point load............................ 78

Figure 3-18: Tension beam on Winkler foundation under concentrated moment .......... 78

Figure 3-19: Seabed profile using tension beam on Winkler elastic foundation ............. 80

Figure 3-20: TDZ shear force using tension beam on Winkler elastic foundation .......... 80

Figure 3-21: TDZ bending moment using tension beam on Winkler elastic

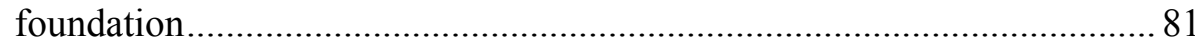

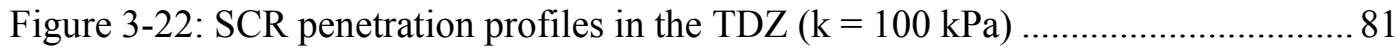

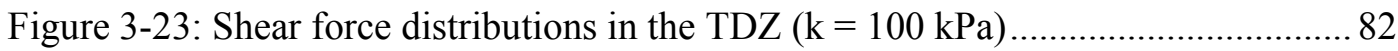

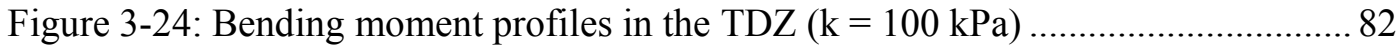

Figure 3-25: Discontinuity of closed form shear force at the TDP $(\mathrm{k}=100 \mathrm{kPa}) \ldots \ldots \ldots . . .83$

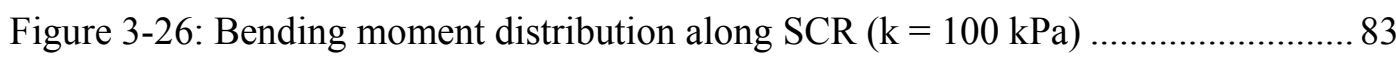

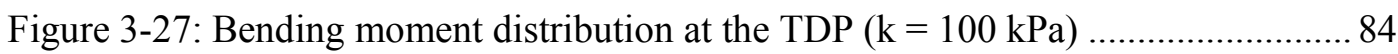

Figure 3-28: SCR configuration, (Pesce et al. 1998a) ................................................. 85

Figure 3-29: Normalised shear force in TDZ, (Pesce et al. 1998a) ................................ 88

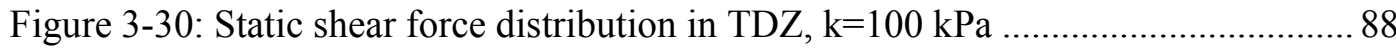

Figure 3-31: Comparison of bending moments along SCR, $\mathrm{k}=100 \mathrm{kPa}$...................... 89

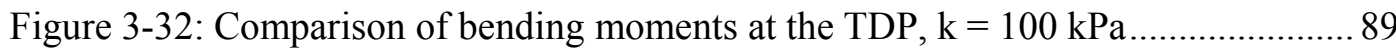

Figure 3-33: Peak shear force at TDZ in different seabed stiffness.............................. 90

Figure 3-34: Shear force distribution in two different vessel locations, produced by

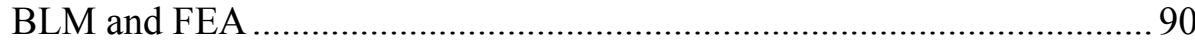

Figure 3-35: Von Mises stress at two different vessel locations, produced by BLM and FEA. 
Figure 3-36: Von Mises stress range between two different vessel locations, from BLM and FEA

Figure 3-37: Stress range variation of stiff and soft seabed at TDP 93

Figure 3-38: Peak fatigue position relative to shear force and other key profiles 94

Figure 4-1: Sample S-N curves for fatigue damage calculations (DNV-RP-C203 2008).

Figure 4-2: The global geometry of SCR modelled by ABAQUS 103

Figure 4-3: Schematic illustration of FE analysis steps 104

Figure 4-4: Spar floating system with helical strakes on hull (Marinetalk 2000). 107

Figure 4-5: Generic truss Spar motion RAO, head sea (Bridge, 2005) ........................ 108

Figure 4-6: Sample RAO with no phase information 109

Figure 4-7: Sample RAO with phase information 109

Figure 4-8: Main flowchart of fatigue analysis using ABAQUS.

Figure 4-9: The flowchart of ABAQUS main procedure for fatigue analysis.

Figure 4-10: Internal flowchart of DISP subroutine for vessel excitation 114

Figure 4-11: Sample fatigue damage distribution through TDZ, seabed stiffness $100 \mathrm{kPa}$ 116

Figure 4-12: Contribution of each sea state to total fatigue damage 117

Figure 4-13: Variation of significant wave height versus sea state ID 117

Figure 4-14: Variation of wave period versus sea state ID. 117

Figure 4-15: Variation of number of applied waves versus sea state ID 118

Figure 4-16: Cumulative fatigue damage for the order of min to max vessel offset .... 118

Figure 4-17: Morphology of fatigue damage distribution

Figure 4-18: Fatigue damage with different number of cycles .................................. 120

Figure 4-19: fatigue damage with different order of sea states................................. 120

Figure 4-20: Fatigue damage variation with seabed stiffness .................................. 121

Figure 4-21: Fatigue life variation with seabed stiffness

Figure 4-22: Percentage of rigid seabed fatigue damage with varying seabed stiffness.

Figure 4-23: Damage rate of various seabeds to rigid seabed (Bridge et al. 2004) ...... 122

Figure 4-24: Fatigue life is decreased as seabed stiffness increased (Randolph and Quiggin 2009) 123

Figure 4-25: Shear force distribution along TDZ in various seabed stiffness .............. 123

Figure 4-26: Seabed reaction along TDZ in various seabed stiffness. 124

Figure 4-27: Proportional fatigue damage obtained from analytical solutions and numerical simulations . 125

Figure 4-28: Fatigue damage prediction flowchart using boundary layer method ....... 127 
Figure 4-29: Comparison of fatigue damage predicted by numerical analysis and closed form solutions

Figure 4-30: Comparison of fatigue life predicted by numerical analysis and closed form solutions 128

Figure 5-1: Non-linear riser-seabed interaction model (Bridge et al. 2004) 131

Figure 5-2:Non-linear Soil model characteristics for different modes (Randolph and Quiggin 2009) 133

Figure 5-3: Example response under complex motion 139

Figure 5-4: Force displacement plot with different $\mathrm{K}_{\max }$ values. 141

Figure 5-5: Influence of $\mathrm{f}_{\text {suc }}$ on peak suction mobilisation 142

Figure 5-6: Influence of $\lambda_{\text {suc }}$ on suction decay and soil softening. 143

Figure 5-7: influence of $\lambda_{\text {rep }}$ on delay of penetration resistance mobilisation 144

Figure 5-8: Nodes at the seabed with various displacement stages at the same time for vessel far offset. 146

Figure 5-9: Schematically location of the sample nodes on final profile of SCR......... 146

Figure 5-10: Full breakout of node 358 in the TDZ .................................................... 147

Figure 5-11: Partial breakout of node 356 in the TDZ ............................................... 147

Figure 5-12: Partial suction of node 354 in the TDZ ................................................... 148

Figure 5-13: Partial suction of node 350 in the TDZ ................................................. 148

Figure 5-14: No suction response of node 344 in the TDZ ......................................... 149

Figure 5-15: Progressive penetration at TDZ under cyclic loading ........................... 150

Figure 5-16: Shear force variation with progressive penetration in the TDZ .............. 150

Figure 5-17: Bending moment variation with progressive penetration in the TDZ ...... 151

Figure 5-18: Shear force and penetration profile at TDZ ….......................................... 152

Figure 5-19: Cyclic contact stress range over 10 load cycles ...................................... 152

Figure 5-20: Contact stress envelope over 10 load cycles ........................................ 153

Figure 5-21: Shear force snapshots along the TDZ over 10 load cycles in extreme far and near offsets compared to the vessel mean position ..................... 153

Figure 5-22: Cyclic bending moment distribution in the TDZ over 10 load cycles ..... 154

Figure 5-23: Embedment geometry and seabed reaction in linear elastic and nonlinear seabed

Figure 5-24: Embedment geometry and shear force in linear elastic and non-linear seabed

Figure 5-25: Embedment geometry and bending moment in linear elastic and nonlinear seabed 156

Figure 5-26: Seabed reaction against normalised penetration depth in linear elastic and non-linear seabed. 
Figure 5-27: Deep trenching by extreme value of non-linear model parameters 157

Figure 5-28: Penetration-resistance of node (N358) at the seabed for deep trenching $\left(\lambda_{\text {rep }}=3.0\right)$

Figure 6-1: Gradual variation and deepening of trench profile under single wave application 162

Figure 6-2: Gradual increase of maximum von Mises stress range with number of wave cycles 162

Figure 6-3: Gradual increase of fatigue damage with trench deepening 163

Figure 6-4: Gradual increase of fatigue damage with trench deepening 163

Figure 6-5: Gradual increase of von Mises stress range variation with trench deepening 164

Figure 6-6: Fatigue damage calculation by increasing number of wave cycles 165

Figure 6-7: Maximum von Mises stress variation range in wave height decreasing order 166

Figure 6-8: Fatigue damage at TDZ with different wave orders in plastic seabed 167

Figure 6-9: Comparison of ultimate stress variation range for sea state II with and without sea state I 168

Figure 6-10: Seabed profiles corresponding to Figure 6-9 168

Figure 6-11: Vessel excitation in descending order of wave severity 169

Figure 6-12: Von Mises stress variation range for full sea state package in descending order of wave severity 170

Figure 6-13: Initial stages of von Mises stress variation range for full sea state package

Figure 6-14: Trench profile at various stages of applying full sea state package ......... 171

Figure 6-15: Vessel surge offset against analysis time in random wave order No.1 .... 172

Figure 6-16 : Detail of vessel surge offset against analysis time for two sea states in random wave order No.1

Figure 6-17: Von Mises stress range variation with random wave order in linear elastic seabed 173

Figure 6-18: Maximum von Mises stress variation range in wave height random order No.1.

Figure 6-19: Comparison of re-shuffled analysis with original random order No.1 .... 174

Figure 6-20: Comparison of re-shuffled analysis with original random order No.1 within range $\mathrm{H}$

Figure 6-21: Vessel surge offset against analysis time in random wave order No.2 $\ldots .175$

Figure 6-22 Vessel surge offset against analysis time in random wave order No.3 ..... 176

Figure 6-23: Maximum von Mises stress variation range in wave height random order No.2. 
Figure 6-24: Maximum von Mises stress variation range in wave height random order No.3.

Figure 6-25: Dependency of fatigue damage on wave orders in non-linear plastic seabed

Figure 6-26: Comparison of von Misses stress range for random order No.1 with and without pre-trench

Figure 6-27 Comparison of von Misses stress range for random order No.2 with and without pre-trench

Figure 6-28 Comparison of von Misses stress range for random order No.3 with and without pre-trench

Figure 6-29: Von Mises stress level increase with pre-trench in random wave order No.1

Figure 6-30: Von Mises stress level increase with pre-trench in random wave order No.2.

Figure 6-31: Von Mises stress level increase with pre-trench in random wave order No.3

Figure 6-32: Fatigue damage with and without pre-trenching in random wave orders of No.1, No.2 and No.3

Figure 6-33: Fatigue damage distribution with various suction force generation

Figure 6-34: Non-logarithmic fatigue damage distribution with various suction force generation.

Figure 6-35: Comparison of final trench profile for various magnitudes of suction ratio.

Figure 6-36: Comparison of average shear force for various magnitudes of suction ratio.

Figure 6-37: Comparison of cyclic bending moment for various magnitudes of suction ratio

Figure 6-38: Comparison of final contact stress with initial contact for various magnitudes of suction ratio

Figure 6-39: Comparison of cyclic contact force for various magnitudes of suction ratio.

Figure 6-40: Comparison of contact force envelope for various magnitudes of suction ratio

Figure 6-41: Comparison fatigue damage for various magnitudes of suction decay.... 189

Figure 6-42: fatigue damage variation with various seabed stiffness 190

Figure 6-43: Fatigue damage variation with various seabed stiffness 190

Figure 6-44: Fatigue life variation with mudline shear strength. 191

Figure 6-45: Fatigue life variation with undrained shear strength gradient 191 
Figure 6-46: Fatigue life variation with mudline shear strength (Randolph and Quiggin 2009)

Figure 6-47: Peak fatigue damage for linear and non-linear seabed with various stiffness values and shear strength gradients.

Figure 6-48: Peak fatigue damage for linear and non-linear seabed with various stiffness values and shear strength gradients (lower range for both).....

Figure 7-1: Allegheny gas export seven month after installation (Bridge and Howells 2007)

Figure 7-2: Allegheny oil export 16 month after installation (Bridge and Howells 2007).

Figure 7-3: Comparison of the SCR trenches from Allegheny oil and gas fields ........ 198

Figure 7-4: Gradual displacement of trench key points with trench deepening ........... 199

Figure 7-5: SCR trench profile in linear elastic seabed 200

Figure 7-6: Mathematical approximation of non-linear seabed trench at cycle number 5 .....

Figure 7-7: Mathematical approximation of non-linear seabed trench at cycle number 1000 202

Figure 7-8: Seabed trenches created by ABAQUS and CARISIMA methods (private communication) 202

Figure 7-9: Quadratic exponential trench with various values of $x_{z-\max } \ldots \ldots \ldots \ldots \ldots \ldots \ldots . . . . . . . . . .203$

Figure 7-10: Quadratic exponential trench with various values of $z_{\max } \ldots \ldots \ldots \ldots \ldots \ldots \ldots . . . . . . . . . .203$

Figure 7-11: Riser-trench contact geometry with various trench locations .................. 204

Figure 7-12: SCR and artificial 1.5D trench profile on 10kPa elastic seabed, case I ... 205

Figure 7-13: SCR and artificial 1.5D trench profile on $10 \mathrm{kPa}$ elastic seabed, case

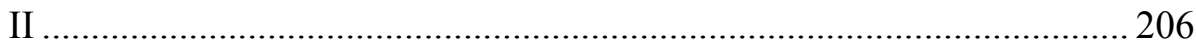

Figure 7-14: Contact pressure in 1.5D, $10 \mathrm{kPa}$ trenched seabed, case I \& II .............. 207

Figure 7-15: Logarithmic fatigue damage in $1.5 \mathrm{D}, 10 \mathrm{kPa}$ trenched seabed, case I \& II 207

Figure 7-16: Non-logarithmic fatigue damage in 1.5D, $10 \mathrm{kPa}$ trenched seabed, case I \& II

Figure 7-17: SCR and artificial 5D trench profile on 10kPa elastic seabed, case I...... 209

Figure 7-18: SCR and artificial 5D trench profile on 10kPa elastic seabed, case II..... 209

Figure 7-19: Contact pressure in 5D, $10 \mathrm{kPa}$ trenched seabed, case I \& II 210

Figure 7-20: Logarithmic fatigue damage in $5 \mathrm{D}, 10 \mathrm{kPa}$ trenched seabed, case I \& II

Figure 7-21: Non-logarithmic fatigue damage in 1.5D, $10 \mathrm{kPa}$ trenched seabed, case I \& II

Figure 7-22: Initial trench profile with various depths 
Figure 7-23: Logarithmic fatigue damage for random wave order No.1 in various pre-trenches

Figure 7-24: Non-logarithmic fatigue damage for random wave order No.1 in various pre-trenches

Figure 7-25: Logarithmic fatigue damage for random wave order No.3 in various pre-trenches

Figure 7-26: Non-logarithmic fatigue damage for random wave order No.3 in various pre-trenches

Figure 7-27: Von Mises stress range level of peak fatigue points for 5.0D trenched and non-trench seabed

Figure 7-28: Fatigue damage distribution in trench and non-trenched seabeds random order No.1 and 3

Figure 7-29: Contact stress envelope with various initial trenches for random wave order No.1.

Figure 7-30: Cyclic contact stress with various initial trenches for random wave order No.1

Figure 7-31: Post trenching shear force at mean vessel location with various trench depths

Figure 7-32: Average shear force with various initial trenches for random wave order No.1.

Figure 7-33: Snapshots of bending moment in vessel far and near offset in 2D trench 220

Figure 7-34: Snapshots of shear force in vessel far and near offset in 2D trench......... 220

Figure 7-35: Snapshots of riser profile in the seabed in vessel far and near offset in $2 \mathrm{D}$ trench 


\section{LIST OF TABLES}

Table 2-1: Typical geotechnical parameters of clay, (DNV-RP-F105 2006) 45

Table 2-2: Fitting coefficients for power law expression (Aubeny et al. 2005)

Table 2-3: Resistance factor for pipeline axial coefficient under fully drained condition (Finch et al. 2000) ................................................................. 59

Table 3-1: Generic SCR parameters for static analysis .............................................. 70

Table 4-1: key dimensions of SCR modelled by ABAQUS ...................................... 103

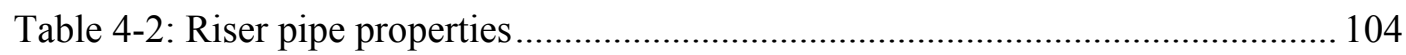

Table 4-3:Manipulated wave scatter diagram for a 30 year operational life, for deep water conditions in Gulf of Mexico ................................................. 111

Table 5-1: Model parameters for example in Figure 5-3 …...................................... 139

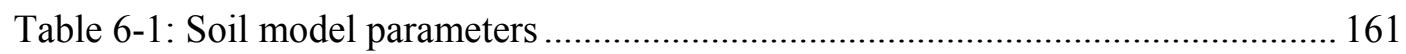

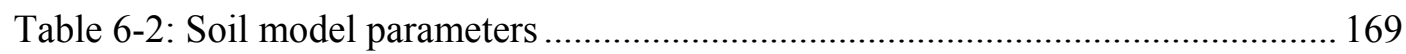

Table 7-1: Non-linear seabed soil parameters for performed fatigue analyses............. 213 


\section{NOTATION}

$\mathrm{A}_{\text {disp }}$ nominal area of the pipe below the seabed tangent plane

$\mathrm{A}_{\mathrm{RP}}$ resistance ratio to ensure correct initial stiffness on load reversal

$\mathrm{A}_{\mathrm{s}} \quad$ steel section area of the riser

$\mathrm{A}_{\mathrm{UL}}$ resistance ratio used within the hyperbolic factor

$\overline{\mathrm{a}} \quad$ intercept of the design $\mathrm{S}-\mathrm{N}$ curve with the $\log \mathrm{N}$ axis

a non-dimensional penetration resistance parameter

a wave amplitude (equation 4-5)

B pipe bearing width limited to external diameter

b non-dimensional penetration resistance parameter

D fatigue damage (equation 4-2)

D outer diameter of the riser steel section

$\mathrm{D}_{\mathrm{T}} \quad$ total fatigue damage (equation 4-3)

E riser steel Young's modulus

EI riser bending stiffness

$E_{R P}(z)$ exponential factor limiting the uplift resistance

$\mathrm{E}_{\mathrm{UL}}(\mathrm{z})$ exponential factor limiting the uplift resistance

$\mathrm{e}_{\mathrm{s}} \quad$ soil void ratio

$\mathrm{F}_{\mathrm{z}} \quad$ applied vertical force per unit length of pipeline

$\mathrm{f}_{\mathrm{b}} \quad$ soil buoyancy factor

$\mathrm{f}_{\text {suc }} \quad$ non-dimensional suction resistance ratio

$\mathrm{f}_{\mathrm{y}} \quad$ steel yield stress

g gravity acceleration

$\mathrm{H}$ horizontal component of the tension in the riser

$\mathrm{H}_{\mathrm{IP}} \quad$ hyperbolic factor for non-linear soil model

$\mathrm{H}_{\mathrm{UL}} \quad$ hyperbolic factor for the uplift mode

$\mathrm{h}_{\mathrm{n}}(\theta(\mathrm{s}))$ lateral components of steady hydrodynamic forces applied on riser 
$\mathrm{h}_{\mathrm{t}}(\theta(\mathrm{s}))$ axial components of steady hydrodynamic forces applied on riser

I second moment of area

$\mathrm{I}_{\mathrm{P}} \quad$ soil plasticity index

i number of a particular constant stress range block in the stress histogram

$\mathrm{K}$ non-dimensional soil rigidity parameter

$\mathrm{K}_{\max } \quad$ normalized maximum stiffness

$\mathrm{k} \quad$ seabed soil stiffness

$\mathrm{k} \quad$ number of stress blocks (equation 4-2)

L fatigue life

$\mathrm{L}_{\text {design }}$ design life of the riser

M bending moment at a given location along the riser

$\mathrm{M}_{\mathrm{R}}$ bending moment at the point of maximum reverse curvature of riser

$\mathrm{m}$ negative inverse slope of S-N curve

$\mathrm{m}_{\mathrm{s}} \quad$ submerged mass per unit length of the riser

$\mathrm{N}$ number of load cycles for fatigue failure

$\mathrm{N}_{\mathrm{c}} \quad$ bearing capacity factor for a strip foundation

$\mathrm{N}_{\mathrm{c}}(\mathrm{z} / \mathrm{D})$ soil bearing factor

$\mathrm{N}_{\mathrm{i}} \quad$ number of stress cycles to failure for the constant stress range $\mathrm{i}$

$\mathrm{n} \quad$ dimensionless visco-elastic rate of soil

$\mathrm{n}_{\mathrm{i}} \quad$ number of stress cycles in stress block i over the design life

P submerged weight per unit length of the riser (equals $\mathrm{m}_{\mathrm{s}} \mathrm{g}$ )

$\mathrm{P}_{0} \quad$ non-dimensional resistance at the start of re-penetration

$\mathrm{P}_{\min }(\mathrm{z})$ limiting displacement for sustained suction

$\mathrm{P}_{\mathrm{u}}(\mathrm{z})$ ultimate penetration resistance at penetration $\mathrm{z}$

$\mathrm{P}_{\mathrm{u} \text {-suc }}(\mathrm{z})$ ultimate suction resistance at penetration $\mathrm{z}$

$\mathrm{Q}_{\mathrm{u}} \quad$ ultimate bearing load per unit length

$\mathrm{q}_{\mathrm{u}} \quad$ bearing capacity of strip footing

$\mathrm{R}$ the RAO amplitude 
s arc length along the catenary from the touchdown point

$\mathrm{s}_{\mathrm{f}} \quad$ actual position of the touchdown point in boundary layer method

$\mathrm{s}_{\mathrm{u}} \quad$ undrained shear strength of soil

$\mathrm{s}_{\mathrm{u}}(\mathrm{z}) \quad$ undrained shear strength at depth $\mathrm{z}$

$\mathrm{S}_{\mathrm{um}} \quad$ undrained shear strength at the mudline

$\mathrm{T}$ tension force at a given location along the riser

$\mathrm{t}$ time (equation 4-5)

$\mathrm{t} \quad$ riser wall thickness

$\mathrm{V}_{\max }$ initial (or static) maximum shear force in the touchdown zone

$\overline{\mathrm{V}} \quad$ average shear force through out of a vessel displacement cycle

$\mathrm{V} \quad$ push in velocity of pipe into the soil

$\mathrm{x}$ horizontal coordinate from the touchdown point to the vessel

$\mathrm{x}_{\mathrm{M} 0}$ distance of the maximum shear force from the touchdown point

$\mathrm{x}_{\text {MR-Max }}$ distance of the maximum reverse curvature from the touchdown point

$\mathrm{y}_{\max }$ section's largest dimension from the neutral axis or the pipe half diameter

$\mathrm{z} \quad$ vertical coordinate upward from the seabed

$\mathrm{z}_{0} \quad$ penetration $\mathrm{z}$ at which the latest episode of the current contact mode started

$\mathrm{z}^{*} \quad$ penetration when the preceding episode of uplift started

$Z_{\max }$ maximum previous penetration depth for a given point of the riser

$\mathrm{Z}_{\mathrm{p}=0} \quad$ largest penetration depth at which suction has started during any uplift

\section{Greek}

$\lambda_{\text {suc }}$ non-dimensional normalised suction decay distance

$\lambda_{\text {rep }}$ non-dimensional re-penetration offset after uplift

$\zeta_{0} \quad$ non-dimensional penetration at start of latest episode of current contact mode

$\zeta \quad$ non-dimensional penetration in units of $\mathrm{D} / \mathrm{K}_{\max }$

$\rho_{\text {soil }} \quad$ saturated density of the soil

$\rho_{\text {sea }} \quad$ seawater density at the seabed origin

$\Delta \sigma_{f_{i}} \quad$ constant stress range in each block of stress histogram 
$\zeta_{0} \quad$ non-dimensional penetration at the start of re-penetration

$\Delta \mathrm{x} \quad$ approximate displacement of the touchdown point

$\Delta \mathrm{x}_{\mathrm{TDP}}$ maximum touchdown point relocation under a vessel perturbation cycle

$\Delta \sigma_{\mathrm{f}} \quad$ factored von Mises stress range

$\Delta \sigma_{\mathrm{v}} \quad$ von Mises stress range

$\gamma^{\prime} \quad$ submerged unit weight of soil

$\eta \quad$ ratio of vertical coordinate to flexural parameter $(\mathrm{z} / \lambda)$

$\eta \quad$ usage factor for fatigue calculation (equation 4-2)

$\theta \quad$ riser slope at any point along the catenary

$\theta_{\text {TOP }} \quad$ riser lay angle

$\lambda \quad$ flexural length parameter

$\lambda_{\mathrm{H}} \quad$ characteristic parameter

$\xi \quad$ ratio of horizontal distance to flexural parameter $(\mathrm{x} / \lambda)$

$\rho \quad$ shear strength gradient of soil with depth

$\rho_{\mathrm{s}} \quad$ steel density

$\sigma_{\mathrm{A}} \quad$ the axial stress in riser section

$\sigma_{R} \quad$ radial stress in riser tubular section

$\sigma_{\mathrm{VM}} \quad$ von Mises stress in riser section

$\sigma_{\mathrm{y}} \quad$ riser steel yield stress in uniaxial tension or von Mises stress

$\sigma_{\theta} \quad$ hoop stress in riser tubular section

$\varphi \quad$ the RAO phase

$\omega \quad$ wave frequency

\section{Abbreviations}

API American petroleum institute

BLM boundary layer method

CoG centre of gravity

DFF damage fatigue factor

DISP displacement boundary condition subroutine 
DNV det norske veritas

ETLP extendable tension leg platform

FD frequency domain

FE finite element

FEA finite element analysis

FPSO floating production storage and offloading unit

ID identification

JIP joint industry project

LDFE large deformation finite element

LTD linear time domain

NTD non-linear time domain

PDF probability density function

RAO response amplitude operator

RHS right hand side vector

RMS root mean square

ROV remote operating vessel

RP recommended practice

SCF stress concentration factor

SCR steel catenary riser

SK station keeping

STRIDE steel catenary risers in deepwater environments

TDP touchdown point

TDZ touchdown zone

TLP tension leg platform

UEL user defined element

VIV vortex induced vibration 


\section{CHAPTER 1. INTRODUCTION}

\subsection{OVERVIEW}

Development of offshore energy fields increasingly in deep water has raised new technical and economic challenges, particularly in respect of transferring the oil and gas products from the seabed, at depths now approaching $3000 \mathrm{~m}$, to floating facilities on the sea surface. Steel catenary risers (SCRs) (see Figure 1-1) - thick-walled steel pipes that hang in a catenary from the floating system to the seabed - provide a technically feasible and commercially efficient solution, especially when high temperatures and pressures are involved, allowing high capacity production from remote wells (Phifer et al. 1994; Quintin et al. 2007).

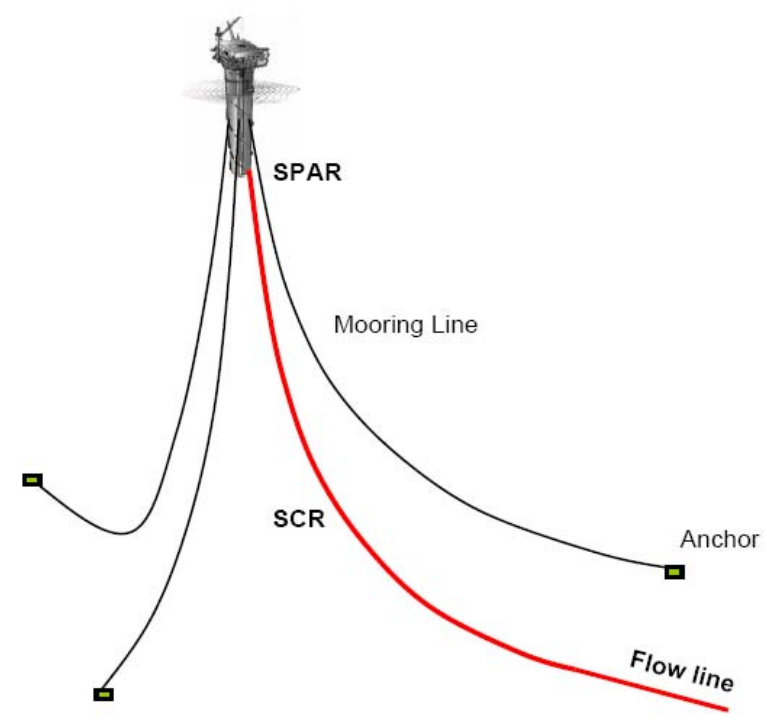

Figure 1-1: Schematic layout of SCR attached to a Spar ( Spar by (Marinetalk 2000))

SCRs are designed to resist the effects of the environmental, fluid and geotechnical loads using numerical models that determine the riser response to static and dynamic loading. They are generally designed using finite element analysis (FEA) due to their complexity and the dynamic nature of the loads and motions, with specialist codes developed in order to model vessel and riser motions together with hydrodynamic loading on the riser itself. Analysis is conducted to calculate the static and extreme stresses, the fatigue life and the probability of the riser clashing with the vessel, mooring lines and neighbouring risers. 


\subsection{SCR FATIGUE PERFORMANCE}

Assessment of the cumulative fatigue damage to SCRs during their operational life is one of the most challenging parts of the design. While SCRs offer numerous benefits, the inability for accurate prediction of their fatigue life using conventional modelling has precluded their use on some ultra deep projects where disproportionate escalation of technical issues is usually unavoidable (Dixon 2009).

New developments in modelling risers, enabling improved assessment of key physical influences, such as the fatigue performance in the region where the SCR makes contact with the seabed, can considerably enhance design calculations, and thus allow more cost-effective riser design options to be established for a particular field development. As an example, in a recent project in West Africa, minor refinements of the pipelineseabed interaction parameters led to the saving of millions of dollars in the cost of the project (Randolph and White 2008b).

The fatigue damage of SCRs is commonly assessed on the basis of contributions from three different types of system motions (API-RP-2RD 1997; Campbell 1999):

1) Wave frequency or first order motions of the vessel under wave action, which is the source of first order fatigue.

2) Low frequency, second order motions or slow drift of the vessel due to swell and light wind, which leads to second order fatigue.

3) Vortex induced vibration (VIV) of SCRs due to hydrodynamic loading by sea currents, which induces VIV fatigue.

Both the touchdown zone and the riser top connection point to the vessel are critical areas for fatigue damage, but different types of motion from different types of floating systems give rise to different magnitude of contributions to the total damage in these critical areas (Carter and Ronalds 1998; Serta et al. 1996; Silva et al. 1994).

It is customary to use different numerical approaches for these different aspects of the fatigue problem. Time-domain analysis of VIV requires sophisticated computational fluid dynamics software, coupled with finite element (FE) analysis of the structural dynamics, but this aspect of the problem is essentially independent of the low frequency fatigue. The latter is generally addressed through specialist software packages such as OrcaFlex (Orcina Ltd 1986) and Flexcom3D (MCS 1994) that model the motion of the supporting vessel and the attached riser under life-time cycles of wave loading. The commercial software packages are relatively strong tools providing appropriate modelling of SCRs from a structural and naval architectural standpoint. However, the seabed-riser interaction, which directly affects the SCR fatigue life in the touchdown zone (TDZ), and is thus one of the most critical factors in the durability of SCRs, is oversimplified in most of these commercial packages through the adoption of linear 
elastic springs. Only recently have some of the package developers taken steps to implement non-linear seabed models for riser-seabed interaction (Orcina Ltd 2009).

\subsection{RISER-SEABED INTERACTION AND FATIGUE DAMAGE IN}

\section{TDZ}

The SCR service life is strongly affected by the fatigue performance in the touchdown zone, the area around the point at which the catenary riser contacts and separates from the seabed due to environmentally induced motions at the top of the riser (see Figure 1-2). It has been found that fatigue near the vessel (or upper support of the SCR) is unaffected by how the interaction with the seabed is modelled, but fatigue in the TDZ is heavily influenced by the riser-seabed interaction. The greatest uncertainty in the SCR analysis comes from this interaction, and recent investigations suggest that modelling the seabed as a rigid or linear elastic medium is a very conservative approach (Theti and Moros 2001). Hence, there is strong incentive from industry to explore this aspect of SCR design in greater detail, with the hope of developing more realistic estimates of fatigue damage. This has made more sophisticated modelling of seabed interaction an area of particular research interest in the last few years (Aubeny and Biscontin 2008; Aubeny et al. 2009; Bostrom et al. 1998; Bridge et al. 2004; Palmer 2000; Randolph and Quiggin 2009).

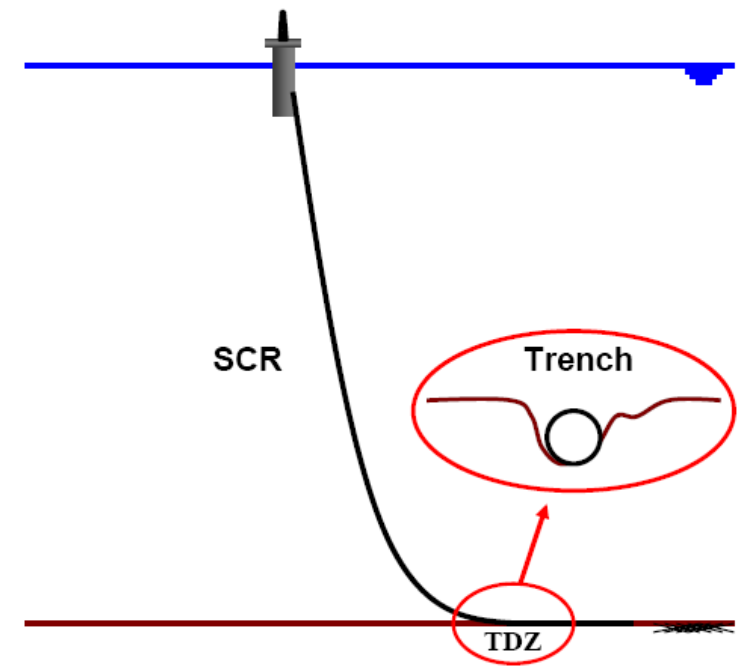

Figure 1-2: Trench formation in touchdown zone for an SCR connected to a Spar

This aspect is particularly important in deep and ultra deep water, where conventional, linear seabed models often do not allow SCRs to satisfy minimum fatigue-life requirements.

The dynamic motions of SCRs cause soil degradation, due to remoulding of the soil under the cyclic motions of the riser and physical erosion of the soil due to alternating sucking in and expulsion of water between the riser and the seabed. The combined 
effects lead to the creation of a trench in the TDZ. Trenches may vary in size, but are generally at least two to three times the riser diameter in depth and may extend over hundreds of diameters in length (Bridge and Howells 2007; Langner 2003). Typical fatigue laws follow a power law relationship in respect of the cyclic stresses, with damage proportional to a high power (equal to the negative inverse slope of S-N curves, typically about 3) of the cyclic stress amplitude (Barltrop and Adams 1991; DNV-RPC203 2008). Most of the fatigue damage in the TDZ comes from the lifting and setting down of the riser, where it undergoes repeated changes in curvature. The changes in distribution of seabed reaction force during these cyclic motions, and consequently the cyclic stress amplitude in the riser wall due to changes in tension and bending moment in the SCR, determine the riser fatigue life. Even small changes in seabed stiffness, although resulting in relatively small changes in the amplitude of the bending stresses, can have a significant effect on the fatigue life because of the steep gradient of the S-N curves.

As the SCR trenches into the seabed, the mean touchdown point (TDP), which tends to be the point of maximum fatigue, changes location with alteration of curvature at the seabed. The actual curvature at the TDP and its position are governing factors in any fatigue differences between a flat and profiled seabed. Changing trench depths and shape may significantly alter the location of the touchdown point, shifting the profile of fatigue damage axially along the riser.

Another aspect of riser-seabed interaction that has caused concern is the potential for suction resistance on the riser during uplift. The magnitude of suction can have a strong influence on the bending moment distribution, and hence the cyclic stress changes (Giertsen et al. 2004).

In summary, non-linear interaction between riser and seabed, the formation and geometry of trenches, and the magnitude of suction resistance, are all aspects that are generally not taken into account at present and need to be captured through more advanced riser-soil interaction models in order to allow more accurate prediction of SCR fatigue damage in the TDZ. This is the area of focus of this thesis, which explores the effect of different soil models, trench geometries and suction resistance on fatigue damage in the TDZ, with the objective of establishing principles to guide design. The research concentrates on one particular riser geometry and design life wave scatter diagram, and is deliberately limited to quasi-static analysis of the riser under the cyclic wave motion in order to develop a clear picture of what aspects of the riser-seabed interaction model most affect estimation of the fatigue life.

The thesis includes a detailed assessment of simple closed form solutions for estimating fatigue of SCRs resting on 'linear' seabeds, and then explores the advantages of a more sophisticated non-linear seabed interaction model. In addition, the thesis investigates the influence of trench formation beneath the riser with a robust methodology that allows the riser to follow its natural catenary shape while the trench is developed. This 
consistent approach is more appropriate than trying to impose a pre-defined trench shape, since that always leads to contact stress hotspots in the touchdown zone and consequently over-estimates fatigue damage. The research also investigates the vessel excitation scheme through a range of waves with various significant heights, periods and the different order of application to the floating system, assessing the impact on local cyclic stress variations. The thesis concludes with various recommendations for practical assessment of SCR fatigue in the touchdown zone for non-linear plastic seabed response.

\subsection{THESIS ORGANISATION}

This thesis has been organised in eight chapters as summarised below:

- Chapter 1: Steel catenary risers (SCRs) are introduced briefly and the significant importance of seabed-riser interaction on their fatigue performance in the touchdown zone is emphasised. The research goals are briefly described and the thesis organisation is explained.

- Chapter 2: The literature relevant to the areas of research is reviewed, including SCRs, seabed soils, pipe-soil interaction, fatigue performance and trench formation beneath the riser.

- Chapter 3: Catenary equations describing the suspended part of the riser are summarised along with a series of analytical closed form solutions for modelling the riser-seabed interaction in the touchdown zone including beams, with and without axial tension, on a Winkler linear elastic foundation and boundary-layer methods. These are used to predict the shear force and bending moment distributions in the touchdown zone and the results are compared with numerical simulations obtained using the finite element package ABAQUS. The significant importance of the shear force distribution in the TDZ is discussed. A simple calculation method is presented, using the shear force and catenary equations obtained from closed form solutions to predict the von Mises stress variation range in the TDZ during cyclic perturbation of the top of the riser. This calculation provides a first step towards estimating the fatigue damage in the TDZ through simple hand calculations instead of computationally intensive numerical simulations.

- Chapter 4: Fatigue performance of steel catenary risers on linear elastic seabeds is investigated through numerical simulation of an SCR using ABAQUS. User defined element, UEL and DISP subroutines in ABAQUS are introduced for coding the seabed model and vessel excitation respectively. The system global configuration, analysis details and convergence aspects are discussed, including vessel excitation mechanisms and riser-seabed interaction modelling. The simple hand calculation methods introduced in Chapter 3 are used to predict the von Mises cyclic stress range in the TDZ and consequently the peak fatigue damage 
under a full package of sea states based on environmental conditions in the Gulf of Mexico. The results are then compared with those from numerical simulations. Results of a series of simulations and comparisons are presented to identify the key parameters influencing fatigue damage distribution in the TDZ.

- Chapter 5: Non-linear seabed interaction models are discussed and the recent hysteretic non-linear seabed model (Randolph and Quiggin 2009) is described in detail including implementation in a UEL subroutine in ABAQUS. A series of numerical simulations are presented, examining the capability of the hysteretic non-linear model in simulating suction resistance and the gradual evolution of moderate and deep trenches underneath the riser through cycles of vessel excitation. The shear force, contact stress and cyclic bending moment distribution in the TDZ, along with trench formation, are discussed and compared with those obtained with a linear elastic seabed.

- Chapter 6: Fatigue performance of steel catenary risers is studied using the hysteretic non-linear seabed model. A comprehensive investigation is presented showing how superposition may be applied, even for a plastic seabed with limited recovery of displacements, in order to assess fatigue damage. The influence of suction resistance and trench generation on fatigue performance is studied in detail. A consistent methodology is introduced for extreme fatigue damage calculations on non-linear seabed, demonstrating how Miner's damage superposition rule may be applied for non-linear seabeds for particular conditions.

- Chapter 7: Typical trench morphology is studied starting from existing ROV survey records fitted using analytical shape functions. The significant influence on the final fatigue damage of how the initial trench profile is developed is investigated using linear elastic and non-linear seabed models. The capability of the hysteretic non-linear model to make deep trenches is shown to provide a consistent and robust technique to model a trench, prior to applying the design wave spectra for the fatigue analysis, according to the calculation methodology introduced in Chapter 6. A series of extreme fatigue analysis calculations are presented using this methodology and the results are discussed.

- Chapter 8: A final chapter reviews the main findings of the research and identifies areas of further development, such as assessing the impact of the dynamic riser response for the non-liner seabed model.

Further details of the UEL and DISP subroutines in ABAQUS are provided in Appendix A. Results from the comprehensive parametric study of the hysteretic non-linear model and fatigue performance are presented in Appendix B. 


\section{CHAPTER 2. LITERATURE REVIEW}

\subsection{OVERVIEW OF STEEL CATENARY RISERS}

\subsubsection{Introduction}

Steel catenary risers, commonly referred to as SCRs, are a relatively new and simple flowline system for transferring hydrocarbon products from the seabed to a floating facility at the sea surface. They comprise a string of conventional steel pipe segments welded together and are continuous with the flowlines on the seabed. The catenary riser is normally connected to the floating system at the top using a special flexible joint (API-RP-2RD 1997) and rests on the seabed at the lower end, Figure 2-1. An anchor is usually required at the seabed to provide the tension necessary to maintain the catenary shape. The Auger export pipeline supported by Shell's tension leg platform (TLP) in a water depth of $872 \mathrm{~m}$ in the Gulf of Mexico is believed to be the first application of a steel catenary riser (Phifer et al. 1994). Several advantages of SCRs have made them a preferred solution for development of deep water fields during the last decade, including the compatibility with different floating systems in various water depths and geographical regions, being able to function in harsher operating environments, and with no need for heave compensation or special subsea connections.

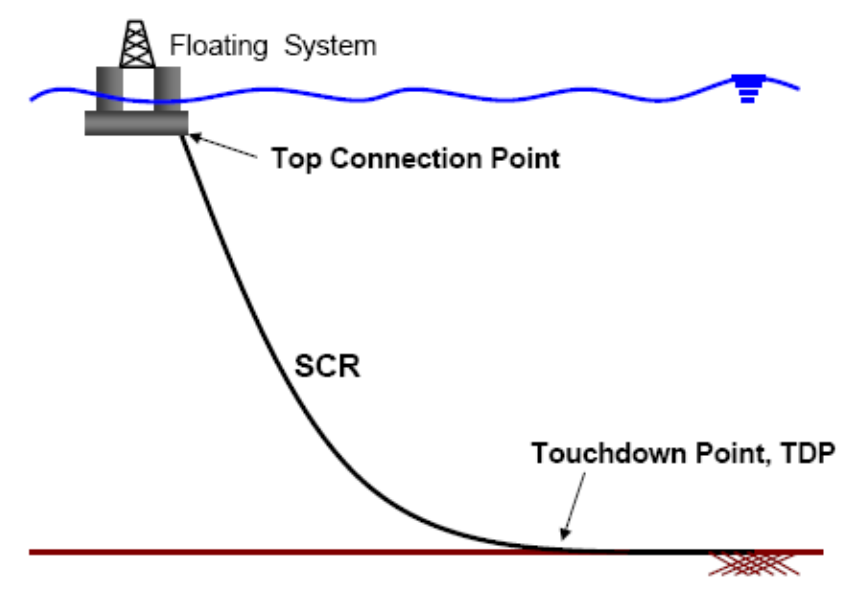

Figure 2-1: Schematic side view of SCR connected to floating system

The popularity of SCRs in the offshore oil and gas industry has encouraged research to focus on geotechnical challenges associated with SCR engineering design such as seabed interaction. The riser-seabed interaction, which is strongly influenced by the seabed properties, can affect the global integrity and design conditions of the SCR in two main categories: 
- Ultimate limit state associated with SCR response to extreme environmental loads, particularly the out-of-plane motions or mooring system failure. This can generate excessive bending and tensile stresses in the riser wall due to lateral soil resistance acting on the riser, either from partial embedment or potentially from the walls of any trench that has been created by the riser motions.

- Fatigue limit state associated with cyclic bending stress variation in the touchdown zone due to vessel motions under environmental loads. The magnitude of the bending moment changes is closely linked to the shear force distribution in the touchdown zone, which in turn is affected by the riser-seabed interaction model. This is the area on which the current thesis has focused.

There are still various non-geotechnical challenging areas in SCR engineering design such as interface management with the floating system, impact of as-built uncertainties on the SCR static configuration, SCR dynamic behaviour, welding requirements and installation aids etc, none of which are of direct interest to this study. As mentioned above, and as will be further discussed later, the fatigue performance of SCR in the touchdown zone is directly affected by the motions of the SCR in that area, which in turn are dictated by the vessel motions. It is therefore important to consider first the motions of the floating system in terms of the applied environmental excitation from waves prior to discussing the main areas of the SCR itself and its interaction with the seabed.

\subsection{SCR SYSTEM BEHAVIOUR}

\subsubsection{Motions of different floating systems}

A floating system can have motions in six degrees of freedom under applied loads: three displacements (surge, sway and heave) and three rotations (roll, pitch and yaw) (Ursell 1964). A real vessel has dynamic motions defined in terms of periods and amplitudes. In general, the main motions of a floating structure can be described in three categories (API-RP-2SK 1996):

- first order, wave frequency motions developed under wave action on the floating system;

- second order, low frequency motions or 'slow drift' motions excited by winds and long period swell waves;

- quasi-static large displacements due to sustained ocean currents or mooring line failure etc.

The vessel response to environmental loads is interactive, in the sense that the structural and naval architectural features of the system and the associated components like mooring and flow lines, also affect the motions of the floating systems (John 1985; Wehausen 1971). Hence, the different types of floating system, such as Spars, truss 
Spars, semi-submersibles, tension leg platforms (TLPs), floating production storage and offloading vessels (FPSOs), will show different responses for a particular degree of freedom under a given wave environment (Murray 2007). The functional requirements of floating systems for a particular development include the drilling, production, riser and well systems, hull compartmentalisation and air gap requirement. These collectively dictate the type of floating system and consequently its motions. However, a primary design objective is to minimise the system response to the environment. Figure 2-2 shows considerable variation of relative hull response (heave natural period with respect to wave energy) to the environment for various floating structures with different mooring systems (FloaTec 2006).

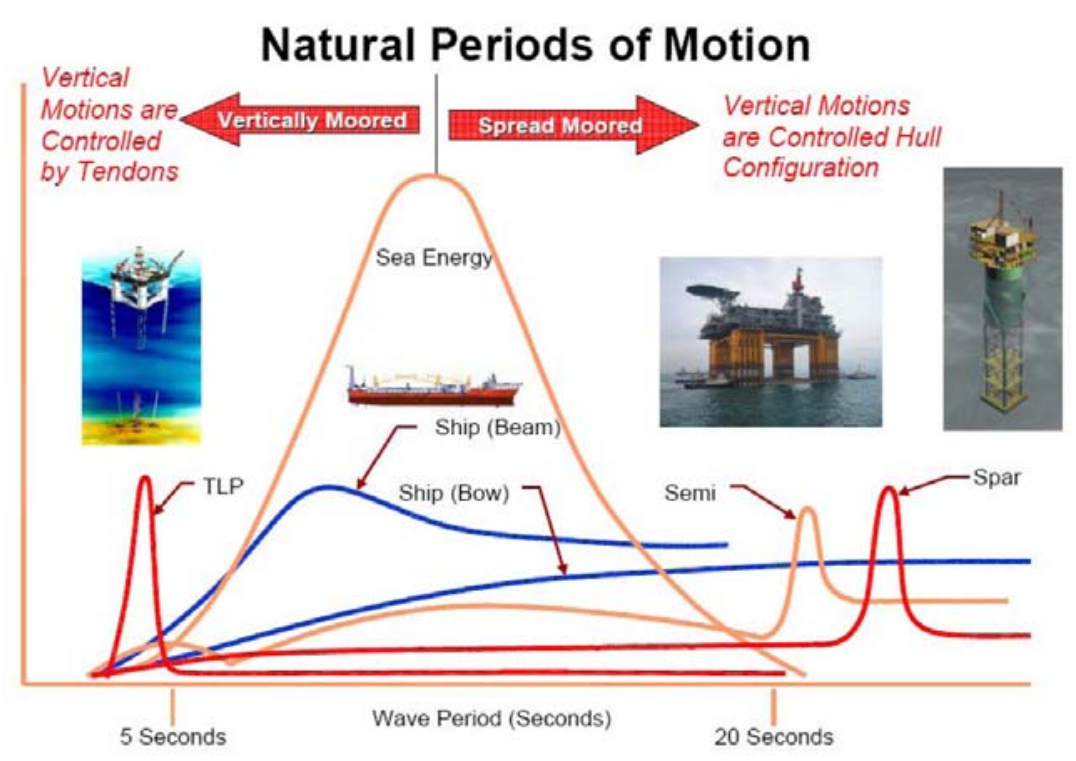

Figure 2-2: The relative hull response of various floating systems to environment (FloaTec 2006)

Figure 2-3 to Figure 2-5 compare the RAOs (response amplitude operators) of three different types of floating systems, including an extendable tension leg platform (ETLP), a dry tree semi-submersible and a Spar system, to in-plane motions, for a normally applicable range of wave periods (Murray 2007). The RAOs of a vessel can be obtained eighther by sophisticated laboratory model tests or with software for analysis of hydrodynamic motion, such as MOSES, in which the response of vessel model to the entire environmental loads package is obtained by means of extensive analyses. The plots show that Spars do not seem to experience any peak values, reflecting a resonance condition, within the normal range of wave periods; in particular, the results show a very low response for higher wave frequencies (periods of 3 to 8 seconds). 


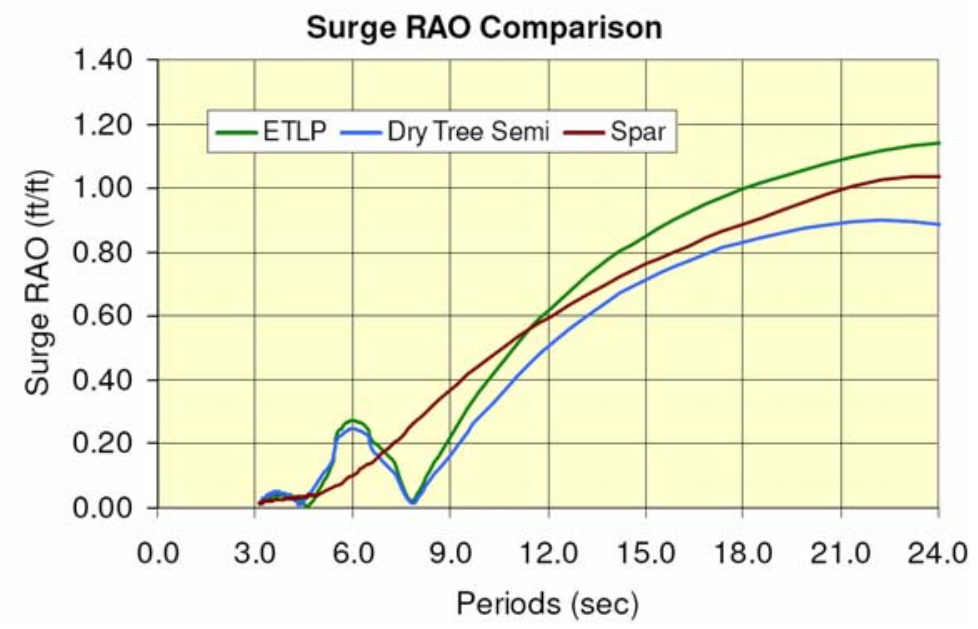

Figure 2-3: Sample surge RAO of various floating system (Murray 2007)

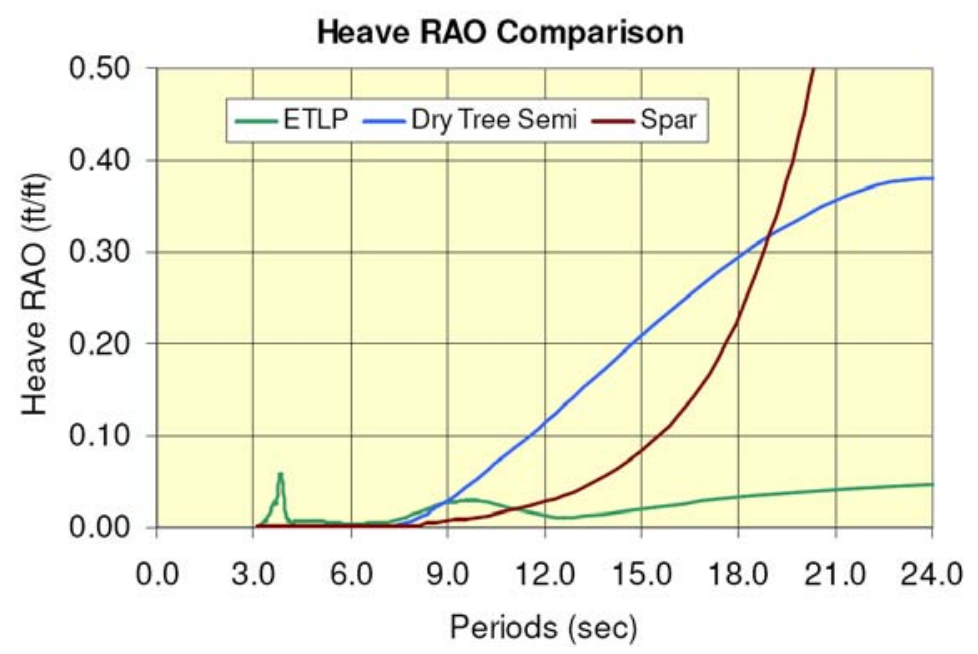

Figure 2-4: Sample heave RAO of various floating system (Murray 2007)

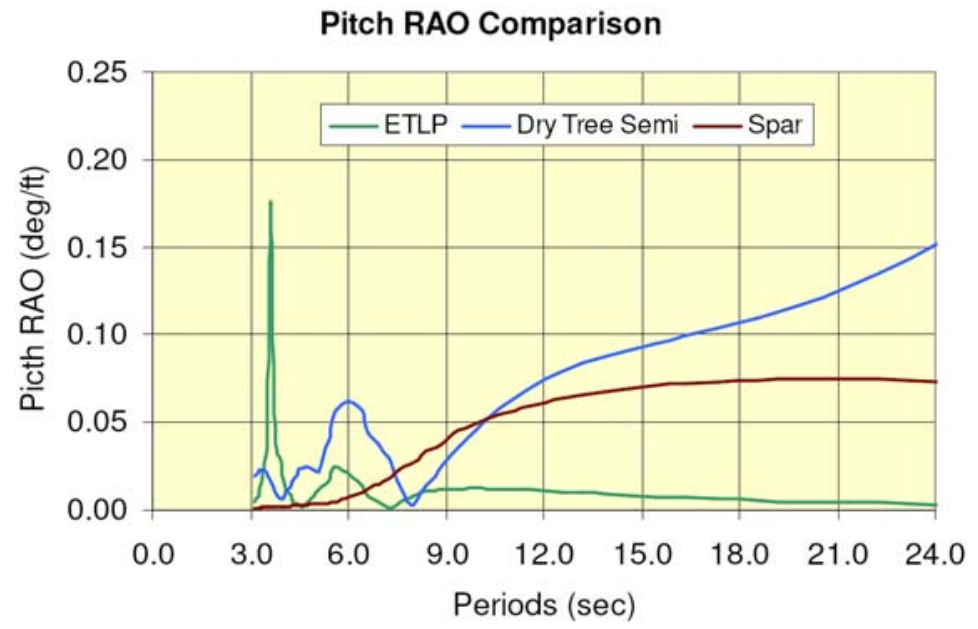

Figure 2-5: Sample pitch RAO of various floating systems (Murray 2007) 
It is worth mentioning here that in a Spar, which has been selected as the floating system in the current dissertation, the centre of gravity is always considerably lower than the centre of buoyancy, which makes the Spar unconditionally stable with limited pitch motions even in complete absence of its mooring system (Rho et al. 2002). In addition, the deep draft of the system helps to minimise heave motions (FloaTec 2006; Murray and Yang 2009; Steen et al. 2004). These are the principal advantages of this system, minimising wave induced motions while still enabling the use of dry trees with direct vertical access to wells from the deck. Spar systems can also be fully configured for drilling, work over and production processing, all of which are facilitated by the relatively small motions. Further advantages of the Spar system and its suitability for the analyses performed within this study will be discussed in Chapter 4 . The response of the TLPs and semi-subs are different from Spars (Murray 2007), but they offer specific benefits that make them the best option for particular cases.

\subsubsection{SCR behaviour}

The vessel motions under wave and wind actions are transmitted to the SCR and cause excitation and relocation of the touchdown point, but these are not the only source of SCR motions in the touchdown zone (TDZ). Hydrodynamic loading of the suspended part of the SCR and more localised vortex induced shedding due to currents, can give rise to significant SCR excitation in the TDZ. Understanding the riser motions in the TDZ, which contribute significantly to riser penetration into the seabed, is important in modelling the riser-seabed interaction and consequently accurately assessing the SCR ultimate embedment. As will be discussed in Chapter 4, the overall impact of the seabed interaction on fatigue performance in the TDZ is the main focus point of the current study, rather than accurate estimation of the fatigue damage. Hence, the VIV and hydrodynamic effects of the SCR will be deliberately ignored and only the vessel motions will be simulated through out the quasi-static analysis. It is worth mentioning that amongst the vessel motions, the heave component causes the maximum TDP excitation and consequently the maximum stress fluctuation at the TDP, which directly affect the fatigue performance of SCR in this area (Chandwani and Larsen 1997; Chaudhury 2001).

Theti and Moros (2001) tried to map the TDZ, reflecting the probabilistic position of the TDP over the service life of an SCR. The study was performed as part of the STRIDE JIP (Steel Catenary Risers in Deepwater Environments) project, and considered a 14" deepwater Gulf of Mexico SCR connected to a Spar. To develop a stochastic map of the TDP location, the authors considered the first and second order vessel motions comprising a full range of day-to-day to extreme waves, along with mean vessel offset and sea state exceedence probability data. The results of this study, illustrated in Figure $2-6$, show that $97 \%$ of the TDP movements occur inside a narrow longitudinal area with \pm 17 m longitudinal and $\pm 0.25 \mathrm{~m}$ transverse offset from the mean TDP position (red and purple areas). 


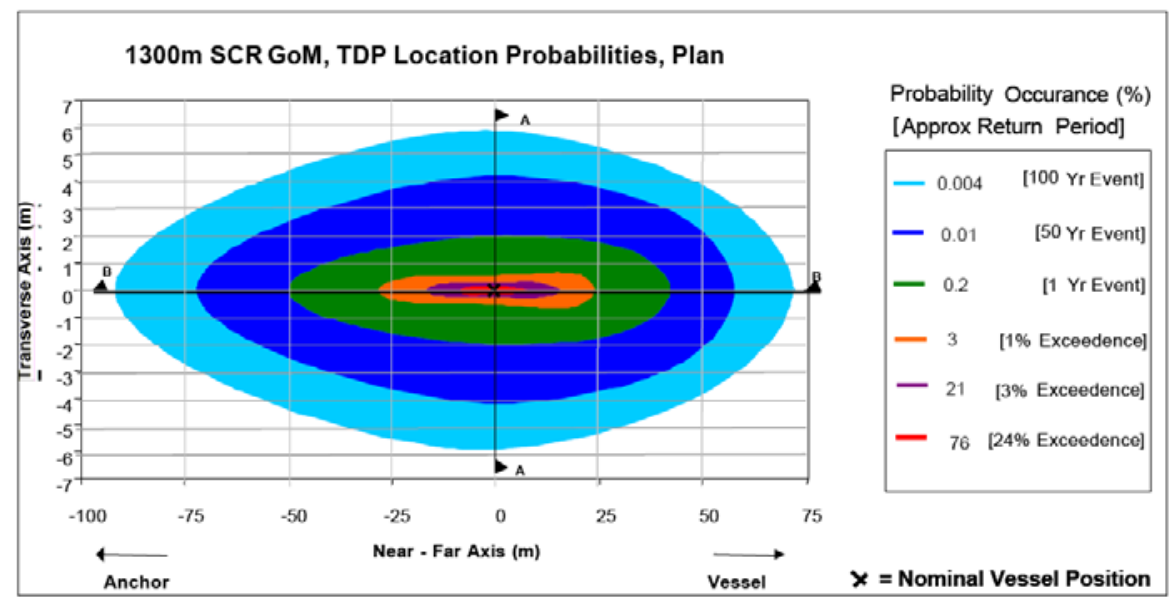

Figure 2-6: SCR/Spar TDP global dynamic movement (Theti and Moros 2001)

In reality, considering the system dynamics, there is no direct and simple relation between the magnitude and direction of the vessel and TDP movements in a given time stage. Every part of the SCR, even in the touchdown area, can move in the same or reverse direction of the vessel movement, and with very different magnitudes. In a quasi-static system, the horizontal direction of the TDP relocation is the opposite with respect to the vessel movement. Thus, if the vessel moves towards the anchor end of the SCR, commonly known as 'near offset', the TDP will move towards the vessel end and vice versa, Figure 2-7. Evidently, the magnitude of TDP and vessel horizontal movements can be quite different but they can be obtained from the simple catenary equation as will be discussed in Chapter 3. The magnitude and direction of the movement, particularly in the touchdown zone, can be very complicated, even for a quasi-static system. As will be shown in the coming chapters, neighbouring elements of the SCR in the touchdown zone can move in opposite vertical directions, and with different magnitudes, for a given vessel movement. 


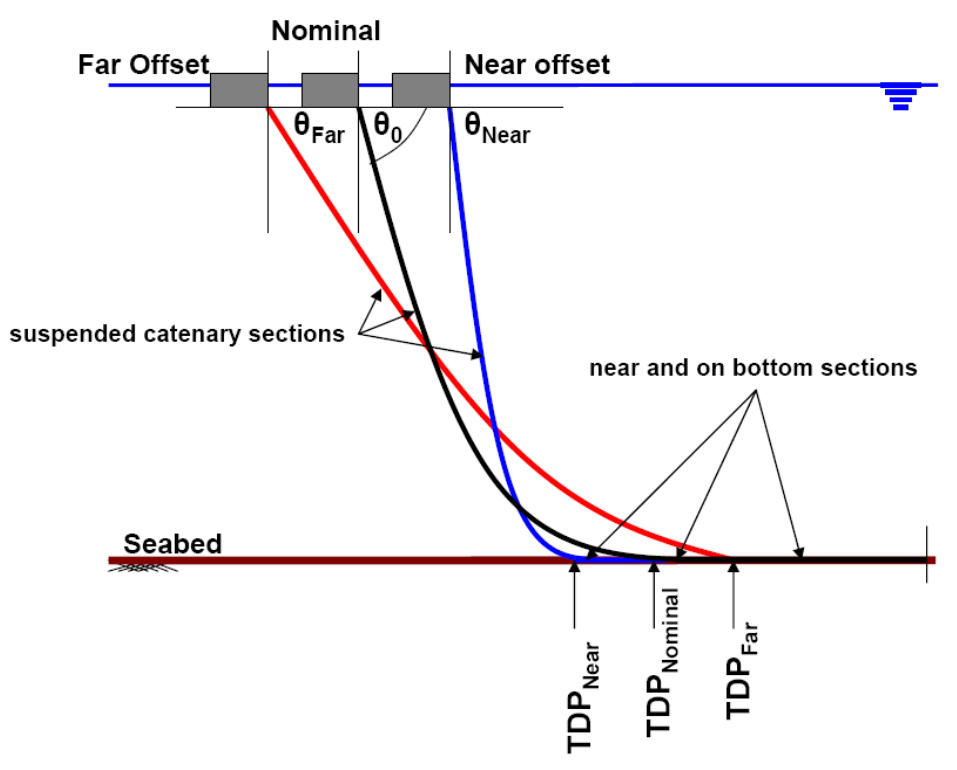

Figure 2-7: Schematic horizontal movement of TDP relative to vessel offset

\subsection{ANALYSIS OF SCR RESPONSE}

\subsubsection{SCR static configuration in linear seabed}

A steel catenary riser may be divided into two main parts as shown in Figure 2-8. The first part is the suspended, or catenary, section between the attachment to the floating system and a point close to the seabed, and the second part is the section close to, and then in contact with, the seabed. Although the boundary between these two parts is not defined, they require different theoretical solutions to describe the geometry and variations of internal forces and moments. Specific consideration must be given to the interface zone near the TDP in order to ensure continuity of the system, with the bending stiffness of the SCR. It is becoming an important factor in the region where the SCR makes contact with the seabed and reaches a point of maximum embedment and then undergoes reversal of curvature as it makes the transition into a horizontal pipeline. The suspended part of the SCR may be treated simply as a catenary and can be solved analytically using the traditional catenary equations (Leibniz 1691a). The second part or the touchdown area, just before contact with the seabed and after contact, can be modelled with separate analytical solutions that take account of the bending stiffness, the (essentially constant) tension in the riser and the contact forces from the seabed. The overall approach is commonly referred to as a boundary layer solution (Lenci and Callegari 2005; Pesce et al. 1998a). In this section the theoretical basis of both the suspended part and seabed part of the SCR will be discussed briefly, including a short review of construction tolerances that can cause some deviations from the analytical configuration assumed. 


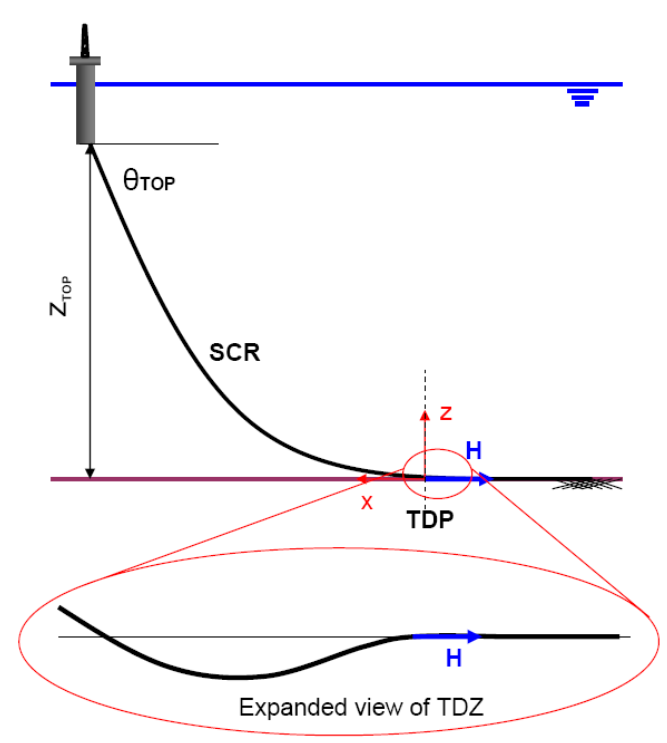

Figure 2-8: Static configuration of SCR connected to a Spar

Traditional catenary equations for suspended section of SCR

A detailed discussion of the classical catenary equations is provided in Chapter 3, where alternative approaches to model the complete SCR system are compared. The high aspect ratio (the ratio of length to diameter) of SCRs in the suspended part through the water column enables application of the fundamental catenary solution developed for a suspended cable with uniform mass and no bending stiffness (Leibniz 1691a). A comparison of the catenary equations with finite element solutions was performed by Pesce et al (1997). Their results showed that the effect of the bending stiffness of SCR is significant only close to the touchdown point. Pesce et al. (1997) introduced nondimensional groups of parameters controlling the SCR configuration, the main one of which is the flexural length parameter, $\lambda$. This parameter quantifies the length scale of the boundary layer and is shown to be the distance between the actual TDP, for example from the FEA (finite element analysis) solution, and the TDP from the simple catenary solution. The basis of the flexural length parameter, $\lambda$, is the work of Love (1892), where he shows that the main effect of the bending stiffness of a beam string suspended between two points occurs at the ends of the beam string. The flexural length parameter defines the extra length of the beam string calculated with no bending stiffness, in order to achieve the same conditions over the central section (see Chapter 3 for details).

The horizontal component, $\mathrm{H}$, of the tension force is constant along the suspended part of the SCR and can be written in terms of geometrical parameters as:

$\mathrm{H}=\mathrm{m}_{\mathrm{s}} \mathrm{gz}_{\mathrm{TOP}} \frac{\cos \left(\theta_{\mathrm{TOP}}\right)}{1-\cos \left(\theta_{\mathrm{TOP}}\right)}$

where

$m_{s} \quad$ is the mass per unit length of the riser 
is the gravity acceleration

$\mathrm{z}_{\mathrm{TOP}}$ is the height of the attachment point above the seabed

$\theta_{\text {TOP }}$ is the lay angle (measured from horizontal)

The presence of external forces will affect the catenary solution. Various authors have assessed the effect of current drag as an external force on catenary structures (Pedersen 1975; Vaz and Patel 2000; Zajac 1957) and the solution given by Pesce et al. (1998a) is valid for low current velocity. Further discussion of the fundamental catenary equations is delayed until Chapter 3.

\section{Boundary-layer solutions for touchdown part of SCRS}

Steel catenary risers are an example of tensioned, long, slender catenary systems with high geometrical non-linearity, where critical design calculations must consider localised boundary layers at the end supports, which perturb the natural catenary shape. For the SCRs these boundaries are mainly confined to the attachment point and the zone of seabed support. Analytical predictions of the stress distribution through these boundary zones for design purposes, particularly in respect of the bending moment and shear force, have been a challenging area of research for the last few decades. In the pipeline and riser industry, many authors have tried to develop analytical predictions for the non-linear behaviour of SCRs, taking into account the boundary layer effects, but most of these have concentrated on the top boundary layer with much less attention to the touchdown zone (Plunkett, 1967; Dixon and Rutledge, 1968; Palmer et al., 1974; Konuk, 1980; Champneys et al., 1997; Guarracino and Mallardo, 1999; Karayaka and $\mathrm{Xu}, 2003)$. Many of these investigations have been based on the asymptotic expansion method introduced originally by Wasow (1956). The top boundary layer or the riserfloater interaction is outside the area of interest in the current research, where attention is focused on the lower boundary layer and the riser-seabed interaction.

Considering only the catenary equations for modelling the SCR is essentially equivalent to assuming a rigid seabed. Investigations show severe discontinuity and inaccuracy in the curvature and higher order derivatives of the SCR shape in the TDZ with this assumption (Pesce et al. 1998b). Hence, the catenary equations alone, which do not account for the bending stiffness of the SCR and the seabed forces acting on it in the TDZ, cannot provide a consistent model for the static configuration of an SCR and more sophisticated solutions are required to be further developed in the touchdown zone. Several authors have proposed appropriate solutions for the TDZ, where the effect of the bending stiffness and seabed stiffness become important (Croll 2000; Lenci and Callegari 2005; Palmer et al. 1974; Pesce et al. 1998a).

The analytical boundary-layer solutions for the static and dynamic curvatures of the SCR in the TDZ were developed in the late 1990s (Aranha et al. 1997; Pesce et al. 1997) where the bending moment was approximated analytically based on a quasi-linear frequency domain solution of a suspended cable $(E I=0$, where $E I$ is the bending 
rigidity of the SCR) assuming an infinitely rigid seabed. This simple asymptotic boundary-layer solution accounted for all the non-linear features that result from the non-linear geometric boundary condition at the seabed. Pesce et al. (1997) also showed that for large vessel motions the SCR bending stiffness in the TDZ needs to be considered, whilst for the small vessel motions the TDP can be assumed as a point of articulation. However, these solutions showed a discontinuity of the shear force (or third derivative of the SCR shape) at the TDP, which was a significant shortcoming of the models.

Pesce et al. (1998a) further developed the static boundary-layer solution assuming a linear elastic seabed. They introduced a non-dimensional soil rigidity, combining the seabed stiffness, $\mathrm{k}$, with the SCR weight per unit length and the flexural length parameter, $\lambda$. The parameter $\lambda$ for an infinitely rigid seabed was properly interpreted as the distance between the actual and ideal (pure catenary) TDP (Aranha et al. 1997), but for the further developed linear elastic seabed (Pesce et al. 1998a), this was refined to be the offset distance of the point of horizontal tangency relative to the TDP attained in the ideal cable solution for a rigid seabed. Pesce et al. (1998a) obtained a typical oscillatory behaviour of the curvature in the TDZ, smoothly matching the catenary solution for the suspended part of the SCR, and with continuous and reasonably accurate shear force distribution at the TDP. Pesce et al. (1998a) also showed that the position of the maximum bending moment in the riser is somewhere close to the TDP, within the suspended section of the SCR, and was not affected by the seabed stiffness. The performance and the accuracy of the model developed by Pesce et al. (1998a) has been examined in detail against results from FEA in Chapter 3. The results show a very good correlation of his proposed solution with the FEA results, which makes it a reliable method for analytical expression of the SCR in the touchdown zone.

Palmer (2000) gave a qualitative description of the forces between the SCR and rigid and elastic seabeds updating his early works on laying configurations for submarine pipelines (Palmer et al. 1974). He emphasised the significant importance of the flexural length parameter, $\lambda$, and asserted that the length of the transition zone between the seabed and catenary suspended part depends on the vertical load-displacement response in the TDZ as well as on the SCR flexural rigidity.

Croll (2000) proposed an incremental boundary layer formulation with an analytically explicit form and tried to simplify the boundary layers to that of the bending of a tensioned straight rod. Although, the simplicity of the method may make it attractive for design engineers, particularly for the upper boundary layer, from a geotechnical standpoint it seems over simplified in the touchdown zone. Croll (2000) did not examine his solution for continuity and accuracy of the shear force prediction at the TDP, which is a vital aspect of seabed interaction.

Another analytical solution from the boundary-layer family was developed by Lenci and Callegari (2005). They proposed a systematic development of a simple analytical 
solution for J-lay problems, starting from the classical catenary equations and incorporating different degrees of realism to improve the model, eventually providing a solution that gives a smooth transition from the catenary to the seabed region, overcoming the stress discontinuity drawback of catenary equations. Lenci and Callegari (2005) started with the one-field model, combining the classical catenary equations for the suspended part of the riser and a rigid seabed in the conventional way. In the second step, they examined a two-field model by incorporating the solution for a tensioned beam on a Winkler linear elastic foundation in the seabed. This trial still showed a discontinuity in the bending moment at the TDP, and also in the shear force. To achieve continuity of the bending moment at the TDP, they proposed a different (third) technique, initially for a rigid seabed, but taking account of the true bending stiffness of the pipe in the neighbourhood of the TDP, where the numerical simulations show that is not negligible. Essentially, they moved the transition point, from the catenary solution (with zero EI) to a solution with finite EI taken into account, into the suspended part of the SCR. This contrasts with most of the classical boundary-layer solutions, which use a singular perturbation theory consisting in superposing two solutions in the boundary layer: the catenary solution and a boundary layer solution just in the vicinity of the boundary. The solution proposed by Lenci and Callegari (2005) seems to be a new methodological approach for investigating the boundary layer phenomenon. In the final step, Lenci and Callegari (2005) proposed a three-field model implementing a new transitional boundary layer solution between the catenary part and seabed part defined in the previous two-field model, but now incorporating a compliant linear seabed. This final development of the model removed any discontinuity in the bending moment and shear force, providing a smooth transition profile at the TDP and very close agreement with FEA results.

Randolph and White (2008b) as an incidental part of their consideration of pipeline embedment in deep water, tried to quantify the consistency and the accuracy of the boundary layer solutions through a group of dimensionless parameters, comparing the results with numerical simulations performed using the commercial (FE-based) software OrcaFlex. The controlling dimensionless group, $\frac{H}{\lambda p}$, may be expressed directly in terms of the SCR geometrical features, including the water depth and lay angle, as:

$$
\frac{\mathrm{H}}{\lambda \mathrm{p}}=\left(\frac{\cos \theta_{\mathrm{TOP}}}{1-\cos \theta_{\mathrm{TOP}}}\right)^{3 / 2}\left(\mathrm{z}_{\mathrm{TOP}}{ }^{3} \frac{\mathrm{p}}{\mathrm{EI}}\right)^{1 / 2}
$$

where $\mathrm{p}=\mathrm{m}_{\mathrm{s}} \mathrm{g}$ is the submerged weight per unit length of the riser. As referred before $\mathrm{z}_{\mathrm{TOP}}$ is the height of the attachment point and $\theta_{\mathrm{TOP}}$ is the lay angle.

EI is the bending rigidity

$\lambda \quad$ is the flexural length parameter 
They showed that, for the typical pipelines where $H / \lambda p \geq 3$, the maximum shear force and contact stress in the TDZ becomes independent of $\mathrm{H} / \lambda \mathrm{p}$, and the results obtained from the simplified analytical solution of Lenci and Callegari (2005) will be sufficiently accurate. Randolph and White (2008b) provided approximate curve fits for the maximum bending moment, shear force and contact stress in the TDZ, through a parametric study and comparisons with the OrcaFlex results. Their investigations showed that the peak bending moment may be estimated with sufficient accuracy for prediction of the maximum stresses in the pipe wall during laying, using the approximate fit:

$\frac{M_{\max }}{\lambda^{2} \mathrm{p}} \approx 1-\frac{1}{1+(\mathrm{H} / \lambda \mathrm{p})^{0.9}}$

The maximum contact stress and consequently the peak shear force were shown to be affected by the seabed stiffness, $\mathrm{k}$ defined as the ratio of the contact force per unit length to penetration. The approximation fits proposed by Randolph and White (2008b) for the maximum shear force, $\mathrm{V}_{\max }$ and the peak contact force (per unit length), $\mathrm{P}_{\max }$, are given by the following expressions:

$$
\begin{aligned}
& \frac{\mathrm{V}_{\max }}{\lambda \mathrm{p}} \approx 1-\mathrm{e}^{-0.4[\log (10 \sqrt{\mathrm{K}})]^{1.5}} \\
& \frac{\mathrm{P}_{\max }}{\mathrm{p}} \approx 0.6+0.4 \mathrm{~K}^{0.25}=0.6+0.4\left(\lambda^{2} \mathrm{k} / \mathrm{H}\right)^{0.25}
\end{aligned}
$$

where $\mathrm{K}$ is the dimensionless stiffness defined as:

$$
\mathrm{K}=\lambda^{2} \mathrm{k} / \mathrm{H}
$$

As implied by equation (2-4), for typical pipelines with $H / \lambda p \geq 3$, the maximum shear force is independent of $\mathrm{H} / \lambda \mathrm{p}$. Figure 2-9 shows example plots produced by Randolph and White (2008b) comparing the results of the boundary layer method proposed by Lenci and Callegari (2005) with numerical simulations performed by OrcaFlex, with the suggested approximation curve fits for maximum shear force in the touchdown zone. 


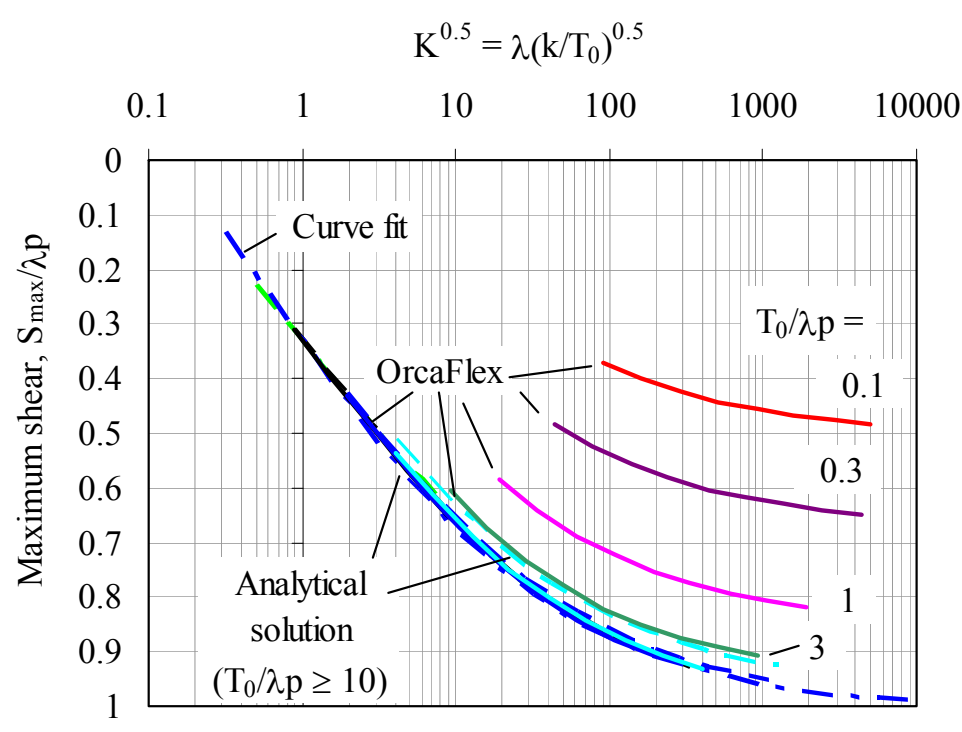

Figure 2-9: Maximum shear force in touchdown zone (Randolph and White 2008b)

From a design perspective, it is important to quantify any variation between analytical solutions such as those discussed here, or numerical simulations, and what happens in reality when the SCR system is installed. This issue and the overall design basis are discussed briefly in the following section.

\section{Impact of construction uncertainties on SCR static configuration}

In an actual SCR construction project, the final static configuration can easily deviate from the initial calculations. The static configuration for a particular SCR is generally obtained as a balance between excessive riser top tension (which reduces curvature in the riser), and the maximum bending stress in the touchdown zone (which increases as the tension is reduced). It is also important to ensure sufficient tension to minimise the risk of the riser clashing with neighbour structures during extreme environmental events. The design and cost of the system mooring, the local reinforcement of the hull at the riser attachment point and the horizontal tension at the touchdown point, which may need external anchors to avoid the riser sliding along the seabed, are all directly affected by the riser top tension. Hence, the design endeavours to minimise the riser top tension, but still satisfying the restrictions on maximum bending moment and extreme motions of the SCR.

There are many uncertainties inherent in the SCR system, from fabrication to installation. Quintin (2007) identified a number of these uncertainties, such as actual thickness and weight of the riser pipe, tolerances in installation of subsea equipment, tolerances for the fabrication of the hang-off support on the hull, tolerances of water depth, tolerances in the as laid length of the riser etc. These can have a significant impact on the final static configuration of the SCR and need to be carefully analysed prior to construction. Hence, the selected static configuration should be robust enough to withstand such uncertainties. 


\subsubsection{SCR dynamic analysis}

Depending on the level of sophistication various analysis methodologies, such as nonlinear time domain (NTD), linear time domain (LTD) and frequency domain (FD), can be used to evaluate the first and second order and VIV fatigue damage along the SCR. The same range of approaches allows analysis of potential interference with neighbouring structures and assessment of the response of the complete SCR system under extreme storm conditions. The extreme storm analyses are performed iteratively to optimise the initial static configuration. This type of analysis is normally conducted by applying regular waves with 1 year, 10 year and 100 year return periods and accidental design cases such as failure of a mooring line.

First order fatigue analysis is performed based on the first order or wave frequency motions of the vessel. Time domain analysis is the best approach to evaluate first order fatigue due to large non-linearities, particularly around the critical touchdown point (Hatton and Willis 1998). Second order fatigue damage is obtained by applying the low frequency or slow drift motions of the vessel. The interaction and combination of the first and second order fatigue damage is an important aspect and cannot be considered by simply adding together the damage profiles obtained separately. A methodology that can be used to combine the first and second order fatigue damage is to perform time domain analysis for stochastic sea states and the calculate fatigue damage from the response time traces using stress cycle counting techniques such as the rain-flow method (Campbell 1999). An alternate approach is the statistical method proposed by Jiao and Moan (1990).

Vortex induced vibration (VIV) of the SCR arises from vortex shedding and is a critical design issue for SCRs in the upper zone where the highest current forces occur (APIRP-2RD 1997). In contrast to first and second order fatigue damage, which is usually most critical in the touchdown zone (depending on the type of floating system), VIV leads to high frequency cyclic stresses in the upper part of the SCR towards the attachment point, with potentially significant fatigue damage (Hatton and Willis 1998). There are various VIV suppression techniques to mitigate its impact, such as helical strakes and fairings (Grant and Patterson 1977; Rogers 1983), but these affect the dynamic response of the system and need to be investigated carefully. Quantifying VIV is a complex area of study, requiring advanced computational fluid dynamics, and is outside the area of interest in the current research. VISFLOW (DNV, 1998) and SHEAR7 (Vandiver and Li 1994) are two popular software packages for VIV analysis of slender elements, and these are widely used in the SCR industry.

Interference analysis is performed to make sure that the SCR will not collide with adjacent umbilicals, risers, mooring lines etc under extreme wave loads and currents. This requires dynamic analysis of the complete floating system and the associated appurtenances, for example using commercial software packages such as those referred 
to below. As for VIV analysis, the results are essentially unaffected by the seabed response, and this aspect of SCR design is not considered further here.

\subsubsection{SCR analysis software}

Various commercial software packages have been developed for analysis of offshore floating structures, with SCR analysis being part of their capabilities. FLEXCOM3D (MCS, 1994), RIFLEX (SINTEF, 1998) and ORCAFLEX (ORCINA, 1986) are the most well-known packages currently used in the offshore industry. All of these are (dynamic) finite element based, using either explicit or implicit time-integration approaches, and they include modules to simulate aspects such as VIV and line interference. They are tailor made for the particular application, providing user-friendly interfaces to help create the relatively complex models and are optimised to minimise computation times.

General purpose finite element software, such as ABAQUS and ANSYS, can also be used for modelling SCRs. Since these packages are not tailor made for SCRs, the analyses tend to be more time consuming to set up and run. They are, however, very useful for detailed modelling of specific aspects of SCR response, which may be simplified in commercial packages. In particular, commercial SCR design packages generally assume a linear elastic seabed, and so are not useful for sophisticated riserseabed interaction studies. Non-linear seabed models are starting to be incorporated, for example in the recent version of ORCAFLEX, (Randolph and Quiggin 2009), but with limited scope to explore specific aspects of the seabed response.

\subsubsection{SCR design guidelines}

Various classification societies have recommended guidelines for design of steel catenary risers with the most well-known of these standards being the API-RP-2RD (1997), API-RP-16Q (1993), 17A (1994), 17B (2001) and 17C (1999) and DNV (APIRP-17C 1999; 1996). A combination of various guidelines is sometimes used in recommended industry approaches. For example, STRIDE (Hatton and Willis 1998) has recommended the use of DNV(1996) for pipe sizing for burst and collapse and API-RP$2 \mathrm{RD}(1997)$ for extreme storm response (Hatton and Willis 1998). The API series of guidelines are usually based on working stress design (WSD) format, whilst the DNV and ISO guidelines adopt a limit state design (LSD) format.

\subsection{FATIGUE PERFORMANCE OF SCR}

\subsubsection{Introduction}

As discussed in general terms above, and now summarised here, steel catenary risers must be analysed for three major kinds of fatigue damage (API-RP-2RD, 1997):

- first order fatigue caused by first order wave frequency motions of the vessel under wave action; 
- second order fatigue caused by second order low frequency and slow drift motions of the vessel under the action of swell and light winds;

- vortex induced vibration (VIV) fatigue caused by current action;

In addition to the above, there are additional minor sources of fatigue that need to be addressed in design, such as shut-down and start-up and installation effects.

The critical areas of SCRs for first and second order fatigue damage are:

- the hang-off point, where the riser is connected to the vessel;

- the touchdown zone, the transition zone between the catenary section and seabed section of the SCR-flowline system.

Two types of approaches are used to assess first and second order fatigue damage:

- deterministic methodology;

- stochastic methodology.

Deterministic fatigue analysis, which is the simplest way to evaluate the damage, uses a constant-amplitude S-N diagram to relate the fatigue life in cycles to failure to the cyclic stress amplitude. In this method the environmental loads are defined as a wave scatter diagram giving the wave heights, wave periods and the number of each wave applied during a given period. In order to account for the damages from all sea states and all directions of loading and yet to constrain the computational effort, the wave scatter diagram is typically manipulated by dividing the wave package into several windows with a single wave (sea state) representing the range from each window. The response transfer function or stress RAO along the riser is obtained by analysing the system for each sea state. Then the riser response is linearised for other sea states in the window by assuming that the response is proportional to wave height and is constant throughout the window. The windowing of the scatter diagram usually starts with a preliminary assessment to identify the sea states that provide the greatest contribution to the total fatigue damage (Bai 2001). The effect of the second order motions can be accounted for as part of first order fatigue by considering appropriate vessel offsets within the linearization (API-RP-2RD 1997). The total fatigue damage is obtained by linear summation, using Miner's rule (Miner 1945; Palmgren 1924) of the relative damage caused by each sea state within the windowed wave scatter diagram.

In stochastic fatigue analysis, which is more accurate and considers the nonlinearities and probabilities associated with the system behaviour, the environmental loads are defined based on a wave spectrum and a probability density function (PDF) (SACS 2009; Vughts and Kinra 1976). The wave spectrum gives the dimensionless spectral density for a range of common wave frequencies and the PDF gives the probability of occurrence of a given wave. The wave spectrum may be expressed in the form of Pierson-Moskovitz (Pierson Jr and Moskowitz 1964) or JONSWAP (Hasselmann et al. 
1973) single peak spectra or a Bi-modal spectrum. The parameters defining these spectra are obtained from observation data.

The first step in stochastic method, after defining the wave spectra, is finding the transfer function (TF) to relate the wave period to a desired output, which can be stress or even cyclic stress range. In fact, the transfer function is essentially similar to the vessel motion RAO but its output can be any required parameter rather than just the wave height (as given in generic RAOs). To derive the transfer function, a wave frequency band with an appropriate width around the peak point of the wave spectrum is chosen and the system is analysed for the selected waves with unit wave height. Then the desired output (e.g. von Mises stress) is plotted versus the wave frequency with the range of the chosen frequency band. This plot acts exactly like a motion RAO in which, for a given wave with a particular frequency and wave height, the system output can be obtained by simply multiplying the output for a unit wave height at the given frequency by the actual wave height.

Using the stress RAO or transfer function, the root mean square of stress ( $\left.\sigma_{\mathrm{RMS}}\right)$ and the mean period is calculated, with the number of applied waves obtained from the probability density function (PDF). Then the fatigue damage is obtained simply by using Miner's rule in the form of an integral over the specified frequency range. The environmental load is sometimes given in the form of a time history of random waves instead of a wave spectrum. In that case, an appropriate time trace (commonly 3 hours (API-RP-2RD 1997)) is taken as representative to generate the wave spectrum using the Fourier transform functions. Other techniques, such as the rain-flow half cycle counting method, may also be used to extract directly the number of applied waves and wave periods from a time history of wave heights.

The deterministic approach to fatigue calculation has always been appealing to industry because of its simplicity. However, the accuracy of the method in comparison with results from a stochastic approach is an area of current research in the offshore industry for various types of structures and elements. In the current study the deterministic method has been chosen due to its simplicity, and because absolute accuracy of the total fatigue damage is not a primary focus since the research is directed at investigating the influence of the seabed interaction model on first order fatigue in the touchdown zone. Also, the method is widely applied in industry and with acceptable range of accuracy. Sheehan et al. (2005) compared the deterministic and stochastic methods for various cases of flexible risers and concluded that the deterministic method is sufficiently accurate provided the wave scatter diagram is defined with an appropriate number of wave blocks.

\subsubsection{Fatigue performance of SCR in touchdown zone}

The area in the vicinity of the touchdown point (TDP) of a steel catenary riser is a hotspot for fatigue damage, since the contact force from the seabed leads to a high gradient of bending moment, and hence large changes in bending moment due to any 
change in position of the TDP. The fatigue performance of the SCR in the touchdown zone (TDZ) is obviously affected by the SCR-seabed interaction. As will be discussed later in this chapter, the dynamic motion of the SCR leads to remoulding and softening of the seabed soil, and even physical erosion due to the pumping action of water from beneath the riser. This can lead to significant embedment of the riser into the seabed and the development of a trench beneath the SCR that may be several diameters deep, as observed from ROV surveys. The trench formation under the riser in turn affects the contact stress distribution and the maximum shear force in the riser. Since the shear force represents the gradient of bending moment, this has a direct consequence for the fatigue damage in the TDZ. Suction generated during the uplift movement of the riser will also affect the shear force and bending moment distributions in the TDZ, and hence will affect the fatigue performance as well. Realistic assessment of fatigue damage in the TDZ therefore needs to account not only for the non-linear response of the seabed, but also suction resistance during uplift and the potential formation of a deep trench through the region where the SCR makes and breaks contact with the seabed.

A simple linear elastic flat seabed is still considered by various design guidelines to model the seabed for design of steel catenary risers, which is obviously a gross simplification from a geotechnical standpoint. After the first experience of SCR technology in the Auger field of the Gulf of Mexico (Phifer et al. 1994), the STRIDE and CARISIMA JIPs (1999) were the first organised attempts to investigate the need for more sophisticated riser-seabed interaction models. These studies included assessment of the influence of suction during uplift, and also the presence of a trench, on the performance of SCRs particularly with respect to fatigue in the TDZ. The studies were wide-ranging, and included full-scale harbour tests, laboratory model tests and numerical simulations. Studies carried out in the STRIDE JIP showed that riser strength and fatigue performance in the TDZ were affected by the seabed soil stiffness and its local geometry, as shown in Figure 2-10 (Theti and Moros 2001). Assuming a rigid seabed maximises the peak fatigue damage compared with an elastic seabed even though the extreme storm stresses (due to the combination of peak bending moment and tension) are essentially insensitive to the seabed stiffness. Theti and Moros (2001) highlighted the significant influence of considering non-linear riser-soil interaction models, accounting for suction during uplift, soil damping, trench formation etc, on the fatigue performance in the TDZ and recommended further exploration of the issue. 


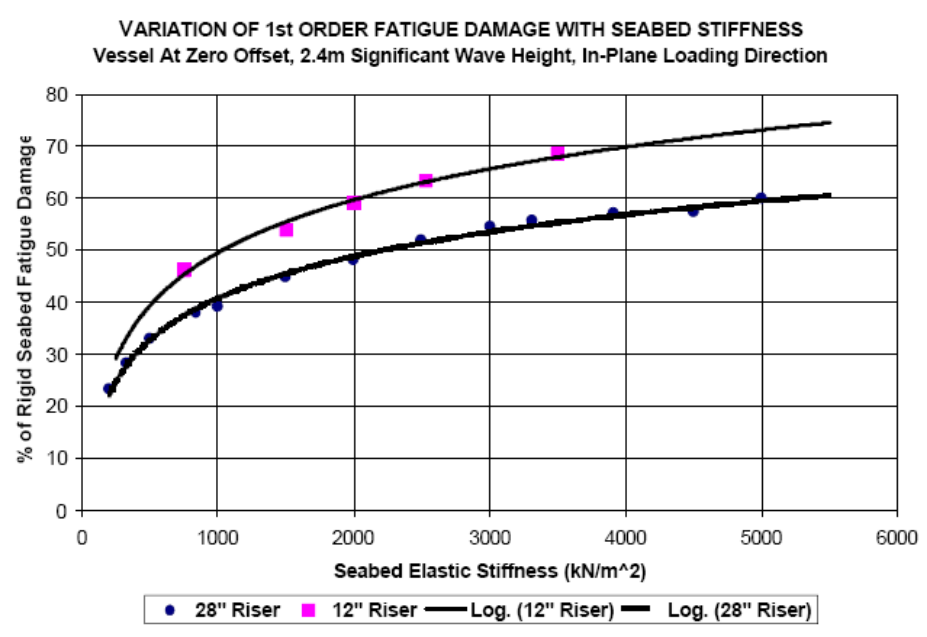

Figure 2-10: Variation of first order fatigue damage with seabed stiffness (Theti and Moros 2001)

Longner (2003) studied the influence of trench development on fatigue performance in the TDZ. He inserted a mathematical trench profile into a rigid seabed beneath the riser and investigated its influence on fatigue damage. The mathematical trench proposed by Langner (2003) was a combination of a circular arc on the catenary side of the trench and a seventh-order polynomial fit to the boundary conditions on the anchor side of the trench. The proposed trench profile is relatively similar to Hahn (2003) who proposed a combination of exponential and sixth-order polynomial curves. Longner then conducted time-domain dynamic analysis by applying irregular waves in addition to quasi-static analysis. He commented, although expressing some doubts in respect of the results, that the presence of the trench appeared to improve the fatigue life in the TDZ in one of the analyses but was inconclusive for the others. He also suggested a need for further investigation of the issue. It is worth mentioning that the results obtained by Langner (2003) are in contradiction with the findings of the current study. A critical issue is the technique used to implement the trench, which can lead to incompatibility with the natural catenary shape of the SCR and totally distort the final results. This is discussed fully in Chapter 7.

Giertsen et al (2004) presented the results of a series of model tests and numerical simulations as part of the CARISIMA JIP. They implemented the CARISIMA riserseabed interaction model including an initial trench insertion (2D trench depth) and performed three case studies with simulations incorporating many irregular waves and motions with different directions and sea states, assessing the accumulated total fatigue damage. The study was planned to capture the influence of suction alone, initial trench alone and simultaneous presence of suction and initial trench. Their results showed that for transverse and diagonal loading relative to the riser plane, the CARISMA soil model increased the fatigue damage; this can be very significant depending on the riser diameter and weight. They also commented that the effect of suction is not critical (about 10\% increase of fatigue damage for SCRs). Giertsen et al (2004) commented that trench formation is favourable for pure in line loading. Leira et al (2004) and Bridge et 
al (2004) presented the results of the same study with further discussion but the same conclusions.

Fontaine (2006) investigated the effect on fatigue performance of the riser-seabed contact and the initial trench shape proposed by Langner (2003) and Hahn (2003). The author defined a global model for varying vertical stiffness along the riser and a local model to describe the compression phase. The local stiffness has been extracted in relation with the experienced local amplitude of the cycles and observed to be governing the fatigue damage. Fontaine (2006) used the DeepLines software, monitoring the soil history during simulation, and adjusting the vertical stiffness of the elastic soil to account for its displacement history. A series of time domain dynamic and quasi-static analyses were performed, leading to very different responses of the SCR. Fontaine described fatigue as a dynamic phenomenon, associated with structural waves initiated by the vessel, travelling through the SCR and being partially reflected, transmitted and absorbed by the seabed soil in the TDZ. He described the decrease in fatigue life with higher seabed stiffness as: "the higher the soil stiffness, the more reflection occurs, leading to stronger variations in bending stress responsible for structural damage". Fontaine recommended a cautious approach to selection of the vertical soil stiffness for design purposes because of the reduced fatigue lives obtained from his DeepLine simulations. His trench investigation showed good correlation in prediction of the trench length with the existing models for riser abrasion (Hahn et al. 2003; Langner 2003), but he did not comment on the trench longitudinal geometry. He strongly recommended monitoring of SCRs at the TDP due to the high level of uncertainties that exist in riser-seabed interaction.

Clukey et al (2007) examined SCR fatigue performance for trenches of various depths and for various soil models. The results showed slightly decreasing fatigue damage for deeper trenches and higher fatigue damage for a stiffer seabed. Clukey et al (2007) also obtained a significant effect on fatigue damage of the suction force in a non-linear soil model, which tended to shift the peak fatigue towards the vessel end of the riser. The authors reported a significant influence of the soil model and trench depth on fatigue performance and recommended further work in this area of study.

Nakhaee and Zhang (2008) investigated the fatigue performance in the TDZ, proposing a penetration-resistance soil model considering trench development under continuos pounding of the riser on the seabed. They modified a numerical code, CABLE3D, to consider stiffness degradation under cyclic loading, and then studied the feedback effect on bending moment along the riser, finding that trenching may decrease the maximum variation of the bending moment in the TDZ and hence improve the fatigue life of SCRs. 


\subsection{SCR-SEABED INTERACTION}

\subsubsection{Introduction}

Pipe-soil interaction is a wide area of pipeline research, which has developed exponentially over the last fifty years, driven by the growth in offshore oil and gas activities. Static embedment, lateral and axial friction mobilisation, on-bottom stability, self-burial, liquefaction around the pipeline, sediment transport, heat transfer, response to submarine slides, ploughing and trenching are the main areas of concerns arising in pipe-soil interaction. In the majority of these areas, the ultimate embedment of the pipeline and the embedment processes are critical issues, which in turn affect many of the pipeline engineering design parameters, particularly in respect of thermal expansion and buckling analysis (Cathie et al. 2005; Randolph and White 2008b). Deeper embedment reduces the convective thermal exchange and thus the heat transfer, which increases the temperature of the fluid inside the pipeline and thus the amount of thermal expansion (Cheuk and White 2008; Randolph and White 2008b). On the other hand, deeper pipeline embedment in the seabed causes larger lateral and axial resistance and thus increases the buckling resistance during thermal expansion (Bruton et al. 2007). The amount of embedment can also affect the stress range variation in the TDZ of SCRs, affecting the fatigue life (Clukey et al. 2007; Fontaine 2006; Langner 2003).

In the following sections, a brief review is provided, focusing on the geotechnical aspects of deepwater SCR interaction with the seabed. Despite the riser resting on the seabed, cyclic motions of the floating system and dynamic effects of the catenary part of the SCR have a strong influence on the ultimate embedment. The review concentrates on vertical interaction rather than lateral and axial, consistent with the $2 \mathrm{D}$ planar analyses performed during the current research. Attention here is restricted to finegrained sediments, as are typically encountered in deep water, with the most critical soil characteristic being the profile of undrained shear strength. Other key aspects of the soil include its unit effective weight (which affects buoyancy resistance to penetration of the pipe), sensitivity of the soil, consolidation parameters and the extent to which suction is sustained during uplift of the SCR.

\subsubsection{Deepwater seabed sediments characterization}

In contrast to offshore foundation and anchoring systems, which usually require relatively deep soil characterisation, the most important depth range relevant for submarine pipelines in a deepwater environment is limited to the upper 0.5 to $1 \mathrm{~m}$ of the seabed, increasing to a depth of 5 to 6 diameters (of the riser) in the touchdown zone of an SCR, where observations show trench development beneath the riser (Bridge and Howells 2007). The geotechnical aspects of deepwater pipelines are relatively distinct from shallow water pipelines in certain characteristics, partly due to reduced current (and wave) loading in deep water and partly due to the nature of the sediments, which tend to become softer and more fine-grained as the water depth increases. These 
differences result in different design challenges in deep water projects more focused on issues associated with the phenomena such as thermal expansion and geohazards rather than pipeline stability, which is more an issue for shallower water.

The soil sediments in deepwater areas, where the steel catenary risers are usually installed, generally comprise very soft silty clays or fine-grained carbonate sediments, which may be characterised by their undrained shear strength, $s_{u}$, plasticity index, $I_{P}$, submerged unit weight, $\gamma^{\prime}$, and void ratio, es (Yen et al., 1975; Dunlap et al., 1990; Baudet and Ho, 2004). Table 2-1 shows the DNV's suggestions for key geotechnical parameters of clay (DNV-RP-F105 2006), with deepwater sediments generally falling in the first category.

Table 2-1: Typical geotechnical parameters of clay, (DNV-RP-F105 2006)

\begin{tabular}{|l|c|c|c|}
\hline Soil type & $\begin{array}{c}\mathbf{S}_{\mathbf{u}} \\
{[\mathbf{k P a}]}\end{array}$ & $\begin{array}{c}\boldsymbol{\gamma}^{\mathbf{\prime}} \\
{\left[\mathbf{k N} / \mathbf{m}^{\mathbf{3}}\right]}\end{array}$ & $\mathbf{e}_{\mathbf{s}}$ \\
\hline Very soft & $<12.5$ & $4-7$ & $1.0-3.0$ \\
\hline Soft & $12.5-25$ & $5-8$ & $0.8-2.5$ \\
\hline Firm & $25-50$ & $6-11$ & $0.5-2.0$ \\
\hline Stiff & $50-100$ & $7-12$ & $0.4-1.7$ \\
\hline Very stiff & $100-200$ & $10-13$ & $0.3-0.9$ \\
\hline Hard & $>200$ & $10-13$ & $0.3-0.9$ \\
\hline
\end{tabular}

Amongst the clay parameters referred to above, the undrained shear strength and, to a lesser extent the submerged unit weight, $\gamma^{\prime}$, are the main parameters required by design engineers. The magnitude of the undrained shear strength generally increases with depth below the mudline and may be expressed as:

$\mathrm{s}_{\mathrm{u}}(\mathrm{z})=\mathrm{s}_{\mathrm{um}}+\rho \mathrm{z}$

where

$$
\begin{aligned}
& \mathrm{S}_{\mathrm{um}} \quad \text { is the undrained shear strength at mudline } \\
& \rho \quad \text { is the shear strength gradient with depth }
\end{aligned}
$$

At the depth scale of foundations or anchoring systems, $s_{u m}$ is typically assumed to be 0 to $15 \mathrm{kPa}$ and $\rho$ is taken as 1 to $2 \mathrm{kPa} / \mathrm{m}$ (Randolph et al. 2007). In the upper $1 \mathrm{~m}$ relevant for pipeline design, $\mathrm{s}_{\mathrm{um}}$ may generally be taken as $\sim 0 \mathrm{kPa}$ (perhaps as high as 1 or $2 \mathrm{kPa}$ ), while the intact strength gradient may be as high as 5 to $20 \mathrm{kPa}$ (Low et al. 2008; Newson et al. 2004).

The accuracy of the undrained shear strength profile is a vital aspect of reliable calculations, particularly for the pipeline penetration depth. The very low strengths of surface sediments necessitate in situ strength measurement such as miniature shear vane or full-flow penetrometer tests, either on the seabed or in a box-core or occasionally in 
large diameter samples (although these tend to disturb the upper $1 \mathrm{~m}$ ); this can usually provide the most reliable input values provided the tests are fully controlled and performed carefully. Full-flow penetrometers (T-bar, ball) have now become the norm for strength measurement for pipeline design. Randolph et al. (2007) developed a time effective manually operated penetrometer, the DMS, for measuring intact and remoulded strength profiles in box-cores. The T-bar or ball penetrometer can be interchanged on the shaft, with the load measured directly behind the T-bar or ball. In addition, the DMS facilitates cyclic penetration and extraction tests in laboratory to assess the soil sensitivity, cyclic remoulding and dynamic affects (Low et al. 2008; Randolph et al. 2007), which is of particular relevance for pipeline and riser design (e.g. for estimating embedment or trenching), Figure 2-11.

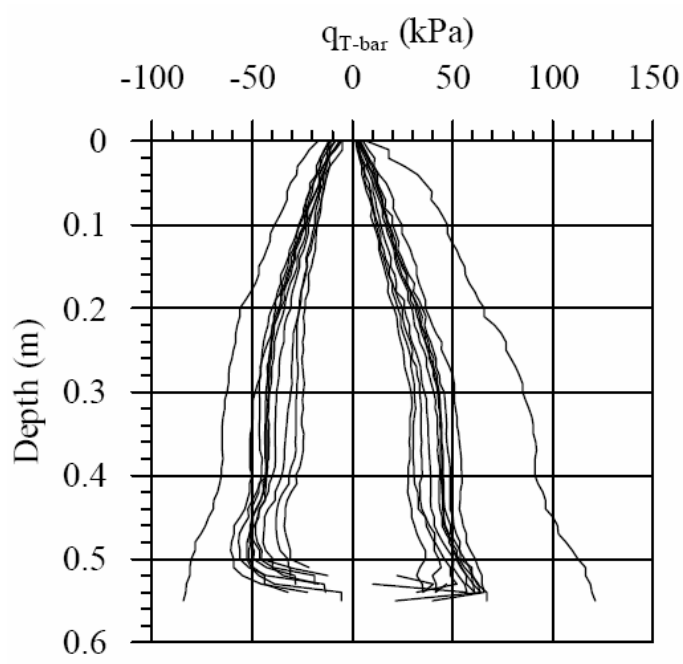

Figure 2-11: Penetration and extraction data for cyclic penetration test (Low et al. 2008)

Research has also been carried out within the last decade to increase the quality of core sampling for deep water applications (Borel et al. 2002; Young et al. 2000) either introducing new sampling techniques such as the static sampler of NGI (Lunne et al. 2008) or even new in-situ testing methods such as the expendable bottom penetrometer (XBP) (Aubeny and Shi 2006).

\subsubsection{Classical static penetration of a cylinder into soil}

Published approaches for estimating the pipeline static embedment include both theoretical and empirically based methods, with the ultimate vertical pipe force per unit length applied on the soil expressed in terms of the undrained shear strength, $\mathrm{s}_{\mathrm{u}}$, soil effective unit weight, $\gamma^{\prime}$, and the pipe diameter, D. Early solutions considered the pipeline as a strip footing with a width taken as the chord length of the embedded part (Bostrom et al. 1998; Jones 1976; Small et al. 1972). It is assumed that the pipe will sink until the bearing resistance equals the submerged pipe weight, leading to: 
$\mathrm{Q}_{\mathrm{u}}=\mathrm{q}_{\mathrm{u}} \mathrm{B}=\left(\mathrm{N}_{\mathrm{c}} \mathrm{s}_{\mathrm{u}}+\gamma^{\prime} \mathrm{z}\right) \mathrm{B}=\mathrm{m}_{\mathrm{s}} \mathrm{g}$

where

$\mathrm{Q}_{\mathrm{u}} \quad$ is the ultimate bearing load per unit length

$\mathrm{q}_{\mathrm{u}} \quad$ is the bearing capacity of a strip footing (Terzaghi 1943)

$\mathrm{B} \quad$ is the pipe bearing width limited to external diameter

$\mathrm{N}_{\mathrm{c}} \quad$ is the bearing capacity factor for a strip foundation, allowing for the embedment depth (Meyerhof 1964; Skempton 1942)

The term $\gamma^{\prime} z$ in Terzaghi's bearing capacity (equation (2-8)) is only applicable to foundations with no backfill material, i.e. in the case of an SCR when the riser is sitting in an open trench. Bridge et al (2005) substituted equation (2-7) into (2-8) ignoring the effect of soil weight $\left(\gamma^{\prime} z=0\right)$ and obtained the static embedment as:

$\mathrm{z}=\frac{1}{\rho}\left[\frac{\mathrm{m}_{\mathrm{s}} \mathrm{g}}{\mathrm{N}_{\mathrm{C}} \mathrm{B}}-\mathrm{s}_{\mathrm{u} 0}\right]$

Murff et al. (1989) presented upper and lower bound plasticity solutions for rough and smooth pipes, with full adhesion and no adhesion respectively. The plasticity solution does not take account of the effects of buoyancy, soil heave and strength increase with depth, though the potential impacts of heave and strength increase with depth are discussed.

Dunlap et al. (1990) proposed an empirical estimation for pipe embedment under monotonic loading based on an experimental study implementing the effect of embedment speed. The suggested formula shows a non-linear relationship between embedment depth and the penetration force and the inverse proportion to the shear strength and pipe diameter as given below:

$$
\frac{\mathrm{z}}{\mathrm{D}}=0.01573\left[\frac{\mathrm{F}_{\mathrm{z}} / \mathrm{D}}{\mathrm{s}_{\mathrm{u}}(\mathrm{V} / \mathrm{D})^{\mathrm{n}}}\right]^{1.7822}
$$

where
$\mathrm{Z} \quad$ is the penetration depth
$\mathrm{F}_{\mathrm{z}} \quad$ is the applied force per unit length
$\mathrm{D} \quad$ is the pipe diameter
$\mathrm{s}_{\mathrm{u}} \quad$ is the soil shear strength
$\mathrm{v} \quad$ is the push in velocity, so that $\mathrm{v} / \mathrm{D}$ represents a nominal shear strain rate 
examined.

$\mathrm{n} \quad$ is the dimensionless visco-elastic rate, taken as 0.03 for the soil

Results of extensive laboratory testing program were presented by Verley and Lund (1995) proposing another empirical equation in clay, expressed as:

$$
\frac{\mathrm{z}}{\mathrm{D}}=0.0071\left(\frac{\mathrm{F}_{\mathrm{z}}}{\mathrm{Ds} \mathrm{s}_{\mathrm{u}}}\left(\frac{\mathrm{s}_{\mathrm{u}}}{\mathrm{D} \gamma_{\mathrm{s}}}\right)^{0.3}\right)^{3.2}+0.062\left(\frac{\mathrm{F}_{\mathrm{z}}}{\mathrm{Ds}}\left(\frac{\mathrm{s}_{\mathrm{u}}}{\mathrm{D} \gamma_{\mathrm{s}}}\right)^{0.3}\right)^{0.7}
$$

An initial trial of this expression was given before for sand under cyclic loading (Verley and Sotberg (1994)). There is no theoretical logic either for the empirical expression itself or for the use of saturated unit weight, $\gamma_{\mathrm{s}}$, rather than the effective unit weight, $\gamma^{\prime}$ in equation (2-11) (Randolph and White 2008b).

Cathie and Jaeck (2005) compared the plasticity solution with the experimental data and Verley and Lund methods (1995). Their comparison shows higher embedment depth for normalised resistance force $\left(\mathrm{F}_{\mathrm{z}} / \mathrm{Ds}_{\mathrm{u}}\right)$ less than 3, Figure 2-12. Cathie and Jaeck (2005) suggested to assume remoulded soil beneath the pipeline with a low soil-pipe adhesion for penetration assessment. This is because of the remoulding of soil locally to the pipe wall during pipelaying because of the effects of wave and current induced motions of the pipeline.

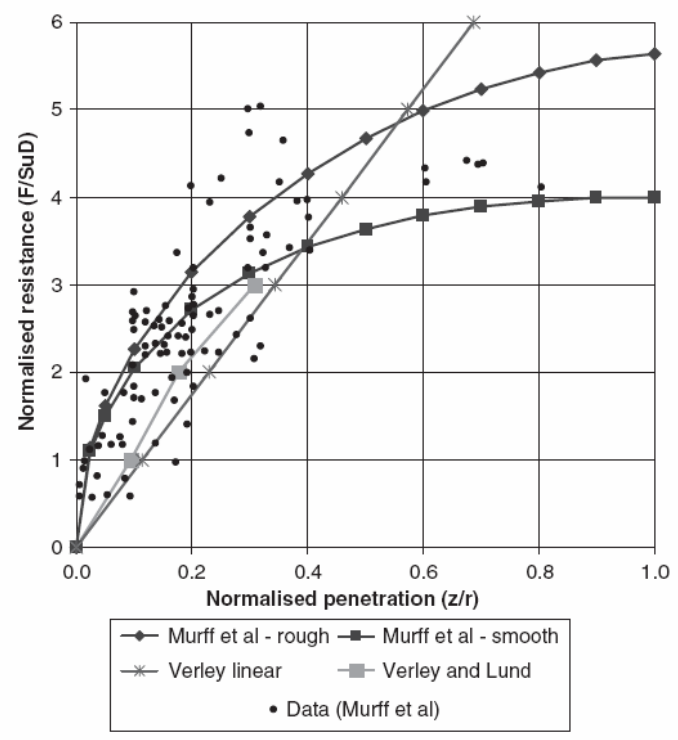

Figure 2-12: Plasticity solutions and empirical approaches for pipe embedment (Cathie et al. 2005)

Aubeny et al (2005) reported numerical simulations of the collapse load for a cylinder embedded at the bottom of a vertically sided trench in purely cohesive soil. They also extended the plasticity solution for a seabed with linearly increasing shear strength with depth. They suggested a power law expression of the normalised load in terms of shear strength at the pipe invert, expressed as: 


$$
\frac{\mathrm{F}_{\mathrm{z}}}{\mathrm{Ds}_{\mathrm{u}, \text { invert }}}=\mathrm{a}\left(\frac{\mathrm{z}}{\mathrm{D}}\right)^{\mathrm{b}}
$$

Fitting coefficients $\mathrm{a}$ and $\mathrm{b}$ for limiting conditions of roughness (perfectly smooth and rough) and limiting shear strength profile were given by authors, as detailed in Table $2-2$.

Table 2-2: Fitting coefficients for power law expression (Aubeny et al. 2005)

\begin{tabular}{|c|c|c|}
\hline Boundary Roughness & $\mathrm{z} / \mathrm{D} \leq 0.5$ & $\mathrm{z} / \mathrm{D}>0.5$ \\
\hline Smooth & $\mathrm{a}=4.97, \mathrm{~b}=0.23$ & $\mathrm{a}=4.88, \mathrm{~b}=0.21$ \\
\hline Rough & $\mathrm{A}=6.73, \mathrm{~b}=0.29$ & $\mathrm{a}=6.15, \mathrm{~b}=0.15$ \\
\hline
\end{tabular}

Bruton et al (2006) proposed another force-displacement response model based on large and small scale tests performed within the SAFEBUCK JIP on deepwater soils from the Gulf of Mexico and West Africa, and also in kaolin. This model is relatively similar to the Verley and Lund (1995) model and is expressed as:

$$
\frac{\mathrm{z}}{\mathrm{D}}=\frac{\mathrm{S}_{\mathrm{t}}}{15}\left(\frac{\mathrm{F}_{\mathrm{z}}}{\mathrm{Ds}_{\mathrm{u}, \text { invert }}}\right)^{2}
$$

where $S_{t}$ is the soil sensitivity. The model does not account for the variation of the soil shear strength with depth and the authors acknowledge the necessity of further improvement of the model in this area. Bruton et al (2006) commented on the discrepancy of the model and the classical plasticity solution (Murff et al. 1989) even for a sensitivity of unity. They suggest that the discrepancy might be due to additional consolidation settlement in the test data or over-estimation of the shear strength at shallow penetration due to softening close to the free surface.

Randolph and White (2008a) presented yield envelopes obtained from upper-bound plasticity solutions for pipeline embedment of up to $0.5 \mathrm{D}$ in clay soil, offering a simple way to estimate the embedment resistance for various combinations of horizontal and vertical loading. They also considered linearly increasing shear strength with depth but ignored the effect of self-weight due to its small role at shallow embedment for common ratios of effective stress to shear strength. The pipeline was also assumed to break away at its trailing edge, based on experimental observations where only transient suction resistance can be maintained at the trailing edge. The results of the upper-bound solution (Randolph and White 2008a) were compared with finite element analysis and experimental data by Merifield et al (2008) and found to be remarkably accurate, Figure $2-13$. 

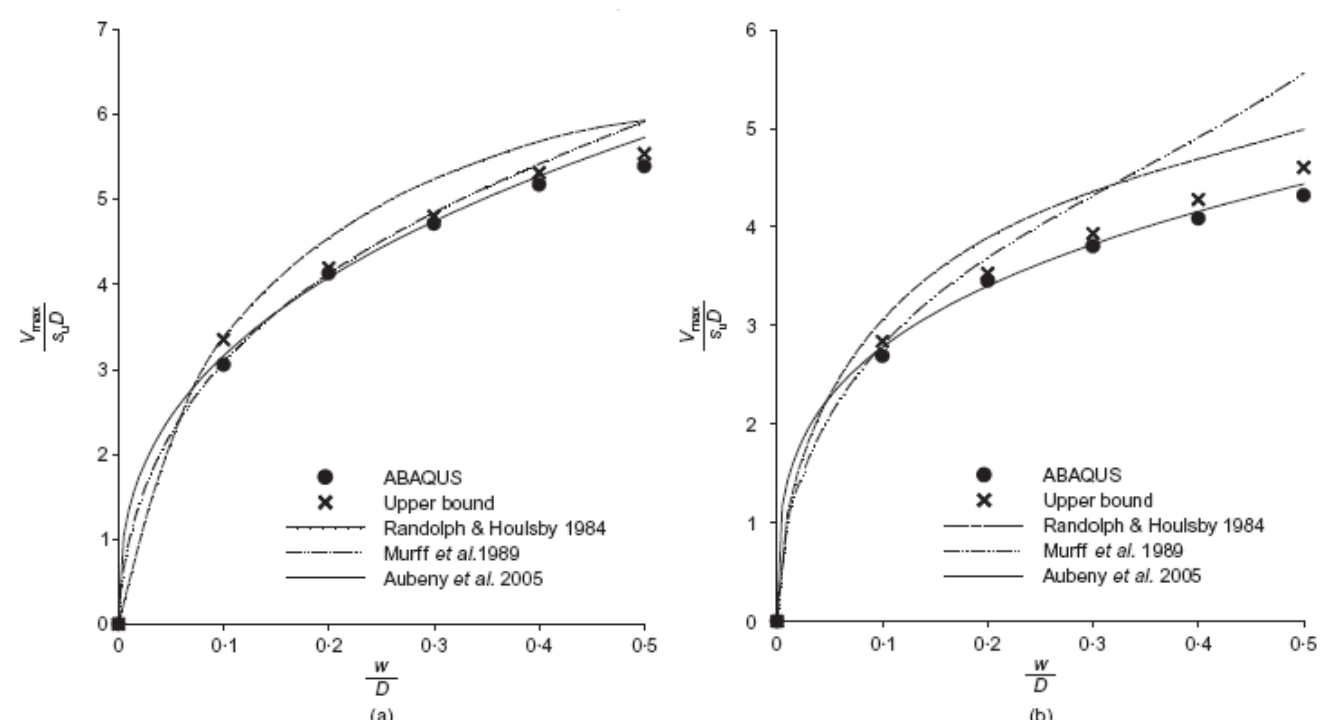

Figure 2-13: Collapse load for vertical pipe embedment (a) rough pipe (b) smooth pipe (Merifield et al. 2008)

Randolph and White (2008b) suggested that for design purpose, the theoretical solutions should be considered as the basic of design in combination with the power law method (Aubeny et al. 2005) with rounded values of parameters a and $b$, for example $a=6, b=$ 0.25 . The authors' suggestion is also supported by various studies incorporating high quality experimental data (Aubeny et al. 2008; Cheuk and White 2008; Dingle et al. 2008).

As the pipeline penetrates into the seabed soil, it experiences buoyancy because of the higher density of the seabed soil relative to seawater. Local heave at the sides of the pipeline will result in buoyancy that is greater than arising from the nominal embedment of the pipe. Merifield et al (2009) studied the heave effect on the response of partially embedded pipes in clay and showed that the heave caused the local pipe embedment to considerably exceed (by $\sim 50 \%$ ) the nominal embedment. This finding was shown to be in agreement with experimental data reported by Dingle et al (2008).

Randolph and White (2008b) discussed the significant importance of buoyancy and local heave on the penetration resistance for a given embedment and compared the results with large deformation finite element (LDFE) analyses (Randolph et al. 2008), Figure 2-14. They showed that the numerical data could be modelled using Equation (2-11), together with a buoyancy term based on the nominal volume or soil displaced by the pipe, multiplied by a buoyancy factor, $\mathrm{f}_{\mathrm{b}}$, of 1.5 . 


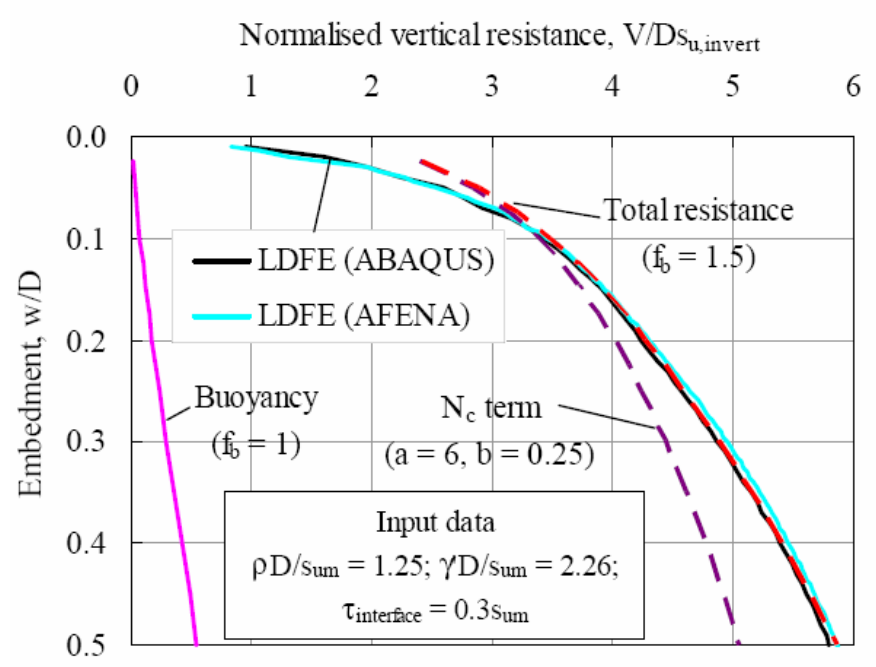

Figure 2-14: Comparison of penetration resistance curves (Randolph and White 2008b)

The solutions and approaches discussed above were developed for a pipe resting on the soil and hence are not directly applicable to SCR static embedment in the touchdown zone. As explained early in this chapter, a local load concentration occurs due to the interrupted natural catenary shape of the SCR at the seabed, and this will cause greater static embedment of the SCR into the seabed than would occur with just the submerged pipe weight. The degree of the over-stress at the seabed depends on the water depth, riser bending stiffness, seabed soil stiffness and the horizontal tension of the riser at the TDP. It may be quantified by a concentration factor, $f_{\text {lay }}$, Then a conventional bearing capacity calculation (from the approaches reviewed above) is used to calculate the maximum static penetration of the SCR in the touchdown zone. The effect of the SCR dynamics is then considered by multiplying the maximum static penetration into the seabed soil (as calculated above) by a dynamic factor, $\mathrm{f}_{\text {dyn }}$ to achieve the predicted final embedment. An alternative approach is to estimate the final embedment by using the remoulded shear strength, rather than the intact shear strength, in the bearing capacity relationships, as suggested by Cathie \& Jaeck (2005)

Further details of these approaches can be found in the work published by Bruton et al (2006) and Hodder et al (2008). The cyclic riser-seabed interaction and the dynamic effect will be discussed in the next section and published work reviewed briefly. It is worth mentioning, before entering the next discussion, that the cyclic behaviour of soft clays is a wide area of study and has been examined by many authors (Andersen 1976; Idriss et al. 1978; Nova 1983; Prévost 1977). The area of interest here is restricted to the cyclic interaction of a pipeline with the seabed soil and the resulting dynamic lay effects on the pipeline as-laid embedment, which is conceptually very close to an SCR configuration in the touchdown zone. 


\subsubsection{Seabed response to system dynamics in TDZ}

Studies of the seabed response under vessel-induced cyclic motions of the SCR in the TDZ have focused on soil stiffness degradation, softening of the soil due to the cyclic loading and further embedment of the riser (including trench formation) due to combined vertical and lateral motion resulting form wave-induced motion of the vessel.

\section{Seabed response to cyclic loading}

Morris et al (1988) carried out laboratory model tests to evaluate pipeline penetration into very soft clay due to horizontal cyclic oscillations. The authors noted that the magnitude of the load or displacement and the duration of the excitation have significant influence on further penetration of the pipeline. Their results showed that the rate of embedment gradually decreases with increasing number of cycles but the embedment does not appear to reach a constant value. Morris et al. (1988) also noted the formation of heave at the sides of the pipeline with increasing penetration depth. Dunlap et al. (1990) performed another experimental study in which a 6 inch (diameter) pipe was penetrated to a depth of 1D into remoulded clay. Cyclic lateral motion was then imposed on the pipe for two hours with the applied vertical force staying within the typical range for mean static load, with no separation of the pipe from the soil. The tests were performed by both load and displacement control and the results were presented in the form of modified backbone curves for a wide range of cycles expressed by a power law equation similar to equation (2-12) (see Figure 2-15). The results showed that cyclic loading with limited load amplitude had minor effect, with the embedment depth increasing slightly, whilst large amplitude cycles with full suction mobilisation ending with separation of the pipe from the soil resulted in much deeper embedment. Such difference is believed to be due primarily to greater remoulding of the soil during the full separation of the pipe element (Cathie et al. 2005). Randolph and White (2008b) comment that water entrainment in the soil as it is remoulded, leading to additional softening, and physical ploughing of the seabed soil due to lateral motion of the pipe, are sources of further embedment during large amplitude motions of the pipe. 


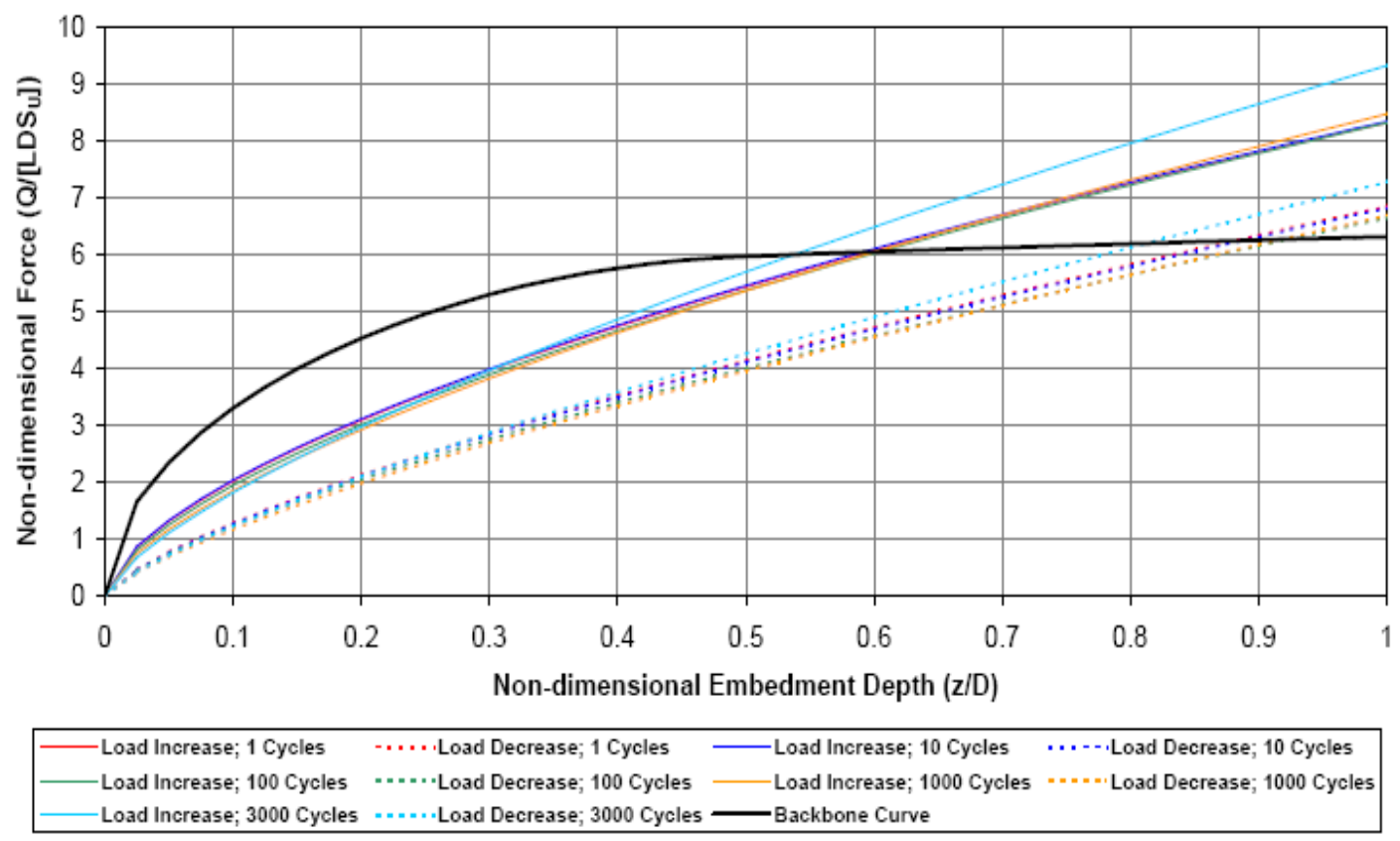

Figure 2-15: Non-dimensional bearing capacity factor, load-controlled tests (Dunlap et al. 1990)

Fontaine et al (2004) introduced a model for seabed response to small amplitude cyclic motions through an experimental study of pipeline segments in soft kaolinite. They evaluated the non-dimensional vertical stiffness and equivalent damping as a function of the pipe diameter, soil shear strength and, particularly, the amplitude of the cyclic motion, which was believed to be the main parameter in characterizing the cyclic pipesoil interaction. The authors then compared their results with other solutions (DNV-RPF105 2006; Dunlap et al. 1990; Verley and Lund 1995) and found it more realistic for soft kaolinite.

A comprehensive study of cyclic pipe-soil interaction was performed under the STRIDE and CARISIMA joint industry projects, with particular attention to suction force mobilisation during the uplift movement of the pipeline (Bridge et al. 2004). The hyperbolic model proposed by Bridge et al (2004) captures various non-linear aspects of soil behaviour characteristics within the applicable displacement stages, including initial penetration, uplift, suction mobilisation, breakout and repenetration. The hyperbolic curve of the model was developed based on the hyperbolic force-displacement interaction curve for sand developed by Audibert et al. (1984) and is similar in form to the hyperbolic pipe-soil interaction curve developed by Hardin and Drnevich (1972) that was originally proposed for clay soils by Kondner (1963). Bridge et al. (2004) then proposed different soil stiffness for small and large static and cyclic displacements of the pipe. The soil suction during uplift was modelled based on the test data obtained from the STRIDE and CARISIMA JIPs. For calculation of the dynamic soil stiffness, the model used the bearing load as opposed to the TDP reaction force. Hence, the model does not account for soil softening due to repeated cycles, resulting in a conservative 
value of dynamic soil stiffness; this is the main drawback of the model as acknowledged by the authors. The model proposed by Bridge et al. (2004) was used in numerical simulations of various SCR projects in the Gulf of Mexico.

Further exploration of soil stiffness degradation due to breakout, particularly under large amplitude cyclic loading, was reported by Clukey et al. (2005) on the basis of a series of vertically loaded pipe tests. The tests were conducted both in load and displacement control on overconsolidated clay with characteristics similar to samples from the Sigsbee Escarpment in deepwater Gulf of Mexico. The results obtained from small and large amplitudes were then compared with the hyperbolic models proposed by Audibert et al. (1984) and Bridge et al. (2004) and showed a good correlation of the measured soil stiffness with the hyperbolic models for uplift-repenetration conditions dominated by compressive loads. For more robust loading, the soil stiffness was significantly degraded below the prediction of hyperbolic models.

Hodder et al. (2008) conducted further investigation of stiffness degradation under cyclic loading, again using soft kaolin. They showed a dramatic decrease of the soil strength (by a factor of 7.5 relative to initial penetration). The authors noted that this degradation was much more significant than the degradation recorded in cyclic T-bar penetration tests and commented that this might be due to water entrainment during pipe separation and re-contact. They also acknowledged the significant effect of the buoyancy component of resistance, for conditions following considerable loss of strength, which was enhanced by local heave of soil adjacent to the pipe. Figure 2-16 shows the banana-shaped cyclic vertical response of the soil and penetration-resistance plot (Hodder et al. 2008). It is also interesting to note that the major part of the trench was developed in only a few early cycles, which is consistent with the findings from the current research as will be shown in Chapter 5.
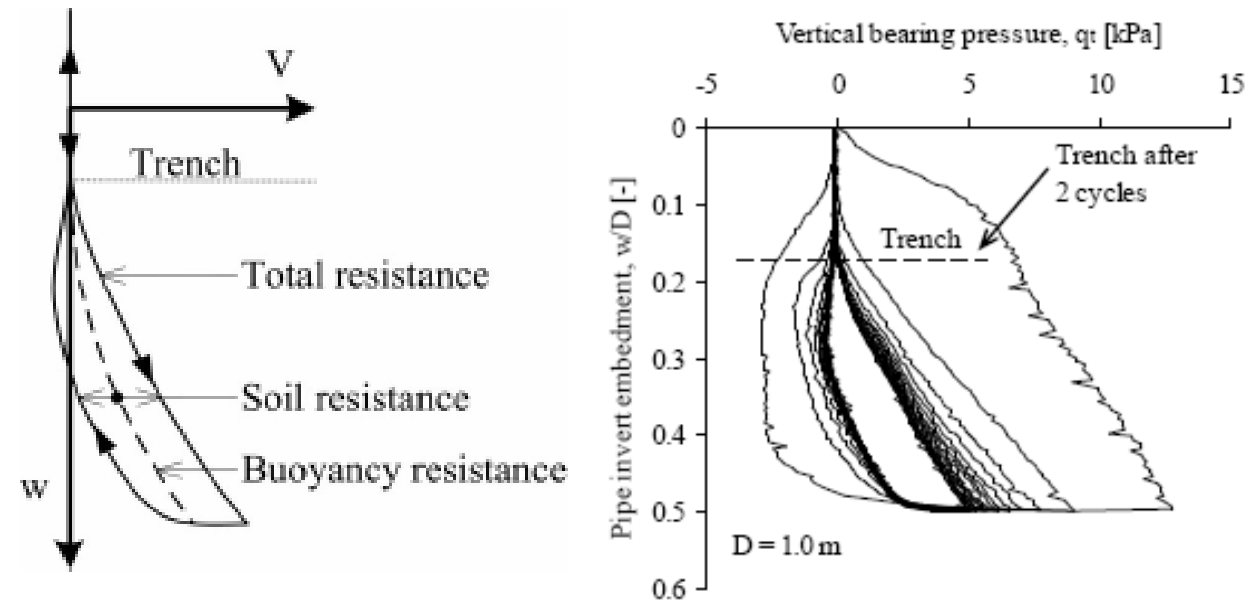

Figure 2-16: Banana-shaped cyclic vertical pipe-soil interaction and vertical resistance (Hodder et al. 2008) 
Aubeny et al. (2008) conducted laboratory floor model tests in soft kaolin to assess the main aspects of pipe-soil interaction including the embedment resistance for initial penetration (static backbone curve), the secant soil stiffness within uplift-repenetration cycles and the ultimate penetration under cyclic loading. Small and large amplitude loading cycles were performed and the soil stiffness was obtained for different displacement amplitudes. The initial penetration resistance was found to correlate well with previously developed backbone curves (Aubeny et al. 2005; Murff et al. 1989). Aubeny et al. (2008) confirmed that the repenetration secant stiffness is much less than the uplift stiffness for a given cyclic displacement $(50 \sim 80 \%$ for no separation conditions). The authors also acknowledged the probable effects of remoulding and consolidation on the cyclic stiffness variation and reported a $25 \%$ reduction in secant stiffness under cyclic loading for uplift and repenetration. They confirmed that the accumulation rate of ultimate embedment under cyclic loading is quite dependent on the cyclic load amplitude, with at least an order of magnitude greater embedment for the large amplitude cycles (range of 1D), which does not reach a limit even after a large number of load cycles. In the small amplitude load cycles, the accumulation rate decreased with increasing number of load cycles. The test results also showed that the penetration-resistance curve approaches the original backbone curve after monotonic penetration of $1 \mathrm{D}$ following the cyclic test. Figure 2-17 shows an example of the reported penetration-resistance plot from their large amplitude test. The model pipe dimensions were $25 \mathrm{~mm}$ diameter by $125 \mathrm{~mm}$ long. The legend "Reference" in Figure 2-17 refers to a reference test establishing the backbone curve for monotonic penetration followed by extraction, which was used as a basis for evaluating the cyclic test data. The results obtained from the experimental study helped the authors to develop a nonlinear seabed interaction model for riser analysis, accounting for soil stiffness degradation and gradual trench formation due to cyclic loading (You et al. 2008).

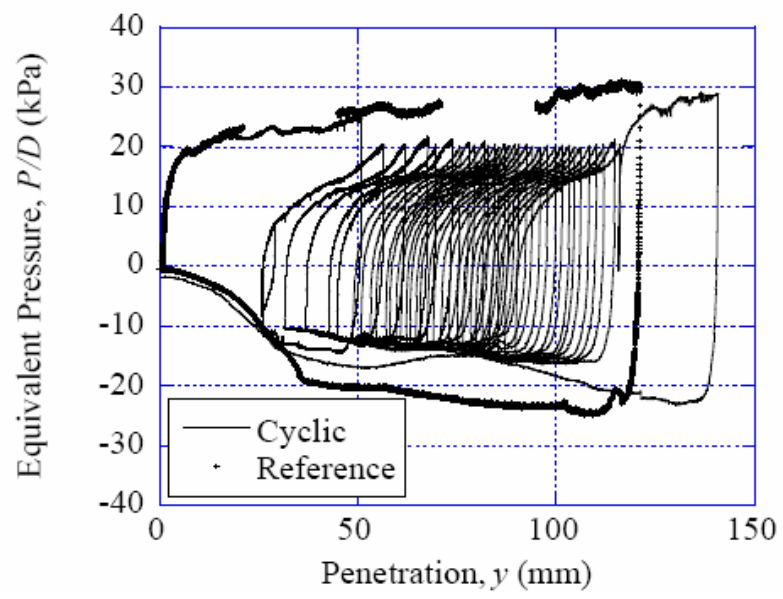

Figure 2-17: Penetration-resistance curves in large amplitude tests (Aubeny et al. 2005; Murff et al. 1989) 
A hysteretic non-linear riser-seabed interaction model was proposed by Randolph and Quiggin (2009) intended for use in practical engineering analysis. The model, which is based on a hyperbolic formulation, was implemented in the commercial software package OrcaFlex and the results obtained were compared with laboratory tests and and a field test conducted in a harbour. The authors also used the new model in OrcaFlex to compare the peak fatigue damage with a linear elastic seabed with various stiffness values. This model has been used in the current thesis for fatigue studies and a comprehensive description of the model can be found in Chapter 5 .

\section{Seabed response to hydrodynamic effect of SCR or dynamic lay effect of pipeline}

Verley and Lund (1995) proposed an empirical equation to predict the maximum penetration depth of a partially buried pipe on clay under hydrodynamic wave and current actions. In addition to assessment of the maximum embedment from a given motion amplitude, the suggested equation intended to evaluate the penetration process as a function of the work conducted by the pipe on the soil:

$$
\left(\frac{\mathrm{z}}{\mathrm{D}}\right)_{\max }=1.1 \frac{\mathrm{F}_{\mathrm{z}}}{\mathrm{Ds}_{\mathrm{u}}}\left(\frac{\mathrm{s}_{\mathrm{u}}}{\mathrm{D} \gamma}\right)^{0.54}\left(\frac{\mathrm{a} *}{\mathrm{D}}\right)^{0.17}
$$

where $\mathrm{a}^{*}$ is the amplitude of horizontal movement. To calibrate the model, the authors collected data from a number of large and small-scale laboratory tests and numerical simulations. Time domain FE analyses were performed to implement the pipe-soil interaction model and the results were compared with full-scale laboratory tests to validate the model. A validity range of $\mathrm{z} / \mathrm{D}<0.3$ was set to cover the range of the data used within the study.

The dynamic effects on pipelines during the lay process were considered further by Lund (2000) along with the cyclic response, investigating the as-laid embedment of a large diameter pipeline (Zeepipe 2B pipeline in the North Sea, $1.2 \mathrm{~m}$ OD). Particular focus was on the effects of the hydrodynamic motions of the vessel and the suspended pipeline, especially the horizontal oscillations. He back-analysed the as-laid embedment of the pipeline and found significant underestimation of the final embedment and then concluded that this was mainly due to lateral cyclic motions of the pipe in the touchdown zone. He noticed that the wave-induced motions of the vessel and hydrodynamic perturbations of the pipeline catenary induce significant horizontal and vertical oscillations of the pipeline in the touchdown zone with the dominant frequency of the movement most related to the natural frequency of the pipeline catenary part, rather than the motion period of the floating system. Lund (2000) multiplied the maximum static penetration, enhanced by the static load concentration factor, by a dynamic touchdown factor, $\mathrm{f}_{\mathrm{dyn}}$, to predict the as-laid embedment, thus accounting for the extra penetration due to dynamic effects. He compared the observed as-laid embedment with the classical static calculations and suggested a dynamic factor between 2 and 10 for the areas with water depths of less than $500 \mathrm{~m}$. This approach was 
followed by Bruton et al. (2006), applying multiplicative adjustments to the static embedment.

Further investigation of dynamic lay effects on the ultimate embedment was reported by Cheuk and White (2008). The authors conducted a series of centrifuge model tests to study the dynamic embedment of an initially unburied pipe segment under smallamplitude horizontal oscillation. They made use of a two-directional actuator, with independent motion control of each axis, to apply combinations of cyclic horizontal displacements and cyclically varying vertical loads, representing vessel-induced motions of the pipeline catenary in the TDZ. They then discussed various mechanisms of dynamic embedment including ploughing (from the lateral motions) and soil softening. They confirmed the significant influence of lay effects on further embedment of the pipeline and showed that a few cycles of small-amplitude oscillation can double or even triple the static embedment; further cycles within the range of what typically occurs during the laying process can make the pipe penetrate into the seabed as much as eight time the initial static embedment. They also concluded that the magnitude of embedment is governed by vertical load level, relative to the bearing capacity, and the amplitude of lateral oscillations. Figure 2-18 shows the results presented by Cheuk and White (2008), emphasizing the influence of the vertical load on the rate of embedment, but also with significant differences for the two types of soil tested.

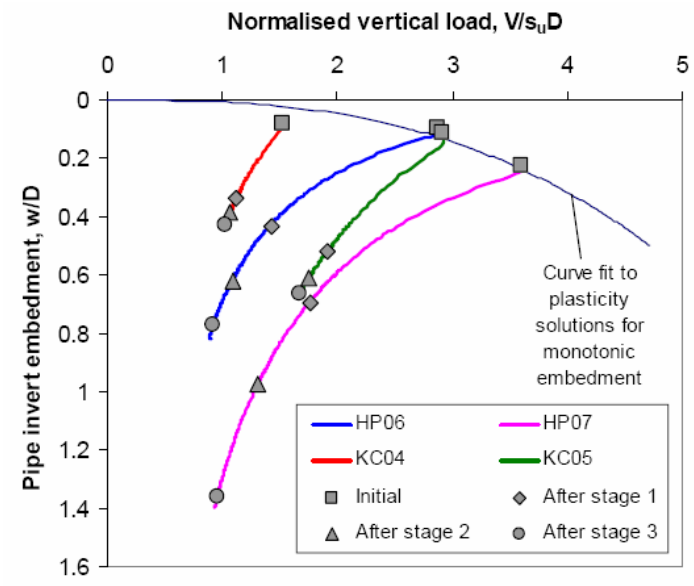

\begin{tabular}{|c|c|c|}
\hline $\begin{array}{c}\text { Lay effect } \\
\text { stage }\end{array}$ & $\begin{array}{c}\text { Horizontal cyclic } \\
\text { amplitude, } \Delta u / D\end{array}$ & $\begin{array}{c}\text { Number of } \\
\text { horizontal cycles } \\
\text { imposed }\end{array}$ \\
\hline 1 & \pm 0.05 & 40 \\
\hline 2 & \pm 0.1 & 40 \\
\hline 3 & \pm 0.2 & 40 \\
\hline 4 & \pm 0.1 & 20 \\
\hline 5 & \pm 0.05 & 20 \\
\hline
\end{tabular}

Figure 2-18: Accumulation of pipe embedment during lay effect model test (Cheuk and White 2008) 
Randolph and White (2008b) presented a keynote lecture on pipeline embedment covering an extensive range of the related aspects, particularly the impact of dynamic lay effects of the hanging part and the cyclic motions of the vessel on additional embedment of the pipeline in the touchdown zone. As part of this study, they performed numerical simulation of the lay effects using the commercial software OrcaFlex with an implemented non-linear seabed response using the model of Randolph and Quiggin (2009). The results showed incremental penetration with each cycle even under purely vertical motions, which were in agreement with model test results. The authors also summarised recent physical modelling studies of dynamic lay effects from various centrifuge model tests, emphasising the significant influence of lay effects on ultimate embedment of the pipeline.

In addition to vertical interaction, pipeline and riser responses also involve lateral and axial interaction with the surrounding soil. Understanding the lateral and axial interaction is important in engineering design of pipelines, particularly for lateral and upheaval buckling due to thermal expansion. Lateral buckling itself is an important area of pipeline research but is not directly related to the area of interest in this dissertation and will not be reviewed here. Axial pipe-soil interaction has a minor relevance to the 2D planar models developed for this research. Hence, the literature review includes a short review of axial pipe-soil interaction in the next section.

\subsubsection{Riser-seabed axial interaction}

Steel catenary risers are normally stretched on the seabed over a relatively short distance and then anchored at the end. Therefore, axial interaction is not a major concern in steel catenary risers. However, since a simple axial frictional response has been considered in all numerical simulations conducted for this research, it is worth reviewing here the fundamentals of axial friction.

The axial resistance of the soil is affected by pipeline embedment and time dependent aspects of the soil response such as thixotropy and consolidation following pipeline installation. Generally, however, the magnitude of the resistance is calculated simply by a Coulomb friction model given as:

$\mathrm{F}_{\mathrm{X}}=\mu \mathrm{W}$

where $\mu$ is the coefficient of axial friction and $\mathrm{W}$ is the (submerged) pipe weight per unit length. Equation (2-15) is strictly only valid for drained conditions, but in practice it is often used for all axial response regardless of the rates of movement. In thermal expansion, which is the main source of changes in axial friction, increasing the temperature takes several hours and the assumption of drained conditions is reasonable (Cathie et al. 2005). The magnitude of the parameter $\mu$ depends on the properties of the pile-soil interface and the soil frictional strength, with values recommended in various guidelines for piles and offshore pipelines, particularly for granular soils. For the soilsteel interface, it is given as: 
$\mu=\tan \left(\phi^{\prime}-5^{\circ}\right) \quad($ API-RP-2A WSD 2000)

$\mu=2 / 3 \tan \left(\phi^{\prime}\right) \quad($ Cathie et al. 2005 )

Finch et al (2000) conducted a series on tests on various types of pipeline surfaces from polypropylene to concrete weight coating and proposed the following equation:

$\mu=\mathrm{f}_{\mathrm{r}} \tan \left(\phi^{\prime}\right)$

with values of the empirical factor, $f_{r}$, based on the coating roughness and soil grain size, as detailed in Table 2-3.

Table 2-3: Resistance factor for pipeline axial coefficient under fully drained condition (Finch et al.

2000)

\begin{tabular}{|l|l|}
\hline Condition & $\mathbf{f}_{\mathbf{r}}$ \\
\hline Granular cohesionless soil, pipe roughness $>\mathrm{D}_{50}$ & 1.0 \\
\hline Granular cohesionless soil, pipe roughness $<\mathrm{D}_{50}$ & $0.75<\mathrm{f}_{\mathrm{r}}<0.9$ \\
\hline Fine-grained cohesive soil, pipe roughness $>\mathrm{D}_{50}$ & 1.0 \\
\hline Clay, pipe roughness $<\mathrm{D}_{50}$ & 0.6 \\
\hline Silt, pipe roughness $<\mathrm{D}_{50}$ & 0.4 \\
\hline
\end{tabular}

For fine-grained soils with a high rate of loading and thus undrained conditions, equation (2-15) may alternatively be written as a function of undrained shear strength as:

$\mathrm{F}_{\mathrm{x}}=\alpha \mathrm{s}_{\mathrm{u}} \mathrm{L}$

where $\alpha$ is a so-called adhesion factor and $\mathrm{L}$ is the arc length of the pipe section partially penetrated in the soil and including local heave. The values of $\alpha$ and $s_{u}$ are different for residual and ultimate axial resistance and can be obtained from laboratory shear tests. In the absence of specific data for very soft clays, $\alpha$ may be assumed to be unity for the ultimate resistance and the inverse of soil sensitivity $\left(1 / \mathrm{S}_{\mathrm{t}}\right)$ for residual resistance (Cathie et al. 2005). Audibert et al. (1984) suggested the following equation to relate the ultimate axial force to the ultimate axial mobilisation displacement:

$\mathrm{F}_{\mathrm{U}}=\pi \mathrm{D} \mu \mathrm{s}_{\mathrm{u}}, \quad \mathrm{x}_{\mathrm{U}}=0.2$ to 0.4 inches $(5$ to $10 \mathrm{~mm}$ )

where $\mu=f_{r} \tan \phi^{\prime}$ in which $\phi^{\prime}$ is the internal friction angle of soil and $f_{r}$ is the resistance factor for pipeline soil axial friction coefficient which depends on the properties of the pipe-soil interface. The value of $f_{r}$ is less than unity and suggested by various design guidelines and authors for different pipe-soil interface conditions (API-RP-2A 2002; Finch et al. 2000). 
Chaudhury (2001) comments that for steel catenary riser analysis the contribution of axial resistance is negligible and can be ignored.

\subsection{SUMMARY AND CONCLUSION}

The geotechnical and naval architectural aspects of steel catenary risers, as affecting the fatigue performance of SCRs in the touchdown zone, have been discussed and relevant published researched has been reviewed briefly. The significant influence of hydrodynamic loading of the suspended part of the SCR on the as-laid embedment has been highlighted and the proposed riser-seabed interaction models to capture the gradual degradation of seabed soil stiffness and trench development under cyclic loading have been discussed. The impact of non-linear riser-seafloor interaction models, including mobilization of suction resistance and trench formation, on the SCR fatigue performance in the touchdown zone has been emphasised and areas where contradictory results have been obtained by different authors have been highlighted.

The literature review has shown that, apart from dynamic effects of the SCR itself, the influence of the longitudinal trench development beneath the riser on fatigue performance, due to gradual remoulding and degradation of the soil under quasi-static cyclic motions of the vessel is still a challenging and important aspect of SCR design. The current dissertation has focused on this area and the complexities associated with the resolving the issue. A primary reason for inconsistent results reported by different authors has been the absence of a sophisticated riser-seabed interaction model enabling modelling of the key relevant features of non-linear soil behaviour. This study has tried to remove part of the uncertainties through the implementation of a newly developed non-linear hysteretic seabed model (Randolph and Quiggin 2009), which results in gradual trench formation with a consistent longitudinal profile, in addition to capturing non-linear aspects of the cyclic soil behaviour and suction mobilisation. This model has the potential to provide more reliable assessment of the influence of non-linear seabed response and trench formation on the fatigue performance of SCRs in the touchdown zone. 


\section{CHAPTER 3. CLOSED FORM SEABED INTERACTION MODELS}

\subsection{INTRODUCTION}

Riser-seabed interaction is the main boundary condition governing the performance of SCRs in the touchdown zone. Design must ensure that the curvature remains well within elastic limits, and that fatigue damage due to stress changes in the pipe wall remains acceptable during the operation life. Closed form solutions, although limited in their accuracy because of idealisations of the riser response, offer a first step in assessing the riser performance. They have the advantage of offering insight into the system mechanics, which cannot be gained through numerical methods such as FEA (finite element analysis). Simplified closed form solutions allow approximate estimation of the maximum stress in the SCR, thus on the one hand providing useful data for design and on the other hand serving as a good reference point for overall checking of the results from FEA. These kind of analytical solutions normally use the basic catenary equations for the hanging part of the riser and a boundary layer approach in the touchdown zone where the importance of the riser bending stiffness and internal shear force becomes significant. This chapter reviews briefly the fundamental catenary equations and analytical closed form solutions, comparing the results with numerical simulation by ABAQUS.

A generic SCR may be simply divided in two different parts:

o Part I : A hanging catenary section

o Part II : A part resting on the seabed

As will be shown later in this chapter, the influence of the theoretical model used for Part II is significant in the final stress distributions and their continuity along the SCR. Over-simplified models give a discontinuity in bending moment and shear force at the TDP, which is the most critical part in the SCR design from a fatigue damage stand point. A comparison of the results of different models is provided with those from finite element analysis using ABAQUS to evaluate the accuracy and consistency of the analytical solutions for initial design assumptions and for fatigue assessment as well. Finally, the significant impact of the maximum shear force in the SCR, and hence the influence of the seabed stiffness, on the fatigue damage will be discussed.

\subsection{STATIC CATENARY RESTING ON RIGID SEABED}

An SCR normally hangs under its own weight between the floating system at the top and the touchdown point (TDP) on the seabed; the rest of the SCR is laid on the seabed (as a flowline or pipeline) from the TDP to an anchored end. Applying only the catenary equations for the hanging part of the SCR, ignoring the bending stiffness and assuming 
a rigid seabed, necessitates an abrupt change in the curvature to zero beyond the touchdown point. Although this is not realistic, it provides the starting point for more realistic solutions. In this section, the catenary equations will be applied to the SCR to examine their consistency for SCR modelling, in comparison to rigorous analysis using ABAQUS.

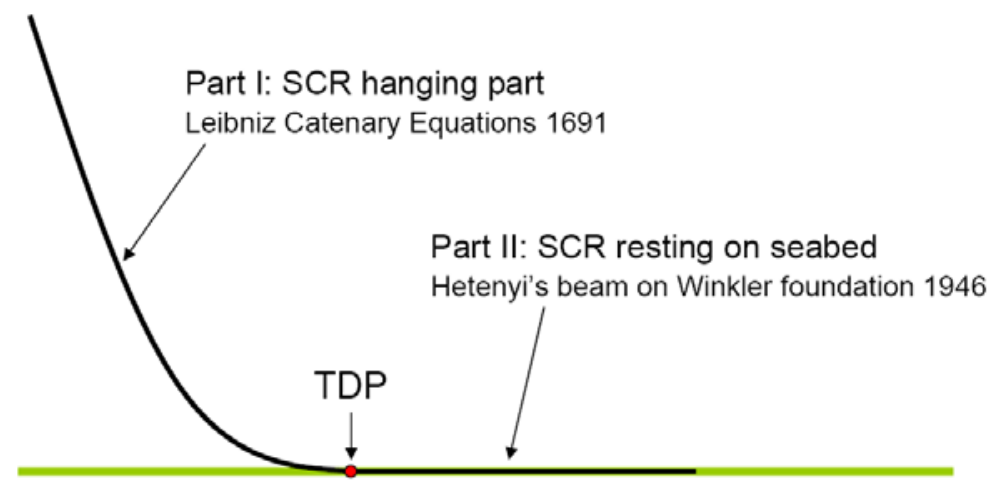

Figure 3-1: Generic SCR overall configuration on rigid seabed

Figure 3-1 shows a generic SCR as it is modelled by the catenary solutions in the suspended part, and assumed zero curvature on the seabed. It is worth mentioning here that sketches of a hanging chain were drawn by Leonardo da Vinci (lived 1452-1519). The word "catenary" is an anglicized form of the Latin term catenaria which itself has been derived from catena meaning "chain". Huygens used this term for the first time in a letter to Leibniz in 1690. Galileo (1564-1642) mistook the shape of the hanging chain as a parabola which was later disproved by Jungius (1669). Jacob Bernoulli first gave the equation of the catenary 49 years after death of Galileo. Huygens, Leibniz and John Bernoulli gave their solutions in reply to the challenge of Jacob Bernoulli posted in Acta Eruditorum to find the actual catenary shape (1690-1691)(Bukowski 2008; Gray and Venit 2002). Leibniz's governing equation for the catenary section, assuming zero bending stiffness, infinite axial stiffness, and with no environmental forces acting is:

$H \frac{d^{2} z}{d x^{2}}=m_{s} g\left[1+\left(\frac{d z}{d x}\right)^{2}\right]^{1 / 2}$

where

$\mathrm{z}$ is the vertical coordinate upward from the seabed

$\mathrm{x}$ is the horizontal coordinate from the TDP to the vessel

$\mathrm{m}_{\mathrm{s}} \quad$ is the submerged mass per unit length of the SCR

$\mathrm{H}$ is the horizontal component of the tension in the SCR

$\mathrm{g} \quad$ is the gravity acceleration 
Note that the horizontal force, $\mathrm{H}$, is constant throughout the length of the SCR, both in the catenary and along the seabed (ignoring friction with the seabed).

The various geometric parameters for the SCR, assumed hanging off a Spar at some depth below the water surface, are indicated in Figure 3-2.

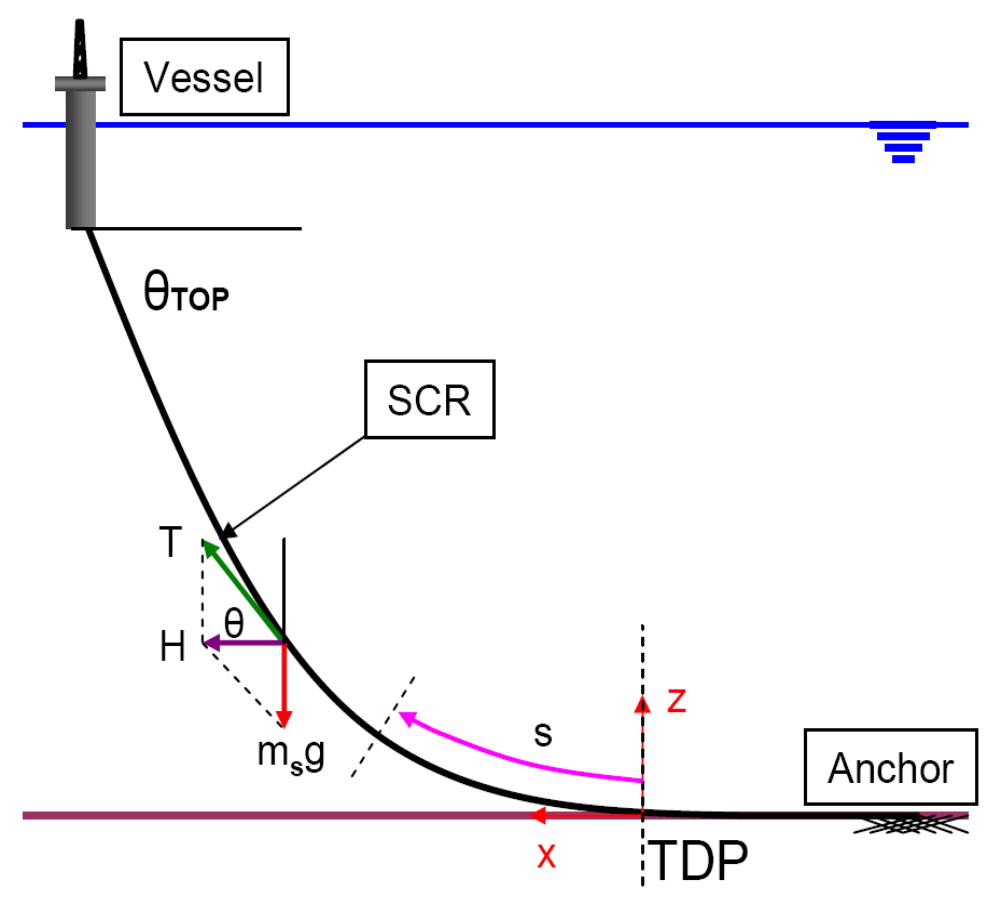

Figure 3-2 : Generic configuration of SCR connected to a Spar

A simple solution of equation (3-1) is given by (Timoshenko 1968):

$\mathrm{z}=\frac{\mathrm{H}}{\mathrm{m}_{\mathrm{s}} \mathrm{g}}\left[\cosh \left(\frac{\mathrm{m}_{\mathrm{s}} \mathrm{gx}}{\mathrm{H}}\right)-1\right]$

Equation (3-2) can be written in terms of geometric parameters, $s, x$ and $\theta$ as:

$\mathrm{z}=\frac{\mathrm{s}}{\tan \theta}\left[\cosh \left(\frac{\mathrm{x} \tan \theta}{\mathrm{s}}\right)-1\right]$

where

$\mathrm{S}$ is the arc length along the catenary from the TDP

$\theta \quad$ is the SCR slope at any point along the catenary 
The length of catenary from the touchdown point to any point along the SCR is expressed as:

$$
\mathrm{s}=\frac{\mathrm{H}}{\mathrm{m}_{\mathrm{s}} \mathrm{g}}\left[\sinh \left(\frac{\mathrm{xm}_{\mathrm{s}} \mathrm{g}}{\mathrm{H}}\right)\right]
$$

The slope of the SCR at any point along the catenary is given by:

$$
\tan \theta=\frac{d z}{d x}=\sinh \left(\frac{x_{s} g}{H}\right)
$$

Re-arranging equation (3-5) the horizontal coordinate from the TDP is:

$$
\mathrm{x}=\frac{\mathrm{H}}{\mathrm{m}_{\mathrm{s}} \mathrm{g}} \operatorname{arcsinh}(\tan \theta)
$$

With further manipulation of equations (3-2) to (3-6), the distance of the touchdown point from the floating system, $\mathrm{x}_{\mathrm{TDP}}$, and the total length of the catenary part of the SCR from the TDP to the vessel, $\mathrm{s}_{\mathrm{R}}$, can be expressed in terms of the height of the attachment point $\mathrm{z}_{\mathrm{A}}$ and the SCR top angle, $\theta_{\mathrm{TOP}}$, as:

$$
\begin{aligned}
& \mathrm{x}_{\mathrm{TDP}}=\mathrm{z}_{\mathrm{A}} \frac{\operatorname{arcsinh}\left(\tan \theta_{\mathrm{TOP}}\right)}{\sqrt{\tan ^{2}\left(\theta_{\mathrm{TOP}}\right)+1}-1}=\mathrm{z}_{\mathrm{A}} \frac{\operatorname{arcsinh}\left(\tan \theta_{\mathrm{TOP}}\right) \cos \theta_{\mathrm{TDP}}}{1-\cos \theta} \\
& \mathrm{s}_{\mathrm{R}}=\mathrm{z}_{\mathrm{A}} \frac{\tan \theta_{\mathrm{TOP}}}{\sqrt{\tan ^{2}\left(\theta_{\mathrm{TOP}}\right)+1}-1}=\mathrm{z}_{\mathrm{A}} \frac{\sin \theta_{\mathrm{TOP}}}{1-\cos \theta_{\mathrm{TOP}}}=\mathrm{x}_{\mathrm{TDP}} \frac{\tan \theta_{\mathrm{TOP}}}{\operatorname{arcsinh}\left(\tan \theta_{\mathrm{TOP}}\right)}
\end{aligned}
$$

The horizontal component of tension may be expressed directly in terms of the water depth and top angle as

$$
\mathrm{H}=\mathrm{z}_{\mathrm{A}} \mathrm{m}_{\mathrm{s}} \mathrm{g} \frac{\cos \theta_{\text {TOP }}}{1-\cos \theta_{\text {TOP }}}
$$

Another important property of the SCR is the tension along the riser at any given point. This may be written as:

$$
\mathrm{T}=\sqrt{\left(\operatorname{sm}_{\mathrm{s}} \mathrm{g}\right)^{2}+\mathrm{H}^{2}}=\mathrm{H} \cosh \left(\mathrm{xm}_{\mathrm{s}} \mathrm{g} / \mathrm{H}\right)
$$

\subsubsection{Catenary equations for vessel's near and far offset}

Depending on the type of vessel and system configuration, the vessel connected to an SCR may be displaced from its initial position by about $5 \%$ of the water depth. Every change in vessel position will cause relocation of the touchdown point, which will affect the SCR profile and stress distribution at the seabed as will be discussed later. Hence, it is useful to apply the catenary equations to evaluate the movement of the TDP for near and far offset of the vessel. In a 2D planar system, the vessel can have vertical (heave) 
and horizontal (surge) displacement and the vessel rotation (pitch) can be converted to equivalent vertical and horizontal displacements (taking account of the position of the attachment point of the riser, relative to the centre of gravity of the vessel). Figure 3-3 shows a schematic configuration of the SCR as the vessel moves from its initial position (i) to a new relocated position (r) under environmental loading. The positive and negative sign of the heave and surge offsets $(a, b)$ are determined based on the defined coordinate system. The catenary length of the hanging part of the SCR, the horizontal distance of the TDP from vessel and the distance of the vessel from the seabed are considered to be always positive.

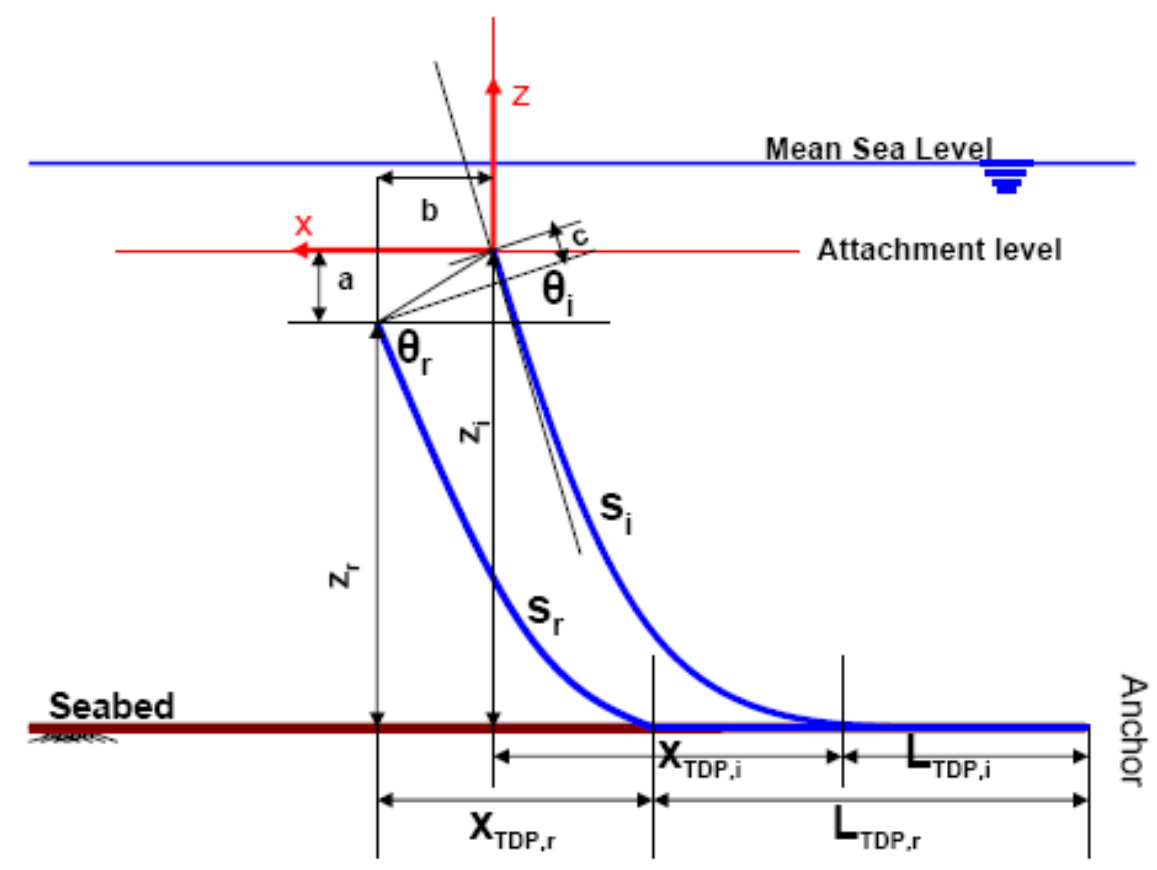

Figure 3-3: Vessel offsets

The total length of the SCR from vessel to the anchor end is a constant value and can be written as:

$\mathrm{s}_{\mathrm{i}}+\mathrm{L}_{\mathrm{TDP}, \mathrm{i}}=\mathrm{s}_{\mathrm{r}}+\mathrm{L}_{\mathrm{TDP}, \mathrm{r}}$

On the other hand, the following equations can be written about the horizontal and vertical distances of the vessel from anchor end and seabed respectively:

$\mathrm{x}_{\mathrm{TDP},{ }_{\mathrm{i}}}+\mathrm{L}_{\mathrm{TDP}, \mathrm{i}}+\mathrm{b}=\mathrm{x}_{\mathrm{TDP}, \mathrm{r}}+\mathrm{L}_{\mathrm{TDP}, \mathrm{r}}$

and 
$\mathrm{z}_{\mathrm{i}}=\mathrm{z}_{\mathrm{r}}+\mathrm{a}$

The sign of vessel offsets a (heave) and b (surge) are defined according to the coordinate system illustrated in Figure 3-3 . Subtracting equation (3-11) from equation (3-12) and rearranging equation (3-13) gives:

$$
\begin{aligned}
& \mathrm{b}=\mathrm{x}_{\mathrm{TDP}, \mathrm{r}}-\mathrm{x}_{\mathrm{TDP}, \mathrm{i}}+\mathrm{s}_{\mathrm{i}}-\mathrm{s}_{\mathrm{r}} \\
& \mathrm{a}=\mathrm{z}_{\mathrm{i}}-\mathrm{z}_{\mathrm{r}}
\end{aligned}
$$

Using equation (3-8) for $s_{i}$ and $s_{r}$ and substituting in equation (3-14) gives, after mathematical simplification:

$$
\mathrm{b}=\mathrm{z}_{\mathrm{r}}\left[\frac{\operatorname{arcsinh}\left(\tan \theta_{\mathrm{r}}\right)-\tan \theta_{\mathrm{r}}}{\sqrt{\tan \theta_{\mathrm{r}}{ }^{2}+1}-1}\right]-\mathrm{z}_{\mathrm{i}}\left[\frac{\operatorname{arcsinh}\left(\tan \theta_{\mathrm{i}}\right)-\tan \theta_{\mathrm{i}}}{\sqrt{\tan \theta_{\mathrm{i}}{ }^{2}+1}-1}\right]
$$

The heave and surge offsets ( $a$ and $b$ ) are known parameters and can be written as ratios of the initial depth $\mathrm{z}_{\mathrm{i}}$ as:

$$
\begin{aligned}
& \mathrm{b}=\overline{\mathrm{b}} \mathrm{z}_{\mathrm{i}} \\
& \mathrm{a}=\overline{\mathrm{a}} \mathrm{z}_{\mathrm{i}}
\end{aligned}
$$

Substituting equation (3-18) into equation (3-15) and rearranging for $z_{r}$ gives:

$$
\mathrm{z}_{\mathrm{r}}=(1+\overline{\mathrm{a}}) \mathrm{z}_{\mathrm{i}}
$$

Substituting equation (3-19) into equation (3-16) and rearranging the known and unknown parameters gives:

$$
\left[\frac{\operatorname{arcsinh}\left(\tan \theta_{\mathrm{r}}\right)-\tan \theta_{\mathrm{r}}}{\sqrt{\tan \theta_{\mathrm{r}}^{2}+1}-1}\right]=\frac{1}{[\overline{\mathrm{a}}+1]}\left[\overline{\mathrm{b}}+\frac{\operatorname{arcsinh}\left(\tan \theta_{\mathrm{i}}\right)-\tan \theta_{\mathrm{i}}}{\sqrt{\tan \theta_{\mathrm{i}}^{2}+1}-1}\right]
$$

The unknown part of the equation (3-20) on the left is referred to as $\mathrm{M}_{\text {unknown }}$. To solve this equation a non-linear regression of the left hand side can be obtained as a function of $\theta_{\mathrm{r}}$ with a high approximation accuracy (with unit correlation factor, $\mathrm{R}^{2}=1$ ), as shown in Figure 3-4. 


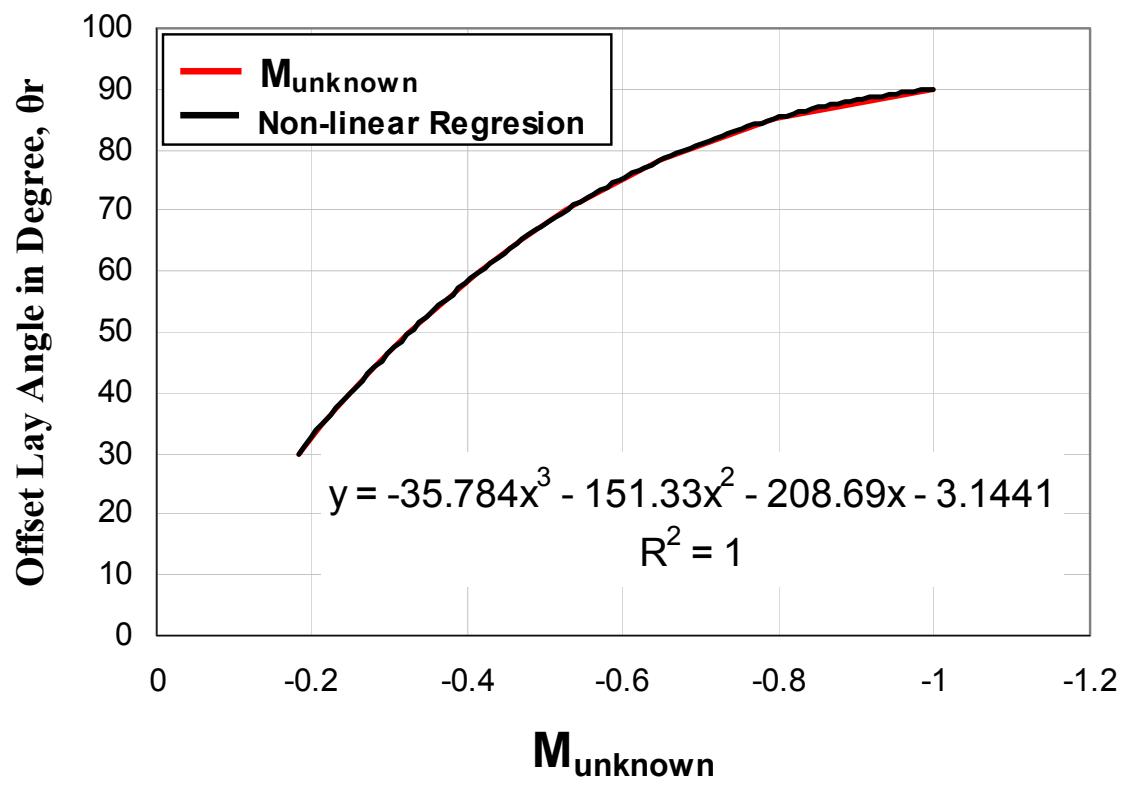

Figure 3-4: Non-linear regression of the left hand side of equation (3-20)

Substituting the known part of equation (3-20) (the right hand part, which is referred to as $\mathrm{M}_{\text {known }}$ ), into the non-linear regression equation shown in Figure 3-20, gives the solution of (3-20) for $\theta_{\mathrm{r}}$ as:

$\theta_{\mathrm{r}} \approx-35.78\left(\mathrm{M}_{\text {known }}\right)^{3}-151.33\left(\mathrm{M}_{\text {known }}\right)^{2}-208.69\left(\mathrm{M}_{\text {known }}\right)-3.14$

Equation (3-21) gives an approximation of the lay angle at the new offset position which can be used in combination with equations (3-7) and (3-8) to give the new configuration of the SCR through the length of the catenary part and the distance of the vessel's new position from the TDP.

The relocation of the touchdown point due to the vessel offset will be simply the difference of the initial and offset catenary lengths:

$\Delta_{\mathrm{TDP}}=\left|\mathrm{s}_{\mathrm{r}}-\mathrm{s}_{\mathrm{i}}\right|$

It will be shown in Chapter 4 that the touchdown point (TDP) relocation calculated above is a key parameter to predict the maximum fatigue damage near the TDP due to vessel excitation under a single wave.

\subsubsection{Bending moment and shear force distribution}

The bending moment of a structural element can be calculated from the curvature, $\kappa$. For a 2D planar non-linear bending element with large deflections, such as the SCR catenary system illustrated in Figure 3-2, the curvature can be written as: 


$$
\kappa=\frac{\frac{d^{2} z}{d x^{2}}}{\left[1+\left(\frac{d z}{d x}\right)^{2}\right]^{1.5}}
$$

Using the standard relationship between the curvature and bending moment and substituting the derivatives of $\mathrm{z}$ from equation (3-3) into equation (3-23) gives the bending moment as:

$$
M=-\kappa E I=-\frac{m_{s} g}{H\left[\cosh \left(\frac{m_{s} g x}{H}\right)\right]^{2}} E I
$$

The shear force along the SCR can be derived by differentiating the bending moment, giving:

$$
V=2\left(\frac{m_{s} g}{H}\right)^{2} \frac{\sinh \left(\frac{m_{s} g x}{H}\right)}{\left[\cosh \left(\frac{m_{s} g x}{H}\right)\right]^{3}} E I
$$

These equations may be used to evaluate conditions at the TDP, setting $\mathrm{x}$ to zero. The shear force will be zero at the TDP, from equation (3-25), while the curvature and bending moment are given by:

$$
\begin{aligned}
& \kappa_{\mathrm{TDP}}=\frac{\mathrm{m}_{\mathrm{s}} \mathrm{g}}{\mathrm{H}} \\
& \mathrm{M}_{\mathrm{TDP}}=-\frac{\mathrm{m}_{\mathrm{s}} \mathrm{g}}{\mathrm{H}} \mathrm{EI}
\end{aligned}
$$

Using equations (3-7) and (3-8), equation (3-27) can be re-written in terms of the catenary length $\mathrm{s}_{\mathrm{R}}$ and the $\mathrm{SCR}$ top angle $\theta_{\mathrm{TOP}}$ as:

$$
\mathrm{M}_{\mathrm{TDP}}=\frac{\tan \theta_{\mathrm{TOP}}}{\mathrm{s}_{\mathrm{R}}} \mathrm{EI}
$$

\subsubsection{Von Mises stress distribution}

An important design consideration for SCRs is the combined bending and tension stress along the riser, especially in the touchdown area where it reaches its maximum value. The von Mises yield criterion is the most commonly used equation to evaluate the combined stress at a tubular section such as the riser for fatigue calculations. The yield criterion is generally expressed in principal stresses as: 


$$
\left(\sigma_{1}-\sigma_{2}\right)^{2}+\left(\sigma_{2}-\sigma_{3}\right)^{2}+\left(\sigma_{3}-\sigma_{1}\right)^{2}=2 \sigma_{\mathrm{y}}{ }^{2}
$$

where

$$
\sigma_{\mathrm{y}} \quad \text { is the yield stress in uniaxial tension or von Mises stress. }
$$

For a tubular pipe section, equation (3-29) can be written as:

$$
\left(\sigma_{\mathrm{R}}-\sigma_{\theta}\right)^{2}+\left(\sigma_{\theta}-\sigma_{\mathrm{A}}\right)^{2}+\left(\sigma_{\mathrm{R}}-\sigma_{\mathrm{A}}\right)^{2}=2 \sigma_{\mathrm{VM}}^{2}
$$

where

$$
\begin{array}{ll}
\sigma_{\mathrm{R}} & \text { is the radial stress } \\
\sigma_{\theta} & \text { is the hoop stress } \\
\sigma_{\mathrm{A}} & \text { is the axial stress } \\
\sigma_{\mathrm{VM}} & \text { is the von Mises stress }
\end{array}
$$

The dominant stress in the touchdown area is caused by the bending moment and, to a lesser extent, the tension. If we ignore the effect of radial stress and hoop stress in evaluating the von Mises stress, equation (3-30) can be written as:

$$
\sigma_{\mathrm{VM}}=\sigma_{\mathrm{A}}=\frac{\mathrm{T}}{\mathrm{A}_{\mathrm{s}}}+\frac{\mathrm{My}_{\max }}{\mathrm{I}}=\frac{\mathrm{T}}{\mathrm{A}_{\mathrm{s}}}+\frac{\mathrm{MD}}{2 \mathrm{I}}
$$

where

$$
\begin{aligned}
& \text { T is the tension force at a given location along the SCR } \\
& A_{s} \quad \text { is the steel section area of the riser } \\
& M \quad \text { is the bending moment at a given location along the SCR } \\
& y_{\max } \text { is the section's largest dimension from the neutral axis or the pipe half } \\
& \text { diameter (ignoring any coating) } \\
& D \quad \text { is the outer diameter of the SCR steel section }
\end{aligned}
$$

The maximum magnitude of the von Mises stress can be obtained by substituting the TDP relevant parameters (from equation (3.27):

$$
\sigma_{\mathrm{VM}, \mathrm{TDP}}=\frac{\mathrm{H}}{\mathrm{A}_{\mathrm{s}}}+\frac{\mathrm{m}_{\mathrm{s}} \mathrm{g}}{2 \mathrm{H}} \mathrm{ED}
$$

The catenary shape and profiles of bending moment, shear force and von Mises stress are shown for a generic SCR for three different lay angles in Figure 3-5 to Figure 3-8, using the parameters given in Table 3-1. These SCR characteristics are used throughout this chapter for example analyses. 
Table 3-1: Generic SCR parameters for static analysis

\begin{tabular}{|c|c|}
\hline Parameter & Magnitude \\
\hline Outer Diameter $\left(\mathrm{D}_{\mathrm{o}}\right)$ & $0.324 \mathrm{~m}$ \\
\hline Wall Thickness $(\mathrm{t})$ & $0.0205 \mathrm{~m}$ \\
\hline In Service Submerged Weight $\left(\mathrm{m}_{\mathrm{s}}\right)$ & $100 \mathrm{~kg} / \mathrm{m}$ \\
\hline Bending Stiffness $(\mathrm{EI})$ & $4.67 \times 10^{7} \mathrm{Nm}^{2}$ \\
\hline Steel Yield Stress $\left(\mathrm{f}_{\mathrm{y}}\right)$ & $448 \mathrm{MPa}$ \\
\hline Seabed Stiffness $(\mathrm{k})$ & $100 \mathrm{kPa}$ \\
\hline Lay Angle $\left(\theta_{\text {TOP }}\right)$ & $78^{\circ}$ \\
\hline Water Depth & $1800 \mathrm{~m}$ \\
\hline Height of attachment point & $1600 \mathrm{~m}$ \\
\hline
\end{tabular}

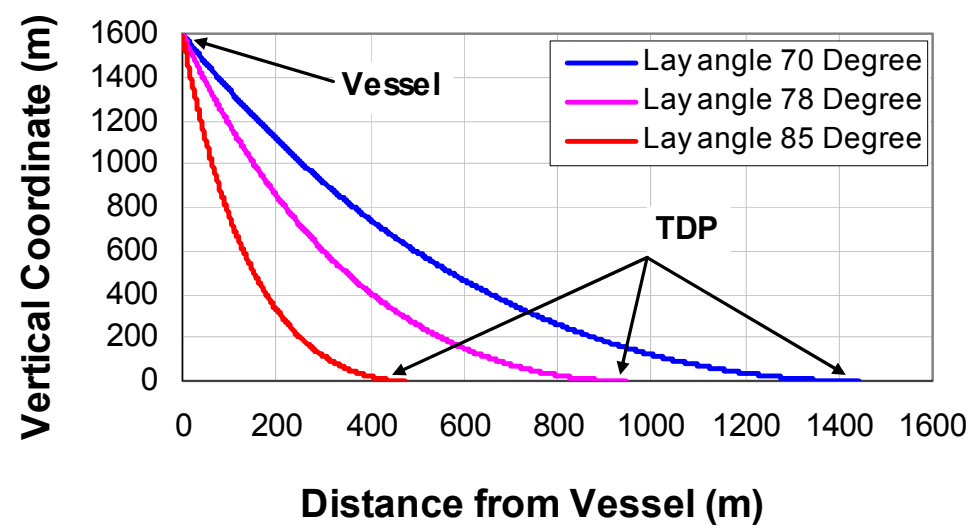

Figure 3-5: SCR catenary shape based on mathematical equations

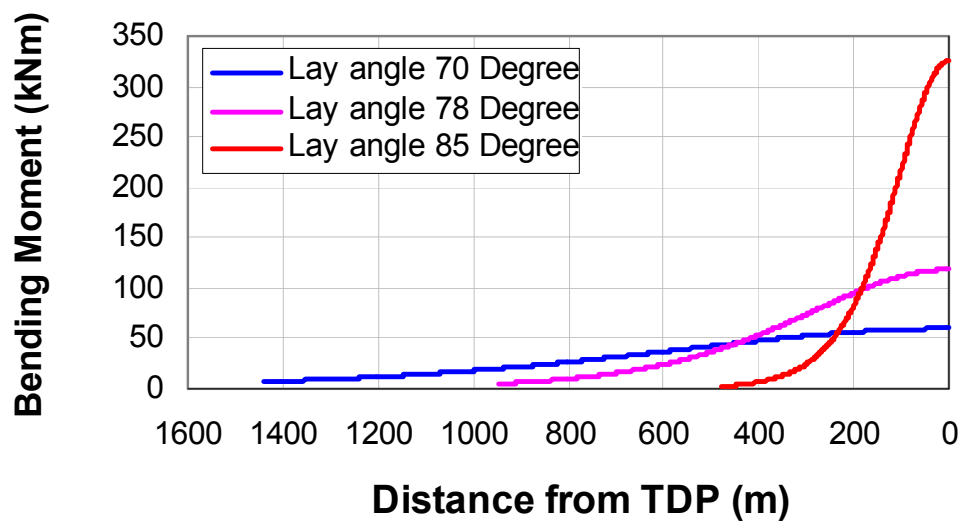

Figure 3-6: Bending moment distribution along SCR based on catenary equations 


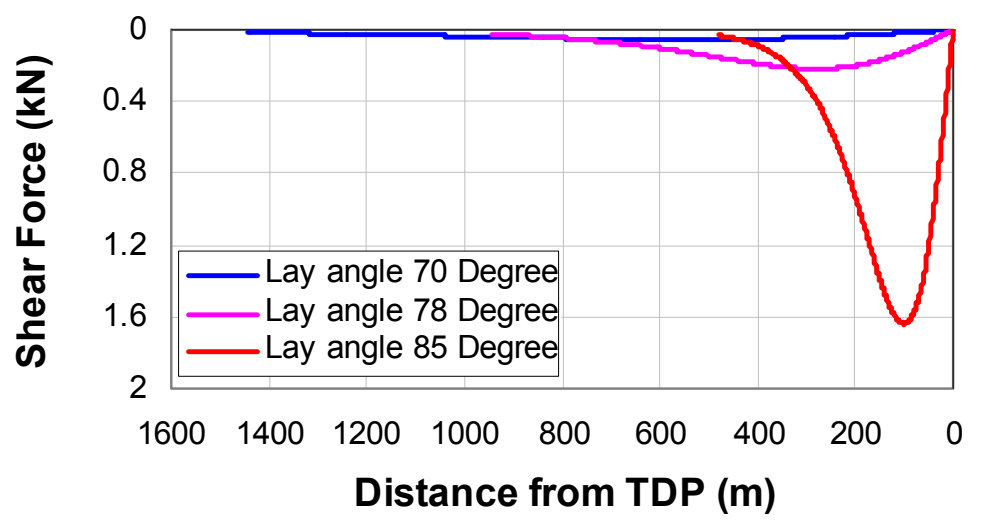

Figure 3-7: Shear force distribution along SCR based on catenary equations

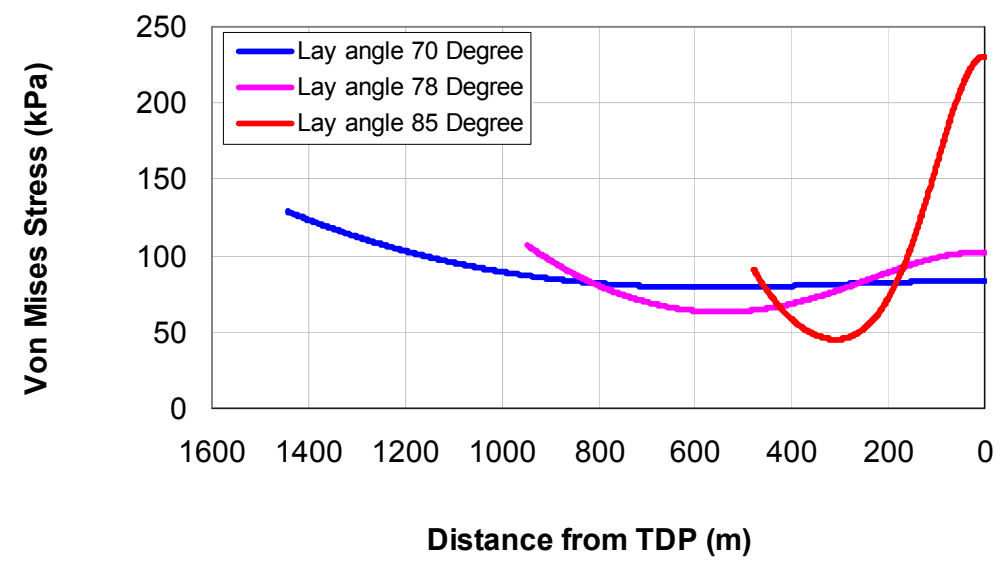

Figure 3-8: Von Mises stress along SCR based on catenary equations

As Figure 3-6 illustrates, the most important drawback of modelling SCRs on a rigid seabed using the catenary equations is the significant discontinuity of bending moment at the touchdown point, where the bending moment immediately drops from its maximum value at the TDP to zero in the remaining part beyond the TDP. This serious disadvantage makes the rigid seabed model impractical for SCR modelling. Hence, a more refined model is needed in the vicinity of the TDP to ensure continuity of (at least) the moment distribution along the SCR. In the next section, the rigid seabed will be replaced by Hetentyi's solutions for a beam on a linear elastic foundation (Winkler model) in order to overcome the bending moment discontinuity at the TDP.

\subsection{STATIC CATENARY RESTING ON ELASTIC SEABED}

\subsubsection{Hetenyi's solutions for beam on Winkler elastic foundation}

Solutions for a semi-infinite beam under simultaneous axial and transverse loading have been derived by Hetenyi (1946). These can provide a simple model for SCR-seabed 
interaction in the touchdown zone as a first approach to overcome the bending moment discontinuity at the TDP. Figure 3-9, shows the global setup of an SCR resting on an elastic seabed. As illustrated in Figure 3-10 for a small element of beam at section A-A of Figure 3-9, the equilibrium condition leads to the following differential equation, to describing the SCR-seabed interaction:

$\mathrm{EI} \frac{\mathrm{d}^{4} \mathrm{z}}{\mathrm{dx}^{4}}-\mathrm{H} \frac{\mathrm{d}^{2} \mathrm{z}}{\mathrm{dx}^{2}}+\mathrm{kz}=0$

where $\mathrm{k}$ is the elastic stiffness of the seabed (force per unit length divided by displacement)

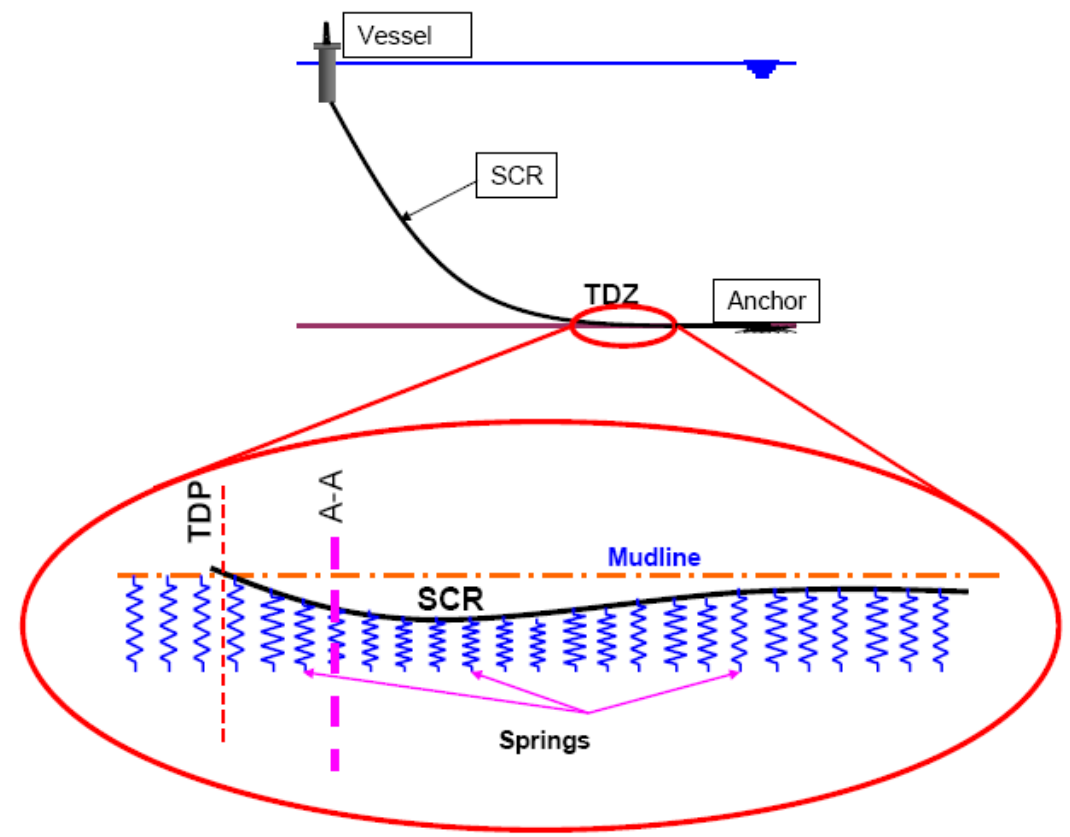

Figure 3-9: Static catenary combination with beam on Winkler elastic foundation
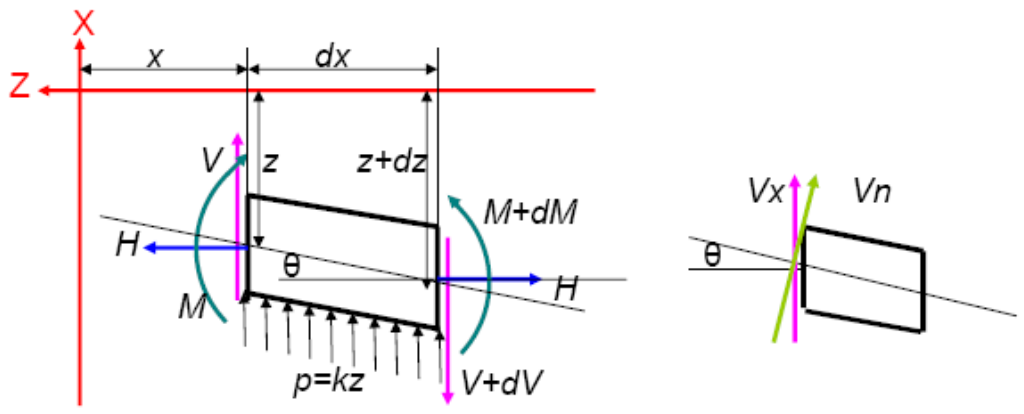

Figure 3-10 : Section A-A, Tension beam on Winkler elastic foundation (Hetenyi 1946) 
Hetenyi (1946) gave solutions to equation (3-33) finding terms of the shear force, bending moment, rotation and vertical displacement along the beam for cases of a concentrated point load and moment at the end of the beam. Solutions show that the existence of a horizontal tension force at the beam end can significantly change the results as will be shown later in this chapter. First, we review the simplest case of a tensionless beam on a Winkler elastic foundation and then a tension is applied at the end of the beam (the point considered as the touchdown point for the SCR).

\subsubsection{Tensionless beam on Winkler elastic foundation}

Neglecting the horizontal force $\mathrm{H}$ at the TDP, the governing differential equation (3-33) can be re-written as:

$\mathrm{EI} \frac{\mathrm{d}^{4} \mathrm{z}}{\mathrm{dx}^{4}}+\mathrm{kz}=0$

Figure 3-11 and Figure 3-12 show this new condition:

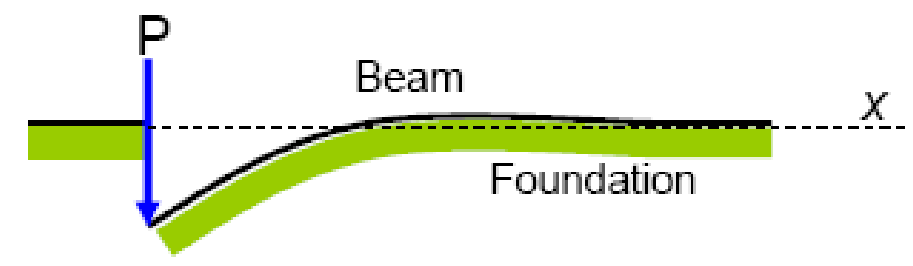

Figure 3-11: Tensionless beam on Winkler foundation under point load

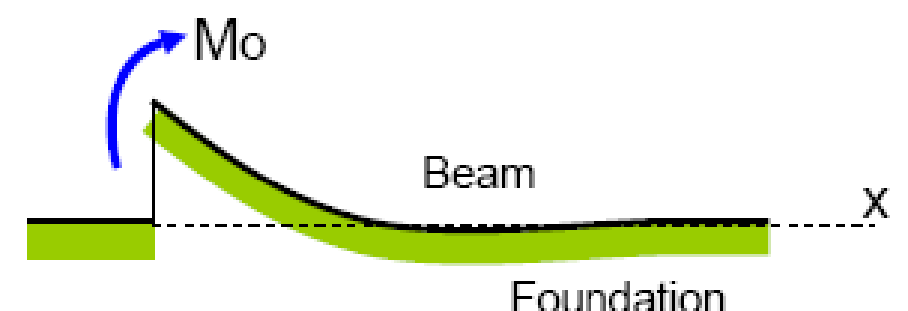

Figure 3-12: Tensionless beam on Winkler foundation under concentrated moment

Hetenyi's solutions for the combined case of an applied point load, $\mathrm{P}$, and concentrated moment, $\mathrm{M}_{0}$ are given below: 


$$
\begin{aligned}
& \mathrm{z}_{\mathrm{X}}=\frac{\mathrm{P}}{2 \mathrm{EI} \lambda_{\mathrm{H}}^{3}} \mathrm{e}^{-\lambda_{\mathrm{H}} \mathrm{x}} \cos \lambda_{\mathrm{H}} \mathrm{x}-\frac{\mathrm{M}_{0}}{2 \mathrm{EI} \lambda_{\mathrm{H}}^{2}} \mathrm{e}^{-\lambda_{\mathrm{H}} \mathrm{x}}\left(\cos \lambda_{\mathrm{H}} \mathrm{x}-\sin \lambda_{\mathrm{H}} \mathrm{x}\right) \\
& \theta_{\mathrm{x}}=-\frac{\mathrm{P}}{2 \mathrm{EI} \lambda_{\mathrm{H}}{ }^{3}} \mathrm{e}^{-\lambda_{\mathrm{H}} \mathrm{x}}\left(\cos \lambda_{\mathrm{H}} \mathrm{x}+\sin \lambda_{\mathrm{H}} \mathrm{x}\right)+\frac{\mathrm{M}_{0}}{\mathrm{EI} \lambda_{\mathrm{H}}} \mathrm{e}^{-\lambda_{\mathrm{H}} \mathrm{x}} \cos \lambda_{\mathrm{H}} \mathrm{x} \\
& \mathrm{M}_{\mathrm{x}}=-\frac{\mathrm{P}}{\lambda_{\mathrm{H}}} \mathrm{e}^{-\lambda_{\mathrm{H}} \mathrm{x}} \sin \lambda_{\mathrm{H}} \mathrm{x}+\mathrm{M}_{0} \mathrm{e}^{-\lambda_{\mathrm{H}} \mathrm{x}}\left(\sin \lambda_{\mathrm{H}^{\mathrm{x}}}+\cos \lambda_{\mathrm{H}} \mathrm{x}\right) \\
& \mathrm{V}_{\mathrm{x}}=-\mathrm{Pe}^{-\lambda_{\mathrm{H}} \mathrm{x}}\left(\cos \lambda_{\mathrm{H}} \mathrm{x}-\sin \lambda_{\mathrm{H}} \mathrm{x}\right)-2 \mathrm{M}_{0} \mathrm{e}^{-\lambda_{\mathrm{H}} \mathrm{x}} \sin \lambda_{\mathrm{H}} \mathrm{x}
\end{aligned}
$$

where

$\lambda_{\mathrm{H}} \quad$ is the characteristic parameter with units of $\left(\mathrm{m}^{-1}\right)$ given by

$$
\lambda_{\mathrm{H}}=\left(\frac{\mathrm{k}}{4 \mathrm{EI}}\right)^{0.25}
$$

The subscript $H$ refers to Hetenyi's name to distinguish it from the flexural length parameter $\lambda$ used in the boundary layer method, which will be discussed later in this chapter.

For modelling the touchdown zone, the boundary condition described in section 3.2 can be written as:

$$
\mathrm{z}=0 ; \mathrm{M}=\mathrm{M}_{0}=\mathrm{M}_{\mathrm{TDP}} \quad \text { at } \mathrm{x}=0
$$

From equation (3-35) and the boundary conditions of equation (3-40):

$$
\begin{aligned}
& \frac{\mathrm{P}}{2 \mathrm{EI} \lambda_{\mathrm{H}}{ }^{3}}-\frac{\mathrm{M}_{\mathrm{TDP}}}{2 \mathrm{EI} \lambda_{\mathrm{H}}^{2}}=0 \quad \text { hence } \\
& \mathrm{P}=-\lambda_{\mathrm{H}} \mathrm{M}_{\mathrm{TDP}}=-\sqrt[4]{\frac{\mathrm{k}}{4 \mathrm{EI}}} \mathrm{M}_{\mathrm{TDP}}
\end{aligned}
$$

Substituting equation (3-42) into equations (3-35) to (3-46) gives the response to the right of the TDP $(x \leq 0$ from Figure $3-2)$ as: 


$$
\begin{aligned}
& \mathrm{z}_{\mathrm{x}}=-\frac{\mathrm{M}_{\mathrm{TDP}}}{2 \mathrm{EI} \lambda_{\mathrm{H}}^{2}} \mathrm{e}^{-\lambda_{\mathrm{H}} \mathrm{x}} \sin \lambda_{\mathrm{H}} \mathrm{x} \\
& \theta_{\mathrm{x}}=\frac{\mathrm{M}_{\mathrm{TDP}}}{2 \mathrm{EI} \lambda_{\mathrm{H}}} \mathrm{e}^{-\lambda_{\mathrm{H}} \mathrm{x}}\left(\sin \lambda_{\mathrm{H}} \mathrm{x}-\cos \lambda_{\mathrm{H}} \mathrm{x}\right) \\
& \mathrm{M}_{\mathrm{x}}=\mathrm{M}_{\mathrm{TDP}} \mathrm{e}^{-\lambda_{\mathrm{H}} \mathrm{x}} \cos \lambda_{\mathrm{H}} \mathrm{x} \\
& \mathrm{V}_{\mathrm{x}}=-\lambda_{\mathrm{H}} \mathrm{M}_{\mathrm{TDP}} \mathrm{e}^{-\lambda_{\mathrm{H}} \mathrm{x}}\left(\sin \lambda_{\mathrm{H}} \mathrm{x}+\cos \lambda_{\mathrm{H}} \mathrm{x}\right)
\end{aligned}
$$

Figure 3-13 to Figure 3-15 show the seabed profile, bending moment and shear force distribution in the touchdown zone (TDZ) beyond the TDP towards the anchor end, as given by equations (3-43) to (3-46) for different values of seabed stiffness. These figures use the SCR parameters given in Table 3-1, with a seabed stiffness range of 1 to $10000 \mathrm{kPa}$. It is worth emphasising that this is a direct solution, so no iteration is needed to solve the shape and stress distributions. Comparison of Figure 3-13 at the TDP $(\mathrm{x}=0)$ with the basic assumptions of the catenary equations which gives zero gradient $(\theta=0)$ at TDP shows an obvious discontinuity of the gradient $(\theta)$ at the touchdown point. The same is true of the shear force as well. Comparison of Figure 3-14 and Figure 3-7 illustrates the discontinuity of the shear force between the beam and the catenary part. Such significant discontinuities in the shear force $(V)$ and the gradient ( $\theta)$ at TDP are basically the main disadvantage of the beam on Winkler's elastic foundation and make the method impractical for accurate assessment of the TDP. This aspect will be explored in more detail within a later section of the current chapter by combining the results of beam and catenary equations.

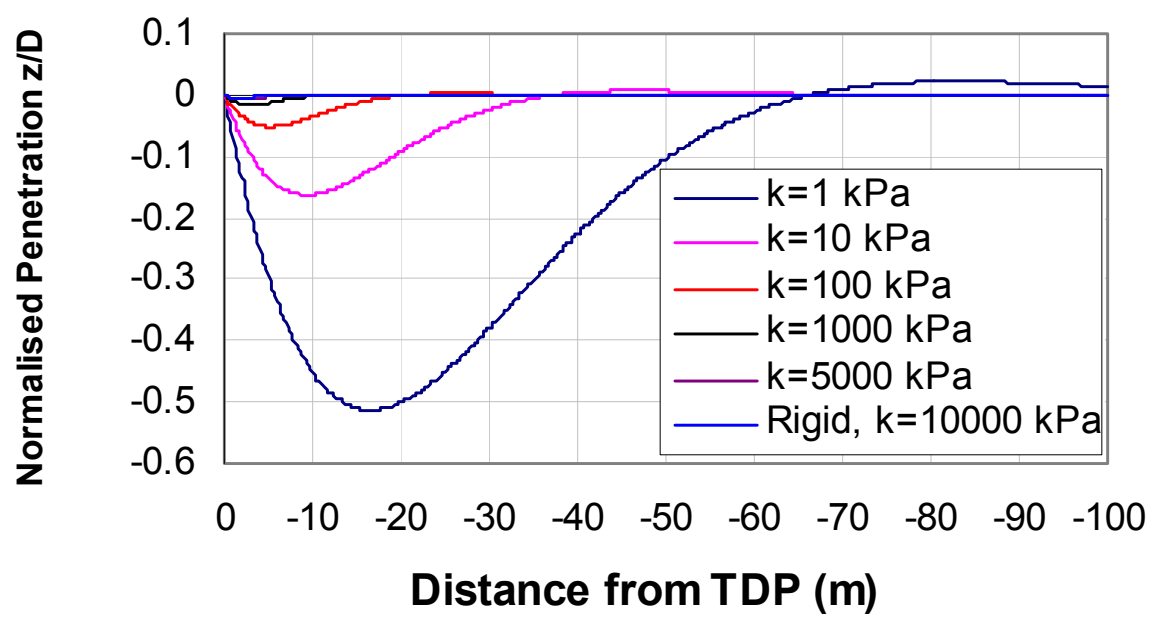

Figure 3-13: SCR profile in TDZ for tensionless beam on Winkler foundation 


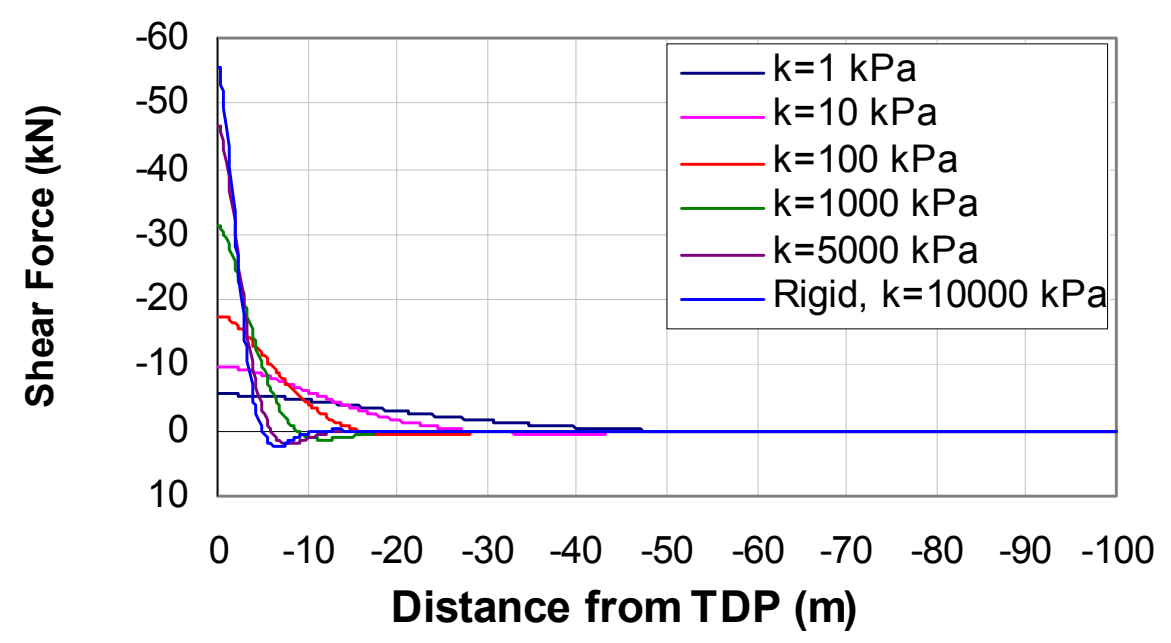

Figure 3-14: SCR shear force in TDZ for tensionless beam on Winkler foundation

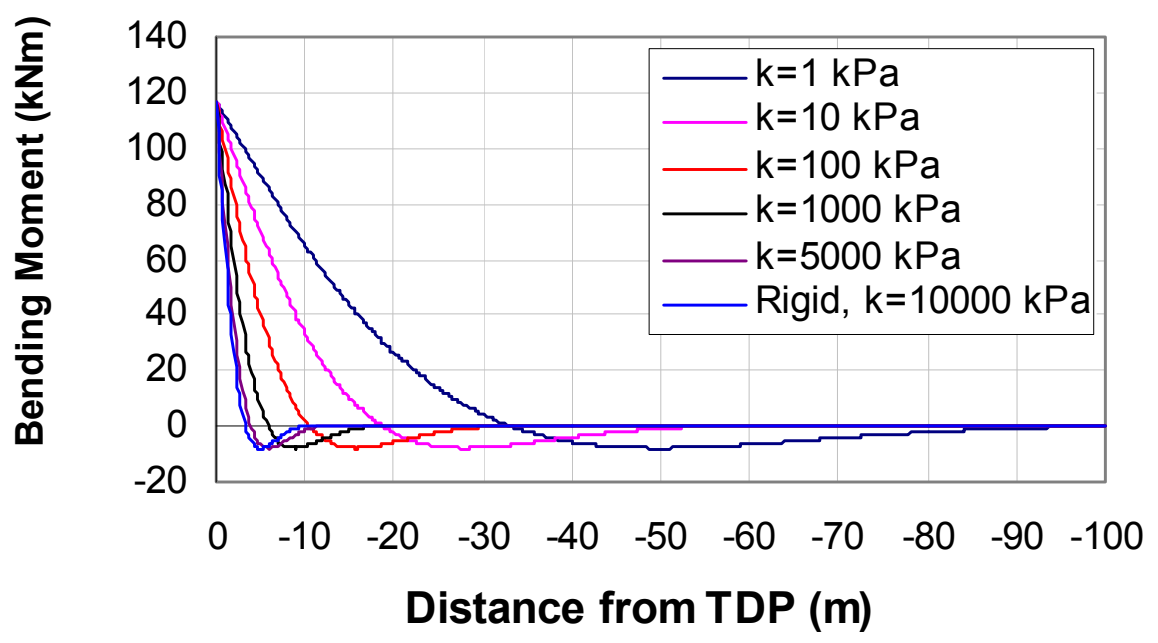

Figure 3-15: SCR bending moment in TDZ for tensionless beam on Winkler foundation

The SCR profile in the touchdown zone is shaped like a ladle, Figure 3-13. The maximum penetration depth is reached at a particular distance from the TDP as described by equations (3-43) to (3-46). The results illustrated in Figure 3-14 and Figure 3-15 show that, within the touchdown zone, both shear force and bending moment change from their maximum values in the catenary zone to a minimum value. The bending moment reaches its minor (negative) peak in reverse curvature at the point where the shear force becomes zero. Then both of them gradually decay to zero in the remaining section of the SCR laid on the seabed. Equations (3-43) to (3-46) can be used to find key parameters of the SCR geometry on the seabed as illustrated in Figure 3-16: 


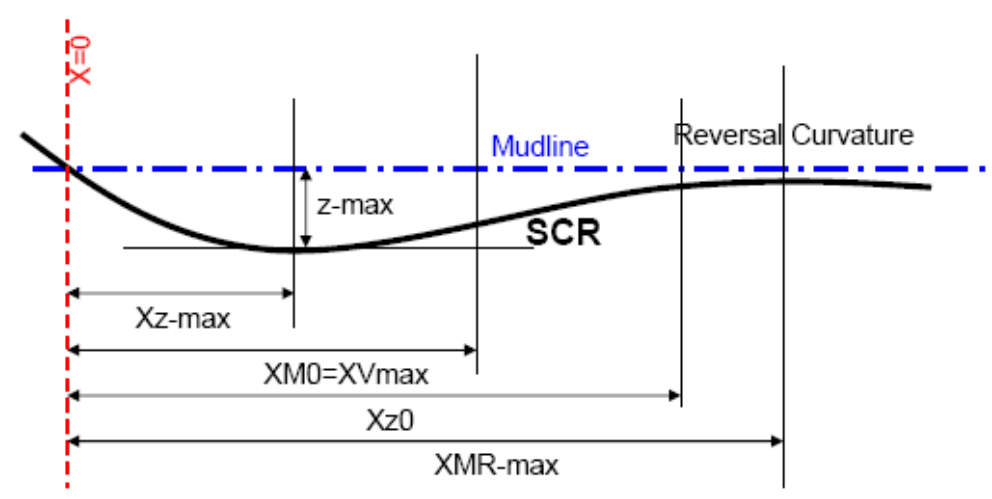

Figure 3-16 : SCR geometry at touchdown zone

$\mathrm{x}_{\mathrm{z}-\max }=\frac{\pi}{4 \lambda_{\mathrm{H}}}$

$\mathrm{x}_{\mathrm{z} 0}=\frac{\pi}{\lambda_{\mathrm{H}}}$

$\mathrm{Z}_{\max }=\frac{\mathrm{e}^{\frac{-\pi}{4}}}{2 \sqrt{2} \mathrm{EI} \lambda_{\mathrm{H}}{ }^{2}} \mathrm{M}_{\mathrm{TDP}}=0.161 \frac{\mathrm{M}_{\mathrm{TDP}}}{\mathrm{EI} \lambda_{\mathrm{H}}{ }^{2}}$

$\mathrm{X}_{\mathrm{Mo}}=\mathrm{X}_{\mathrm{V} \max }=\frac{\pi}{2 \lambda_{\mathrm{H}}}$

$\mathrm{X}_{\mathrm{MR}-\mathrm{Max}}=\frac{3 \pi}{4 \lambda_{\mathrm{H}}}$

$\mathrm{M}_{\mathrm{R}}=-\frac{\mathrm{e}^{-\frac{3 \pi}{4}}}{\sqrt{2}} \mathrm{M}_{\mathrm{TDP}}=-0.134 \mathrm{M}_{\mathrm{TDP}}$

where

$\mathrm{x}_{\mathrm{M} 0} \quad$ is the distance of the maximum shear force from the TDP

$\mathrm{X}_{\text {MR-Max }}$ is the distance of the maximum reverse curvature from the TDP

$\mathrm{M}_{\mathrm{R}} \quad$ is the bending moment at the point of maximum reverse curvature

\subsubsection{Tension beam on Winkler elastic foundation}

The simplified tensionless beam on Winkler elastic foundation can be used for simple calculations, particularly in the case where the horizontal force is low by comparison with the seabed stiffness (strictly, H/EI is low by comparison with $\lambda_{\mathrm{H}}{ }^{2}$, as detailed later), but for more accurate modelling the tension force at the TDP should be 
implemented in the analysis. As for the last model, a point load and concentrated bending moment are considered to act at the free end of SCR, as shown in Figure 3-17 and Figure 3-18.

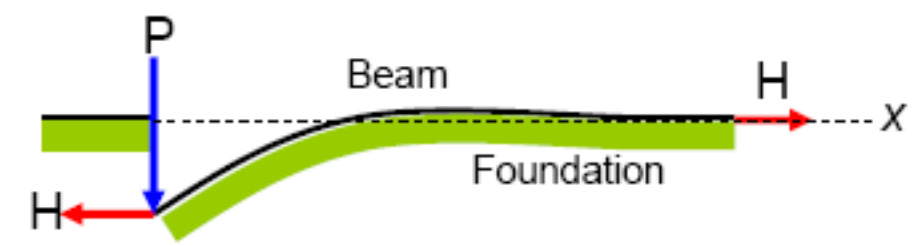

Figure 3-17: Tension beam on Winkler foundation under point load

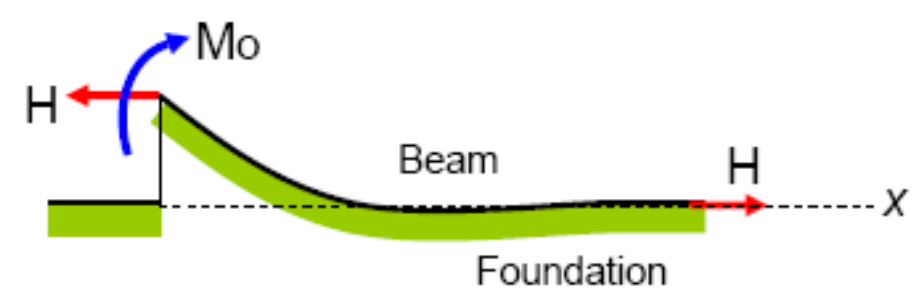

Figure 3-18: Tension beam on Winkler foundation under concentrated moment

Hetenyi (1946) gives the follow solution for this problem:

$$
\begin{aligned}
& \mathrm{z}_{\mathrm{x}}=\frac{1}{\beta} \frac{\mathrm{e}^{-\alpha \mathrm{x}}}{3 \alpha^{2}-\beta^{2}}\left\{\begin{array}{l}
\mathrm{P} \frac{2 \lambda_{\mathrm{H}}^{2}}{\mathrm{k}}\left[2 \alpha \beta \cos \beta \mathrm{x}+\left(\alpha^{2}-\beta^{2}\right) \sin \beta \mathrm{x}\right] \\
-\frac{\mathrm{M}_{0}}{\mathrm{EI}}[\beta \cos \beta \mathrm{x}-\alpha \sin \beta \mathrm{x}]
\end{array}\right\} \\
& \theta_{\mathrm{x}}=\frac{1}{\beta} \frac{\mathrm{e}^{-\alpha \mathrm{x}}}{3 \alpha^{2}-\beta^{2}}\left\{\begin{array}{l}
-\frac{\mathrm{P}}{\mathrm{EI}}[\beta \cos \beta \mathrm{x}+\alpha \sin \beta \mathrm{x}] \\
+\frac{\mathrm{M}_{0}}{\mathrm{EI}}\left[2 \alpha \beta \cos \beta \mathrm{x}-\left(\alpha^{2}-\beta^{2}\right) \sin \beta \mathrm{x}\right]
\end{array}\right] \\
& \mathrm{M}_{\mathrm{x}}=\frac{1}{\beta} \frac{\mathrm{e}^{-\alpha \mathrm{x}}}{3 \alpha^{2}-\beta^{2}}\left\{\begin{array}{l}
-2 \mathrm{P} \lambda_{\mathrm{H}}{ }^{2} \sin \beta \mathrm{x} \\
+\mathrm{M}_{0}\left[\left(3 \alpha^{2}-\beta^{2}\right) \beta \cos \beta \mathrm{x}-\left(\alpha^{2}-3 \beta^{2}\right) \alpha \sin \beta \mathrm{x}\right]
\end{array}\right\} \\
& \left.\mathrm{V}_{\mathrm{x}}=\frac{1}{\beta} \frac{\mathrm{e}^{-\alpha \mathrm{x}}}{3 \alpha^{2}-\beta^{2}}\left\{\begin{array}{l}
-\mathrm{P}\left[\left(3 \alpha^{2}-\beta^{2}\right) \beta \cos \beta \mathrm{x}-\left(3 \beta^{2}-\alpha^{2}\right) \alpha \sin \beta \mathrm{x}\right]+ \\
\mathrm{M}_{0}\left[-4\left(\alpha^{2}-\beta^{2}\right) \alpha \beta \cos \beta \mathrm{x}+\left(\alpha^{4}-6 \alpha^{2} \beta^{2}+\beta^{4}\right) \sin \beta \mathrm{x}\right.
\end{array}\right]\right\}+P \theta_{\mathrm{x}}
\end{aligned}
$$

The parameters $\alpha$ and $\beta$ are defined as follows, depending on the dominancy of either bending stiffness or tension: 


$$
\begin{array}{lll}
\text { for } \mathrm{H}<2 \sqrt{\mathrm{kEI}} & \alpha=\sqrt{\lambda_{\mathrm{H}}{ }^{2}+\frac{\mathrm{H}}{4 \mathrm{EI}}} \quad \beta=\sqrt{\lambda_{\mathrm{H}}{ }^{2}-\frac{\mathrm{H}}{4 \mathrm{EI}}} \\
\text { for } \mathrm{H}=2 \sqrt{\mathrm{kEI}} & \alpha=\sqrt{\frac{\mathrm{H}}{4 \mathrm{EI}}} \quad \beta=0 \\
\text { for } \mathrm{H}>2 \sqrt{\mathrm{kEI}} & \alpha=\sqrt{\lambda_{\mathrm{H}}{ }^{2}+\frac{\mathrm{H}}{4 \mathrm{EI}}} \quad \beta=i \bar{\beta}, \bar{\beta}=\sqrt{\frac{\mathrm{H}}{4 \mathrm{EI}}-\lambda_{\mathrm{H}}{ }^{2}} \\
\cos i \bar{\beta} \mathrm{x}=\operatorname{Cosh} \bar{\beta} \mathrm{x} & , \quad \sin \mathrm{i} \bar{\beta} \mathrm{x}=\mathrm{iSinh} \bar{\beta} \mathrm{x}, & \operatorname{Coshi} \bar{\beta} \mathrm{x}=\cos \bar{\beta} \mathrm{x}, \\
\operatorname{Sinhi} \bar{\beta} \mathrm{x}=\mathrm{i} \sin \bar{\beta} \mathrm{x} &
\end{array}
$$

The solution for $\mathrm{H}=2 \sqrt{\mathrm{kEI}}$ can be expressed as a limit of the case $\mathrm{H}<\sqrt{\mathrm{kEI}}$ by substituting into equation (3-57) from which we get:

$\alpha \rightarrow \sqrt{\frac{\mathrm{H}}{4 \mathrm{EI}}}=\mathrm{a}_{1}$ and $\beta \rightarrow 0$

By substitution of these values for $\alpha$ and $\beta$ in the formulations previously derived they can be directly transformed for use where $\mathrm{H}=2 \sqrt{\mathrm{kEI}}$. Then the penetration profile for the concentrated load $(\mathrm{P})$ and moment $\left(\mathrm{M}_{0}\right)$ can be given as:

$$
\begin{aligned}
& z_{x}=\frac{P}{4 k} a_{1} e^{-a_{1} x}\left(1+a_{1} x\right) \\
& z_{x}=\frac{M_{0} a_{1}^{3}}{4 k} e^{-a_{1} x} x
\end{aligned}
$$

Other parameters can be obtained by the same approach.

As before, the boundary condition is given by

$$
\mathrm{z}=0 ; \mathrm{M}=\mathrm{M}_{0}=\mathrm{M}_{\mathrm{TDP}} \quad \text { at } \mathrm{x}=0
$$

Substituting these into equation (3-53) leads to:

$$
\begin{aligned}
& \mathrm{P} \frac{2 \lambda_{\mathrm{H}}{ }^{2}}{\mathrm{k}} 2 \alpha \beta=-\frac{\mathrm{M}_{\mathrm{TDP}}}{\mathrm{EI}} \beta \quad \text { hence } \\
& \mathrm{P}=-\frac{\lambda_{\mathrm{H}}{ }^{2}}{\alpha} \mathrm{M}_{\mathrm{TDP}}
\end{aligned}
$$

Figure 3-19 to Figure 3-21 show the results from equations (3-53) to (3-65) for a range of seabed stiffness giving the seabed profile, bending moment and shear force distribution beyond the TDP towards the anchor end for the same SCR configuration as before (see Table 3-1). 


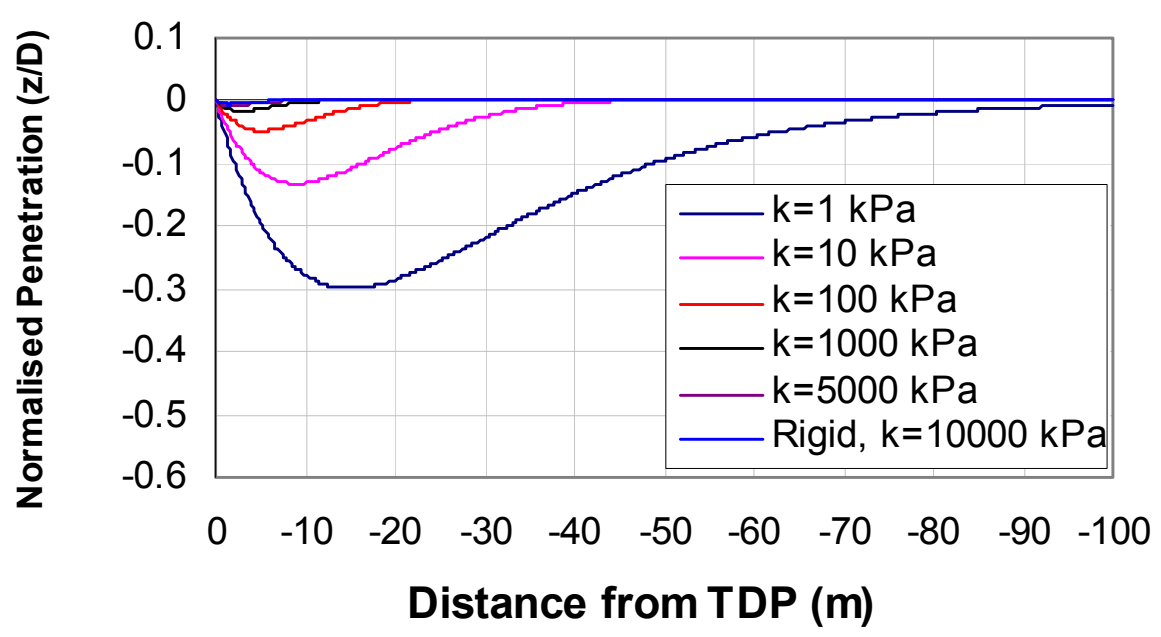

Figure 3-19: Seabed profile using tension beam on Winkler elastic foundation

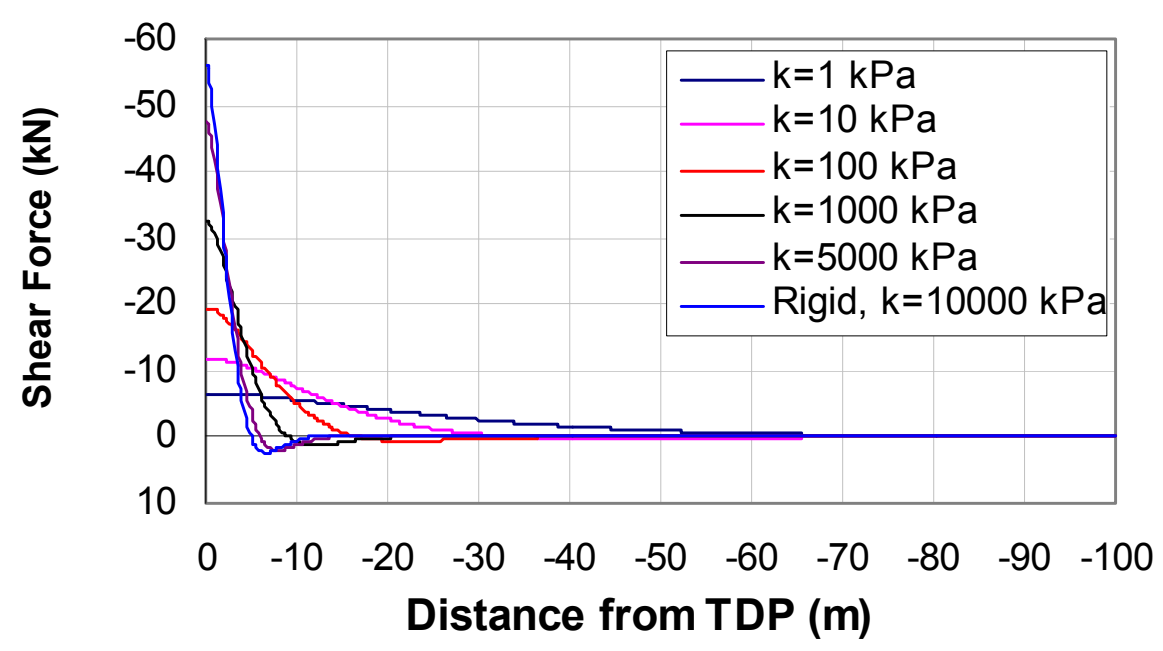

Figure 3-20: TDZ shear force using tension beam on Winkler elastic foundation 


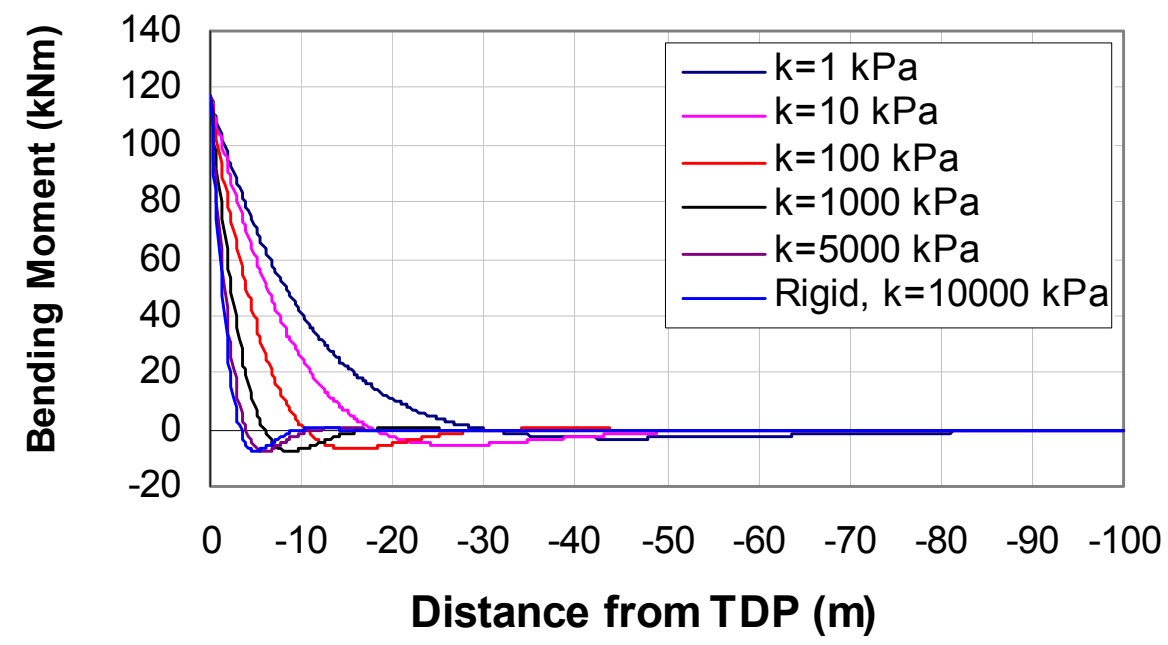

Figure 3-21: TDZ bending moment using tension beam on Winkler elastic foundation

The results for a tensioned and tensionless beam are compared in Figure 3-22 to Figure 3-24 for a seabed with a stiffness of $100 \mathrm{kPa}$. The results show almost exact agreement in respect of the bending moment and slight differences in penetration depth and shear force distribution. As expected, a horizontal tension applied to the TDP slightly reduces the penetration and changes the SCR profile on the seabed,.

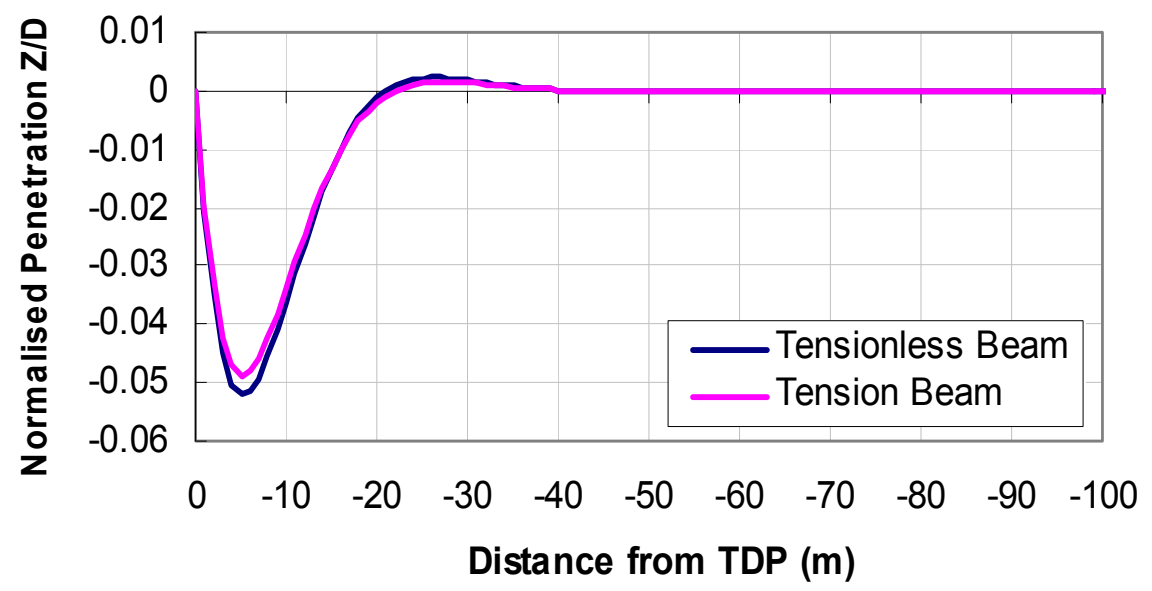

Figure 3-22: SCR penetration profiles in the $\mathrm{TDZ}(\mathrm{k}=100 \mathrm{kPa})$ 


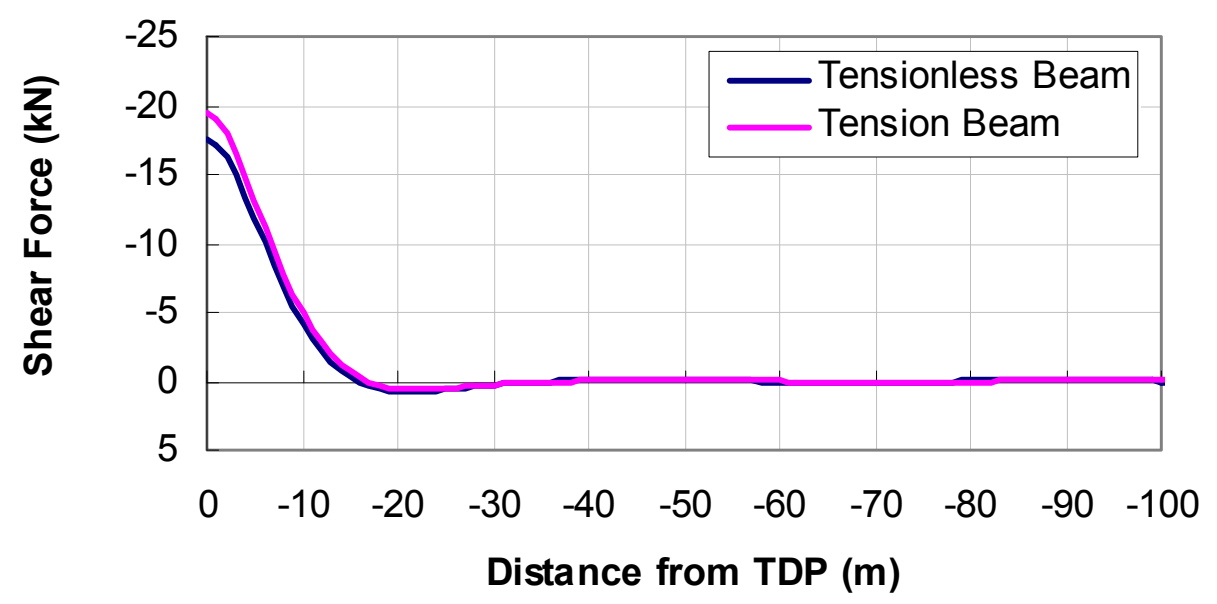

Figure 3-23: Shear force distributions in the $\operatorname{TDZ}(\mathrm{k}=100 \mathrm{kPa})$

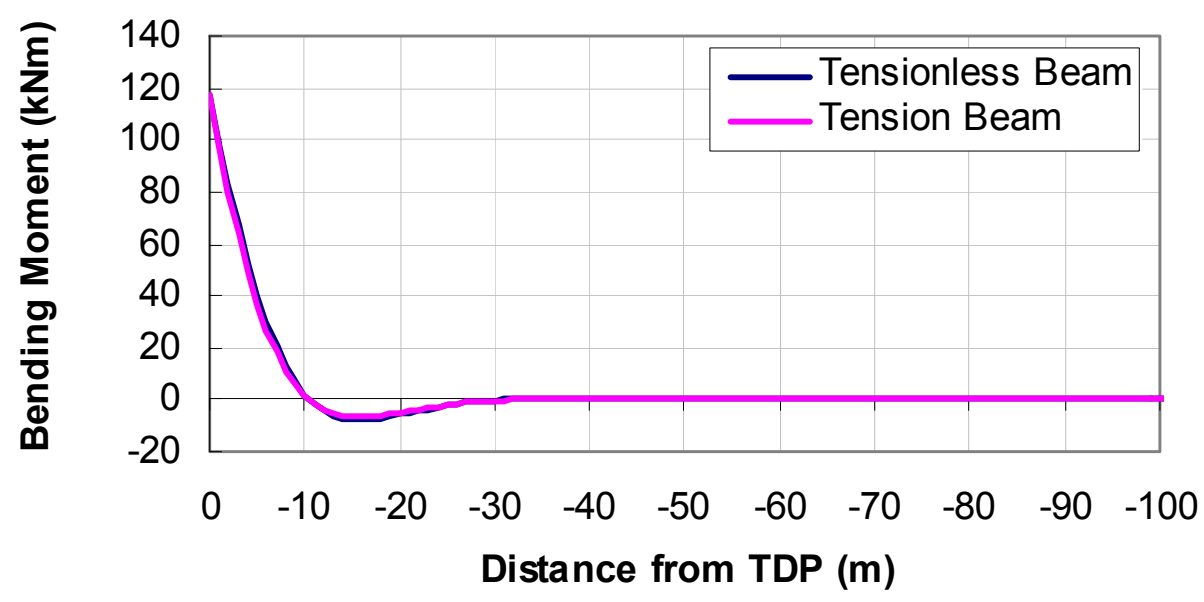

Figure 3-24: Bending moment profiles in the $\operatorname{TDZ}(\mathrm{k}=100 \mathrm{kPa})$

Combining the catenary equations and solutions for a beam on Winkler elastic foundation allows the overall profile and stress distributions along the entire SCR from the hanging part to the part resting on the seabed to be determined. The resulting profiles of shear force and bending moment are illustrated in Figure 3-25 to Figure 3-27 for the case of a seabed stiffness of $100 \mathrm{kPa}$ and 12 degree of lay angle, and compared with results obtained from FE analysis using ABAQUS (full details of which are given in the following chapter). Leaving aside the accuracy of the moment predicted by analytical solutions, the discontinuity of bending moment at the TDP has obviously been removed, although the bending moment at the seabed matches closely the catenary bending moment generated by the catenary equations without the gradual transition. In addition, the maximum value is close to the maximum obtained from the FE analysis, 
although slightly offsets along the SCR (Figure 3-27). Thus it seems the catenary equations are sufficiently accurate for an overall integrity check and sizing of the SCR in initial design stages. Unfortunately, there is still a major drawback in the model, which makes it impractical for fatigue calculations, where the shear force distribution plays a vital role. As commented earlier, and shown in Figure 3-25, there is a discontinuity in the shear force along the SCR at the TDP, and the values calculated from the beam on elastic foundation solutions are far too high. This discontinuity and overestimation of the shear force (and thus the gradient of bending moment) at the most vital part of the SCR, has a major effect in evaluation of the stress changes and consequently the fatigue damage due to motion of the SCR.

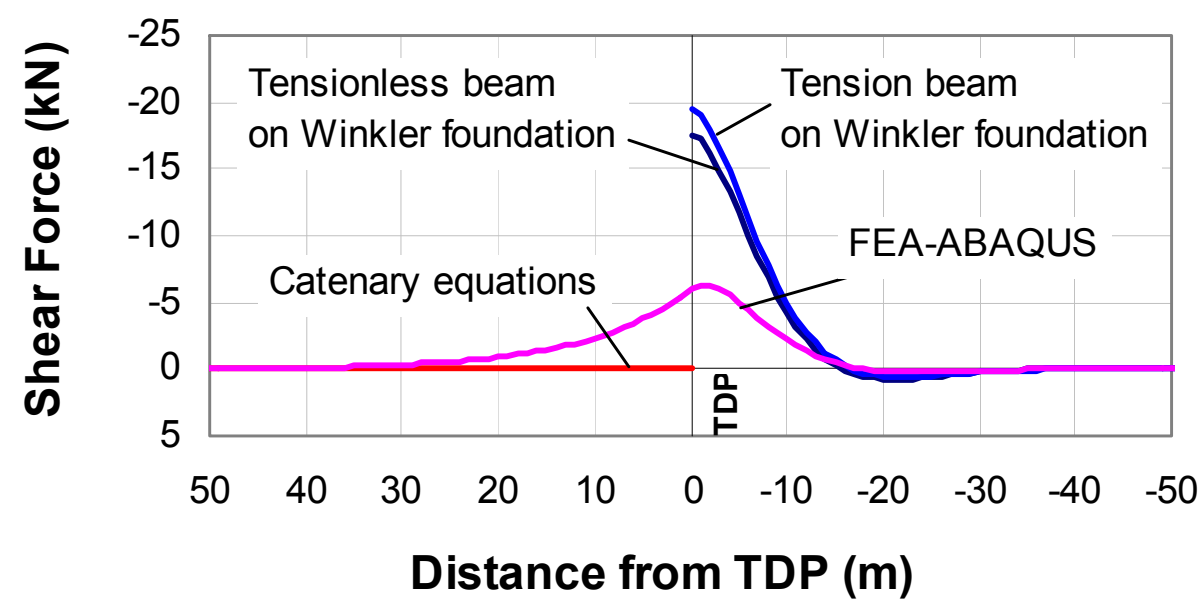

Figure 3-25: Discontinuity of closed form shear force at the TDP $(k=100 \mathrm{kPa})$

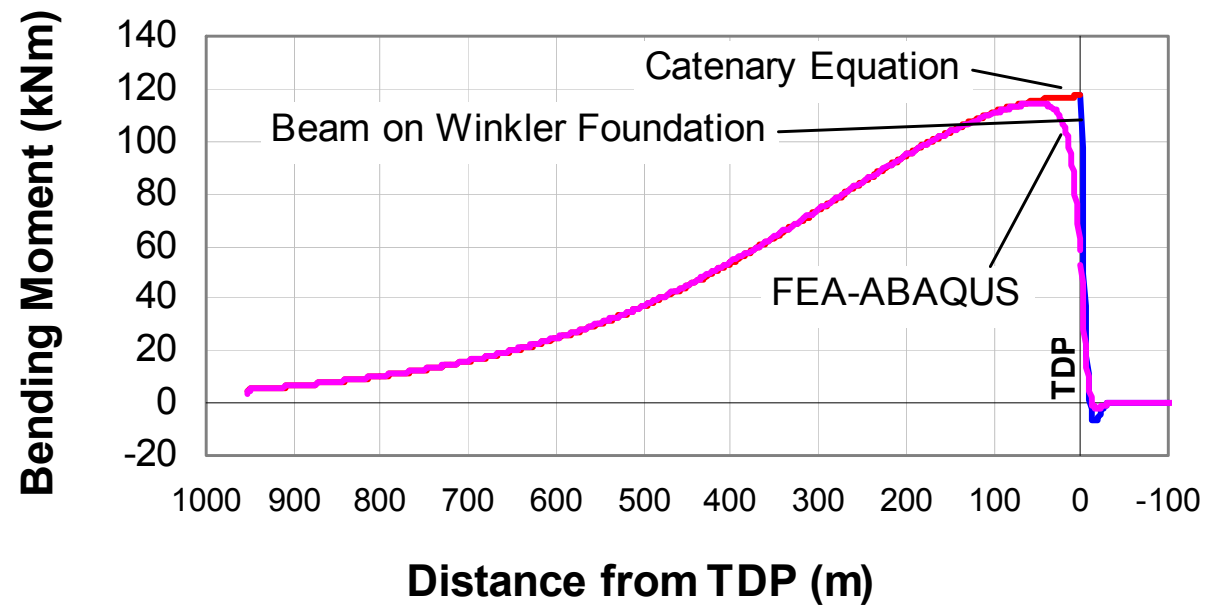

Figure 3-26: Bending moment distribution along SCR $(k=100 \mathrm{kPa})$ 


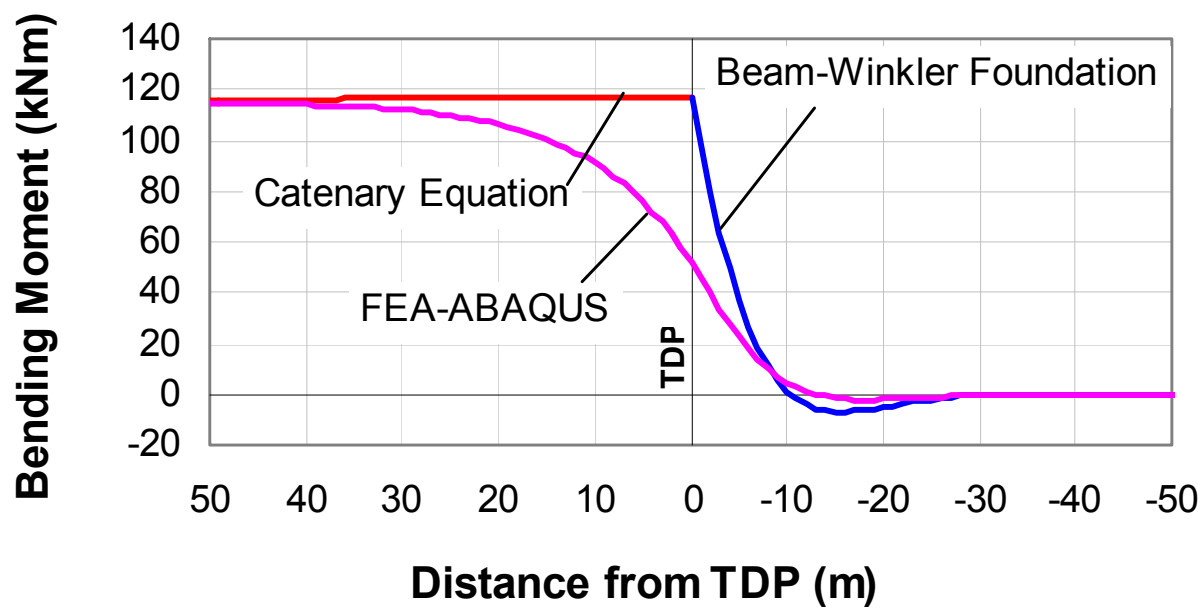

Figure 3-27: Bending moment distribution at the TDP $(k=100 \mathrm{kPa})$

To overcome this remaining drawback, more refined models are needed to capture the gradual transition of shear force at the TDP, ensuring continuity of all significant quantities between the hanging catenary section and the part on the seabed. So-called 'boundary layer' methods have been developed by various authors to solve this problem. In the next section, one of these models will be reviewed as an example and the results will be compared with FE analysis to examine the consistency of boundary layer methods.

\subsubsection{Boundary layer method for seabed interaction modelling}

Removing the discontinuity of shear force and achieving a more accurate response in the touchdown zone need local improvement of the seabed interaction model at the TDP, and defining more extensive boundary conditions there. Various authors have presented boundary layer methods to overcome this problem (Croll 2000; Lenci and Callegari 2005; Palmer et al. 1974; Pesce et al. 1998a). All of these models endeavour to give a precise evaluation of the SCR curvature in the touchdown zone, and to ensure continuity of displacement, gradient and shear force. As an example of these methods, the results of the model presented by Pesce et al. (1998a) are reviewed, comparing results with those from the beam on elastic foundation solutions and from finite element analysis. The system setup defined by Pesce et al. (1998a)is shown in Figure 3-28. 


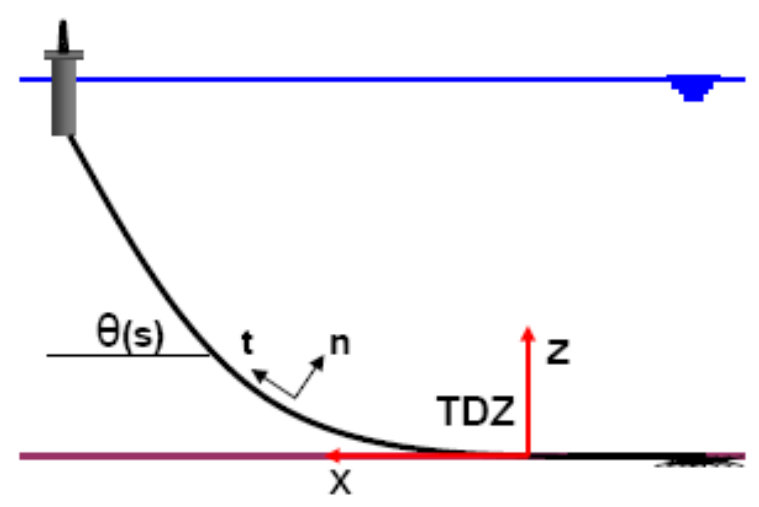

Figure 3-28: SCR configuration, (Pesce et al. 1998a)

Pesce et al. (1998a) applied a standard boundary layer technique to construct local consistent analytical solutions for the static and dynamic curvature problems (Aranha et al. 1997), with the aim to match smoothly the corresponding 'outer' ideal cable solution. A non-dimensional soil rigidity parameter is defined as (Pesce et al. 1998a):

$$
\mathrm{K}=\mathrm{k} \lambda^{4} / \mathrm{EI}=\mathrm{k} \lambda^{2} / \mathrm{H}=\mathrm{kEI} / \mathrm{H}^{2}
$$

where

$\mathrm{k} \quad$ is the soil rigidity per unit area

E is the SCR Young's Modulus

I is the SCR second moment of area

$\mathrm{H} \quad$ is the horizontal tension force at the TDP

$\lambda$ is a flexural length parameter that provides the boundary layer length scale, playing a significant role in local solution and being properly interpreted as the distance between the actual and ideal cable TDP; it is given by:

$\lambda=\sqrt{\mathrm{EI} / \mathrm{H}}$

Caution is needed in respect of the dimensions of $\lambda$, which is a length (m) and differs from the characteristic parameter $\lambda_{\mathrm{H}}$ defined by Hetenyi (1946) in equation (3-39), which has units of the inverse of length $\left(\mathrm{m}^{-1}\right)$.

For the suspended part of the SCR, Clebsch-Love equations (Clebsch 1883; Love 1892) have been written as: 


$$
\begin{aligned}
& \frac{d T}{d s}-V \frac{d \theta}{d s}+f_{t}=0 \\
& \frac{d V}{d s}+T \frac{d \theta}{d s}+f_{n}=0 \\
& E I \frac{d^{2} \theta}{d s^{2}}+V=0
\end{aligned}
$$

where $\mathrm{V}$ is the shear force, EI is the bending stiffness, assumed invariant, and

$$
\begin{aligned}
& \mathrm{f}_{\mathrm{t}}=-\mathrm{m}_{\mathrm{s}} \mathrm{g} \sin \theta+\mathrm{h}_{\mathrm{t}}(\mathrm{s}) \\
& \mathrm{f}_{\mathrm{n}}=-\mathrm{m}_{\mathrm{s}} \mathrm{g} \cos \theta+\mathrm{h}_{\mathrm{n}}(\mathrm{s})
\end{aligned}
$$

are the external forces in the tangential and normal directions, being $\mathrm{m}_{\mathrm{s}} \mathrm{g}$ the immersed weight and $h_{t}(\theta(s)), h_{n}(\theta(s))$ the components of steady hydrodynamic forces, all per unit length.

Combining the equations (3-68) to (3-72) gives a second order non-linear differential equation in the variable $\theta(\mathrm{s})$, which captures the effects of hydrodynamic forces on the SCR. The parameters s and $\theta$ are the arc length of the ideal SCR and the angle from the horizontal of the tangential direction at any given point of the ideal SCR respectively. This equation is written as:

$$
\begin{aligned}
& \text { EI } \frac{d^{2} \theta}{d s^{2}} \sec \theta+m_{s} s-\int_{s}\left(h_{n} \sec \theta+\sec ^{2} \theta\left(\frac{d \theta}{d s}\right) \int_{s}\left(h_{n} \sin \theta-h_{t} \cos \theta\right) d \xi\right) d s \\
& =H \tan \theta-V_{0}
\end{aligned}
$$

In this equation, $\mathrm{V}_{0}$ and $\mathrm{H}$ are respectively the shear force and tension at the point where $\theta=0$ and $\xi=\mathrm{x} / \lambda$.

This is a classical singular perturbation problem of the boundary layer type. The solution has been given by neglecting the flexural rigidity or dropping the first term in Equation (3-73), (Kevorkian and Cole 1981), which leads to a discontinuity in the static curvature at the TDP which in turn is considered as a drawback of the catenary equations.

If not only flexural rigidity is neglected but also hydrodynamic forces are supposed to be absent, equation (3-73) reduces to the familiar catenary equation:

$$
\frac{\mathrm{m}_{\mathrm{s}} \mathrm{s}}{\mathrm{H}}=\tan \theta
$$

Pesce et al. (1998a) take into account the effect of flexural rigidity by keeping the first term in the governing equation (3-73) and defining $\mathrm{s}=\mathrm{s}_{\mathrm{f}}$ as the actual position of the TDP to locally improve the sudden variation of curvature within the local length scale 
defined by $\lambda$. They tried to simplify the governing equation (3-73) by appropriate assumptions and re-presented it for a linear elastic seabed in the following form:

$$
\frac{\mathrm{d}^{4} \mathrm{z}}{\mathrm{dx}^{4}}-\frac{1}{\lambda^{2}} \frac{\mathrm{d}^{2} \mathrm{z}}{\mathrm{dx}^{2}}+\frac{\mathrm{k}}{\mathrm{EI}} \mathrm{z}=-\frac{\mathrm{m}_{\mathrm{s}}}{\mathrm{EI}}
$$

or in non-dimensional form with $\xi=x / \lambda ; \eta=z / \lambda$, by

$$
\eta^{\mathrm{IV}}-\eta^{\prime \prime}+\mathrm{K} \eta=-\frac{\mathrm{m}_{\mathrm{s}} \lambda}{\mathrm{H}}
$$

where the non-dimensional soil rigidity parameter, $\mathrm{K}$, has been defined in equation (3-66).

This solution gives a typical oscillatory behaviour for the curvature on the supported part of the SCR, matching smoothly the catenary solution along the suspended part. Using the governing equations above, Pesce et al. (1998a) gave the following equations for the shear force distribution in the TDZ:

$$
\begin{aligned}
& \mathrm{V}=\frac{\mathrm{m}_{\mathrm{s}} \mathrm{g} \lambda \cdot \mathrm{K}^{0.25}}{\sqrt{2}+\mathrm{K}^{0.25}} \exp \left(\frac{\mathrm{K}^{0.25}}{\sqrt{2}}\left(\xi-\xi_{\mathrm{f}}\right)\right) \times\left\{\cos \left(\frac{\mathrm{K}^{0.25}}{\sqrt{2}}\left(\xi-\xi_{\mathrm{f}}\right)\right)-\sin \left(\frac{\mathrm{K}^{0.25}}{\sqrt{2}}\left(\xi-\xi_{\mathrm{f}}\right)\right)\right\} \\
& \text { if } \xi \leq \xi_{\mathrm{f}}
\end{aligned}
$$

or

$$
\mathrm{V}=\frac{\mathrm{m}_{\mathrm{s}} \mathrm{g} \lambda \cdot \mathrm{K}^{0.25}}{\sqrt{2}+\mathrm{K}^{0.25}} \exp \left(-\left(\xi-\xi_{\mathrm{f}}\right)\right)
$$

if $\xi \geq \xi_{\mathrm{f}}$

where $\xi_{\mathrm{f}}$ is the position of the TDP, defined as:

$$
\xi_{\mathrm{f}}=\frac{1}{\sqrt{2}+\mathrm{K}^{0.25}}\left(\frac{1}{\mathrm{~K}^{0.25}}-\mathrm{K}^{0.25}\right)
$$

The maximum value of shear force can be obtained by differentiation of equations $(3-77)$ and (3-78) and occurs, as expected, at $\xi=\xi_{\mathrm{f}}$; the magnitude is expressed as:

$$
\mathrm{V}_{\max }=\frac{\mathrm{m}_{\mathrm{s}} \mathrm{g} \lambda \mathrm{K}^{0.25}}{\sqrt{2}+\mathrm{K}^{0.25}}
$$

Figure 3-29 shows profiles of shear force through the TDZ, obtained from this boundary layer solution, for a range of non-dimensional seabed rigidity values from 1 to 10000 for the same SCR configuration as used elsewhere in this chapter. (Note the $\mathrm{K}$ is approximately 0.27 times the numerical value of $\mathrm{k}(\mathrm{kPa})$, for the particular SCR parameters, so the above range corresponds to $\mathrm{k} \sim 4$ to 40000), The results in Figure 3-29 demonstrate removal of the shear force discontinuity at the TDP, and also a shift of the TDP towards the anchor end of the SCR compared with the catenary solution. 


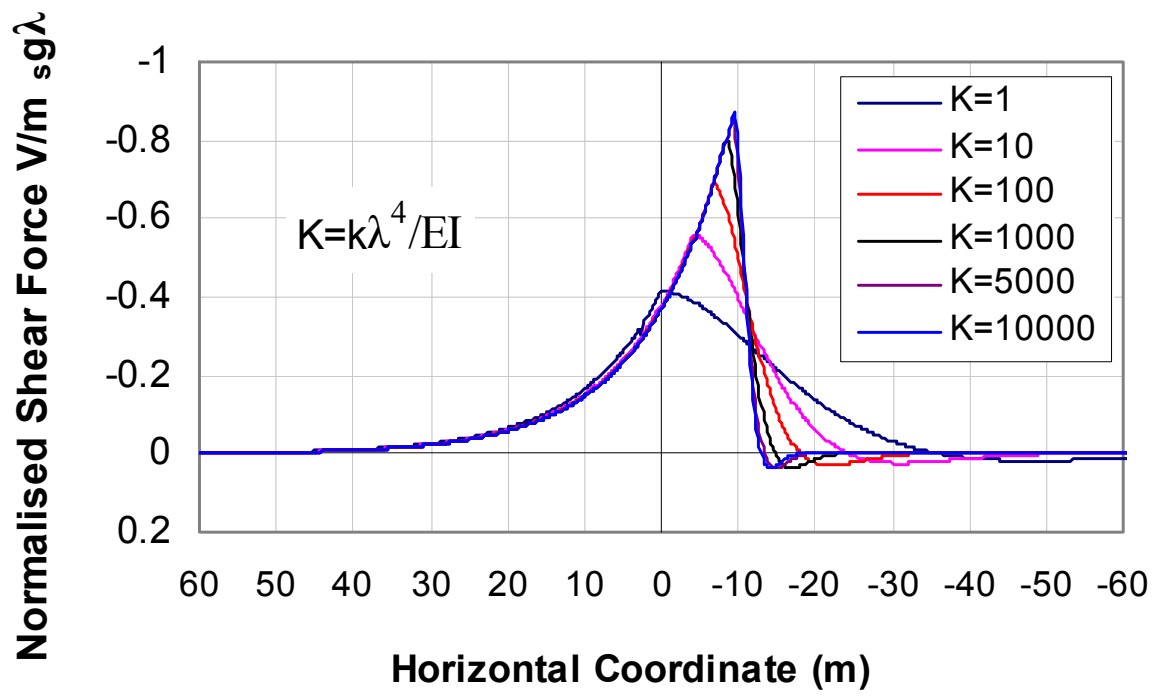

Figure 3-29: Normalised shear force in TDZ, (Pesce et al. 1998a)

A comparison of shear force prediction by the boundary layer method (Pesce et al. 1998a), beam on Winkler foundation and finite element analysis (FEA) for a seabed stiffness of $100 \mathrm{kPa}$ is illustrated in Figure 3-30. The magnitude of the boundary layer shear force shows good agreement with the FEA results with a minor difference in the TDP position.

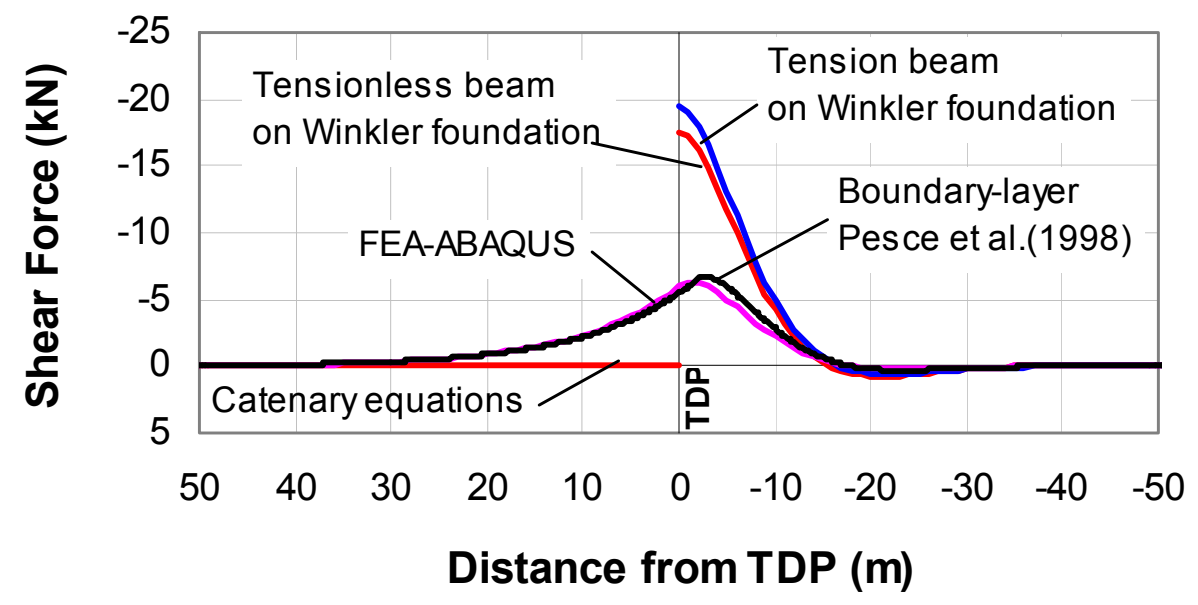

Figure 3-30: Static shear force distribution in $\mathrm{TDZ}, \mathrm{k}=100 \mathrm{kPa}$

The bending moment generated by the boundary layer method has been compared with the results from the beam on Winkler foundation solutions, catenary equations and finite element analysis. The comparison shows an appropriate smooth transition between the seabed and catenary parts, with only slight differences from the FEA results, as shown in Figure 3-31 (bending moments) and Figure 3-32 (maximum von Mises stresses). 


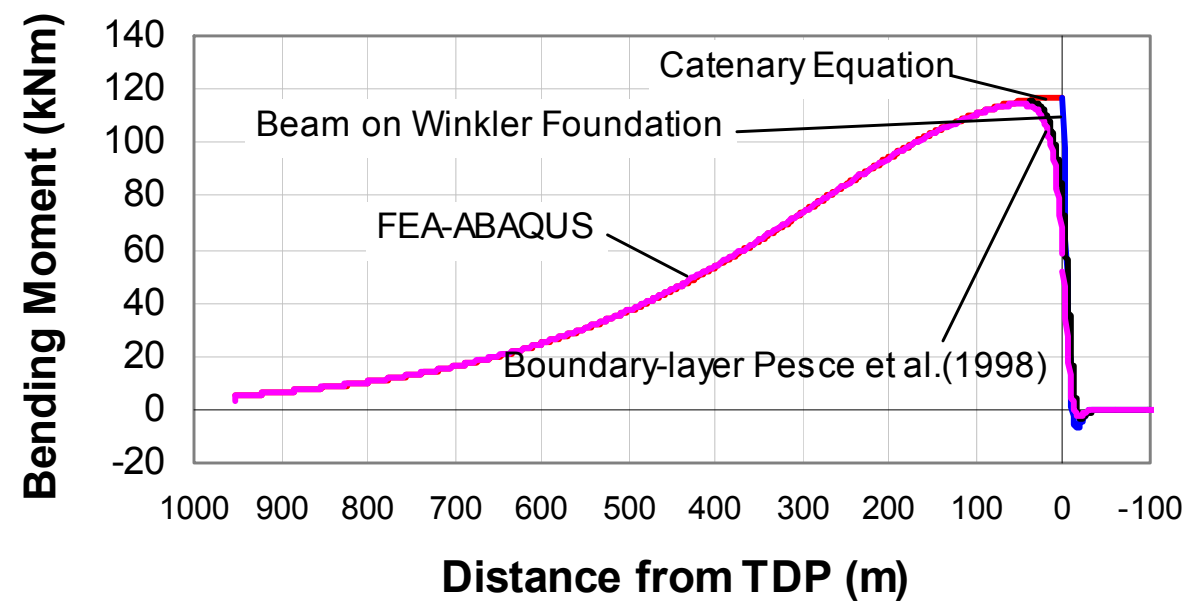

Figure 3-31: Comparison of bending moments along SCR, $k=100 \mathrm{kPa}$

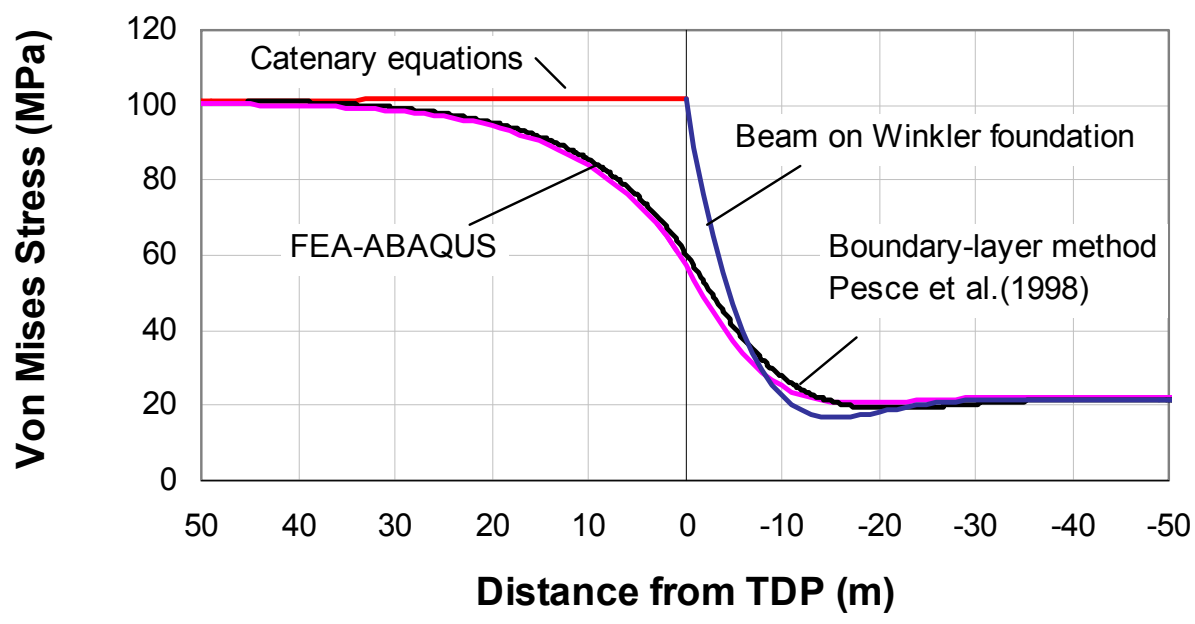

Figure 3-32: Comparison of bending moments at the TDP, $k=100 \mathrm{kPa}$

The results of various seabed interaction models demonstrate the significant effect of seabed stiffness on stress distribution along the SCR particularly in the TDZ. Figure 3-33, compares the maximum shear force in the touchdown zone for a range of seabed stiffness. The results of FEA and the boundary-layer method (Pesce et al. 1998a) show good agreement, but the beam on Winkler foundation, both tensionless and tensioned beam, overestimate the peak shear force by a factor of about 5.5. 


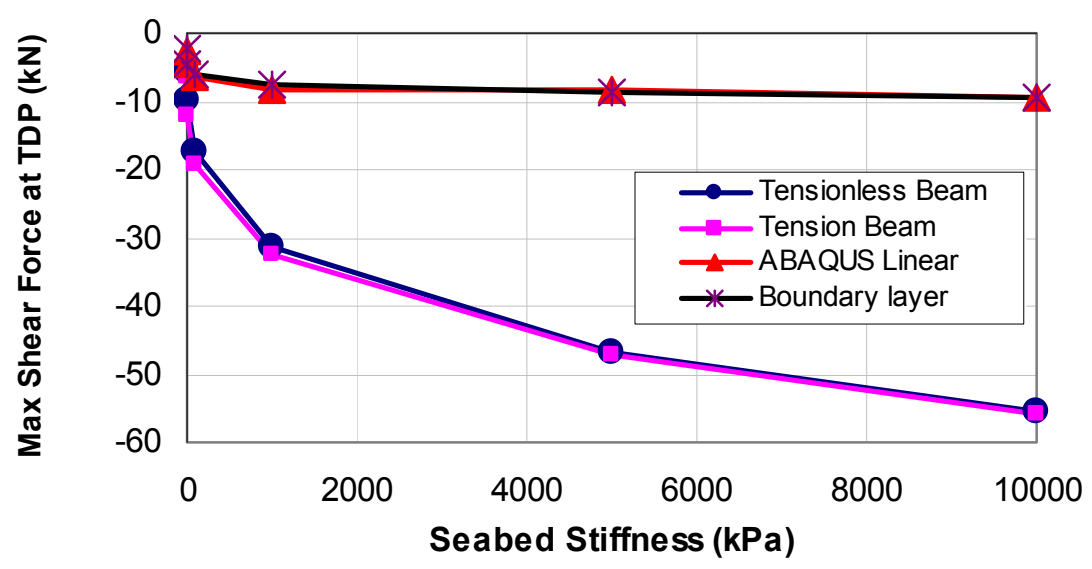

Figure 3-33: Peak shear force at TDZ in different seabed stiffness

\subsubsection{Fatigue damage prediction at TDZ by boundary-layer method}

The von Mises stress range in a perturbation cycle is the basic input to calculate the fatigue damage of SCRs treated as tubular sections. The boundary layer method (BLM) has been examined to evaluate the von Mises stress range and the results have been compared with finite element analysis. For this purpose, the vessel supporting the SCR has been moved horizontally from its initial position to an offset point inside the normal range for a Spar displacement for conditions in deep water Gulf of Mexico. Then the shear force, bending moment, von Mises stress and von Mises stress range have been evaluated and compared with FEA results. The results are presented in Figure 3-34 to Figure 3-36.

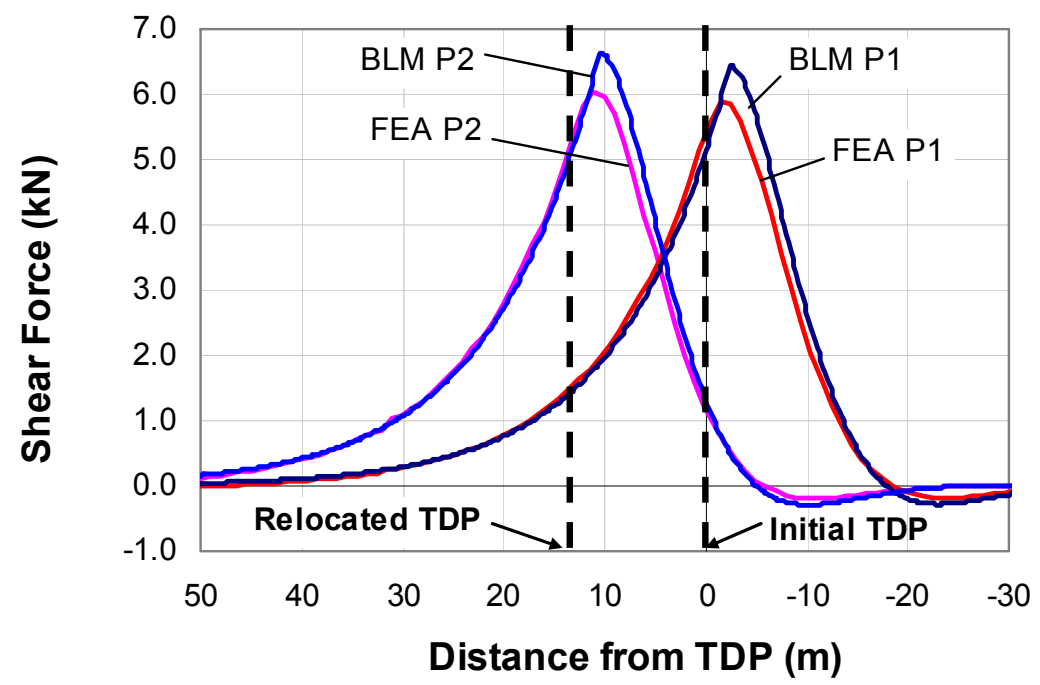

Figure 3-34: Shear force distribution in two different vessel locations, produced by BLM and FEA 


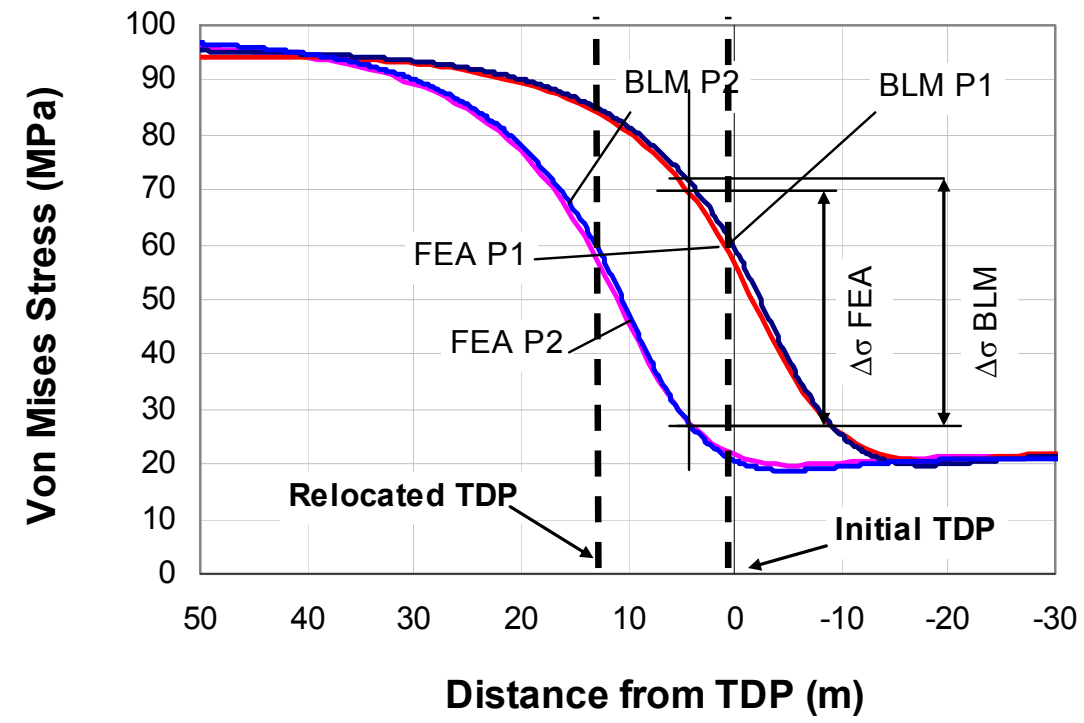

Figure 3-35: Von Mises stress at two different vessel locations, produced by BLM and FEA

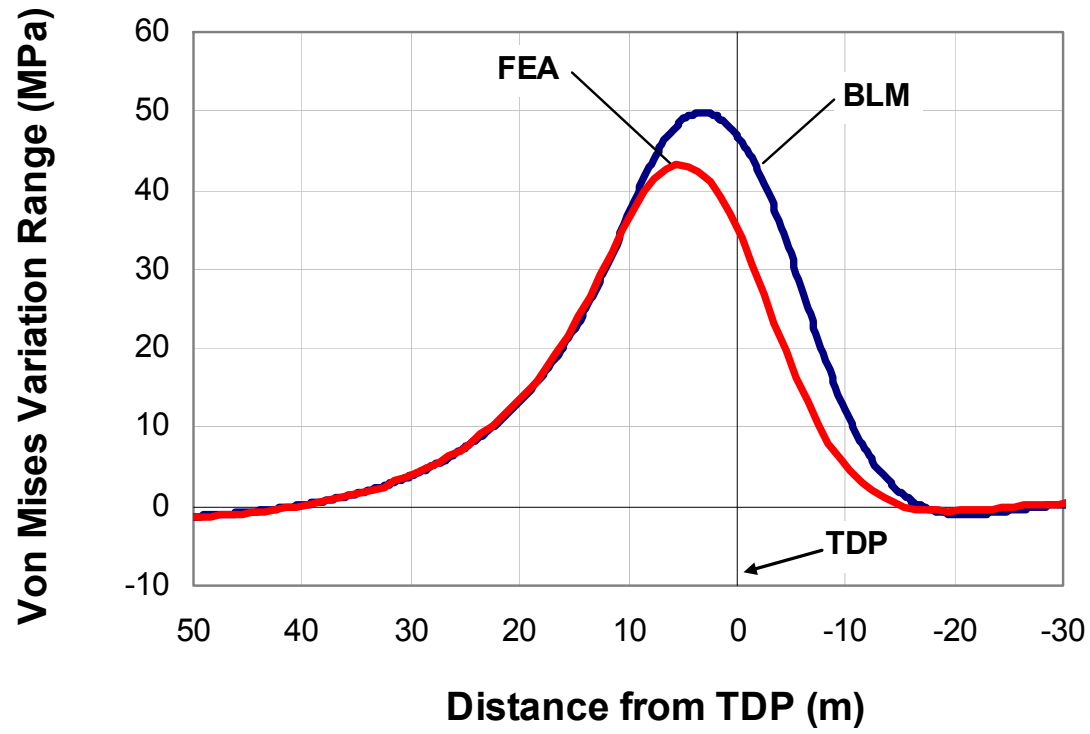

Figure 3-36: Von Mises stress range between two different vessel locations, from BLM and FEA

A simple investigation of the plots above shows the direct relationship between the maximum shear force and von Mises stress variation range and consequently the fatigue damage. Using equation (3-31) and ignoring the small change in horizontal force, the von Mises stress range variation can be written as: 
$\Delta \sigma_{\mathrm{V}}=\sigma_{\mathrm{V} 1}-\sigma_{\mathrm{V} 2}=\frac{\mathrm{M}_{1} \cdot \mathrm{D} / 2}{\mathrm{I}}-\frac{\mathrm{M}_{2} \mathrm{D} / 2}{\mathrm{I}}=\frac{\mathrm{D}}{2 \mathrm{I}} \Delta \mathrm{M}$

where $\mathrm{M} 1$ and $\mathrm{M}_{2}$ are the bending moments in a section of the SCR at the two different vessel positions, $\mathrm{D}$ is the riser diameter and $\mathrm{I}$ is the second moment of area. Replacing the average shear force $\bar{V}$ in equation (3-81) as the gradient of bending moment gives:

$\Delta \sigma_{\mathrm{V}}=\frac{\mathrm{D}}{2 \mathrm{I}} \overline{\mathrm{V}} \Delta \mathrm{x}$

where $\Delta \mathrm{x}$ approximates the displacement of the TDP.

From Figure 3-34, the shear force from the BLM is about $5.5 \mathrm{kN}$ and the TDP relocation is about $13 \mathrm{~m}$. Substituting these values and the riser diameter and the second moment of area in (3-82) gives:

$\Delta \sigma_{\mathrm{V}}=\left(\frac{0.324}{2 \times 0.000226} \times 5.5 \times 13.13\right) / 1000=51.8 \mathrm{MPa}$

The value $51.8(\mathrm{MPa})$ is in very good agreement with Figure 3-36, where the stress range variation from the BLM is about $50(\mathrm{MPa})$. This simple calculation shows the direct relationship of shear force and the von Mises stress (or bending moment) range. On the other hand, equation (3-80) shows the direct relationship of shear force with the non-dimensional seabed stiffness. Hence, the von Mises stress variation arising from movement of the TDP is also strongly influenced by the seabed stiffness. Although $\mathrm{K}$ (and hence, the stiffness, $k$ ) appears to the power of 0.25 in equation (3-80), it will be shown in Chapter 4, that the fatigue damage has direct relationship with the von Mises stress variation to the power of 3 to 4 (Barltrop and Adams 1991). Thus a small change in seabed stiffness can result in considerable impact on fatigue damage. The motivation behind the simple calculation method above is the ability to arrive at an approximate estimate of the fatigue life by considering the shear force, which in turn is governed by the stiffness of the linear elastic seabed. This provides a more direct approach than calculation of the von Mises stress changes through the BLM method.

Figure 3-37 provides illustration of the above point, showing the distribution of normalised von Mises stress for two different positions of the TDP, for a soft and stiff seabed. As illustrated graphically, the normalised von Mises stress range, $\Delta \sigma_{\mathrm{vm}} / f_{\mathrm{y}}$, for a given point of the SCR in the TDZ is much larger for a seabed with high stiffness than for the same point for a softer seabed. Thus as will be further discussed in the following chapters a stiff seabed causes considerably higher fatigue damage than a soft seabed. 


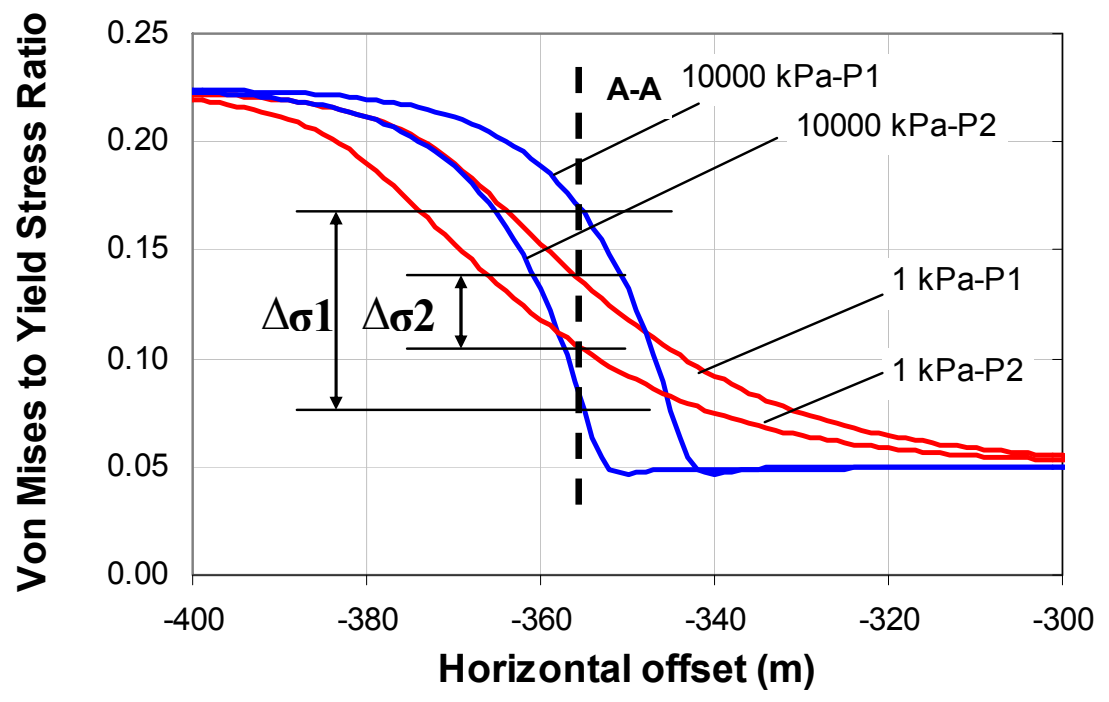

Figure 3-37: Stress range variation of stiff and soft seabed at TDP

As will be discussed in the coming chapters, for a linear elastic seabed, depending on the magnitude of seabed stiffness, the peak fatigue damage occurs in the same location as the peak shear force at a location between the TDP and the position of the maximum bending moment. This is exactly the area of discontinuity encountered in the solutions for a beam on Winkler foundation. Figure 3-38 compares the position of the peak fatigue with the shear force, contact stress, bending moment and catenary shape diagrams for a linear elastic seabed with a stiffness of $100 \mathrm{kPa}$. Although a full examination of the fatigue profile predicted by the boundary layer method requires application of a series of different sea states (and hence movements of the TDP), the simple check on the von Mises stress variation presented above highlights the good agreement of the boundary layer method and FEA results and the potential for the closed form boundary layer solution to give preliminary estimates of fatigue damage. 


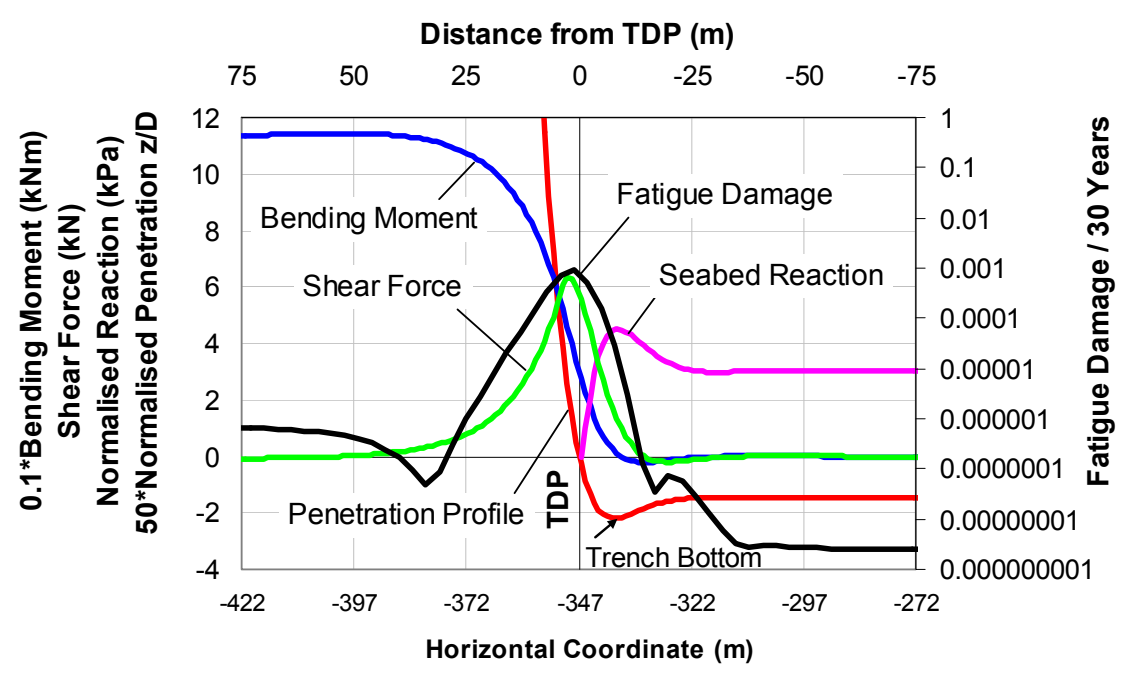

Figure 3-38: Peak fatigue position relative to shear force and other key profiles

\subsection{SUMMARY AND CONCLUSIONS}

Various methodologies for modelling the interaction of an SCR with the seabed, including the tensionless and tensioned beams on a Winkler elastic foundation (Hetenyi 1946) and boundary layer methods (Pesce et al. 1998a), have been examined in combination with catenary equations to evaluate the consistency of these modelling techniques in generating the stress distribution along the SCR in the TDZ. The results have been compared with those from numerical finite element analysis performed using ABAQUS.

Applying only the static catenary equations is equivalent to considering a rigid seabed with zero curvature beyond the touchdown point. The results for a rigid seabed show significant discontinuity in bending moment distribution at the TDP, although the maximum bending moment predicted close to the TDP is reasonably accurate. This discontinuity, and the necessary assumption of a rigid seabed, limits this method to making preliminary calculations of the maximum bending strains in the SCR.

The beam on Winkler elastic foundation does not improve the prediction of the bending moment from the catenary equations, whilst the significant disadvantage of this method is the discontinuity in the shear force at the TDP within the transition area between the catenary part and the supported part of the riser, together with gross overestimation of the maximum shear force at the TDP. The inaccuracy in the shear force, and hence the gradient of bending moment and the resulting variation in von Mises stress as the TDP moves, makes this model unhelpful in calculation of fatigue damage in the TDZ, which is the most challenging issue for the SCR design. Overall, beam on Winkler elastic foundation seems not to be an attractive analytical solution for SCR. 
An example of the boundary layer methods (Pesce et al. 1998a) has been reviewed and comparison of the results with those from FE analysis demonstrates that the approach removes any discontinuity in the shear force and bending moment profiles at the TDP, and gives good agreement in respect of the peak shear force and bending moments. The position of the touchdown point with this method shows a minor offset towards the anchor end of the SCR. The continuity of shear force (and thus bending moment gradient) at the TDP enables fatigue damage calculation in the touchdown zone. Likewise, the variation of von Mises stress produced by the boundary layer method shows good agreement with FEA results in predicting the magnitude and position of the maximum fatigue in the TDZ.

A simple calculation has been presented to demonstrate the direct relationship of fatigue damage and shear force and the rationale has been explained regarding the impact of seabed stiffness on shear force and consequently on fatigue damage. It has been shown, both graphically and algebraically, that a stiffer seabed causes higher fatigue damage in the TDZ than for a soft seabed, because of the higher gradient of bending moment and thus greater von Mises stress variation under cyclic loading. 


\section{CHAPTER 4. FATIGUE PERFORMANCE OF SCRS ON LINEAR ELASTIC SEABED}

\subsection{INTRODUCTION}

The prediction of fatigue damage in the touchdown zone (TDZ) under environmental loads is the most challenging area in design of steel catenary risers (SCRs). The current design codes and standards for SCRs (e.g (API-RP-2RD 1997)) have been developed based on a linear elastic seabed response, due to the relative complexity and uncertainty of the actual non-linear riser-soil interaction. The absence of robust models for the nonlinear response, suitable for incorporating in riser analysis software, is another reason for simplification of riser-seabed interaction as linear elastic, even though it is now widely accepted that the appropriate stiffness depends on the amplitude of the cyclic displacements (Aubeny et al. 2008) and that the stiffness value has a significant effect on the fatigue performance of an SCR.

Although the focus of this research is to investigate the effect of a non-linear seabed on the fatigue of SCRs, it is first necessary to establish how the fatigue damage is affected by the seabed stiffness for a linear seabed response in order to provide a basis for the subsequent assessment of the non-linear model. This chapter quantifies the fatigue performance of SCRs on a linear elastic seabed and the influence of the seabed stiffness on the stress distribution and fatigue damage in the TDZ. It thus provides an introduction to the following chapter, which will study the effect of non-linear riserseabed interaction on fatigue damage. As discussed in Chapter 2, the total fatigue damage is the product of first order, second order and VIV fatigue damages which is analysed dynamically by means of time or frequency domain analyses. Since the focus point of this study is the influence of the seabed interaction on fatigue performance rather than high accuracy evaluation of fatigue damage, the fatigue analyses have been simplified, ignoring the dynamic effects of the SCR by considering only the first order motions of the vessel through quasi-static analyses in a deterministic approach.

\subsection{THE BASIC CONCEPT OF FATIGUE}

The gradual damage and localised failure of a material under cyclic loading for stress values less than the ultimate stress is called 'fatigue'. The inverse of fatigue damage, which is the number of loading cycles required for material failure, is the 'fatigue life'. The first article on fatigue was published by Wilhelm Albert (1838), reporting the failure of iron mine-hoist chains arising from repeated small loading. The fatigue life is influenced by several factors, including but not limited to, material type, surface quality, geometry, residual stresses, cyclic stress state, temperature, external loading direction, material grain size and microstructure, and environmental conditions (Schijve 2001). Because of the uncertainties involved in predicting the fatigue life, it is customary to 
adopt a very high factor of safety on fatigue life. Typically, for SCR design, the estimated fatigue life should exceed 10 times the operating life for a component of major importance to structural integrity and inaccessible for inspection, and 3 times the operating life for an accessible component for inspection and maintenance (API-RP2RD 1997)

For high-cycle fatigue calculations, where the number of cycles required to failure is more than $10^{4}$, the performance of the material is characterised by a graph called a Wohler or S-N curve, which shows the cyclic stress (S) against the logarithmic scale of number of cycles to failure $(\mathrm{N})$. S-N curves are commonly generated experimentally, by conducting a series of tests under controlled laboratory conditions, applying sinusoidal stresses on a sample of the material and counting the cycles to failure.

Design codes and standards usually provide S-N curves for various materials associated with recommended practices developed for specific areas. Figure 4-1 shows a sample SN curve provided by DNV-RP-C203 (2008) for a range of material classes assuming immersion in seawater with cathodic protection.

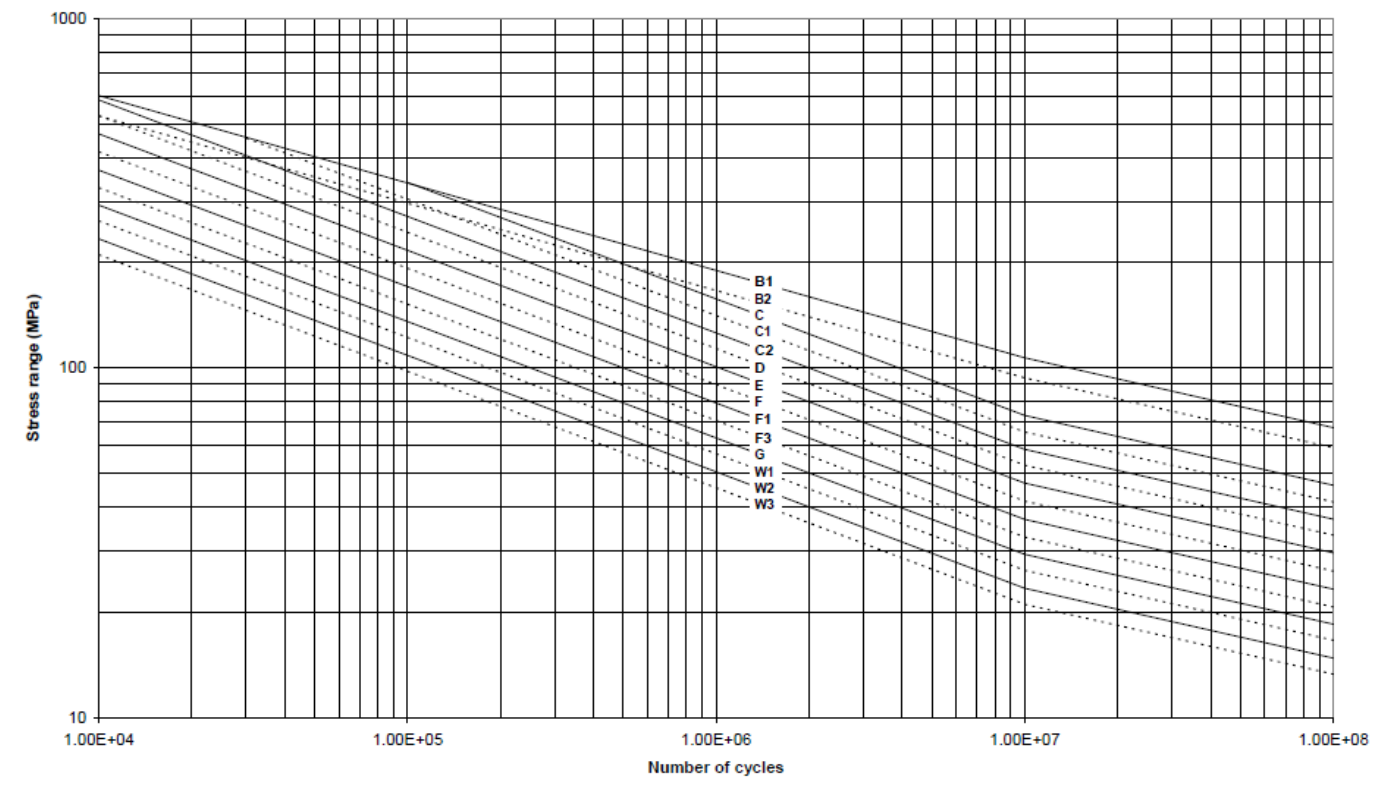

Figure 4-1: Sample S-N curves for fatigue damage calculations (DNV-RP-C203 2008)

Barltrop \& Aams (1991) described a standard form of S-N curves for marine structures to be used for fatigue calculations as:

$\mathrm{N}=\overline{\mathrm{a}} \Delta \sigma_{\mathrm{f}}{ }^{-\mathrm{m}}$

where

$\mathrm{N}$ is the number of cycles to failure

$\overline{\mathrm{a}} \quad$ is an empirical coefficient (equivalent to the fatigue life for $\Delta \sigma_{\mathrm{f}}=1 \mathrm{MPa}$ ) 
$\Delta \sigma_{\mathrm{f}}$ is the factored stress variation range in $\mathrm{MPa}$

$\mathrm{m} \quad$ is the inverse slope of the S-N curve (typically around 3).

In a standard fatigue calculation, for an operating life that incorporates many millions of cycles of various stress ranges, as expressed by a stress histogram, the accumulated fatigue damage can be calculated by using a linear cumulative damage (Palmgren 1924), according to the method presented by Miner (1945), commonly known as Miner's rule:

$\mathrm{D}=\sum_{\mathrm{i}=1}^{\mathrm{k}} \frac{\mathrm{n}_{\mathrm{i}}}{\mathrm{N}_{\mathrm{i}}}$

where

$\mathrm{D}$ is the total accumulated fatigue damage

$\mathrm{k}$ is the number of stress blocks

$i$ is the number of a particular constant stress range block in the stress histogram

$\mathrm{n}_{\mathrm{i}}$ is the number of stress cycles in stress block i over the design life

$\mathrm{N}_{\mathrm{i}}$ is the number of stress cycles to failure for the constant stress range $\mathrm{i}$, equation (4-1)

Equation (4-2) simply superposes the fatigue damage under the various cyclic stress blocks, and implicitly assumes that the order in which the cyclic stresses are applied is irrelevant in terms of the cumulative damage.

The fatigue calculation is straightforward for a linear system, where chaotically ordered cyclic perturbations can first be sorted into blocks of a similar size that will result in a similar size cyclic stress range on the fatigue target, before applying Miner's rule. However, if the system involves materials with a non-linear response, such as the hysteretic non-linear seabed model (Randolph and Quiggin 2009) adopted in the present application, the stress changes in the SCR will depend on the hierarchy of the various sea states or, even if these are sorted into a spectrum of similar sized waves, on the order in which the wave packages are applied. Thus for non-linear seabed response, some modification must be made to the manner in which the cyclic stresses in the SCR are obtained from the applied sea states, as will be discussed in Chapter 6.

However, for a linear system, it is straightforward to evaluate the cyclic stress range associated with a given magnitude of perturbation. The fatigue damage criterion can then be written as (DNV-RP-C203 2008):

$\mathrm{D}_{\mathrm{T}}=\sum_{\mathrm{i}=1}^{\mathrm{k}} \frac{\mathrm{n}_{\mathrm{i}}}{\mathrm{N}_{\mathrm{i}}}=\frac{1}{\overline{\mathrm{a}}} \sum_{\mathrm{i}=1}^{\mathrm{k}} \mathrm{n}_{\mathrm{i}}\left(\Delta \sigma_{\mathrm{f}_{\mathrm{i}}}\right)^{\mathrm{m}} \leq \eta$

where 
$\bar{a} \quad$ is the intercept of the design S-N curve with the $\log \mathrm{N}$ axis (i.e. for $\Delta \sigma_{\mathrm{f}}=$ $1 \mathrm{MPa})$

$\Delta \sigma_{f i}$ is the constant stress range in each block of stress histogram

$\mathrm{m} \quad$ is the negative inverse slope of S-N curve

$\eta \quad$ is the usage factor, normally between 0.7 and 3.0 and for design purposes is normally taken as 1 .

The usage factor, $\eta$, is defined as the inverse of the damage fatigue factor (DFF), (DNV-OS-C101 2008) which itself is a parameter applied to reduce the probability for fatigue failure. This parameter is suggested depending on the accessibility and inspection intervals of the structure and is conservatively considered as unity for most design purposes.

For SCR fatigue design the stress range, $\Delta \sigma_{f_{i}}$ is factored by a stress concentration factor SCF, which is recommended by design codes and standards based on the geometric and finished properties of riser pipe connections. The magnitude of SCF can vary from unity to large values greater than 10 depending on the conditions mentioned above and an extensive part of the fatigue guidelines are usually allocated to recommendations for SCF for various components. In the current study for E class pipe the magnitude of SCF is taken as 1.13 (DNV-RP-C203 2008).

Defining the fatigue life as the time required for failure, it can be written as:

$\mathrm{L}=\frac{\mathrm{L}_{\text {design }}}{\mathrm{D}_{\mathrm{T}}} \eta$

where $\mathrm{L}_{\text {design }}$ is the design life of the riser in years, which has been taken as 30 years through out of the current dissertation.

\subsection{FATIGUE DAMAGE OF STEEL CATENARY RISERS}

The prediction of fatigue damage in SCRs is a complicated process involving many factors under various loading conditions, but can be mainly be considered in three categories (API-RP-2RD 1997; Campbell 1999):

1. First order fatigue arising from wave frequency or first order motions of the vessel under wave action.

2. Second order fatigue arising from low frequency, second order motions or slow drifts of the vessel due to swell and light wind.

3. VIV fatigue arising from Vortex Induced Vibration (VIV) of the SCR under sea current loads. 
The maximum fatigue damage is determined by combining the first and second order fatigue damage with the fatigue damage from VIV. The VIV fatigue damage is essentially independent of the other categories and is customarily simulated by sophisticated computational fluid dynamics software, coupled with finite element (FE) structural dynamic analysis. The first and second order fatigue can be calculated by analysing the riser either under random sea states defined by a series of irregular waves. The sea states are chosen as a statistical representation of individual waves within a given period of time (say 3 hours) or under individual regular waves. Sea states are defined by a wave scatter diagram giving the wave characteristics and number of applied cycles. The calculated fatigue life is scaled by a safety factor and the final value is checked against the production life of the SCR.

If the SCR is assessed to fail, depending on the area of damage and the category of dominant contributor to fatigue (defined above), different fatigue reduction techniques may be applied, such as strakes and fairings for VIV fatigue (particularly in the upper areas of SCRs), or slight relocation of the floating system and consequently TDP for first and second order fatigues.

VIV fatigue is not part of the current research, which focuses on fatigue in the touchdown zone due to motion of the riser originating from the supporting floating facility. The affected area is around the mean touchdown point (TDP) at which the catenary riser separates from the seabed. It has been found that fatigue near to the vessel attachment point (or upper support of the SCR) is unaffected by how the interaction with the seabed is modelled (STRIDE JIP, 1997), but fatigue in the TDZ is heavily influenced by the riser-seabed interaction and causes the greatest uncertainty in the SCR analysis.

The SCR motion, even under the first order motions of the vessel, is still a dynamic event, and the stress changes in the riser at the TDZ under a given wave amplitude are affected by the period of the wave. However, in order to simplify the study, allowing displacement-controlled analysis and focusing on how the seabed properties affect the stress changes due to first order oscillations of the riser that lead to in-plane movement of the TDP, the analyses have been conducted in a quasi-static mode. Therefore, only the first order motions of the vessel have been implemented and the second order motions, VIV and dynamic effects of the SCR itself (inertia, drag and added mass) have been ignored. Although this will underestimate the true stress changes and thus the fatigue damage, it is sufficient to achieve the main objectives of evaluating the influence of the seabed model on fatigue, and how to conduct fatigue studies for a non-linear seabed response.

It is well known (Bridge et al. 2004; Campbell 1999; Clukey et al. 2007), and will be confirmed by results presented here, that the fatigue damage increases with the magnitude of the soil reaction force, which increases with soil stiffness. The full fatigue calculation process is quite complicated, even for a linear elastic seabed, and is 
generally carried out with purpose developed software. The analyses here are restricted to a single SCR and Spar system, and a wave scatter diagram typical of deep water Gulf of Mexico. The external loading considered in all the analyses comprises the Spar motions resulting from the first order wave actions. Therefore, the Spar's harmonic motion due to its natural frequency and the second order slow drift motions have not been accounted for.

\subsection{FATIGUE ANALYSIS SETUP}

\subsubsection{ABAQUS finite element analysis program}

ABAQUS is a popular commercial package for finite element analysis due to its strong capabilities in numerical modelling of a wide range of materials with complex behaviour. For simple modelling of problems with high non-linearity such as steel catenary risers on rigid or elastic seabed, in particular for dynamic transient and quasistatic analyses, the explicit module of ABAQUS, which uses an explicit integration scheme, is the most appropriate option. In that case the existing element library of ABAQUS can be used to model the riser-seabed interaction, using the springs and connector elements that are included in the element library.

Alternatively, the riser-seabed interaction can be modelled by a user defined element, which is coded in FORTRAN as a subroutine within the ABAQUS user element library (UEL). The UEL subroutine is not a part of ABAQUS explicit, so the system must then be modelled in the ABAQUS standard module, which is a general-purpose solver and uses the traditional implicit integration scheme to solve the finite element problem. The present research has used this latter approach, developing UELs initially for a linear elastic seabed, allocating linear seabed stiffness in user-defined springs, and later for the non-linear model described in the following chapter.

\subsubsection{Fatigue analysis methodology}

The deterministic fatigue analysis method has been applied using a realistic wave scatter diagram from the Gulf of Mexico containing the wave period, significant wave height and the probability of occurrence.

Displacement-controlled quasi-static analyses have been performed with the vessel excitation based on generic RAOs (response amplitude operators), which quantify the dynamic response of the floating system to different sea states. For this purpose the DISP subroutine of ABAQUS has been used to update the boundary conditions including the vessel position at every increment. A generic Spar RAO from the Gulf of Mexico has been used for the analyses conducted in this thesis. Hydrodynamic effects for the SCR (inertia, drag and added mass) have been ignored in the quasi-static analyses. The analyses comprise three steps in which the first and second steps are used to configure and set up the SCR system and the third step incorporates the time history of vessel movements that simulate the environmental loading on the floating system. 
The seabed has been modelled by user-defined elements through the UEL, which is called by ABAQUS for every user element at every increment of an analysis step to provide the element's stiffness matrix and its contribution to the global right-hand-side vector (RHS) or system external forces.

The von Mises stress given by ABAQUS is used to calculate the cyclic stress variation range in the riser pipe-wall. Taking into account the $2 \mathrm{D}$ planar nature of the analyses, two section integration points, one at the top and the other at the bottom of the riser cross section, have been taken as the fatigue calculation reference points at each location along the SCR. Since ABAQUS itself does not support any specific module for fatigue analysis, an Excel spreadsheet and macro have been developed for postprocessing the results from ABAQUS in order to calculate the fatigue damage through the touchdown zone.

\subsubsection{Global configuration of model}

Figure 4-2, shows the key dimensions of the SCR system for fatigue analysis on a linear elastic seabed. The spring spacing and the mechanical properties of the riser pipe along with the S-N curve parameters for fatigue calculations are given in Table 4-1 and Table 4-2 respectively. The S-N curve parameters presented in Table 4-2 have been applied for all of the analyses throughout the thesis. The riser pipe itself has been modelled as a Timoshenko (shear flexible) beam using element B21 from the ABAQUS element library, with a total number of 828 nodes, starting from node No. 1 at the anchor end and ending with node No. 828 at the vessel attachment point. This kind of element can be used for slender pipes assuming linear elastic transverse shear behaviour. The length of beam elements in the hanging part is $5 \mathrm{~m}$ (except for the last element connected to the vessel which is $3 \mathrm{~m}$ in length) and $1 \mathrm{~m}$ in the remaining part on the seabed within a length of $450 \mathrm{~m}$ as seen in Figure 4-2. The touchdown point position depends on the SCR profile on the seabed, which in turn depends on the seabed stiffness, but approximately, for the configuration illustrated in Figure 4-2 and with a seabed stiffness of $100 \mathrm{kPa}$, the TDP is close to node No. 347. 


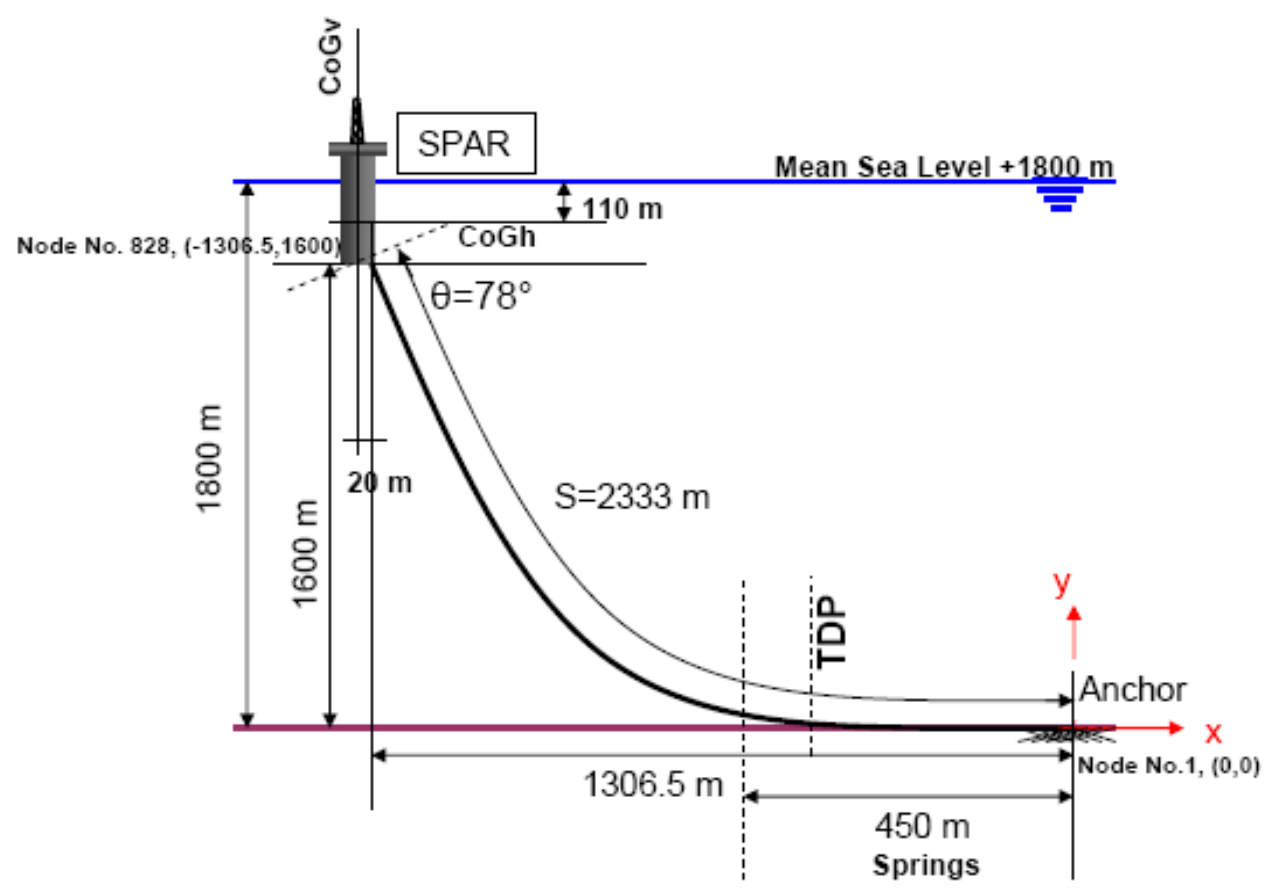

Figure 4-2: The global geometry of SCR modelled by ABAQUS

Table 4-1: key dimensions of SCR modelled by ABAQUS

\begin{tabular}{|l|c|}
\hline \multicolumn{1}{|c|}{ Dimension } & Value \\
\hline Vessel type & SPAR \\
\hline Water depth & $1800 \mathrm{~m}$ \\
\hline Height of attachment point & $1600 \mathrm{~m}$ \\
\hline SCR length & $2333 \mathrm{~m}$ \\
\hline Vessel distance from anchor end & $1306.5 \mathrm{~m}$ \\
\hline Lay angle & $78^{\circ}$ \\
\hline Attachment point offset from CoG & $20 \mathrm{~m}$ \\
\hline Length of zone of seabed springs & $450 \mathrm{~m}$ \\
\hline Spacing of seabed springs & $1.0 \mathrm{~m}$ \\
\hline
\end{tabular}


Table 4-2: Riser pipe properties

\begin{tabular}{|l|c|}
\hline \multicolumn{1}{|c|}{ Dimension } & Value \\
\hline Outer diameter, $\mathrm{D}_{\mathrm{o}}$ & $0.324 \mathrm{~m}\left(12^{3 / 4}{ }^{\mathrm{n}}\right)$ \\
\hline Wall thickness, $\mathrm{t}$ & $0.0205 \mathrm{~m}$ \\
\hline Second moment of area, I & $2.26 \times 10^{-4} \mathrm{~m}^{4}$ \\
\hline Steel Young's Modulus & $2.07 \times 10^{11} \mathrm{~N} / \mathrm{m}^{2}$ \\
\hline Steel density, $\rho_{\mathrm{s}}$ & $7850 \mathrm{~kg} / \mathrm{m}^{3}$ \\
\hline In service submerged weight, $\mathrm{m}_{\mathrm{s}}$ & $100 \mathrm{~kg} / \mathrm{m}$ \\
\hline Fatigue S-N curve & $\begin{array}{l}\mathrm{DNV}(2008), \mathrm{E} \text { Class } \\
\text { weld, } \overline{\mathrm{a}}=1.05 \times 10^{-12} \\
\mathrm{~m}=3.0, \mathrm{SCF}=1.13\end{array}$ \\
\hline
\end{tabular}

\subsubsection{Numerical analysis steps}

As for any finite element software, ABAQUS allows the user to define various steps for accurate modelling of the system behaviour during different stages of loading. Three steps have been defined to model the SCR connected to the floating system as follows:

\section{- Step 1: pipe end lift-up}

The SCR is initially modelled as a straight pipe, laid on the seabed, partially supported by linear elastic, tensionless springs, with a simple support at the vessel end and fixed at the anchor end. At this stage, no gravity loading has been applied on the pipe. At the start of Step 1, the submerged weight is applied to the riser and the vessel end of the riser is lifted up simultaneously to the level equal to the height of the attachment point, to a position where the SCR is completely clear of the seabed, Figure 4-3.

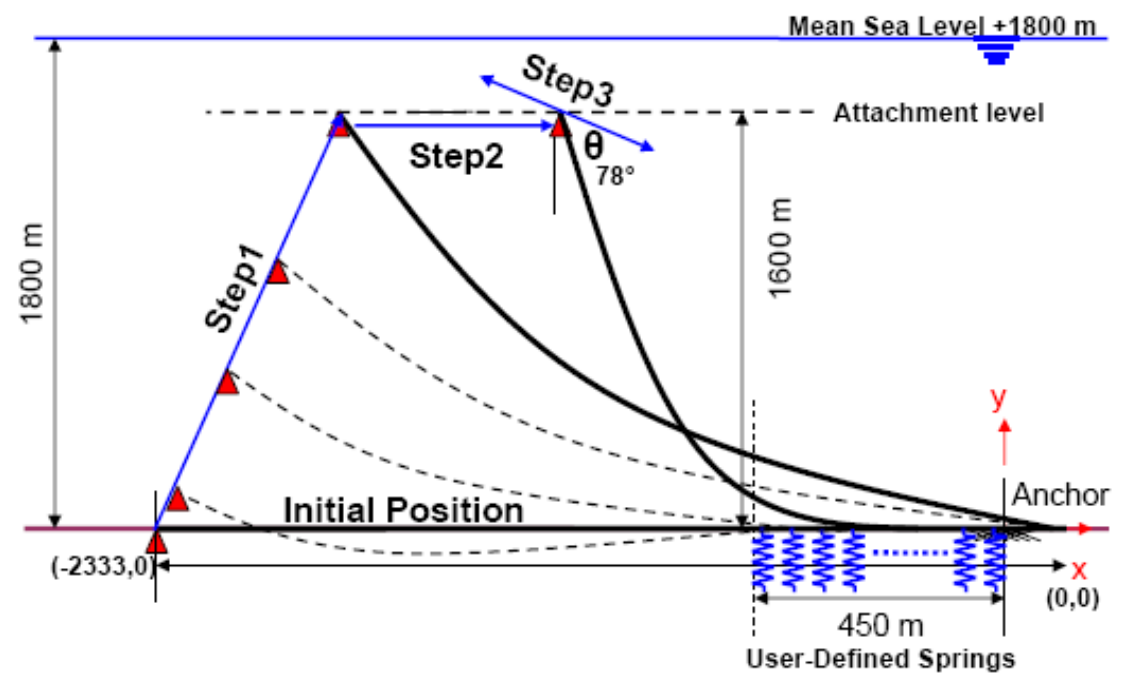

Figure 4-3: Schematic illustration of FE analysis steps 


\section{- Step 2: vessel positioning}

In Step 2, the vessel is shifted from its position at the end of Step 1 to the vessel's nominal position towards the anchor end to achieve the targeted lay angle ( 78 degrees in this example). During the translation of the vessel to its nominal position, the SCR touches the seabed springs gradually and the springs are activated. At the end of Step 2, the SCR reaches its global configuration, developing the interaction with the seabed through the user-defined linear springs, coded within the UEL subroutine, (Figure 4-3)

\section{- Step 3: vessel excitation}

In Step 3, the vessel is perturbed to simulate the effects of wave action according to the predetermined wave sequence and RAOs in a time domain analysis. Each new position of the vessel is generated by the DISP subroutine of ABAQUS, which processes the applied sea state data and vessel RAO at every increment of analysis. The surge, heave and pitch of the vessel at its centre of gravity $(\mathrm{CoG})$ is transferred to the attachment point by subroutine DISP, causing the riser to be lifted-up and lowered sequentially in the touchdown zone through the cycles of loading and unloading. Each spring may experience a different state of displacement and movement direction at any given time stage, depending on the longitudinal position of the spring along the riser.

\subsubsection{Convergence of the analyses}

The extreme geometric non-linearity of the model, in addition to the large displacement applied to the riser during the initial lift-up of the vessel end from the seabed in the first step, can make the static analysis unstable. Hence, in the first step of the analysis, during the riser lift-up, an automatic stabilisation technique of ABAQUS has been used, applying an artificial damping to the riser. It is assumed that the problem is stable at the beginning of the step but that instabilities may develop in the course of the step. While the model is stable, viscous forces, and therefore the viscous energy dissipated, are very small. Thus, the additional artificial damping has no effect. If a local region goes unstable, the local velocities increase and, consequently, part of the strain energy then released is dissipated by the applied damping. ABAQUS can reduce the time increment to permit the process to occur without any unstable response causing very large displacements. The artificial damping has not been applied in Steps 2 and 3, enabling a direct link between the vessel and TDP displacements.

To achieve convergence with an optimized accuracy and efficient solution, it was found necessary to adjust some of the convergence control parameters in ABAQUS. In difficult cases with high non-linearity like the SCR, the solution procedure may not converge with the default controls or may use an excessive number of increments and iterations. The solution control parameters can be used to control the time increment adjustment and the accuracy of the non-linear equation solution as well as defining the tolerances for field equations such as rotation and displacement. The word "field" refers to the basic variables of the system, such as the component of the displacement in a 
continuum stress analysis. Other algorithms like "line search" can be used to scale the solution correction to prevent divergence of the solution in the early iterations for cases such as the SCR with strong non-linearity. For instance, $R^{\alpha}{ }_{n}$ is the convergence criterion for the ratio of the largest residual to the corresponding average flux norm. The word "flux" means the variable whose discretised equilibrium is being sought such as force and moment. The default value of $\mathrm{R}^{\alpha}{ }_{\mathrm{n}}$ is $0.5 \%$ which is rather strict by engineering standards but usually guarantees an accurate solution. In highly complex non-linear problems the default value of $\mathrm{R}^{\alpha}{ }_{\mathrm{n}}$ can lead to extensive computational effort and unnecessary convergence difficulties. In the analyses conducted here the value of $\mathrm{R}^{\alpha}{ }_{\mathrm{n}}$ was set to $5 \%$ for particular stages where convergence difficulties were encountered. This helped to achieve a much smoother analysis with reduced computational effort and with minor effect on the results, particularly for full fatigue analysis with a non-linear seabed as will be discussed in Chapter 6 .

\subsection{FLOATING SYSTEM EXCITATION}

\subsubsection{Overview}

The vessel motions have been simulated using a generic RAO for a particular type of vessel (in this case a Spar), which gives the vessel response in six degrees of freedom for a range of waves with various periods and unit significant wave height. These degrees of freedom include three translations (surge, sway and heave) and three rotations (roll, pitch and yaw). In the analysis, a series of waves are applied to the vessel and the RAO responses are scaled for each wave at every increment of analysis, updating the vessel's new position for that specific increment. All of this process is generated and managed by the DISP subroutine of ABAQUS and will be further discussed in coming sections.

\subsubsection{Selection of Spar vessel as floating system}

Steel catenary risers are used with various kinds of supporting floating systems such as FPSO, Spar, semi-submersible etc. Field developments in recent years have tended to favour the Spar concept as the most viable option for deep water developments, partly because it reduces the motions applied to the riser compared with a conventional FPSO or semi-submersible (e.g. (Ghosh et al. 2005)). 


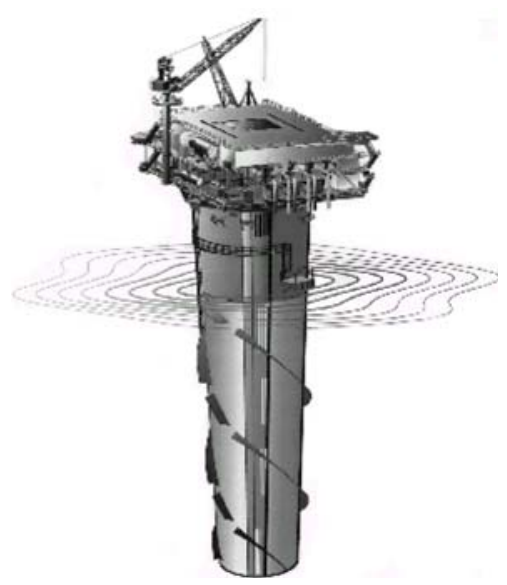

Figure 4-4: Spar floating system with helical strakes on hull (Marinetalk 2000)

A Spar commonly consists of a dry topside carried by a long cylindrical floating hull, Figure 4-4. Ballasting of the Spar, which is common practice, shifts its centre of gravity well bellow the centre of buoyancy, presenting a very stable system against toppling motions. The Spar vessel is normally kept in position by catenary or semi-taut mooring lines.

Selection of a Spar as the supporting system has some benefits for the present study. The Spar response to environmental loads is almost insensitive to water depth since it is mainly a floating cylinder and its heave natural period is only a function of the draft (Mekha et al. 1996). The geometry and naval architectural design of Spars makes them very stable, even with no mooring line; the pitch motions are essentially negligible and can be ignored in vessel perturbations. As seen in Figure 4-2, the vertical offset of the CoG (Centre of Gravity) and attachment point for our case is $90 \mathrm{~m}$; a simple hand calculation using the data in Figure 4-5 shows that the contribution of the peak pitch motion for a mean environment to the heave and surge offsets of the Spar vessel is less than $3 \%$, which is quite negligible. In addition, generic RAOs for Spar systems show no peak value of surge or heave within the relevant range of wave periods (less than 25 seconds), Figure 4-5. This means that no resonance occurs in the normal window of wave periods in Spar dynamic motions. This at least decreases the degree of simplification between dynamic and quasi-static analyses but the dynamic effects of the SCR itself will still exist (although ignored here) and will influence the actual fatigue performance.

A generic 2D planar response amplitude operator (RAO) of a truss Spar vessel containing three active degrees of freedom, surge, heave and pitch is illustrated in Figure 4-5 (Bridge et al. 2004) and has been applied for all of the analysis performed for this research. As an example, Figure 4-5 shows that when a single regular wave with 1.0 m significant wave height and a 20 second period hits the Spar from a head sea, the centre of gravity (CoG) of the Spar will be displaced $0.65 \mathrm{~m}$ horizontally (surge), $0.35 \mathrm{~m}$ vertically (heave) and rotate $0.013 \mathrm{deg} / \mathrm{m}$ in its peak wave amplitude. Now if a 
single wave with $15 \mathrm{~m}$ wave height (and hence $7.5 \mathrm{~m}$ wave amplitude) and the same wave period hit the Spar in the same direction, then the vessel will be displaced $9.75 \mathrm{~m}$ $(15 \times 0.65)$ horizontally (surge), $5.25 \mathrm{~m}(15 \times 0.35)$ vertically (heave) and rotate 0.195 degrees $(15 \times 0.013)$ at the peak wave amplitude. Then, in this case, taking into account the vertical offset of the $\mathrm{CoG}$ and attachment point $(90 \mathrm{~m}$, Figure 4-2) the resulting surge and heave motions incorporating the pitch effect will be $10.06 \mathrm{~m}$ and $5.252 \mathrm{~m}$ respectively.

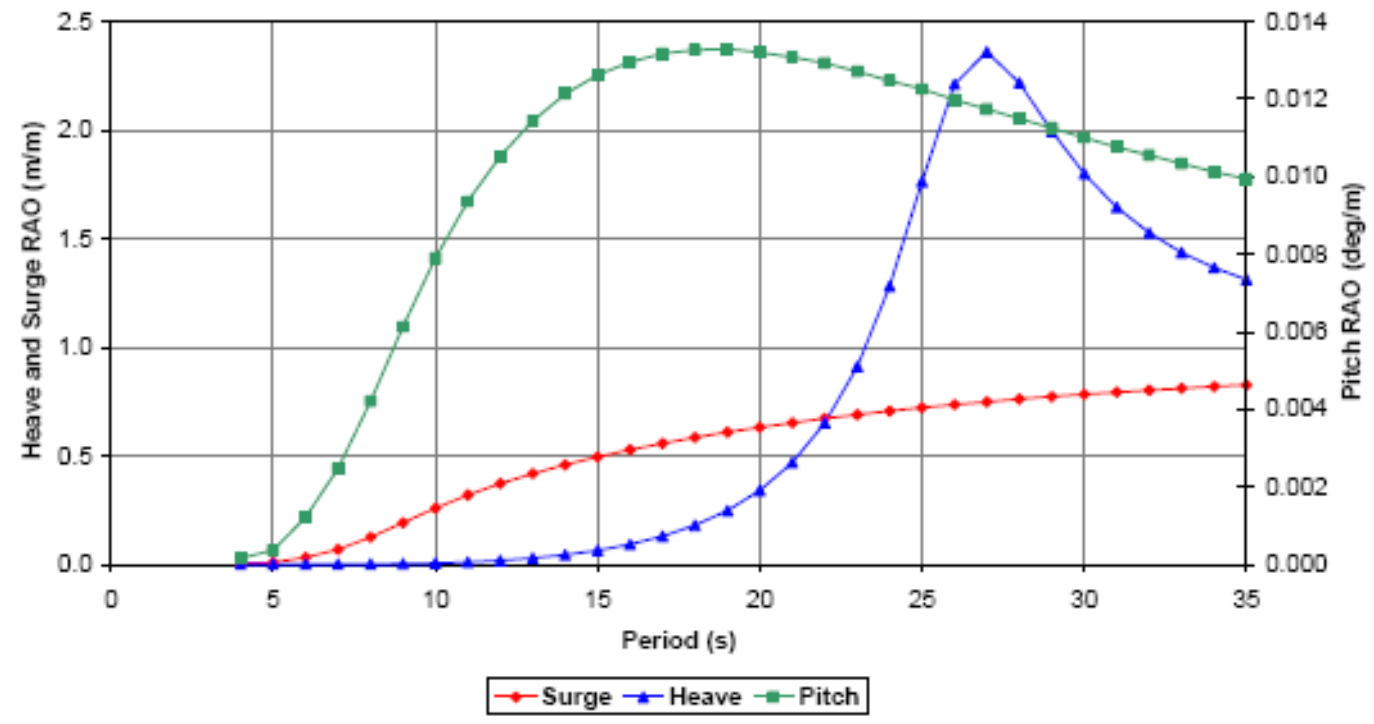

Figure 4-5: Generic truss Spar motion RAO, head sea (Bridge, 2005)

The vessel displacement under a particular regular wave can be determined using its RAO in the following form (Orcina Ltd, 2008):

$\mathrm{x}=\mathrm{Ra} \cos (\omega \mathrm{t}-\varphi)$

where

$\mathrm{x}$ is the vessel displacement (in length units for translations and degrees for rotations)

$\mathrm{R}$ is the $\mathrm{RAO}$ amplitude

$\mathrm{a}$ is the wave amplitude ( in length units)

$\omega$ is the frequency (in radian/second)

$\mathrm{t}$ is the time (in seconds)

$\varphi$ is the RAO phase

A generic RAO, as has been used for all of the analyses here, does not include any information about the timing of the vessel motion relative to the wave, which is commonly referred to as 'phase', $(\varphi)$. Model tests or hydrodynamic analysis software 
are used to determine the RAOs of a particular vessel including the phase information, which is not part of the current study. Hence, it has been assumed here that the peak values of various degrees of freedom will occur simultaneously and the geometrical position of the excited vessel will be linear. Figure 4-6, shows a sample of such excitation. Although, the assumption does not affect the accuracy of the analyses within the area of interest, a more realistic simulation of the vessel movements should include the phase information to account for the timing of vessel motions relative to the wave. In such case the vessel position will follow an elliptical path in the vertical plane, as shown in Figure 4-7.

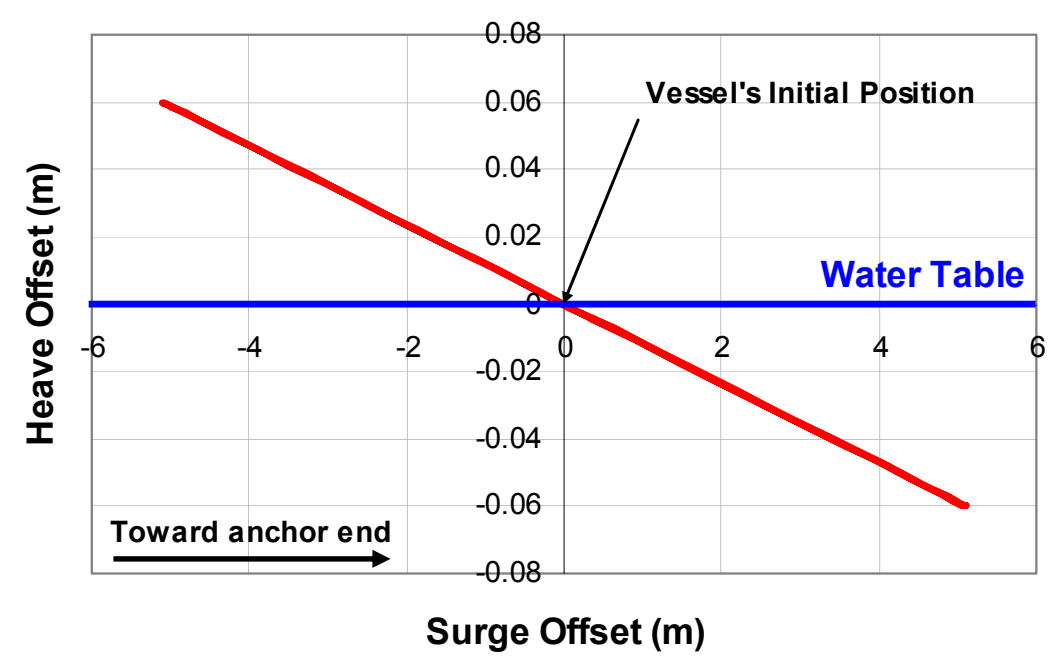

Figure 4-6: Sample RAO with no phase information

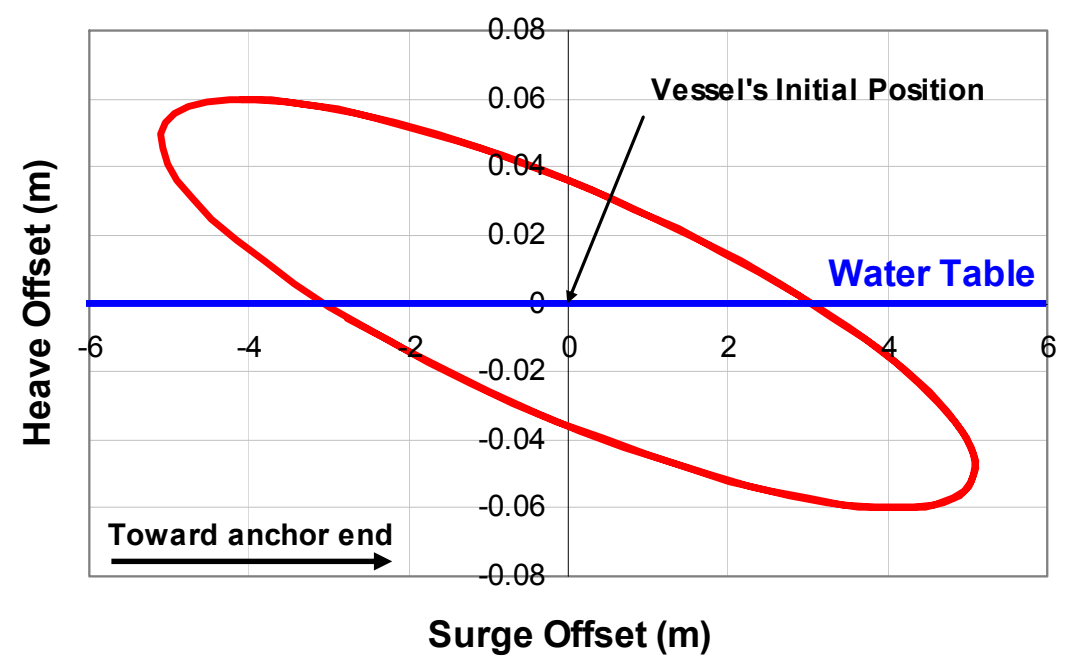

Figure 4-7: Sample RAO with phase information 
The RAO of a vessel is normally given at the centre of gravity $(\mathrm{CoG})$, while the SCR will be connected to the Spar at a point that is offset horizontally and vertically from the CoG. Therefore, the displacements of the attachment point need to be extracted from the RAO at the $\mathrm{CoG}$, in the present case taking account of pitch of the Spar. For the example Spar considered here, the attachment point has been assumed to be at a distance of $90 \mathrm{~m}$ below the CoG. All of this process has been coded inside the DISP subroutine of ABAQUS. The schematic flowchart of DISP subroutine is explained in section 4.6.3.

As illustrated in Figure 4-6, the direction of excitation is set to be aligned with the SCR orientation at the attachment point. This maximises the perturbation of the riser in the touchdown zone, allowing better evaluation of the influence of the seabed response.

\subsubsection{Environmental data / wave scatter diagram}

The wave scatter diagram applied for the fatigue analyses is drawn from typical Gulf of Mexico conditions (provided courtesy of BP America) and the resulting wave heights, periods and values for a 30 year design life are given in Table 4-3. The total number of sea states is 30 and their ID identifies the hierarchy of the waves. The table gives details of the significant wave heights, Hs, and the wave periods, Tz. The number of applied waves has been calculated from a table of probabilities of occurrence, considering 30 years service life for the SCR. As an example, Table 4-3 indicates that the $3^{\text {rd }}$ sea state in the table, with a significant wave height of $1.5 \mathrm{~m}$ and a wave period of $5.0 \mathrm{~s}$, may hit the vessel 48,449,608 times during 30 years of system service life. This is the parameter $n_{i}$, in equation (3-1). As Table 4-3 shows, the number of expected wave decreases as the severity of the waves is increased, as indicated by higher significant wave heights and longer periods. The fatigue damage depends on both the stress variation range (which increases with increasing wave height) and the number of cycles; thus moderate waves with average heights and periods, but a high number of cycles, will give a significant contribution to the total fatigue damage and inversely the fatigue life of the SCR; this will be shown later in this chapter. 
Table 4-3: Manipulated wave scatter diagram for a 30 year operational life, for deep water conditions in Gulf of Mexico

\begin{tabular}{|l|l|l|l|l|l|l|l|}
\hline Sea State ID & Hs $(\mathbf{m})$ & Tz(s) & n applied & Sea State ID & Hs $(\mathbf{m})$ & Tz(s) & $\mathbf{n}$ applied \\
\hline 1 & 0.5 & 4.2 & 18011291 & 16 & 8.0 & 9.1 & 3389 \\
\hline 2 & 1.0 & 4.6 & 71370445 & 17 & 8.5 & 9.3 & 3011 \\
\hline 3 & 1.5 & 5.0 & 48449608 & 18 & 9.0 & 9.5 & 1822 \\
\hline 4 & 2.0 & 5.4 & 25187856 & 19 & 9.5 & 9.7 & 1395 \\
\hline 5 & 2.5 & 5.8 & 13529335 & 20 & 10.0 & 9.9 & 1070 \\
\hline 6 & 3.0 & 6.1 & 7473660 & 21 & 10.5 & 10.1 & 1246 \\
\hline 7 & 3.5 & 6.5 & 3080495 & 22 & 11.0 & 10.2 & 566 \\
\hline 8 & 4.0 & 6.9 & 1631014 & 23 & 11.5 & 10.4 & 928 \\
\hline 9 & 4.5 & 7.3 & 583770 & 24 & 12.0 & 10.6 & 544 \\
\hline 10 & 5.0 & 7.7 & 363725 & 25 & 12.5 & 10.7 & 813 \\
\hline 11 & 5.5 & 8.0 & 114700 & 26 & 13.0 & 10.9 & 712 \\
\hline 12 & 6.0 & 8.4 & 33676 & 27 & 13.5 & 11.0 & 877 \\
\hline 13 & 6.5 & 8.5 & 16907 & 28 & 14.0 & 11.2 & 262 \\
\hline 14 & 7.0 & 8.7 & 10864 & 29 & 14.5 & 11.3 & 343 \\
\hline 15 & 7.5 & 8.9 & 5421 & 30 & 15.0 & 11.5 & 420 \\
\hline
\end{tabular}

\subsection{FATIGUE ANALYSIS FLOWCHART USING ABAQUS}

The presence of various steps in fatigue analyses performed using ABAQUS, including developing the global model in ABAQUS, calling the DISP subroutine for vessel excitation, calling the UEL subroutines for seabed interaction modelling, post processing of the field and history outputs, and processing the fatigue calculation in macro-driven spreadsheets, is quite a complex process. Flowcharts for the different stages of the analysis are given below to facilitate understanding of the various loops and their functions. The flowcharts include only the main actions. Figure 4-8 illustrates the global loops and steps for analysis, where the central part is the main ABAQUS analysis. The flowchart of the main ABAQUS procedure itself is shown in Figure 4-9 and the internal flowchart for the DISP subroutine for vessel excitation is given in Figure 4-10.

\subsubsection{The global flowchart of analysis setup}

Upon starting the analysis, ABAQUS reads the input file and checks the model consistency and connections with defined links to subroutines, see Figure 4-8. Steps 1, 2 and 3 have been described previously, and comprise raising the SCR clear of the seabed after first applying the self-weight of the SCR while it lies on the seabed, repositioning of the suspended end of the SCR to give the correct hang-off angle (and thus tension in the riser), and then carrying out the time series analysis of the vessel perturbations. As can be seen in Figure 4-8, the DISP subroutine is inactive during Step 1 and Step 2; ABAQUS realises this from the input file, where the modified boundary conditions are 
directly given in Step 1 and 2, but the user-defined boundary condition (DISP subroutine) is called in Step 3. The UEL subroutine is active from the beginning of the analysis, providing the springs' stiffness matrix and the contribution to external forces in the right hand side vector (RHS). In Step 3, the vessel starts to be excited as controlled through the DISP subroutine and ABAQUS calls the DISP subroutine for the updated position of the vessel at each time increment.

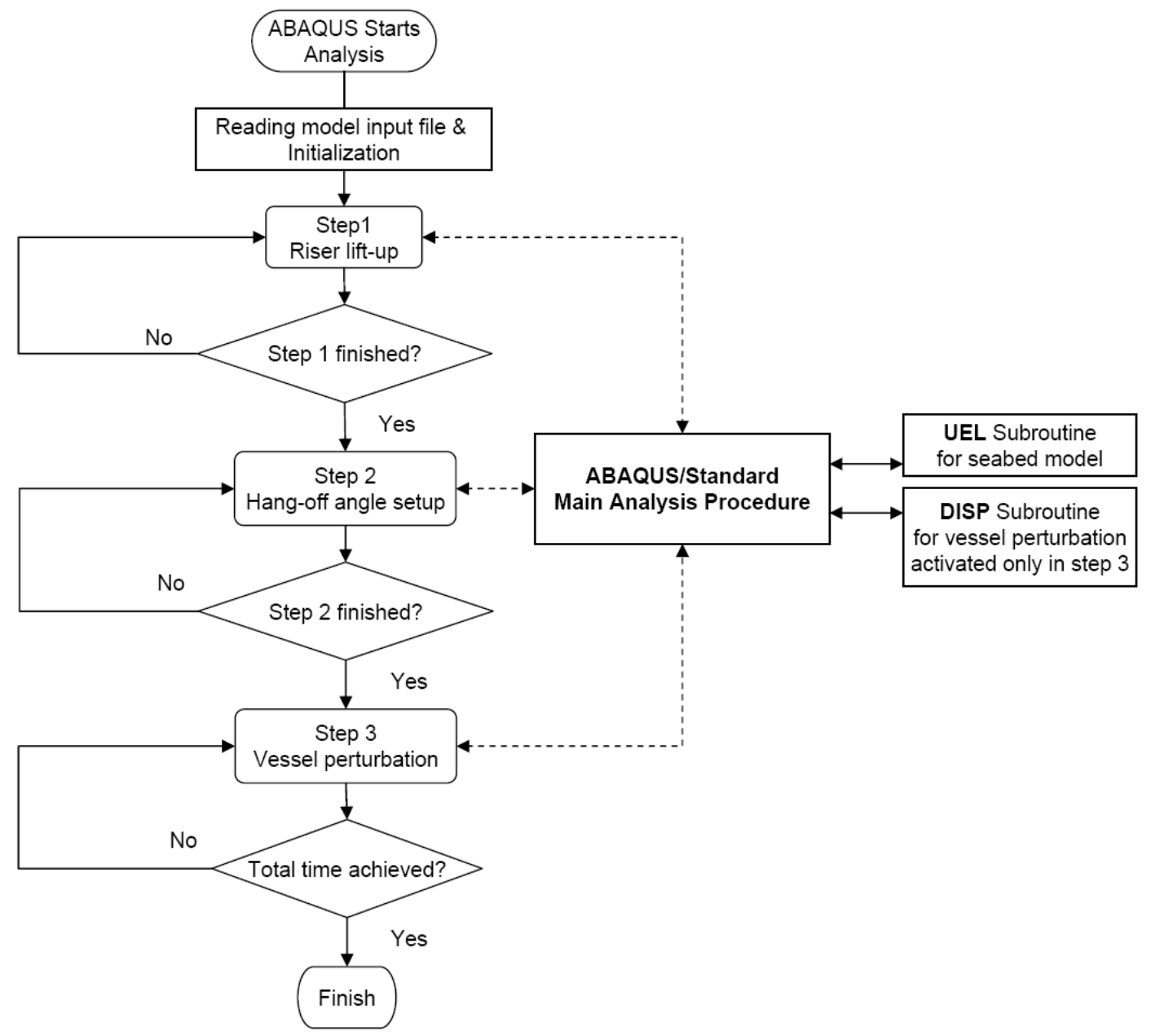

Figure 4-8: Main flowchart of fatigue analysis using ABAQUS

\subsubsection{Main calculation flowchart}

Figure 4-9 shows the internal activities of the main analysis procedure for Step 2 and Step 3, which follow after raising the SCR clear of the seabed in Step 1. On starting an analysis step, ABAQUS identifies the type of analysis and defines the time incrementation scheme based on suggested values defined in the input file, updating the boundary conditions accordingly. At this stage, in Steps $1 \& 2$, ABAQUS receives the final destination of the vessel at the end of the step from the input file along with any external loads (for example, the self-weight of the SCR in Step 1). Then ABAQUS schedules the vessel incremental displacement to achieve the final destination at the end 
of the step; this schedule is updated and optimised during the analysis. In Step 3, the displacement boundary condition at the vessel end of the SCR is obtained from the DISP subroutine for each time step (as described later) and ABAQUS then optimises the incremental displacement of the vessel to the position instructed by the DISP subroutine. It is worth mentioning that at least two iterations are applied by ABAQUS to achieve the equilibrium in every increment. These iterations are not controlled by the user but are performed automatically by ABAQUS itself.

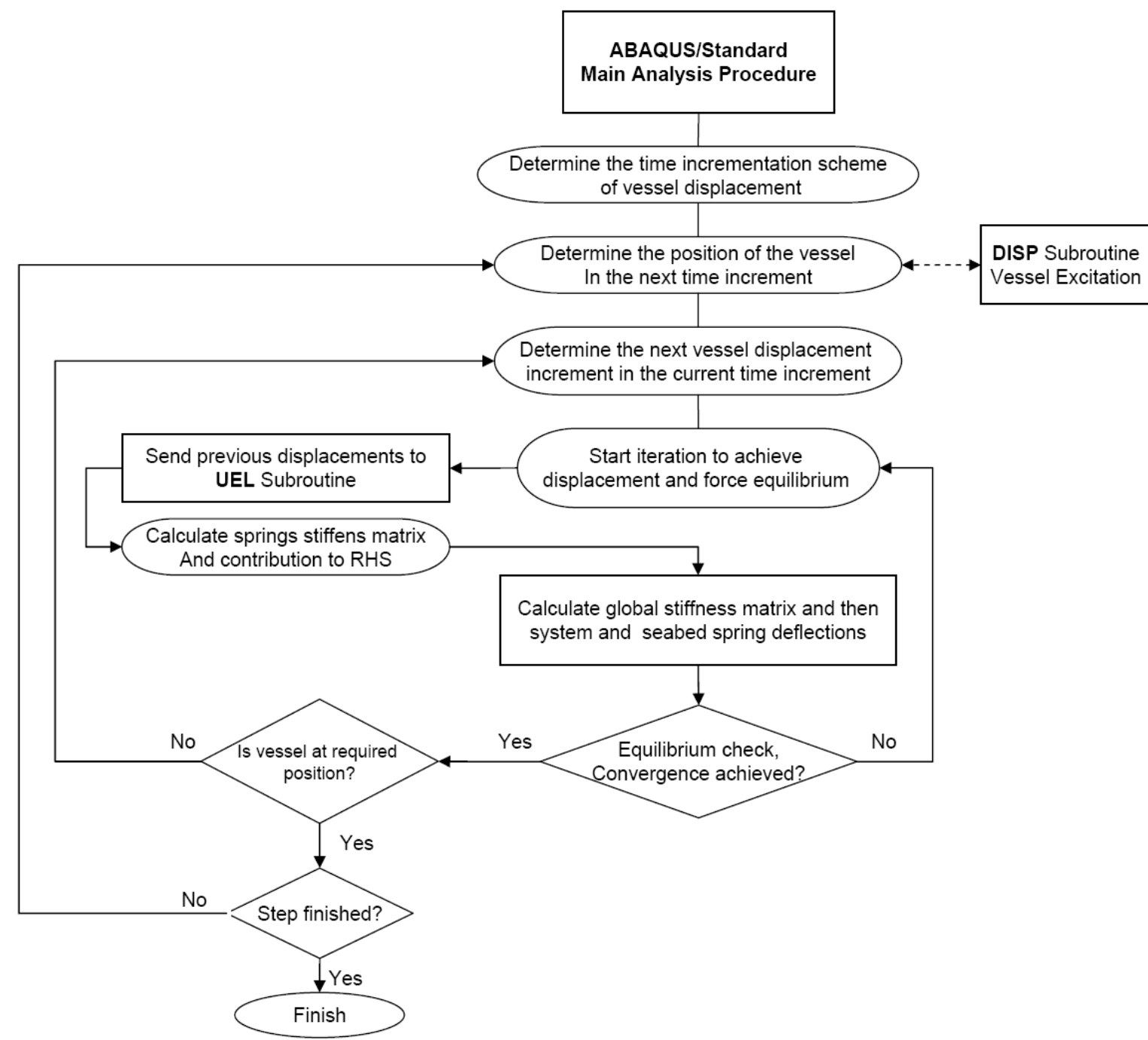

Figure 4-9: The flowchart of ABAQUS main procedure for fatigue analysis

After defining the incrementation, iterations are undertaken for each increment until force equilibrium is satisfied. ABAQUS needs to determine the global stiffness matrix for each iteration, for which it first passes the displacement history and other key data to the relevant UEL subroutine, which then supplies the seabed spring stiffness (for a nonlinear seabed response) and contribution to the RHS force vector. Upon achieving equilibrium in an increment, depending on the vessel position, another increment is 
started or a new position is requested from the DISP subroutine. This process is continued until the end of Step 3.

\subsubsection{Vessel excitation internal flowchart}

Figure 4-10 shows the steps taken inside the DISP subroutine to define the new position of the vessel for the ABAQUS main procedure. The environmental data, including the characteristics of every sea state indicated in Table 4-3, is provided to the DISP subroutine as input data. Prior to analysis, the hierarchy or the order of sea states and the number of cycles to be applied in each sea state hitting the vessel is defined manually inside the DISP subroutine, then the total step time for Step 3 is set to the total time for all the applied sea states, allowing for the number of cycles. The frequency of calling the DISP subroutine is equal to number of time increments, which is automatically decided by ABAQUS depending on the key time incrementation parameters defined in the input file including initial time increment, time period of the step and minimum and maximum time increments allowed.

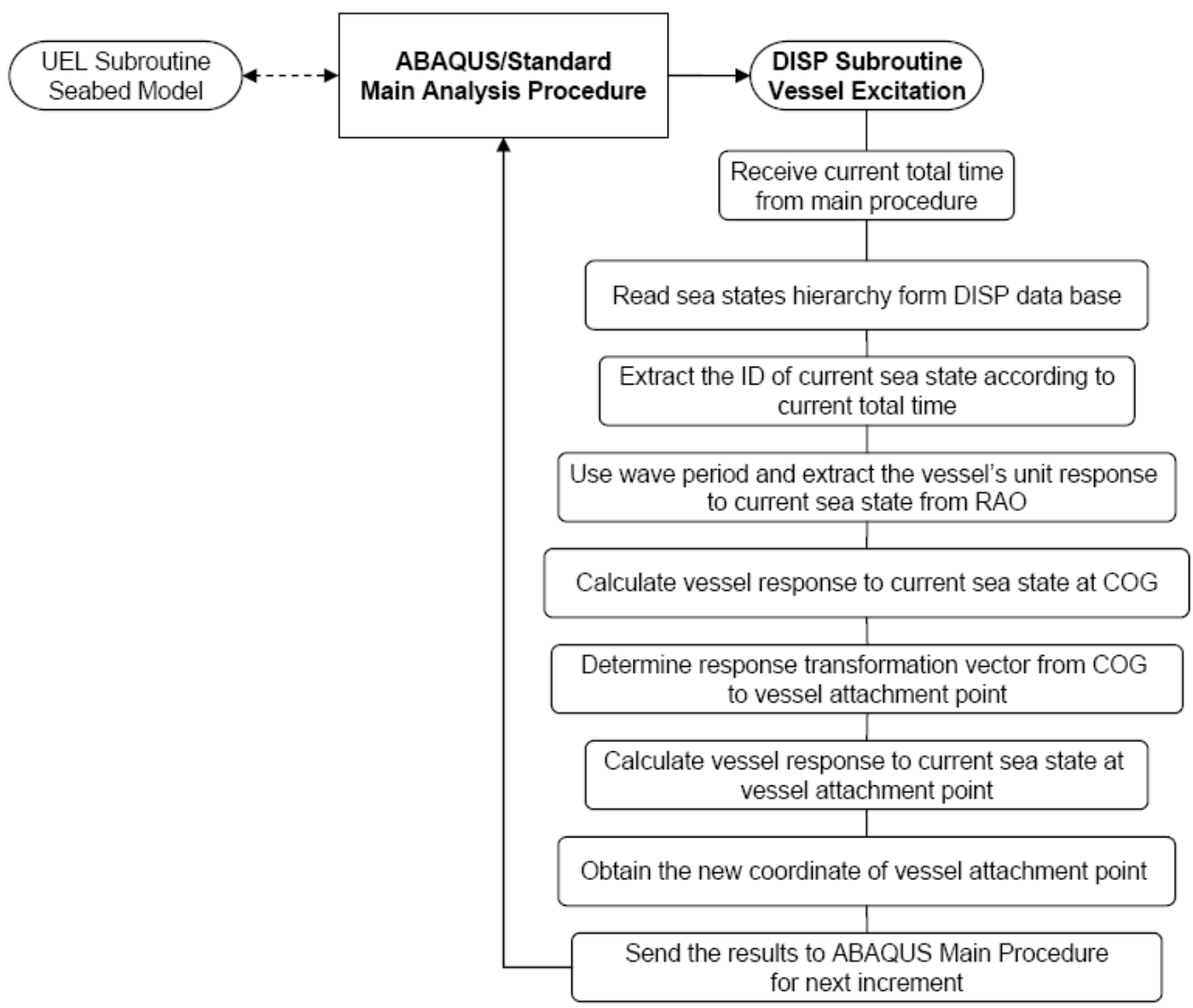

Figure 4-10: Internal flowchart of DISP subroutine for vessel excitation 
As an example, suppose the user wants to apply sea states 4, 8 and 29 to the vessel, with the number of cycles in each sea state being 10,100 and 60 respectively; then, considering the wave period in each sea state the total time for Step 3 can be calculated as:

$\mathrm{t}_{\text {total }}=5.4 \times 10+6.9 \times 100+11.3 \times 60=1422 \mathrm{~s}$

During Step 3, ABAQUS calls the DISP subroutine and passes the current analysis time step to the subroutine as the main input data, along with some other information to be used in calculations. The first action taken by the subroutine is to read the hierarchy of sea states defined by the user. Then the subroutine takes the ID of the current sea state and searches inside the data base to find the period, height and the number of cycles to be applied. The period of the wave is then compared with the RAO data base and the unit response of the vessel to that specific sea state is extracted. The subroutine now calculates the vessel response to the sea state at the $\mathrm{CoG}$, and uses the transformation matrix to calculate the corresponding response of the SCR attachment point. The response is then changed to the global coordinate system of ABAQUS and the new coordinates for the (vessel) end node of the SCR are output to the ABAQUS main procedure. This process continues according to the user-defined wave hierarchy until the end of Step 3.

\subsection{SAMPLE RESULTS IN LINEAR ELASTIC SEABED}

After finishing the analysis, the von Mises stresses through the touchdown zone are extracted from the ABAQUS output file (.odb) which provides the stress information for every increment of the analysis for the requested springs in the TDZ. This database is inserted in a dedicated worksheet of an Excel spreadsheet developed for fatigue analysis. Running the macro, the responses are separated for every sea state considering the hierarchy of sea states and global analysis time. The macro calculates the stress variation range and plots the fatigue damage distribution in the TDZ along with the stress variation history at the specified nodes. The spreadsheet is also able to accept various S-N curves and any combination of sea states with any number of cycles defined by the user. Another capability of the macro is the calculation of fatigue damage in various cycles, which is very useful in studying the effect of trench formation on fatigue performance and the application procedure of fatigue calculation for non-linear seabed response, as will be discussed in the next chapter. Figure 4-11 shows example results for the fatigue damage distribution through the TDZ. The y-axis in this plot shows the fatigue damage calculated over the 30 years, a common service life of the SCR. This analysis has been performed by applying the sea state package presented in Table 4-3 on a seabed with stiffness of $100 \mathrm{kPa}$. The fatigue damage for a linear elastic seabed is independent of the number of waves applied in the analysis to represent the sea states (as shown later in this chapter); but to make sure that the potential irregular stress change will not affect the stress range calculation during the initial stages of 
transferring from one sea state to another, the number of wave cycles applied is taken as 5 .

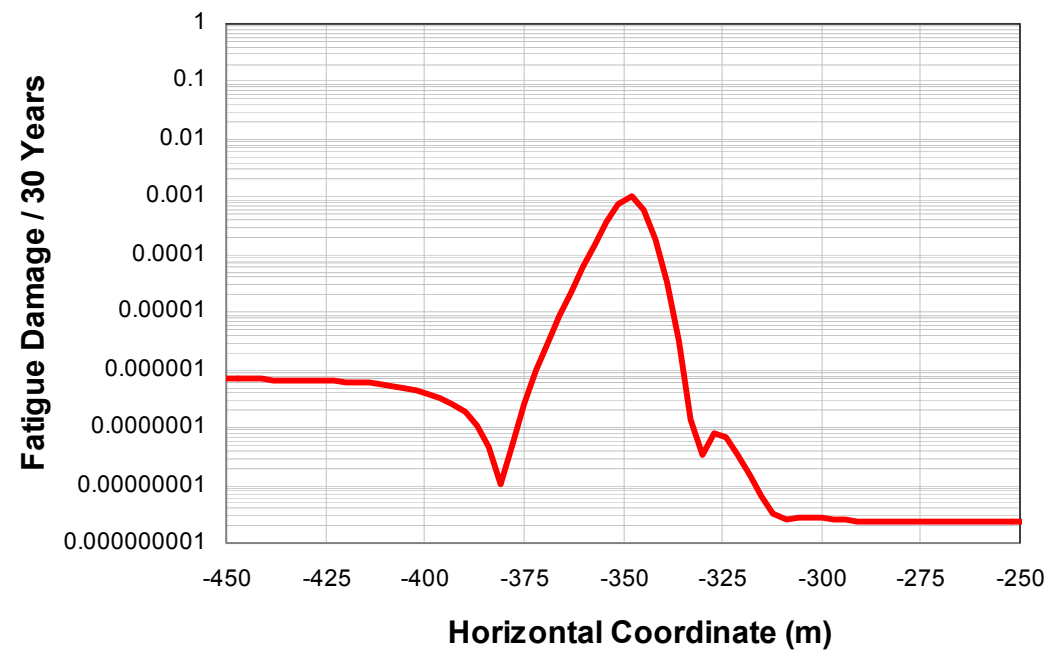

Figure 4-11: Sample fatigue damage distribution through TDZ, seabed stiffness $100 \mathrm{kPa}$

Figure 4-12 illustrates the contribution of each sea state to the total fatigue damage at the peak damage point. As mentioned before in section 4.4.2, the S-N diagram (i.e the parameters $\bar{a}$ and m) used for the fatigue damage assessed in Figure 4-12 and all of the analyses throughout the thesis are as per the values presented in Table 4-2. This plot shows that sea state number 10 (Table 4-3), with a moderate magnitude of significant wave height and period and considerable number of applied waves, has the greatest contribution to the total fatigue damage. The interesting part of the plot is the considerable fluctuation for the sea states beyond number 20. Given the relatively linear variation of significant wave height and the period (see Figure 4-13 and Figure 4-14 ), such significant fluctuation appears to be due to the minor irregularities in the number of applied waves for these sea states, as seen in Figure 4-15. This emphasises the significance of the number of applied waves for the sea states of greater significant wave heights, which is directly affected by defining the windows of sea states to linearise the system response. The windows in the wave scatter diagram applied in this study (BP sea states, Table 4-3) are defined taking the $\Delta \mathrm{H}_{\mathrm{s}}$ as $0.5 \mathrm{~m}$. The cumulative fatigue damage for the waves ordered from minimum to maximum height is shown in Figure 4-16. 


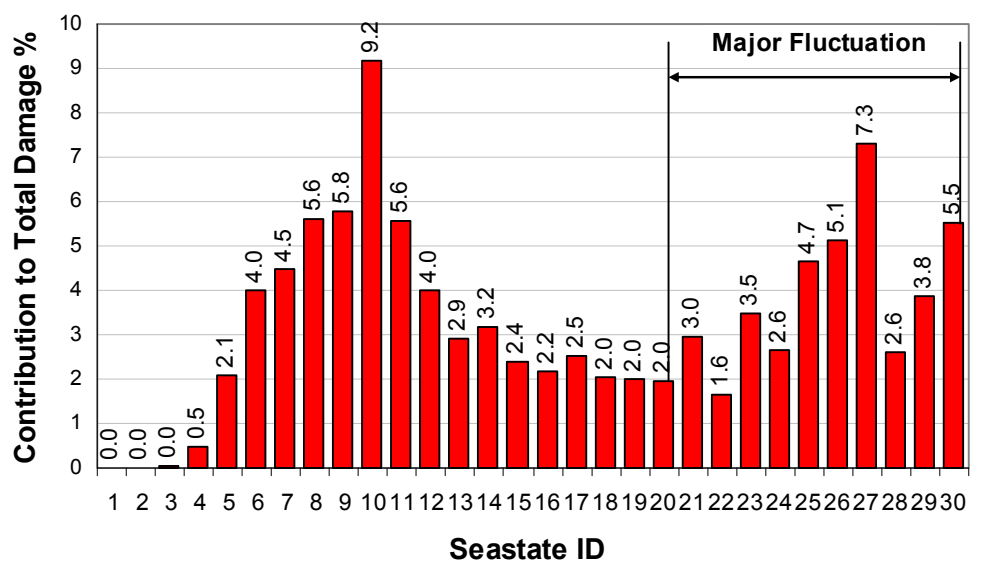

Figure 4-12: Contribution of each sea state to total fatigue damage

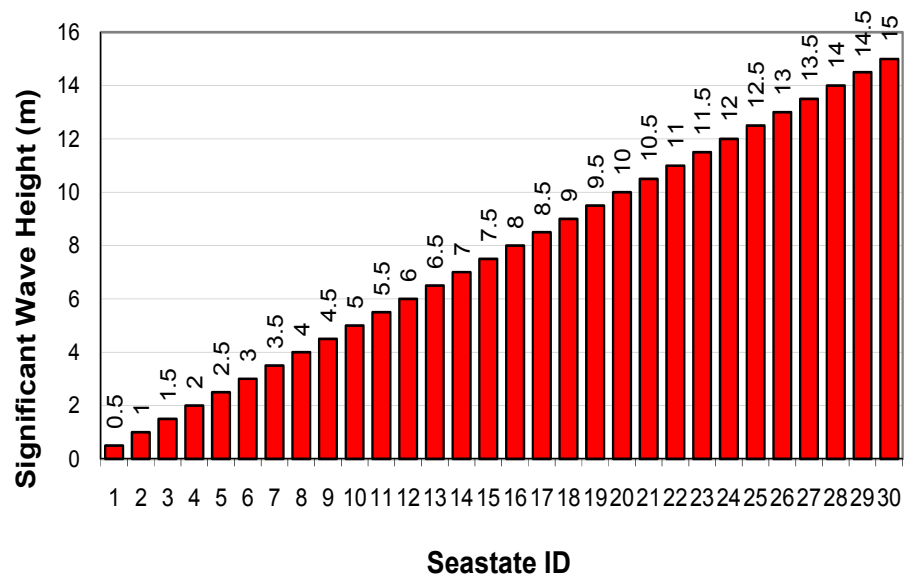

Figure 4-13: Variation of significant wave height versus sea state ID

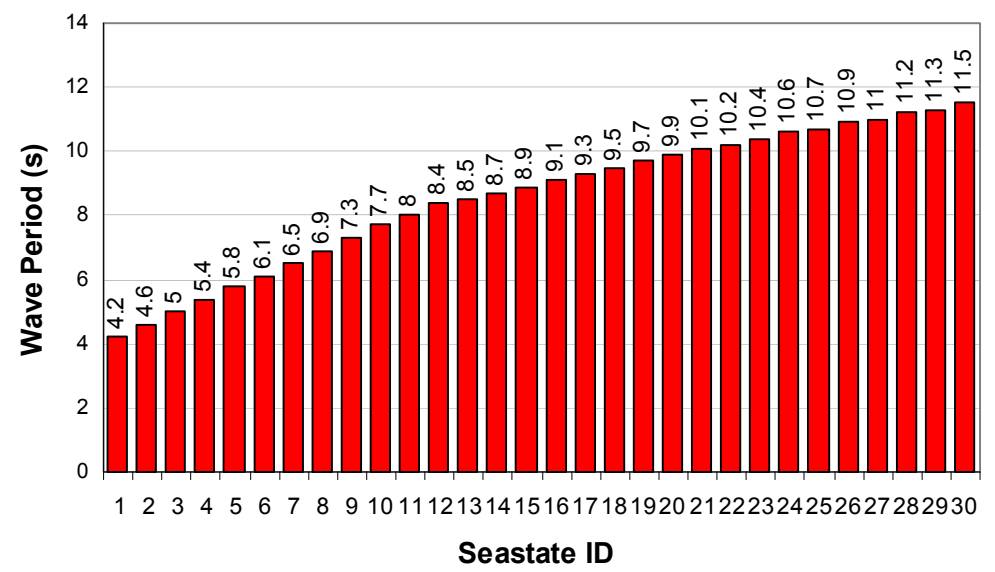

Figure 4-14: Variation of wave period versus sea state ID 


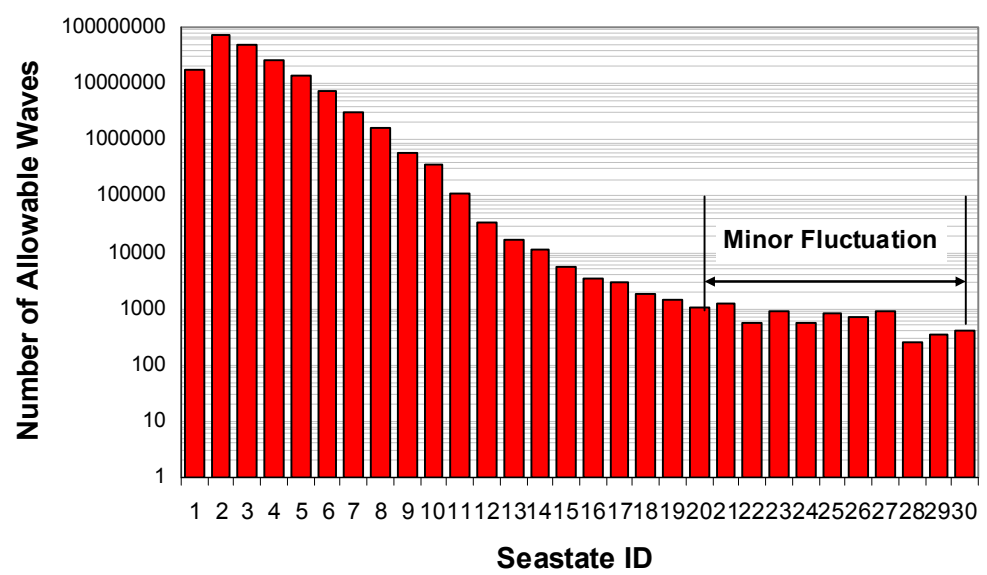

Figure 4-15: Variation of number of applied waves versus sea state ID

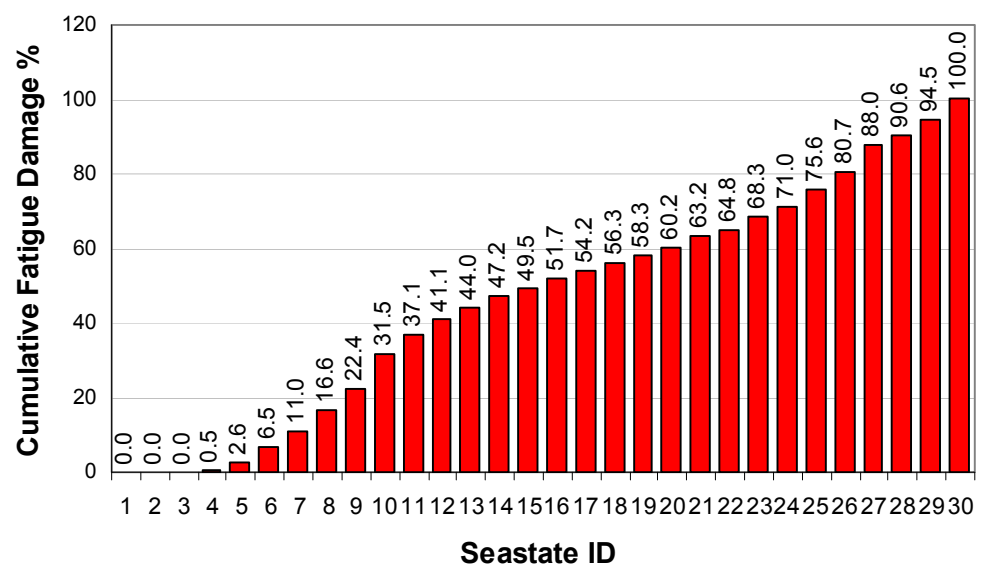

Figure 4-16: Cumulative fatigue damage for the order of min to max vessel offset

\subsubsection{Fatigue damage distribution morphology}

Figure 4-17 illustrates the geometrical position of the fatigue damage distribution in comparison with the static (unperturbed) SCR seabed profile, bending moment, shear force and seabed reaction diagrams for a linear elastic seabed with a stiffness of 100 $\mathrm{kPa}$. The fatigue damage distribution shows two peak values of damage in the TDZ, a major peak on the vessel side, where the bending moments are positive and the critical fatigue position is at the invert of the SCR, and a minor peak on the anchor side, resulting from negative bending moments with the critical fatigue position being on top of the SCR. Fatigue results from the cumulative changes in von Mises stress, which in turn is dominated by the change in bending moment. The change in bending moment results primarily from the lateral shift of the TDP (in x-direction) and thus the bending moment profile, so the change in bending moment is closely linked with the shear force, which is the gradient of bending moment (Bridge et al. 2004). Because of this, the overall shape of the fatigue damage distribution is extremely similar to the shear force 
distribution, highlighting the significance of the shear force distribution in the TDZ. A wide range of analyses show that depending on the magnitude of the seabed stiffness, the peak fatigue damage occurs at some point between the TDP and the peak bending moment location, but closer to the TDP as shown in Figure 4-17. The peak shear force and peak fatigue damage are located at the same position due to the direct relationship between them, as described above. The seabed reaction increases from zero at the TDP to a peak value located at the deepest penetration of the SCR. It is also seen that the sign of the fatigue damage gradient changes close to the point of curvature change.

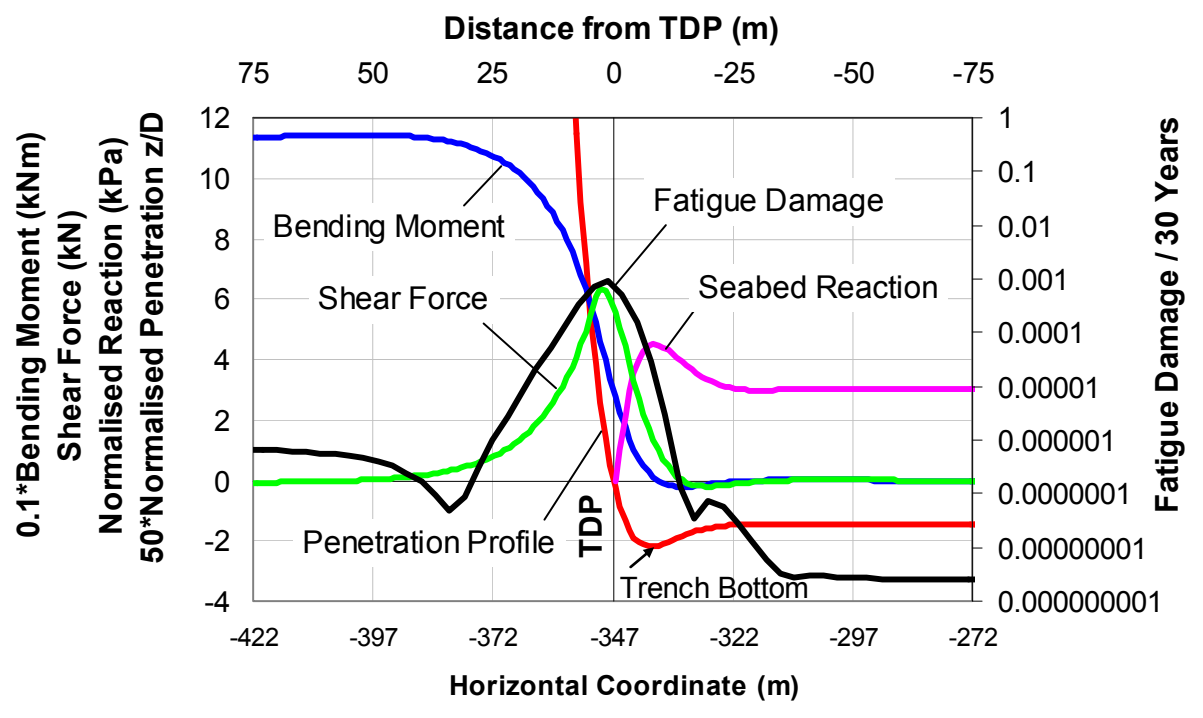

Figure 4-17: Morphology of fatigue damage distribution

\subsubsection{Influence of wave orders and wave number of cycles}

Modelling the seabed as a linear elastic medium means that neither the order of sea states nor the number of applied cycles for each sea state affect the fatigue damage (assuming that the damage from each sea state is scaled according to the true number of cycles). In Figure 4-18, two different analyses with different number of cycles have been compared, applying the sea states of Table 4-3. In the first analysis the fatigue damage has been calculated by applying 5 cycles of each sea state and then the analysis has been repeated by applying 10 cycles of each sea state. In both analyses, the number of allowable cycles has been calculated based on the stress range variation within the last cycle of each sea state. As is seen, the results are identical. Figure 4-19 shows a comparison of two analyses with different ordering of the applied sea states. In the first analysis, the sea states have been put in order of descending values of amplitude and wave period. Then, the same analysis has been repeated for a random order of sea states. The results show perfect coincidence of two curves for 5 and 10 cycles of each sea state. 


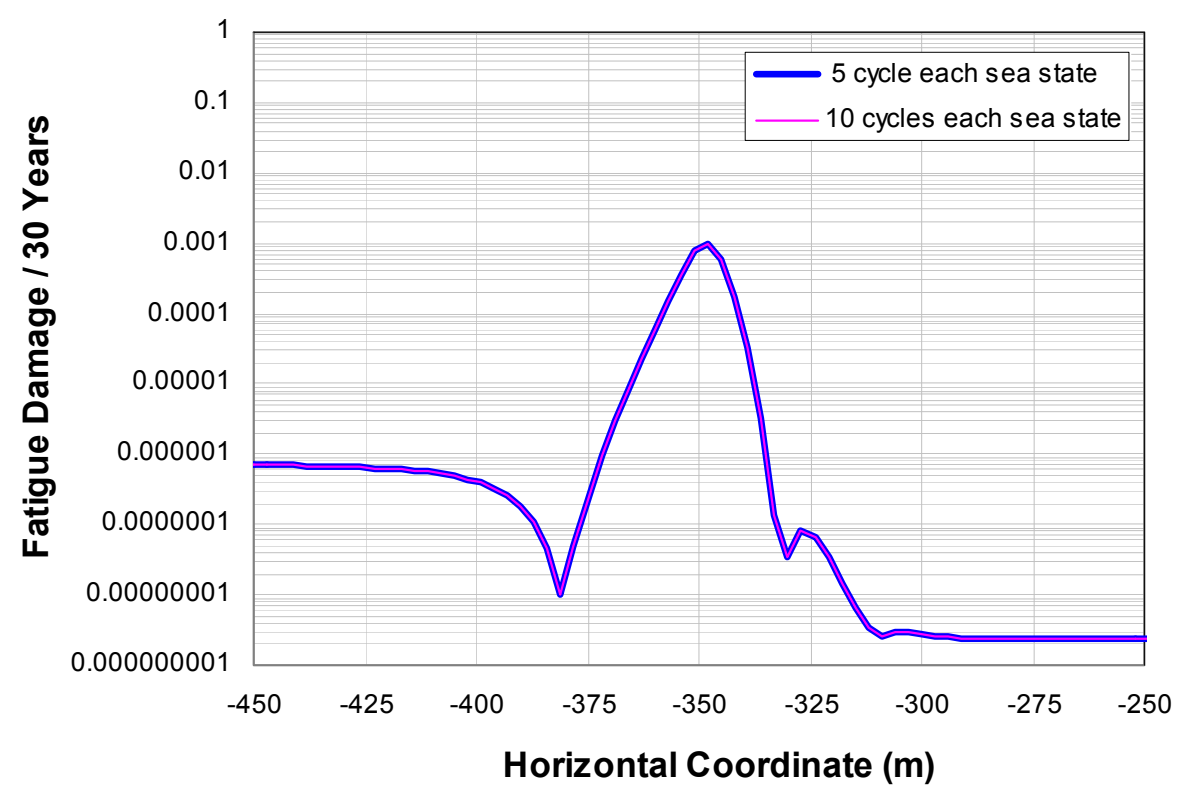

Figure 4-18: Fatigue damage with different number of cycles

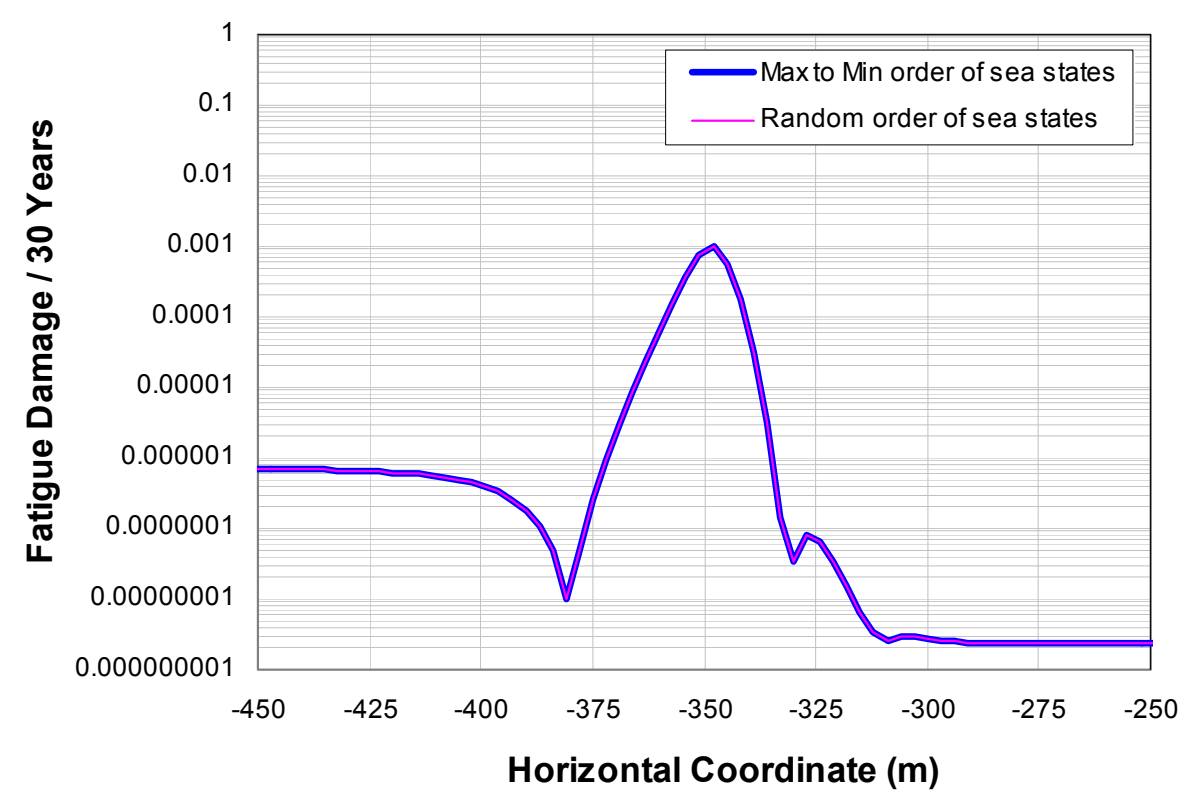

Figure 4-19: fatigue damage with different order of sea states

\subsubsection{Influence of seabed stiffness on fatigue}

A series of fatigue analyses were performed for various values of seabed stiffness. As is well known and illustrated in Figure 4-20 and Figure 4-21, the stiffer the seabed the greater is the fatigue damage and the shorter the fatigue life (a safety factor of 10 has been applied). As the seabed stiffness decreases, the peak fatigue damage moves slightly towards the vessel end of the SCR. 


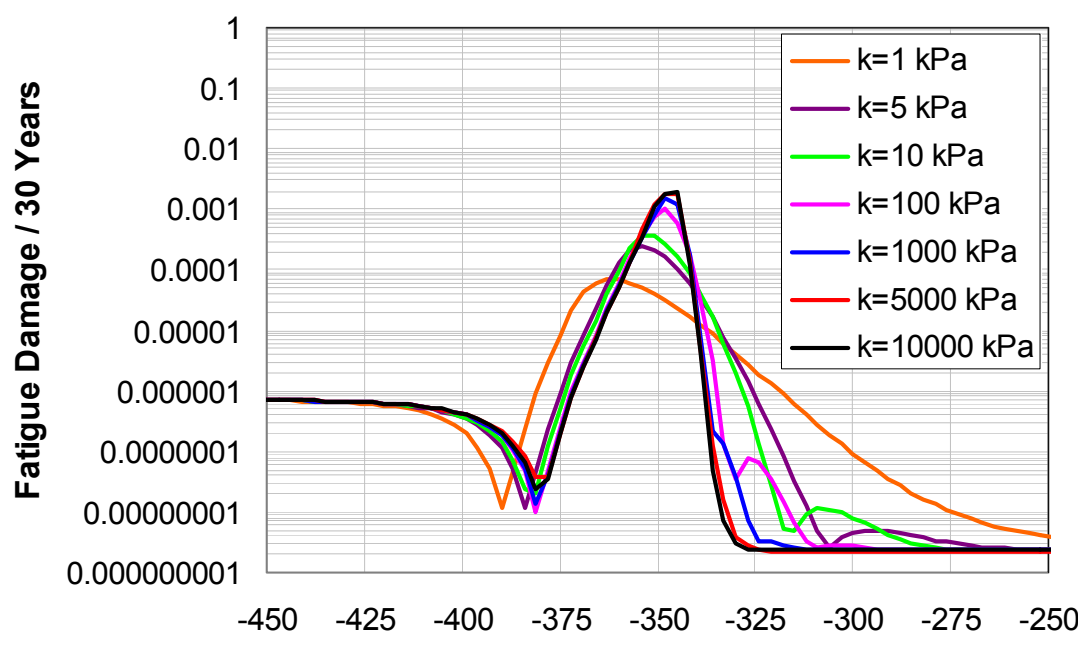

Horizontal Coordinate $(\mathrm{m})$

Figure 4-20: Fatigue damage variation with seabed stiffness

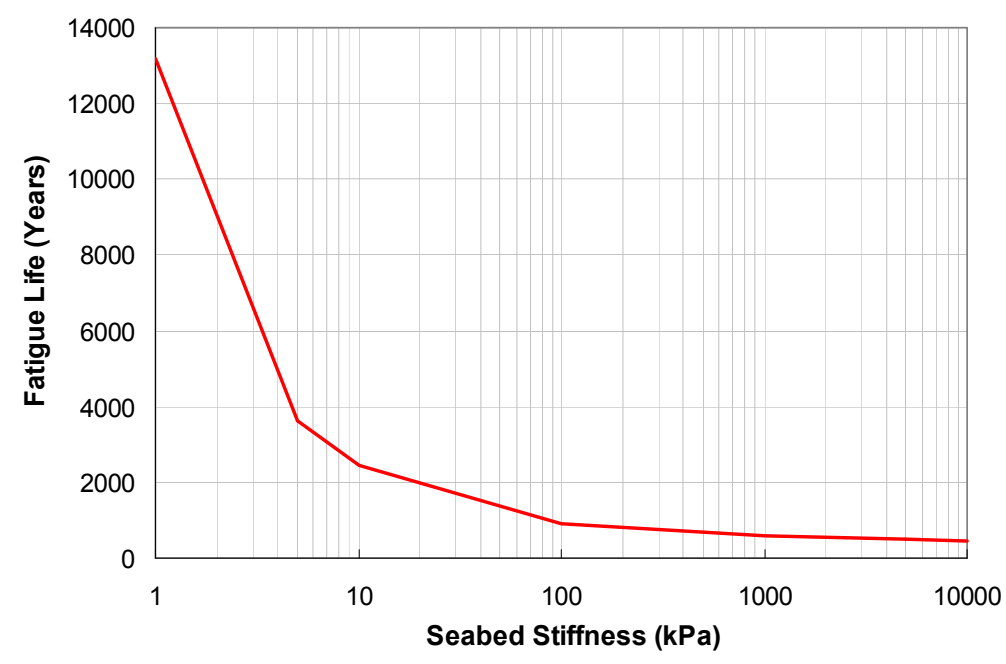

Figure 4-21: Fatigue life variation with seabed stiffness

Figure 4-22 illustrates the relative fatigue damage for different seabed stiffness compared with a very stiff seabed $(\mathrm{k}=10,000 \mathrm{kPa})$. The global trend agrees well with the results reported by Bridge (2004), Figure 4-23, with minor differences due to different wave scatter diagrams and SCR configuration. 


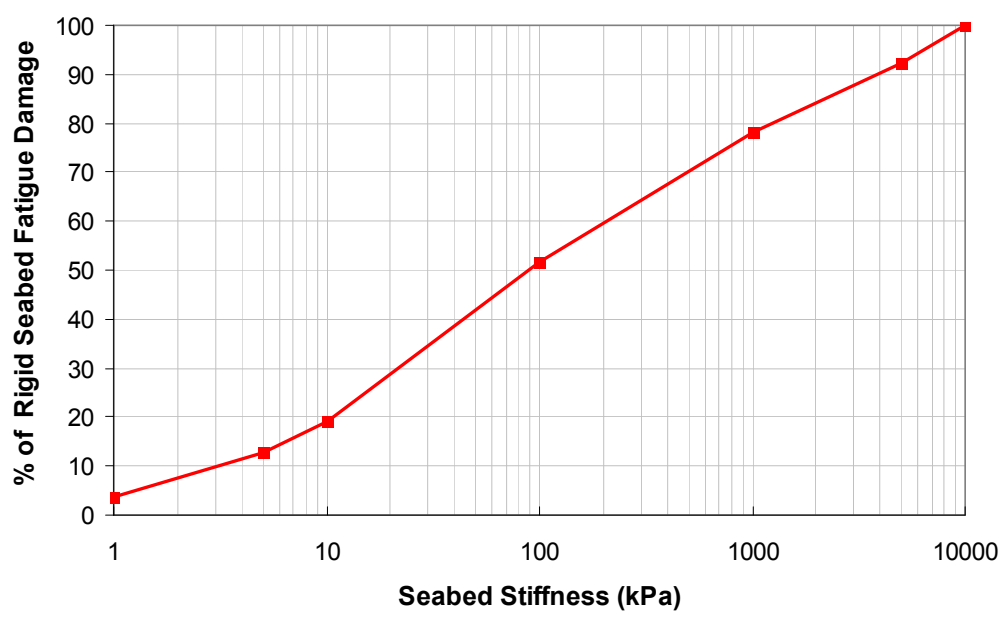

Figure 4-22: Percentage of rigid seabed fatigue damage with varying seabed stiffness

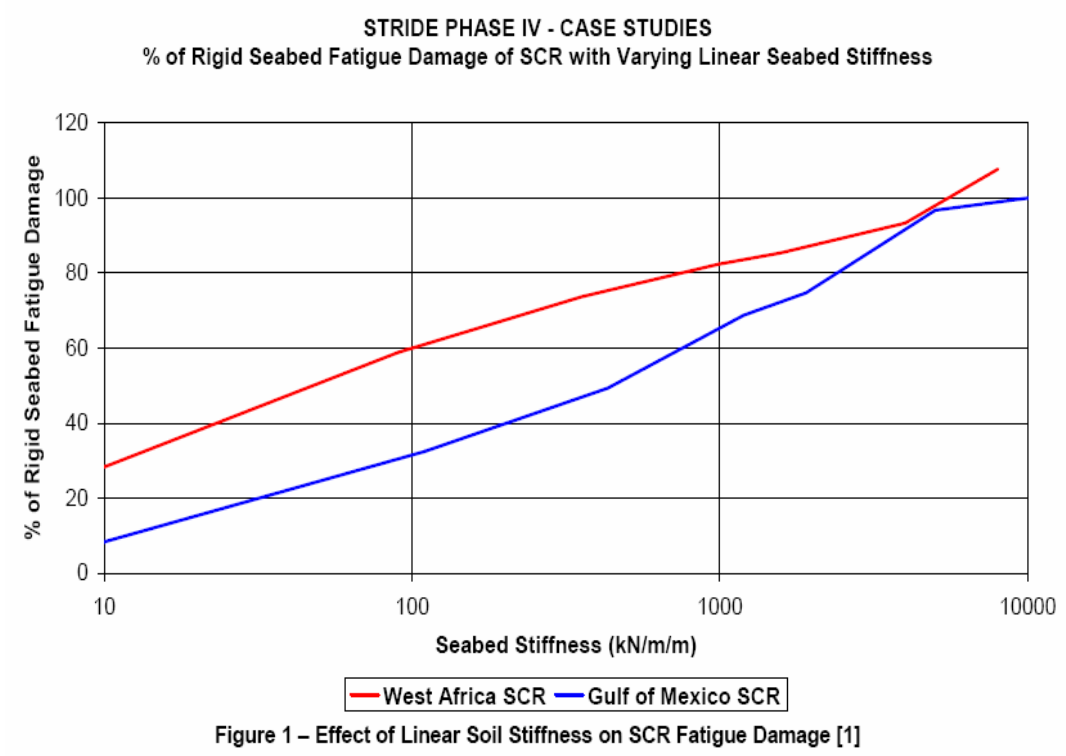

Figure 4-23: Damage rate of various seabeds to rigid seabed (Bridge et al. 2004)

Spot checks of the results presented in Figure 4-21 also show good agreement in the overall trends with results obtained using OrcaFlex, reported by Randolph and Quiggin (2009), Figure 4-24. Again, the SCR configuration and wave scatter diagram were not the same as in the present analysis and some differences are expected. 


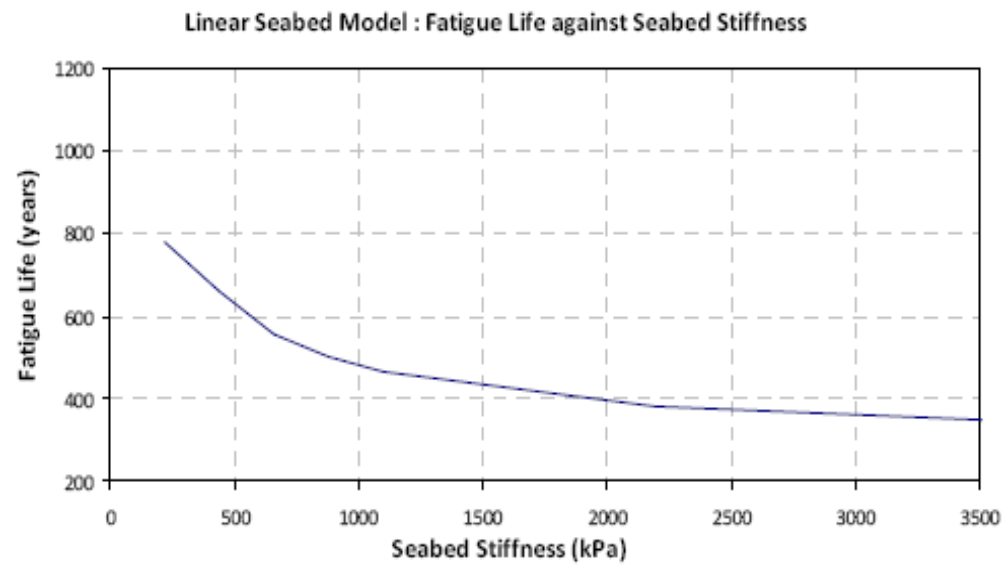

Figure 4-24: Fatigue life is decreased as seabed stiffness increased (Randolph and Quiggin 2009)

As the seabed stiffness increases, the maximum seabed reaction and shear force increase and the position moves towards the anchor end, Figure 4-25 and Figure 4-26 .

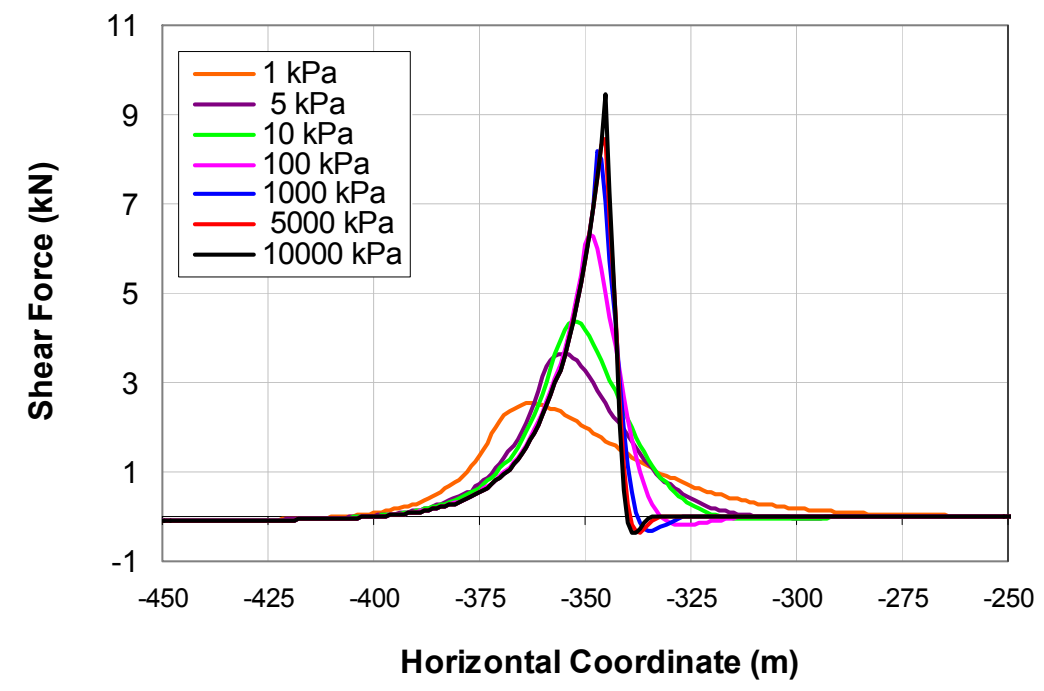

Figure 4-25: Shear force distribution along TDZ in various seabed stiffness 


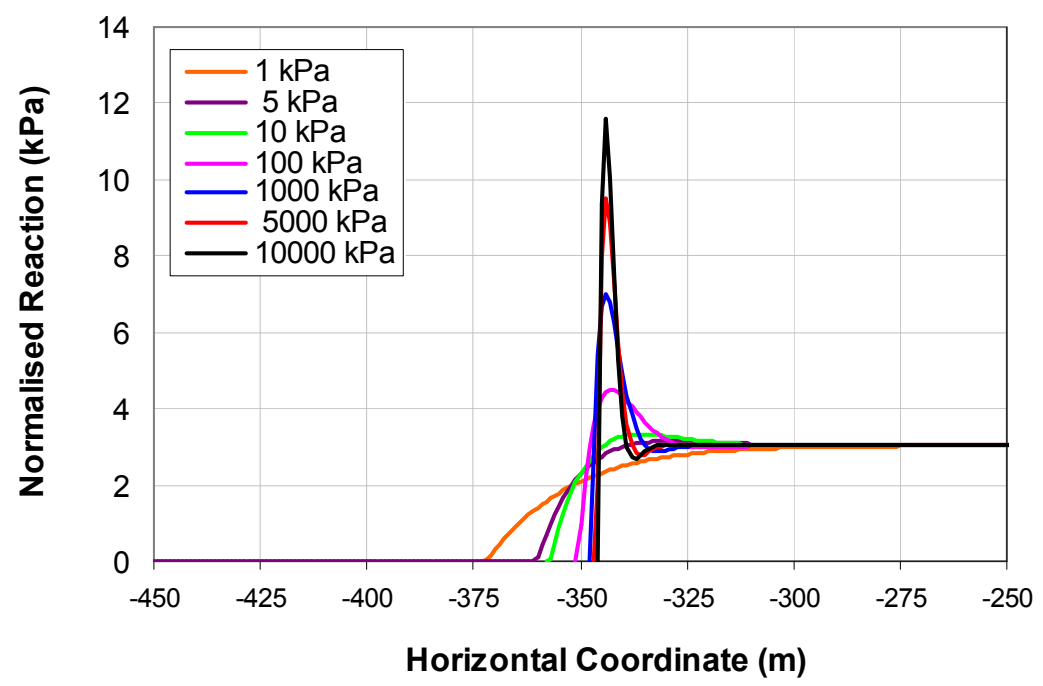

Figure 4-26: Seabed reaction along TDZ in various seabed stiffness

A comparison between the peak fatigue damage for seabeds with stiffness of $1 \mathrm{kPa}$ and $10 \mathrm{kPa}$ in Figure 4-20 shows that the fatigue damage for the stiffer seabed is $563 \%$ higher than for the softer seabed $(0.0004 / 0.000071=5.63)$, highlighting the significant influence of seabed stiffness on fatigue damage, as the seabed becomes softer. The difference in fatigue damage can be explained approximately by theory as follows.

From equation (4-1), taking $\mathrm{m}=3$ (see Figure 4-1), the relative fatigue damage for two different stress ranges $\sigma_{\mathrm{f} 2}$ and $\sigma_{\mathrm{f} 1}$ is:

$\frac{\mathrm{D}_{1}}{\mathrm{D}_{2}}=\left(\frac{\sigma_{\mathrm{f} 2}}{\sigma_{\mathrm{f} 1}}\right)^{3.0}$

It was shown that the stress range is directly related to the shear force and, using the BLM, the shear force may be expressed as a function of the normalised seabed stiffness (see equation (3.80)), so that

$\frac{\mathrm{D}_{2}}{\mathrm{D}_{1}}=\left(\frac{\mathrm{K}_{2}^{0.25}}{\sqrt{2}+\mathrm{K}_{2}^{0.25}} \frac{\sqrt{2}+\mathrm{K}_{1}^{0.25}}{\mathrm{~K}_{1}^{0.25}}\right)^{3.0} \quad$ where $\mathrm{K}=\mathrm{k} \frac{\mathrm{EI}}{\mathrm{H}^{2}} \approx 0.274 \mathrm{k}$

Substituting the actual stiffness, $\mathrm{k}_{1}$ and $\mathrm{k}_{2}$ gives:

$$
\frac{\mathrm{D}_{2}}{\mathrm{D}_{1}}=\left(\frac{\mathrm{k}_{2}}{\mathrm{k}_{1}}\right)^{3 / 4}\left(\frac{\sqrt{2}+\left(0.274 \mathrm{k}_{1}\right)^{0.25}}{\sqrt{2}+\left(0.274 \mathrm{k}_{2}\right)^{0.25}}\right)^{3}
$$

Substituting the actual values of stiffness, $\mathrm{k}_{1}=1 \mathrm{kPa}$ and $\mathrm{k}_{2}=10 \mathrm{kPa}$, gives: 
$\frac{\mathrm{D}_{2}}{\mathrm{D}_{1}}=2.80$

This shows good agreement with the results of Figure 4-20, as described above. Figure 4-27 illustrates the accuracy of the calculated proportional fatigue damage using the maximum shear force predicted by analytical solutions against the damage ratio obtained from numerical simulation. The results show relatively good correlation for the linear elastic seabed.

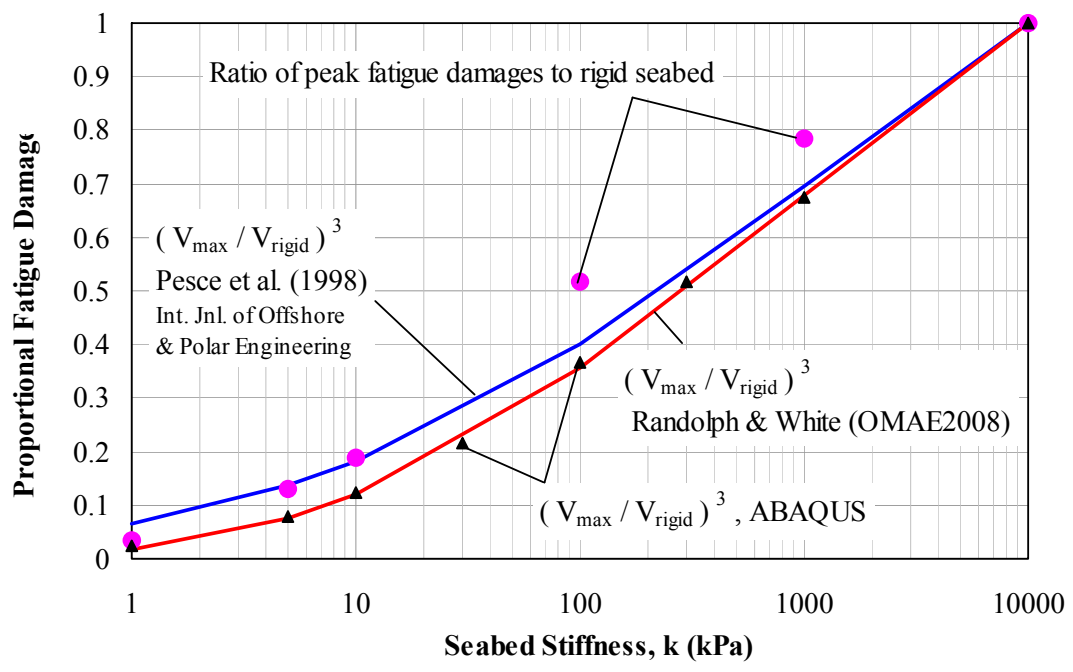

Figure 4-27: Proportional fatigue damage obtained from analytical solutions and numerical simulations

These results highlight the vital impact of the seabed interaction model on prediction of fatigue damage and consequently fatigue life.

\subsection{FATIGUE DAMAGE PREDICTION BY CLOSED FORM SOLUTIONS}

Examination of closed form solutions in modelling the seabed interaction in Chapter 3 showed a good correlation between the boundary layer method and numerical analysis in prediction of the maximum shear force in the touchdown zone. Now, the findings regarding stress distributions obtained by the boundary layer methods in Chapter 3 are used here to propose a simple methodology for fatigue damage calculation. Since fatigue damage occurs mainly through changes in bending stress in the SCR, and since the change in bending moment is due to lateral shift of the TDP (in x-direction) and thus bending moment profile, the fatigue damage is closely linked to the shear force (since this is the gradient of the bending moment). As such, the catenary equations linked with a suitable boundary layer method may be used to estimate the maximum shear force, and hence to approximate the maximum fatigue damage for a given seabed stiffness. 
As described in Chapter 3, the maximum von Mises stress can be written as follow:

$$
\sigma_{f-\operatorname{Max}}=\frac{H}{A_{s}}+\frac{M_{M a x} D}{2 I}
$$

As shown in equation (4-1), the fatigue damage is calculated using the von Mises stress range $\Delta \sigma_{\mathrm{f}}$ within a load cycle (a cycle of vessel perturbation). Using equation (4-11) and ignoring the minor change in horizontal force in the TDZ due to relocation of the touchdown point, $\Delta \sigma_{\mathrm{f}}$ can be approximated as:

$$
\Delta \sigma_{\mathrm{f}-\mathrm{Max}} \approx \frac{\Delta \mathrm{M}_{\mathrm{Max}} \mathrm{D}}{2 \mathrm{I}}
$$

Figure 4-17 shows that the maximum fatigue damage occurs in the vicinity of the TDP, so that equation (4-12) can be written approximately in terms of the initial maximum shear force and the TDP relocation, as:

$$
\Delta \sigma_{\mathrm{f}-\mathrm{Max}} \approx \frac{\mathrm{V}_{\max } \Delta \mathrm{x}_{\mathrm{TDP}} \mathrm{D}}{2 \mathrm{I}}
$$

where

$$
\begin{aligned}
& \Delta \mathrm{x}_{\mathrm{TDP}} \text { is the maximum TDP relocation under a vessel perturbation cycle } \\
& \mathrm{V}_{\max } \text { is the initial (or static) maximum shear force in the TDZ. }
\end{aligned}
$$

The accuracy of equation (4-13) was examined in Chapter 3. The results of numerical analyses show that the maximum shear force on a given seabed for the various vessel positions corresponding to the imposed sea states does not change much, and the value can be estimated using a boundary layer method as described in Chapter 3. Substituting the value of $V_{\max }$ into equation (4-13) and using this value of $\Delta \sigma_{\mathrm{f}-\mathrm{Max}}$ in equation (4-1) allows estimation of the fatigue damage per cycle. Figure 4-28 summarises the recommended calculation flowchart for fatigue damage using catenary equations and the boundary layer method for seabed interaction. 


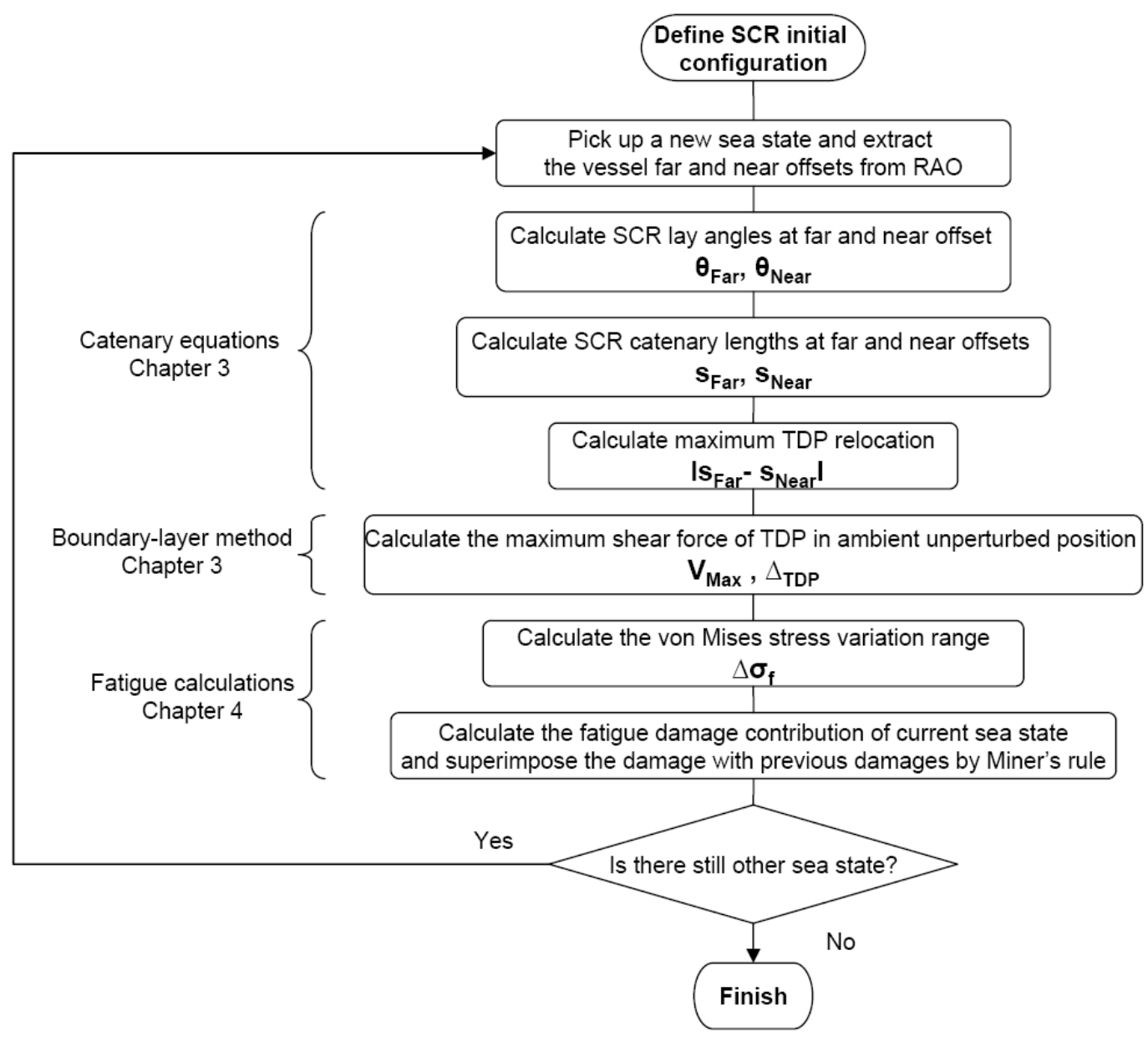

Figure 4-28: Fatigue damage prediction flowchart using boundary layer method

Figure 4-29, compares the maximum fatigue damage in the TDZ for various seabed stiffness values, as obtained from numerical analysis and the above closed form prediction. The vessel excitation has been evaluated based on the sea states presented in Table 4-3. The good agreement of the results is very interesting and valuable for the riser industry, enabling a quick estimation of SCR fatigue life based on seabed stiffness without running time-consuming numerical fatigue analyses and simulations. Figure 4-30 show a comparison of fatigue life predicted by both methods in a non-logarithmic scale. The results show very good correlation for seabed stiffness about $100 \mathrm{kPa}$, which is the normal range assumed for the seabed stiffness in deepwater sediments. Even for a soft seabed, the discrepancy is relatively small, and the closed form result lies on the conservative side. 


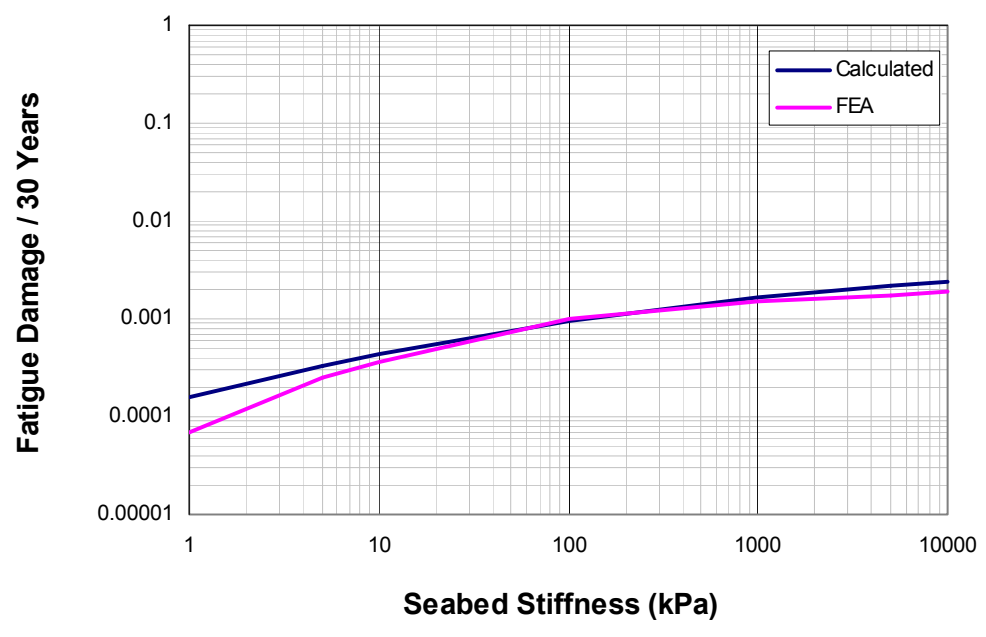

Figure 4-29: Comparison of fatigue damage predicted by numerical analysis and closed form solutions

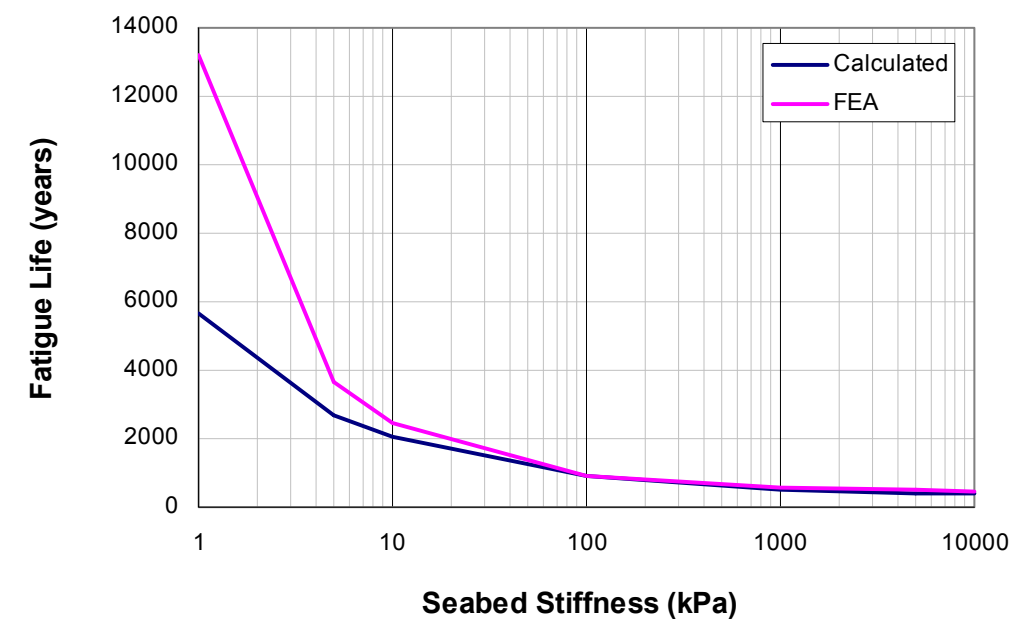

Figure 4-30: Comparison of fatigue life predicted by numerical analysis and closed form solutions

\subsection{SUMMARY AND CONCLUSIONS}

The fatigue performance of SCRs on a linear elastic seabed, which is the current standard industry approach, has been discussed in detail and the fundamentals have been reviewed. This serves as an introduction to fatigue analysis for non-linear plastic seabed response, which will be discussed in the following chapters.

The ABAQUS finite element software has been used to model the SCR system, supported by a Spar vessel and resting on a linear elastic seabed. The seabed has been modelled using user-defined linear elastic springs coded through a UEL subroutine in ABAQUS. Evaluation of the vessel excitation under various sea states, which is the 
primary input into fatigue analysis, has been described and an internal procedure coded in a DISP subroutine in ABAQUS to provide the time series of perturbations at the SCR attachment point to the Spar. Post processing of the analysis results in macro-driven Excel spreadsheets has been described, where the representative wave cycles of each sea state for calculation of the stress range are extracted from a continuous time series analysis and the damage encountered by each sea state is calculated to obtain the cumulative fatigue damage at various points along the SCR. The macro-driven Excel spreadsheet also provides the possibility of parametric study along with other useful plots such as the stress range variation history for a given point on the SCR.

A series of fatigue calculations have been performed to study the influence of seabed stiffness on the distribution and magnitude of fatigue damage and the results has been compared with other published data. The profile of fatigue damage has been studied in relation to profiles of shear force, seabed reactions, bending moment and SCR embedment in the TDZ and the significant importance of the shear force distribution and seabed reaction (or stiffness) has been highlighted. Likewise, the insensitivity of fatigue damage to the order of sea states and the number of applied cycles analysed in order to evaluate the stress ranges has been confirmed.

As anticipated, the results showed that stiffer seabeds give higher fatigue damage and shorter fatigue life, with the location of the maximum damage moving towards the vessel end of the SCR as the seabed stiffness decreases. The peak fatigue damage occurs somewhere between the ambient TDP and the point of maximum bending moment, but closer to the TDP. Considerable decrease in fatigue damage occurs as the stiffness reduces below $10 \mathrm{kPa}$, which is an important indicator of the potential influence of a non-linear seabed interaction model on the estimated fatigue performance of SCRs. The significant dependence of fatigue damage on shear force distribution and seabed contact stress, which in turn are affected by the SCR profile in the TDZ and the seabed stiffness, suggests that a non-linear seabed model may lead to much lower fatigue damage in the TDZ, as will be discussed in the following chapters.

A simple procedure was introduced to allow a quick estimation of the maximum fatigue damage through a combination of catenary equations and boundary layer solution for seabed interaction. Comparison of the results of this method and numerical fatigue analysis for various seabed stiffness shows reasonably good agreement. Even though the analysis was limited to static response of the SCR, ignoring inertia-driven dynamic effects, this suggests that the method might prove very valuable in the initial stages of SCR design, allowing an appropriate estimation of the fatigue life by means of a simple hand calculation without the need for computationally complex fatigue analyses. 


\section{CHAPTER 5. NON-LINEAR SCR-SOIL INTERACTION THEORY}

\subsection{INTRODUCTION}

As noted in the previous chapter, the stiffness of the riser-seabed interaction response has a significant effect on the SCR system, especially the fatigue life in the touchdown zone. Simple linear elastic springs are currently used to model the contact between the SCR and the seabed whilst, in reality, the appropriate stiffness varies through the touchdown zone, depending on the amplitude of cyclic displacements (Clukey et al. 2005; Clukey et al. 2008). The linear elastic simplification leads to over-conservative predictions of fatigue damage in the TDZ, as will be discussed in the next chapter. Nonlinear constitutive riser-soil interaction models are needed, able to capture the non-linear force-displacement relationship, taking into account different aspects of soil behaviour, such as high unload-reload stiffness, limiting penetration and uplift resistance, hysteretic effects of cycling loading with incremental penetration and trench formation etc in order to arrive at more accurate predictions of system behaviour, in particular for SCR fatigue performance. Randolph and Quiggin (2009), described a mathematical non-linear hysteretic seabed model for catenary pipeline contact encompassing many of these aspects. The model has been adopted in this thesis and implemented into ABAQUS, through the coding of a user defined element (UEL) in FORTRAN, to study the model performance and its influence on SCR fatigue life in the TDZ. In this chapter, the hysteretic non-linear soil model will be discussed in detail and its performance will be examined using ABAQUS to investigate the effect of the seabed model and its influence on trench formation and on the contact stress distribution along the SCR, which in turn affects the fatigue performance in the TDZ.

\subsection{PRINCIPLES OF NON-LINEAR SOIL MODELS}

As illustrated in Figure 5-1, a basic non-linear soil model should incorporate a few key features as follows (Aubeny and Biscontin 2006; Bridge et al. 2004)

- a non-linear backbone curve for initial loading;

- a non-linear unloading curve with high initial stiffness;

- a breakout resistance due to suction between the riser and the soil;

- an initially convex re-loading curve transient from soft to stiffening response. 


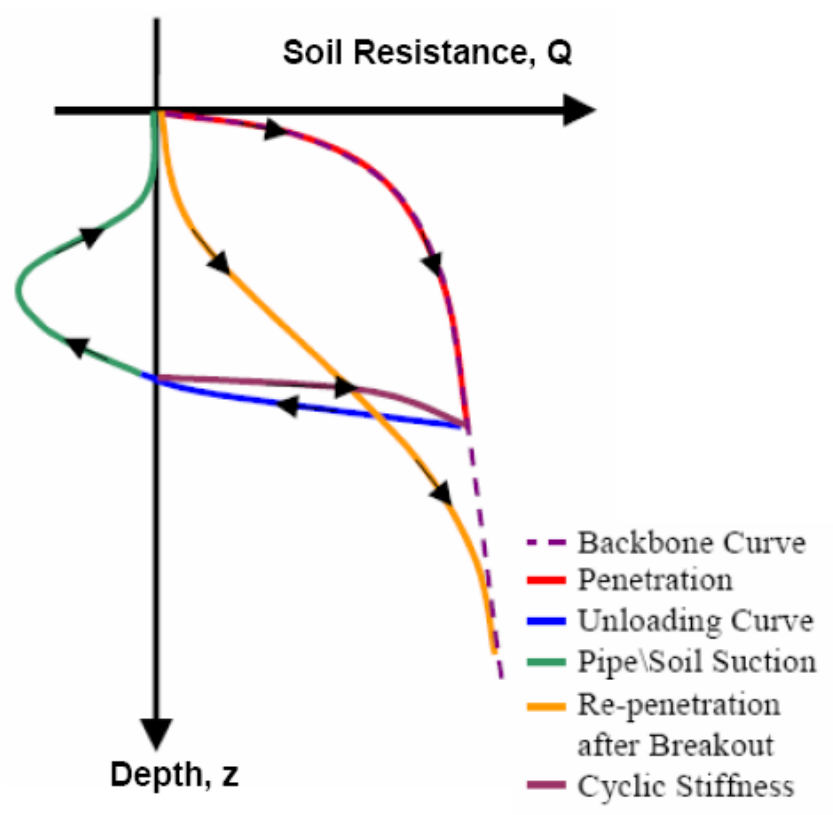

Figure 5-1: Non-linear riser-seabed interaction model (Bridge et al. 2004)

During initial penetration, the riser penetrates to a depth where the soil resistance balances the penetration force, following a backbone curve. Uplift of the riser causes the soil resistance to reduce rapidly to zero over a relatively small displacement. As the uplift continues, a tensile force is developed due to suction between the soil and the riser. The suction force first increases to a maximum value and then reduce to zero over a particular displacement. With further uplift, the riser breaks out of the soil. When the riser is pushed down again, the riser re-penetrates into the existing trench created during initial penetration; the initial value of re-penetration resistance is zero when the riser enters in the trench. The re-penetration resistance then rises (slowly at first) until it rejoins the backbone curve at a penetration depth beyond the previous penetration; further penetration then follows the backbone curve. A significant feature that is not captured by this model is the effect of cyclic loading on the soil response, particularly for cycles that cause the net load between riser and soil to oscillate through zero.

Randolph and Qiggin (2009) presented a mathematical non-linear soil model incorporating the above features, and also able to model incremental penetration during cyclic displacements, and hence trench formation. The model was intended for use in practical engineering analysis and has been implemented in a commercial riser analysis program, OrcaFlex (Orcina 2008). The model's main features are as follows:

- The seabed normal resistance is modelled using four penetration modes, namely Not-in-Contact, Initial penetration, Uplift and Re-penetration.

- In each penetration mode the seabed reaction force per unit length, $\mathrm{P}(\mathrm{z})$, is modelled using an analytic function of the non-dimensional penetration $z / D$, where $\mathrm{z}$ is the penetration (pipe invert) and $\mathrm{D}$ is the pipe diameter. 
- For each mode, the analytical formulae use a hyperbolic term, which provides a high stiffness response for small reversals of motions, but ensures that the resistance $\mathrm{P}(\mathrm{z})$ asymptotically approaches the soil ultimate penetration resistance (for penetration) or ultimate suction resistance (for uplift), as the penetration $\mathrm{z}$ increases or decreases from its value when this episode of penetration or uplift started (Randolph and Quiggin 2009).

\subsection{RANDOLPH AND QUIGGIN'S NON-LINEAR HYSTERETIC SOIL MODEL}

\subsubsection{Penetration modes}

The model uses the current penetration depth, $z$, and the direction of displacement (upward or downward) relative to the previous time step to define four different penetration modes.

\section{- Not In Contact Mode}

At any stage of the analysis, if any part of the riser is above the seabed, that part will be in "Not in Contact" mode with zero penetration or suction resistance.

\section{- Initial Penetration Mode}

At the start of the analysis, the penetration mode is set to "Initial Penetration Mode" for the parts of the riser penetrating into the seabed $(+v e)$. This initial assumption does not allow for the effect any of uplift and re-penetration that might have occurred during first installation. In other words, the initial static penetration is assumed to occur as a single progressive penetration. Other parts of the riser above the seabed are allocated a "Not in Contact" mode as explained before.

For dynamic analysis, the mode continues as "Initial Penetration" from the point reached during the static initialization step, until the riser starts to lift up at which point it changes to "Uplift" mode (described below).

\section{- Uplift Mode}

Upward displacement (-ve) of the riser is defined as "Uplift Mode". The uplift mode may either continue until the absolute penetration depth is zero, in which case the riser breaks out of the soil and the mode is changed to "Not in Contact", or else may be interrupted by a reversal of displacement direction, in which case the mode changes to "Re-penetration" (described below). The same mode is used for re-penetration following break out.

\section{- Re-penetration Mode}

The penetration mode for every downward displacement (+ve) into the seabed, increasing the current penetration depth, is set to "Re-penetration Mode" if that part of 
the riser has experienced at least one "Uplift" mode during its displacement history, even if the penetration occurs after a break out. This is because the model assumes that initial penetration followed by uplift has disturbed that area of the seabed, and so subsequent contacts are made into disturbed soil.

\subsubsection{Model features}

The riser-seabed resistance for an SCR oscillating up and down in the TDZ is shown schematically in Figure 5-2, (Randolph and Quiggin 2009).

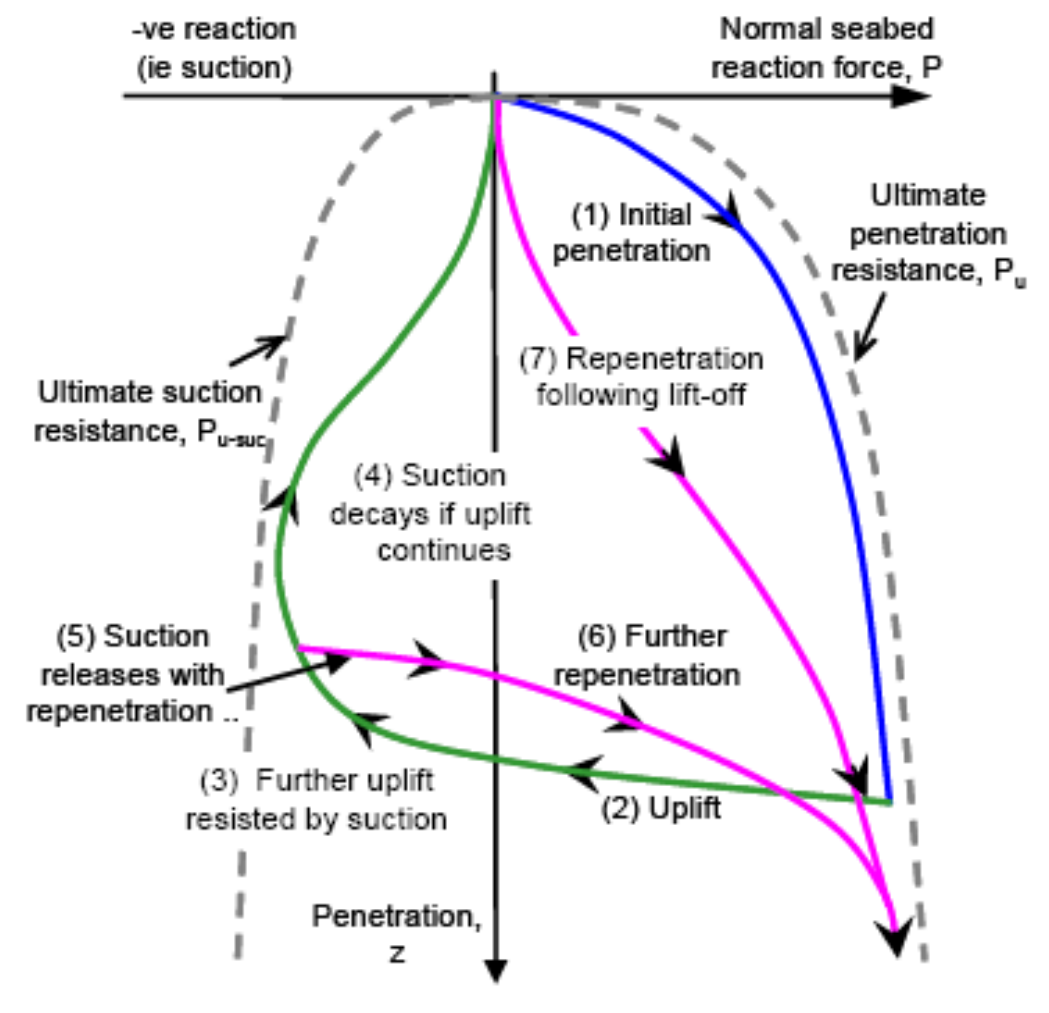

Figure 5-2:Non-linear Soil model characteristics for different modes (Randolph and Quiggin 2009)

The model starts in "Initial Penetration" mode as shown by the blue line in Figure 5-2. Increasing the penetration depth, the resistance asymptotically approaches the ultimate penetration resistance $\mathrm{P}_{\mathrm{u}}$. By reversing the riser penetration direction, the model enters uplift mode and the reaction force decreases rapidly with a high initial secant stiffness, $\mathrm{K}_{\max }$ (note 2, Figure 5-2). Further uplift takes the model into the suction region with negative reaction force. The suction resistance reaches its maximum value approaching the ultimate suction resistance, $\mathrm{P}_{\mathrm{u}-\text { suc }}$, (note 3 , Figure 5-2). The magnitude of ultimate suction resistance is a fraction of the ultimate penetration resistance controlled by 
parameter $\mathrm{f}_{\text {suc }}$. With further uplift, the suction resistance decays to zero over a distance controlled by parameter $\lambda_{\text {suc }}$ (note 4 , Figure 5-2). Re-penetration after an entire break out follows an initially convex curve reflecting the soil softening beneath the riser during uplift (note 7, Figure 5-2). The re-penetration resistance approaches the ultimate penetration resistance at a penetration depth greater than the previous maximum penetration depth, as controlled by parameter $\lambda_{\text {rep }}$. Re-penetration may also occur when the suction resistance is partially mobilised (note 5, Figure 5-2). In such a case, the suction resistance reduces rapidly and the model enters the positive reaction region. Further cycles of uplift and re-penetration would give further episodes of Uplift and Repenetration modes and so give hysteresis loops of seabed resistance with incremental penetration at each cycle (Randolph and Quiggin 2009).

\subsection{FORMULAE FOR HYSTERETIC NON-LINEAR SOIL MODEL}

\subsubsection{Ultimate resistance limits}

Randolph and Quiggin (2009) devised resistance formulae to ensure that the penetration and suction resistance asymptotically approached the ultimate penetration resistance and ultimate suction resistance respectively (dashed grey lines, Figure 5-2). These ultimate limits are given by a combination of soil shear strength and soil buoyancy as:

$$
\begin{aligned}
& P_{u}(z)=N_{c}(z / D) s_{u}(z) \cdot D+f_{b} A_{\text {disp }}\left(\rho_{\text {soil }}-\rho_{\text {sea }}\right) g \\
& P_{u-\text { suc }}(z)=-f_{\text {suc }} P_{u}(z)
\end{aligned}
$$

where

$\mathrm{Z} \quad$ is penetration depth

$\mathrm{D} \quad$ is the riser outer diameter

$\mathrm{f}_{\mathrm{b}} \quad$ is the soil buoyancy factor, a model parameter

$\mathrm{A}_{\text {disp }} \quad$ is the nominal area of the pipe below the seabed tangent plane

$\rho_{\text {soil }} \quad$ is the saturated density of the soil

$\rho_{\text {sea }} \quad$ is the seawater density at the seabed origin

g is the acceleration due to gravity

$\mathrm{P}_{\mathrm{u}}(\mathrm{z}) \quad$ is the ultimate penetration resistance at penetration $\mathrm{z}$, which is the highest soil shear reaction force per unit length of pipe that the seabed can give.

$\mathrm{f}_{\text {suc }} \quad$ is a model parameter giving the non-dimensional suction resistance ratio 
$\mathrm{P}_{\mathrm{u} \text {-suc }}(\mathrm{z})$ is the ultimate suction resistance at penetration $\mathrm{z}$, which is the highest uplift resistance or suction force per unit length of pipe that can be sustained.

$\mathrm{N}_{\mathrm{c}}(\mathrm{z} / \mathrm{D})$, is the soil bearing factor and is defined as:

$\mathrm{N}_{\mathrm{c}}(\mathrm{z} / \mathrm{D})=\mathrm{a}(\mathrm{z} / \mathrm{D})^{\mathrm{b}} \quad$ for $\quad \mathrm{z} / \mathrm{D} \geq 0.1 \quad$ (Aubeny et al. 2005)

$\mathrm{N}_{\mathrm{c}}=\mathrm{N}_{\mathrm{c}}(0.1) \sqrt{10 \mathrm{z} / \mathrm{D}} \quad$ for $\quad \mathrm{z} / \mathrm{D}<0.1 \quad$ (Randolph and White 2008a)

where

$\mathrm{a}$ and $\mathrm{b}$ are non-dimensional penetration resistance parameters (Aubeny et al. 2005).

The undrained shear strength at depth $\mathrm{z}, \mathrm{s}_{\mathrm{u}}(\mathrm{z})$, is idealised as:

$\mathrm{s}_{\mathrm{u}}(\mathrm{z})=\mathrm{s}_{\mathrm{um}}+\rho \mathrm{z}$

where
$\mathrm{s}_{\mathrm{um}} \quad$ is the undrained shear strength at the mudline
$\rho \quad$ is the shear strength gradient with depth.

The factor $f_{b}$ in equation (3-1) is greater than 1 and increases the standard buoyancy force $A_{\text {disp }} \cdot\left(\rho_{\text {soil }}-\rho_{\text {sea }}\right)$.g by about $50 \%$; this is because when the seabed soil is displaced by the riser it tends to heave locally around the riser rather than dispersing thinly across the seabed plane (Merifield et al. 2009; Randolph and White 2008b),

\subsubsection{Initial penetration mode}

For the initial penetration (blue line, Figure 5-2), the resistance is given by:

$\mathrm{P}(\mathrm{z})=\mathrm{H}_{\mathrm{IP}}(\zeta) \cdot \mathrm{P}_{\mathrm{u}}(\mathrm{z})$

where

$\zeta=\mathrm{z} /\left(\mathrm{D} / \mathrm{K}_{\max }\right)$

and

$\mathrm{H}_{\mathrm{IP}}(\zeta)=\zeta /[1+\zeta]$

$\zeta$ is the non-dimensional penetration in units of $\mathrm{D} / \mathrm{K}_{\max }$, where $\mathrm{K}_{\max }$ is the normalized maximum stiffness parameter of the model. $\mathrm{K}_{\max }$ is essentially the pipe-soil stiffness, $\mathrm{dP} / \mathrm{dz}$, normalized by the ultimate net bearing pressure at that depth, with typical values in the range 150 to 250 (Bridge et al. 2004; Clukey et al. 2005). 
$\mathrm{H}_{\mathrm{IP}}$ is a hyperbolic factor, providing a high initial stiffness $\left(\mathrm{dP} / \mathrm{dz}=\mathrm{K}_{\max } \mathrm{P}_{\mathrm{u}} / \mathrm{D}\right)$ while ensuring that the penetration resistance $\mathrm{P}(\mathrm{z})$ rises smoothly from zero at first contact and asymptotically approaches the ultimate penetration resistance, $\mathrm{P}_{\mathrm{u}}(\mathrm{z})$. The magnitude of this hyperbolic factor at the initial penetration $(\zeta=0)$ is zero and asymptotically approaches 1 as the penetration increases compared to $\mathrm{D} / \mathrm{K}_{\max }$.

\subsubsection{Uplift mode}

The penetration resistance formula for uplift mode is given by:

$\mathrm{P}(\mathrm{z})=\mathrm{P}_{0}-\mathrm{H}_{\mathrm{UL}}\left(\zeta_{0}-\zeta\right)\left(\mathrm{P}_{0}-\mathrm{P}_{\mathrm{u}-\text { suc }}(\mathrm{z})\right)$

where

$\zeta_{0} \quad$ is the non-dimensionalised penetration at which the latest episode of the current contact mode started and can be written as:

$$
\zeta_{0}=\mathrm{z}_{0} /\left(\mathrm{D} / \mathrm{K}_{\max }\right)
$$

where

$\mathrm{z}_{0} \quad$ is the penetration $\mathrm{z}$ at which the latest episode of the current contact mode started.

The hyperbolic factor for the uplift mode is given by:

$$
\mathrm{H}_{\mathrm{UL}}\left(\zeta_{0}-\zeta\right)=\left(\zeta_{0}-\zeta\right) /\left[\mathrm{A}_{\mathrm{UL}}(\mathrm{z})+\left(\zeta_{0}-\zeta\right)\right]
$$

where

$\mathrm{A}_{\mathrm{UL}}$ is the resistance ratio used within the hyperbolic factor to ensure correct initial stiffness on load reversal, namely $\mathrm{K}_{\max } \mathrm{P}_{\mathrm{u}}(\mathrm{z}) / \mathrm{D}$ and is given by:

$$
\mathrm{A}_{\mathrm{UL}}(\mathrm{z})=\left(\mathrm{P}_{0}-\mathrm{P}_{\mathrm{u}-\mathrm{suc}}(\mathrm{z})\right) / \mathrm{P}_{\mathrm{u}}\left(\mathrm{z}_{0}\right)
$$

At the beginning of the uplift mode (i.e $\zeta=\zeta_{0}$ ), the hyperbolic factor will equal zero. When the non-dimensional uplift $\zeta-\zeta_{0}$ increases and becomes large in comparison with the resistance ratio, $A_{U L}(z)$, the magnitude of the hyperbolic factor asymptotically approaches 1 and the uplift resistance approaches the ultimate suction resistance (green curve, Figure 5-2).

The uplift resistance is limited to a negative lower bound $\mathrm{P}_{\min }(\mathrm{z})$ to model the limiting displacement over which suction resistance can be sustained (Bridge et al. 2004). The adjusted uplift resistance is expressed as:

$$
\mathrm{P}_{\min }(\mathrm{z})=\mathrm{E}_{\mathrm{UL}}(\mathrm{z}) \mathrm{P}_{\mathrm{u}-\mathrm{suc}}(\mathrm{z})
$$

where 


$$
\begin{gathered}
\mathrm{E}_{\mathrm{UL}}(\mathrm{z}) \quad \text { is an exponential factor limiting the uplift resistance, given by: } \\
\mathrm{E}_{\mathrm{UL}}(\mathrm{z})=\operatorname{Exp}\left(\operatorname{Min}\left(0,\left(\mathrm{z}-\mathrm{z}_{\mathrm{p}=0}\right) /\left(\lambda_{\mathrm{suc}} \mathrm{z}_{\max }\right)\right)\right)
\end{gathered}
$$

where

$\mathrm{Z}_{\max } \quad$ is the maximum previous penetration depth for that point of the riser

$\mathrm{z}_{\mathrm{p}=0} \quad$ is the largest penetration depth at which suction has started during any uplift

$\lambda_{\text {suc }}$ is a model parameter representing a non-dimensional normalized suction decay distance.

The exponential factor always lies between zero and unity; it takes the value 1 when the penetration depth is larger than $\mathrm{z}_{\mathrm{p}=0}$, but decays towards 0 if the penetration depth is less than $\mathrm{Z}_{\mathrm{p}=0}$. The suction is therefore limited to $\mathrm{P}_{\mathrm{u} \text {-suc }}(\mathrm{z})$ when the first uplift starts and is further reduced when the pipe is lifted up relative to $z_{p}=0$.

\subsubsection{Re-penetration mode}

The penetration resistance formula for re-penetration mode is given by:

$$
\mathrm{P}(\mathrm{z})=\mathrm{P}_{0}+\mathrm{H}_{\mathrm{RP}}\left(\zeta_{0}-\zeta\right)\left(\mathrm{P}_{\mathrm{u}}(\mathrm{z})-\mathrm{P}_{0}\right)
$$

where

$\zeta_{0}$ and $\mathrm{P}_{0} \quad$ are the non-dimensional penetration and resistance at the start of this re-penetration.

The hyperbolic factor for re-penetration mode is given by:

$$
\mathrm{H}_{\mathrm{UL}}\left(\zeta-\zeta_{0}\right)=\left(\zeta-\zeta_{0}\right) /\left[\mathrm{A}_{\mathrm{RP}}(\mathrm{z})+\left(\zeta-\zeta_{0}\right)\right]
$$

where

$A_{R P} \quad$ is the resistance ratio used within the hyperbolic factor to ensure correct initial stiffness on load reversal, given by:

$$
\mathrm{A}_{\mathrm{RP}}(\mathrm{z})=\left(\mathrm{P}_{\mathrm{u}}(\mathrm{z})-\mathrm{P}_{0}\right) / \mathrm{P}_{\mathrm{u}^{*}}
$$

where

$$
\begin{array}{lll}
\mathrm{P}_{\mathrm{u}^{*}}=\mathrm{P}_{\mathrm{u}}(\mathrm{z}) & \text { if } & \mathrm{P}_{0} \leq 0 \\
\mathrm{P}_{\mathrm{u}^{*}}=\mathrm{P}_{\mathrm{u}}\left(\mathrm{z}^{*}\right) & \text { if } & \mathrm{P}_{0}>0
\end{array}
$$

where

$\mathrm{z}^{*} \quad$ is the penetration when the preceding episode of uplift started. 
At the beginning of this re-penetration (i.e $\zeta=\zeta_{0}$ ), the hyperbolic factor will equal zero. When the non-dimensional re-penetration $\zeta-\zeta_{0}$ increases and becomes large in comparison with the resistance ratio, $A_{R P}(z)$, the magnitude of the hyperbolic factor asymptotically approaches 1 and the re-penetration resistance approaches the ultimate penetration resistance $\mathrm{P}_{\mathrm{u}}(\mathrm{z})$. (purple curve, Figure 5-2).

The re-penetration resistance after a large uplift movement is reduced until the previous maximum penetration is approached (Bridge et al. 2004). This is modelled by limiting the re-penetration resistance to an upper bound $\mathrm{P}_{\max }(\mathrm{z})$ given by:

$\mathrm{P}_{\max }(\mathrm{z})=\mathrm{E}_{\mathrm{RP}}(\mathrm{z}) \mathrm{P}_{\mathrm{IP}}(\mathrm{z})$

where

$$
\begin{gathered}
\mathrm{E}_{\mathrm{RP}}(\mathrm{z}) \quad \text { is an exponential factor limiting the uplift resistance and given by: } \\
\mathrm{E}_{\mathrm{RP}}(\mathrm{z})=\operatorname{Exp}\left(\operatorname{Min}\left(0,\left(\mathrm{z}-\mathrm{z}_{\mathrm{p}=0}\right) /\left(\lambda_{\text {suc }} \mathrm{z}_{\text {max }}\right)-\lambda_{\text {rep }}\right)\right)
\end{gathered}
$$

where

$$
\begin{array}{ll}
Z_{\max } & \text { is the maximum previous penetration depth for that point of the riser } \\
\mathrm{z}_{\mathrm{p}=0} & \text { is the largest penetration depth at which suction has started during any } \\
& \text { uplift } \\
\lambda_{\text {suc }} & \text { is a model parameter giving the non-dimensional normalized suction } \\
& \text { decay distance } \\
\lambda_{\text {rep }} & \text { is a model parameter giving the non-dimensional re-penetration offset } \\
& \text { after uplift. }
\end{array}
$$

The exponential factor is always between zero and unity, and so limits the repenetration resistance to be less than the ultimate penetration resistance $\mathrm{P}_{u}(\mathrm{z})$ until the penetration depth exceeds $z_{p=0}$ by a certain amount. The term $\lambda_{\text {rep }}$ models the incremental penetration of the riser under cyclic loading by delaying the point where the response rejoins the limiting resistance curve beyond the previous maximum penetration; hence $\lambda_{\text {rep }}$ controls the additional penetration with each cycle. The magnitude of $\lambda_{\text {rep }}$ can vary between zero for no incremental penetration to perhaps 0.3 for nominal incremental penetration of about $0.3 \mathrm{D}$.

\subsubsection{Example response of the model}

The model performance was evaluated by developing an Excel spreadsheet in which an artificial displacement history can be simulated. Figure 5-3 shows the response of a single spring of the hysteretic non-linear model over a few arbitrary cycles of uplift and 
re-penetration. The input values and model parameters implemented in the calculation are summarised in Table 5-1.

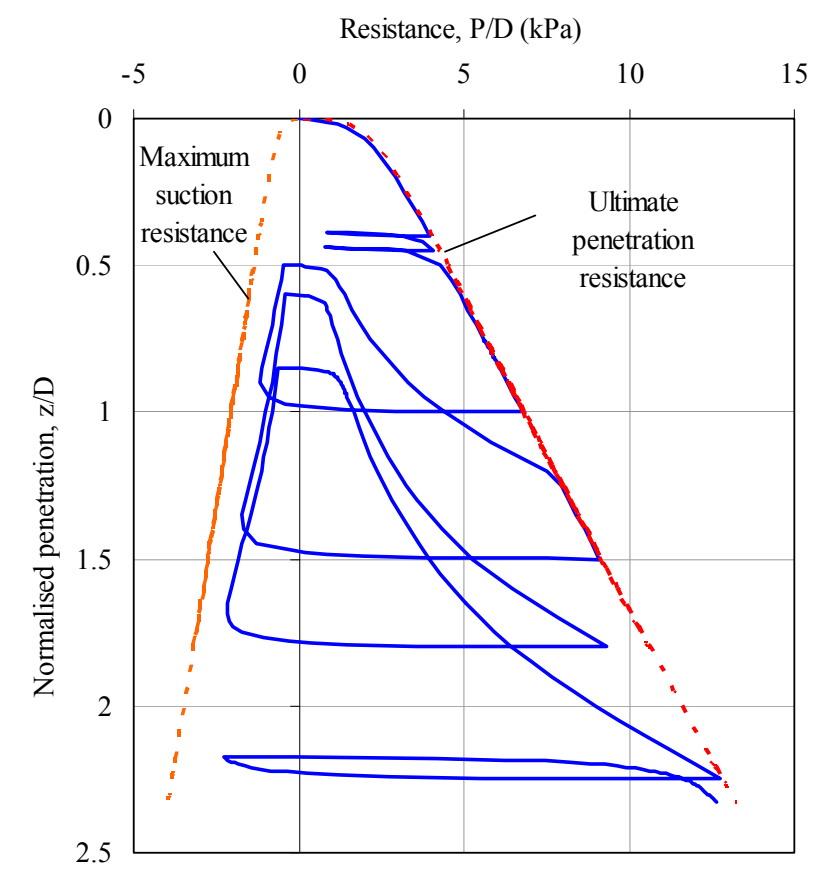

Figure 5-3: Example response under complex motion

Table 5-1: Model parameters for example in Figure 5-3

\begin{tabular}{|l|c|c|}
\hline \multicolumn{1}{|c|}{ Parameter } & Symbol & Value \\
\hline Pipe diameter & $\mathrm{D}$ & $0.324 \mathrm{~m}$ \\
\hline Mudline shear strength & $\mathrm{S}_{\mathrm{um}}$ & $0.65 \mathrm{kPa}$ \\
\hline Shear strength gradient & $\rho$ & $1.5 \mathrm{kPa} / \mathrm{m}$ \\
\hline Power law parameter & $\mathrm{a}$ & 6 \\
\hline Power law parameter & $\mathrm{b}$ & 0.25 \\
\hline Normalized maximum stiffness & $\mathrm{K}_{\mathrm{max}}$ & 200 \\
\hline Suction ratio & $\mathrm{f}_{\text {suc }}$ & 0.3 \\
\hline Suction decay parameter & $\lambda_{\text {suc }}$ & 0.5 \\
\hline Re-penetration parameter & $\lambda_{\text {rep }}$ & 0.5 \\
\hline
\end{tabular}




\subsubsection{Typical values for model parameters}

Randolph and Quiggin (2009) divide the model parameters in two categories. The first category contains the parameters with reasonably assumed default values. These parameters are the buoyancy factor $f_{b}$, maximum normalized stiffness $K_{\max }$ and the power law coefficients $(a, b)$ for the bearing factor $N_{c}$. The second category includes the parameters that can be adjusted by the user to suit particular conditions. These parameter are the maximum suction parameter $f_{\text {suc }}$, suction decay parameter $\lambda_{\text {suc }}$ and repenetration resistance mobilization delay parameter $\lambda_{\text {rep. }}$

\section{o Category $I: \mathbf{f}_{\mathbf{b}}, K_{\max },(\mathbf{a}, \mathbf{b})$ of $\mathbf{N}_{\mathrm{c}}$}

The buoyancy factor can never be less than 1 due to Archimedes principle. The buoyancy component of resistance is usually much less than the soil shear resistance, except for fully softened conditions. From large deformation finite element analysis of pipe penetration, an $f_{b}$ value of about 1.5 was found to give an appropriate match to analysis results (Randolph and White 2008b).

Experimental investigations of the normalized secant stiffness $(\Delta \mathrm{P} / \Delta \mathrm{z}) /\left(\mathrm{D} / \mathrm{P}_{\mathrm{u}}\right)$, i.e. the effective riser-soil stiffness since the last penetration reversal or since penetration started, showed the following range of values for $\mathrm{K}_{\max }$ (Aubeny et al. 2008; Bridge et al. 2004; Clukey et al. 2008):

for $\quad \Delta \mathrm{z} \sim 0.001 \mathrm{D},(\Delta \mathrm{P} / \Delta \mathrm{z}) /\left(\mathrm{D} / \mathrm{P}_{\mathrm{u}}\right) \sim 150$ to 400

for $\quad \Delta \mathrm{z} \sim 0.005 \mathrm{D},(\Delta \mathrm{P} / \Delta \mathrm{z}) /\left(\mathrm{D} / \mathrm{P}_{\mathrm{u}}\right) \sim 100$

for $\quad \Delta \mathrm{z} \sim 0.025 \mathrm{D},(\Delta \mathrm{P} / \Delta \mathrm{z}) /\left(\mathrm{D} / \mathrm{P}_{\mathrm{u}}\right) \sim 40$

Randolph and Quiggin (2009) recommend $\mathrm{K}_{\max }=200$ in the model, which provides the following values of normalised secant stiffness $(\Delta \mathrm{P} / \Delta \mathrm{z}) /\left(\mathrm{D} / \mathrm{P}_{\mathrm{u}}\right)$, showing appropriate agreement with experimental results referred above:

for $\quad \Delta \mathrm{z} \sim 0.001 \mathrm{D},(\Delta \mathrm{P} / \Delta \mathrm{z}) /\left(\mathrm{D} / \mathrm{P}_{\mathrm{u}}\right)=167$

for $\quad \Delta \mathrm{z} \sim 0.005 \mathrm{D},(\Delta \mathrm{P} / \Delta \mathrm{z}) /\left(\mathrm{D} / \mathrm{P}_{\mathrm{u}}\right)=100$

for $\quad \Delta \mathrm{z} \sim 0.025 \mathrm{D},(\Delta \mathrm{P} / \Delta \mathrm{z}) /\left(\mathrm{D} / \mathrm{P}_{\mathrm{u}}\right)=40$

Figure 5-4, illustrates the effect of $\mathrm{K}_{\max }$ value on the force-displacement behaviour of a point at the pipe invert, over two load cycles. Higher values of $\mathrm{K}_{\max }$ give higher penetration resistance and lower suction force for a given penetration depth, with the maximum difference occurring in the late re-penetration and early suction stages. 


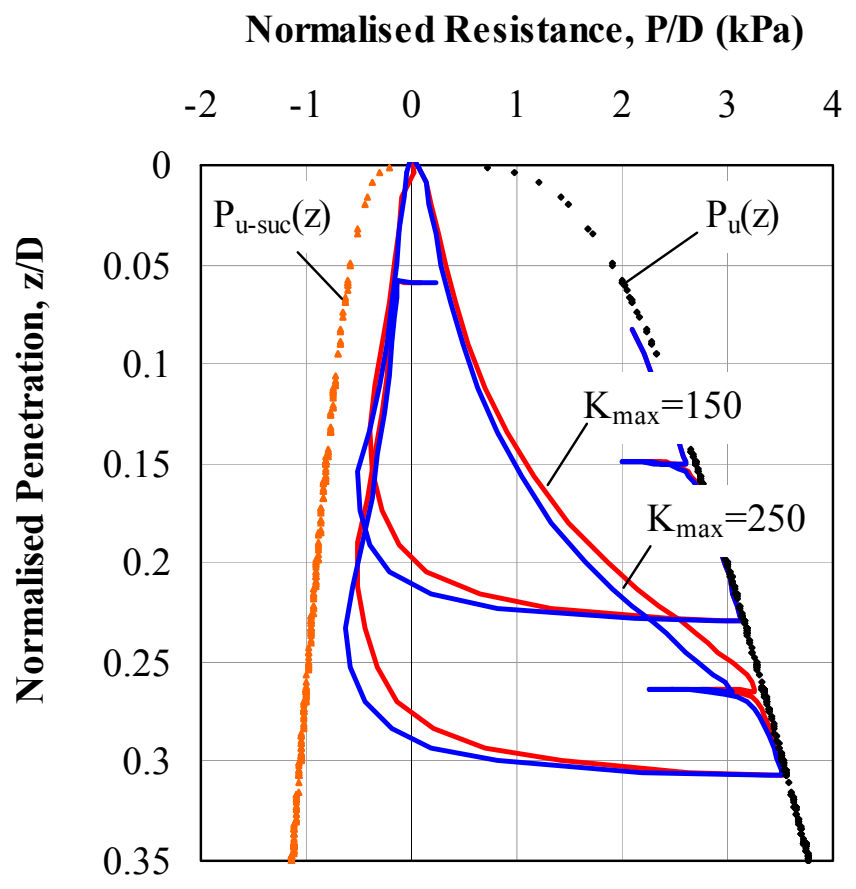

Figure 5-4: Force displacement plot with different $K_{\max }$ values

For the power law coefficients of the bearing factor $(a, b)$, a typical range of $(5,0.2)$ to $(6.5,0.3)$ has been reported by Aubeny et al (2005). The values depend on the pipe roughness and the shear strength profile with depth. Rounded values of $(6,0.25)$ were suggested by Randolph and White (2008b).

\section{o Category II : $\lambda_{\text {suc }}, \lambda_{\text {rep }}, \mathbf{f}_{\text {suc }}$}

The amount of suction resistance in uplift is controlled by $f_{\text {suc }}$. The magnitude of this parameter can lie between zero for no suction resistance to unity for maximum suction resistance equal to the ultimate penetration resistance. Randolph and Quiggin (2009) suggest a more realistic upper limit of 0.7 for $\mathrm{f}_{\text {suc }}$. Since the suction force appears to reduce rapidly to a low level within a few cycles (Bridge and Willis 2002), for single uplift of a riser, a value of 0.5 to unity is considered to be suitable whilst for fatigue studies, or other applications with many cycles, the recommended range is 0 to 0.3 . In reality, various factors affect the magnitude of suction resistance, such as the lift-up rate of the riser, the time over which the uplift motion is sustained and the recent history of cyclic motion (Bridge et al. 2004). However, considering the very limited published data, Randolph and Quiggin (2009) have adopted a constant (but user-defined) value of $\mathrm{f}_{\text {suc }}$. Figure 5-5 shows the effect of $\mathrm{f}_{\text {suc }}$ on the peak suction mobilised, and the following re-penetration resistance. Higher values of $\mathrm{f}_{\text {suc }}$ give higher suction resistance and a slower mobilisation of the re-penetration resistance. Since $f_{\text {suc }}$ does not control the suction decay distance, larger values of $\mathrm{f}_{\text {suc }}$ give a higher gradient of suction decay. 


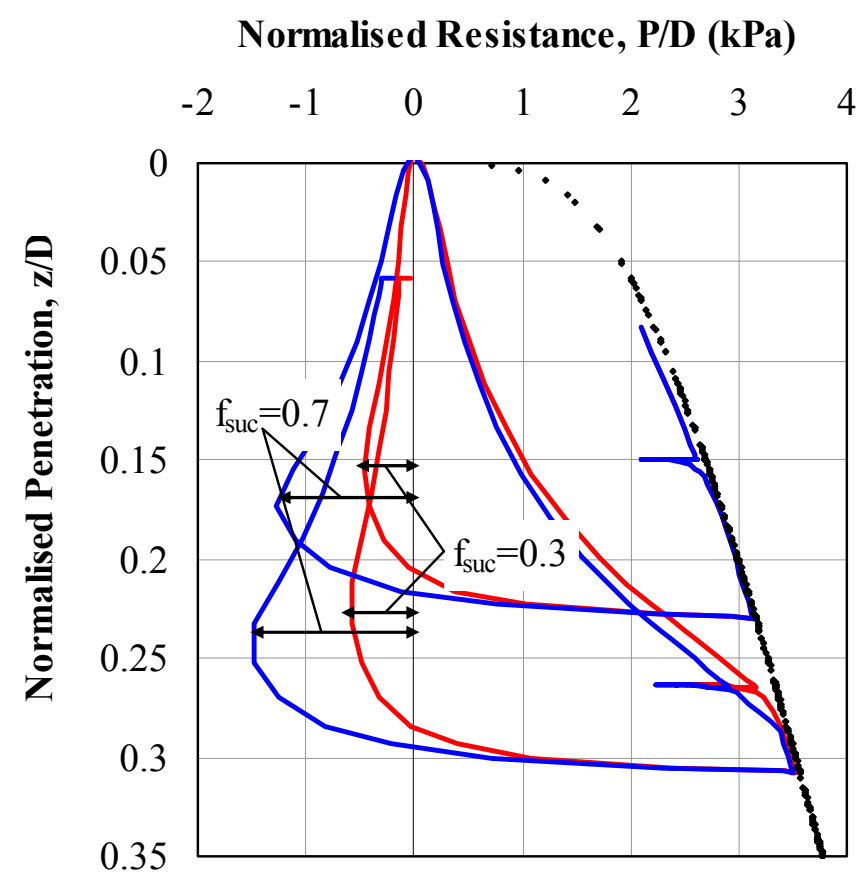

Figure 5-5: Influence of $f_{\text {suc }}$ on peak suction mobilisation

The suction decay distance in the model is adjusted by the parameter $\lambda_{\text {suc. }}$ Randolph and Quiggin (2009) consider a value range of 0.2 to 0.6 for $\lambda_{\text {suc }}$, which leads to small residual suction resistance after an uplift displacement of about 1 diameter. This matches experimental results showing a suction decay distance in the order of the riser diameter or less, although the response will also be affected by the rate of motion and the previous displacement history (Aubeny et al. 2008; Bridge et al. 2004). Figure 5-6 illustrates the extension of the suction decay distance with higher values of $\lambda_{\text {suc }}$ which in turn affect the peak suction mobilisation and the soil softening (or reduced resistance) in the initial stages of re-penetration. Lower values of $\lambda_{\text {suc }}$ give a reduced suction decay distance, lower peak suction mobilisation and a softer response in the initial stages of re-penetration (i.e. lower resistance for a given re-penetration depth). 
Normalised Resistance, P/D (kPa)

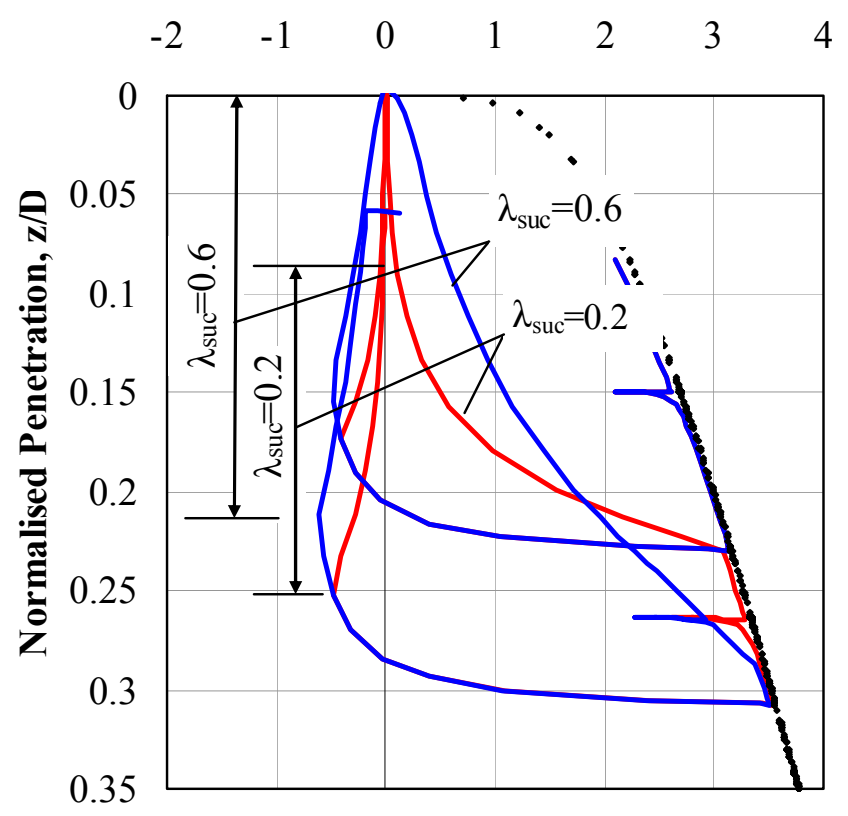

Figure 5-6: Influence of $\lambda_{\text {suc }}$ on suction decay and soil softening

The model parameter, $\lambda_{\text {rep, }}$ simulates the progressive penetration with cumulative loadcontrolled cyclic movements, delaying the mobilisation of ultimate re-penetration resistance. Randolph and Quiggin (2009) recommend a value in the range 0.1 to 0.5 , which shows reasonable correlation with experimental results. Figure 5-7 shows the delay in ultimate resistance mobilisation by adopting a higher value of $\lambda_{\text {rep. }}$. 


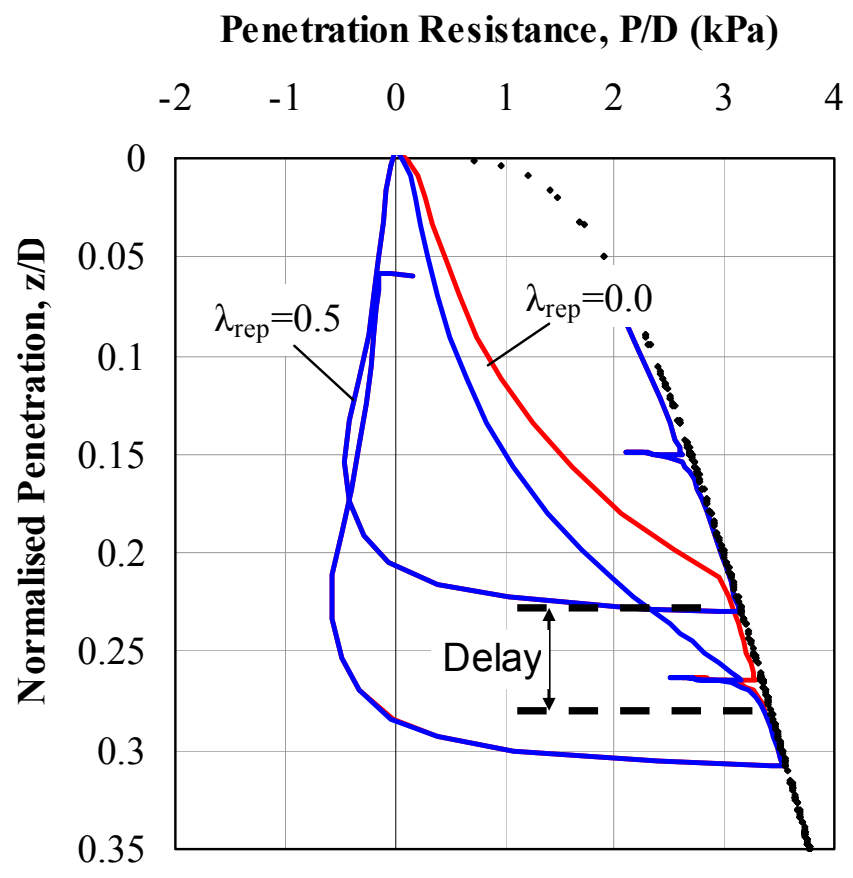

Figure 5-7: influence of $\lambda_{\text {rep }}$ on delay of penetration resistance mobilisation

Randolph and Quiggin (2009) have compared the model simulation capabilities with the laboratory test results reported by Aubeny et al. (2008) following implementation of the model into the OrcaFlex software. The reported tests covered three different loading paths: 'monotonic', 'small amplitude cycles' and 'large amplitude cycles'. The model simulation showed a very good correlation with the test results.

The non-linear soil model implemented in OrcaFlex has also been examined by Randolph and Quiigin (2009) with respect to two fully instrumented full scale tests of an SCR in the touchdown zone, at Watchet harbour, UK, as reported by Bridge et al. (2004). The tests consisted of raising the top of the riser with a particular velocity followed by lowering the top end again at the same velocity. The results of the tests, which studied the variations of bending moment and tension at the TDP due to the imposed top end motions, showed the significant influence of suction resistance during the riser lift-up after a period of rest. The simulation results of Randolph and Quiggin (2009) showed some effect of suction resistance during uplift, with reasonable correlation with the test results but with less effect of the suction resistance on the bending moment ranges.

\subsection{NON-LINEAR SOIL MODEL IMPLEMENTED IN ABAQUS}

The UEL coded in Chapter 4, for a linear elastic seabed, was further developed to model the hysteretic non-linear seabed. Then the same global configuration described in 
Chapter 4 and the same vessel excitation technique was used to apply a few cycles of loading to check the functionality of the non-linear hysteretic seabed model.

Similar to the linear elastic seabed, the user subroutine is called by ABAQUS for every user element at every increment of an analysis step to provide the element's stiffness matrix and its contribution to the right-hand-side vector (RHS). The soil and model parameters considered for FE analysis are as per Table 5-1 and the SCR properties and the global configuration are as defined in Chapter 4 (Tables 4-1 and 4-2).

\subsubsection{Numerical analysis steps}

The same three steps described in Chapter 4 have been defined to model the SCR connected to the floating system with a few changes in Steps 2 and 3 to model the displacement stage for the non-linear seabed.

At the beginning of Step 2 the linear elastic springs at the seabed are replaced with userdefined non-linear elasto-plastic elements and initialised to respond in "Mode 1" of displacement, the initial penetration mode, described in section 5.4.2. In other words, during the horizontal translation of the vessel to its nominal position, the SCR contacts the seabed springs gradually and the springs respond in "Mode 1". If the initial displacement mode is left to be automatically selected by the model, some minor numerical inverse displacements and fluctuations in the contact force appear among the nodes resting on the seabed. The initialization of the analysis by setting the "Mode 1" removes the unrealistic stress fluctuations, leading to a smooth analysis.

In the Step 3, first the forced "Mode 1" of the springs is relaxed, so that each spring is free to respond in any displacement mode, depending on the movement direction and position of the riser element, for the rest of analysis. Then, the vessel is excited in the same way through the DISP subroutine. Depending on the longitudinal position of every spring beneath the riser, each spring may experience a different state of displacement and movement direction at any given time stage.

\subsubsection{Load-displacement response in quasi-static analysis}

Example spring responses for locations with various penetration states from partial suction to full breakout are shown below. Figure 5-8 shows schematically the movement directions for different groups of seabed nodes on the displaced SCR, corresponding to displacement of the vessel away from the SCR. This causes the TDP to move towards the anchor end (as shown in Figure 2-4 of Chapter 2). The initial and displaced profiles are numbered as 1 and 2 respectively. Certain nodes towards the vessel end of the TDZ undergo upward movements, while nodes further towards the anchor end of the SCR undergo further penetration. The Figure 5-8 is just a schematic presentation of different modes of movement in different parts of the riser. In the hysteretic non-linear model (Randolph and Quiggin 2009) the suction is started when the mobilised penetration resistance in the last stage is quickly decayed and the riser 
proceeds with further lift up. Then the breakout is started when the mobilised suction force is entirely decayed.

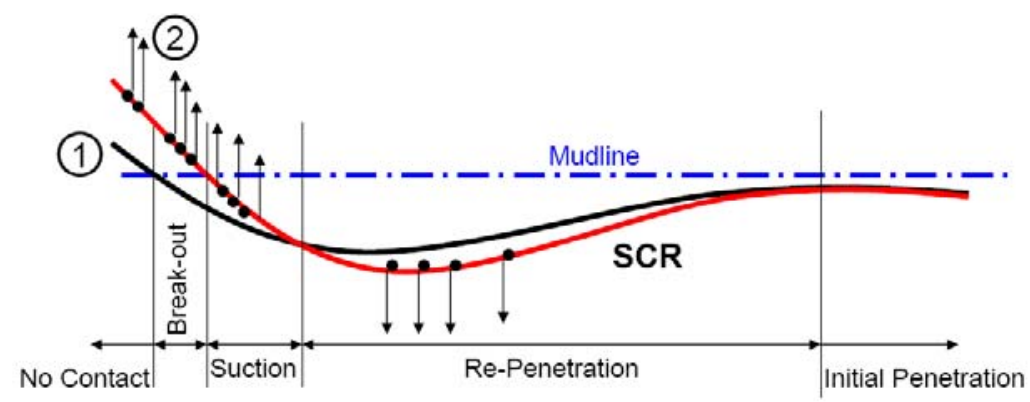

Figure 5-8: Nodes at the seabed with various displacement stages at the same time for vessel far offset

Example nodes at various locations indicated approximately in Figure 5-8 have been selected to illustrate the response of the numerical model for different movement stages. The force-displacement plots are presented below.

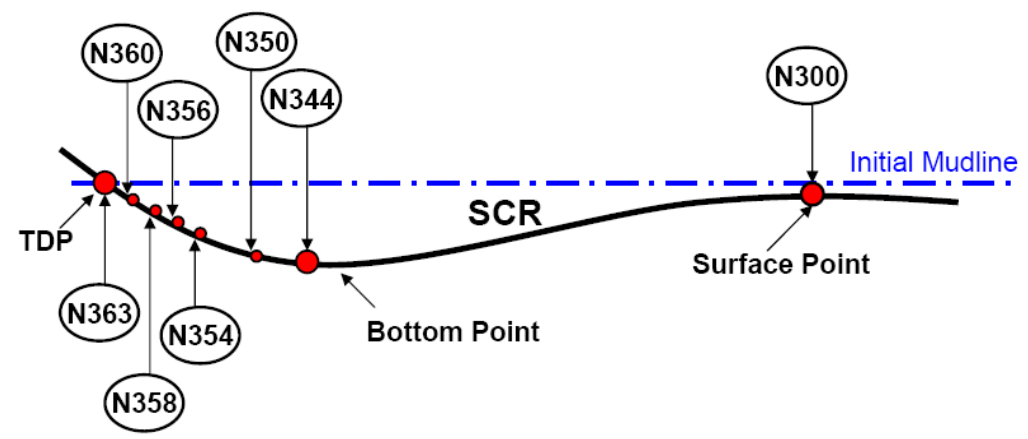

Figure 5-9: Schematically location of the sample nodes on final profile of SCR

Figure 5-10 illustrates the hysteretic load-displacement of node 358 near the TDP. The node experiences penetration, full suction and full breakout over 10 load cycles where the Spar end of the riser and the TDP moved respectively by $\pm 5.13 \mathrm{~m}$ and $\pm 3.8 \mathrm{~m}$. The penetration depth increases from the initial monotonic penetration of $\mathrm{z} / \mathrm{D}=0.15$ to a final normalised depth of 0.32 at the end of the $10^{\text {th }}$ cycle. This implies that a slight trench is formed beneath the riser under cyclic loading. As shown, incremental penetration under cyclic loading has been appropriately captured through the gradual soil softening by the hysteretic non-linear soil model (Randolph and Quiggin 2009). Figure 5-11 to Figure 5-14 show the hysteretic load-displacement response of nodes $356,354,350$ and 344 as well, spanning positions from the TDP towards the anchor end of the touchdown zone. The results presented for nodes 356 and 354 seem similar but are different; in contrast with node 354, node 356 experiences breakout. Since the spring spacing is $1 \mathrm{~m}$ throughout the region on the seabed, the node ID reflects the node distance from the anchor end. For this analysis the initial TDP is about node 356 and 
transfers to about node 363 after 10 cycles of excitation. In these plots, the penetration has been considered positive ignoring the signs of local coordinate system in analysis. A complete parametric study of model response to various parameters is presented in Appendix C.

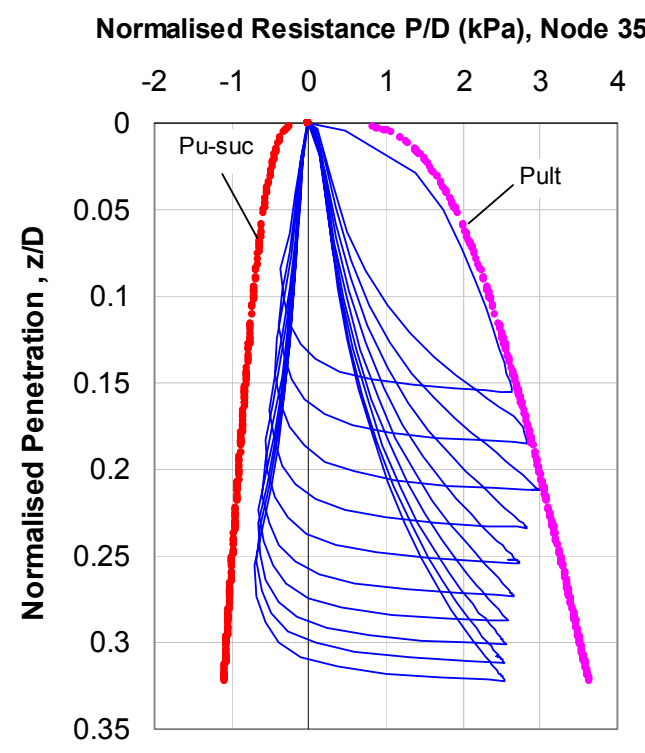

Figure 5-10: Full breakout of node 358 in the TDZ

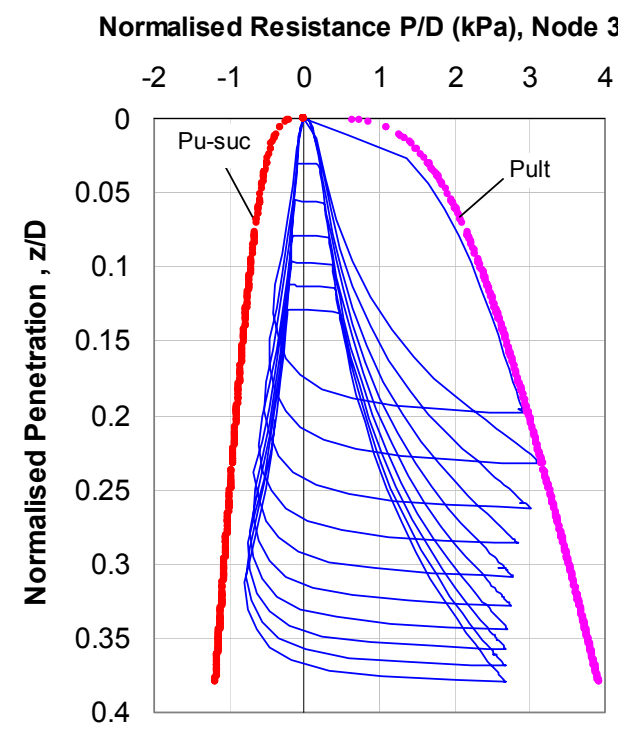

Figure 5-11: Partial breakout of node 356 in the TDZ 


\section{Normalised Resistance P/D (kPa), Node 354}

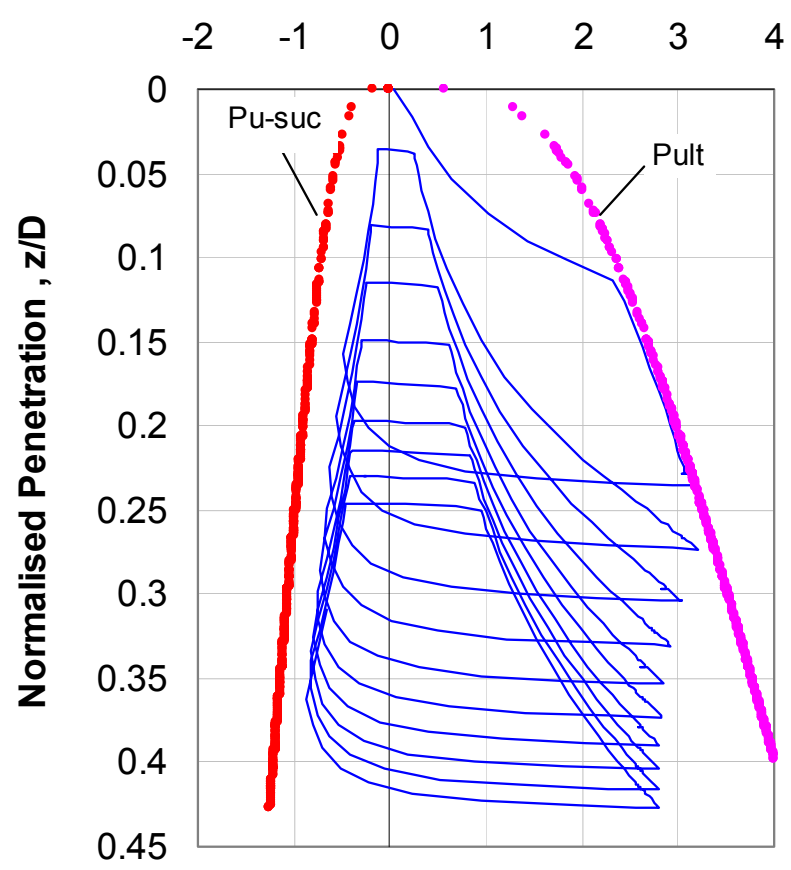

Figure 5-12: Partial suction of node 354 in the TDZ

Normalised Resistance P/D (kPa), Node 350

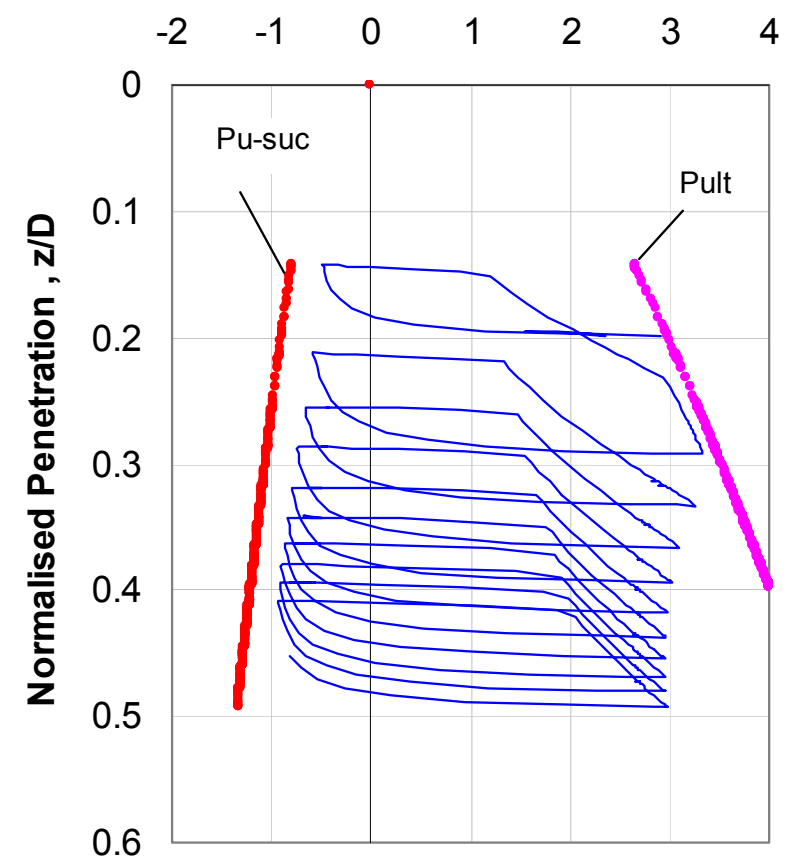

Figure 5-13: Partial suction of node 350 in the TDZ 


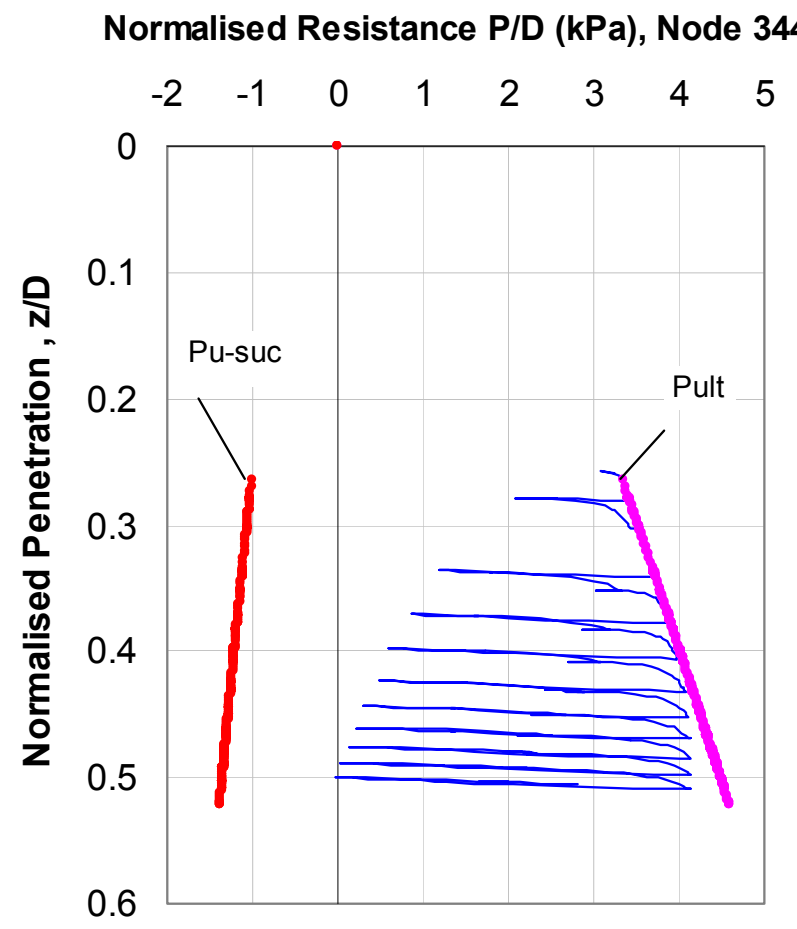

Figure 5-14: No suction response of node 344 in the TDZ

\subsubsection{Gradual embedment and stress distribution}

The differences in the final depth for various nodes after 10 cycles, Figure 5-10 to Figure 5-14, and the increasing penetration depth in every new cycle indicate the potential to model trench formation under cyclic loading. The gradual development of the trench is shown in Figure 5-15 based on the maximum penetration depth of all nodes in the TDZ at the end of every cycle. The general trench geometry is like a ladle. As the trench is deepened, the TDP moves towards the vessel end whilst the point where the riser profile joins the straight part at the seabed, referred to here as the 'surface point' dose not move significantly. Figure 5-16 shows the shear force variation along the SCR in the touchdown zone as the embedment gradually increases. The maximum shear force is mobilised at a point between the deepest point or 'maximum embedment' and the TDP. The peak point is initially close to the maximum embedment and gets closer to the TDP at the end of 10 cycles.

Figure 5-16 shows that the peak shear force shows a sharp increase during the first cycle but then gradually decreases over the remaining cycles. This might be due to forcing the penetration mode in the non-linear model to be the 'initial penetration mode' in the initialisation stage of the analysis. Indeed, in the second step of the analysis, when the vessel is moved to the correct position and the riser gradually makes contact with the seabed, the ladle shape profile (in the touchdown zone) is gradually moved from the anchor end towards the vessel. This means that parts of the riser in the bottom profile keep slightly moving upward and straightening whilst the penetration mode is forced to 
remain as 'initial penetration' rather than 'uplift' to initialise the analysis. This causes the riser to setup immediately upon starting the vessel excitation, assigning the correct penetration modes in the seabed, which in turns cause a slight jump in the shear force diagram.

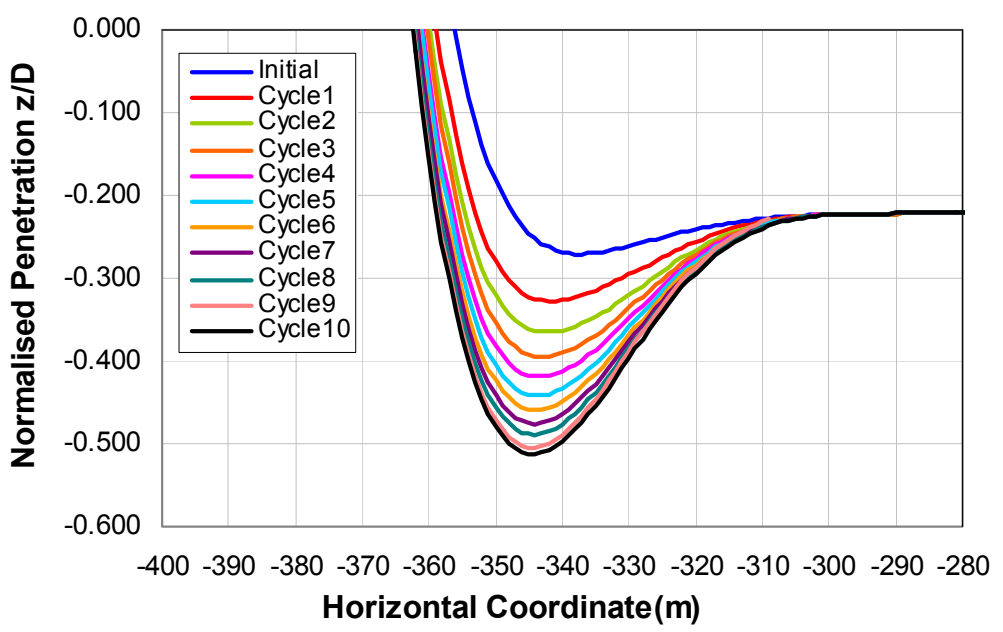

Figure 5-15: Progressive penetration at TDZ under cyclic loading

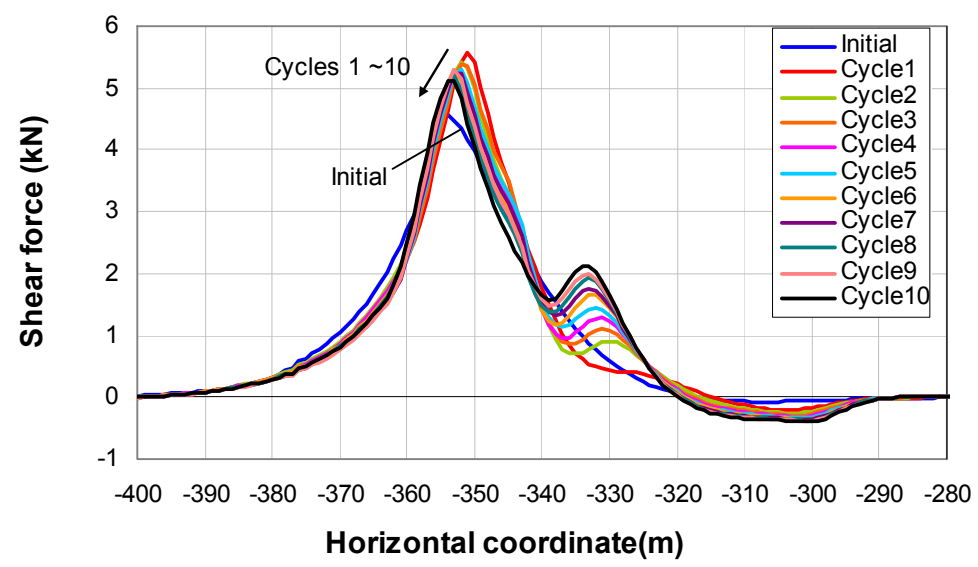

Figure 5-16: Shear force variation with progressive penetration in the TDZ 


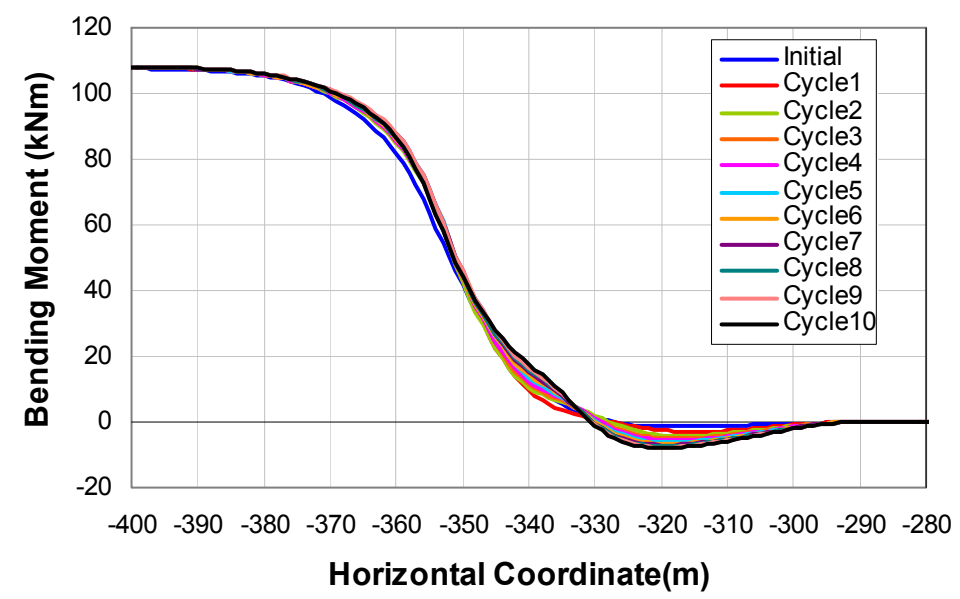

Figure 5-17: Bending moment variation with progressive penetration in the TDZ

The shear force is the gradient of bending moment and bending moment itself results in the dominant stress in the touchdown zone that leads to the von Mises stress variation range under cyclic loading and which is responsible for the fatigue damage. As will be shown in next chapter the distribution of shear force along the TDZ is interestingly similar to the distribution of fatigue damage. Hence, the shear force, and to a lesser extent the contact force, distribution and variation are vital in the fatigue performance of SCRs in the TDZ. As seen in Figure 5-17, a second peak appears in the shear force diagram as the number of cycles increase. When the vessel moves towards the near offset with increasing number of cycles and consequently embedment, the riser is stuck in the generated trench and the contact pressure at the shoulder of the profile in area number 2 (see Figure 5-18) is increased, thus causing the second peak. \#s illustrated in Figure 5-18, the touchdown area can be divided into three important zones from the stress distribution standpoint. The border of zones 2 and 3 is the peak negative curvature of the riser in the TDZ, where the shear force becomes zero; the surface point is located in zone 3 . The border of zones 1 and 2 is the maximum embedment point; the TDP and the peak shear force are located in zone 1. 


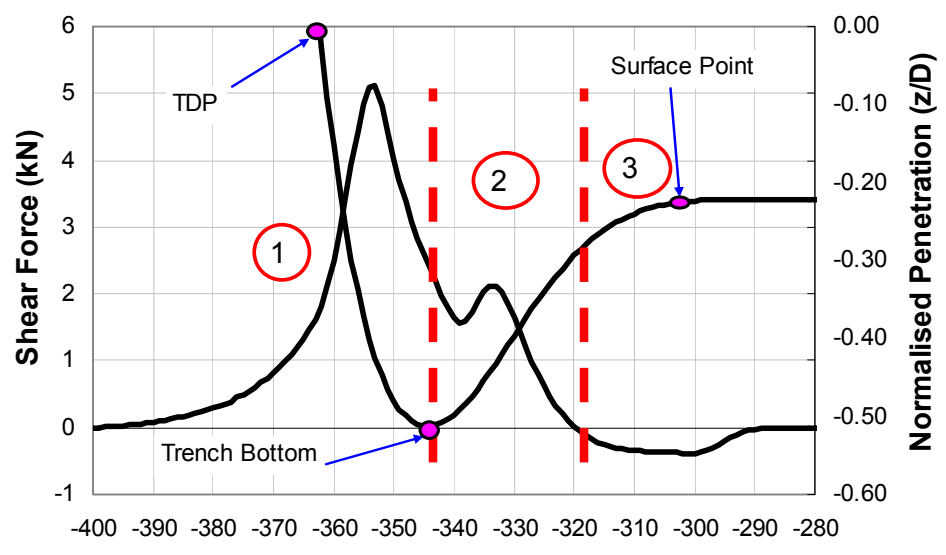

Horizontal Coordinate(m)

Figure 5-18: Shear force and penetration profile at TDZ

For better understanding of the variations of shear force, contact stress and bending moment using the non-linear hysteretic seabed, it is interesting to plot the stress variations in terms of their cyclic ranges, i.e. the difference of the maximum and minimum values experienced by the riser. Figure 5-19 to Figure 5-22 show the cyclic contact stress $(\mathrm{P} / \mathrm{D})$, contact stress envelope, shear force and the cyclic bending moment along the riser in the TDZ.

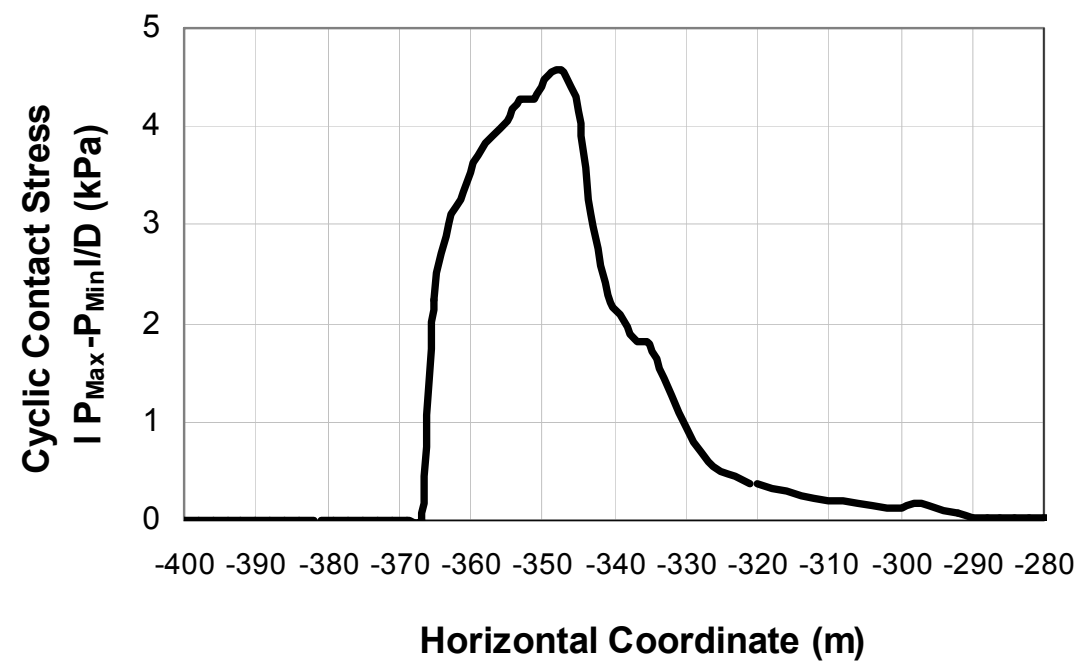

Figure 5-19: Cyclic contact stress range over 10 load cycles 


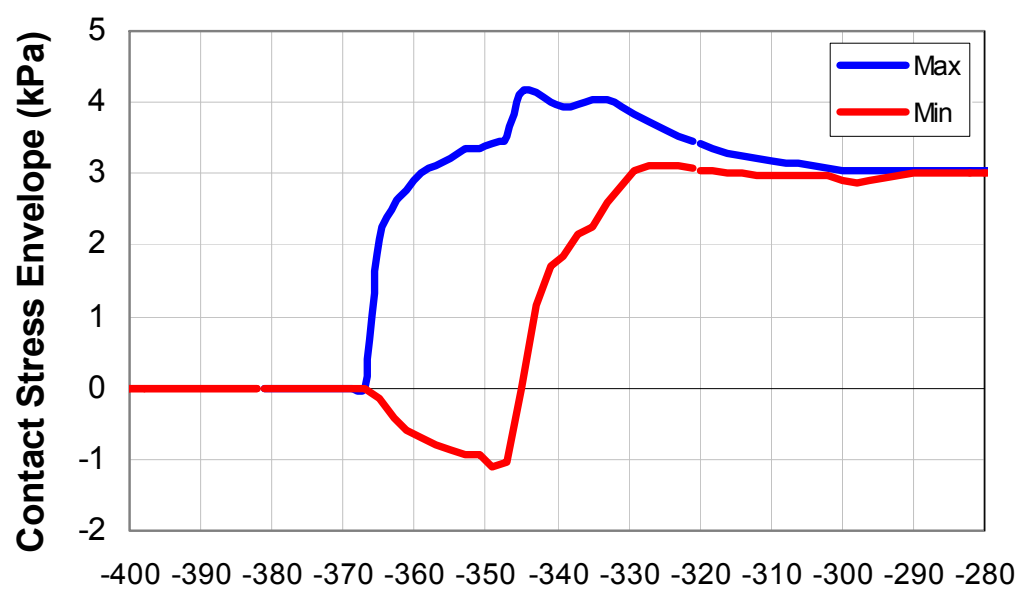

Horizontal Coordinate(m)

Figure 5-20: Contact stress envelope over 10 load cycles

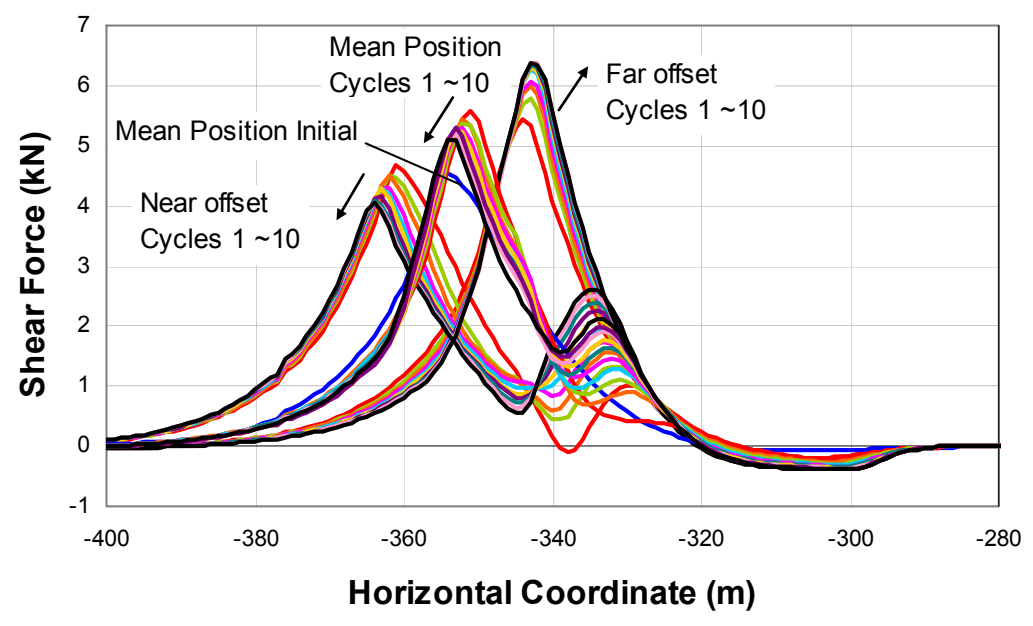

Figure 5-21: Shear force snapshots along the TDZ over 10 load cycles in extreme far and near offsets compared to the vessel mean position 


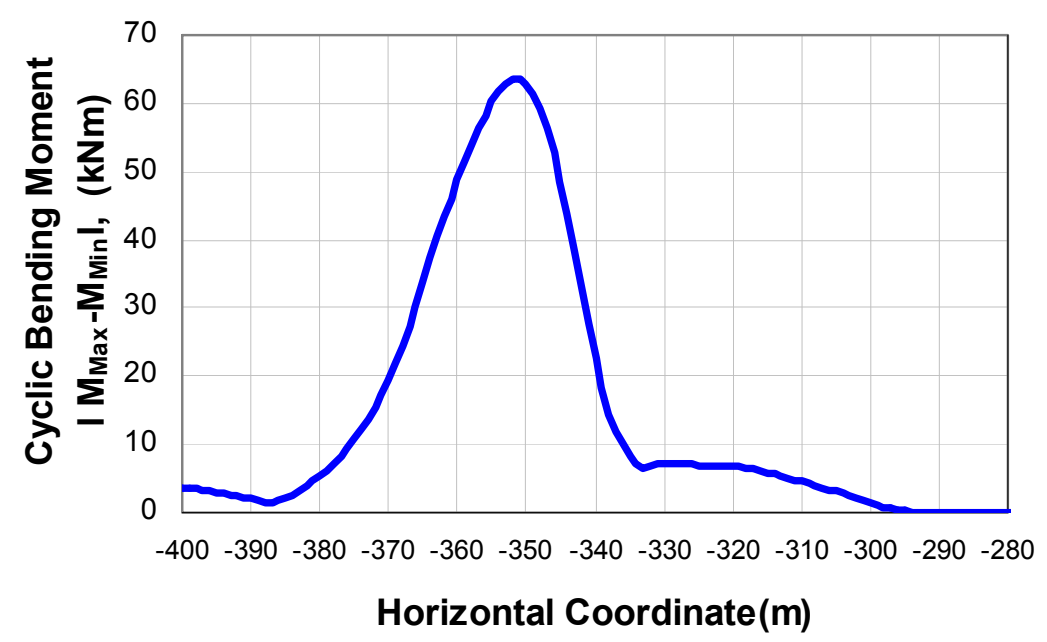

Figure 5-22: Cyclic bending moment distribution in the TDZ over 10 load cycles

\subsubsection{Comparison with linear elastic seabed in an in-place static analysis}

Results using the hysteretic non-linear model in a seabed with $1.3 \mathrm{kPa}$ undrained shear strength intercept and $1.5 \mathrm{kPa} / \mathrm{m}$ undrained shear stress gradient are compared here with those obtained using a linear elastic seabed with stiffness of $100 \mathrm{kPa}$, for a static inplace analysis with no vessel excitation. The overall configuration of the SCR and the other mechanical and structural parameters are as described in Chapter 4 (Table 4-1 and Table 4-2). Figure 5-23 shows the relative shapes of the SCR profile on the seabed, including TDP, magnitude and position of maximum embedment and the profile of contact stress for the two different soil models. 


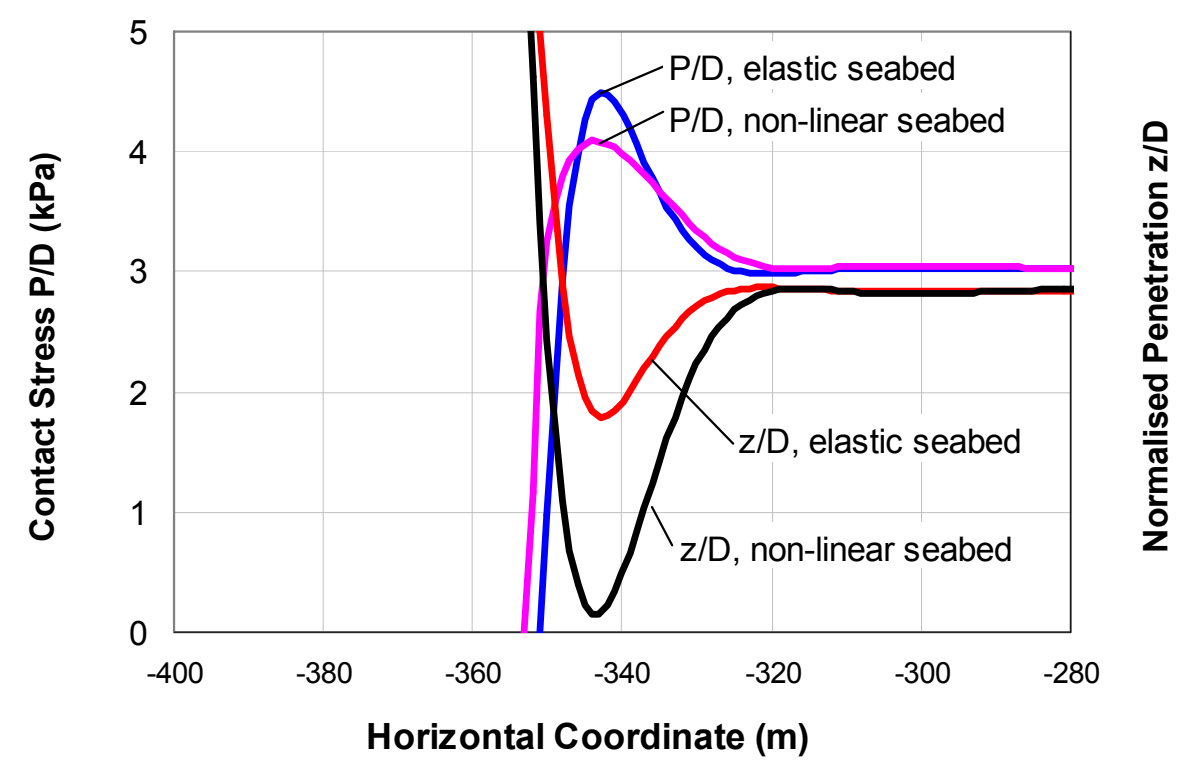

Figure 5-23: Embedment geometry and seabed reaction in linear elastic and non-linear seabed

Comparing the shear force and bending moment distribution along the TDZ for the linear elastic and non-linear seabed models shows how different seabed models can lead to different shear force and bending moment distribution as shown in Figure 5-24 and Figure 5-25.

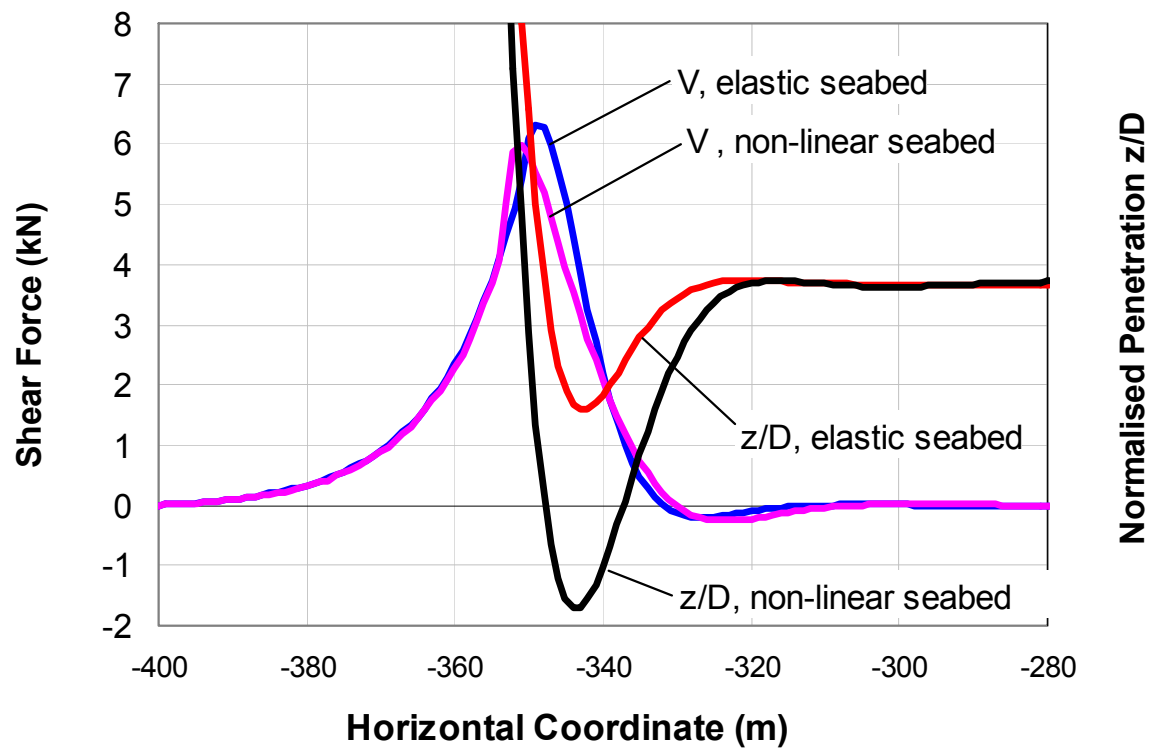

Figure 5-24: Embedment geometry and shear force in linear elastic and non-linear seabed 


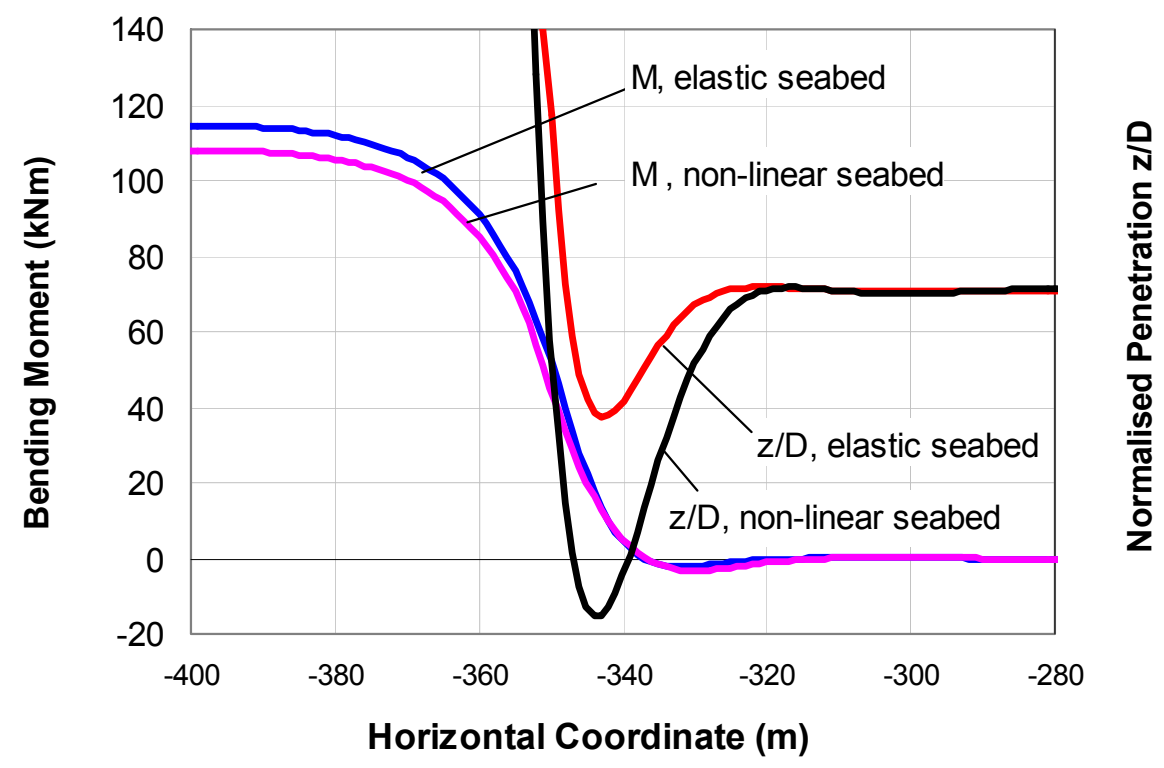

Figure 5-25: Embedment geometry and bending moment in linear elastic and non-linear seabed

Figure 5-26, shows the relation of penetration depth and reaction force in $100 \mathrm{kPa}$ linear elastic and two non-linear seabed with different shear strength.

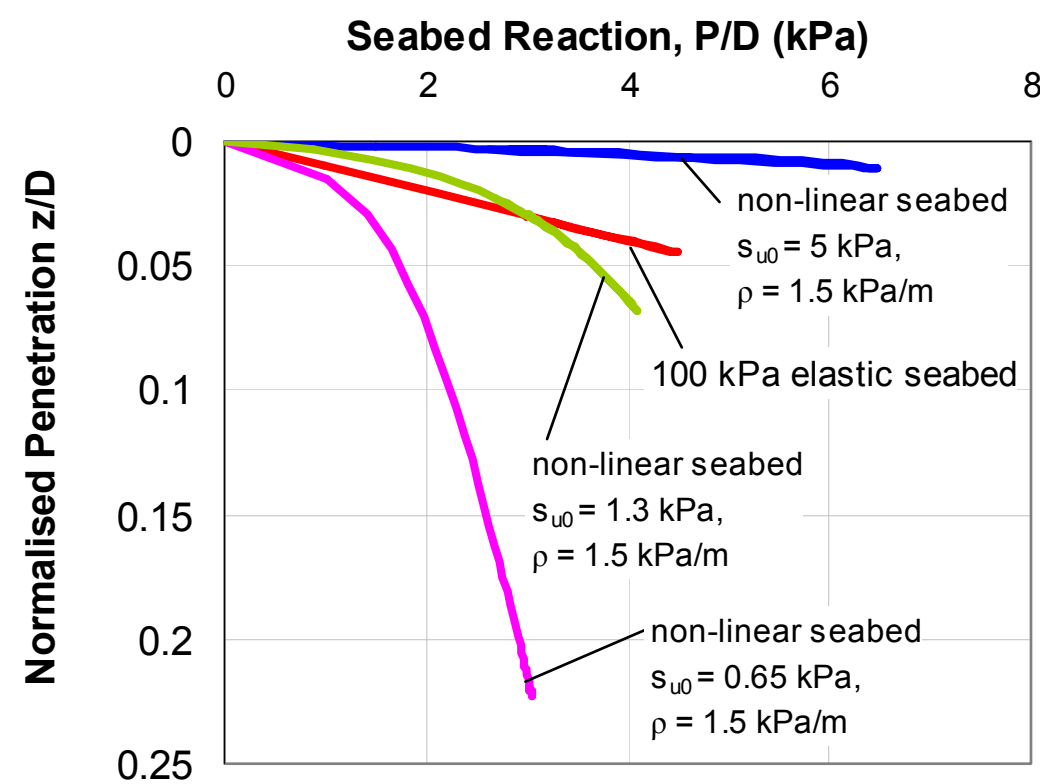

Figure 5-26: Seabed reaction against normalised penetration depth in linear elastic and non-linear seabed 


\subsubsection{Deep trenching by hysteretic non-linear seabed model}

The penetration profile generation illustrated in Figure 5-15 shows a gradual stabilisation in embedment depth. It seems that the trench deepens quickly during the early cycles and but reaches a stable maximum as the number of cycles increases. The final stabilised trench depth in the example of Figure 5-15 is somewhere between $0.5 \mathrm{D}$ and 0.6D. ROV surveys have shown final trench depths as large as 3D to 5D (Bridge et al. 2004), which is clearly higher than the range achieved in the example of Figure 5-15. The impact of the trench depth on the fatigue performance of SCRs is considered in detail in Chapter 7, but it is convenient here to examine the capability of the non-linear seabed model to simulate the formation of relatively deep trenches, similar to the range reported from ROV surveys. Trials showed that using extreme values for the repenetration delay parameter $\lambda_{\text {rep }}$ (higher than 1) can generate very deep trenches within only a few cycles of vessel displacement within the normal range of applicable sea states. As an example, Figure 5-27 shows results from a series of analyses illustrating deep trench generation by applying extreme values of re-penetration delay parameter $\lambda_{\text {rep }}$ and small values of suction ratio $\left(f_{\text {suc }}=0.05\right)$. Only three cycles of a very large amplitude has been applied, outside the normal range of applicable sea states $( \pm 37.5 \mathrm{~m}$ horizontal motion applied to the suspended end of the SCR causing $\pm 33.5 \mathrm{~m}$ horizontal motion of the TDP). This is just to evaluate the model capability for deep trenching. For refined fatigue analysis in the presence of deep trenches, more realistic amplitudes will be used within the range of applicable sea states and appropriate number of cycles, as will be shown later in Chapter 7.

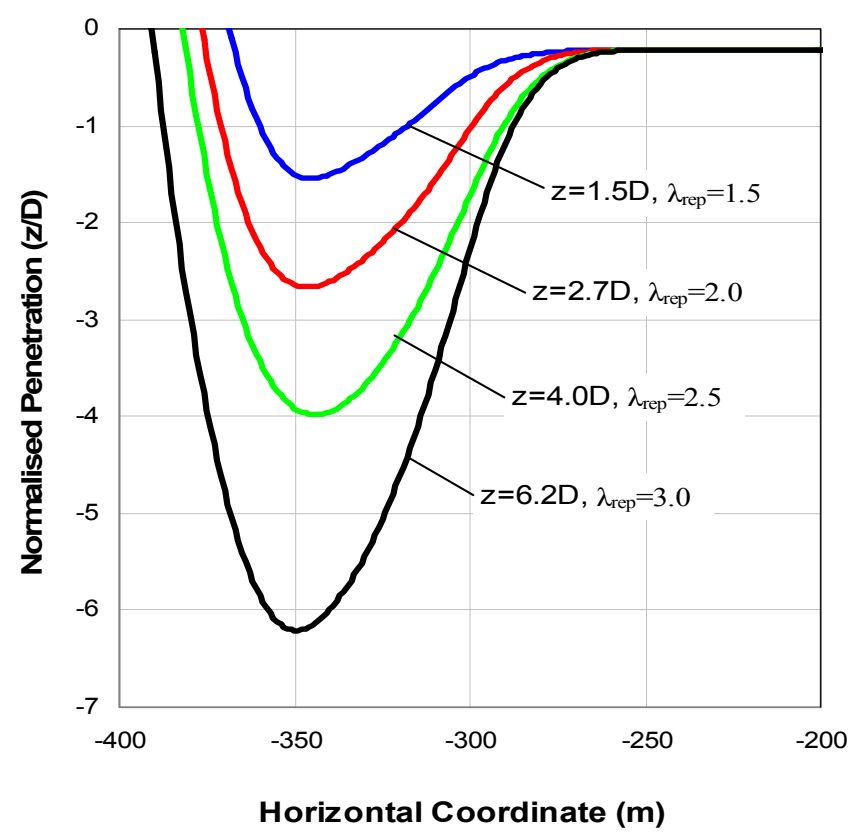

Figure 5-27: Deep trenching by extreme value of non-linear model parameters 
Figure 5-28 shows the penetration-resistance of a sample node (node 358). The riser reaches an ultimate trench depth in only a few cycles by using an extreme value of 3 for the re-penetration delay parameter $\lambda_{\text {rep. }}$. This leads to considerably decreased gradient of the re-penetration resistance curve along with rapid incremental penetration of the riser into the seabed.

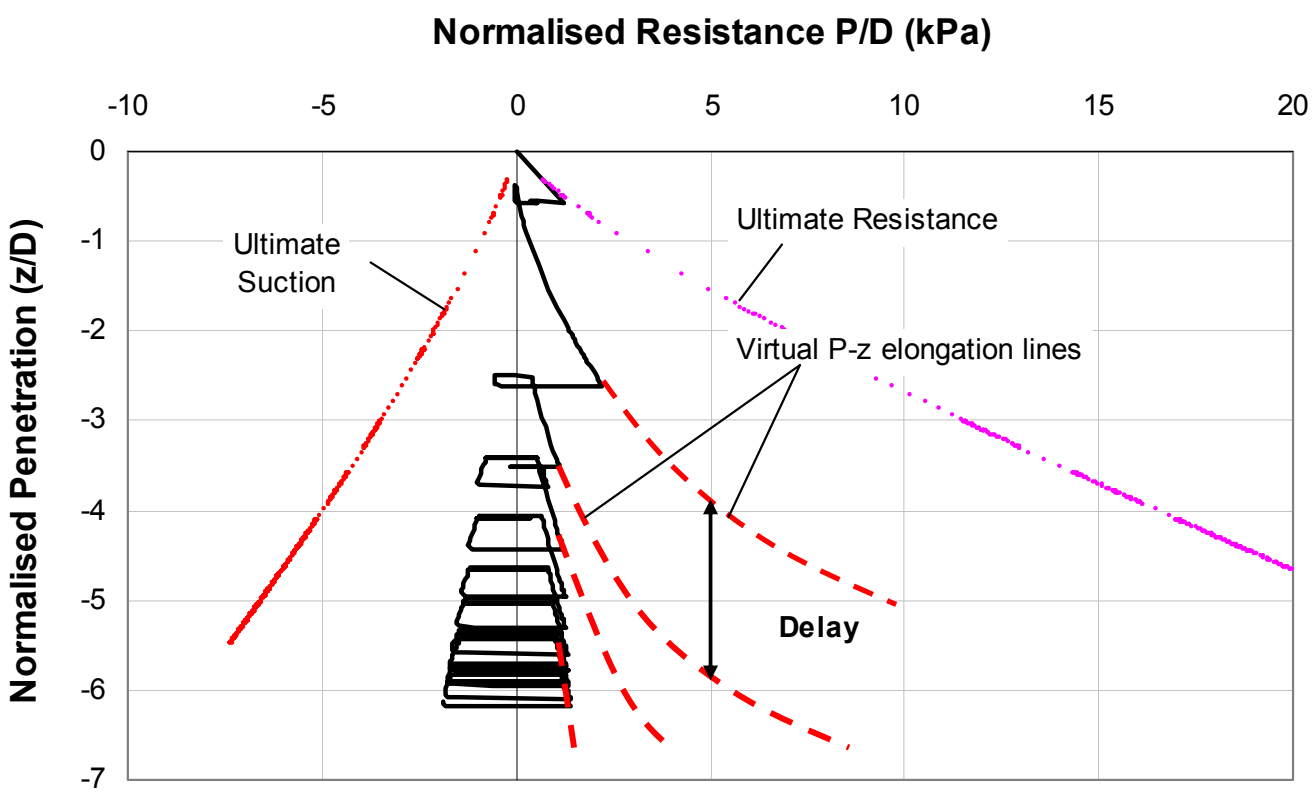

Figure 5-28: Penetration-resistance of node (N358) at the seabed for deep trenching $\left(\lambda_{\text {rep }}=3.0\right)$

\subsection{SUMMARY AND CONCLUSIONS}

The requirements for non-linear seabed interaction models have been reviewed and the hysteretic non-linear model recently developed by Randolph and Quiggin (2009) has been discussed in detail. This model was coded as a user-defined element in FORTRAN through the UEL subroutine of ABAQUS. The UEL was implemented to model the riser-seabed interaction for a generic steel catenary riser.

In-place static analysis was performed using ABAQUS to study the influence of the non-linear seabed interaction model on the stress and bending moment distributions along the SCR particularly through the touchdown zone and the results were compared with those for a linear elastic seabed. Quasi-static cyclic analysis was performed simulating horizontal perturbation of the vessel under an extreme sea state. The capability of the model to simulate progressive trench formation, due to cyclic penetration and lift-up of the SCR in the TDZ as a result of the vessel motion, was explored. The effect of certain model parameters on the response at a typical location on the SCR in the TDZ was investigated.

The preliminary study showed that trench formation under cyclic loading using the hysteretic non-linear seabed model has little effect on the overall bending moment 
distribution along the SCR or its maximum value in the touchdown zone. By contrast, the shear force and contact stress distribution in the TDZ were more influenced by the gradual deepening of the trench beneath the riser. This will play a vital role in the fatigue performance of the SCR in the TDZ, where the maximum shear force in the $\mathrm{SCR}$ is a key parameter affecting the development of fatigue damage, as will be discussed in the next chapter.

The capability of the non-linear model for simulating trenches several diameters deep, as observed in practice from ROV surveys, was demonstrated by using extreme values of the model parameter $\lambda_{\text {rep }}$. It is anticipated that this capability of the non-linear model will be very useful in investigating the effect of deep trenches on SCR fatigue calculations.

In the present chapter, the study of the hysteretic non-linear model was limited to applying 10 harmonic cycles of vessel motion, with the purpose of exploring the potential of the model. More extensive application of the model to fatigue analysis will be described in Chapter 7, where parametric studies will be presented investigating the influence of key features, such as suction force generation and trench formation, on SCR fatigue performance in the TDZ. 


\section{CHAPTER 6. FATIGUE PERFORMANCE OF SCR ON HYSTERETIC NON-LINEAR SEABED}

\subsection{INTRODUCTION}

As discussed in Chapter 2, the stiffness of the seabed soil beneath the riser is a function of the load level and displacement path followed at that point of the riser. The soil is also continually remoulded by the cyclic motion of the riser, leading to a gradual reduction in stiffness and strength. This is quite far from the simplified assumptions in current design practice, where the seabed is generally simulated by a series of linear elastic springs. In addition, survey results from ROV inspections of SCRs show that trenches several diameters deep can form beneath the riser in the touchdown zone (Bridge and Howells 2007), a fact that is also ignored in design because of general uncertainties associated with non-linear riser-seabed interaction and lack of quantitative guidance on trench shapes and depths. Although design practice is still mostly limited to linear seabed response, in recent years, there has been increasing awareness of the consequences of non-linear riser-seabed interaction, and the need to capture the actual soil response more closely (Clukey et al. 2008; Giertsen et al. 2004; Hodder et al. 2009). In this chapter, the influence of non-linear seabed model on fatigue performance in TDZ has been studied by implementing the hysteretic non-linear riser-seabed interaction model (Randolph and Quiggin 2009) described in Chapter 5. From a practical fatigue calculation standpoint, the impact of a non-linear seabed response is not limited just to the soil behaviour, but also has consequences for the application of Miner's rule for superposition of damage due to different wave packages. Therefore the study may be divided into two main aspects:

- the influence of a hysteretic non-linear seabed model on the stress range variation in the SCR;

- the effect of ordering of wave packages and number of wave cycles modelled in each package in applying Miner's superposition rule for fatigue damage calculations.

The hysteretic non-linear seabed model (Randolph and Quiggin 2009) influences the stress distribution and consequently the stress range variation in the TDZ through primary and secondary effects as below:

- Primary effect: variation of SCR geometric profile and consequently contact stress in TDZ throughout the gradual trench formation

- Secondary effect: Suction force mobilisation during riser uplift 
From a damage calculation point of view, the SCR fatigue damage under various sea states cannot be simply superposed using Miner's rule for the non-linear seabed response, since the damage calculation will be affected by the ordering of the sea states and the number of wave cycles applied. Hence, this chapter will also investigate appropriate procedures for fatigue calculation using the non-linear seabed model.

Initially, the influence on fatigue performance of progressive embedment of the SCR and suction force mobilisation will be investigated for a single sea state, focusing on the stress variation in the TDZ. Then the entire sea states package presented in Chapter 4 will be applied, under various cycles and hierarchies, to investigate how best to apply Miner's rule for cumulative damage calculations. Finally, the importance of trench formation on fatigue performance will be highlighted as an introduction to a study of pre-trenching the SCR artificially, which will be considered in detail in the next chapter.

\subsection{FATIGUE DAMAGE AT TDZ UNDER SINGLE WAVE}

In order to investigate application of Miner's rule for a non-linear seabed response, a single sea state, with significant wave height of $4.5 \mathrm{~m}$ and period of $13.0 \mathrm{~s}$, has been taken for vessel excitation. The study focuses on the influence of gradual increase of the riser embedment depth on the stress range variation and consequently on the fatigue damage in the TDZ. The riser diameter $(\mathrm{D}=0.324 \mathrm{~m})$, overall system setup, vessel's RAO and global coordinate system are as per Chapter 4, as will be used for all the analyses performed in this thesis. Note that the horizontal coordinate is the distance from the anchored end of the SCR laid on the seabed and is shown by negative values. The parameters adopted for the non-linear hysteretic seabed model are given in Table 6-1.

Table 6-1: Soil model parameters

\begin{tabular}{|l|c|c|}
\hline \multicolumn{1}{|c|}{ Parameter } & Symbol & Value \\
\hline Mudline shear strength & $\mathrm{s}_{\text {um }}$ & $10 \mathrm{kPa}$ \\
\hline Shear strength gradient & $\rho$ & $1.5 \mathrm{kPa} / \mathrm{m}$ \\
\hline Power law parameter & $\mathrm{a}$ & 6 \\
\hline Power law parameter & $\mathrm{b}$ & 0.25 \\
\hline Normalised maximum stiffness & $\mathrm{K}_{\max }$ & 200 \\
\hline Suction ratio & $\mathrm{f}_{\text {suc }}$ & 0.3 \\
\hline Suction decay parameter & $\lambda_{\text {suc }}$ & 0.5 \\
\hline Repenetration parameter & $\lambda_{\text {rep }}$ & 0.5 \\
\hline
\end{tabular}

The chosen single wave was applied to the vessel with a large number of cycles. The analysis was deliberately stopped after 1539 cycles due to achieving a stabilised embedment profile in the seabed. The trench profile, von Mises stress range variation and fatigue damage in the TDZ were captured after different numbers of cycles. The 
gradual embedment of the riser into the seabed in the touchdown zone is shown in Figure 6-1.

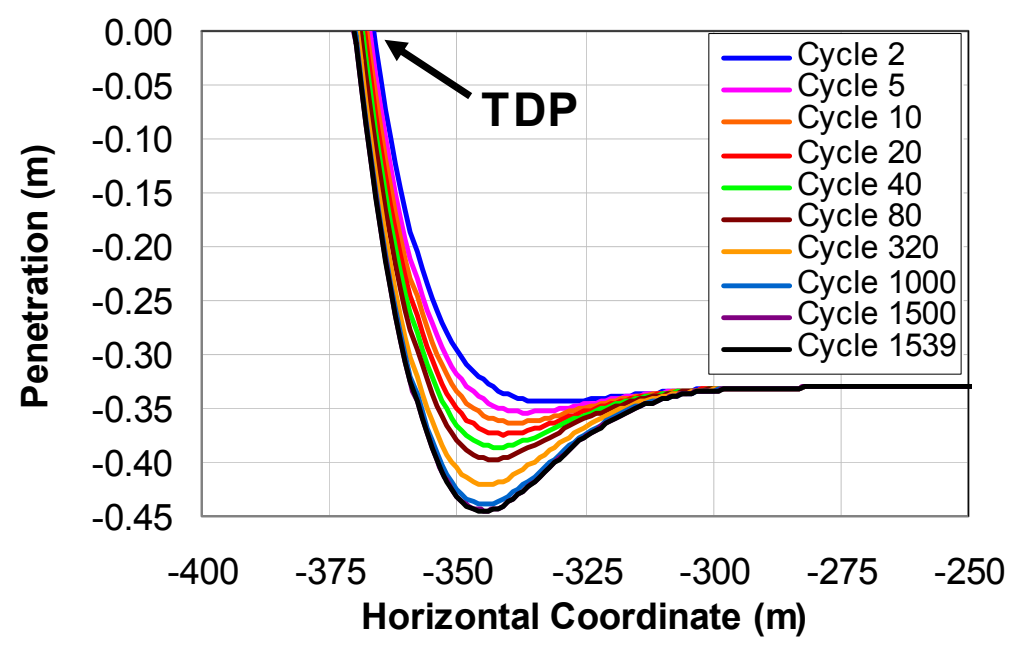

Figure 6-1: Gradual variation and deepening of trench profile under single wave application

From Figure 6-1, it is clear that the hysteretic non-linear soil model (Randolph and Quiggin 2009) leads to gradual trench deepening under cyclic motion of the riser. This leads to gradual increase of the maximum von Mises stress range level (see Figure 6-2) and consequently fatigue damage in the touchdown zone.

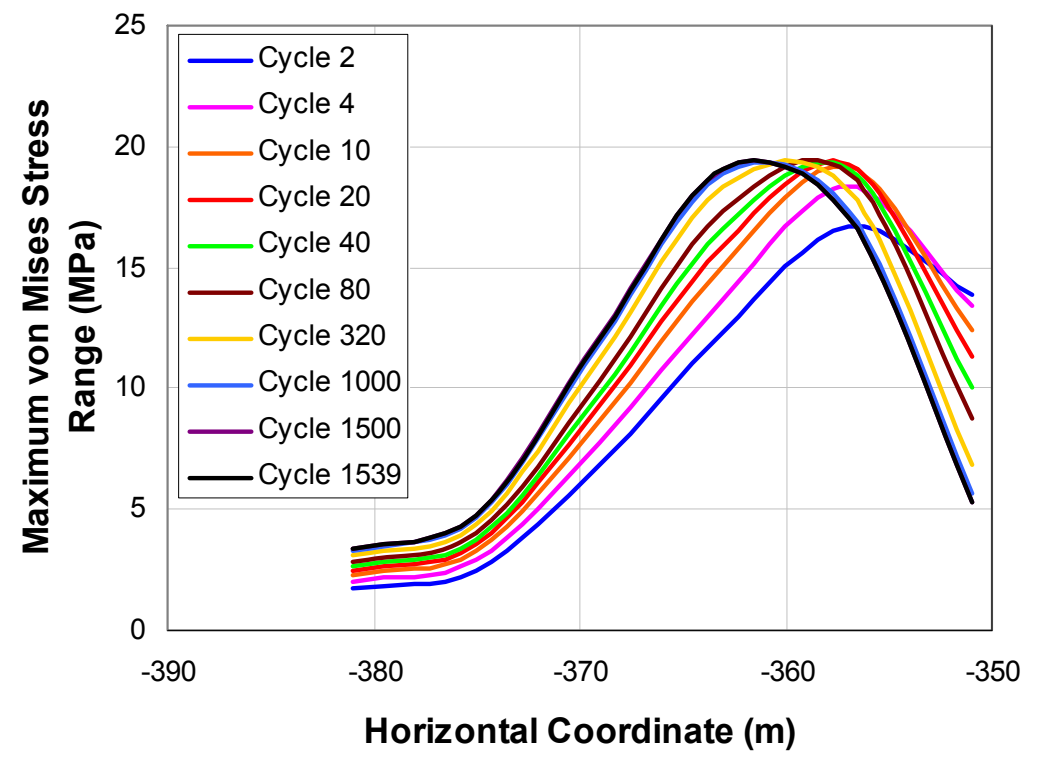

Figure 6-2: Gradual increase of maximum von Mises stress range with number of wave cycles

The fatigue damage distribution has been calculated and plotted in Figure 6-3. The low level of fatigue damage is due to applying only one sea state with a limited number of waves. 


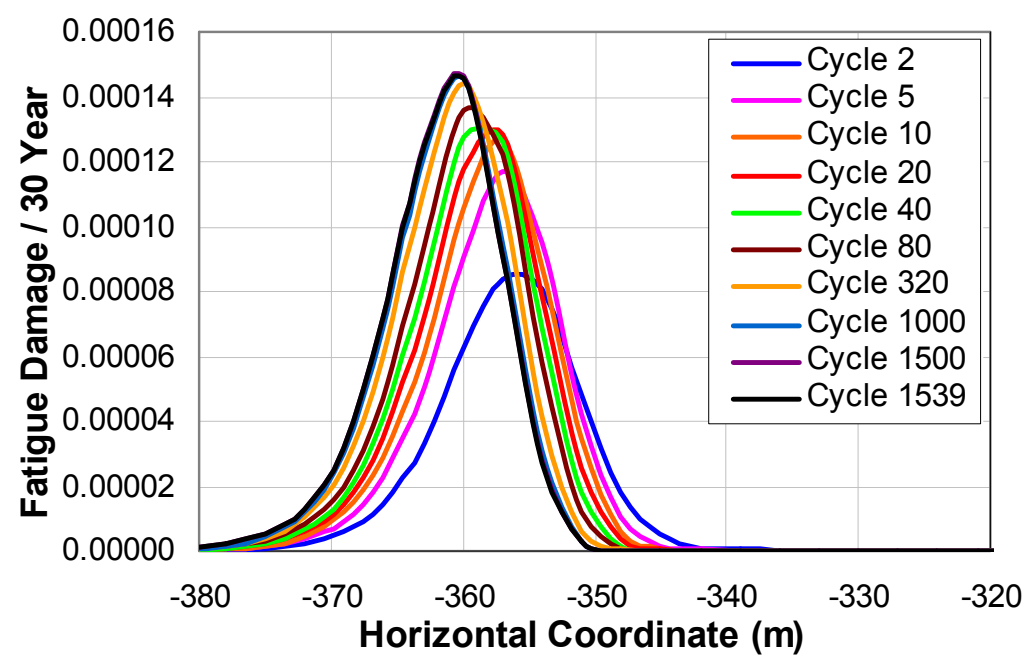

Figure 6-3: Gradual increase of fatigue damage with trench deepening

To better understand the overall profile of fatigue damage in TDZ, the fatigue damage distribution has been plotted in logarithmic scale in Figure 6-4.

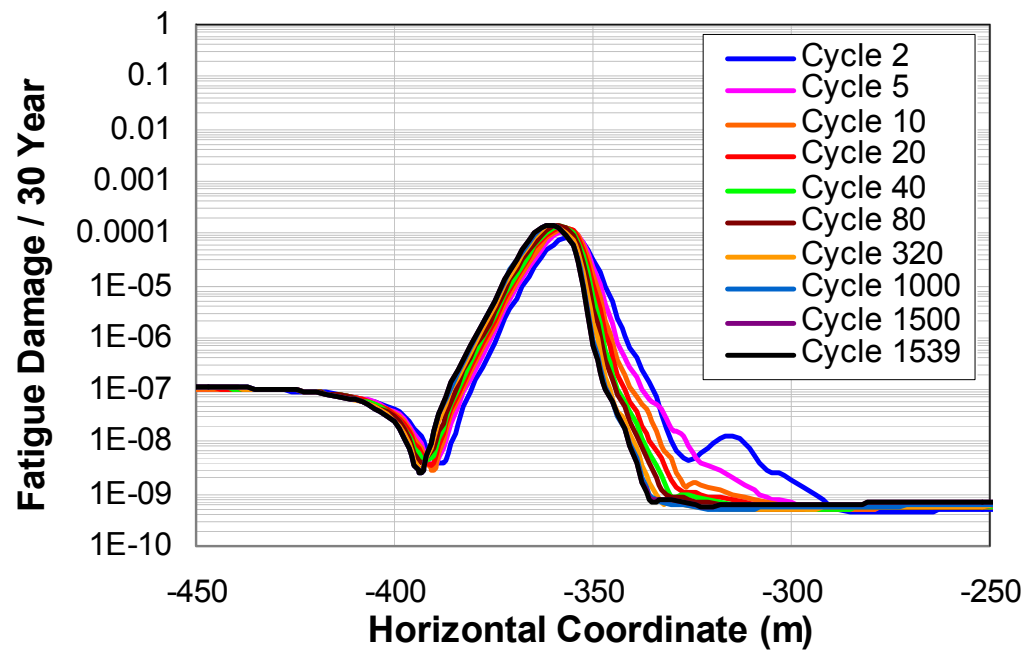

Figure 6-4: Gradual increase of fatigue damage with trench deepening

As discussed before in Chapter 4, the main parameter in calculation of fatigue damage is the von Mises stress range and any change in fatigue damage is directly related to this parameter. Figure 6-5 shows the gradual increase of von Mises stress range level with increasing number of cycles (and riser embedment). The stress range gradually becomes constant as the embedment depth stabilises. 


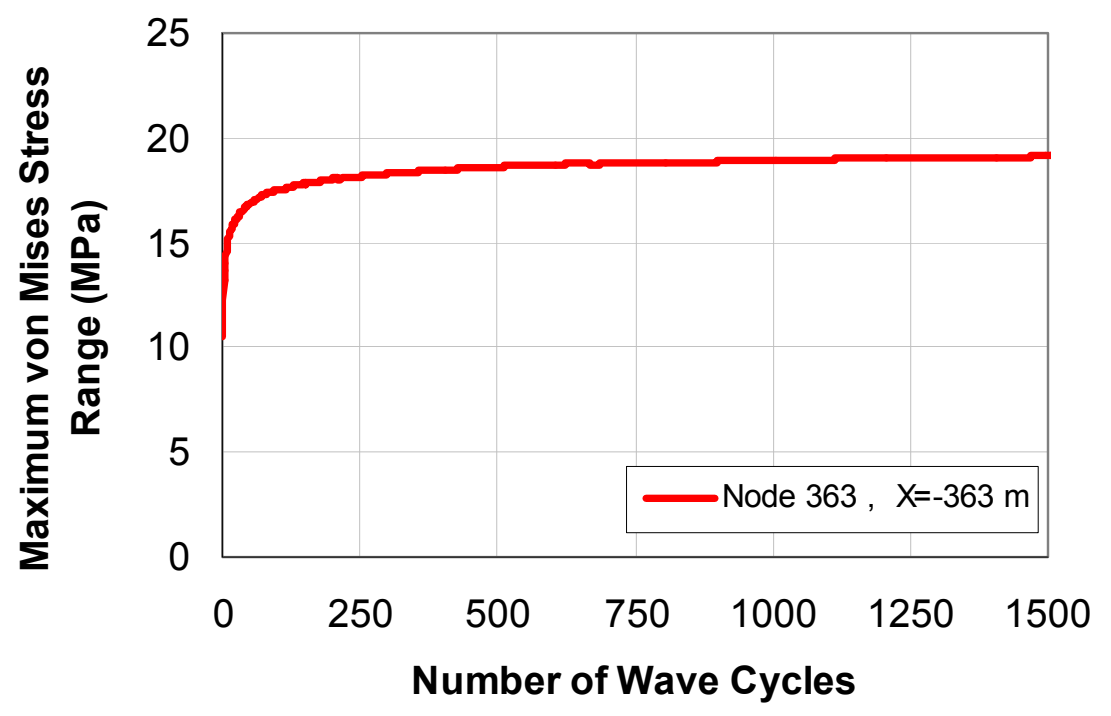

Figure 6-5: Gradual increase of von Mises stress range variation with trench deepening

This raises the first question about fatigue damage calculation using the non-linear seabed model, namely the number of wave cycles that need to be applied in analysis to give a true representation of the von Mises stress range for that particular sea state. It was shown in Chapter 4 that increasing the number of applied wave cycles and changing the hierarchies of sea states had no effect on fatigue damage for an elastic seabed. Thus for calculating the fatigue damage for a linear elastic seabed, only a single cycle of each wave need be applied (or a few cycles to avoid stress fluctuations in the transition between sea states). By contrast, for the non-linear seabed response, Figure 6-5, shows that the von Mises stress range variation and consequently the fatigue damage in the TDZ depends directly on the number of applied wave cycles. Thus the fatigue damage calculated using fewer applied wave cycles will be less than the fatigue damage obtained by applying the full number of wave cycles, due to the different von Mises stress range. This is shown in Figure 6-6, where the final fatigue damage for a nominal SCR life of 1539 cycles has been estimated from the stress range obtained after applying 5, 80 or 1500 cycles. 


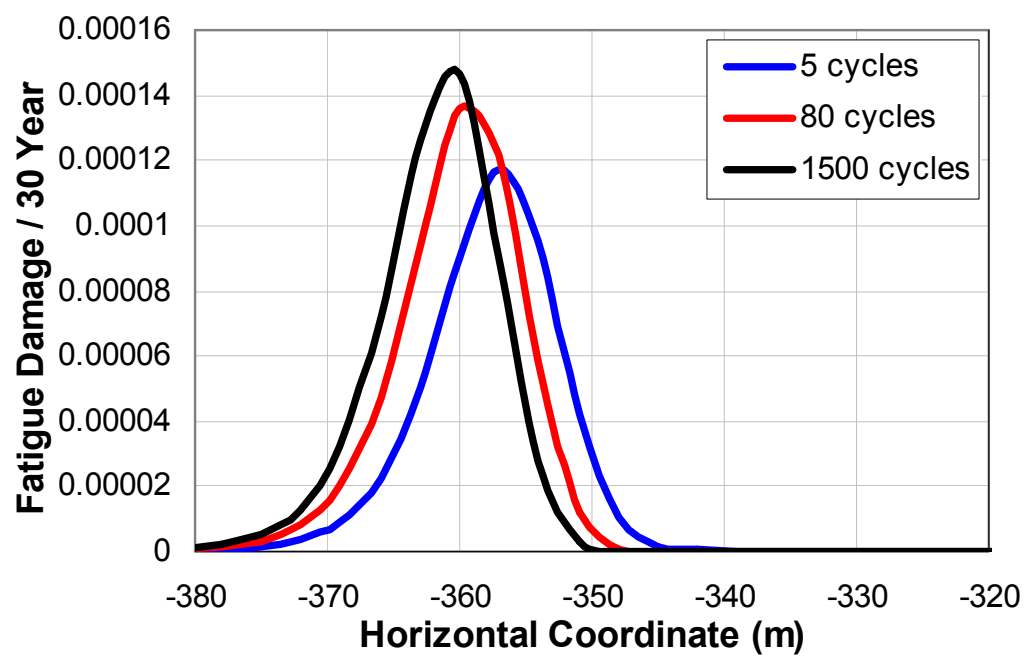

Figure 6-6: Fatigue damage calculation by increasing number of wave cycles

The results show that, for the non-linear model, it is important that a sufficient number of wave cycles for any given wave package is applied to allow the von Mises stress range level to stabilise, becoming approximately constant with application of further wave cycles.

The second challenging issue is the application of Miner's rule to superpose the fatigue damage caused by different sea states, in order to accomplish a complete fatigue analysis. The embedment depth and consequently the von Mises stress range variation will depend on the hierarchy of sea states. In another words, different sea state ordering will lead to different embedment depths, different von Mises stress ranges and different fatigue damage. Hence, it is no longer possible to apply Miner's rule by considering the fatigue damage contribution from each different wave package, considered in isolation. Instead, the full sequence of wave packages needs to be considered, with appropriate choices for (a) the hierarchy of the separate wave packages, and (b) the number of wave cycles analysed for each package.

These issues are investigated in the next sections, where the impact of different sea states hierarchies is investigated and strategies are developed for choosing the required number of wave cycles to be analysed for a given package, depending on the previous history of wave packages.

\subsection{VON MISES STRESS RANGE VARIATION BY WAVE HIERARCHY}

\subsubsection{Two single waves with different orders}

Two different sea states were applied to the vessel and separate analyses performed with different ordering of the waves. In the first analysis the larger wave (sea state I, 
$\mathrm{H}_{\mathrm{s}}=16.0 \mathrm{~m}, \mathrm{~T}_{\mathrm{z}}=11.0 \mathrm{~s}$ ) was applied first, followed by the smaller wave (sea state II, $\mathrm{H}_{\mathrm{s}}=4.5 \mathrm{~m}, \mathrm{~T}_{\mathrm{z}}=13.0 \mathrm{~s}$ ), both with 200 wave cycles. The second analysis was the same as the first but reversing the order of the sea states, with sea state II followed by sea state I. The maximum von Mises stress range for a sample node (Node 363, $x=363 \mathrm{~m}$ ) and the fatigue damage distribution in the TDZ are compared in Figure 6-7.

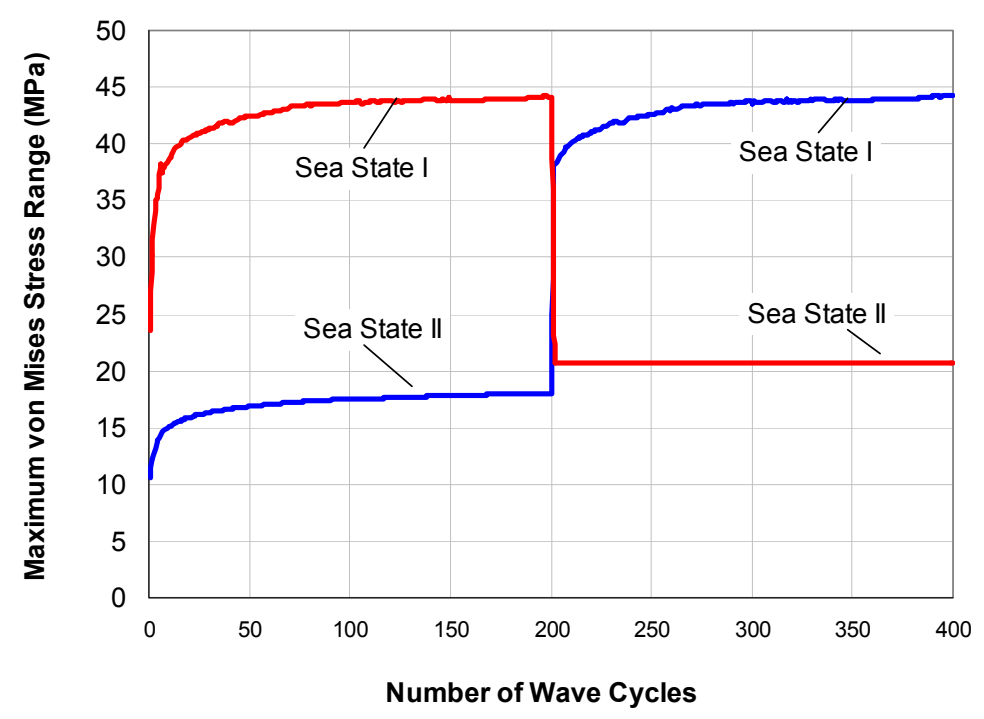

Figure 6-7: Maximum von Mises stress variation range in wave height decreasing order

As seen in Figure 6-7, in the first analysis the von Mises stress range within sea state I gradually increased with increasing number of wave cycles, while for sea state II it remained essentially constant from the start of application. By contrast in the second analysis, the stress range gradually increased with increasing number of wave cycles for both sea states. Thus in the first analysis, the von Mises stress range for sea state II was insensitive to the number of wave cycles applied and the analysis could have been performed with only one or a few cycles, just as for a linear elastic seabed. This is an important finding and can be considered as the first clue for answering the questions raised in the previous section regarding the number of wave cycles for different wave hierarchies. It is also noteworthy that the von Mises stress range for sea state II is greater in the first case, where it follows the larger sea state I, than in the second case. Figure 6-8 shows the difference in fatigue damage distribution through the TDZ due to different order of the sea states showing anticipated differences for the different order of sea states due to the non-linear response of the seabed. Since the von Mises stress range for sea state I is much the same, regardless of the order, while that for sea state II is greater in the first analysis when it comes second, the cumulative fatigue damage is higher for the first analysis. 


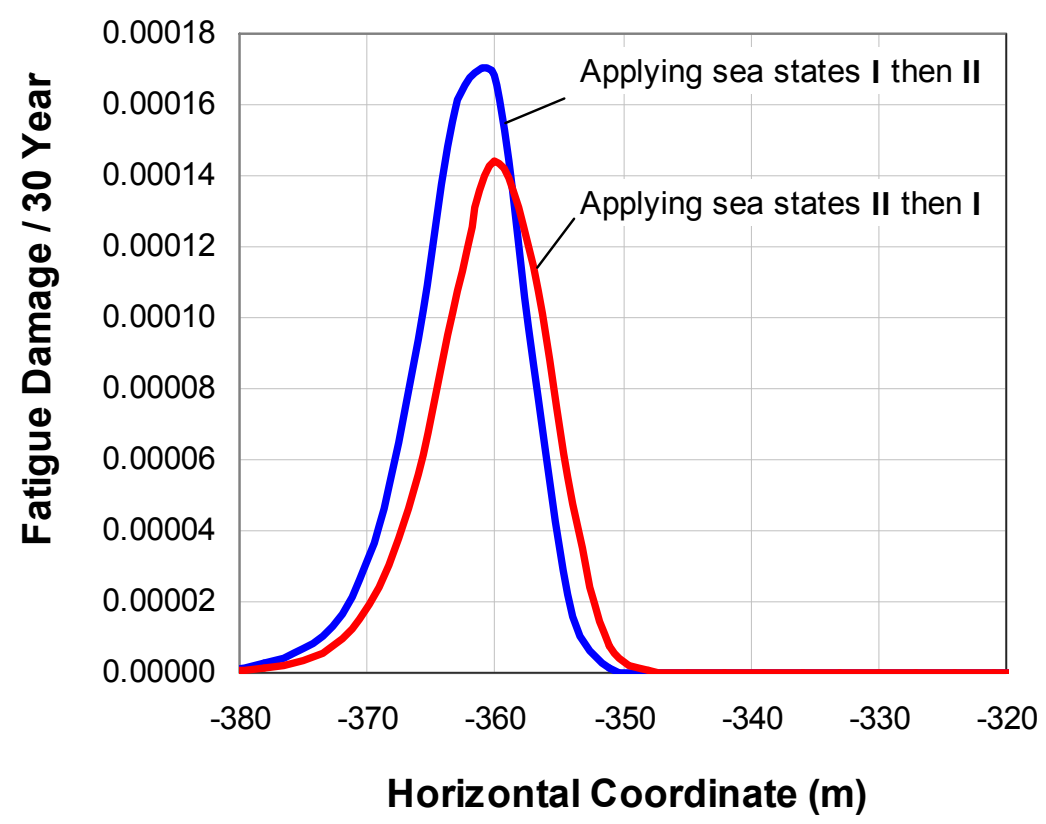

Figure 6-8: Fatigue damage at TDZ with different wave orders in plastic seabed

The explanation for the specific variation of von Mises stress range is that in the first analysis, sea state II starts in a condition in which the seabed has already experienced a more severe wave and consequently more severe soil deflections than imposed by this sea state. However, in the second analysis, the smaller wave hits the vessel first and the second (more severe) sea state is still able to further deepen the embedment induced by the smaller wave, resulting in a gradually increasing von Mises stress variation range (though note that this starts from a slightly higher value than without the preconditioning by sea state II).

Therefore, a working hypothesis is that:

- In a quasi-static system, if the vessel has already experienced a sea state more severe than the currently applied sea state, the von Mises stress range in the current sea state will be independent of the number of wave cycles.

If this hypothesis is shown to be true for more general sea states, it means that, if the most severe sea state hits the vessel first, then the von Mises stress ranges (and calculated fatigue damage) for subsequent sea states will be independent of the wave hierarchies and the number of wave cycles applied for each sea state. This would greatly facilitate application of Miner's rule for superposition of fatigue damage from different wave packages.

In addition, comparing the results of Figure 6-7 and Figure 6-5 (see Figure 6-9), shows that applying a more severe wave in advance (sea state I) has increased the maximum von Mises stress range for sea state II beyond that reached after 1500 cycles, due to the 
greater initial embedment of the riser (see Figure 6-9). This raises another hypothesis that development of deeper riser embedment (or trenches) will cause an increase in the von Mises stress range and consequently fatigue damage in the TDZ. If this hypothesis is substantiated, then the maximum fatigue damage in the TDZ can be obtained by initially establishing an 'ultimate' design trench at the start of the analysis, although the methodology for making such a trench is itself a challenging issue as will be discussed in the next chapter.

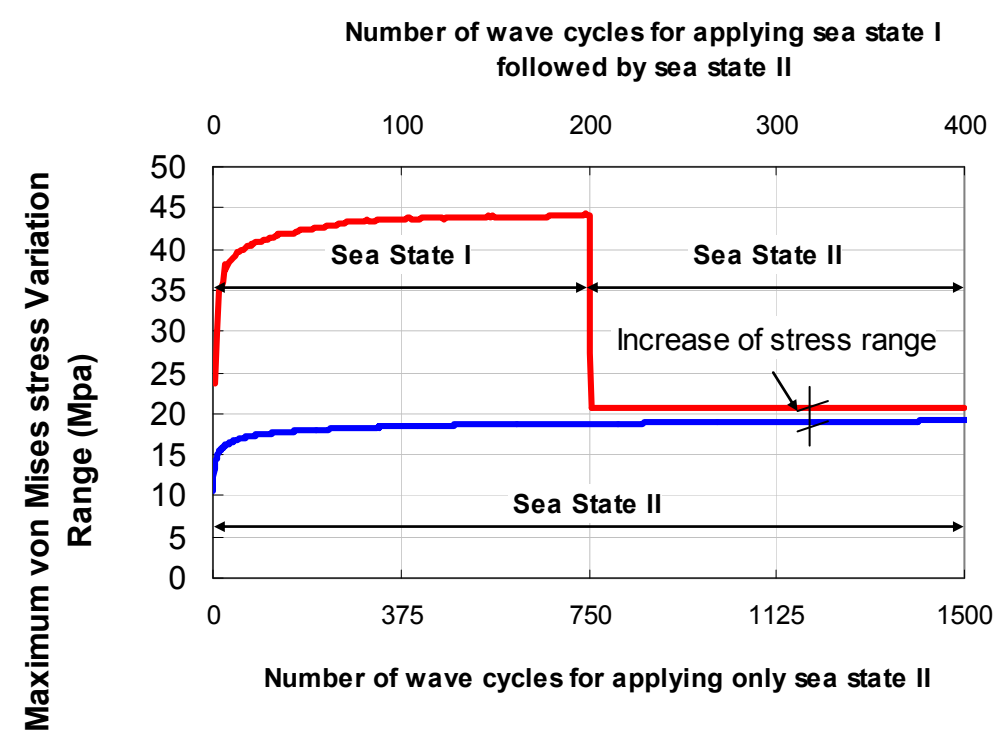

Figure 6-9: Comparison of ultimate stress variation range for sea state II with and without sea state I

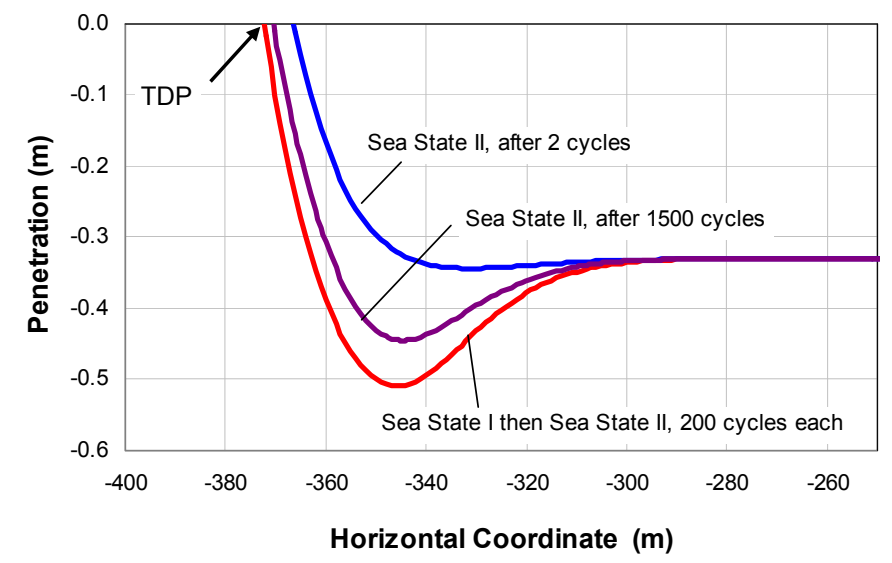

Figure 6-10: Seabed profiles corresponding to Figure 6-9

The preliminary findings from applying one or two sea states will now be examined by applying the full package of sea states. 


\subsubsection{Full package of waves with regular order}

A regular ordering of full sea states from Table 4-3 (Chapter 4), associated with maximum to minimum vessel offset has been applied to the vessel using the non-linear plastic seabed model with parameters defined in Table 6-2. Initially, the response to 10 cycles of each wave state is analysed.

Table 6-2: Soil model parameters

\begin{tabular}{|l|c|c|}
\hline \multicolumn{1}{|c|}{ Parameter } & Symbol & Value \\
\hline Mudline shear strength & $\mathrm{s}_{\mathrm{um}}$ & $0.65 \mathrm{kPa}$ \\
\hline Shear strength gradient & $\rho$ & $1.5 \mathrm{kPa} / \mathrm{m}$ \\
\hline Power law parameter & $\mathrm{a}$ & 6 \\
\hline Power law parameter & $\mathrm{b}$ & 0.25 \\
\hline Normalized maximum stiffness & $\mathrm{K}_{\max }$ & 200 \\
\hline Suction ratio & $\mathrm{f}_{\text {suc }}$ & 0.3 \\
\hline Suction decay parameter & $\lambda_{\text {suc }}$ & 0.5 \\
\hline Repenetration parameter & $\lambda_{\text {rep }}$ & 0.5 \\
\hline
\end{tabular}

Figure 6-11 shows a representation of the time base surge offset of the vessel for the descending order of applied waves.

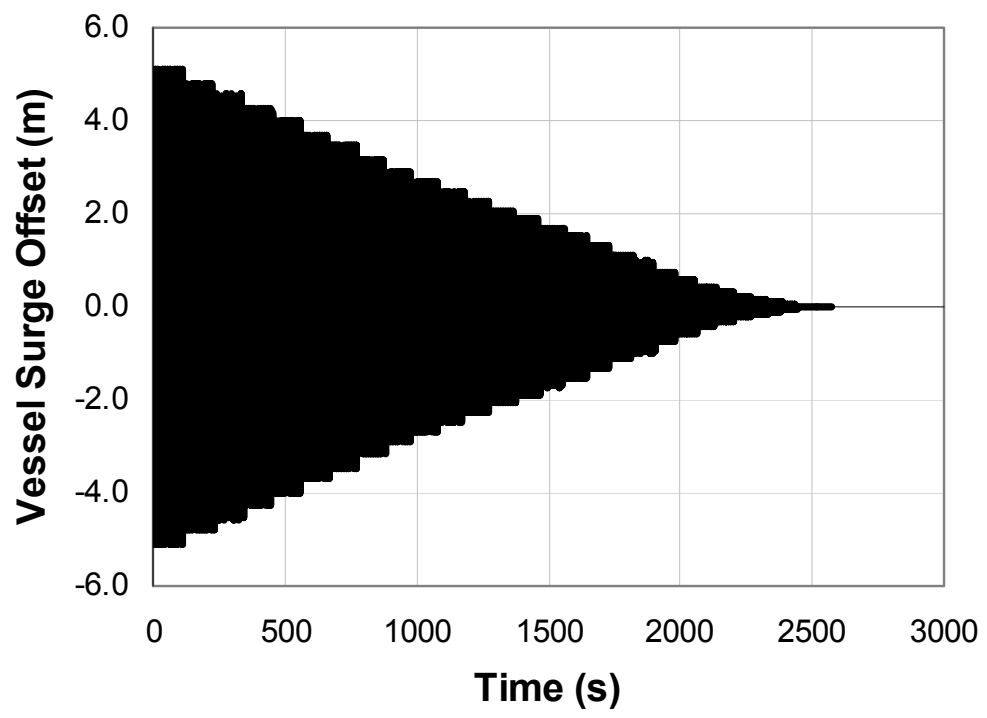

Figure 6-11: Vessel excitation in descending order of wave severity

The von Mises stress range variation calculated for a sample node on the SCR (Node.363) is illustrated in Figure 6-12, showing the rapid stabilisation of stress range level for all sea states where the system has experienced a more severe wave previously. 
This confirms the initial results obtained from two single waves, presented in Figure 6-7.

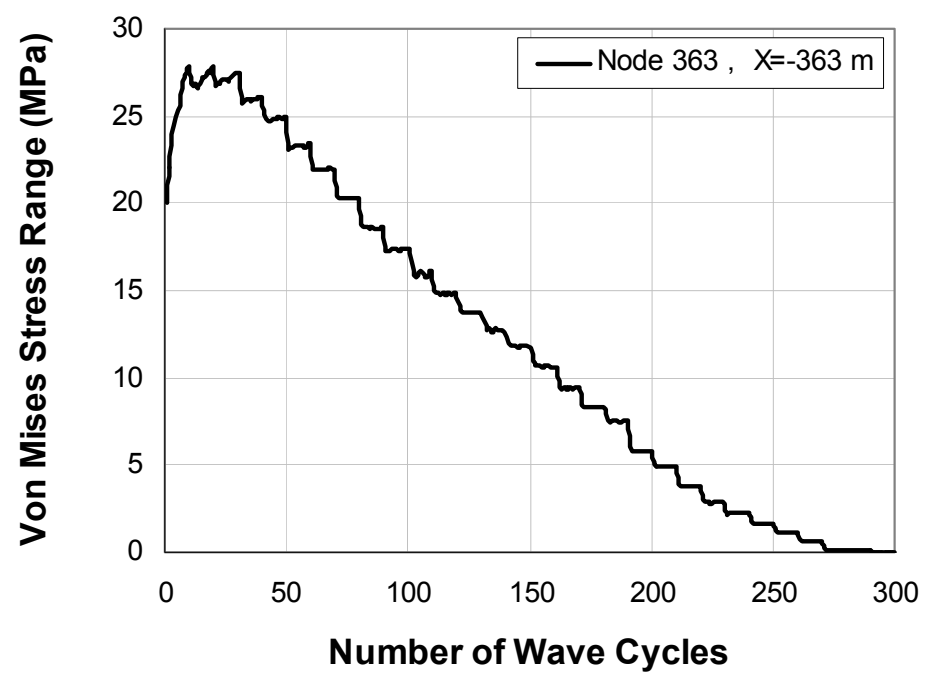

Figure 6-12: Von Mises stress variation range for full sea state package in descending order of wave severity

A zoomed view of Figure 6-12, for the early stages, has been plotted in Figure 6-13. This plot shows an increasing regime of von Mises stress variation range for the first four sea states of the analysis with the total number of wave cycles under 40. Beyond this point, the von Mises stress variation range shows rapid stabilisation to constant values during the load cycles, just as for a linear elastic seabed, allowing the Miner's rule to be applied to superpose fatigue damage from individual waves.

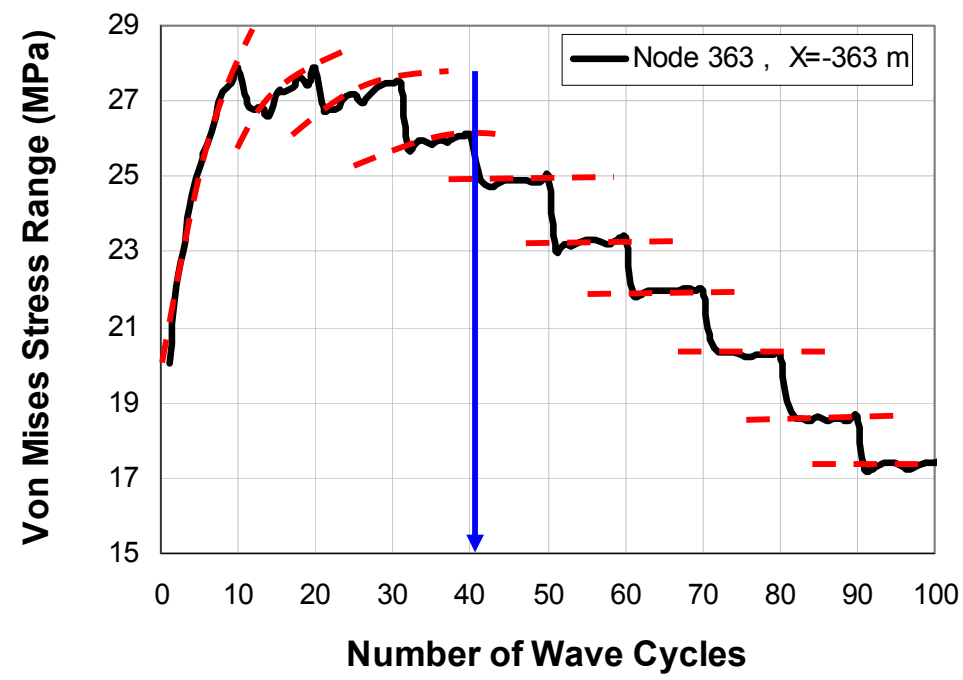

Figure 6-13: Initial stages of von Mises stress variation range for full sea state package 
The rapid stabilisation of the von Mises stress range during a few early cycles has a direct relation with the embedment response showed in Figure 6-14. A high proportion, some $75 \%$, of the final embedment depth has been developed by the first sea state alone and the embedment reaches more than $90 \%$ of its final depth by the end of the third sea state.

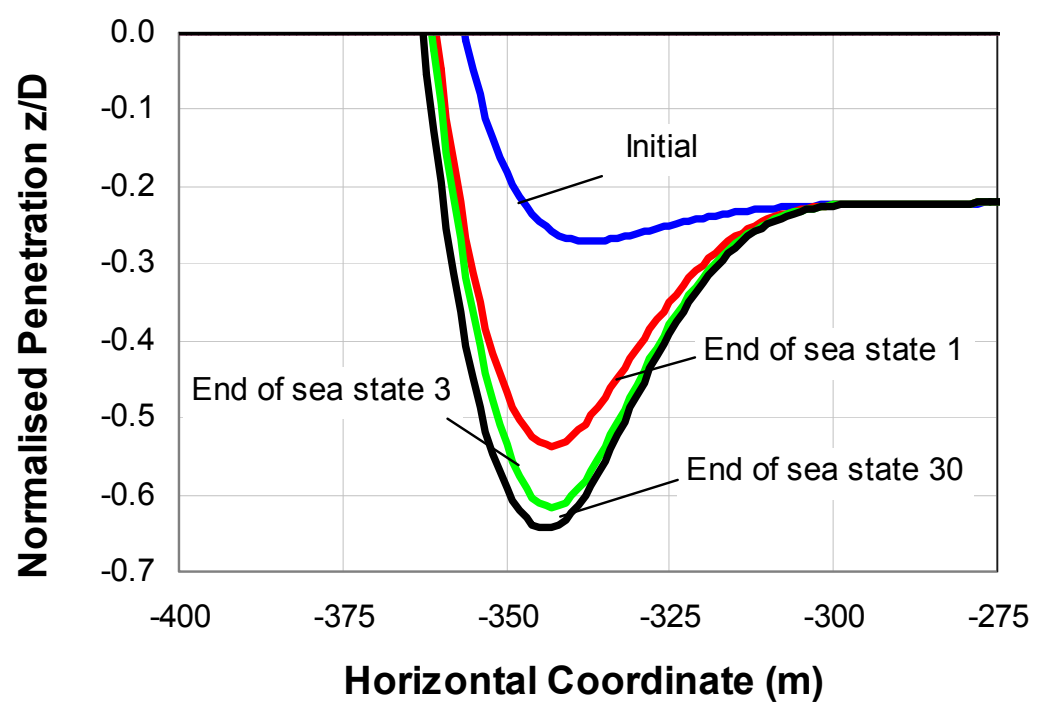

Figure 6-14: Trench profile at various stages of applying full sea state package

Before concluding, it is worth examining the von Mises stress range variation for irregular random ordering of the waves in order to be confident that the application of a particular sea state will stabilise the von Mises stress range for all subsequent smaller waves.

\subsubsection{Full package of waves with various random orders}

This section considers random ordering of the wave packages detailed in Table 4-3, initially for a linear elastic seabed as further demonstration of the independence of the von Mises stress range from the number of wave cycles analysed. A random order (No.1) was applied to the floating system with the same setup as previously and with 10 cycles of each wave applied. Figure 6-15, illustrates the random wave application order through the vessel's surge offset during the total analysis time and a partial window of wave application for two sea states is presented in Figure 6-16. 


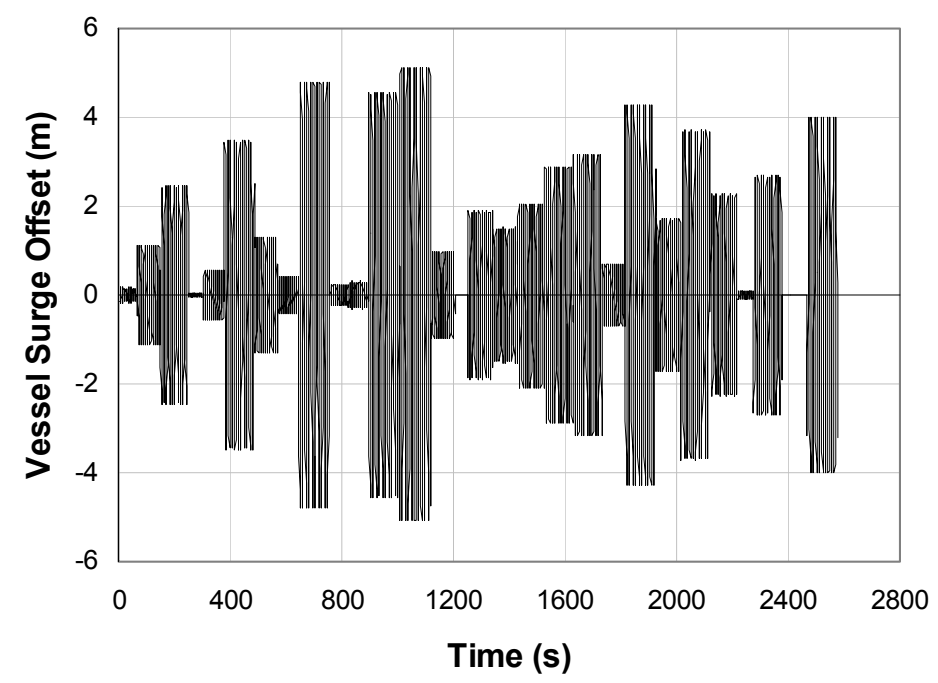

Figure 6-15: Vessel surge offset against analysis time in random wave order No.1

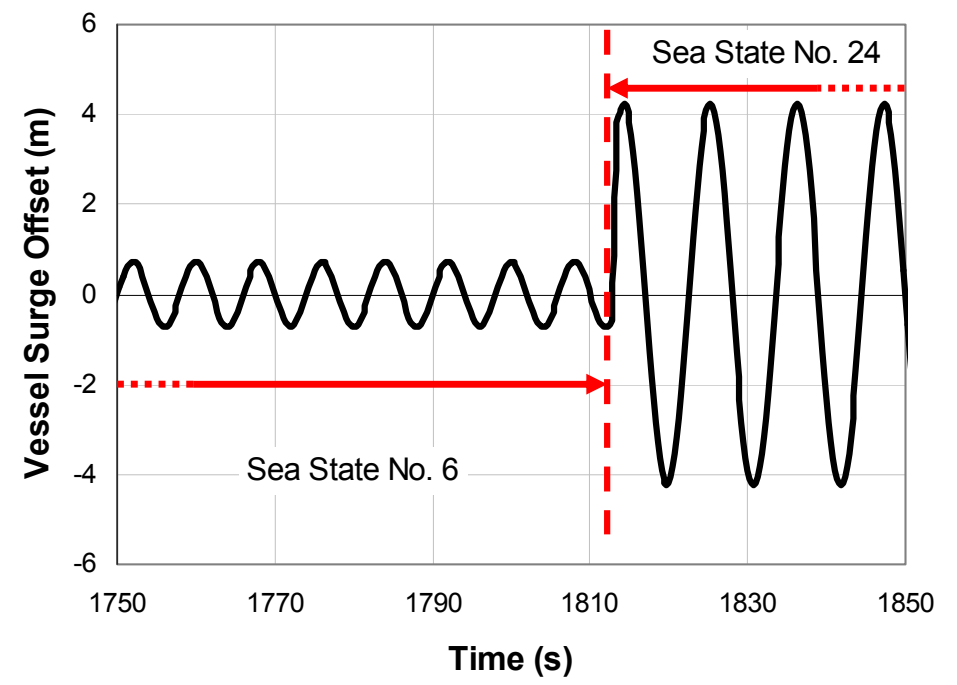

Figure 6-16 : Detail of vessel surge offset against analysis time for two sea states in random wave order No.1

Figure 6-17 shows the von Mises stress range variation for this analysis. The straight horizontal lines of von Mises stress range variation shows the independency from the number of applied wave cycles of each sea state, and also the hierarchy of sea states, allowing application of Miner's rule for superposition of fatigue damage from individual sea states. 


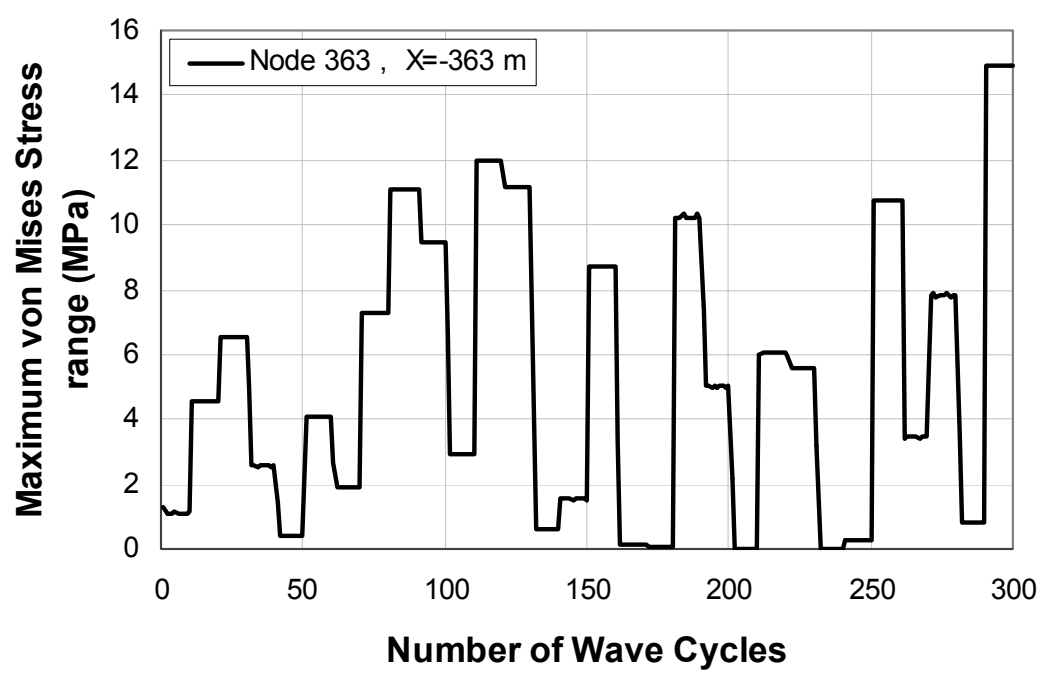

Figure 6-17: Von Mises stress range variation with random wave order in linear elastic seabed

Now the same order of sea states and the same analysis is repeated for the hysteretic non-linear seabed with model parameters as shown in Table 6-2, again applying 10 cycles of each sea state. Before discussing the results, it is necessary to remember that we are not concerned here with comparison of the von Mises stress ranges and final fatigue damage magnitude between the linear elastic and non-linear plastic seabed models. The focus is just on the effect of the non-linear seabed interaction on calculation of the fatigue performance.

Figure 6-18 shows the maximum von Mises stress variation range against the number of wave cycles for random order No.1, confirming again the earlier hypothesis. The sea states in sections A, C, E and G in Figure 6-18, show a gradual increase of von Mises stress range with the number of applied wave cycles. These are sections in which the current sea state exceeds any previous sea state. Thus in these sections the von Mises stress variation range and consequently the fatigue damage depend on the number of wave cycles and the hierarchy of sea states, In order to apply Miner's rule, these sea states would need to be applied over many more cycles, until the von Mises stress rang had stabilised, and also an appropriate order to the sea states would need to be determined. By contrast, for the sea states in sections B, D, F and all sea states in the range $\mathrm{H}$ show constant von Mises stress variation ranges just as for a linear elastic seabed, insensitive to the number of applied waves and the hierarchy of sea states, because of the prior application of more severe sea states. In these sections, linear superposition of fatigue damage using Miner's rule is straightforward, extrapolating the damage contribution from a few cycles to the full life-time number of cycles.

To illustrate the insensitivity of the maximum von Mises stress range to the hierarchy of sea states in the ranges like B, D, F and $\mathrm{H}$, the order of sea states in range $\mathrm{H}$ has been reshuffled and the analysis has been repeated swapping the place of sea states $5,11,18$ 
and 21. The maximum von Mises ranges have then been compared with those for the original hierarchy in Figure 6-19 and Figure 6-20. As expected, the comparison shows the same level of von Mises stress range in the re-shuffled analysis, demonstrating the insensitivity of sea states in range $\mathrm{H}$ to the hierarchy of sea states due to presence of more severe sea states in advance (ranges A, C, E and G).

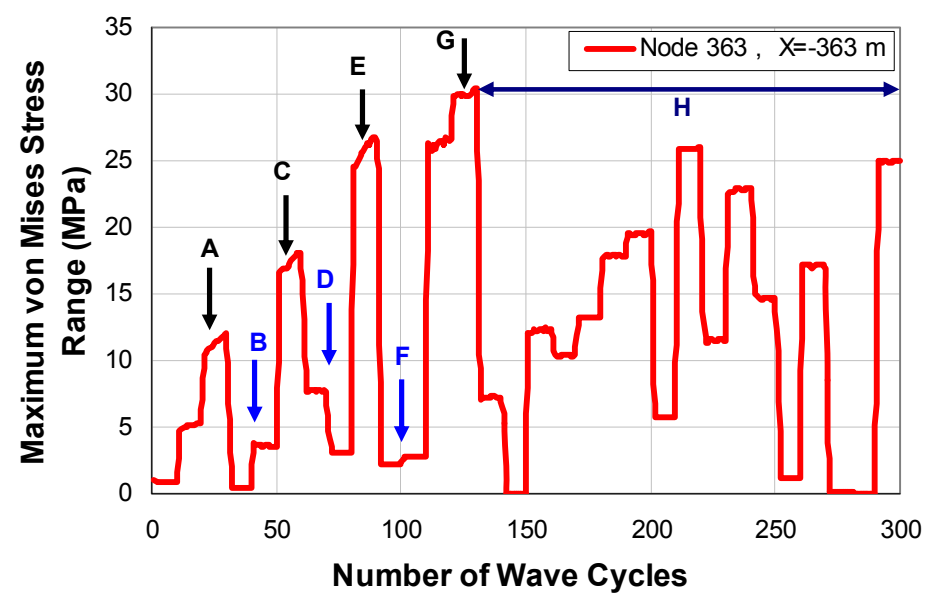

Figure 6-18: Maximum von Mises stress variation range in wave height random order No.1

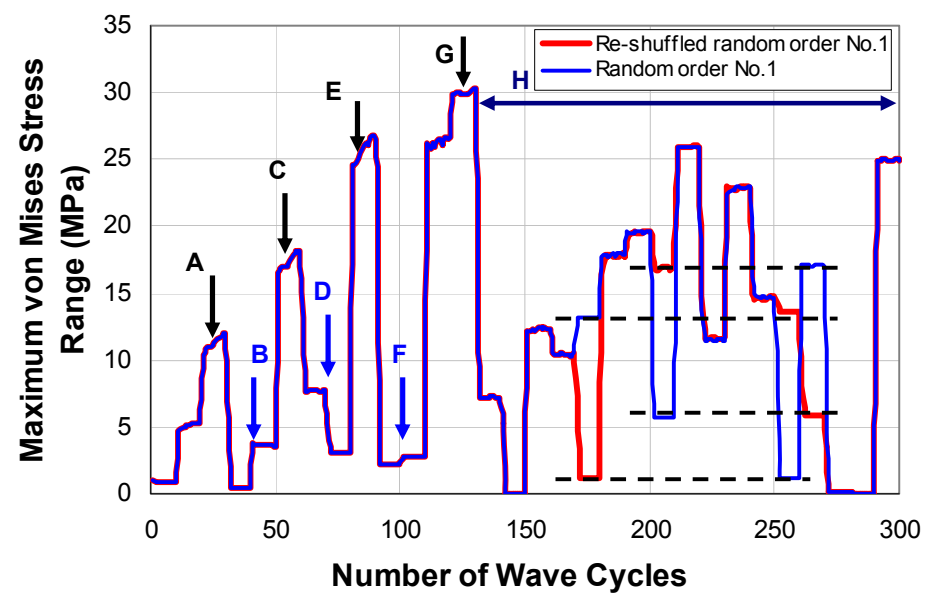

Figure 6-19: Comparison of re-shuffled analysis with original random order No.1 


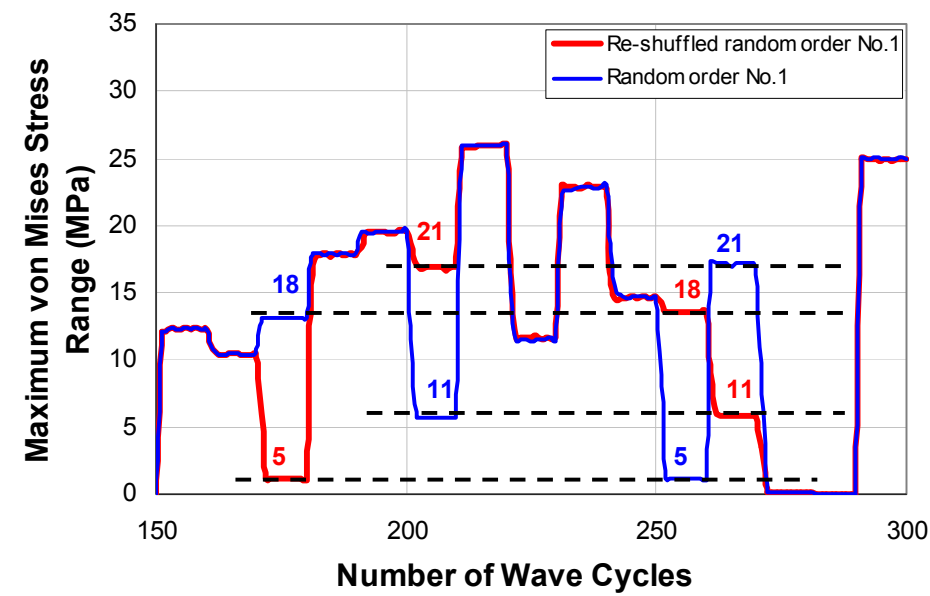

Figure 6-20: Comparison of re-shuffled analysis with original random order No.1 within range $\mathbf{H}$

Two further random order of waves, referred to as random Order 'No.2' and 'No.3' have been developed, and the analysis repeated with the same seabed model parameters. Figure 6-21 and Figure 6-22 illustrate the surge offset of the vessel with random order No.2 and No.3.

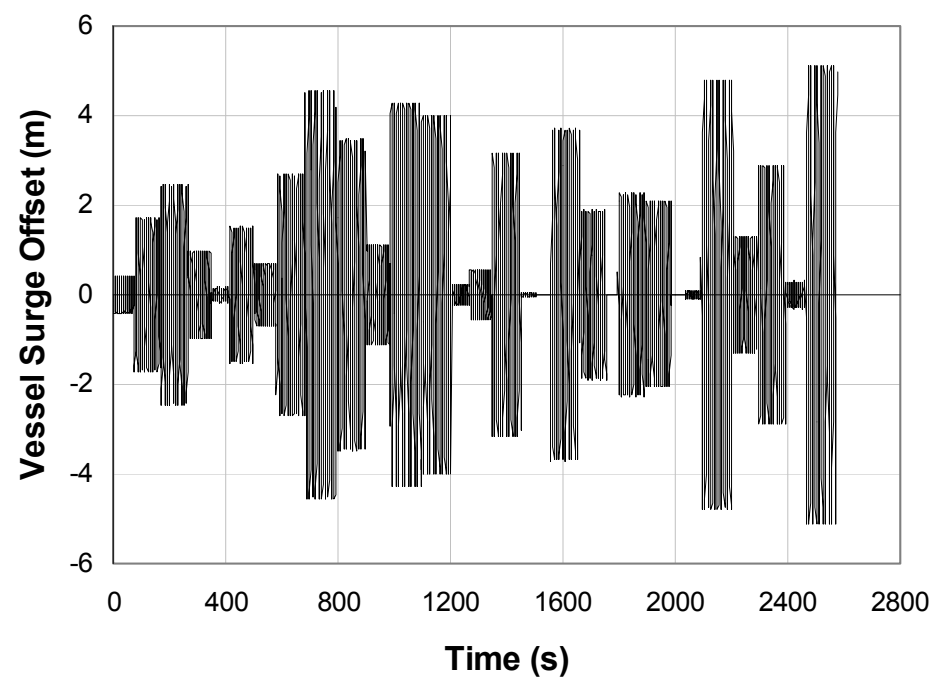

Figure 6-21: Vessel surge offset against analysis time in random wave order No.2 


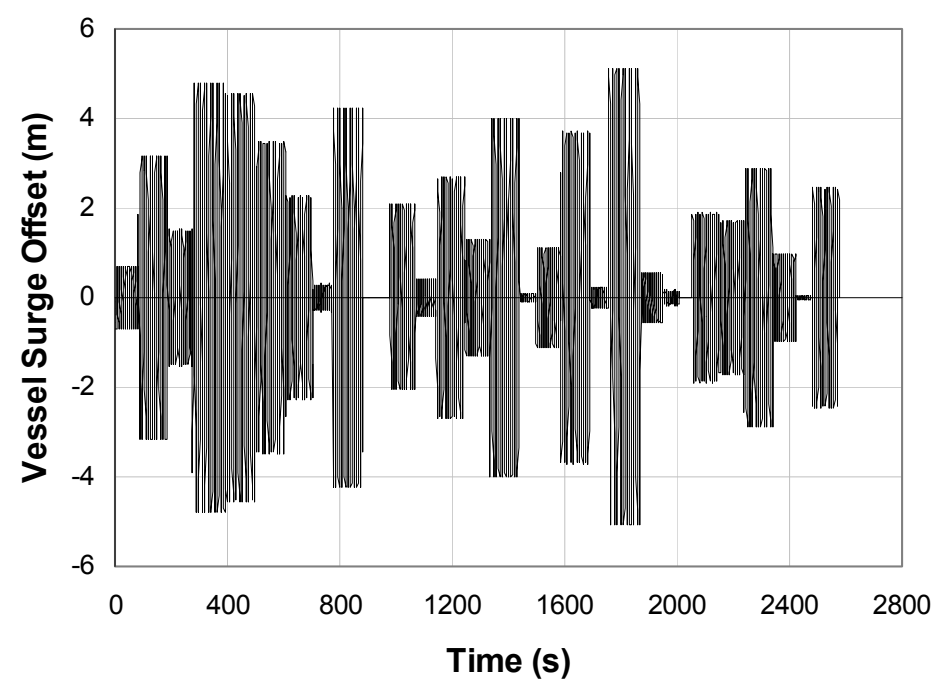

Figure 6-22 Vessel surge offset against analysis time in random wave order No.3

The von Mises stress range variation for the same sample node (Node 363) for both analyses are presented in Figure 6-23 and Figure 6-24. A review of these plots shows correlation with the findings of the first analysis, random order No.1. In random order No.2, Figure 6-23, sections A, B, C, D and E are sea states that exceed all previous sea states and show stress ranges that increase with the number of wave cycles. Conversely, sections $\mathrm{F}, \mathrm{G}$ and $\mathrm{H}$, which are all less severe than previous sea states, show stress range increase, independent of the number of wave cycles or the hierarchy of sea states; these sections are similar to what can be seen for the linear elastic seabed results presented in Figure 6-17. Similar results can be seen in Figure 6-24 for the analysis with random wave order No.3, with changing von Mises stress ranges for sections A, B and C and stable zones D and E.

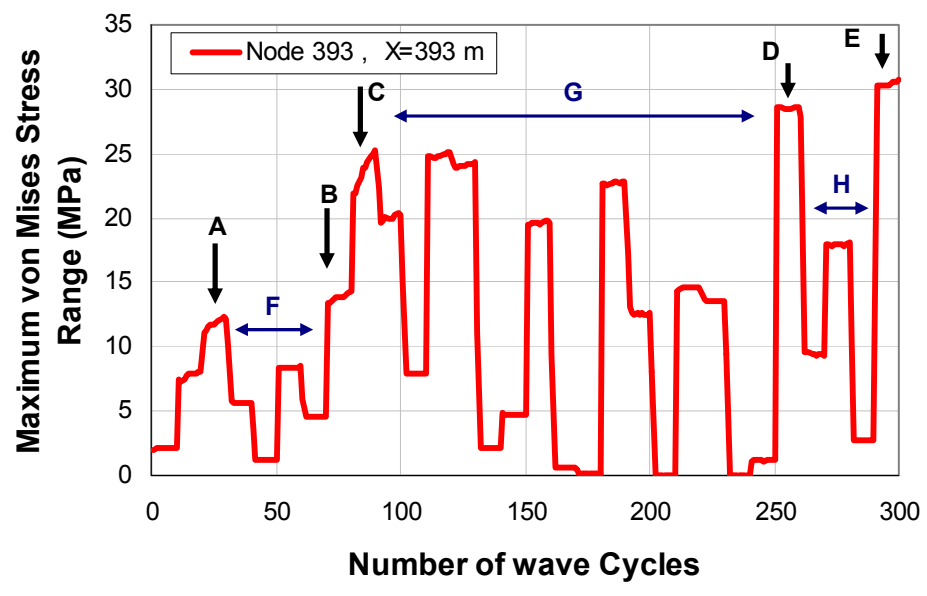

Figure 6-23: Maximum von Mises stress variation range in wave height random order No.2 


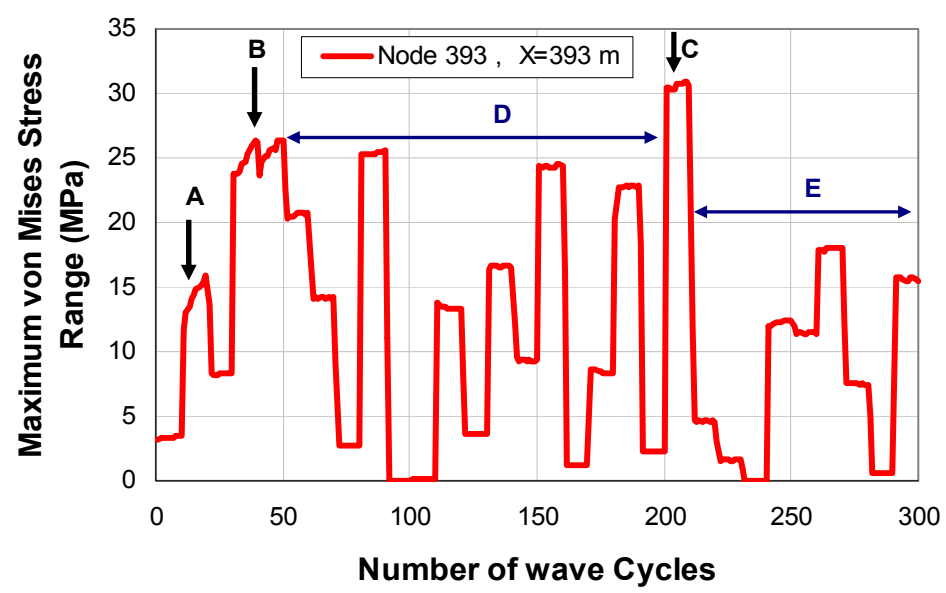

Figure 6-24: Maximum von Mises stress variation range in wave height random order No.3

The results also show that apart from consideration of the wave amplitude alone, stabilisation of the von Mises stress range occurs more rapidly for deeper embedment and dependency of the stress range on the number of wave cycles decreases gradually for sea states later in the sequence (e.g. D and E in Figure 6-23 or C in Figure 6-24) even though they exceed all previous sea states.

In the examples discussed above, the results show that Miner's rule may be applied for fatigue damage calculation, provided the ordering of the waves, or the number of wave cycles for sea states that exceed previous sea states, is chosen appropriately. To obtain a preliminary assessment of the sensitivity of the Miner's rule procedure, ignoring the fact that certain sea states have not reached a stable von Mises stress range, the total fatigue damage has calculated for the three random orders of sea states, and the results are shown in Figure 6-25. Note that the fatigue damage is based on the stress range obtained in the final (i.e. tenth) cycle of each sea state. The results of analyses with random order No.1 and no.2 are very similar, which appears due to the relatively similar distribution of the sea states. 


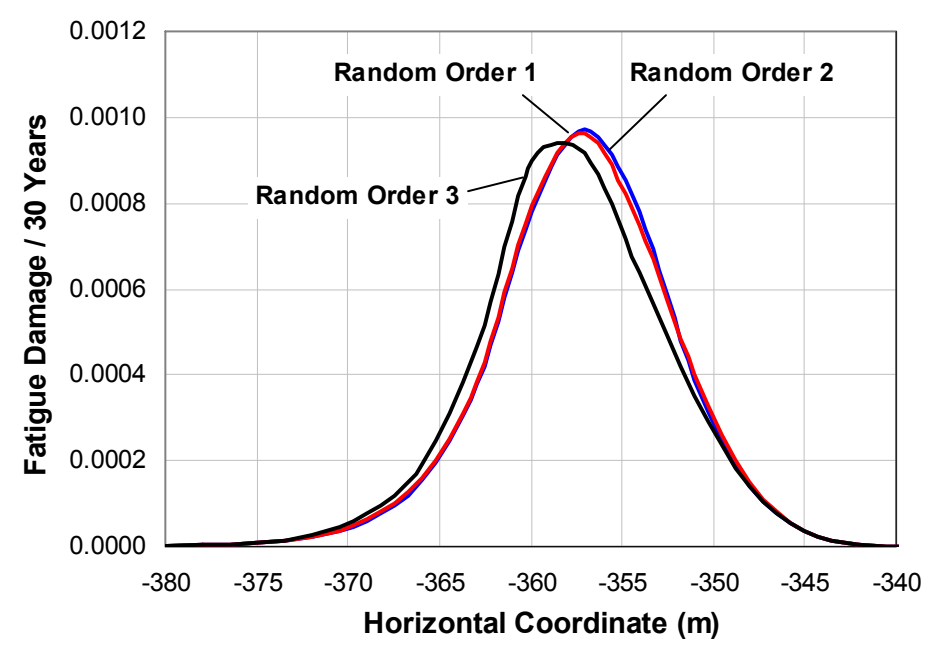

Figure 6-25: Dependency of fatigue damage on wave orders in non-linear plastic seabed

The next stage is to explore the influence on the von Misses stress range and final fatigue damage of having the most severe sea state at the beginning of the analysis. The results of such investigation can also form the basis for calculation of maximum fatigue damage by deliberate creation of a deep trench consistent with ROV observations (3 5D, (Bridge and Howells 2007)), which will be investigated in the following chapter.

\subsubsection{Full package of waves with various random orders and most severe wave in start}

The three analyses with random sea state orders performed in the previous section have been repeated, but applying 50 cycles of the most severe sea state (No.30 from Table 4$3)$ as a pre-trenching phase. The resulting variations of von Mises stress range are compared with the previous results in Figure 6-26 to Figure 6-28. As expected, application of the most severe sea state at the beginning of the analysis has stabilised all of the stress ranges making all of them independent of the hierarchy and the number of applied wave cycles for each sea state. There are some minor fluctuations of the stress range, mainly at the start of each new sea state, but these do not affect the stress range at the end of each batch of 10 cycles and hence the final fatigue damage. As shown initially in Figure 6-9 and seen in all of the results presented below, the severe wave at the start of the analysis, or the initial pre-trenching phase, has increased the global level of the von Mises stress range for every sea state. As discussed before in Chapter 4, fatigue damage varies according to the von Mises stress range to a power of 3 to 5; as such, the slight increase in the maximum von Mises stress level will increase the final fatigue damage considerably. In turn this suggests a significant influence of trench development on fatigue performance. For random wave order No. 1 (see Figure 6-26) the von Mises stress is still increasing after 10 cycles, even with pretrenching. As the amplitude of the applied wave approaches the amplitude of pretrenching sea state, the rate of the von Mises stress stabilisation is decreased. Although, its effect on fatigue 
damage is quite negligable, it can be easily sorted out by adding a few more cycles to related sea states. Even in cases where the von Mises stress changes have stabilised within 10 cycles, the last cycle is taken as the representative cycle.

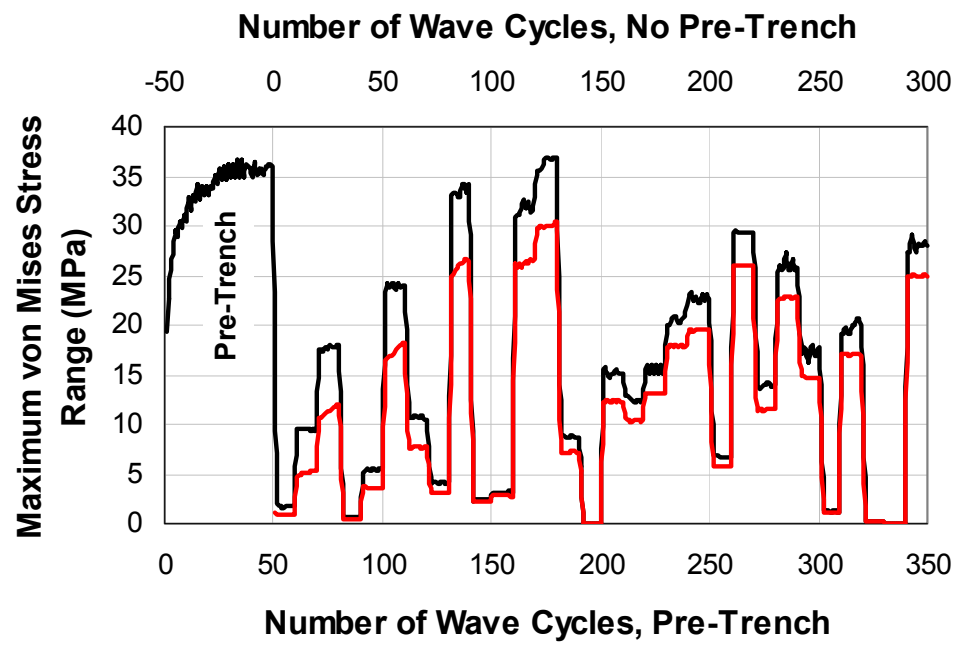

Figure 6-26: Comparison of von Misses stress range for random order No.1 with and without pretrench

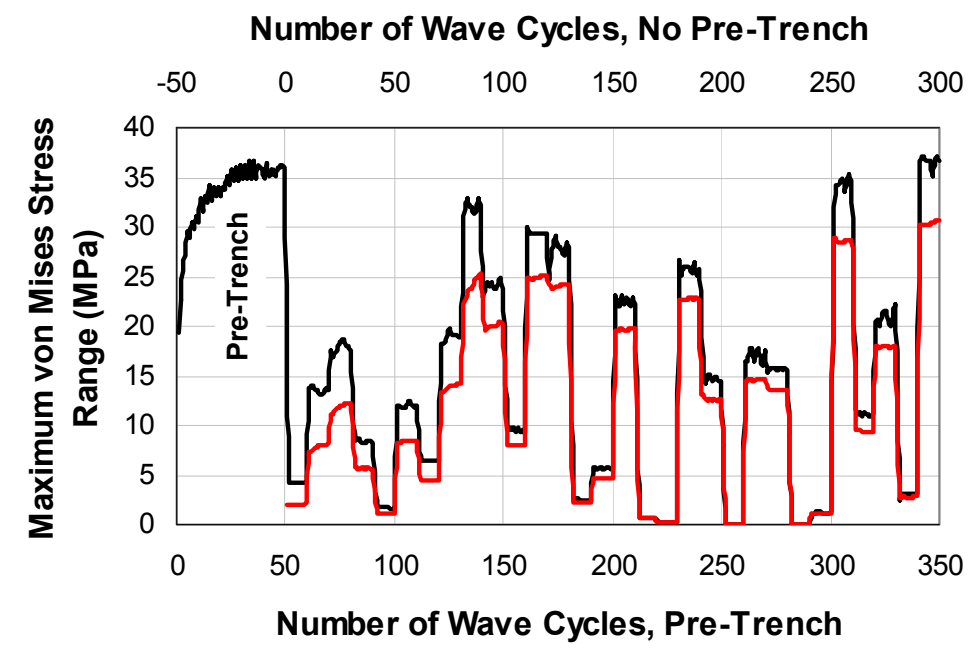

Figure 6-27 Comparison of von Misses stress range for random order No.2 with and without pretrench 


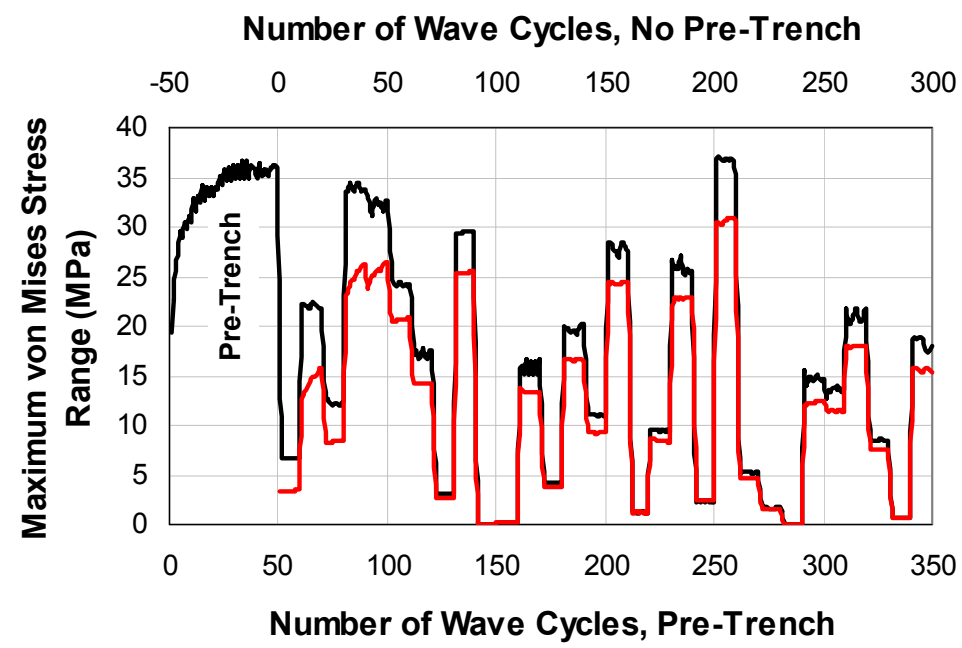

Figure 6-28 Comparison of von Misses stress range for random order No.3 with and without pretrench

More detailed results for the early stages of the analysis are shown in Figure 6-29 to Figure 6-31 to allow a better assessment of the changes in von Mises stress range for the first few sea states. It seems the magnitude of stress level increase (due to pre-trenching) is linked to the severity of the sea state. Specifically, larger waves tend to show greater increase in stress level than the smaller ones. As shown in Chapter 4, fatigue damage is affected by a combination of the significant wave height and the number of applied waves, where a minor increase in the number of waves of higher significant wave heights can increase the damage dramatically. Increasing the von Mises stress range for the larger waves, for a fixed number of wave cycles, would have a similar marked effect, hence the significant importance of the initial trenching on the calculated fatigue damage. 


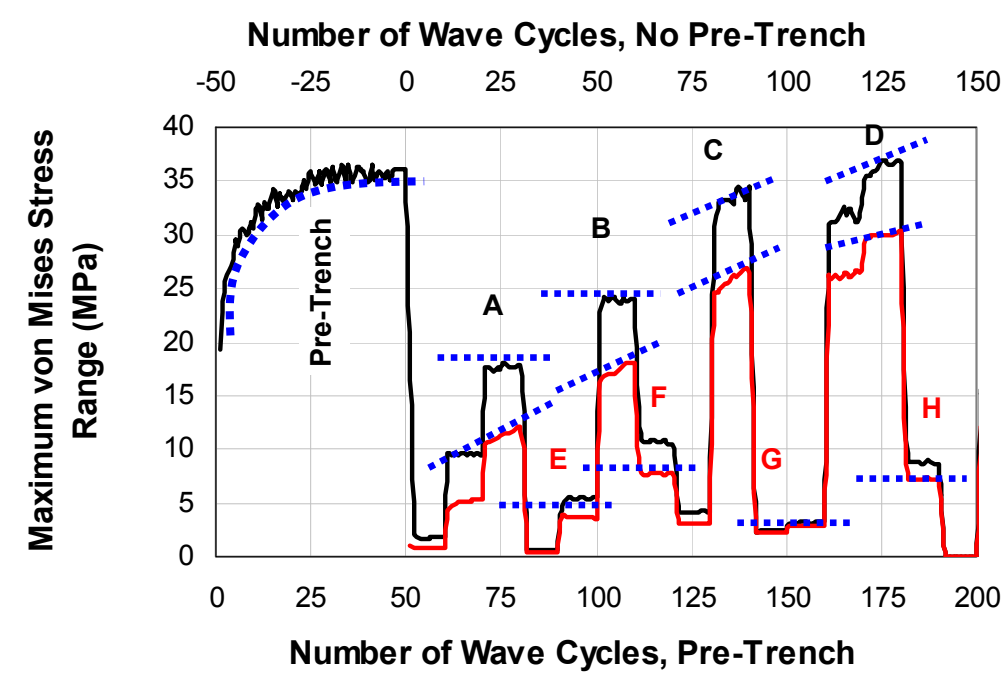

Figure 6-29: Von Mises stress level increase with pre-trench in random wave order No.1

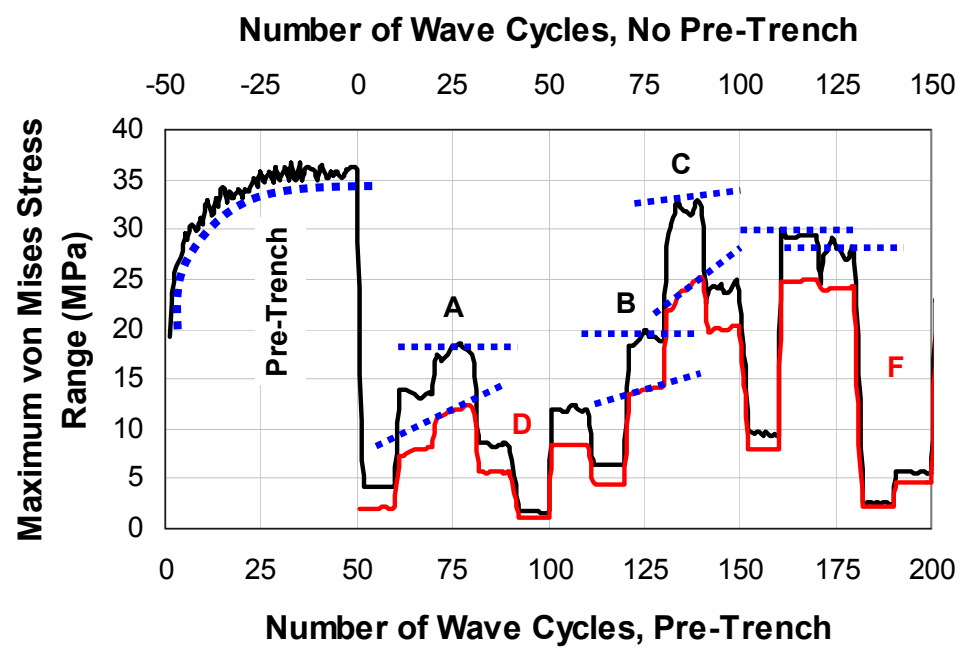

Figure 6-30: Von Mises stress level increase with pre-trench in random wave order No.2 


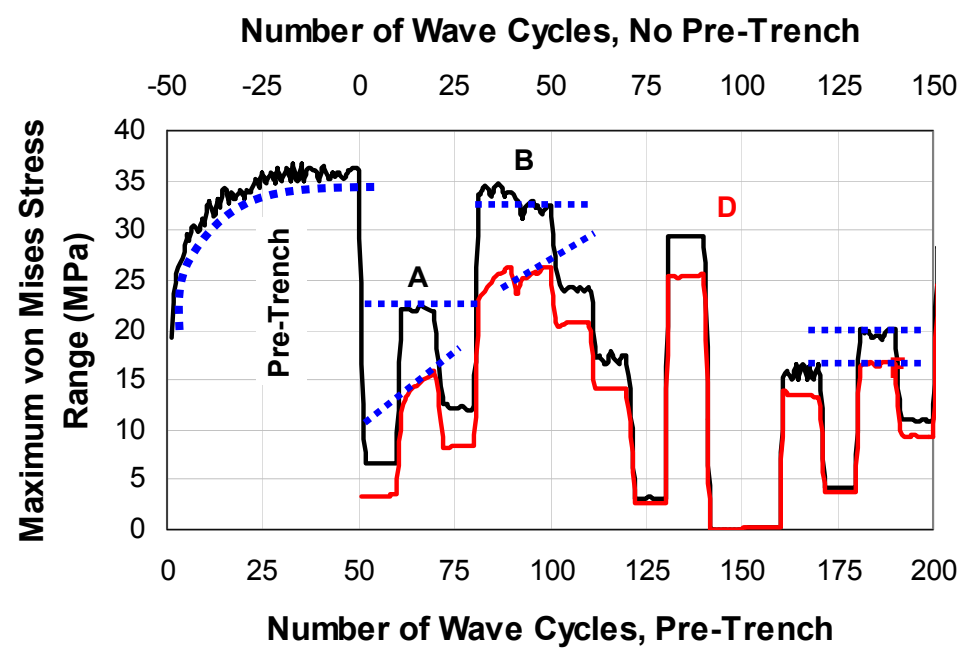

Figure 6-31: Von Mises stress level increase with pre-trench in random wave order No.3

The total fatigue damage calculated from these analyses, applying Miner's rule to superpose the fatigue damage from each sea state, based on the stress range from the final (tenth) cycle, is shown in Figure 6-32. This figure also shows the previous profiles of fatigue damage obtained without pre-trenching. It is interesting to see that the initial pre-trench has increased the peak fatigue damage to the same level from all three random orders, again confirming independency of the final fatigue damage from the hierarchy of the applied waves. The magnitude of fatigue damage is also some 25 to 30 $\%$ greater than without pre-trenching, with the position of maximum damage shifted a few metres towards the vessel (but still on the anchor side of the mean TDP). 


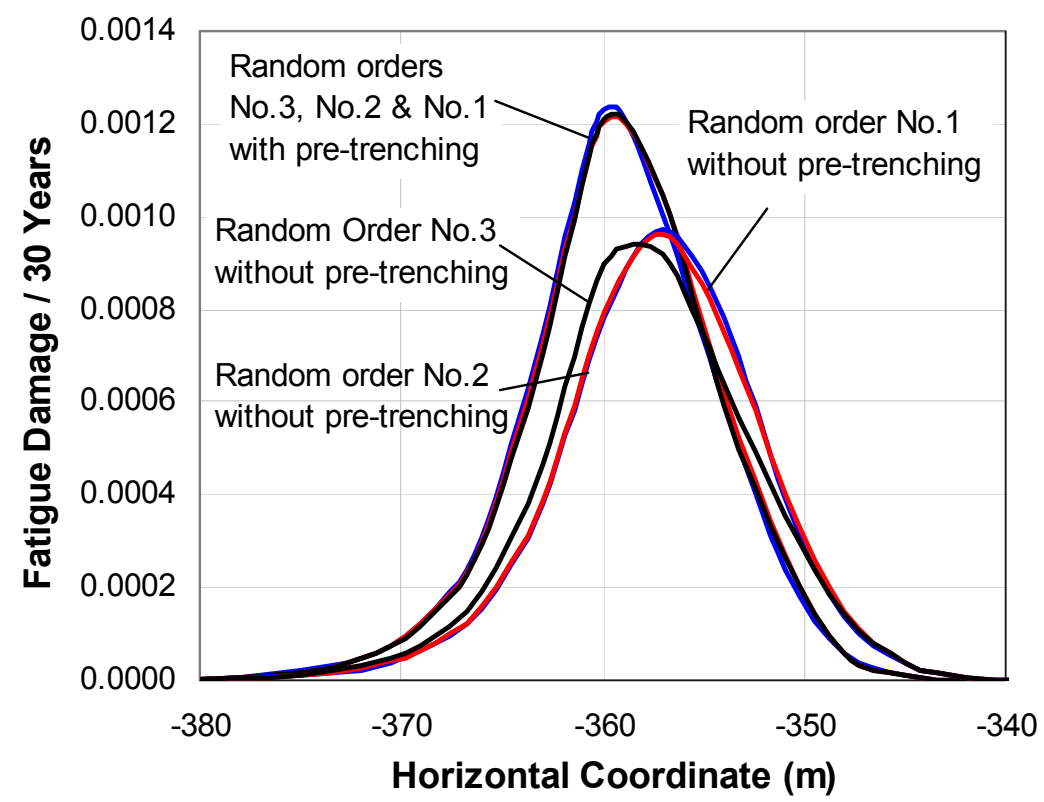

Figure 6-32: Fatigue damage with and without pre-trenching in random wave orders of No.1, No.2 and No.3

It should be emphasised that the resulting fatigue damage following pre-trenching may still be lower than might be expected in practice over the service life of the SCR, because the trench depth generated by these analyses using the normal range of seabed model parameters is similar to that shown in Figure 6-1 (i.e. 0.5D) and in the range of trench depth reported from ROV surveys ( $3 \sim 5 \mathrm{D}$, (Bridge and Howells 2007)). The effect of much deeper trenches will be discussed in detail in Chapter 7.

As discussed in previous chapters, the shear force profile in the SCR, as the gradient of bending moment, plays a vital role in the overall fatigue damage distribution along the SCR. It was also noted earlier in this chapter that a secondary impact of the hysteretic non-linear seabed model on fatigue damage occurs through the generating of suction force on the SCR during uplift movements. The presence of suction resistance tends to increase the magnitude of the cyclic bending moment. The other parameter that may affect the fatigue calculation is the maximum stiffness assigned to the hyperbolic soil model. Hence, before summarising a proposed procedure for fatigue calculation using the non-linear seabed model, the effect of both of these aspects on the shear force, contact stress and cyclic bending moment profiles in the TDZ will be explored.

\subsection{INFLUENCE OF SUCTION FORCE ON FATIGUE DAMAGE}

In order to concentrate on the impact of suction resistance on fatigue damage, a single sea state only, No. 30 from Table 4-3, has been applied to excite the vessel over 20 cycles. The resulting fatigue damage has been calculated by increasing the number of applicable waves over the SCR life to 10000. Although this is unrealistically high 
(compared with the realistic 420 in Table 4-3), it brings the level of fatigue damage to a similar level as applying the full 30 sea states. As described in Chapter 4, the suction generated by the model is controlled by two parameters, suction ratio, $\mathrm{f}_{\text {suc }}$, and suction decay parameter, $\lambda_{\text {suc }}$. The magnitude of the ultimate suction resistance is mainly controlled by parameter $f_{\text {suc }}$. Figure 6-33 compares the fatigue damage for the no suction case with the cases for various suction ratios. The non-logarithmic form of the damage is illustrated in Figure 6-34, which shows more clearly the increase in fatigue damage with increasing suction resistance by increasing parameter $f_{\text {suc. }}$. The results also show a shift of peak fatigue damage towards the vessel.

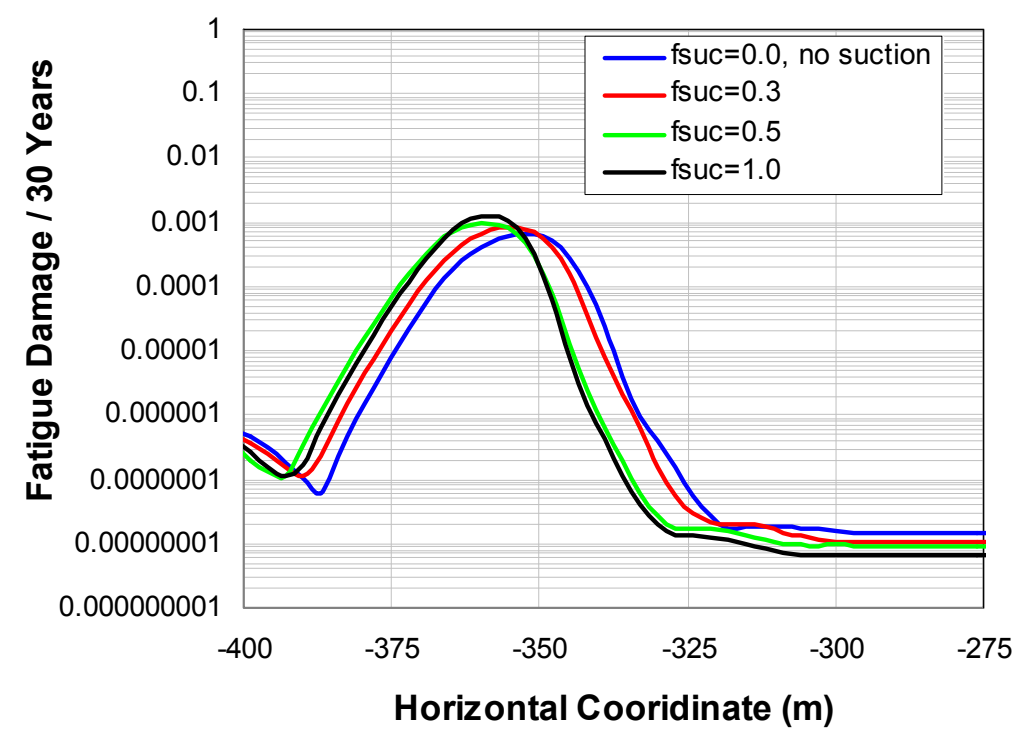

Figure 6-33: Fatigue damage distribution with various suction force generation 


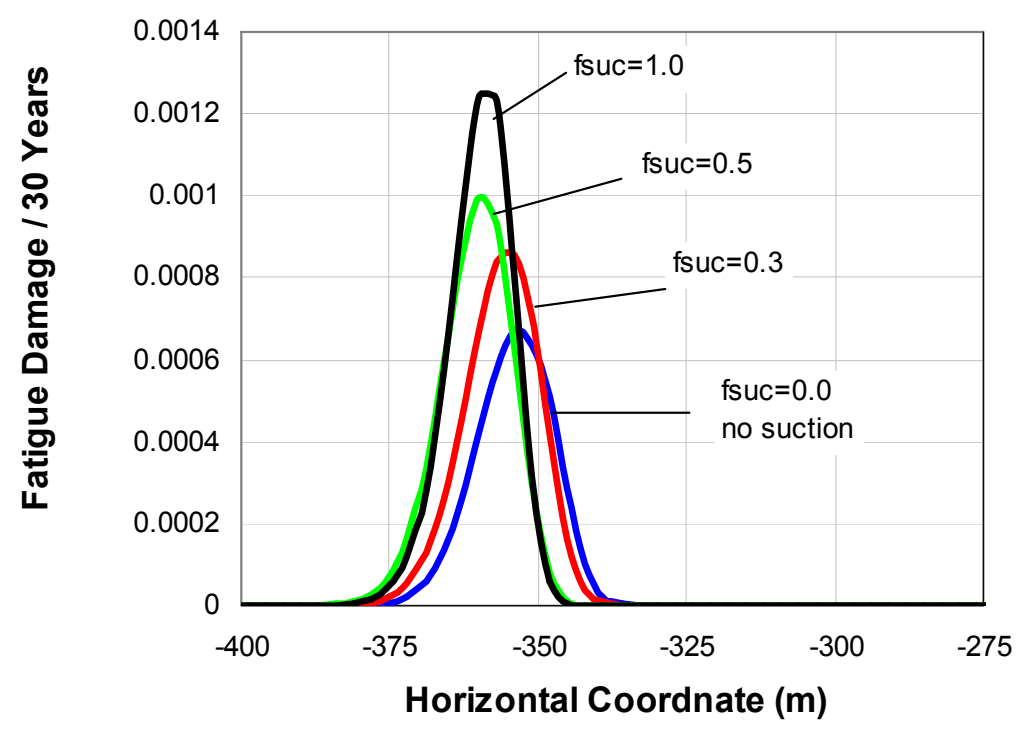

Figure 6-34: Non-logarithmic fatigue damage distribution with various suction force generation

Comparison of the final SCR profile in Figure 6-35 shows that increasing the suction ratio in the model parameters leads to deeper embedment for the given load condition and number of waves. Comparison of Figure 6-34 and Figure 6-35 suggests a direct relationship between trench depth and fatigue damage as observed before for the pretrenching study.

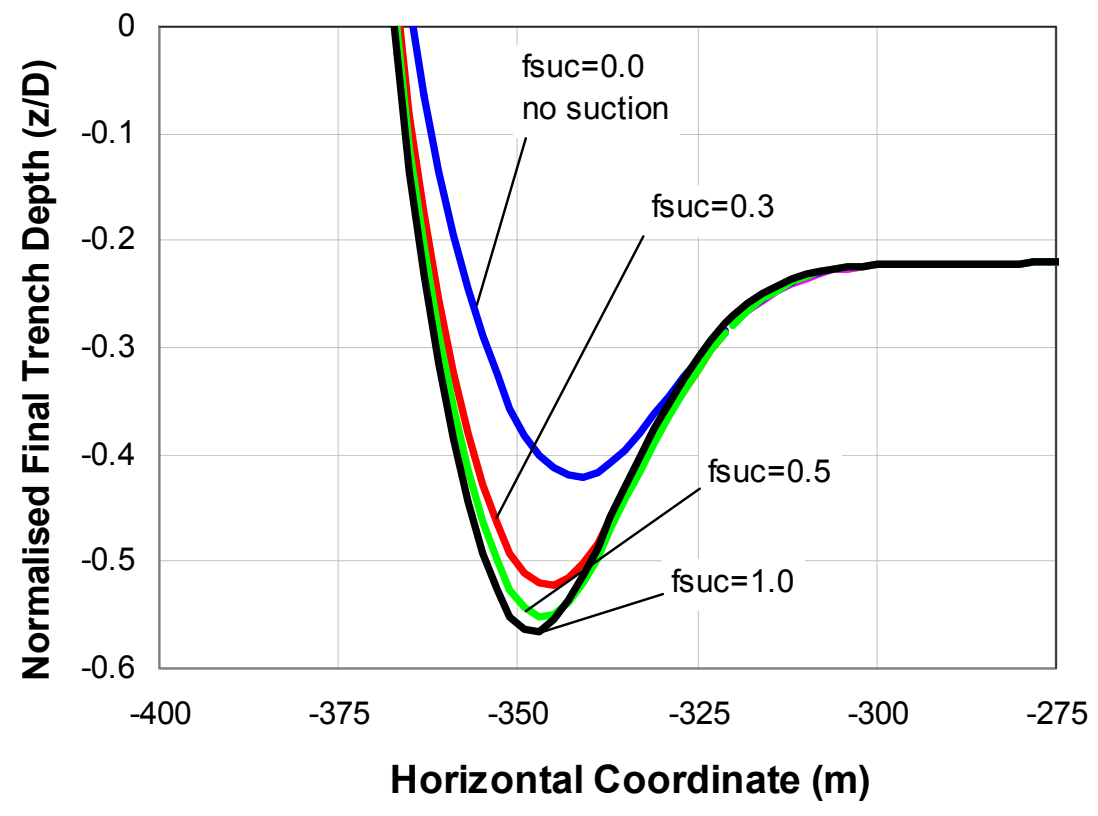

Figure 6-35: Comparison of final trench profile for various magnitudes of suction ratio 
The shear force distributions at mid-cycle (i.e. mean TDP) at the end of the cyclic analyses are compared in Figure 6-36 for the seabeds with different suction ratio. The profiles show relatively irregular variations and further investigation of this issue requires full fatigue analysis, which will be discussed in the coming sections.

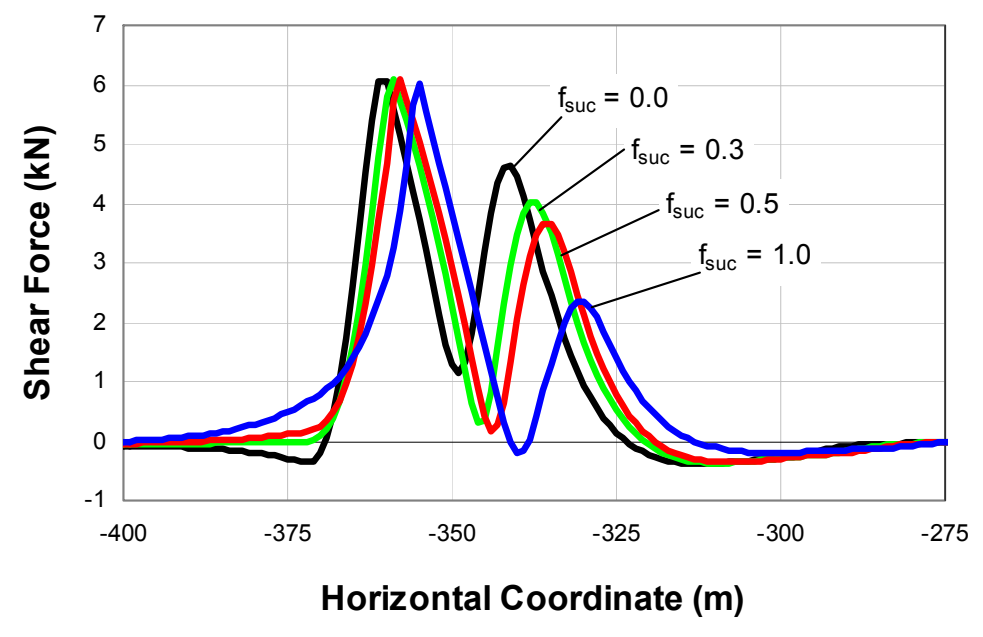

Figure 6-36: Comparison of average shear force for various magnitudes of suction ratio

The profiles of cyclic bending moment, which is the absolute value of the difference of maximum and minimum bending moment experienced by the SCR during the time history of analysis, shows increasing maximum values with increasing value of suction ratio. This is entirely consistent with increase in fatigue damage in the TDZ presented in Figure 6-34. Thus the increase in cyclic bending moment between extremes of $\mathrm{f}_{\text {suc }}=0$ and 1 is about $28 \%$, resulting in an increase in fatigue damage by $1.283 \sim 2$ (where the power 3 is that used in the fatigue damage relationship).

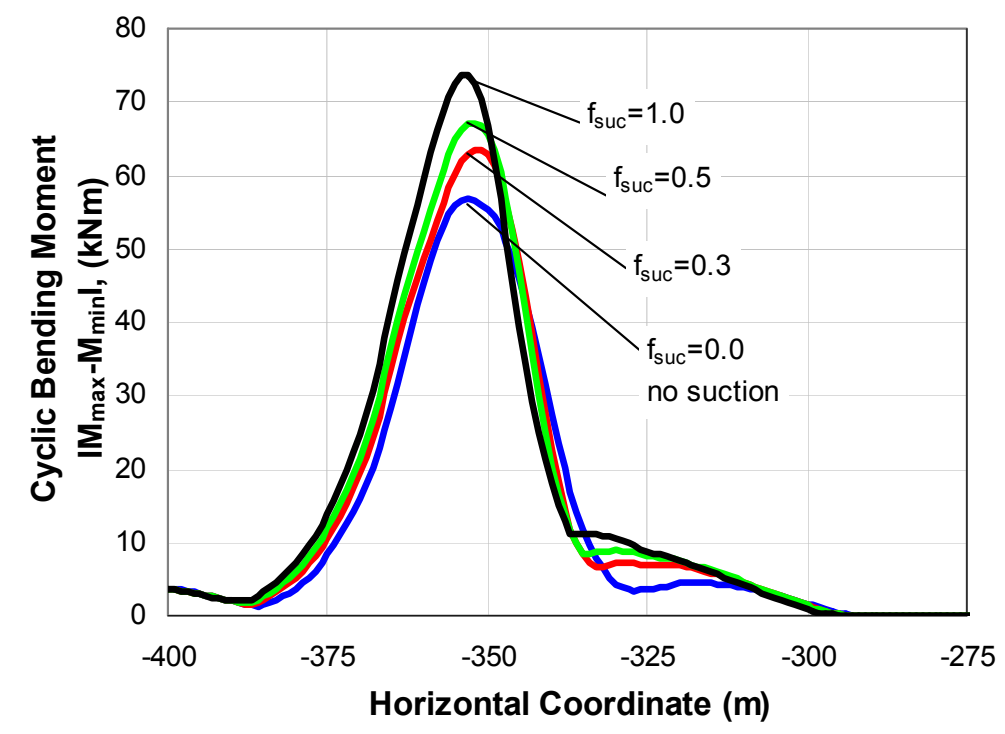

Figure 6-37: Comparison of cyclic bending moment for various magnitudes of suction ratio 
The magnitude of suction resistance will obviously affect the contact stress in the TDZ, just as for the shear force and bending moment. The final contact stress distributions for theses analyses are plotted in Figure 6-38. This figure shows that the suction resistance is mostly generated in the area close to the mean touchdown point $(\sim-350 \mathrm{~m})$, as expected since the maximum cyclic perturbation occurs at this point. Unlike the initial, relatively smooth, distribution of contact stress before any perturbation occurs, the final contact stress distributions are irregular following the cyclic vessel excitation, and will be affected by many details of the non-linear model.

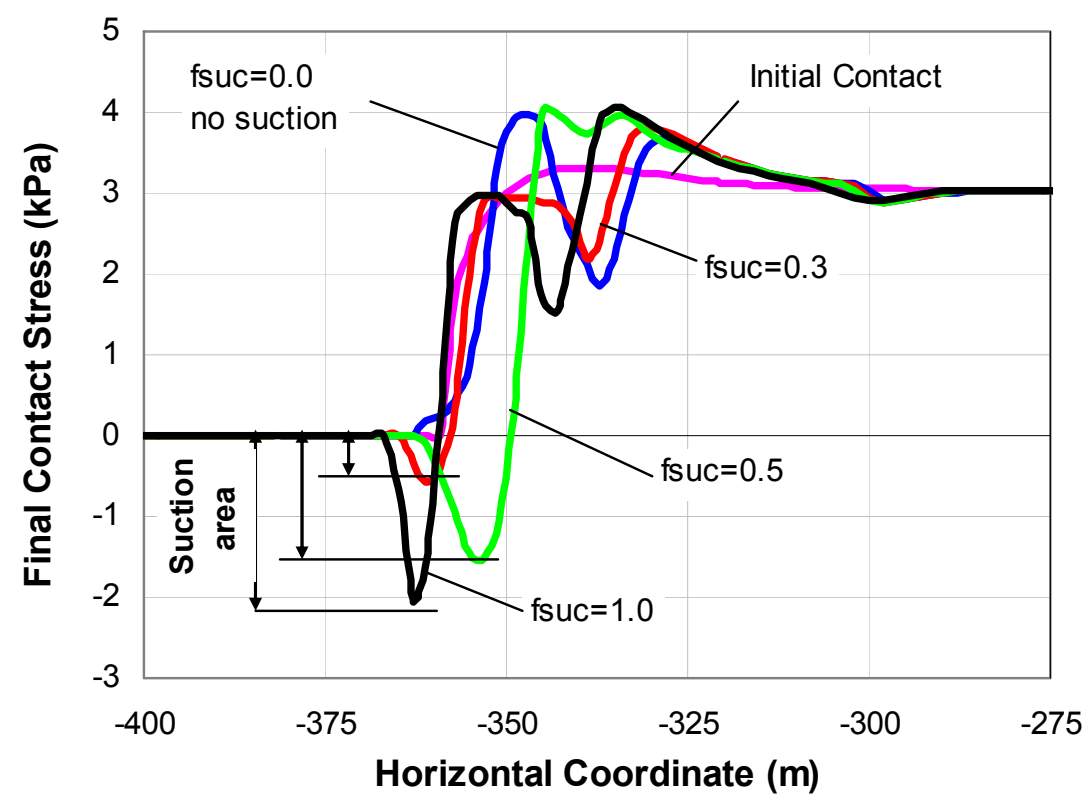

Figure 6-38: Comparison of final contact stress with initial contact for various magnitudes of suction ratio

To have a better view of what happens in respect of the contact stress, the contact stress envelope and the cyclic contact stress are shown in Figure 6-39 and Figure 6-40 respectively. The cyclic contact stress is the absolute value of the difference of maximum and minimum contact force per unit length, $\mathrm{P}$, during the time history of the analysis, normalised by the riser diameter. The contact stress envelope shows the upper and lower bounds of contact stress during the time history of the analysis. Figure 6-39 shows that the peak cyclic contact stress occurs somewhere between the trench bottom and the (mean) TDP, gradually increasing for larger values of suction ratio and getting closer to the TDP for deeper trenches (higher suction ratio). This is in good agreement with Figure 6-34, where the peak fatigue damage migrates further from the trench bottom, and shows the direct impact of suction force generation on fatigue damage. These results show the important role of suction force generation using the hysteretic non-linear seabed model, and also the effect of trench deepening on fatigue damage through the TDZ. 


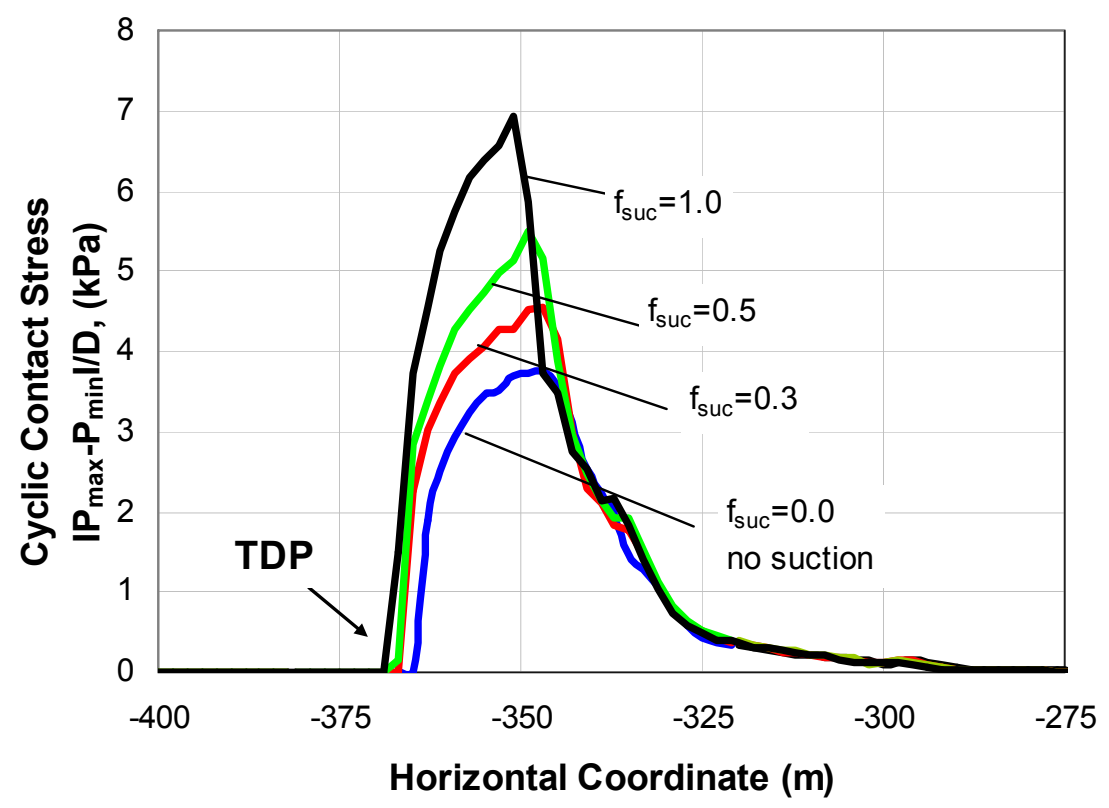

Figure 6-39: Comparison of cyclic contact force for various magnitudes of suction ratio

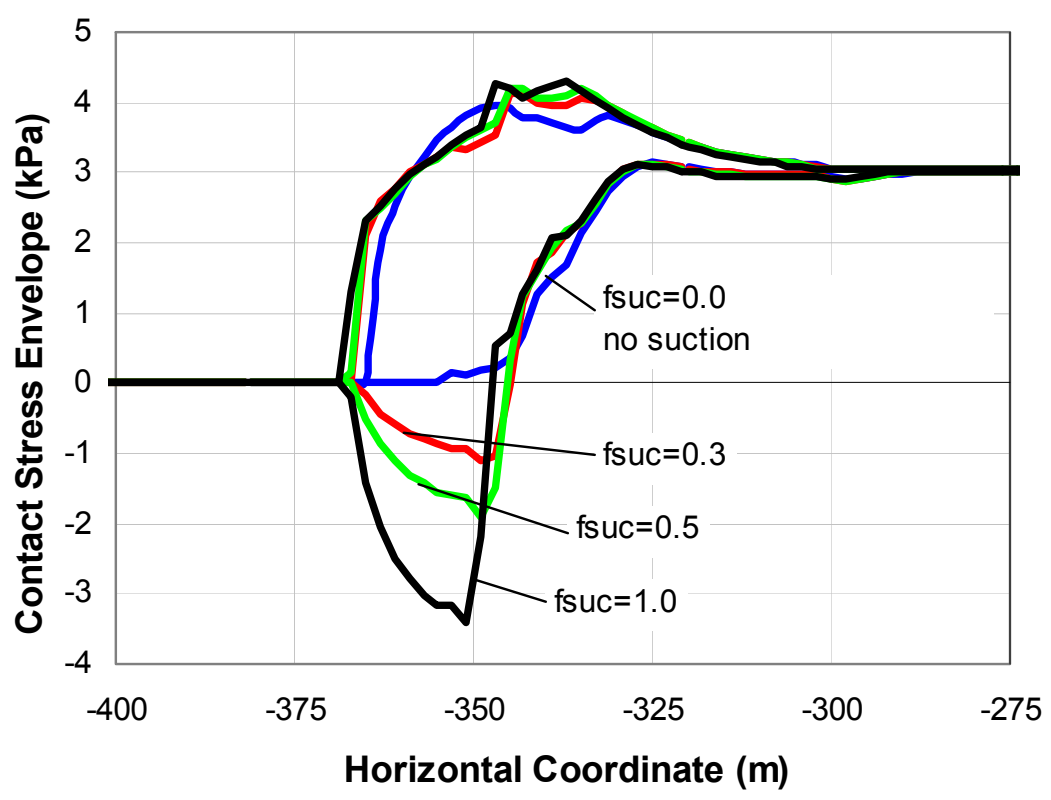

Figure 6-40: Comparison of contact force envelope for various magnitudes of suction ratio

Although, it is not expected that $\lambda_{\text {suc }}$, the suction decay parameter in the hysteretic nonlinear seabed model, would have significant influence on the fatigue damage, the effect of this parameter has been explored as well. Three analyses with different suction decay parameters, all with $\mathrm{f}_{\text {suc }}=0.3$, were carried out and the results are plotted in Figure 
6-41. This confirms that there is negligible influence of this parameter on fatigue damage in the TDZ.

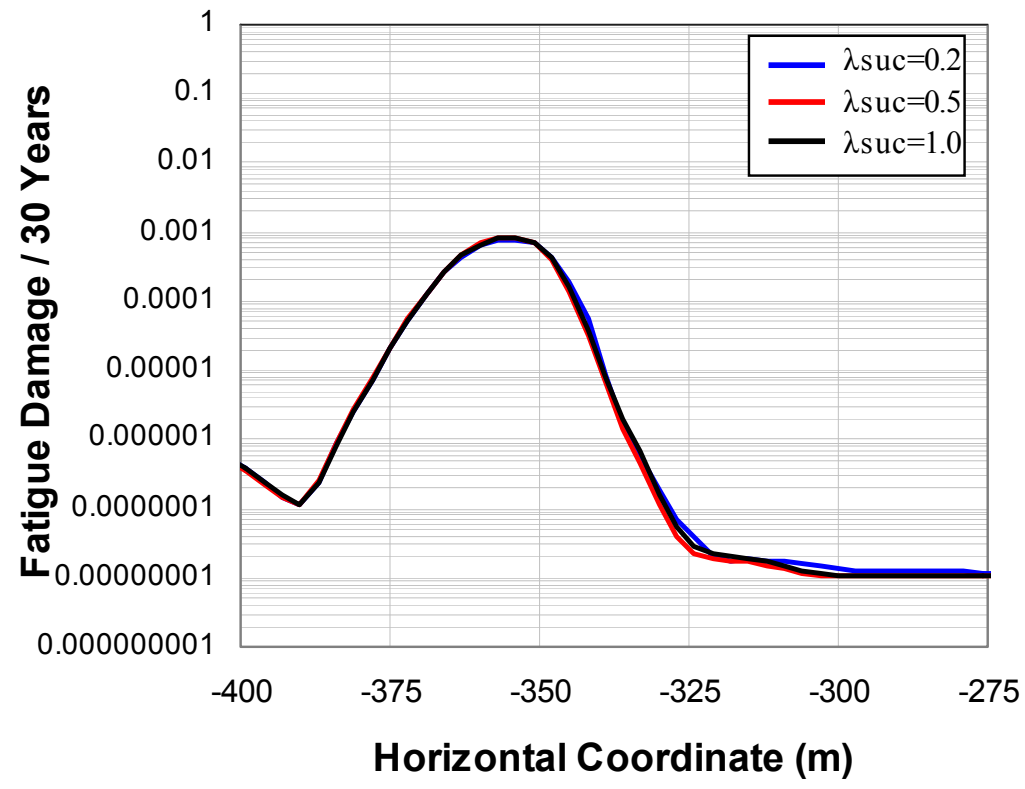

Figure 6-41: Comparison fatigue damage for various magnitudes of suction decay

\subsection{INFLUENCE OF SEABED STIFFNESS ON FATIGUE DAMAGE}

As discussed in Chapter 4 the hysteretic non-linear soil model (Randolph and Quiggin 2009) is based on the undrained shear strength of soil, $s_{u m}$, and the shear strength gradient of the soil, $\rho$, using a hyperbolic formulation that gives a maximum stiffness during reversals of movement direction. The maximum stiffness is expressed as a multiplier, $\mathrm{K}_{\max }$, times the ultimate penetration resistance at the current penetration. The influence of the soil stiffness in the non-linear soil model on fatigue damage in the TDZ, is explored here by varying the strength parameters, since these essentially control the stiffness for a given displacement change. For this purpose, a series of fatigue analyses were performed and values of fatigue damage calculated using Miner's rule for superposition of damage using the last cycle from each sea state, ignoring any slight increases in von Mises stress range that might still be occurring. The full package of sea states presented in Table 4-3, were applied to the vessel in the order of minimum to maximum severity with 10 cycles for each sea state. The gradient of undrained shear strength for the analyses was taken as $1.5 \mathrm{kPa} / \mathrm{m}$, except for the set of analyses where it was the primary variable.

Figure 6-42 illustrates the gradual increase in fatigue damage in the TDZ, and the movement of the peak point towards the anchor end, as the seabed strength intercept, $\mathrm{s}_{\mathrm{um}}$, is increased, corresponding to a stiffer seabed. This result is entirely in agreement 
with the linear elastic seabed results presented in Chapter 5. Note that the fatigue damage increases by a factor of 3 between $s_{u m}$ values of zero and $100 \mathrm{kPa}$.

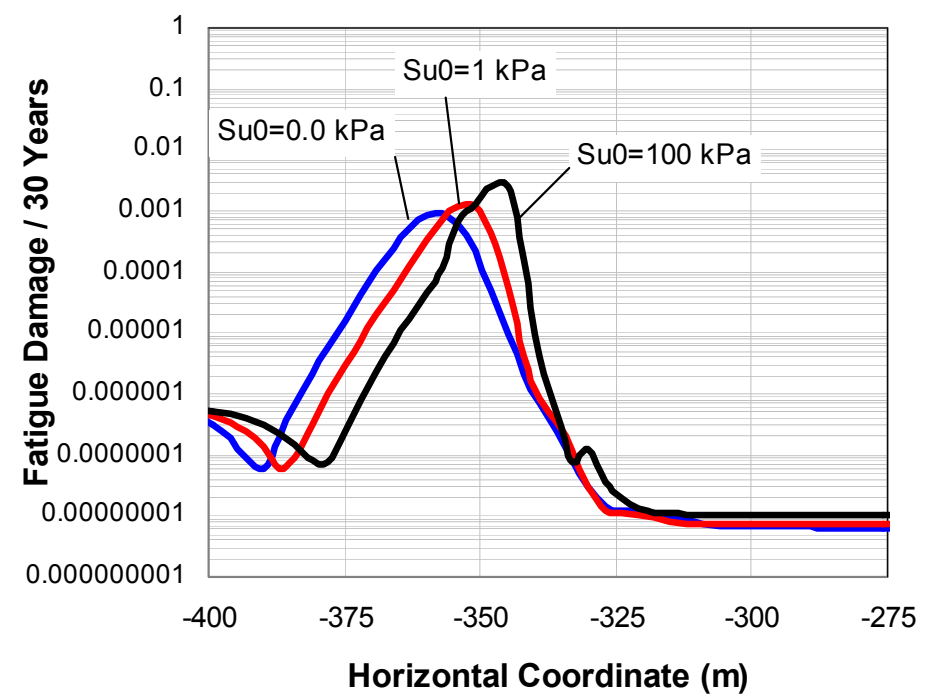

Figure 6-42: fatigue damage variation with various seabed stiffness

The influence of the gradient of undrained shear strength, $\rho$, on fatigue damage was examined by the series of analyses with the same setup as above and the results are presented in Figure 6-43. In these series of analyses, the undrained shear strength at surface was taken as zero. Although, a higher gradient of undrained shear strength results in higher fatigue damage, again with the peak point moving towards the anchor end, it's the influence on fatigue damage is much less than for the undrained shear strength intercept at the seabed.

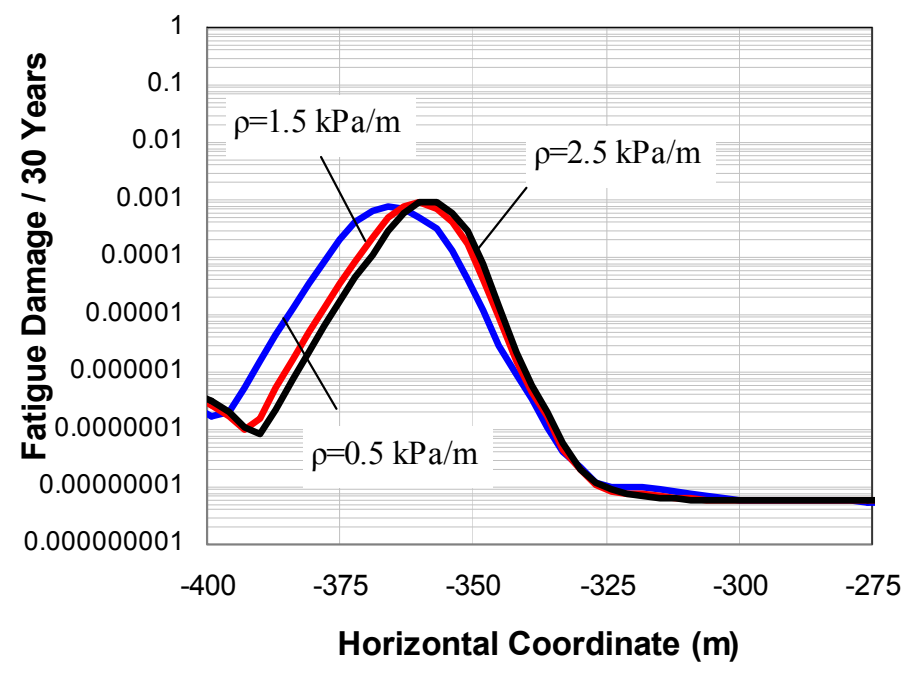

Figure 6-43: Fatigue damage variation with various seabed stiffness 
The peak values presented in terms of fatigue damage in Figure 6-42 and Figure 6-43, have been expressed in terms of fatigue life and are shown in Figure 6-44 and Figure $6-45$.

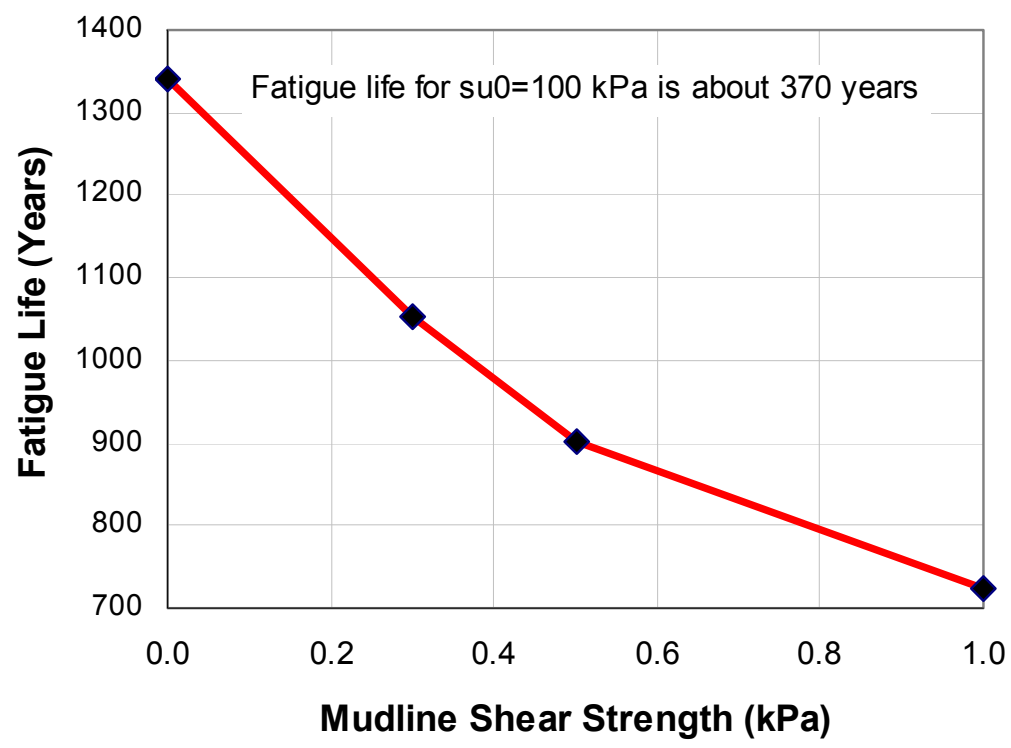

Figure 6-44: Fatigue life variation with mudline shear strength

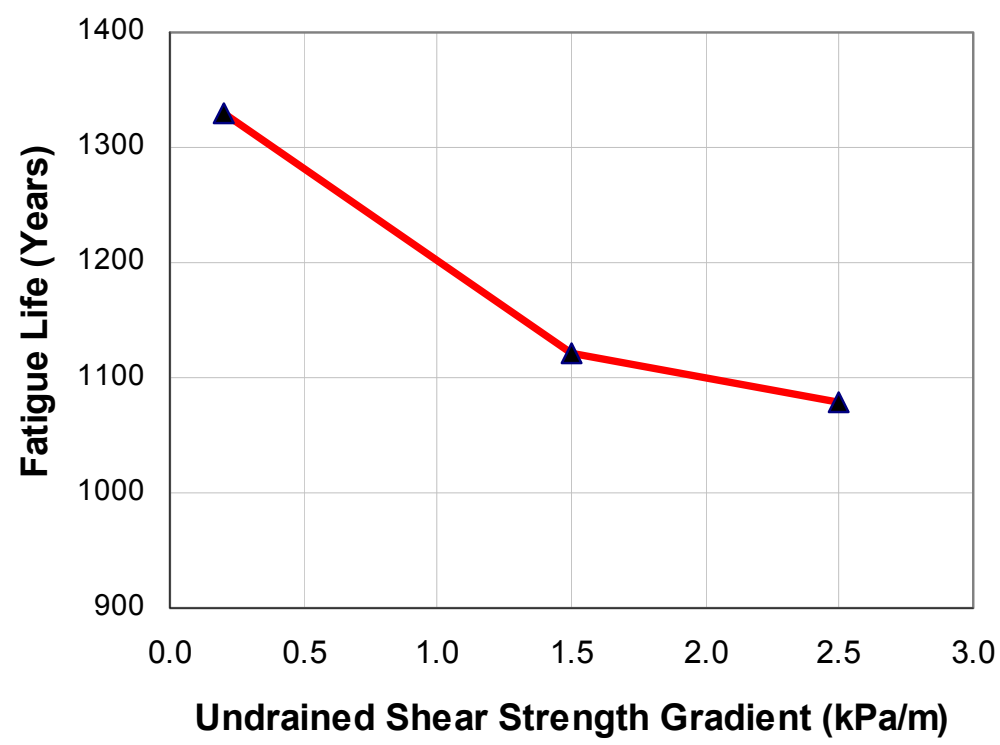

Figure 6-45: Fatigue life variation with undrained shear strength gradient

The results above are in good agreement with the overall trend of the results reported by Randolph and Quiggin (2009). Figure 6-46 shows the results of their parametric study on mudline shear strength. The difference between the two sets of values is due to 
differences in the SCR systems examined and in the applied environmental loads, but the trends and general magnitude of fatigue life are similar.

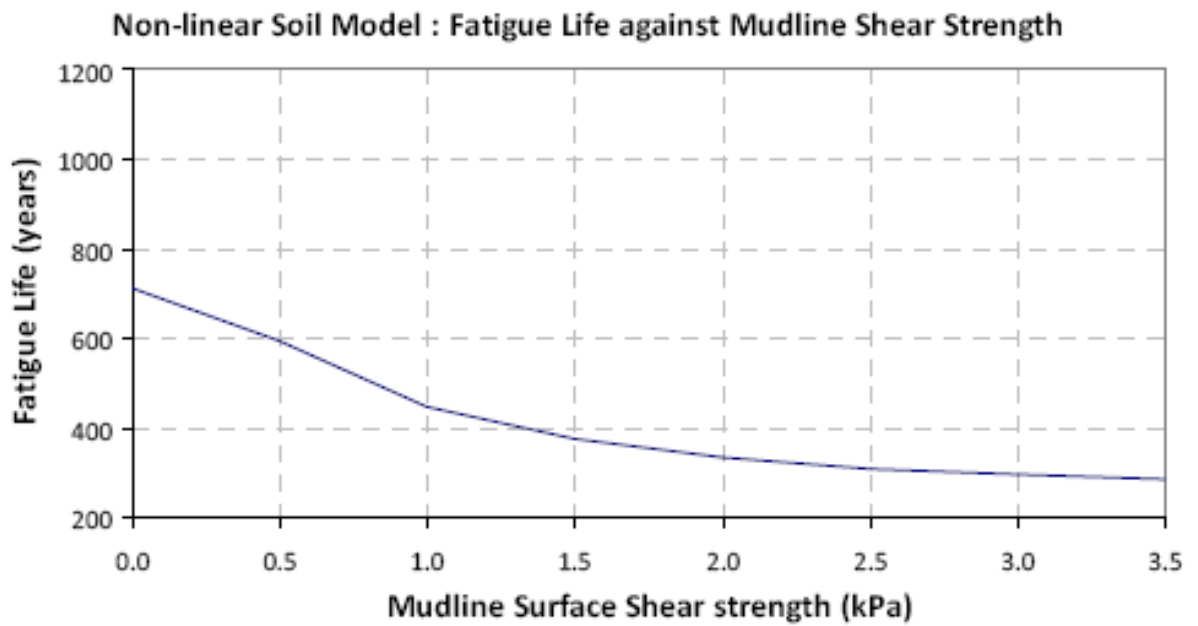

Figure 6-46: Fatigue life variation with mudline shear strength (Randolph and Quiggin 2009)

Figure 6-47 compares the maximum fatigue damage obtained for a range of undrained shear strength gradient in the non-linear hysteretic seabed with the results presented in Chapter 4 for the linear elastic seabed for different stiffness values. The value of undrained shear strength at the mudline has been set to zero in the non-linear seabed and the remaining properties and analysis features are as described in Chapter 4. As may be seen in Figure 6-48, focusing on low range of stiffness, the same fatigue damage is achieved for the linear elastic seabed at a low stiffness of $\mathrm{k} \sim 67 \mathrm{kPa}$.

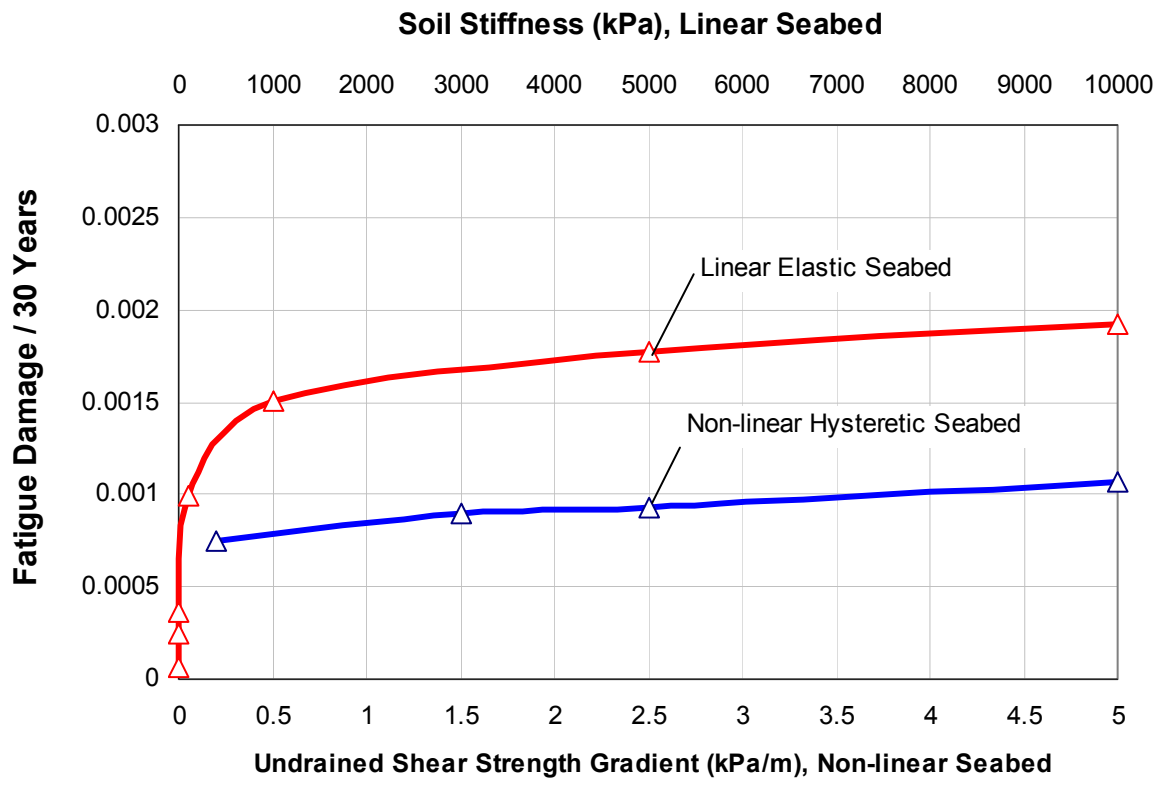

Figure 6-47: Peak fatigue damage for linear and non-linear seabed with various stiffness values and shear strength gradients 


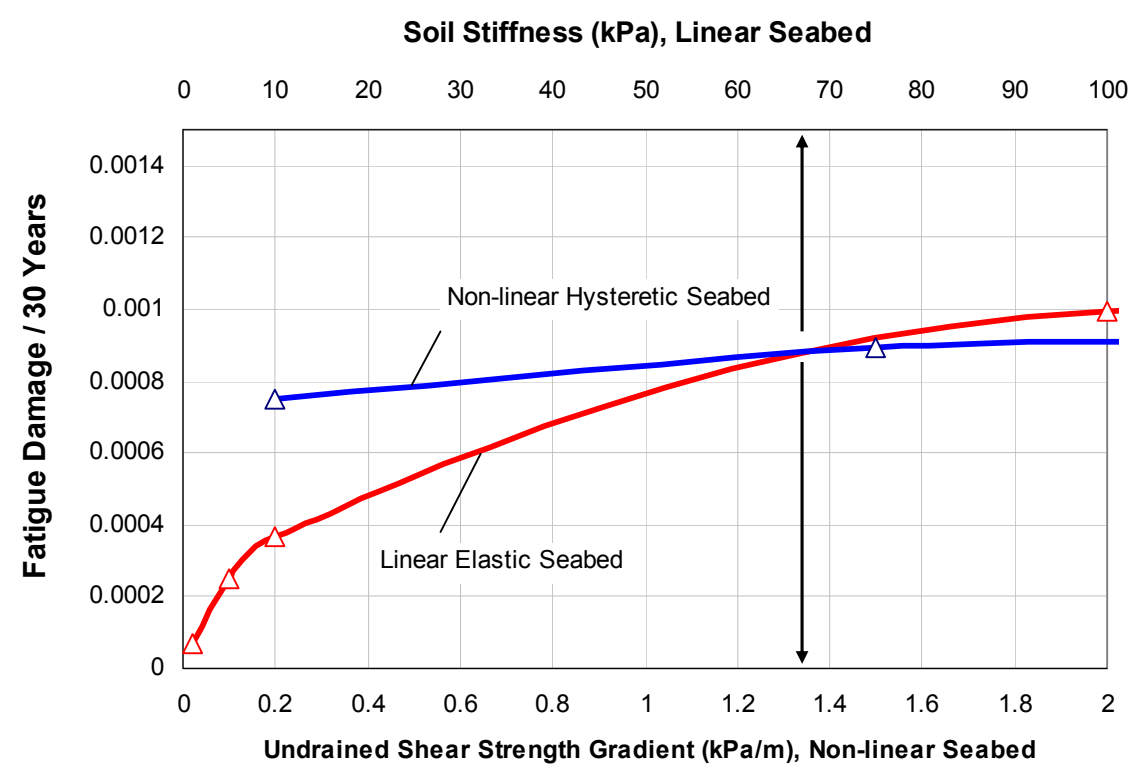

Figure 6-48: Peak fatigue damage for linear and non-linear seabed with various stiffness values and shear strength gradients (lower range for both)

\subsection{FATIGUE CALCULATION PROCEDURE FOR PLASTIC SEABED}

The main results of the studies performed on a non-linear seabed in the previous sections can be summarised in the following two statements:

1) The gradual embedment of a riser into the seabed, or trench development beneath the riser, along with mobilisation of suction resistance during cyclic loading, increases the von Mises stress range and consequently the fatigue damage in the TDZ.

2) The von Mises stress range for a given applied sea state will be constant and essentially independent of the number of wave cycles and wave hierarchies, if a more severe sea state has already been applied to the vessel for a reasonable number of cycles. In such case, Miner's rule may be applied in a straightforward manner for superposition of the fatigue damage due to the different sea states.

To recommend a fatigue calculation procedure for non-linear seabed response, it is really necessary to gain more experience from real SCR projects, particularly in respect of the evolution and magnitude of trench development. There have only been limited ROV seabed survey reports published but these suggest that the main trench formation underneath the riser occurs in the early stages of the SCR life (within, say, the first two years) (Bridge and Howells 2007). This is consistent with the analyses presented here, where the maximum trench depth stabilises with increasing numbers of wave cycles and 
sea states. The survey results show that, depending on the seabed, the type of floating vessel and the local environmental conditions, the trench depth beneath an SCR may extend to 3 to 5 times the riser diameter, although with significant variations in some cases (Bridge et al. 2004; Fontaine et al. 2004; Langner 2003). If an approximate estimate of the ultimate trench depth can be made then, in combination with the two statements above, the following fatigue damage calculation procedure for non-linear seabeds is suggested:

1) Create a trench in the TDZ to the required ultimate depth by applying cycles of the most severe sea state from the full environmental package. This can be achieved using extreme values of the seabed model parameters as will be further discussed in Chapter 7.

2) Excite the vessel with a few cycles of each sea state from the full environmental package; the order of sea states is no longer relevant.

3) Superpose linearly the fatigue damage from each sea state using Miner's rule to obtain the maximum total fatigue damage distribution throughout the TDZ.

Although application of this procedure only gives the ultimate fatigue damage distribution along the SCR in the TDZ rather than the development of fatigue damage at any given stage in the SCR life, it is still very valuable from a design standpoint, which must focus on the most adverse conditions.

The first step of the procedure mentioned above has significant impact on the final fatigue result, and this issue will be investigated in further detail in Chapter 7, where various methods to create a trench in the TDZ will be studied, exploring the detailed mechanism of trench formation and the final SCR profile along the seabed.

\subsection{SUMMARY AND CONCLUSION}

The hysteretic non-linear seabed model has been implemented in a global analysis of SCR response to examine the development of fatigue damage. The extent to which the non-linear response is sensitive to the ordering of sea states and the number of wave cycles analysed for each sea state was investigated, and a new fatigue analysis procedure proposed.

The initial trials showed that gradual embedment of the riser and trench deepening, along with increasing suction resistance in the TDZ, led to an increase in fatigue damage, with the position of the peak damage moving towards the vessel end of the SCR. This demonstrated the potential significance of the non-linear riser-seabed interaction model in predicting SCR fatigue performance.

The analysis results interestingly showed that the riser embedment tended to stabilise within the early stages of analysis after applying relatively few cycles. This is consistent 
with ROV survey results (Bridge and Howells 2007), which showed that trench formation beneath the riser occurs early in the SCR life, typically within the first one or two years. This stabilisation of trench depth in turn causes stabilisation of the maximum von Mises stress range, just as for a linear elastic seabed. This stabilisation suggests the potential to apply Miner's rule for superposition of the fatigue damage from separate seas states in a straightforward manner, even for a non-linear seabed response. The proposed procedure involves a pre-trenching phase, using a number of cycles of the most severe sea state in the design wave spectrum, before undertaking the fatigue analysis. This led to essentially constant values of von Mises stress ranges, independent of the sea state hierarchy or the number of wave cycles analysed for each sea state.

The analyses showed that the riser embedment depth quickly stabilised even for the most severe sea states, leading to maximum embedment depths, with normal values of model parameters, of between $0.5 \mathrm{D}$ and 1D. Therefore, creation of deeper trenches, such as the 3 to $5 \mathrm{D}$ observed in practice for some developments, needs another technique as will be discussed in the next chapter. 


\section{CHAPTER 7. ULTIMATE TRENCH INSERTION AND INFLUENCE ON FATIGUE PERFORMANCE}

\subsection{INTRODUCTION}

The impact on SCR fatigue performance of gradual embedment of the riser into the seabed in the TDZ was explored in the last chapter through a series of fatigue analyses performed using a hysteretic non-linear seabed model. It was noticed that, using the normal range of seabed model parameters, even extreme wave conditions applied over a large number of wave cycles resulted in trenches of less than 1D deep, compared with the $3 \sim 5 \mathrm{D}$ observed in practice from ROV surveys (Bridge and Howells 2007). Therefore, In order to study the influence of deep trenches on fatigue performance, consideration needs to be given to providing a consistent method for creation of deep trenches at the beginning of the fatigue analysis. In Chapter 6, a fatigue damage calculation procedure was introduced and examined for the non-linear seabed model. In the current chapter, the maximum fatigue damage over the SCR service life will be obtained after first creating deep trenches in the TDZ. The trench will be developed using a consistent methodology by adopting extreme values for the repenetration offset parameter of the seabed model. It will be also shown that the manner in which the trench is created can have a significant impact on the fatigue damage results and can even lead to contradictory conclusions. Interestingly, this has already been observed in the literature, where some authors report an increase in fatigue damage due to trench creation (Giertsen et al. 2004; Leira et al. 2004) while others suggest fatigue damage reduction (Clukey et al. 2007; Langner 2003; Nakhaee and Zhang 2008). As part of the study, the morphology of the seabed trench for linear elastic and non-linear plastic seabeds will also be reviewed, comparing them with the ROV observations. A mathematical expression for the seabed trench profile was developed for this purpose.

\subsection{THE MORPHOLOGY OF SCR TRENCH PROFILE}

\subsubsection{SCR trench profile observed by ROV surveys}

Assessment of actual trench profiles developed beneath SCRs is only possible through subsea surveys carried out by remotely operated vehicles (ROVs). These video recordings are normally obtained by field owners but are rarely made available publicly. Only a few authors have reported results from deep-water surveys of SCR trenches. Bridge and Howells (2007) have analysed ROV trench surveys from the Allegheny fields in the Gulf of Mexico, with surveys seven months (gas export riser) and sixteen months (oil export riser) after the SCR installation. Although the trench width and depth has been assessed in detail for different cross-sections, the trench length and shape 
along a longitudinal 2D profile of the trenches have not been evaluated accurately. In addition, the published results do not provide any information about the position of the trench mouth and its horizontal distance from the point of maximum trench depth, the dimensions of which have significant influence on the trench longitudinal geometry. The lack of such key information makes it difficult to check theoretical approximations of the trench profile against actual results. Figure 7-1 and Figure 7-2 illustrate the only information published about the longitudinal profile of trenches.

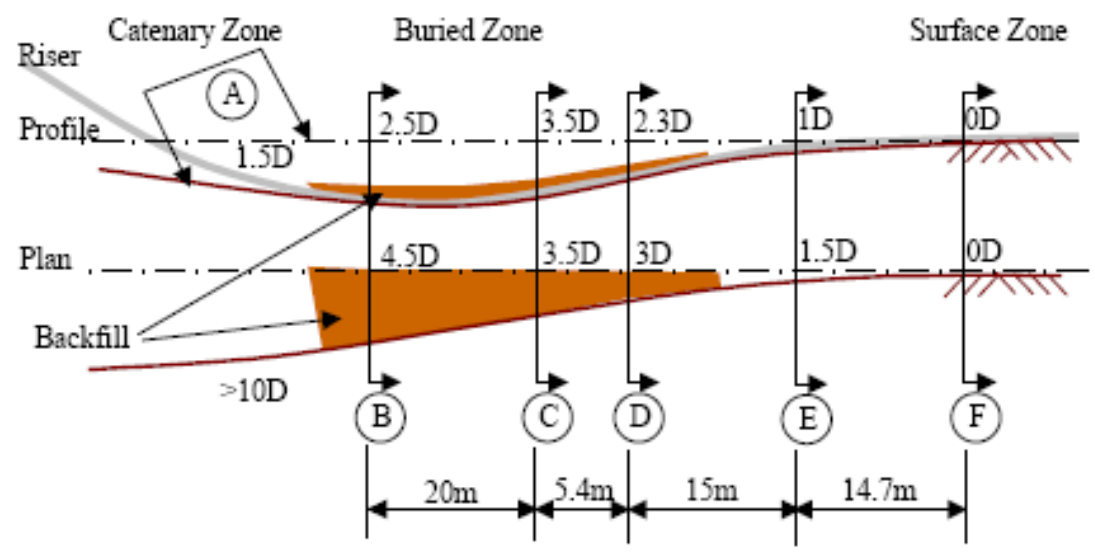

Figure 7-1: Allegheny gas export seven month after installation (Bridge and Howells 2007)

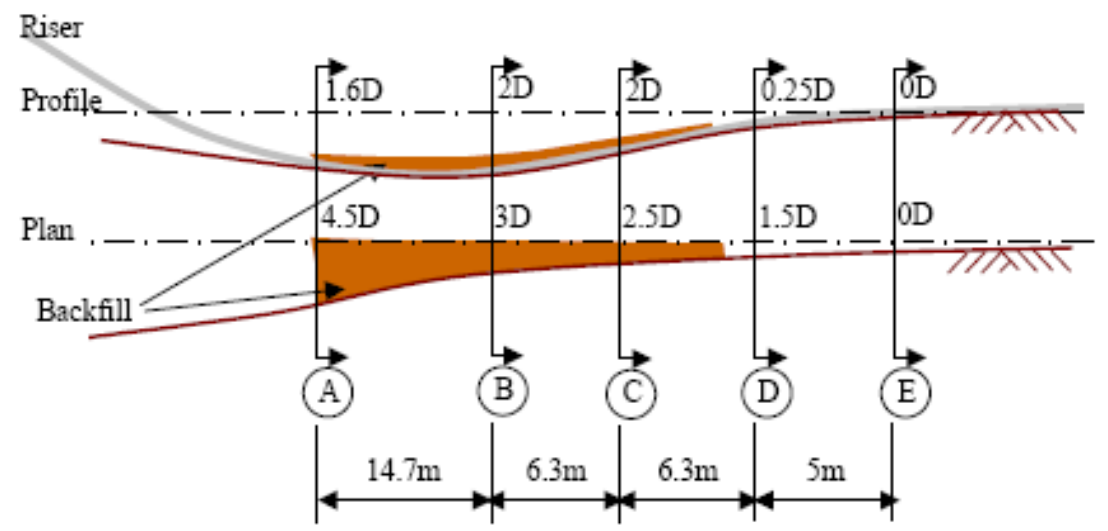

Figure 7-2: Allegheny oil export 16 month after installation (Bridge and Howells 2007)

The dimensions indicated on the schematic plots have been transferred to Excel and shown in Figure 7-3. 


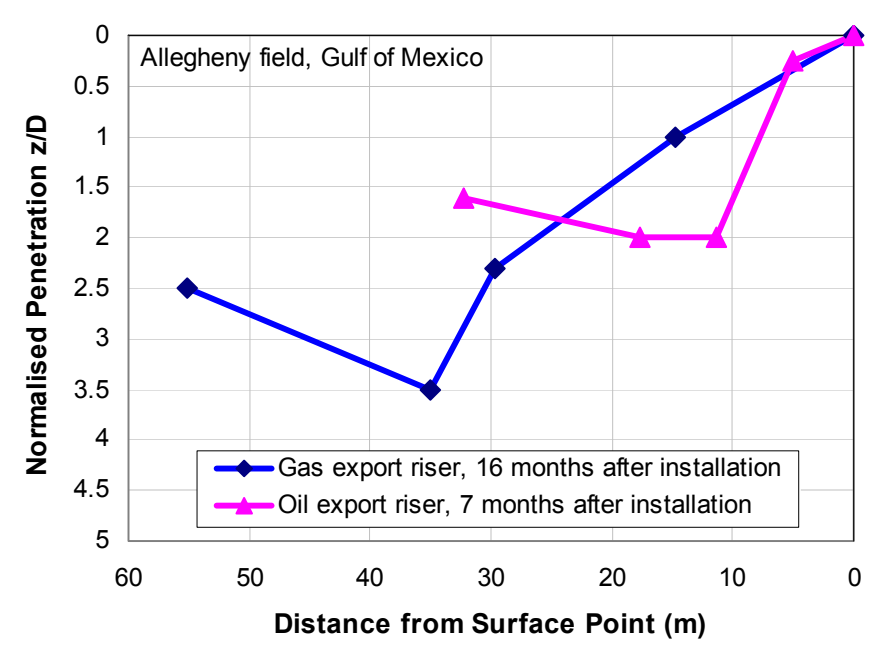

Figure 7-3: Comparison of the SCR trenches from Allegheny oil and gas fields

The trenches appear to have a rather irregular profile, as seen in Figure 7-3, but this may be due to inaccuracies in estimating the longitudinal position of the various crosssections from the ROV log. Overall, though, the shape of the trench is like a ladle with a gradual deepening starting from the anchored end of the riser, but a rather steeper gradient on the vessel side of the deepest point.

\subsubsection{Mathematical approximation of SCR trench}

In view of the potential importance of trench formation on the fatigue performance of SCRs in the touchdown zone, there have been attempts to insert a trench shape in the seabed and assess its impact on fatigue performance. This requires mathematical approximations of the trench profile, which is then used to specify the neutral (zero contact force) position for each seabed spring beneath the riser. The most well-known mathematical trench formulation in the industry arose from the CARISIMA project, which is referred to as a third-order polynomial function (Giertsen et al. 2004), although no details of the formulation have been published in the literature. Langner (2003) has also suggested a trench profile with detailed expressions, assuming a circular arc on the SCR side of the trench and a seventh-order polynomial fit to the boundary conditions on the anchor side of the trench. This expression has been evaluated and compared with results of the current numerical simulation, later in this chapter.

The theoretical trench shape depends on three key points that essentially define the trench geometry:

- touchdown point (TDP), where the SCR reaches the nominal level of the seabed;

- trench bottom point (TBP), where the trench reaches its maximum depth;

- trench surface point (TSP), where the SCR reaches essentially zero gradient towards the anchored end of the riser. 
Figure 7-4 shows an example of the gradual embedment by 1500 cycles of loading obtained using the hysteretic non-linear seabed model. The choice of 1500 cycles was somewhat arbitrary, but was adopted to ensure that the trench had stabilised, which occurs well within the 1500 cycles. The surface point appears to remain unchanged, while both the trench bottom point and the touchdown point move towards the vessel as the maximum trench depth increases. The vertical and horizontal position of these two points have a significant effect on the SCR curvature and consequently the contact stress distribution with the seabed, which in turn will affect the fatigue damage in the TDZ, as discussed in Chapter 4. The penetration depth beyond the surface point towards the anchored end is quite limited (less than 0.1D), so it is sufficient for the mathematical expressions for the trench to assume zero penetration in this area. This will have a minor effect on the horizontal offset of the TDP (since that is now defined in terms of the point where the SCR is at the same level as the TSP), but this should not be significant.

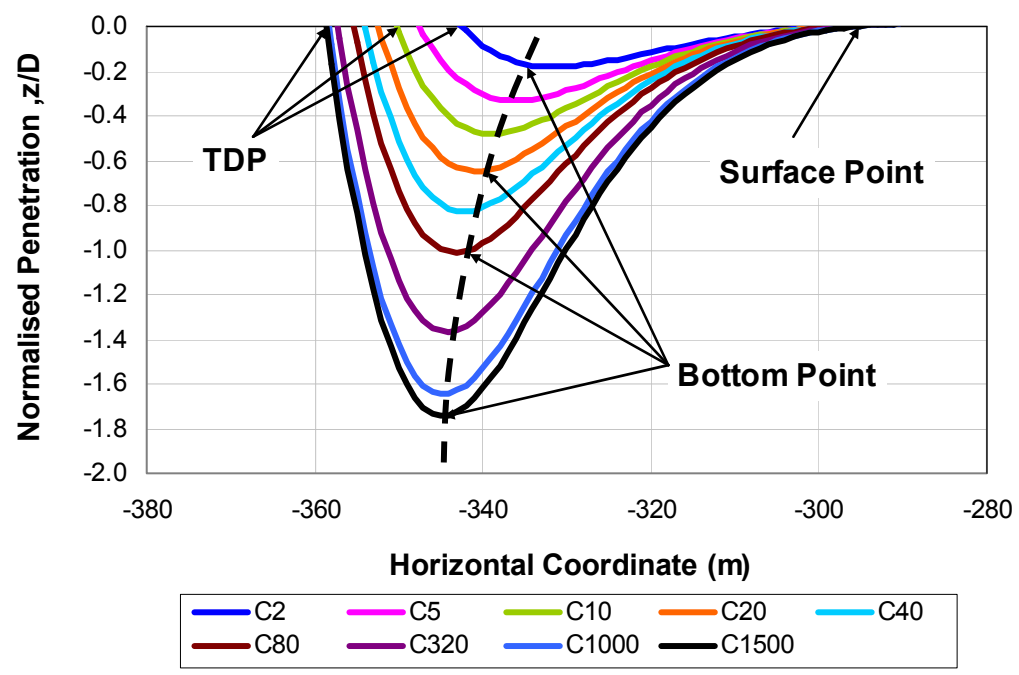

Figure 7-4: Gradual displacement of trench key points with trench deepening

In summary, as seen in Figure 7-4, the main features of the gradual trench deepening for the non-linear seabed are:

1) The location of the surface point does not change significantly.

2) The trench bottom point and touchdown point move gradually towards the vessel.

\section{Linear and quadratic exponential trenches}

Studying a wide range of mathematical functions and comparing with the results of numerical simulations suggest that a linear or quadratic exponential formula with the key parameters illustrated in Figure 7-5 might provide a simple basis for the trench profile in the seabed. 


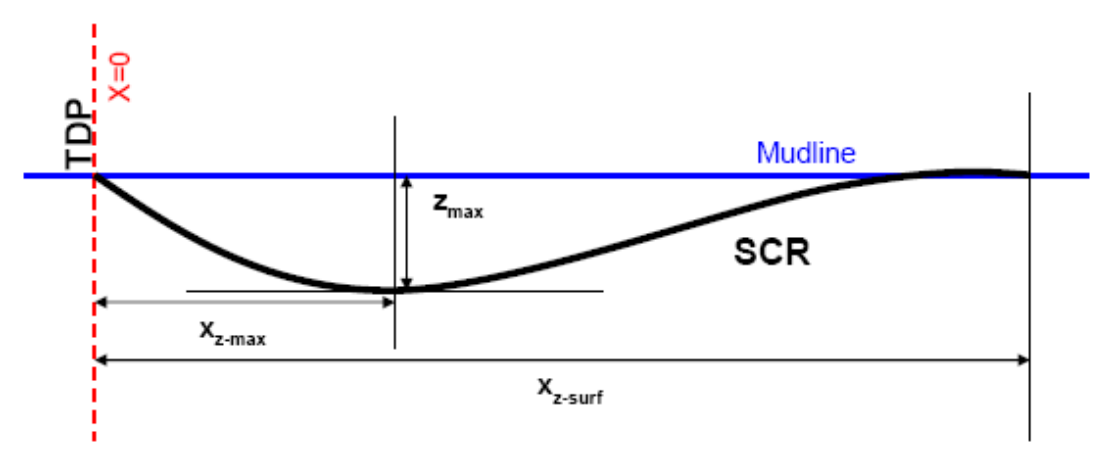

Figure 7-5: SCR trench profile in linear elastic seabed

The linear exponential expression can be written as, considering the TDP as the reference point of coordinate system:

$$
\mathrm{z}=-\mathrm{c}_{1} \mathrm{x} \cdot \mathrm{e}^{-\mathrm{c}_{2} \mathrm{x}}
$$

where $c_{1}$ and $c_{2}$ are coefficients and $\mathrm{x}$ is the horizontal distance from the TDP.

The derivative of equation (7-1) leads to the maximum trench depth, $z_{\max }$ and its horizontal position, $\mathrm{x}_{\mathrm{z}-\max }$, given by:

$$
\begin{aligned}
& \mathrm{z}_{\text {max }}=\frac{\mathrm{c}_{1}}{\mathrm{c}_{2}} \exp (-1) \\
& \mathrm{x}_{\mathrm{z}-\text { max }}=\frac{1}{\mathrm{c}_{2}}
\end{aligned}
$$

Rearranging equation (7-3) and substituting into equation (7-2) gives the values of $c_{1}$ and $\mathrm{c}_{2}$ :

$$
\begin{aligned}
& \mathrm{c}_{1}=\frac{\mathrm{z}_{\text {max }}}{\mathrm{x}_{\mathrm{z}-\max }} \exp (1) \\
& \mathrm{c}_{2}=\frac{1}{\mathrm{x}_{\mathrm{z}-\max }}
\end{aligned}
$$

Equations (7-4), (7-5) and (7-1) allow generation of a trench profile with prescribed values of $\mathrm{z}_{\max }$ and $\mathrm{x}_{\mathrm{z}-\max }$.

The trench profile can also be expressed in quadratic form as:

$$
\mathrm{z}=-\overline{\mathrm{c}}_{1} \mathrm{x}^{2} \mathrm{e}^{-\overline{\mathrm{c}}_{2} \mathrm{x}}
$$

The key dimensions and the constant coefficients for the quadratic formulation can be written as: 
$\mathrm{z}_{\max }=\frac{4 \overline{\mathrm{c}}_{1}}{\overline{\mathrm{c}}_{2}^{2}} \exp (-2)$

$\mathrm{x}_{\mathrm{z}-\max }=\frac{2}{\overline{\mathrm{c}}_{2}}$

$\overline{\mathrm{c}}_{1}=\frac{\mathrm{z}_{\text {max }}}{\mathrm{x}_{\mathrm{z}-\max }^{2}} \exp (2)$

$\bar{c}_{2}=\frac{2}{\mathrm{x}_{\mathrm{z}-\max }}$

The trench shown in Figure 7-4 has been approximated mathematically in the early and late stages of deepening using the linear and quadratic exponential formulae, as shown in Figure 7-6 and Figure 7-7. The results are compared with the trench expression proposed by Langner (2003) and the results of the finite element analysis. It is clear that the quadratic exponential expression shows a better correlation with FEA results, particularly after 1000 cycles, although the minor discrepancies with respect to the finite element results may have a significant impact on the accuracy on fatigue damage prediction using the analytical trench shape. The results show that quadratic exponential formula gives a better approximation for deeper trenches as well. The plots also show that the trench profile given by Langner (2003) overestimate the gradient of the profile in the anchor side for low cycles or shallow trenches Figure 7-6 and underestimate the gradient of profile in vessel side for high cycles or deeper trenches Figure 7-7.

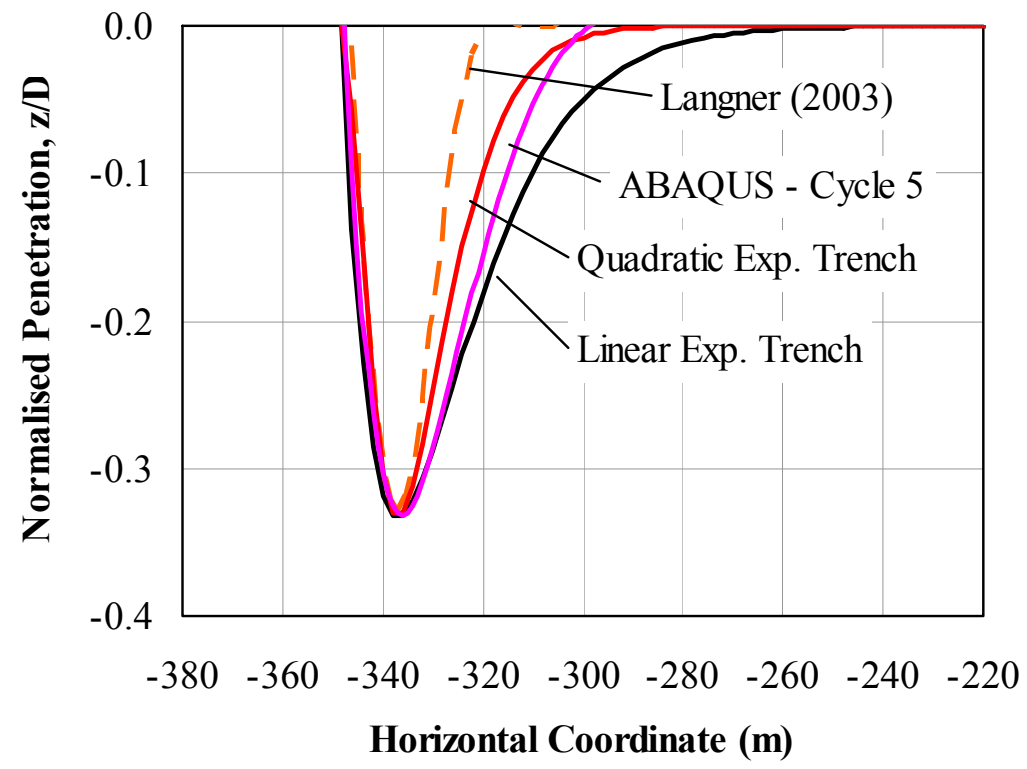

Figure 7-6: Mathematical approximation of non-linear seabed trench at cycle number 5 


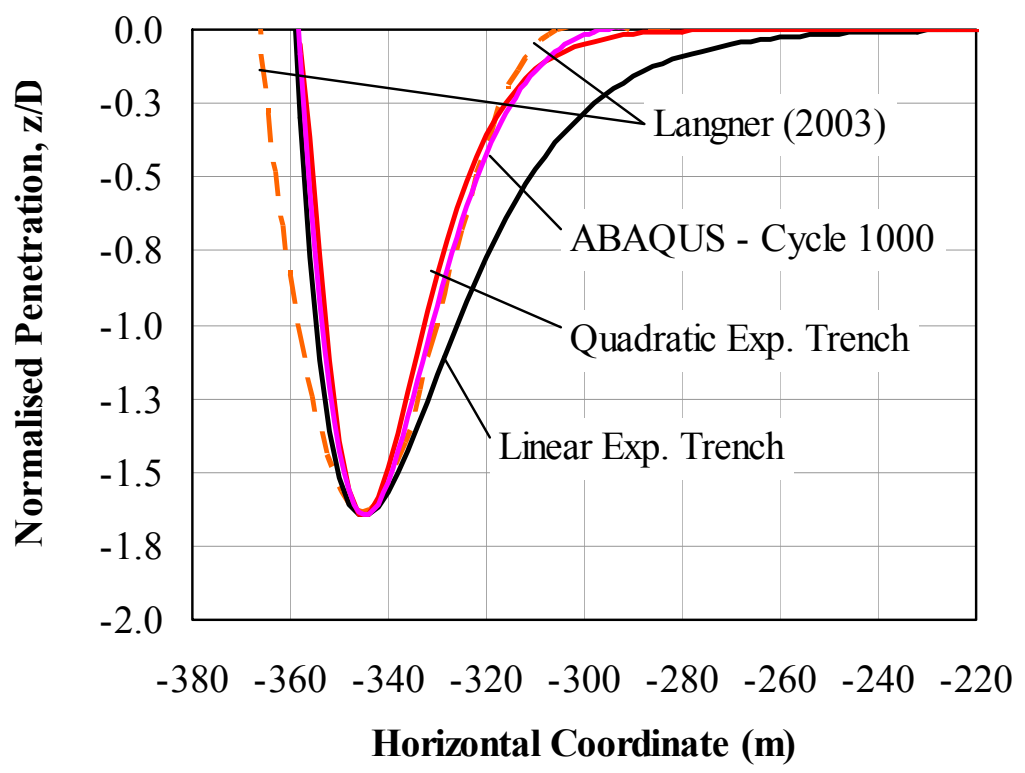

Figure 7-7: Mathematical approximation of non-linear seabed trench at cycle number 1000

Figure 7-8 shows results obtained by private communication with individuals in industry, evaluating trenches predicted using the shape recommended from the CARISIMA project method (legends starting with CAR) compared with trenches obtained using a proprietary approach implemented in ABAQUS (legends starting with ABA). Comparing this plot with Figure 7-6 and Figure 7-7 shows that the quadratic (and even linear) exponential formulae give much better correlations with finite element computations than the (unpublished) CARISIMA shapes.

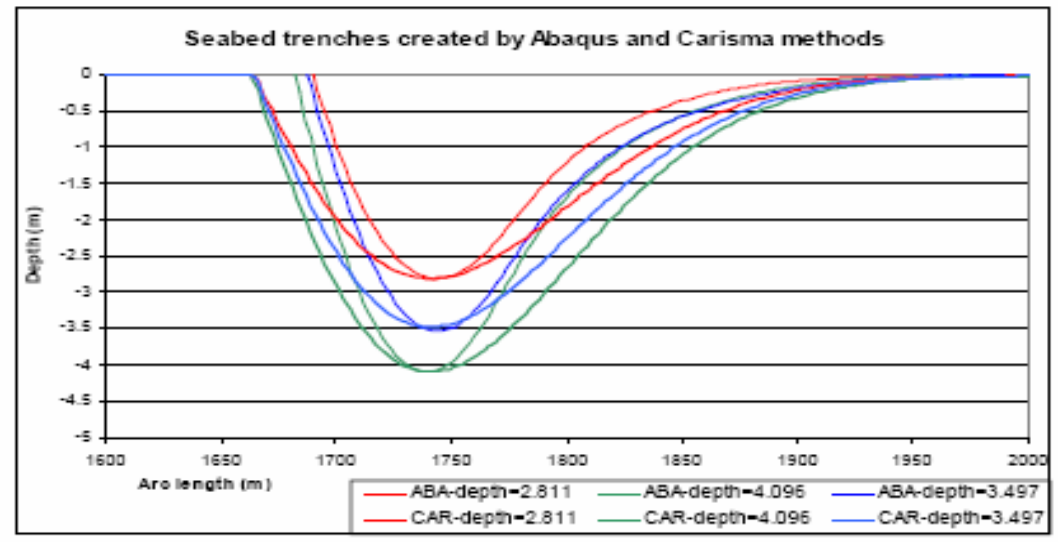

Figure 7-8: Seabed trenches created by ABAQUS and CARISIMA methods (private communication)

The quadratic exponential trench shape can be expressed in non-dimensional form as: 


$$
\frac{\mathrm{z}}{\mathrm{z}_{\max }}=\left[\left(\frac{\mathrm{x}}{\mathrm{x}_{\mathrm{z}-\max }}\right) \mathrm{e}^{\left(\mathrm{x} / \mathrm{x}_{\mathrm{z}-\max }-1\right)}\right]^{2}
$$

Defining the trench surface point as where $\mathrm{z}$ falls to $1 \%$ of $\mathrm{z}_{\max }$, the relative distances $\mathrm{x}_{\mathrm{z}-\mathrm{max}}$ and $\mathrm{x}_{\mathrm{z} \text {-surf }}$ may be expressed approximately as

$$
\mathrm{x}_{\mathrm{z}-\max } \approx \frac{1}{5} \mathrm{x}_{\mathrm{z}-\text { surf }}
$$

Example shapes for the quadratic exponential trench are shown in Figure 7-9 (for a given $z_{\max }$ ) and Figure 7-10 (for a given $x_{z-\max }$ ).

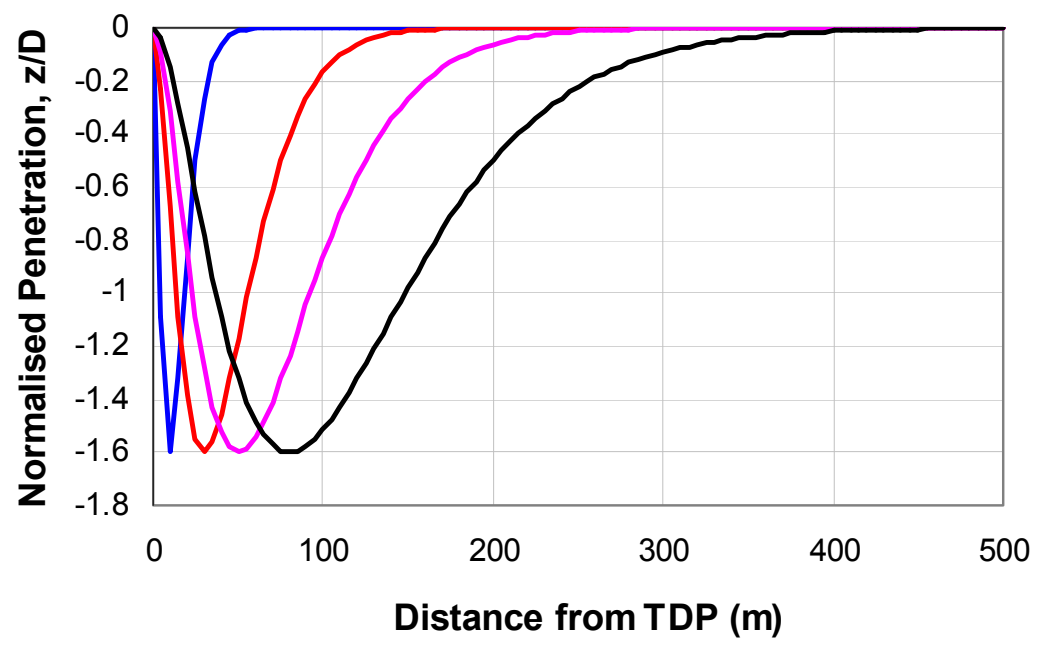

Figure 7-9: Quadratic exponential trench with various values of $x_{z-\max }$

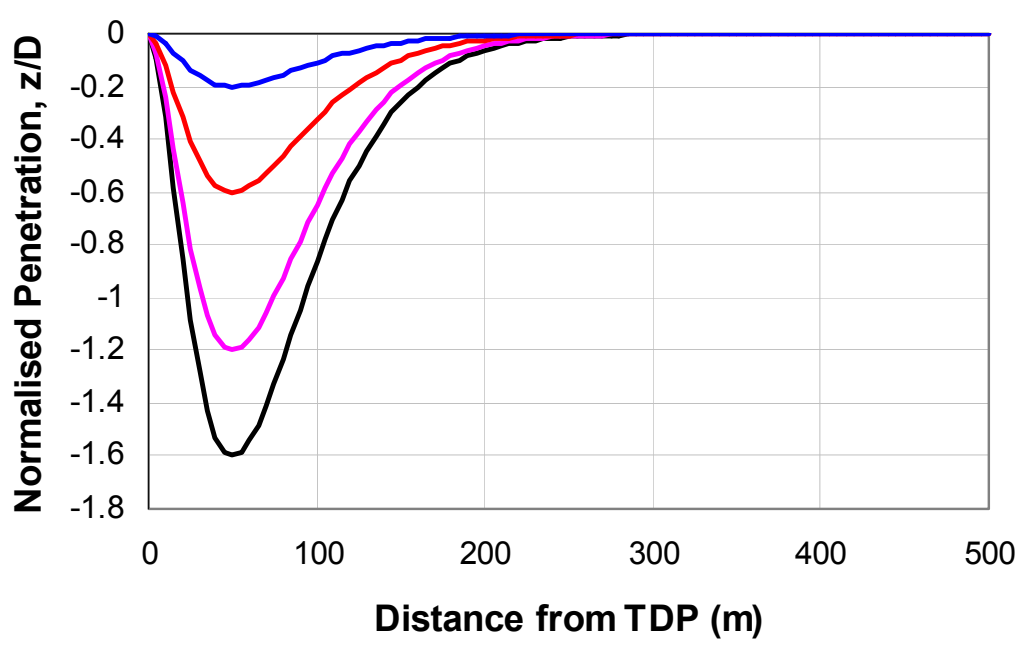

Figure 7-10: Quadratic exponential trench with various values of $z_{\max }$ 


\subsection{ARTIFICIAL TRENCH INSERTION AND FATIGUE PERFORMANCE}

Various methodologies have been used by authors to insert a trench beneath the riser and study the impact on fatigue performance. Typically a mathematical trench profile has been inserted through the TDZ for a linear or non-linear seabed model. These kinds of studies have led to contradictory results as referred in the introduction of this chapter. It appears that this is due to inconsistencies between the defined profile and the natural catenary shape of the riser in the touchdown zone. Such inconsistencies can alter the stress distribution in the TDZ, giving rise to contact pressure hotspots as indicated in Figure 7-11. It will be shown later in this chapter that even a minor change in the geometry of an artificially created trench can result in substantially different outcomes, with increasing, decreasing or shifting of the peak fatigue damage.

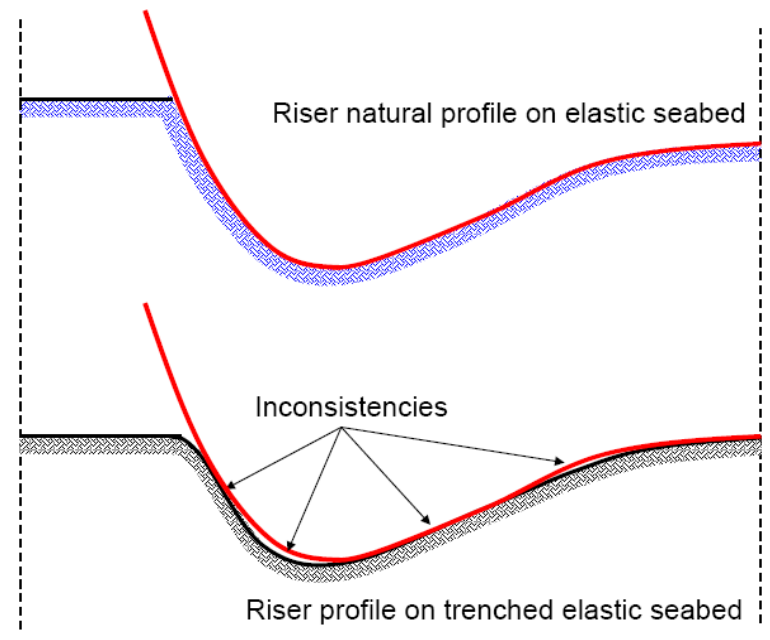

Figure 7-11: Riser-trench contact geometry with various trench locations

In the next sections, the fatigue performance will be examined by initial insertion of moderate and deep trenches generated by the quadratic exponential formula for an elastic seabed, where it will be shown that the fatigue damage obtained by artificial trench insertion is not reliable and can be severely distorted even by minor changes of the inserted trench geometry.

\subsubsection{Ultimate quadratic exponential trench insertion in elastic seabed}

A series of fatigue analyses were performed for an elastic seabed with initial insertion of moderate and deep trenches (1.5D and 5D maximum depths). The SCR configuration and analysis setup is as per defined in Chapter 4. To show the difficulties of trench insertion, a step by step approach is described here. Figure 7-12 and Figure 7-13 show two different cases of a riser and inserted trench profile in comparison with the nontrenched condition for a relatively soft $10 \mathrm{kPa}$ elastic seabed. For the assumed submerged pipe weight of $0.98 \mathrm{kN} / \mathrm{m}$, a seabed of this stiffness leads to a penetration of 
about $0.3 \mathrm{D}$ under the pipe weight alone, as indicated by the SCR initial profile for the untrenched situation. This large self-weight penetration leads to slight difficulties in implementing the trench.

The horizontal coordinates of the surface point and TDP of SCR on non-trenched seabed (purple plot) are $-300 \mathrm{~m}$ and $-364 \mathrm{~m}$ respectively. In Figure 7-12, a 1.5D artificial quadratic exponential trench has been inserted in the seabed. To be consistent with the main findings of trench deepening using a non-linear seabed model, the surface point of the inserted trench has been kept the same as the SCR surface point ( $\mathrm{x} \sim-$ $300 \mathrm{~m}$ ), but the TDP has been moved slightly towards the vessel (to about $-384 \mathrm{~m}$ ). This gives a relative horizontal distance of the TDP and surface point of about $84 \mathrm{~m}$. The corresponding values of the deepest point of the trench are $\mathrm{z}_{\max }=1.5 \mathrm{D}=0.486 \mathrm{~m}, \mathrm{x}_{\mathrm{z}-\max }$ $\sim \mathrm{X}_{\mathrm{z} \text {-surf }} / 5=-384+84 / 5=-367 \mathrm{~m}$

The quadratic trench generated by these parameters (adjusted to global coordinate system) and the updated SCR profile in the trenched seabed are shown in Figure 7-12 (green and red plots respectively). The black plot, overlying the SCR profile to the right of its deepest point and the artificial trench profile beyond that point shows the overall seabed profile after trenching, which is the combination of the SCR and inserted trench profiles. The SCR follows the predefined trench shape where there is contact between the SCR and trench. In other areas the SCR has its own natural shape. Thus the SCR profile (black line) in the seabed is obtained from a combination of the defined trench profile and the natural SCR shape in the contact areas. As is evident, the SCR only makes contact with the trenched seabed over the first part of the trench, and the deepest point is only just over $0.8 \mathrm{D}$ below the nominal seabed.

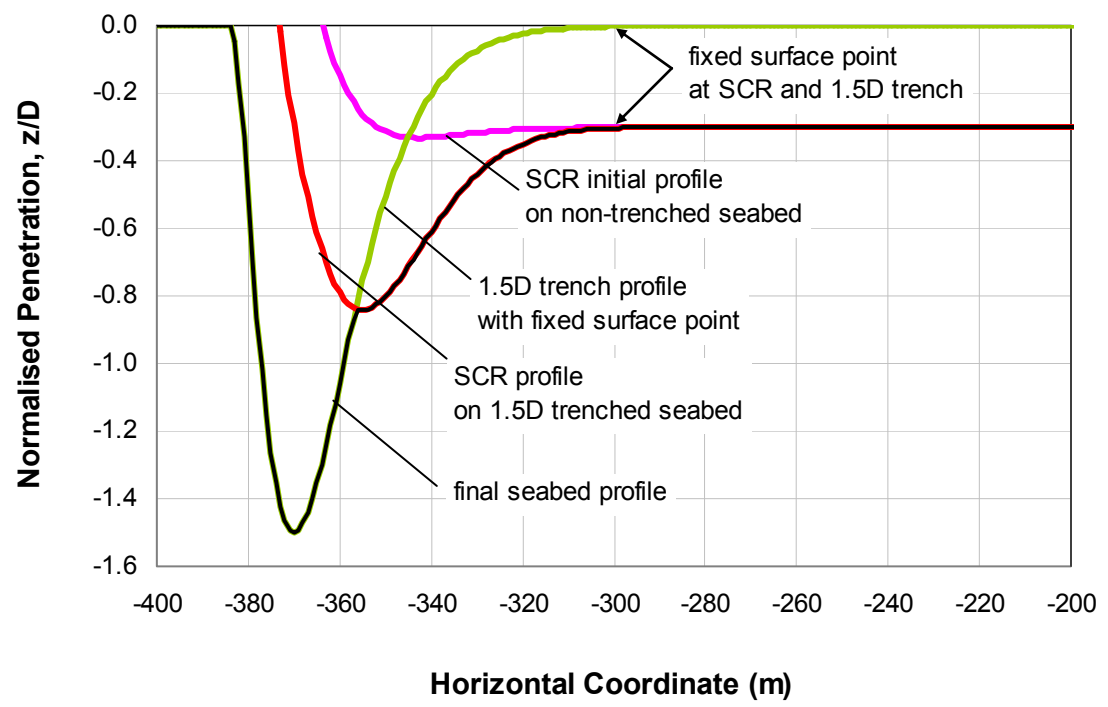

Figure 7-12: SCR and artificial 1.5D trench profile on 10kPa elastic seabed, case I 


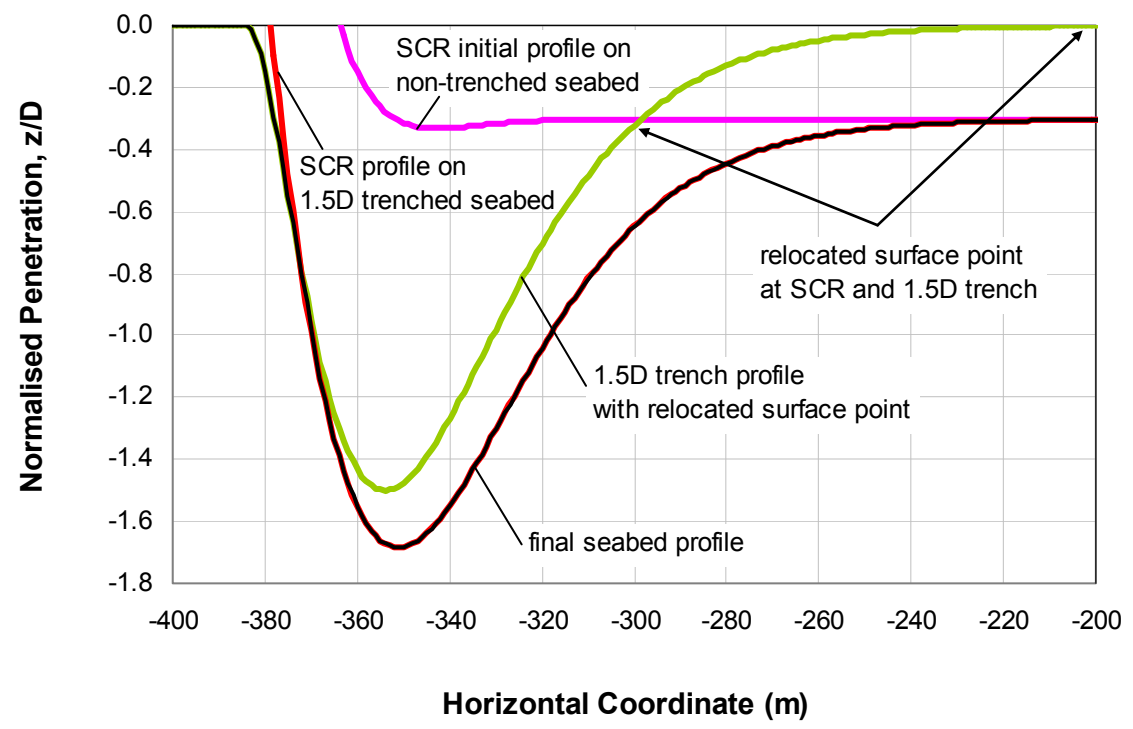

Figure 7-13: SCR and artificial 1.5D trench profile on $10 \mathrm{kPa}$ elastic seabed, case II

In the other trial, the fact that the surface point does not change significantly during trench deepening has been ignored. Instead the trench bottom point has been moved towards the anchor end (to about $\mathrm{x}=-354 \mathrm{~m}$ ) with the same TDP and trench depth. The updated trench surface point is then about $x=-200 \mathrm{~m}$ as shown in Figure 7-13. This trial appears to give a more reasonable geometry, with the SCR now maintaining contact over most of the inserted trench, and with a maximum penetration of just under 1.7D below the nominal seabed.

These two different cases have been investigated to study the impact of changes in the inserted trench profile on the contact stress profile and fatigue performance. Figure 7-14 compares the contact stress in the TDZ for these two different cases with the nontrenched profile.

It is worth mentioning that the enlarged length of the seabed profile is in the scale of metres which is much less than the catenary length in scale of kilometres; the horizontal component of the tension is constant along the catenary part and is not affected by such minor changes in the TDZ. 


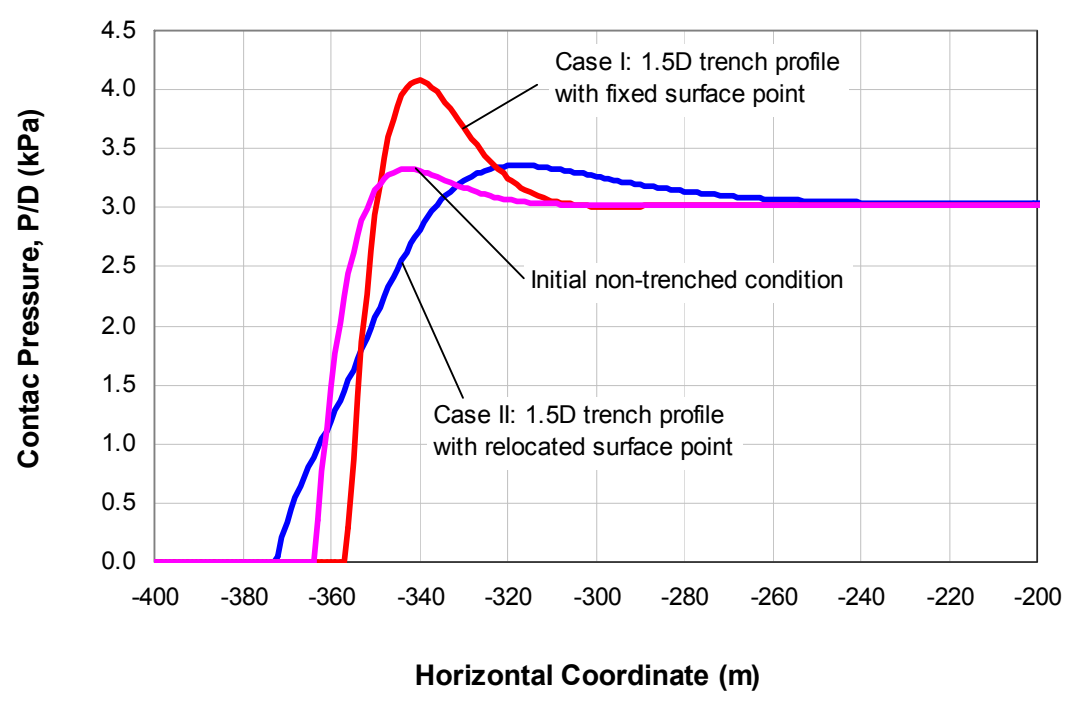

Figure 7-14: Contact pressure in $1.5 \mathrm{D}, 10 \mathrm{kPa}$ trenched seabed, case I \& II

The logarithmic and non-logarithmic distribution of fatigue damage are shown in Figure 7-15 and Figure 7-16, where the peak fatigue damage has decreased (Case I) and increased (Case II) slightly for the two trench shapes, both of which have the same intended maximum depth and minor changes in the longitudinal profile. It may be considered that both of these trenches could represent reasonable approximations to real trenches depending on the environmental loads experienced by the system. The examples show some influence of the trench on fatigue damage although the magnitude of impact is quite limited (about $10 \%$ ).

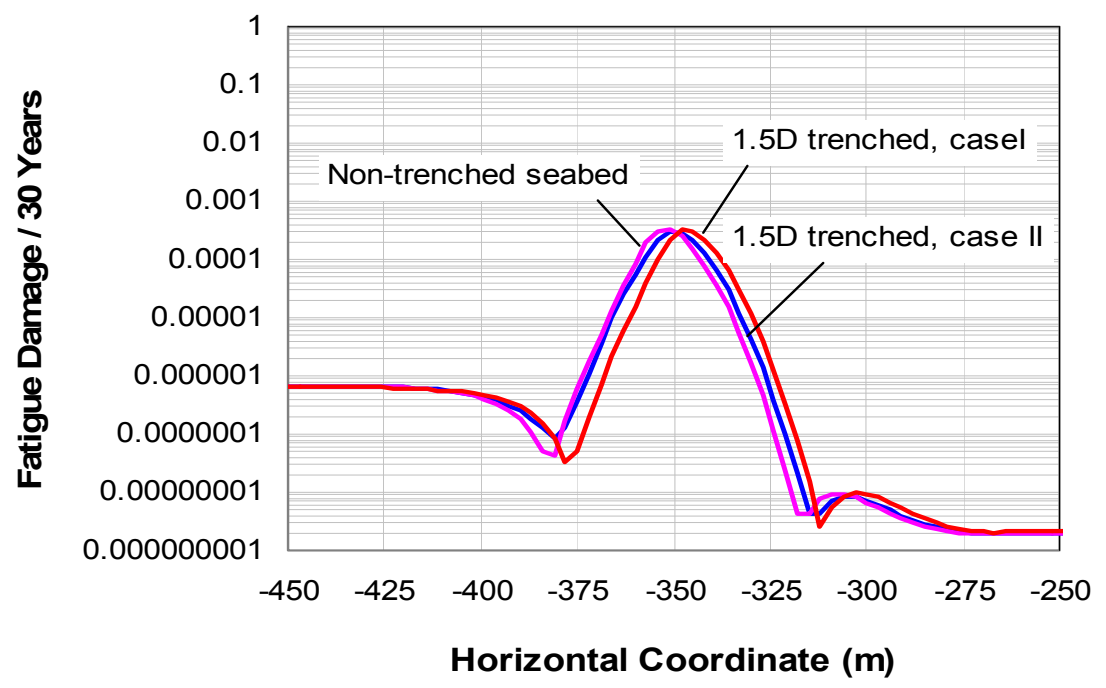

Figure 7-15: Logarithmic fatigue damage in 1.5D, $10 \mathrm{kPa}$ trenched seabed, case I \& II 


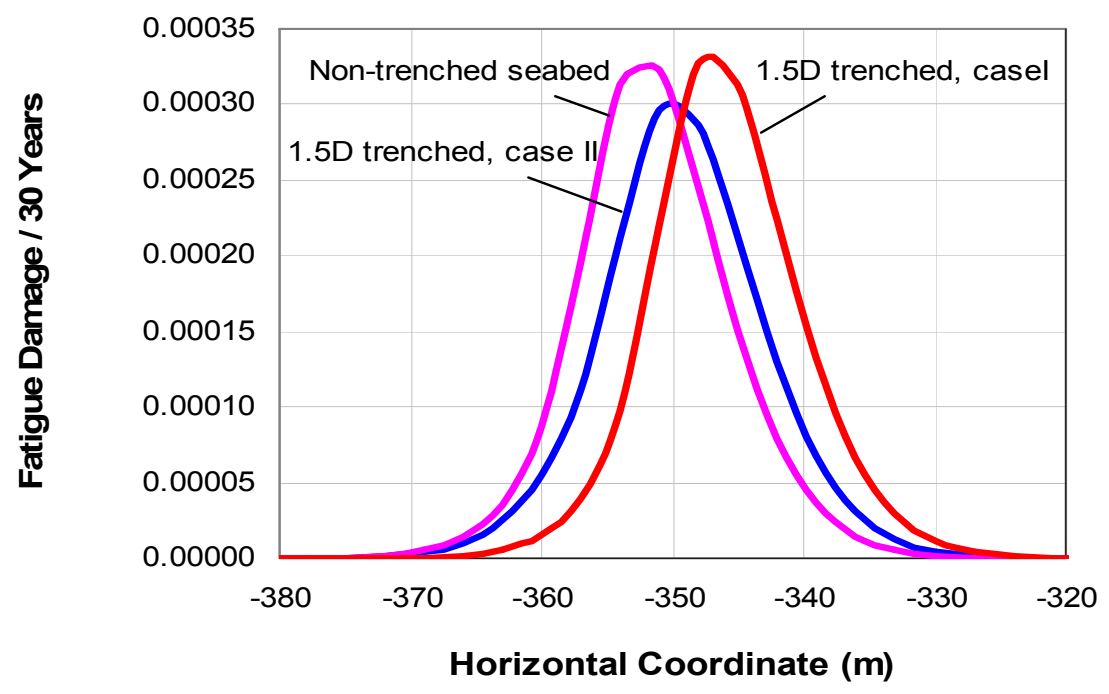

Figure 7-16: Non-logarithmic fatigue damage in 1.5D, $10 \mathrm{kPa}$ trenched seabed, case I \& II

These analyses have been repeated for the same coordinates of the trench key points for Cases I and II respectively, but with a nominal trench depth of 5D. Figure 7-17 shows a very similar contact condition for the $1.5 \mathrm{D}$ trench to that illustrated in Figure 7-12, and so no marked change of fatigue damage relative to the $1.5 \mathrm{D}$ trench is expected. For the case II geometry of the 5D trenched seabed, the SCR ends up suspended over the deepest part of the trench profile, but with a separate contact zone occurring towards the vessel end of the trench, close to the TDP (see Figure 7-18). This inconsistency of the SCR and inserted trench profiles leads to discontinuities in the profile of contact stress (Figure 7-19), and ultimately to an irregular distribution of fatigue damage as shown in Figure 7-20 (see curve for 5D, case II). For this case, the fatigue damage has increased relative to the untrenched case (see Figure 7-21), with the peak damage moving significantly towards the vessel, and occurring close to the TDP of the artificially inserted trench. 


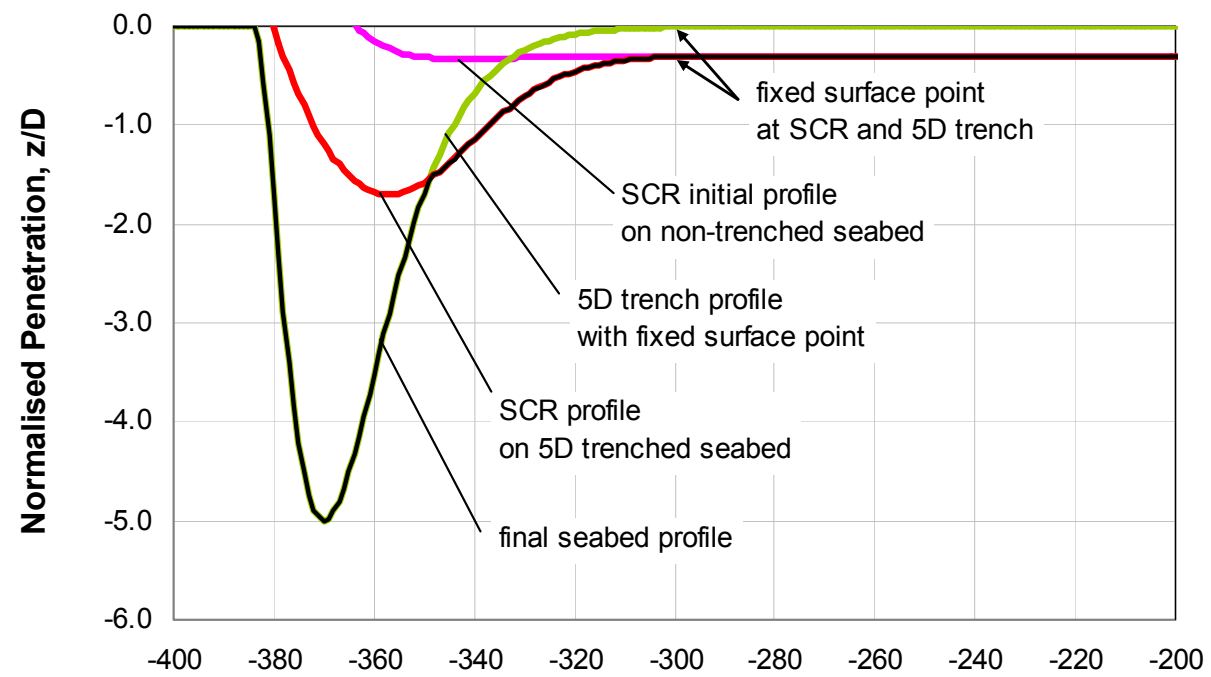

Horizontal Coordinate (m)

Figure 7-17: SCR and artificial 5D trench profile on 10kPa elastic seabed, case I

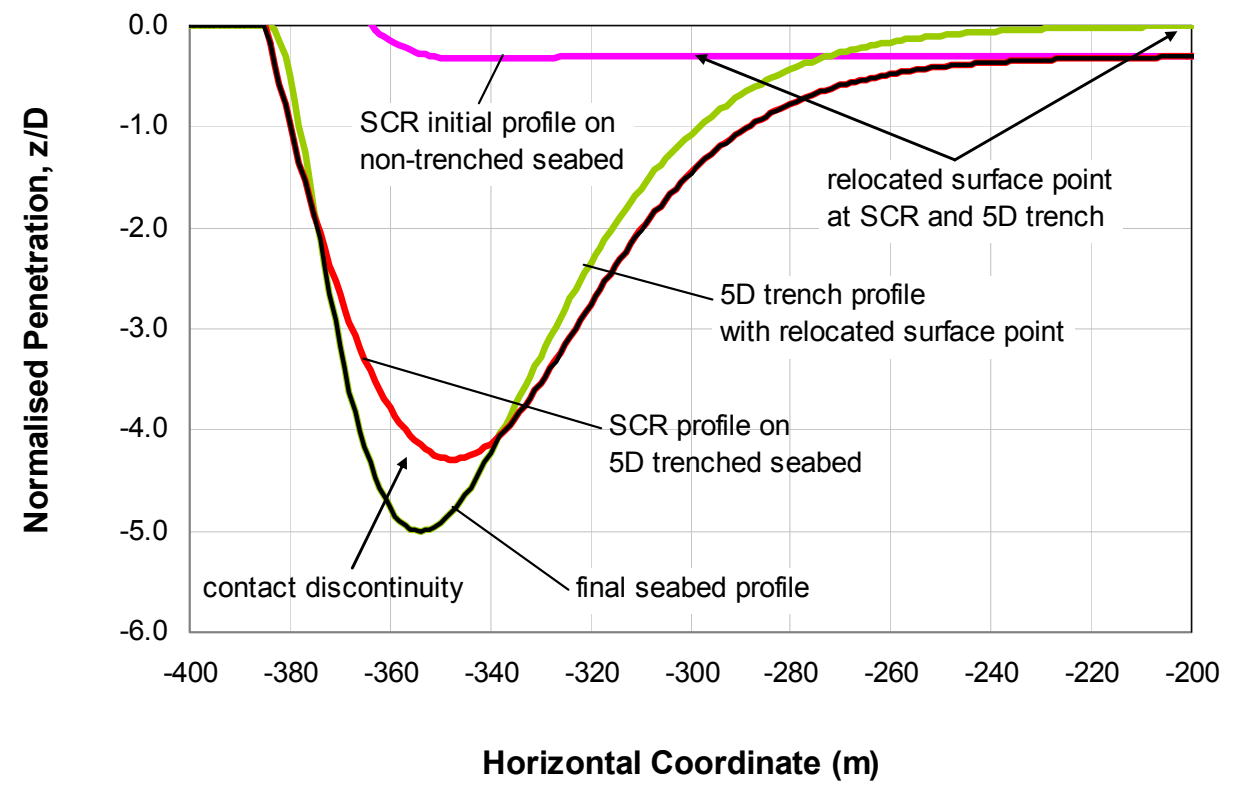

Figure 7-18: SCR and artificial 5D trench profile on 10kPa elastic seabed, case II 


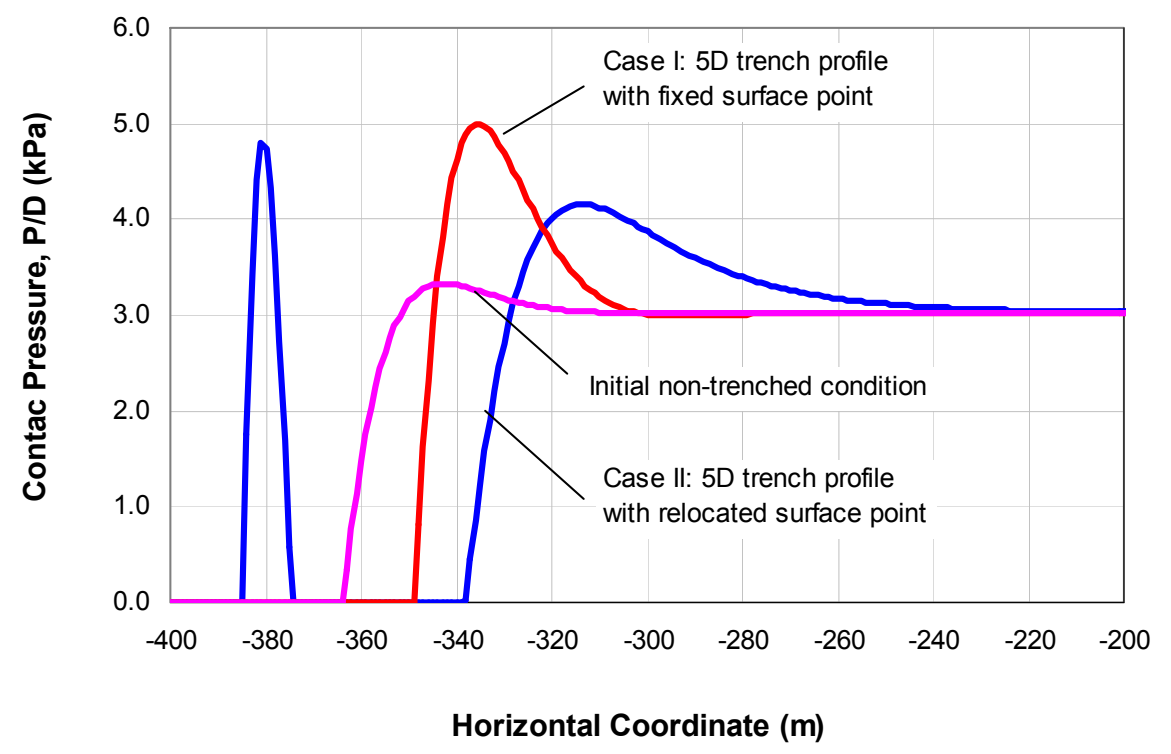

Figure 7-19: Contact pressure in 5D, $10 \mathrm{kPa}$ trenched seabed, case I \& II

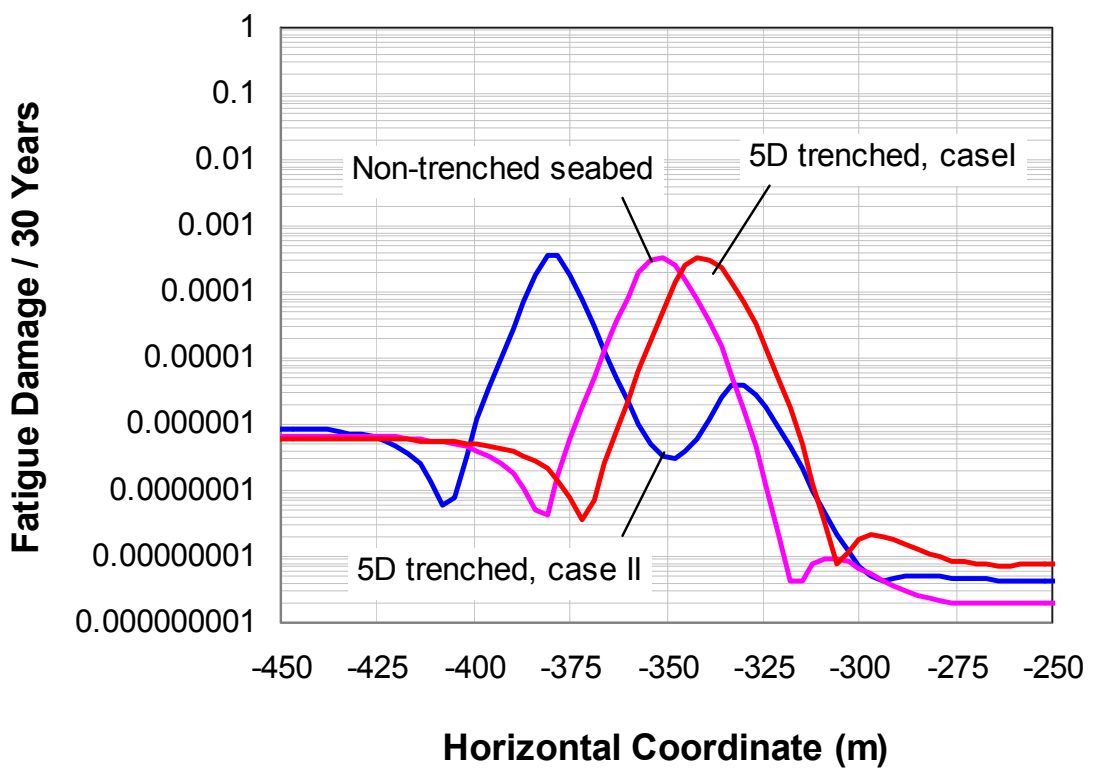

Figure 7-20: Logarithmic fatigue damage in 5D, $10 \mathrm{kPa}$ trenched seabed, case I \& II 


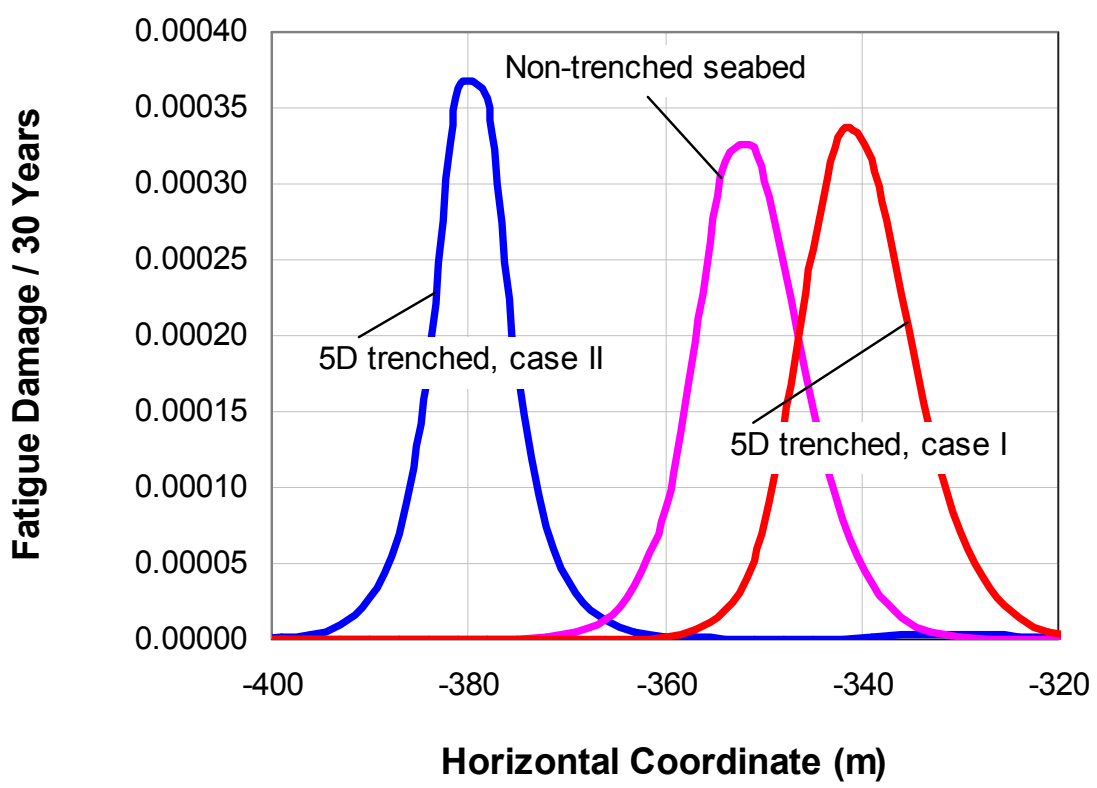

Figure 7-21: Non-logarithmic fatigue damage in $1.5 \mathrm{D}, 10 \mathrm{kPa}$ trenched seabed, case I \& II

These analyses show that the contact condition and fatigue performance in an artificially trenched seabed depends significantly on the consistency of the natural SCR profile and the inserted trench shape. As shown above, minor changes in trench geometry can give rather different results, increasing or decreasing the maximum fatigue damage and shifting its position (in the last case significantly). This is presumably why different authors have reported contradictory results for the effect of the SCR trench on fatigue damage.

The profiles studied above show that contact between the SCR and the trench may occur only on the anchor side of the trench bottom (Case I profiles), or may extend well past the trench bottom (1.5D Case II), or may occur in separate regions either side of the trench bottom point (5D Case II). When a deep trench shape is inserted in an elastic seabed, it might be expected that for low to moderate sea states, contact will be primarily towards the anchor side of the trench bottom, while for more severe sea states the zone of contact will expand towards the vessel and in extreme cases will extend over the full trench profile. The situation where separate contact zones occur appears unrealistic, although it is difficult to ensure that the imposed trench geometry will match the SCR profile for different sea states. Inevitably, spurious hot spots of fatigue damage may arise, as noted above for the 5D Case II situation.

Considering this, artificial trench insertion in an elastic seabed appears likely to lead to unpredictable and inaccurate assessment of the effect of a trenched seabed on the fatigue damage. As an alternative approach, the capabilities of the hysteretic non-linear model will be used to create deep trench profiles, but where the inconsistency of the $\mathrm{SCR}$ and trench profiles is no longer an issue because of the gradual mechanism of trench formation in the non-linear seabed. 


\subsubsection{Ultimate trench insertion by hysteretic non-linear seabed model}

It was demonstrated in Chapter 5 that the hysteretic non-linear soil model is able to generate deep trenches by adopting extreme values of the model parameter $\lambda_{\text {rep }}$, the repenetration delay parameter. To encourage rapid penetration of the riser deep into the seabed, the magnitude of maximum suction mobilisation should also be reduced by assigning a low value to the parameter $\mathrm{f}_{\text {suc }}$. The simple sequence described below has been followed to implement the fatigue calculation procedure introduced in Chapter 6 for studying the influence of the trench on the SCR fatigue performance in the TDZ:

a) Define two different sets of the model parameters $\lambda_{\text {rep }}$ and $f_{\text {suc }}$ are adopted:

- Initial set: with extreme value of $\lambda_{\text {rep }}$ and small value of $f_{\text {suc }}$.

- Second set: with normal 'operational' range of values as suggested in Chapter 5.

b) Set the model parameters to the initial values.

c) Apply the most severe sea state with an appropriate number of cycles to generate a trench with the desired maximum depth. (Note, the number of applicable waves is set to zero for this sea state to eliminate its contribution to fatigue damage in the post-processing spread sheet.)

d) Switch the model parameters to the second set.

e) Apply the full package of waves including the most severe sea state.

f) Calculate fatigue damage as per the procedure explained in Chapter 4.

This procedure first uses the unrealistic initial set of model parameters to create a trench of the required depth. For this purpose it is considered appropriate to use the most severe sea state to speed up trench formation. Since this part of the analysis is not intended as a realistic model of trench formation, which in reality will occur over several months (or even years) of operation with a range of sea states, its contribution to fatigue damage should be set to nil; this is achieved by considering zero number of applicable waves for this sea state in the macro-driven spread sheet calculations. After trench generation, the normal fatigue analysis can be performed by switching the model parameters to the second set, within what is viewed as a practical range, and applying the full package of sea states; this time the most severe sea state has its own contribution to the total fatigue damage as determined through the applicable number of waves of that magnitude.

The random wave orders No.1 and No.3 defined in Chapter 6 have been used to perform a series of fatigue analyses with initial trench creation to various depths from moderate to deep. The initial trenches have been created by applying a sea state in the range of the most severe waves of the package with 30 to 50 cycles and extreme values of re- 
penetration delay parameter $\lambda_{\text {rep. }}$ The model parameters implemented in these analyses are shown in Table 7-1.

Table 7-1: Non-linear seabed soil parameters for performed fatigue analyses

\begin{tabular}{|l|c|c|}
\hline \multicolumn{1}{|c|}{ Parameter } & Symbol & Value \\
\hline Mudline shear strength & $\mathrm{s}_{\text {um }}$ & $0.65 \mathrm{kPa}$ \\
\hline Shear strength gradient & $\rho$ & $1.5 \mathrm{kPa} / \mathrm{m}$ \\
\hline Power law parameter & $\mathrm{a}$ & 6 \\
\hline Power law parameter & $\mathrm{b}$ & 0.25 \\
\hline Normalized maximum stiffness & $\mathrm{K}_{\text {max }}$ & 200 \\
\hline Suction ratio & $\mathrm{f}_{\text {suc }}$ & 0.3 \\
\hline Suction decay parameter & $\lambda_{\text {suc }}$ & 0.5 \\
\hline Regular Repenetration parameter & $\lambda_{\text {rep }}$ & 0.5 \\
\hline Number of Cycles for Fatigue & -- & 10 \\
\hline Parameter for 1.1D pre-trench & Cycles, $\lambda_{\text {rep }}, \mathrm{f}_{\text {suc }}$ & $30,1.5,0.05$ \\
\hline Parameter for 2.0D pre-trench & Cycles, $\lambda_{\text {rep }}, \mathrm{f}_{\text {suc }}$ & $30,3.0,0.05$ \\
\hline Parameter for 3.6D pre-trench & Cycles, $\lambda_{\text {rep }}, \mathrm{f}_{\text {suc }}$ & $30,5.0,0.05$ \\
\hline Parameter for 5.0D pre-trench & Cycles, $\lambda_{\text {rep }}, \mathrm{f}_{\text {suc }}$ & $50,5.0,0.05$ \\
\hline
\end{tabular}

The profiles of trenches created using the parameter values presented in Table 7-1 are compared with the no pre-trench profile in Figure 7-22. The surface point moves slightly away from the vessel and the touchdown point and trench bottom point move in a similar manner to the trend presented in Figure 7-4.

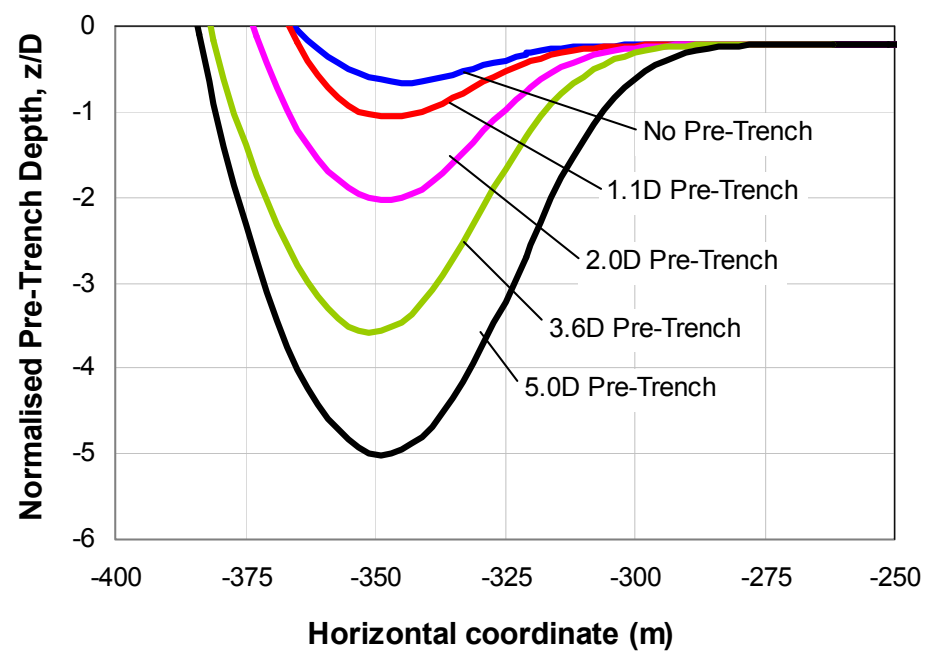

Figure 7-22: Initial trench profile with various depths 
Logarithmic and non-logarithmic fatigue damage profiles for the analyses with random wave order No.1 and the various pre-trench depths are shown in Figure 7-23 and Figure 7-24. As expected from the findings of Chapter 6 , it is seen that the initial trench considerably increases the fatigue damage, with more than $78 \%$ increase for the deepest trench (5D). It is also clear that the peak fatigue damage shifts further towards the vessel the deeper the trench.

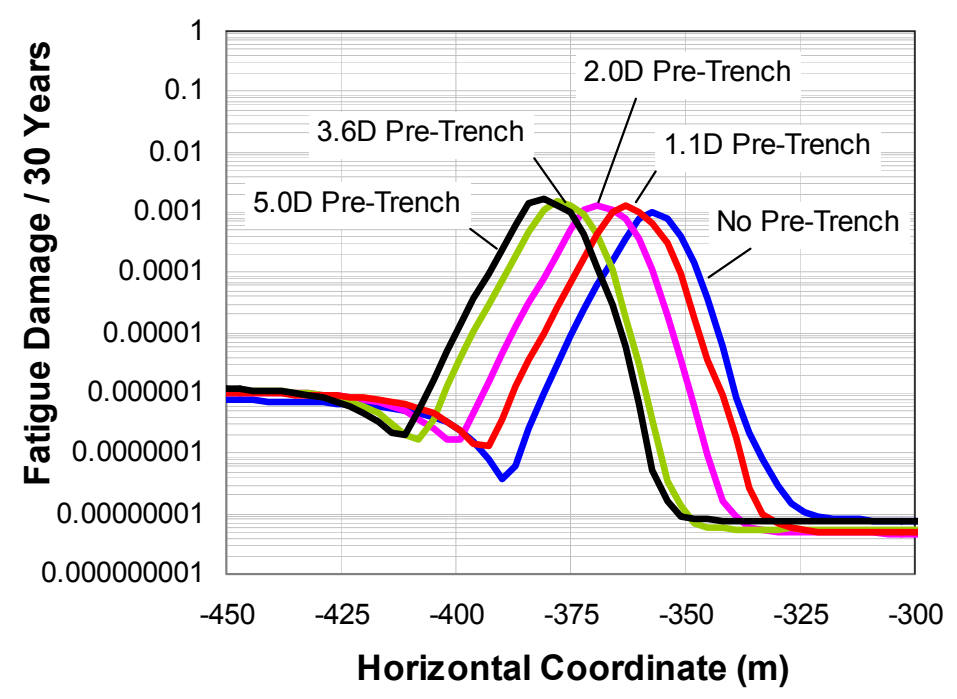

Figure 7-23: Logarithmic fatigue damage for random wave order No.1 in various pre-trenches

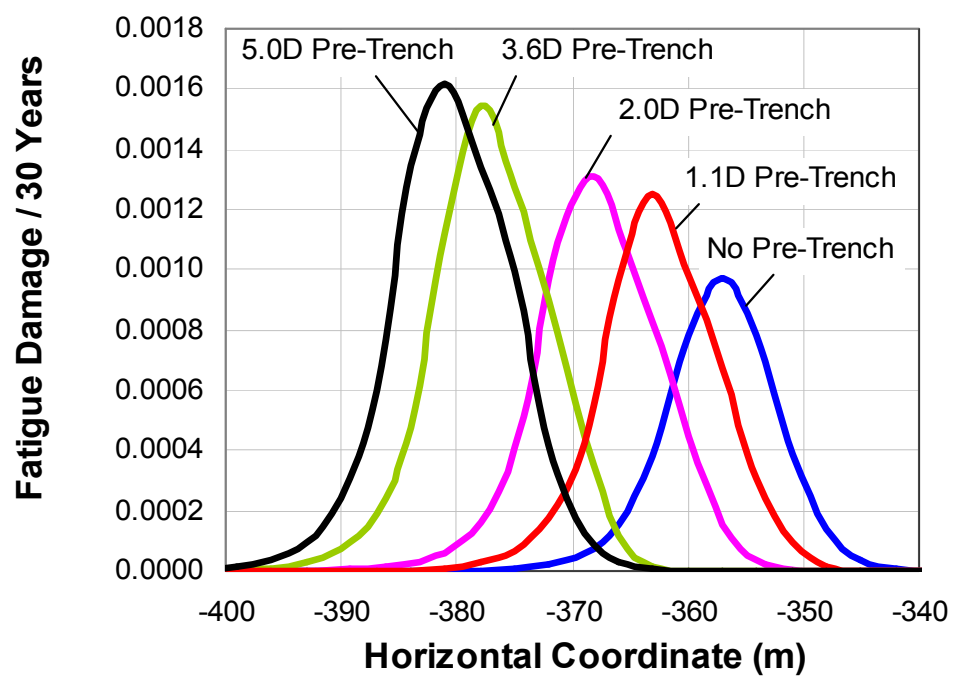

Figure 7-24: Non-logarithmic fatigue damage for random wave order No.1 in various pre-trenches

Corresponding results for random wave order No.3 are presented in Figure 7-25 and Figure 7-26. 


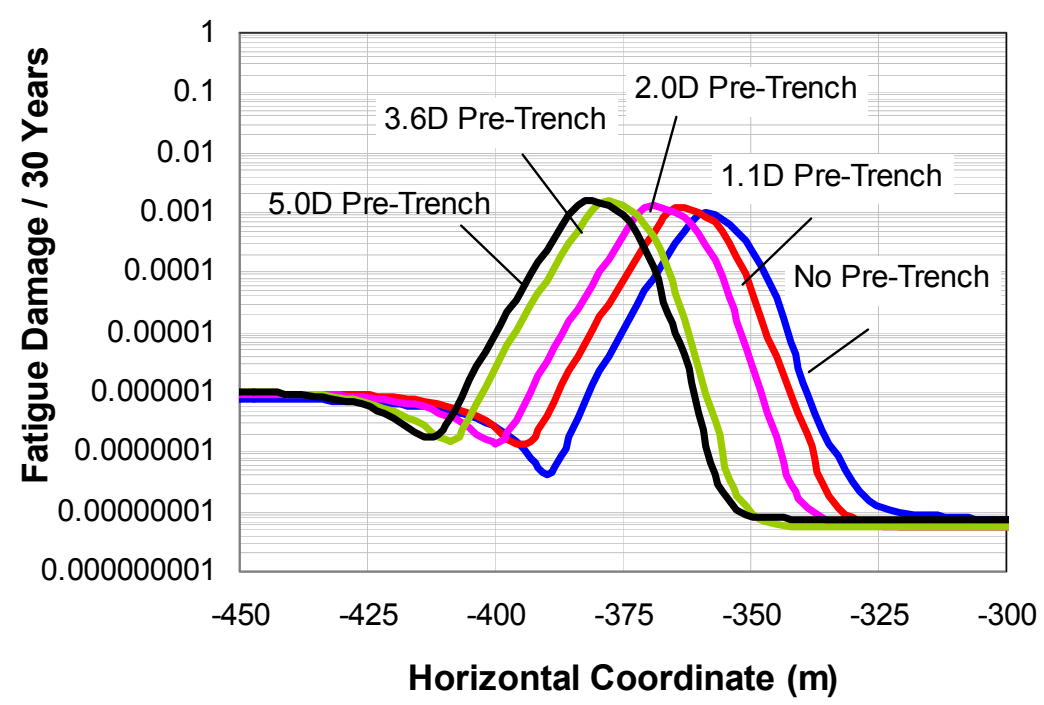

Figure 7-25: Logarithmic fatigue damage for random wave order No.3 in various pre-trenches

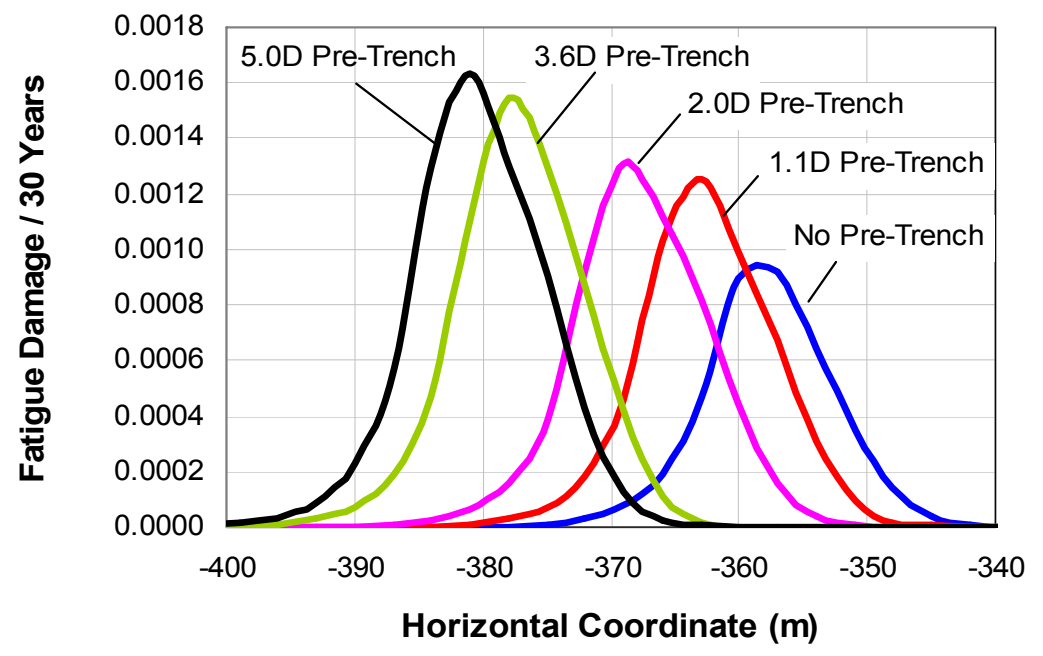

Figure 7-26: Non-logarithmic fatigue damage for random wave order No.3 in various pre-trenches

The increase in fatigue damage with deeper initial pre-trenches is due to the increase of the von Mises stress range variation. Figure 7-27 compares the magnitudes of the von Mises stress ranges for each sea state, at the locations of the maximum fatigue damage for the untrenched case (Node 363) and for a 5D trench (Node 381), for random wave order No.1. The results are entirely consistent with those presented in Chapter 6, showing the significant influence of the trench in rising the von Mises stress range level and consequently the final fatigue damage. In view of the significant differences in the von Mises stress range, which exceeds $50 \%$ for some sea states, it might be expected that the fatigue damage (which is proportional to the cube power of the stress range) would be affected much more. However, the $78 \%$ increase reflects the fact that the 
main contribution to fatigue damage is from intermediate sea states, for which the von Mises stress ranges for the untrenched and trenched cases are less different.

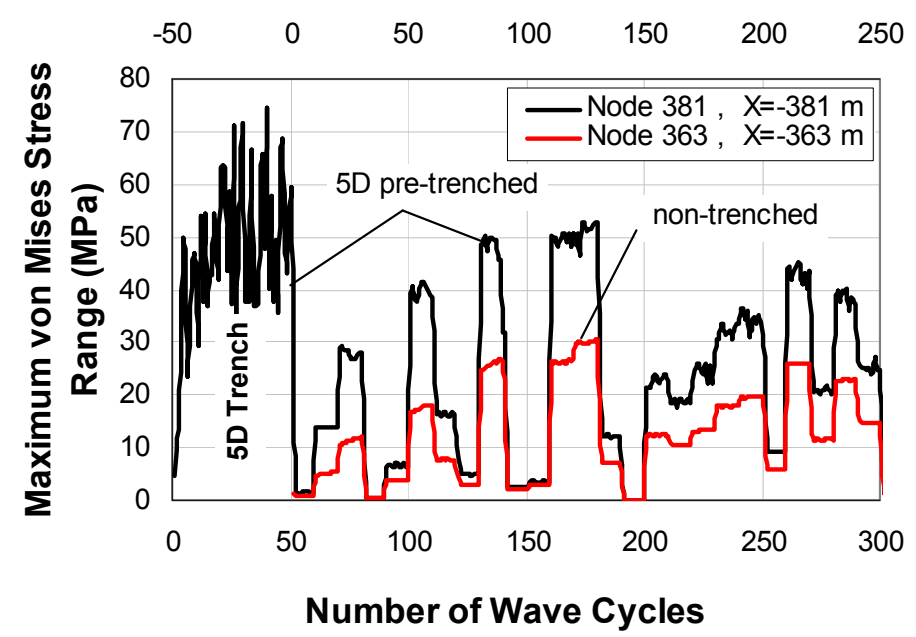

Figure 7-27: Von Mises stress range level of peak fatigue points for 5.0D trenched and non-trench seabed

A comparison of the results obtained using random wave orders No.1 and 3is presented in Figure 7-28. Similar to the example shown in Chapter 6 for small pre-trenches, creation of deep trenches prior to the fatigue analysis has removed any appreciable differences in the fatigue damage distribution for the two different wave orders. Only the fatigue damage profiles for the untrenched condition (blue plots) show slight differences arising from the different orders of the applied waves. The independence of the fatigue damage on the wave order for the remaining analyses confirms the validity of the proposed procedure for calculation of the fatigue damage for a non-linear plastic seabed. In other words, as described in Chapter 6, creation of initial trench using the most severe sea state makes the final fatigue damage independent of the order of the waves on the one hand, and the number of applied wave cycles on the other hand, which is the basis of the proposed procedure for fatigue damage calculations. 


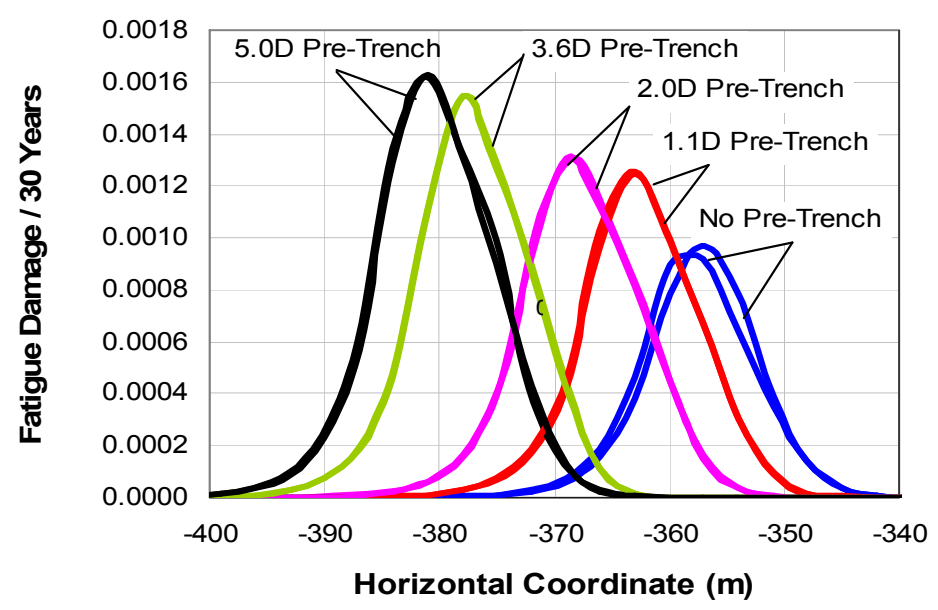

Figure 7-28: Fatigue damage distribution in trench and non-trenched seabeds random order No.1

and 3

Figure 7-29 to Figure 7-30 show the contact stress envelope, cyclic contact stress distribution in the TDZ for random wave order No.1 with various initial trenches of moderate to deep depth. Although the plots shows relatively irregular variations because of the changes in the contact surface, trench profile and suction force, the overall trends are quite interesting, particularly in combination with Figure 7-22, where the SCR profiles are presented. The same areas No.1, No.2 and No.3 discussed in Chapter 5 have been shown in Figure 7-29 for better assessment. No.1 is the area beyond the trench bottom point towards the vessel including the touchdown point; No.2 is the part of the area between the trench bottom point and the surface point adjacent to the bottom point; and No.3 is the area beyond No.2 towards the anchor end, including the surface point. Figure 7-29 shows that increasing the trench depth, leads to an increase in the maximum and minimum contact stress in areas No.1 and No.3, but with a more confused picture with no clear trends in area No.2. This plot shows that the maximum contact stress variation occurred in area No.1, beyond the bottom point towards the vessel. 


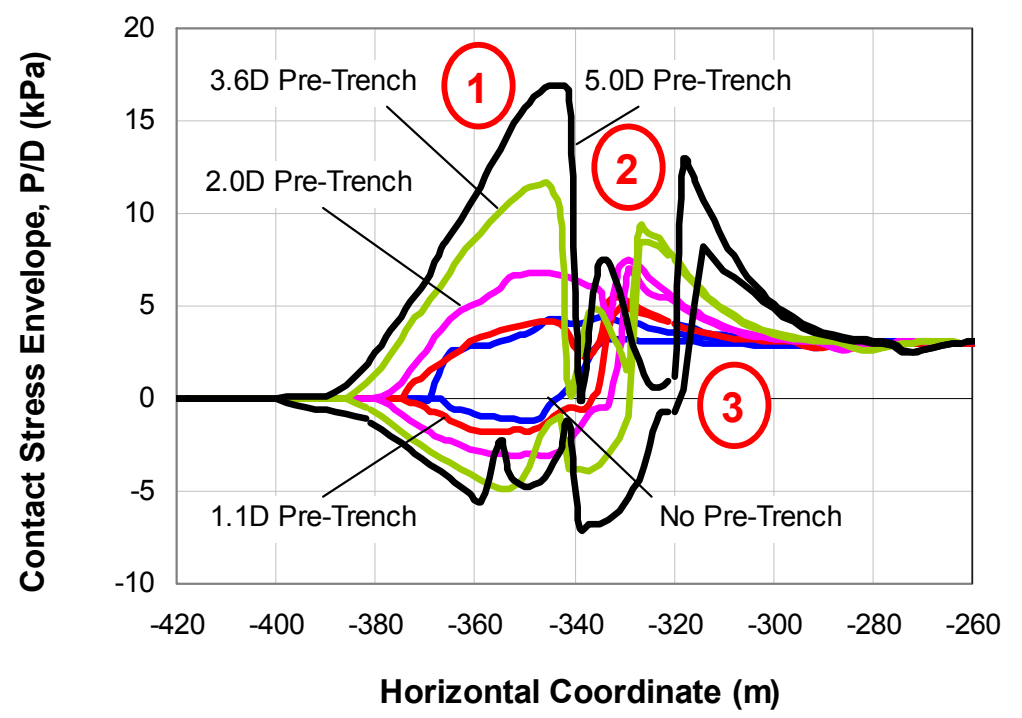

Figure 7-29: Contact stress envelope with various initial trenches for random wave order No.1

The cyclic contact stress shown in Figure 7-30 gives a better presentation of the variation of peak values, which mainly occur in area No.1.

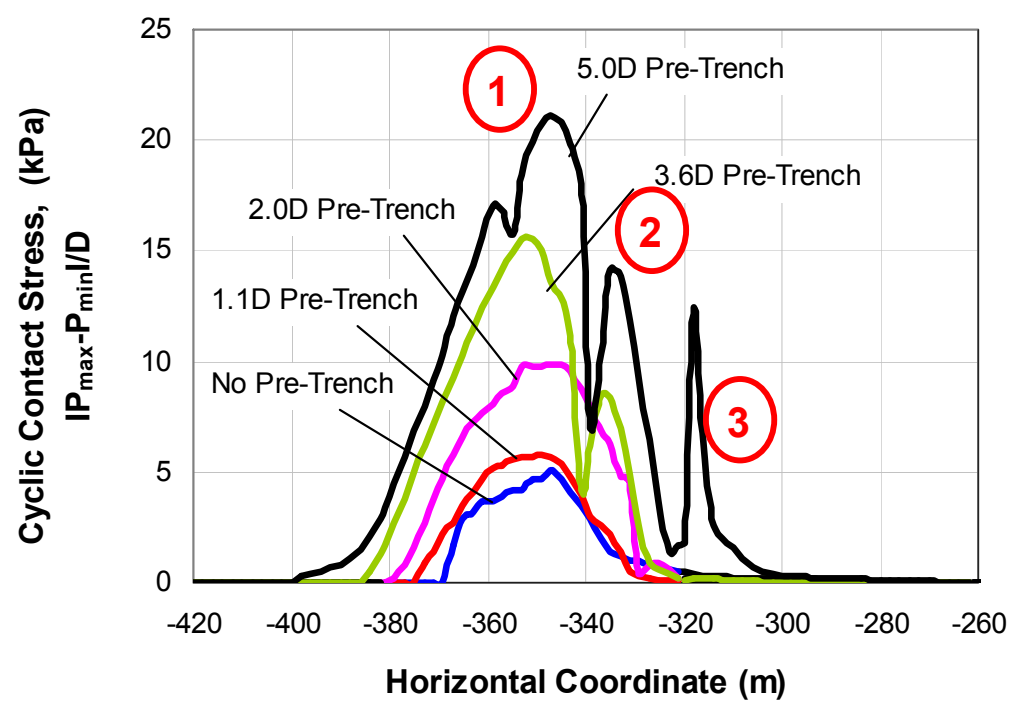

Figure 7-30: Cyclic contact stress with various initial trenches for random wave order No.1

The 'static' shear force variation for the vessel at its mean (calm sea) position is shown in Figure 7-31 following the creation of the different trenches. The average shear force over the whole of the analysis has also been plotted in Figure 7-32. The results show that the peak values of shear force for both of the plots moves towards the anchor end for deeper trenches, whilst the peak fatigue damage shifts towards the vessel end (Figure 7-26). This shows that the peak fatigue damage is no longer controlled by the peak shear force, as was true for untrenched conditions. The analyses confirm that all 
the action (i.e. movement of the SCR, changes in bending moment) occurs at the leading edge of the trench, so the maximum shear force (which occurs towards the trailing edge) is no longer an indicator of the maximum fatigue damage for trenched conditions in a non-linear seabed. The perturbations are quite complex, but it appears that fatigue is driven by the response in the vicinity of the trench edge nearest the vessel.

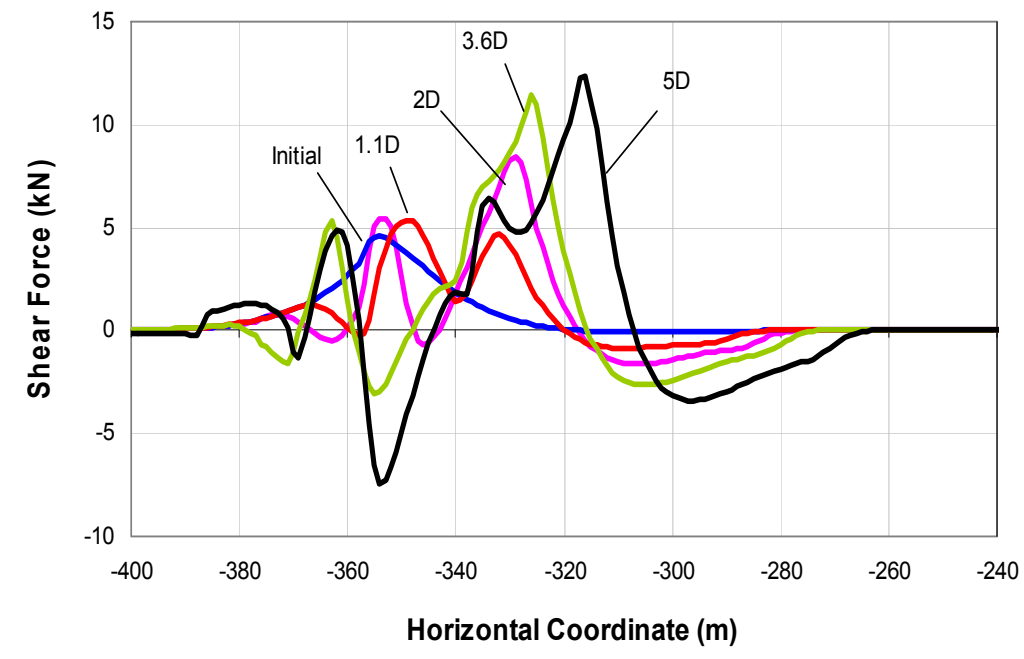

Figure 7-31: Post trenching shear force at mean vessel location with various trench depths

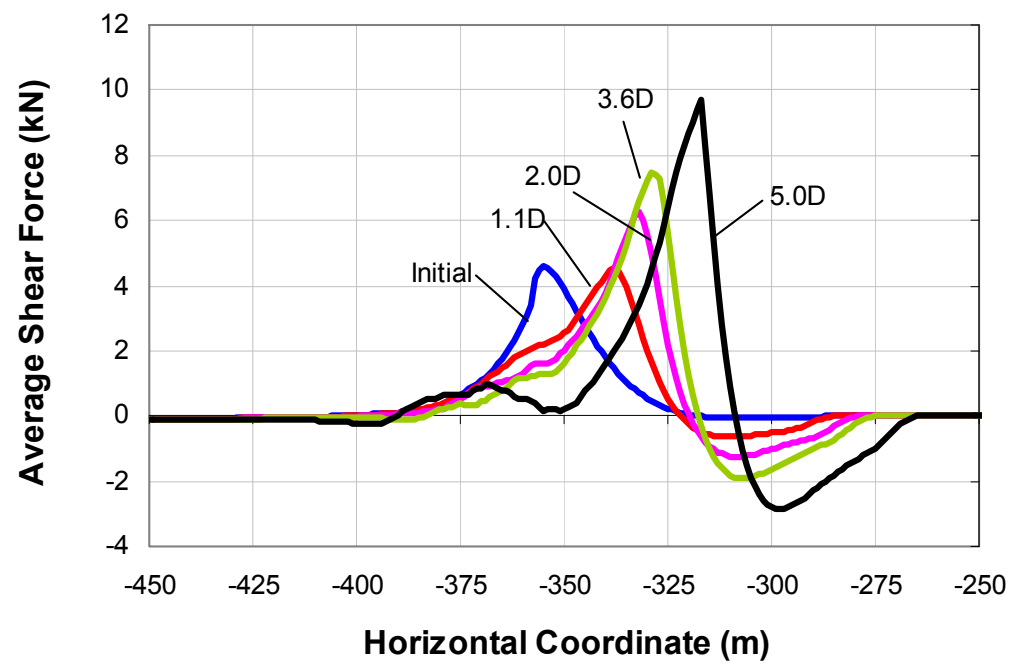

Figure 7-32: Average shear force with various initial trenches for random wave order No.1

To further explore the mechanism of fatigue damage development in the presence of a deep trench, and its relation with shear force and bending moment variations, an analysis has been performed applying 14 cycles of a single sea state (sea state No.30) with a $2 \mathrm{D}$ depth initial trench. Then the snapshots of shear force, bending moment and riser profile in the seabed in the far and near offset have been compared with the fatigue 
damage distribution. The number of applicable wave cycles for sea state No.30 has been as artificially increased to 10000 to have a more realistic level of fatigue damage. The bending moment distribution in Figure 7-33 shows that, as expected, the peak fatigue damage occurs exactly at the section with maximum variation of bending moment over the load cycles.

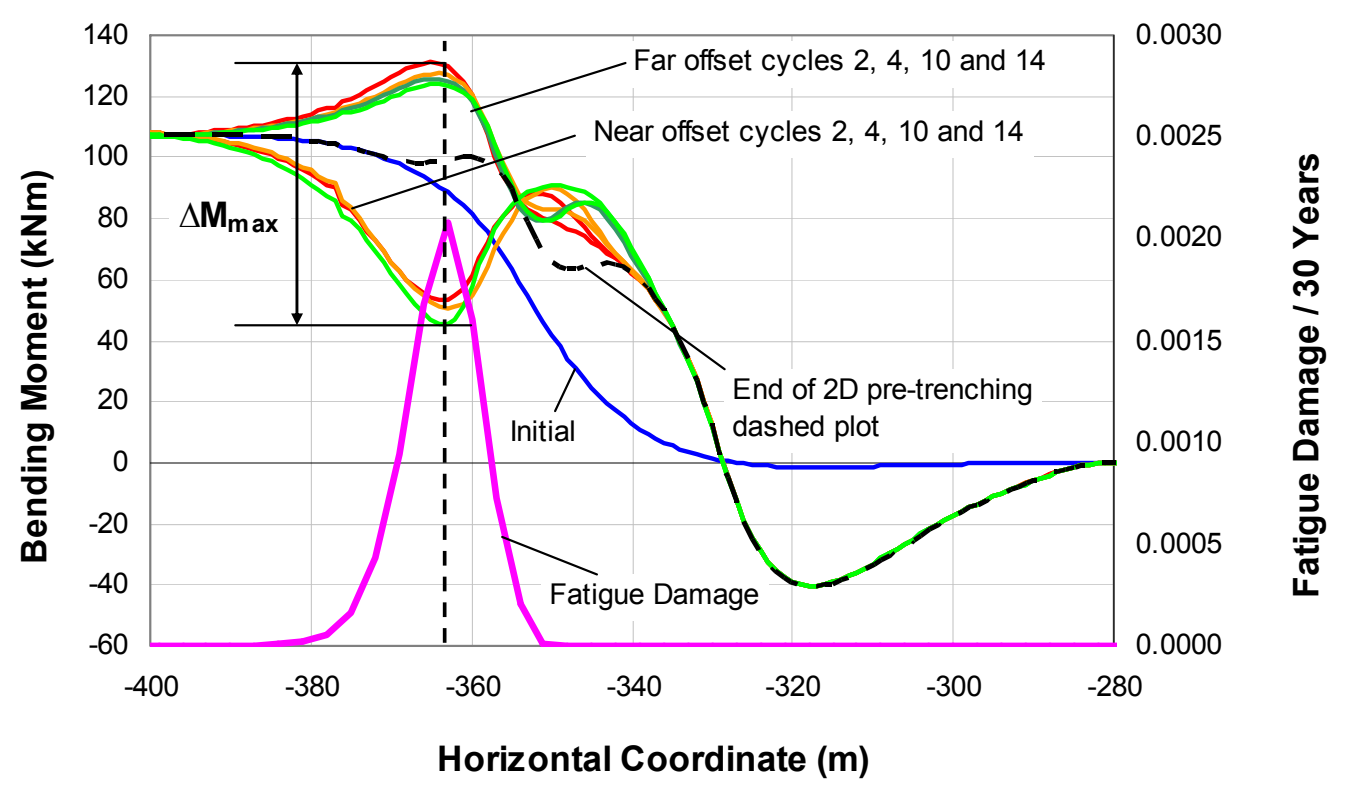

Figure 7-33: Snapshots of bending moment in vessel far and near offset in 2D trench

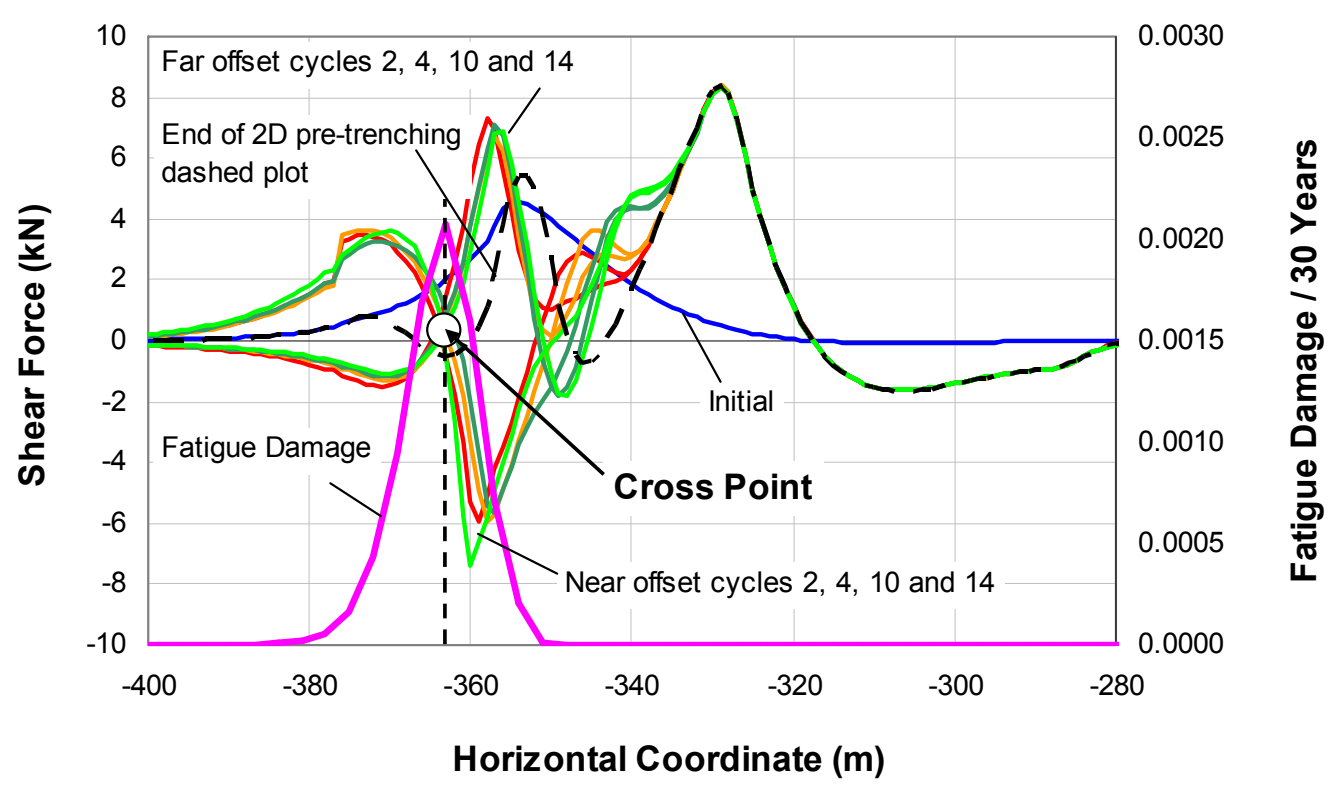

Figure 7-34: Snapshots of shear force in vessel far and near offset in 2D trench 
Figure 7-34 shows that the location of peak fatigue damage is in the section at which the shear force profiles for the far and near offset cross each other, with gradients of opposite sign but with the same value of shear force. This is quite reasonable and can be simply explained by considering that the fatigue damage is obtained from the von Mises stress range over the load cycles, which in turn is derived from the bending moment variation which can be written as:

$$
\Delta \mathrm{M}=\mathrm{M}_{\text {far }}-\mathrm{M}_{\text {near }}
$$

To find the maximum value of $\Delta \mathrm{M}$, the derivative of equation (7-13) should be equal to zero, that is:

$\frac{\mathrm{d}\left(\mathrm{M}_{\mathrm{far}}-\mathrm{M}_{\text {near }}\right)}{\mathrm{dx}}=0$

Hence

$\mathrm{dM}_{\text {far }} / \mathrm{dx}=\mathrm{dM}_{\text {near }} / \mathrm{dx}$

and thus

$\mathrm{V}_{\text {far }}=\mathrm{V}_{\text {near }}$

Equation (7-16) corresponds directly to the cross-over point illustrated in Figure 7-34

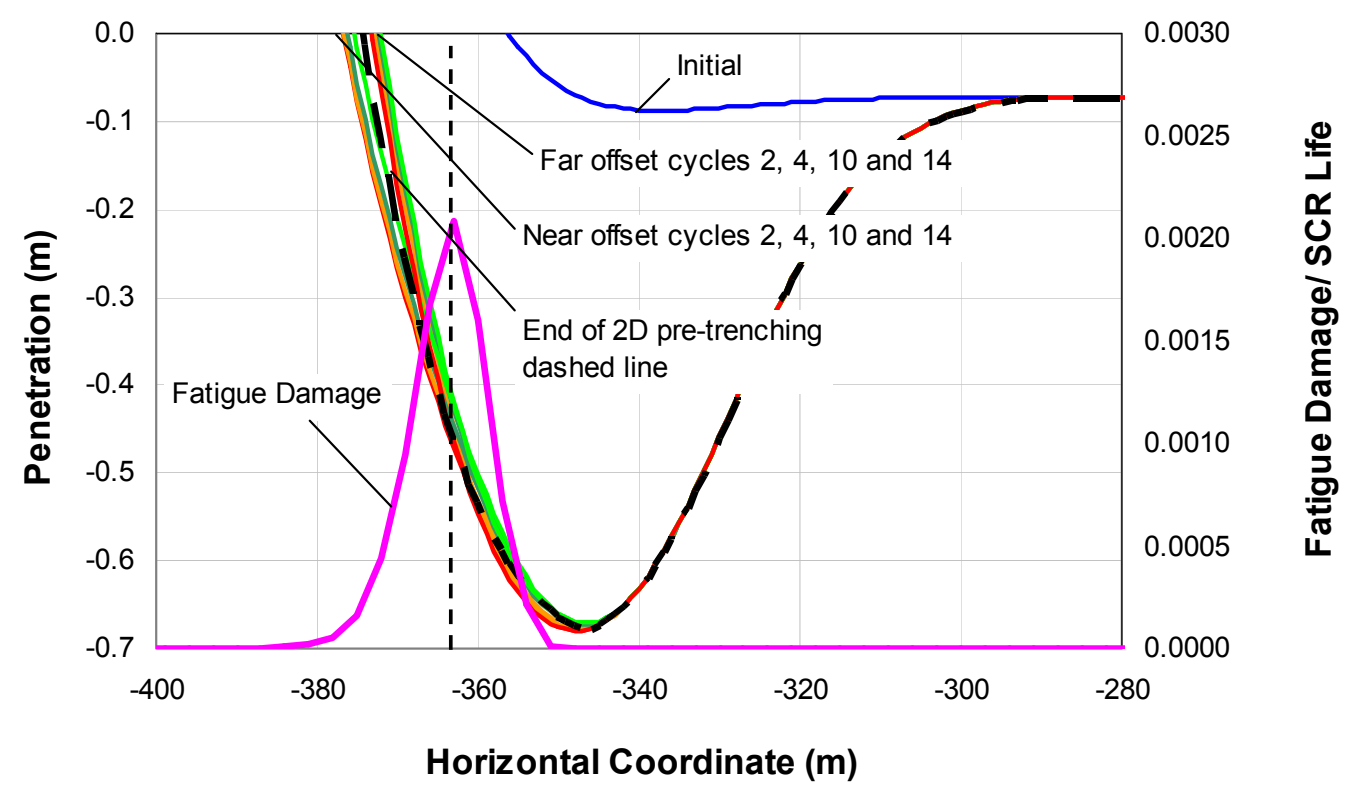

Figure 7-35: Snapshots of riser profile in the seabed in vessel far and near offset in $2 D$ trench 
The relative position of the peak fatigue damage to the riser profile in the seabed is plotted in Figure 7-35 for near and far offsets in the 14 cycles applied, which is in a point between the trench bottom point and touchdown point.

\subsection{SUMMARY AND CONCLUSIONS}

Results from ROV surveys of seabed trenches (Bridge and Howells 2007) giving information about the longitudinal profiles have been reviewed and discussed in relation to analytical trench models. A linear and quadratic exponential expression for the seabed trench has been introduced and compared with numerical simulations. Relatively good agreement was obtained between the quadratic exponential formula and the FEA results.

A series of fatigue analyses were performed in a linear elastic seabed after initial insertion of various artificial trenches through the TDZ. These analyses showed inconsistent results in respect of the magnitude and position of the maximum fatigue damage, depending on minor changes in the geometrical features of the inserted trenches. This appears due to the difficulty in obtaining a trench shape that is compatible with the SCR profile, and is reflected in the literature through papers suggesting contradictory beneficial or adverse effects of a trench. It was shown that inconsistency of the artificially inserted trench shape with the natural curvature of the SCR in the TDZ will give hot spots of contact pressure, distorting estimates of fatigue damage. It was concluded that it would be preferable to create trench profiles in the seabed using an approach consistent with the SCR shape, rather than simply adopting a mathematical expression for the seabed profile. Creating the trench profile by means of the SCR, in conjunction with a suitable non-linear model for the seabed should provide a more reliable way to assess the impact of the trench development on SCR fatigue performance in the TDZ.

The capabilities of the hysteretic non-linear seabed model proposed by (Randolph and Quiggin 2009) were used, adopting extreme values of the repenetration delay parameter, $\lambda_{\text {rep}}$, to create initial trenches in a manner consistent with the SCR shape. A severe sea state comparable with the most severe applicable waves for the design conditions was applied, choosing an appropriate number of wave cycles to generate initial trenches from moderate to deep maximum depths; then the normal fatigue analysis was continued through the full package of sea states with various random orders of the waves, after first resetting the repenetration delay parameter to a more typical value. The results showed that the fatigue damage considerably increased, and the most critical point moved towards the vessel, as the depth of the initial trench was increased.

Maximum fatigue damage is produced by creation of the 'design' trench at the start of the analysis, after which the fatigue calculation procedure introduced in Chapter 6 was successfully applied. It was demonstrated again that the final fatigue damage was independent of the order of the applied waves and the number of applied wave cycles, 
provided the trench was created initially using the consistent method. This in turn means that Miner's rule for superposition of the damage from individual sea states could be applied in a straightforward manner, following the calculation procedure introduced in Chapter 6. Finally, the contact stress variation along with shear force and bending moment distributions were reviewed, demonstrating that the peak fatigue damage is no longer controlled by the maximum shear force once a trench is created initially, because the maximum shear force occurs well to the anchor side of the maximum trench depth, in a region where wave-induced cyclic variations are small. Instead, fatigue damage is driven by the response at the trench edge nearest the vessel, in the one where the contact stress and shear force profiles are extremely complex. 


\section{CHAPTER 8. SUMMARY AND CONCLUSIONS}

\subsection{INTRODUCTION}

The influence of the riser-seabed interaction on fatigue performance of steel catenary risers in the touchdown zone is now widely accepted. Due to uncertainties associated with the complex nature of riser-seabed interaction, existing design codes and recommendations, and most commercial software, consider only a linear elastic flat seabed, which is a gross simplification from a geotechnical point of view. More sophisticated non-linear hysteretic seabed models have recently been introduced, simulating gradual embedment of the riser into the seabed and trench development beneath the riser under cyclic perturbations of the floating vessel supporting the SCR. Trench formation of several diameters deep beneath the riser, in the early stages of the SCR life, has been observed from ROV surveys.

This dissertation has focused on the effects of non-linear seabed response and trench development on the fatigue performance of SCRs in the touchdown zone, which is considered to be one of the most critical questions in SCR design. The study has been carried out using a hysteretic non-linear seabed model in time domain quasi-static 2D planar analyses. Design wave spectra relevant for Gulf of Mexico conditions have been simulated using generic response amplitude operators (RAOs) appropriate for a Spar vessel. The seabed model automatically captures the variation of seabed stiffness through the touchdown zone that arises from variations in the cyclic displacements. The effect of gradual embedment of the riser to a moderate depth (less than 1 diameter) in the seabed, and also the creation of deep trenches of up to 5 diameters, have been studied in terms of the stress distributions along the riser in the touchdown zone and consequently the fatigue performance in the touchdown zone.

A number of aspects associated with the main research focus were investigated during the study, including: Catenary equations in combination with a boundary layer method for modelling the seabed, shear force, bending moment and contact stress distribution through the TDZ; numerical simulation techniques of SCRs; SCR fatigue performance for linear elastic seabeds with various stiffness; fundamentals of the non-linear hysteretic seabed model; strategies for superposition of the fatigue damage from individual wave packages for a non-linear seabed response; SCR fatigue performance for the non-linear seabed model; and the influence on SCR fatigue performance of different degrees of trench development. 


\subsection{LINEAR ELASTIC SEABED AND SCR FATIGUE PERFORMANCE}

The applicability and accuracy of traditional catenary equations for design of SCRs was examined initially, starting from the idealisation of a rigid seabed with zero curvature of the SCR beyond the touchdown point. Significant discontinuity of the bending moment and shear force distribution at the touchdown point showed the inappropriateness of this method within the vicinity of the TDP, even for initial riser calculations.

In the next step, the seabed was modelled by various simple methodologies including tensionless and tensioned beams on a Winkler elastic foundation (Hetenyi 1946) and boundary layer methods (Pesce et al. 1998a). These seabed models were combined with the catenary equations to evaluate their consistency in prediction of stress distributions in the TDZ, with the accuracy of the different models examined through comparisons with finite element numerical simulations performed using ABAQUS. The results showed that using the theory of a beam on a Winkler elastic foundation, still does not remove critical discontinuities at the TDP. However, the approach may be considered for initial sizing of the riser and the overall static configuration of the SCR. The approach also does not give an accurate prediction of the peak shear force at the TDP, which is an important disadvantage. Inaccuracy in the shear force distribution, which is the gradient of bending moment, results in incorrect prediction of the von Mises stress variations at the TDP during cyclic perturbation of the SCR, which in turn is the basis for calculation of fatigue damage. Thus it was concluded that the beam on elastic foundation solutions, in conjunction with the traditional catenary equations, cannot be used for fatigue damage prediction in the TDZ.

Further investigation was carried out by modelling the seabed using the boundary layer method of (Pesce et al. 1998a). This seabed model was combined with the catenary equations and its performance examined through comparison with FEA results. It was found that the model overcomes the discontinuity of the shear force and bending moment distribution at the TDP, with accurate prediction of the peak values with respect to the FEA results. This shows that the boundary layer method in combination with catenary equations can provide a fundamental basis for fatigue damage prediction in the TDZ. Therefore, further investigation was carried out to find a simple way to predict the peak fatigue damage in the TDZ. This was achieved by estimating the von Mises stress range from bending moment changes expressed as the product of the peak shear force obtained from boundary layer method and the TDP relocation found from the catenary equations. The peak fatigue damage obtained using this calculation method was compared with FEA results for various seabed stiffness, showing relatively close agreement, particularly for the range of seabed stiffness normally relevant for deep water where SCR technology is used. This valuable finding provides a simple and quick design approach, yielding an initial relatively accurate evaluation of SCR fatigue life for a linear elastic seabed. Caution is needed, however, since it was found that this simple 
method does not apply for non-linear seabed response where a deep trench has been created, and for which the peak shear force occurs on the pipeline side of the trench in a zone with minimal wave-induced cyclic bending moments. For these conditions, the fatigue performance is mostly driven by the riser perturbations on the vessel side of the trench, in zone where the shear force profile is quite complex.

\subsection{NON-LINEAR HYSTERETIC RISER-SEABED INTERACTION}

The principles of non-linear seabed interaction models were reviewed and a versatile hysteretic non-linear model recently developed by Randolph and Quiggin (2009) was discussed in detail. The non-linear seabed model was coded in FORTRAN through the UEL subroutine of ABAQUS and integrated into a finite element simulation of an SCR system supported by a Spar vessel. The DISP subroutine of ABAQUS was coded to excite the vessel cyclically as per the design wave scatter diagram, based on conditions in the Gulf of Mexico, and generic Spar response amplitude operators (RAOs).

The influence of the non-linear seabed interaction model on stress distribution along the SCR, particularly in the TDZ, was studied for static conditions and the results compared those for a linear elastic seabed. Displacement-controlled quasi-static analyses were performed simulating cyclic excitation of the vessel under environmental loads. Progressive trench development beneath the riser, and the load-displacement response of individual nodes in the TDZ, were captured at various stages of displacement cycles and the model response was examined against key input parameters for the model.

The capability of the non-linear model in developing deep trenches, of the scale observed from ROV surveys, was explored using extreme values of the model's repenetration delay parameter $\lambda_{\text {rep }}$. This capability of the model is considered an excellent tool for initial creation of trenches of various depths, providing a consistent shape that matches the natural SCR catenary profile. This approach was subsequently used to assess the influence of the trenches of different depths on the SCR fatigue performance, as discussed in the next section.

\subsection{SCR FATIGUE IN NON-LINEAR HYSTERETIC SEABED}

Implementation of the non-linear hysteretic seabed model renders the final fatigue damage dependent on the number of wave cycles applied for a given sea state, and also the hierarchy of the applied sea states. This leads to a lack of uniqueness when applying Miner's rule for superposition of fatigue damage from the individual sea states.

To resolve this, a number of numerical simulations were performed with different numbers of applied wave cycles and different order of the waves, observing the effects on the calculated von Mises stress ranges since these are the main input into the fatigue damage calculations. The results of the study led to the development of a robust fatigue 
calculation methodology for non-linear seabed response. The main observations were as follows:

1) The von Mises stress range induced by a particular sea state immediately stabilises to a constant value if the system has already experienced sufficient cycles of a more severe sea state. This means that only a few cycles of that wave need be applied in order to evaluate a representative stress range for the given sea state since further cycles will not change the stress range (just as for a linear elastic seabed).

2) If the system experiences sufficient cycles of the most severe sea at the beginning of an analysis, the von Mises stress ranges obtained from the rest of the sea states will immediately stabilise to constant values. The fatigue damage will therefore be independent of the hierarchy of the sea states and Miner's rule can be applied in a straightforward manner, in spite of the non-linear seabed response.

The results of the analyses showed that the overall level of von Mises stress range is increased by gradual development of a trench beneath the riser; the deeper trench the higher von Mises stress range level and consequently the greater the fatigue damage.

Taking these points into consideration, a consistent procedure for calculating the maximum fatigue damage in the presence of non-linear hysteretic seabed response comprises the following steps:

1. Insert an initial trench in the TDZ of the required design depth by applying appropriate cycles of the most severe sea state from the full design wave spectrum.

2. Excite the vessel with a few cycles of each sea state from the full design wave spectrum; the order of sea states is no longer relevant.

3. Superpose linearly the fatigue damage from individual sea states using Miner's rule in order to obtain the total fatigue damage distribution in the TDZ.

The first step of the above procedure was carried out using the capability of the hysteretic non-linear seabed model in developing deep trenches by assigning extreme values of the repenetration offset parameter $\lambda_{\text {rep }}$, as referred to in the previous section. Initially, the seabed model parameters are set to allow trenches of the design depth to be developed over a moderate number of displacement cycles of the SCR. The design wave spectrum is then applied, after switching the model parameters to more realistic values.

Fatigue analyses were performed for a range of initial trench depths. This study showed that the fatigue damage is significantly affected by the presence of the trench beneath the riser, with the maximum damage increasing with increasing depth of the initially created trench. It was also observed that the location of the peak fatigue damage gradually moved towards the vessel side of the trench. The contact stress variation along 
with shear force and bending moment distributions were investigated in the presence of deep trenches and it was noticed that the peak shear force increased for deeper trenches but moved gradually in the reverse direction, towards the anchor (or pipeline) end of the SCR. The peak shear force at the mean (calm water) position of the vessel can therefore no longer be considered as an index for peak fatigue damage, since it occurs in a region where cyclic perturbations of the riser (and changes in bending moment) are small. Instead, the fatigue is driven by conditions towards the leading (vessel) side of the trench, where the shear force distributions become very complex for deep trenches.

It was also shown through a series of fatigue analyses that the influence of a trench on the fatigue performance of SCRs is sensitive to the methodology by which the artificial trench is introduced, and hence can lead to contradictory conclusions as found in the literature. It was argued that this emanates from inconsistency between the mathematical trench profile and the natural catenary shape of the riser, leading to hotspots of contact pressure that distorts the stress distribution along the riser. This was demonstrated by introducing a quadratic exponential formula for the trench profile, which provided a good match to be obtained with profiles reported in the literature, and also with numerically created trench profiles. However, when the analytical trench profile was artificially inserted in a linear elastic seabed, inconsistent results were obtained from minor changes in the trench geometry. It is considered that the only reliable approach to studying the influence of trench development beneath the riser on fatigue performance seems to be to use a consistent method such as described above, with the trench developed using the natural catenary shape of the SCR.

\subsection{RECOMMENDATION FOR FUTURE WORK}

The thesis has deliberately simplified analysis of SCR fatigue in the touchdown zone by restricting the analysis to quasi-static, 2D planar conditions. This allowed the influence of the seabed model to be investigated in detail, but also means that the more realist analyses are required before the results can be generalised. Further investigations in the following areas are therefore needed:

- Assessing the impact of the dynamic riser response for the non-linear hysteretic seabed model. Incorporating the inertial response of the riser and drag forces with respect to the water will affect the TDP fluctuations and trench development and consequently the fatigue performance.

- Performing three dimensional analysis accounting for transverse riser-seabed interaction and its impact on fatigue performance in the touchdown zone.

- Examining the findings of the thesis against results from commercial packages. At present, the the particular non-linear seabed model is only available in Orcaflex, although non-linear seabed models have been developed for other software. It is recommended that a series of fatigue analyses are undertaken 
using Orcaflex allowing comparisons of the von Mises stress ranges and fatigue performance with results from ABAQUS.

- Further targeted ROV surveys to provide better data on trench development with more accurate information in respect of the trench longitudinal geometry.

- Further development of the non-linear hysteretic seabed model implementing rate effects with velocity dependent resistance, particularly in respect of uplift. Enhancement of the seabed model can give more realistic evaluation of the seabed response, although additional complexity of the model may make fatigue analysis more complicated and with higher computational costs.

- Further investigations to develop simple recommendations for industry allowing use of existing commercial design packages in more effective ways, with improved guidance on choice of linear elastic springs with varying stiffness along the SCR in the touchdown zone.

- Performing time domain analyses with random waves for a window of a few hours as representative of the lifetime history, comparing the results with the deterministic analyses performed during the current research. This can further verify the robustness of the procedure proposed for fatigue damage calculations using a non-linear seabed model. 


\section{REFERENCES}

Albert, W. A. J. (1838). "'Über Treibseile am Harz"." Mineralogie Geognosie Bergbau und Hüttenkunde, 10, 215-34.

Andersen, K. H. (1976). "Behaviour of subjected to undrained cyclic loading." Publikasjon - Norges Geotekniske Institutt(114), 33-44.

API-RP-2A. (2002). "Recommended Practice for Planning, Designing and Constructing Fixed Offshore Platforms." American Petroleum Institute, Washington, DC, USA.

API-RP-2A WSD. (2000). "Recommended Practice for Planning, Designing and Constructing Fixed Offshore Platforms-Working Stress Design." American Petroleum Institute, Washington, DC, USA.

API-RP-2RD. (1997). "Recommended practice for design of risers for floating production systems." American Petroleum Institute, Washington, DC, USA.

API-RP-2SK. (1996). "Recommended Practice for Design and Analysis of Stationkeeping Systems for Floating Structures." American Petroleum Institute, Washington DC, USA.

API-RP-16Q. (1993). "Recommended practice for design, selection, operation and maintenance of drilling riser system." American Petroleum Institute, Washington, DC, USA.

API-RP-17A. (1994). "Design and operation of subsea production systems." American Petroleum Institute, Washington, DC, USA.

API-RP-17B. (2001). "Recommended practice for flexible pipes." American Petroleum Institute, Washington, DC, USA.

API-RP-17C. (1999). "Recommended practice for TFL (Through Flowline) systems." American Petroleum Institute, Washington, DC, USA.

Aranha, J., Martins, C., and Pesce, C. (1997) "Analytical Approximation for the Dynamic Bending Moment at the Touchdown Point of a Catenary Riser." Int. Journal of Offshore and Polar Engineering, 293-300.

Aubeny, C., and Biscontin, G. (2008). "Interaction Model for Steel Compliant Riser on Soft Seabed." SPE Projects, Facilities \& Construction, 3(3), 1-6.

Aubeny, C., Gaudin, C., and Randolph, M. F. (2008), "Cyclic tests of model pipe in kaolin." Offshore Technology Conf., Houston, USA OTC19494.

Aubeny, C. P., and Biscontin, G. (2006). "Seafloor Interaction with Steel Catenary Risers." No.9/06A17,, Offshore Technology Research Center, Texas A\&M University, Texas, USA.

Aubeny, C. P. and Biscontin, G. (2009). "Seafloor-Riser Interaction Model." International Journal of Geomechanics, 9 (3), 133-141.

Aubeny, C. P., and Shi, H. (2006). "Interpretation of impact penetration measurements in soft clays." Journal of Geotechnical and Geoenvironmental Engineering, 132, 770.

Aubeny, C. P., Shi, H., and Murff, J. D. (2005). "Collapse loads for a cylinder embedded in trench in cohesive soil." International Journal of Geomechanics, ASCE, 5(4),320-325.

Audibert, J. M. E., Nyman, D. J., and O'Rourke, T. D. (1984). "Differential ground movement effects on buried pipelines." Guidelines for the Seismic Design of Oil and Gas Pipeline Systems, ASCE publication, 150-177. 
Bai, Y. (2001). Pipelines and risers, Elsevier Science.

Barltrop, N., and Adams, A. (1991). Dynamics of Fixed Marine Structures, Atkins Oil \& Gas Engineering Limited, Epsom, UK.

Borel, D., Puech, A., and de Ruijter, M. (2002) "High quality sampling for deep water geotechnical engineering: the STACOR experience." Proc. Conf. on Ultra Deep Engineering and Technology (UDET), Brest, France.

Bostrom, B., Svano, G., Eiksund, G., Leira, B. J., and Bjorset, A. (1998) "Response effects of riser-soil interaction modelling." Proceedings of the International Conference on Offshore Mechanics and Arctic Engineering - OMAE1998, Lisbon, Portugal, 8.

Bridge, C. (2005). "Effects of Seabed Interaction on Steel Catenary Riser," PhD Thesis, University of Surrey.

Bridge, C., and Howells, H. (2007) "Observations and modeling of steel catenary riser trenches." The Seventeenth International Offshore and Polar Engineering Conference, ISOPE2007, Lisbon, Portugal, 803-813.

Bridge, C., Laver, K., Clukey, E., and Evans, T. (2004), "Steel Catenary Riser Touchdown Point Vertical Interaction Models." Offshore Technology Conference, Houston, Texas, USA, OTC16628.

Bridge, C., and Willis, N. (2002), "Steel Catenary Risers-Results and Conclusions from Large Scale Simulations of Seabed Interaction"." 14 th Annual Conference Deep Offshore Technology, Cape Town, South Africa, 40-60.

Bruton, D., Carr, M., and White, D. J. (2007), "The influence of pipe-soil interaction on lateral buckling and walking of pipelines: the SAFEBUCK JIP." Proceedings of the 6th International Conference on Offshore Site Investigation and Geotechnics, London, UK, 133-150.

Bruton, D., White, D., Cheuk, C., Bolton, M., and Carr, M. (2006), "Pipe/Soil Interaction Behavior During Lateral Buckling, Including Large-Amplitude Cyclic Displacement Tests by the SAFEBUCK JIP." Offshore Technology Conference, Houston, Texas, USA, OTC17944.

Bukowski, J. (2008). "Christiaan huygens and the Problem of the Hanging Chain." The College Mathematics Journal, 39(1), 2-11.

Campbell, M. (1999), "The Complexities of Fatigue Analysis for Deepwater Risers." Deepwater Pipeline Conference, New Orleans, USA.

Carter, B. A., and Ronalds, B. F. (1998). "Deepwater Riser Technology." SPE Asia Pacific Oil and Gas Conference and Exhibition, 1998 Copyright 1998, Society of Petroleum Engineers Inc., Perth, Australia.

Cathie, D. N., Jaeck, C., Ballard, J. C., and Wintgens, J. F. (2005), "Pipeline geotechnics-State of the Art." Proceedings of the international symposium on frontiers in offshore geotechnics, Perth, Australia, 95-114.

Champneys, A. R., Van der Heijden, G. H. M., and Thompson, J. M. T. (1997). "Spatially complex localization after one-twist-per-wave equilibria in twisted circular rods with initial curvature." Philosophical Transactions: Mathematical, Physical and Engineering Sciences, 2151-2174.

Chandwani, R., and Larsen, I. (1997) "Design of Flexible Risers." COPPE/UFRJ, Rio de Janeiro, Brazil.

Chaudhury, G. (2001), "Analysis and design of SCR for platforms in deep and ultra deep waters." Proc. of the Int. Conf. on Deep Offshore Technology, Rio de janeiro, Brazil. 
Cheuk, C. Y., and White, D. J. (2008), "Centrifuge modelling of pipe penetration due to dynamic lay effects." Proceedings of the 27th International Conference on Offshore Mechanics and Arctic Engineering, Estoril, Portugal, 15-20.

Clebsch, A. (1883). Theorie de l'Elasticit6 des Corps Solides. Translated by B. DE Sarntvenant and Flaman, Dunod, Paris.

Clukey, E., Ghosh, R., Mokarala, P., and Dixon, M. (2007), "Steel Catenary Riser (SCR) design issues at touchdown area." International Offshore and Polar Engineering Conference, Lisbon, Portugal, 814-819.

Clukey, E. C., Haustermans, L., and Dyvik, R. (2005), "Model tests to simulate risersoil interaction effects in touchdown point region." Int. Symp. on Frontiers in Offshore Geotechnics - ISFOG, Perth, Australia, 651-658.

Clukey, E. C., Young, A. G., Dobias, J. R., and Garmon, G. R. (2008), "Soil response and stiffness laboratory measurements of SCR pipe/soil interaction." Offshore Technology Conference, Houston, USA, OTC 19303.

Croll, J. (2000). "Bending boundary layers in tensioned cables and rods." Applied Ocean Research (22), 241-253.

Dingle, H. R. C., White, D. J., and Gaudin, C. (2008). "Mechanisms of pipe embedment and lateral breakout on soft clay." Canadian Geotechnical Journal, 45(5), 636652.

Dixon, D. A., and Rutledge, D. R. (1968). "Stiffened catenary calculations in pipeline laying problem." ASME J. Engng. Indust, 90, 153-160.

Dixon, M. (2009). "Modelling advances enhance riser fatigue prediction accuracy." Online Oil \& Gas Engineer Magazine-Expolaration Drilling, London, UK.

DNV-OS-C101. (2008). "Design of Offshore Steel Structure." Det Norske Veritas, Hovik, Norway.

DNV-RP-C203. (2008). "Fatigue Design of Offshore Steel Structures." Det Norske Veritas, Hovik, Norway.

DNV-RP-F105. (2006). "Recommended Practice for Free spanning Pipelines." Det Norske Veritas, Hovik, Norway.

DNV. (1996). "Rules for submarine pipeline systems." Det Norske Veritas, Hovik, Norway.

Dunlap, W., Bhojanala, R., and Morris, D. (1990), "Burial of vertically loaded offshore pipelines in weak sediments." Offshore Technology Conference, Houston, Texas, USA, OTC6375.

Finch, M., Fisher, R., Palmer, A., and Baumgard, A. (2000), "An Integrated Approach to Pipeline Burial in the 21 st Century." Deep Offshore Technology (DOT), New Orleans, LA, USA.

FloaTec. (2006). "Internal Note: An overview of deepwater floating facilities." Houston, USA.

Fontaine, E. "SCR-Soil Interaction: Effect on Fatigue Life and Trenching. (2006), " Proceedings of the Sixteenth (2006) International Offshore and Polar Engineering Conference, San Francisco, CA, USA, 2.

Fontaine, E., Nauroy, J. F., Foray, P., Roux, A., and Gueveneux, H. (2004), "Pipe - Soil interaction in soft kaolinite : Vertical stiffness and damping." Proceedings of the International Offshore and Polar Engineering Conference, Toulon, France, 517524.

Ghosh, R., Finn, L. D., and Halkyard, J. (2005). "Coupled analysis of a Spar structure: Monte Carlo and statistical linearization solutions." Journal of Offshore Mechanics and Arctic Engineering, 127, 11-17. 
Giertsen, E., Verley, R., and Schroder, K. (2004), "CARISIMA a catenary riser/soil interaction model for global riser analysis." Proceedings of the International Conference on Offshore Mechanics and Arctic Engineering, OMAE2004, Vancouver, BC, Canada, 633-640.

Grant, R., and Patterson, D. (1997), "Riser fairing for reduced drag and vortex supression." Offshore Technology Conference, Houston, Texas, USA, OTC2923.

Gray, S., and Venit, S. (2002). "National Curve Bank, Catenary, Chain." Beckman Foundation.

Guarracino, F., and Mallardo, V. (1999). "A refined analytical analysis of submerged pipelines in seabed laying." Applied Ocean Research, 21(6), 281-293.

Hahn, G. D., Shanks, J., and Masson, C. M. (2003), "An Assessment of Steel Catenary Riser Trenching." Deep Offshore Technology Conference(DOT) Marseille, France

Hardin, B. O., and Drnevich, V. P. (1972). "Shear Modulus and Damping in Soils: Measurement and Parameter Effects (Terzaghi Leture)." Journal of the soil mechanics and foundations division, 98(6), 603-624.

Hasselmann, K., Barnett, T. P., Bouws, E., Carlson, H., Cartwright, D. E., Enke, K., Ewing, J. A., Gienapp, H., Hasselmann, D. E., and Kruseman, P. (1973). "Measurements of wind-wave growth and swell decay during the Joint North Sea Wave Project (JONSWAP)." Dtsch. Hydrogr. Z. Suppl. A, 8(12), 289-300.

Hatton, S. A., and Willis, N. (1998), "Steel catenary risers for deepwater environments." Offshore Technology Conference, Houston, TX, USA, OTC8607.

Hetenyi, M. (1946). Beams on elastic foundation, Univ Michigan Press, Ann Arbor, Mich, United States.

Hodder, M., White, D., and Cassidy, M. (2009), "Effect of Remolding and Reconsolidation on the Touchdown Stiffness of a Steel Catenary Riser: Guidance from Centrifuge Modelling." Offshore Technology Conference, Houston, Texas, USA, OTC19871.

Hodder, M. S., White, D. J., and Cassidy, M. J. (2008), "Centrifuge Modelling of RiserSoil Stiffness Degradation in the Touchdown Zone of an Steel Catenary Riser." 27th International Conference on Offshore Mechanics and Arctic Engineering, $O M A E$, Estoril, Portugal, 57302.

Idriss, I. M., Dobry, R., and Singh, R. D. (1978). "Nonlinear behavior of soft clays during cyclic loading." Journal of the Geotechnical Engineering Division, 104(12), 1427-1447.

Jiao, G., and Moan, T. (1990). "Probabilistic analysis of fatigue due to Gaussian load processes." Probabilistic Engineering Mechanics, 5(2), 76-83.

John, F. (1985). "On the Motion of Floating Bodies. I." Fritz John: Collected Papers, 385.

Jones, W. (1976), "On-Bottom Pipe line Stability In Steady Water Currents." Offshore Technology Conference, Houston, Texas, USA, OTC2598.

Karayaka, M., and Xu, L. "Catenary Equations for Top Tension Riser Systems." Proceedings of the International Offshore and Polar Engineering Conference, Honolulu, HI, United States, 638-644.

Kevorkian, J., and Cole, J. D. (1981). "Perturbation Methods in Applied Mathematics." Applied Mathematical Science, 34, 558.

Kondner, R. L. (1963). "Hyperbolic stress-strain response; cohesive soils." ASCE -Proceedings -- Journal of the Soil Mechanics and Foundations Division, 89(SM1, Part 1), 115-143. 
Konuk, I. (1980). "Higher-order approximations in stress analysis of submarine pipelines." J. Energy Resour. Technol, 102(4), 190-196.

Langner, C. G. (2003), "Fatigue Life Improvement of Steel Catenary Risers due to SelfTrenching at the Touchdown Point." Offshore Technology Conference, Houston, Texas, USA, OTC15104.

Leibniz. (1691a). "Solutions to the Problem of the Catenary, or Funicular Curve, Proposed by M. Jacques Bernoulli in the Acta of June 1691." Acta Eruditorum, Leipzig, Germany.

Leira, B. J., Karunakaran, D., Giertsen, E., Passano, E., and Farnes, K.-A. (2004), "Analysis guidelines and application of a riser-soil interaction model including trench effects." Proceedings of the International Conference on Offshore Mechanics and Arctic Engineering, OMAE2004, Vancouver, BC, Canada, 955962.

Lenci, S., and Callegari, M. (2005). "Simple analytical models for the J-lay problem." Acta Mechanica, 178(1-2), 23-39.

Love, A. (1892). A Treatise on Mathematical Theory of Elasticity, Cambridge University Press.

Low, H. E., Randolph, M., Rutherford, C., Bernard, B., and Brooks, J. (2008), "Characterization of Near Seabed Surface Sediment." Offshore Technology Conference, Houston, Texas, OTC19149.

Lund, K. M. (2000), "Effect of Increase in Pipeline Penetration from Installation." 19th Conference on Offshore Mechanics \& Arctic Engineering, OMAE, New Orleans, USA.

Lunne, T., Tjelta, T., Walta, A., and Barwise, A. (2008), "Design and Testing Out of Deepwater Seabed Sampler." Offshore Technology Conference, Houston, Texas, USA, OTC19290.

Marinetalk. (2000). "Spar Alliance Dissolved " Marintalk, your gateway to the global marine industry, www.marinetalk.com

MCS. (1994). User Manual for FLEXCOM3D, a taylor-made finite element programe for non-linear time domian analysis of offshore structures, MCS Advanced Subsea Engineering, Galway, Ireland.

Mekha, B. B., Weggel, D. C., Johnson, C. P., and Roesset, J. M. (1996), "Effects of second order diffraction forces on the global response of spars." 273-280.

Merifield, R., White, D. J., and Randolph, M. F. (2008). "The ultimate undrained resistance of partially embedded pipelines." Geotechnique, 58(6), 461-470.

Merifield, R. S., White, D. J., and Randolph, M. F. (2009). "Effect of surface heave on response of partially embedded pipelines on clay." Journal of Geotechnical and Geoenvironmental Engineering, 135(6), 819-829.

Meyerhof, G. G. (1964). "Some recent research on the bearing capacity of foundations." Canadian Geotechnical Journal, 1(1), 16-26.

Miner, M. A. (1945). "Cumulative damage in fatigue." Journal of applied mechanics, 12(3), 159-164.

Morris, D., Webb, R., and Dunlap, W. (1998), "Self-burial of laterally loaded offshore pipelines in weak sediments." Offshore Technology Conference, Houston, Texas, USA, OTC5855.

Murff, J. D., Wagner, D. A., and Randolph, M. F. (1989). "Pipe penetration in cohesive soil." Geotechnique, 39(2), 213-229. 
Murray, J. (2007), "A comparison of top tension riser response on a tension leg platform, semi-submersible and a spar." Dry Tree \& Riser Forum, Houston, Tx, USA.

Murray, J., and Yang, C. (2009), "SS: Spar Technology: A Comparison of the Spar and Single Column Floater in an Arctic Environment." Offshore Technology Conference, Houston, Texas, USA, OTC19797.

Nakhaee, A., and Zhang, J. "Effects of the Interaction with the Seafloor on the Fatigue Life of a SCR." International Offshore and Polar Engineering Conference, Vancouver, BC, Canada, 87-93.

Newson, T. A., Bransby, M. F., Brunning, P., and Morrow, D. R. "Determination of undrained shear strength parameters for buried pipeline stability in deltaic soft clays." 14th International Offshore and Polar Engineering Conference, Toulouse, France, 38-43.

Nova, R. (1983). "Mathematical modelling of cyclic behaviour of soils." Soil Mechanics, , 47-59.

Orcina Ltd. (1986). "Orcaflex User Manual." Cumbria, UK.

Orcina Ltd. (2009). "Orcaflex User Manual, Version 9.2e." Cumbria, UK.

Palmer, A. (2000), "Catenary Riser Interaction with the Seabed at the Touchdown Point." Deepwater Pipeline \& Riser technology Conference, Houston , Texas, USA.

Palmer, A., Hutchinson, G., and Ells, J. (1974). "Configuration of Submarine Pipelines during Laying Operation." ASME J. Engng. Industry(96), 1112-1118.

Palmgren, A. G. (1924). "Die Lebensdaur von Kugellagern (Life Length of Roller Bearings. In German)." Zeitschrift des Vereines Deutscher Ingenieure (VDI Zeitschrift), 68(14), 339-341.

Pedersen, P. T. (1975). "Installation of cables and pipelines." International Shipbuilding Progress, 22, 399-408.

Pesce, C. P., Aranha, J. A. P., and Martins, C. A. (1998a), "The Soil Rigidity Effect in the Touchdown Boundary-Layer of a Catenary Riser: Static Problem." Eighth International Offshore and Polar Engineering Conference, Montreal, Canada, 207-213.

Pesce, C. P., Aranha, J. A. P., Martins, C. A., Ricardo, O. G. S., and Silva, S. (1998b). "Dynamic curvature in catenary risers at the touchdown point region: An experimental study and the analytical boundary-layer solution." International Journal of Offshore and Polar Engineering, 8(4), 303-310.

Pesce, C. P., Aranha, J. A. P., Martins, G. A., Ricardo, O. G. S., and Silva, S. (1997), "Dynamic curvature in catenary risers at the touchdown point: An experimental study and the analytical boundary-layer solution." ISOPE, Honolulu, HI, USA, 656-665.

Phifer, E. H., Frans, K., Swanson, R. C., Allen, D. W., and Langner, C. G. (1994), "Design And Installation Of Auger Steel Catenary Risers." Offshore Technology Conference, Houston, Texas, USA, OTC7620.

Pierson Jr, W. J., and Moskowitz, L. (1964). "A proposed spectral form for fully developed wind seas based on the similarity theory of SA Kitaigorodskii." Journal of Geophysical Research, 69(24), 5181-5190.

Plunkett, R. (1967). "Static bending stresses in catenaries and drill strings." Journal of Engineering for Industry, Transactions of the ASME, 89(1), 31-36. 
Prévost, J. H. (1977). "Mathematical modelling of monotonic and cyclic undrained clay behaviour." International Journal for Numerical and Analytical Methods in Geomechanics, 1(2).

Quintin, H., Legras, J. L., Huang, K., and Wu, M. (2007), "Steel Catenary Riser Challenges and Solutions for Deepwater Applications." Offshore Technology Conference, Houston, Texas, USA, OTC19118.

Randolph, M. F., Low, H. E., and Zhou, H. F. (2007), "In situ testing for design of pipeline and anchoring systems." Proceeding of 6th International Conference on Offshore Site Investigation and Geotechnics, Confronting New Challenges and Sharing Knowledge, London, UK, 251-262.

Randolph, M. F., and Quiggin, P. (2009), "Non-linear hysteretic seabed model for catenary pipeline contact" 28th International Conference on Ocean, Offshore and Arctic Engineering OMAE 2009, Honolulu, Hawaii, USA, 79259.

Randolph, M. F., Wang, D., Zhou, H., Hossain, M. S., and Hu, Y. (2008), "Large Deformation Finite Element Analysis for Offshore Applications." The 12th International Conference of International Association for Computer Methods and Advances in Geomechanics (IACMAG), Goa, India, 3307-3318.

Randolph, M. F., and White, D. J. (2008a). "Upper bound yield envelopes for pipelines at shallow embedment in clay." Géotechnique, 58(4), 297-301.

Randolph, M. F., and White, D. J. (2008b) "Pipeline embedment in deep water: processes and quantitative assessment." Offshore Technology Conf. , Houston, USA, OTC 19128.

Rho, J. B., Choi, H. S., Lee, W. C., Shin, H. S., and Park, I. K. (2002), "Heave and pitch motions of a spar platform with damping plate." Proceedings of the 12th International Offshore and Polar Engineering Conference, Kitakyshu, Japan, 198-201.

Rogers, A. (1983), "An assessment of vortex suppression devices for production risers and towed deep ocean pipe strings." Offshore Technology Conference, Houston, texas, USA, OTC4594.

SACS. (2009). "The manual of Structural Analysis Computer system program (SACS)." Engineering Dynamic Inc, Release 5, Rev 3.

Schijve, J. (2001). Fatigue of structures and materials, Kluwer Academic Pub, Dordrecht, Netherlands.

Serta, O. B., Mourelle, M. M., Grealish, F. W., Harbert, S. J., and Souza, L. F. A. (1996), "Steel catenary riser for the Marlim Field FPS P-XVIII." Proceedings of the Annual Offshore Technology Conference, Houston, Texas, USA, 359-366.

Sheehan, J. M., Grealish, F. W., Smith, R. J., and Harte, A. M. (2005). "Characterisation of the Wave Environment in the Fatigue Analysis of Flexible Risers." Proceedings of OMAE2005, Paper, 67507.

Silva, R., Scherer, I., Sert, O., and Andrade, B. (1994), "An Alternative For LargeDiameter Flexible Riser." Offshore Technology Conference, Houston, Texas, USA, OTC7597.

Skempton, A. W. (1942). "An investigation of the bearing capacity of a soft clay soil." Journal of the Institution of Civil Engineers, 18, 307-321.

Small, S. W., Tamburello, R. D., and Piaseckyj, P. J. (1972). "Submarine pipeline support by marine sediments." JPT, Journal of Petroleum Technology, 24, 317322. 
Steen, A., Kim, M., and Irani, M. (2004), "Prediction of Spar Responses: Model Tests vs. Analysis." Offshore Technology Conference, Houston, Texas, USA, OTC16583.

Terzaghi. (1943). Theoretical Soil Mechanics, John Wiley and Sons, New York.

Theti, R., and Moros, T. (2001). "Soil interaction effects on simple-catenary riser response." Pipes and Pipelines International, 46(3), 15-24.

Timoshenko. (1968). Theory of Structures, McGraw-Hill International Book Company, Auckland.

Ursell, F. (1964). "The decay of the free motion of a floating body." J. Fluid Mech, 19, 305-319.

Vandiver, J. K., and Li, L. (1994). "SHEAR7 program theory manual." Department of Ocean Engineering, Massachusetts Institute of Technology.

Vaz, M. A., and Patel, M. H. (2000). "Three-dimensional behaviour of elastic marine cables in sheared currents." Applied Ocean Research, 22(1), 45-53.

Verley, R., and Lund, K. M. (1995), "Soil resistance model for pipelines placed on clay soils." Copenhagen, Den, 225-232.

Verley, R. L. P., and Sotberg, T. (1994). "A soil resistance model for pipelines placed on sandy soils." Journal of Offshore Mechanics and Arctic Engineering, 116, 145.

Vughts, J. H., and Kinra, R. K. (1976), "Probabilistic fatigue analysis of fixed offshore structures." Offshore Technology Conference, Dallas, Texas, OTC2608.

Wasow, W. (1956). "Singular perturbations of boundary value problems for nonlinear differential equations of the second order." Communications on Pure and Applied Mathematics, 9(1).

Wehausen, J. V. (1971). "The motion of floating bodies." Annual Review of Fluid Mechanics, 3(1), 237-268.

You, J., Biscontin, G., and Aubeny, C. P. (2008), "Seafloor interaction with steel catenary risers." Proceedings of the International Offshore and Polar Engineering Conference, Vancouver, BC, Canada, 110-117.

Young, A., Honganen, C., Silva, A., and Bryant, W. (2000), "Comparison of geotechnical properties from large-diameter long cores and borings in deep water Gulf of Mexico." Offshore Technology Conference, Houston, Texas, OTC12089.

Zajac, E. E. (1957). "Dynamics and kinematics of the laying and recovery of submarine cable." Bell System Technical Journal, 36(5), 1129-1207. 


\section{APPENDIX A. UEL AND DISP SUBROUTINES OF ABAQUS}

\section{A.1 INTRODUCTION}

ABAQUS, a general-purpose finite element program, has been used in the current research to perform the numerical simulations. This widely used software provides a set of powerful and flexible tools, including user defined subroutines for advanced users to enhance the functionality of the program for analyses where the usual data input methods alone may be too restrictive. These subroutines are typically coded in FORTRAN and linked to the main model constructed in ABAQUS. As discussed in Chapters 4 to 7, the UEL and DISP subroutines have been chosen and coded to model the riser-seabed interaction and the vessel excitation scheme respectively. This Appendix briefly reviews the function of these subroutines in relation to the SCR simulations performed. The details of programming and theoretical aspects of these subroutines can be found in the ABAQUS user manual.

\section{A.2 UEL SUBROUTINE FOR SCR-SEABED INTERACTION}

For numerical simulation, the steel catenary riser constructed in ABAQUS is assumed to sit on user-defined springs distributed along the seabed. The behaviour of these springs is defined and coded inside the UEL subroutine. At every time increment, the UEL subroutine is called by ABAQUS for the elements identified as a user-defined element. ABAQUS then passes key variables to the UEL, including updated displacements of the nodes. The UEL subroutine uses the historical displacements of the nodes and calculates the stiffness matrix of each element and its contribution to external forces through the right hand side vector (RHS). Here the UEL is based on the hysteretic non-linear seabed interaction model described in Chapter 5. This information is returned to ABAQUS to calculate the global stiffness matrix and update the variables to be passed to the UEL again.

\section{A.2.1 Utilised Key Variables Passed into UEL by ABAQUS}

A large number of variables, including arrays and scalar parameters, can be passed to the UEL for information, depending on the analysis to be performed. Some of the key variables that have been used in this study are summarised below:

- PROPS : A floating point array containing the property values defined for use with spring elements. These properties include every input parameter for the analysis, such as the riser diameter, seabed horizontal friction coefficient and all of the hysteretic non-linear soil model parameters including the undrained shear strength at the seabed surface, the gradient of undrained shear strength, suction 
decay and repenetration delay parameters etc. The magnitude of these variables is defined in the ABQUS input file and can be changed for any analysis if required.

- COORDS: An array containing the original coordinates of the nodes of the elements. COORDS(K1,K2) is coordinate K1 of node K2 of the element. The updated position of each node connected to the user-defined elements can be obtained from this array variable. The magnitude of each variable in the array is reported based on the global coordinate system defined in the ABAQUS model; in the simulations here, using the coordinate system defined in Chapter 4, a vertically negative value of the COORDS variable shows that the node is below the mudline.

- U, DU: Arrays containing the current estimates of the basic solution variables, depending on the degree of freedom at the nodes of the element at the end of the current increment. For the current study with the defined coordinate system, $\mathrm{U}(1)$ and $\mathrm{U}(2)$ show the total value of the horizontal and vertical displacement of each node respectively. Likewise, DU $(2,1)$ shows the incremental values of the vertical displacement for the current increment. $\mathrm{DU}(2,1)$ is the basic variable to define the various displacement modes in the non-linear model discussed in Chapter 5. Thus, taking into account the defined coordinate system, a positive DU shows that the node is moving upward and thus experiencing a reduction in resistance and potentially suction resistance. Conversely, a negative DU value means that the node is moving downward, hence penetration or repenetration. The combination of U and DU or COORDS and DU reveals the current position of the node (below the mudline or above it) and the direction that it is moving. The UEL subroutine uses this to decide whether to apply uplift or repenetration formulae for calculation of the stiffness matrix and right-hand-side vector.

- LFLAGS: An array containing the flags that define the current solution procedure and analysis type. As an example, LFLAGS(2)=1 means Largedisplacement analysis with nonlinear geometric effects included in the step.

- KSTEP: Current step number.

- KINC: Current increment number.

- JELEM: User-assigned element number

\section{A.2.2 Key Variables to be Calculated by UEL and Given back to ABAQUS}

The main variables calculated in the UEL and returned to the main ABAQUS system are as follows:

- RHS: An array containing the contributions of this element (connected to a single node in the ABAQUS model) to the right-hand-side vectors or the external force of the overall system. For most nonlinear analysis procedures, 
there is only one right-hand-side vector and RHS should contain the residual vector. The RHS calculation method depends on the LFLAGS parameters obtained from the ABAQUS input file. In the simulations performed, RHS is the negative of the residual force, which in turn equates to the penetration resistance formulated in Chapter 5 for the non-linear seabed model.

- AMATRX: An array containing the contribution of this element to the Jacobian (stiffness) or other matrix of the overall system. The particular matrix required at any time depends on the entries in the LFLAGS array. All nonzero entries in AMATRX should be defined, even if the matrix is symmetric. In the simulations performed, AMATRIX is a two dimensional matrix with a linear elastic component for horizontal displacements to model riser-seabed friction and a vertical secant stiffness component formulated using the non-linear seabed model described in Chapter 5.

- SVARS: An array containing the values of the solution-dependent state variables associated with this element. The number of such variables and their meaning is defined by the user. For a general non-linear step it is passed into the UEL containing the values at the start of the current increment, and the solutiondependent variables are then updated to the values at the end of increment. Breaking down the calculation formulae within the UEL to a number of solution-dependent variables was found to be helpful in verifying the correctness of the calculations in the subroutine.

\section{A.2.3 UEL Internal Calculation Flowchart}

Figure A-1 illustrates the local flowchart of calculation inside the UEL, where the stiffness matrix and right-hand-side vector is calculated to feed back to the ABAQUS main procedure at every time increment. The parameters indicated in the flowchart have been defined in Chapter 5. 


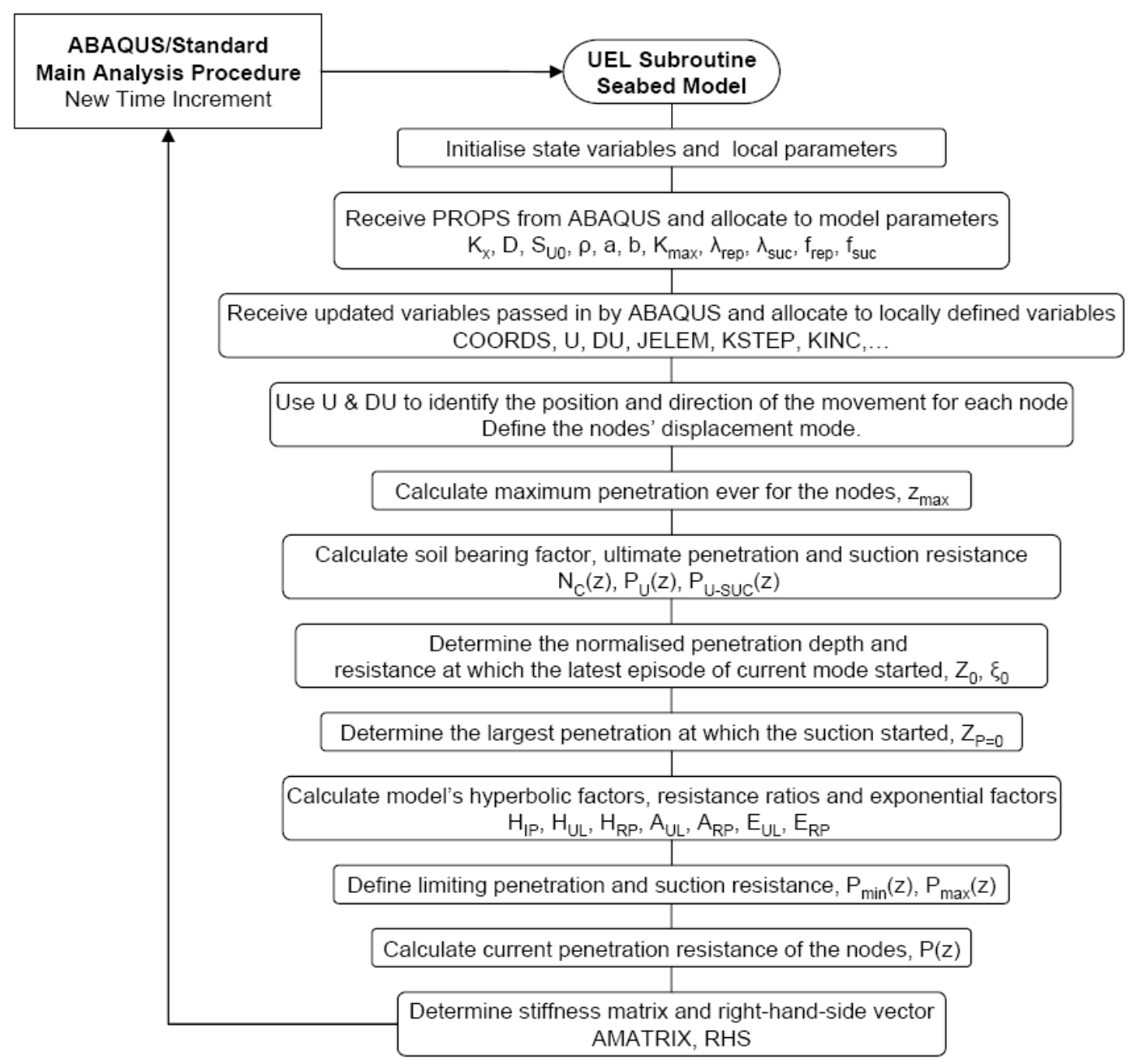

Figure A-1: UEL subroutine internal flowchart

\section{A.3 DISP SUBROUTINE FOR VESSEL EXCITATION}

To perform an integrated fatigue analysis using the non-linear seabed model, for which the hierarchy of the applied sea states on the vessel is important, the DISP subroutine of ABAQUS has been coded to excite the vessel for a full package of sea states. As described in Chapter 4, all of the analyses performed within this study are displacementcontrolled analysis, where the position of the vessel has been continuously updated based on the vessel RAO and applied sea state. The DISP subroutine is basically called for all degrees of freedom listed in the user-subroutine-defined boundary condition and defines the magnitudes of prescribed boundary conditions at each time step.

\section{A.3.1 Utilised Key Variables Passed into DISP Subroutine by ABAQUS}

A number of variables are passed to the DISP subroutine, covering a wide range of applications, but only two of them have been used in the simulations performed in the current study. These variables are as follows: 
- TIME(2): Current value of total time. This variable in combination with the applied wave periods has been used to determine the ID of the sea state that is currently applied on the vessel The ID parameter is linked to all of the sea state characteristics stored in the databank of DISP subroutine; hence by determining the wave ID, its period, height and number of cycles to be applied can be extracted to combine with the RAOs and obtain the vessel's new position.

- JDOF: Degree of freedom. This variable is utilised to calculate the transformation vector of heave and surge amplitudes from the centre of buoyancy, to which the RAOs are referenced, to the riser attachment point where the excitation is intended.

\section{A.3.2 Key Variable to be Calculated by DISP and Given back to ABAQUS}

There is only one variable to be delivered back to the main ABAQUS procedure:

- U(1): Total value of the prescribed variable at this point. In the current simulations, since a displacement-controlled analysis is performed, this variable is displacement. For other kinds of analysis, the variable may be displacement, rotation, pore pressure, temperature, etc., depending on the degree of freedom constrained. In cases where the analysis procedure requires the time derivatives of prescribed variables such as velocity and acceleration in dynamic analyses, as well as the value of the variable, the first and second derivatives of the variable can be defined as $\mathrm{U}(2)$ and $\mathrm{U}(3)$; these were not relevant for the simulations performed within the current study.

\section{A.3.3 DISP Internal Calculation Flowchart}

For completeness, although the internal calculation flowchart of the DISP subroutine has been presented in Chapter 4, it is provided here again, Figure A-2. 


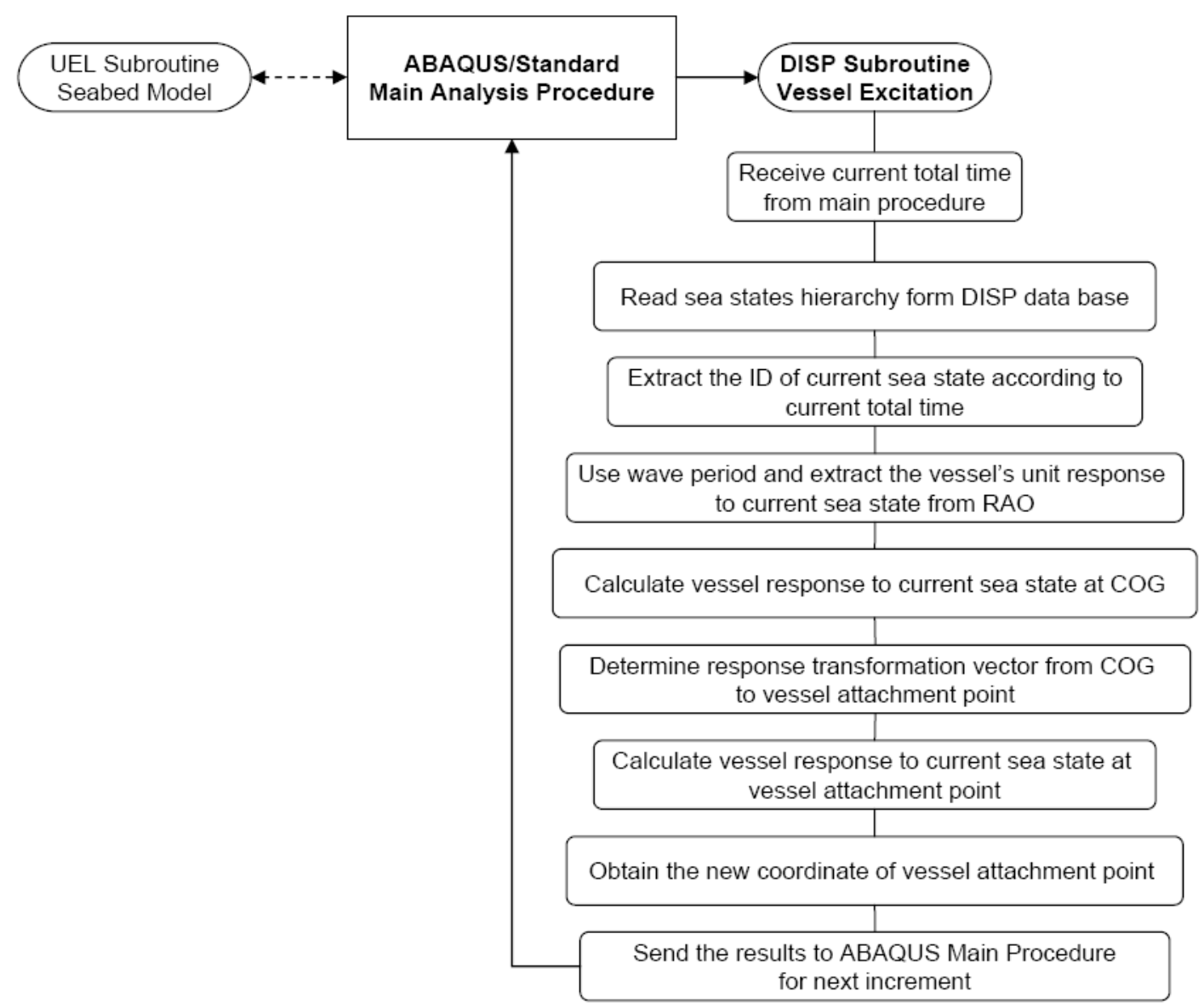

Figure A-2: DISP subroutine internal flowchart 


\section{APPENDIX B.}

\section{PARAMETRIC STUDY OF STRESS DISUTRIBUTION AND FATIGUE PERFORMANCE}

\section{B.1 INTRODUCTION}

The fatigue performance of steel catenary risers was studied through a comprehensive parametric study investigating the influence of various key parameters on the stress distribution and consequently the fatigue performance in the touchdown zone. The global configuration of the SCR system is defined in Chapter 4 and is shown in Figure B-1. The default values of the non-linear seabed model parameters and the riser structural and mechanical characteristics are given in Table B-1 to TableB-3. The remaining sections of this appendix provide results from all the analyses undertaken, some of which have also been presented in the body of the thesis.

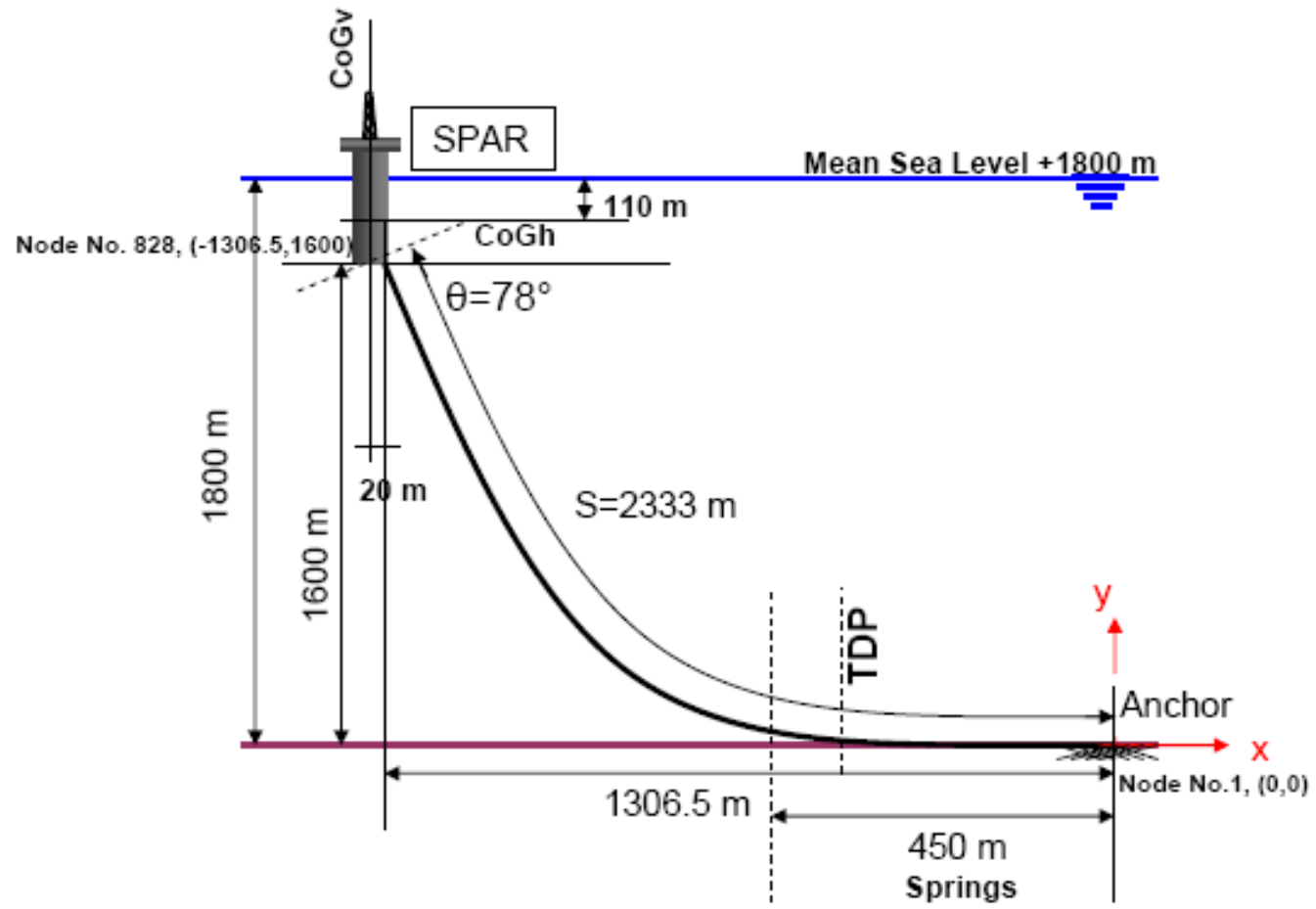

Figure B-1: The global geometry of SCR modelled by ABAQUS 
Table B-1: Default values of non-linear hysteretic model parameters

\begin{tabular}{|l|c|c|}
\hline \multicolumn{1}{|c|}{ Parameter } & Symbol & Value \\
\hline Mudline shear strength & $\mathrm{s}_{\text {um }}$ & $0.65 \mathrm{kPa}$ \\
\hline Shear strength gradient & $\rho$ & $1.5 \mathrm{kPa} / \mathrm{m}$ \\
\hline Power law parameter & $\mathrm{a}$ & 6 \\
\hline Power law parameter & $\mathrm{b}$ & 0.25 \\
\hline Normalized maximum stiffness & $\mathrm{K}_{\max }$ & 200 \\
\hline Suction ratio & $\mathrm{f}_{\text {suc }}$ & 0.3 \\
\hline Suction decay parameter & $\lambda_{\text {suc }}$ & 0.5 \\
\hline Repenetration parameter & $\lambda_{\text {rep }}$ & 0.5 \\
\hline
\end{tabular}

Table B-2: key dimensions of SCR modelled by ABAQUS

\begin{tabular}{|l|c|}
\hline \multicolumn{1}{|c|}{ Dimension } & Value \\
\hline Water depth & $1800 \mathrm{~m}$ \\
\hline Height of attachment point & $1600 \mathrm{~m}$ \\
\hline SCR length & $2333 \mathrm{~m}$ \\
\hline Vessel distance from anchor end & $1306.5 \mathrm{~m}$ \\
\hline Lay angle & $78^{\circ}$ \\
\hline Attachment point offset from COG & $20 \mathrm{~m}$ \\
\hline Length of springs zone at seabed & $450 \mathrm{~m}$ \\
\hline Spacing of seabed springs & $1.0 \mathrm{~m}$ \\
\hline
\end{tabular}

TableB-3: Riser pipe properties

\begin{tabular}{|l|c|}
\hline \multicolumn{1}{|c|}{ Dimension } & Value \\
\hline Outer Diameter, $\mathrm{D}_{\mathrm{o}}$ & $0.324 \mathrm{~m}\left(12^{3 / 4}\right.$ “ $)$ \\
\hline Wall Thickness, $\mathrm{t}$ & $0.0205 \mathrm{~m}$ \\
\hline Second Moment of Inertia, $\mathrm{I}$ & $2.26 \times 10^{-4} \mathrm{~m}^{4}$ \\
\hline Steel Density, $\rho_{\mathrm{s}}$ & $7850 \mathrm{~kg} / \mathrm{m}^{3}$ \\
\hline In service Submerged Weight, $\mathrm{m}_{\mathrm{s}}$ & $100 \mathrm{~kg} / \mathrm{m}$ \\
\hline Fatigue S-N Curve & DNV(2008), E Class weld, $\overline{\mathrm{a}}=1.05 \times 10^{-12}$ \\
\hline
\end{tabular}




\section{B.2 TENSIONLESS BEAM ON WINKLER ELASTIC FOUNDATION}

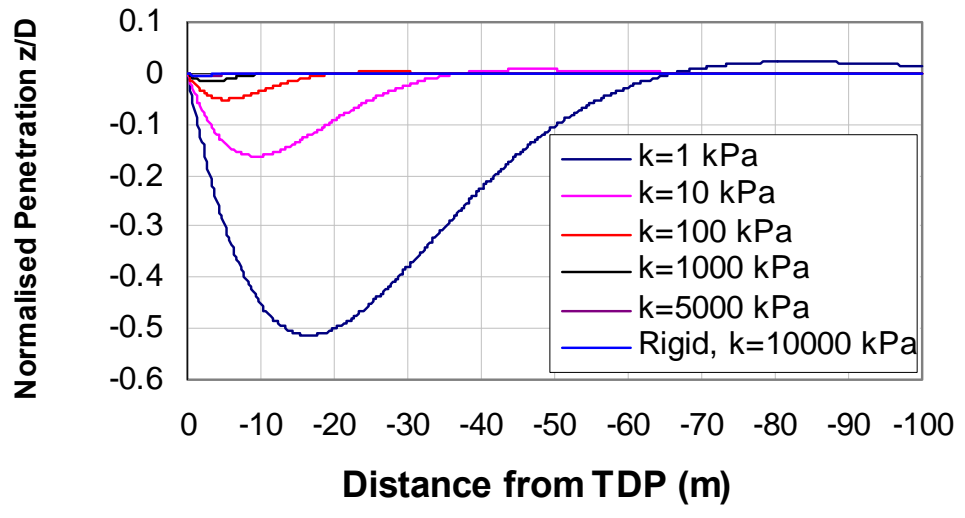

Figure B-2: SCR seabed profile varying with seabed stiffness

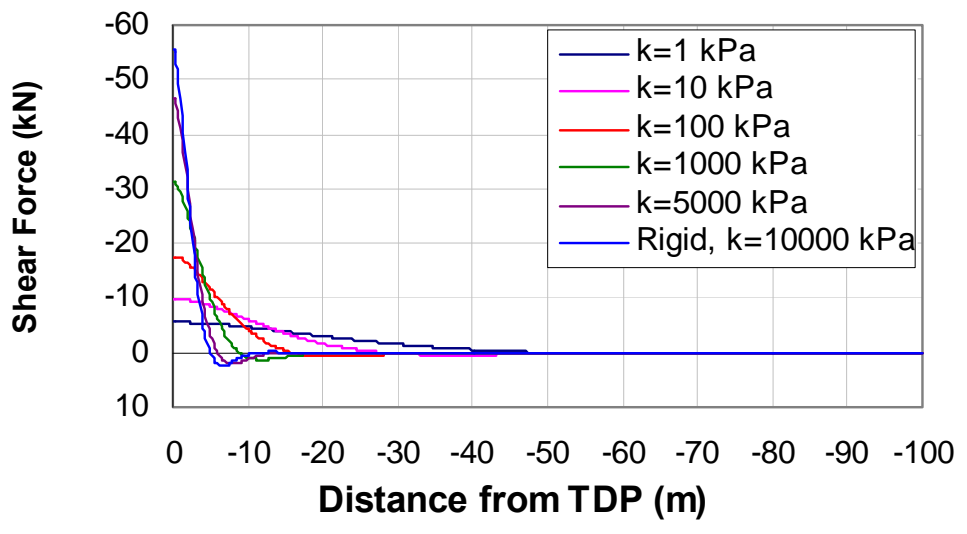

Figure B-3: Shear force diagram of SCR in seabed varying with seabed stiffness 


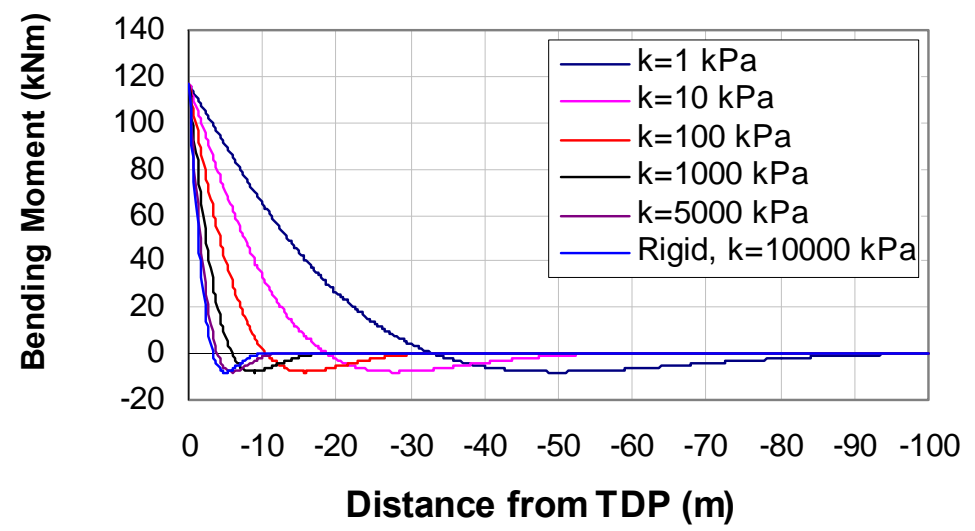

Figure B-4: Bending moment diagram of SCR in seabed varying with seabed stiffness

\section{B.3 TENSION BEAM ON WINKLER ELASTIC FOUNDATION}

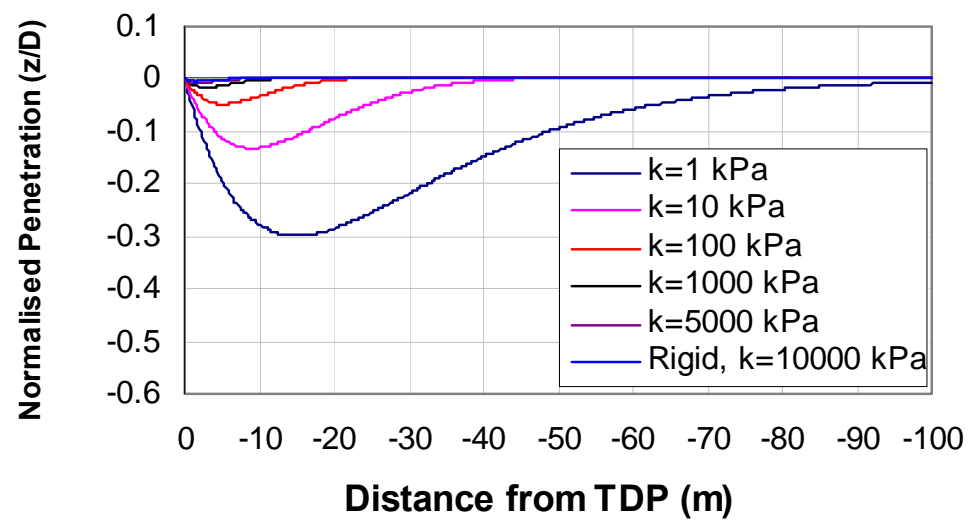

Figure B-5: SCR seabed profile varying with seabed stiffness

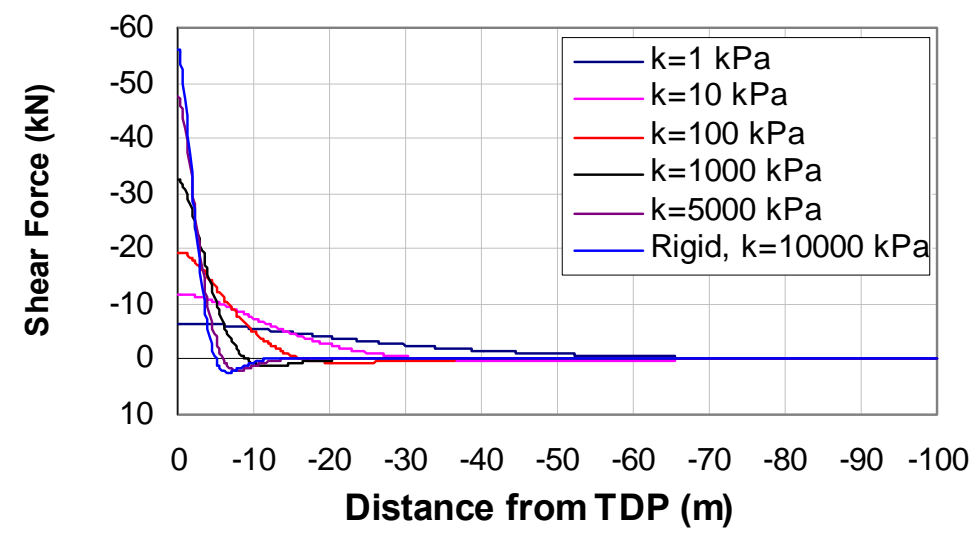

Figure B-6: Shear force diagram of SCR in seabed varying with seabed stiffness 


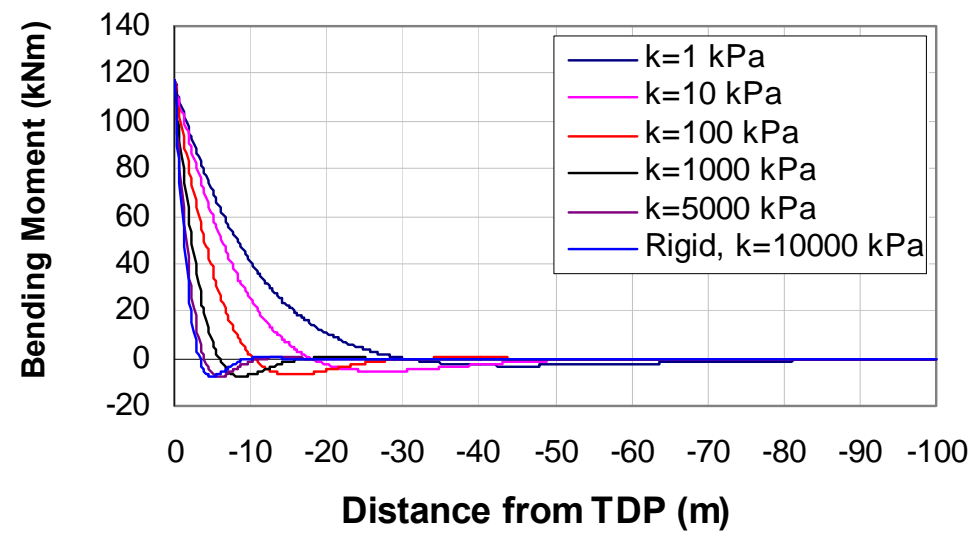

Figure B-7: Bending moment diagram of SCR in seabed varying with seabed stiffness

\section{B.4 BOUNDARY-LAYER METHOD (PESCE ET AL 1998)}

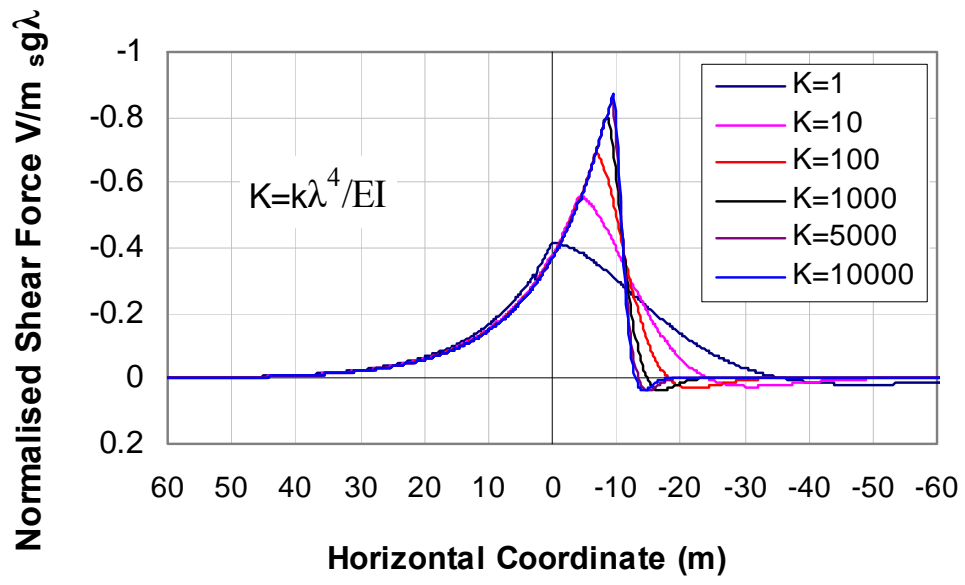

Figure B-8: Shear force diagram of SCR in seabed varying with seabed stiffness 


\section{B.5 FATIGUE PERFORMANCE IN LINEAR ELASTIC SEABED}

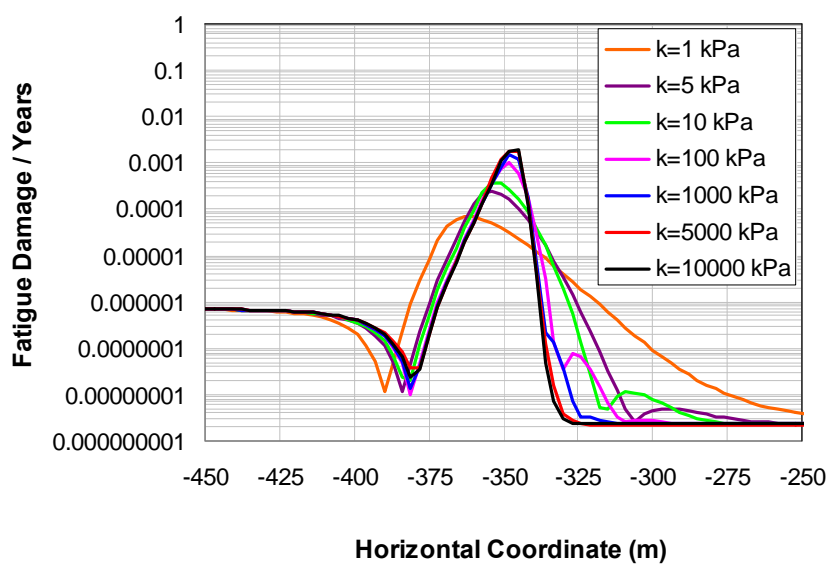

Figure B-9: Fatigue damage distribution in TDZ varying with seabed stiffness

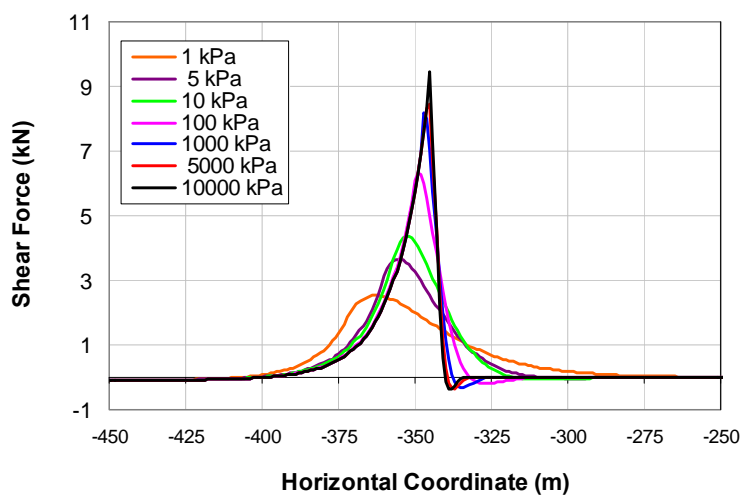

Figure B-10: Shear force distribution in TDZ varying with seabed stiffness

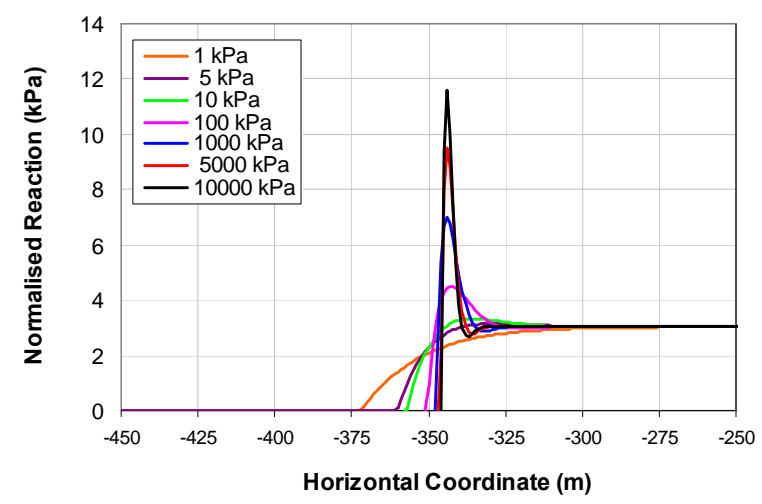

Figure B-11: Bending moment distribution in TDZ varying with seabed stiffness 


\section{B.6 HYSTERETIC NON-LINEAR SEABED MODEL}

B.6.1 Repenetration Offset Parameter, $\lambda_{\text {rep }}$

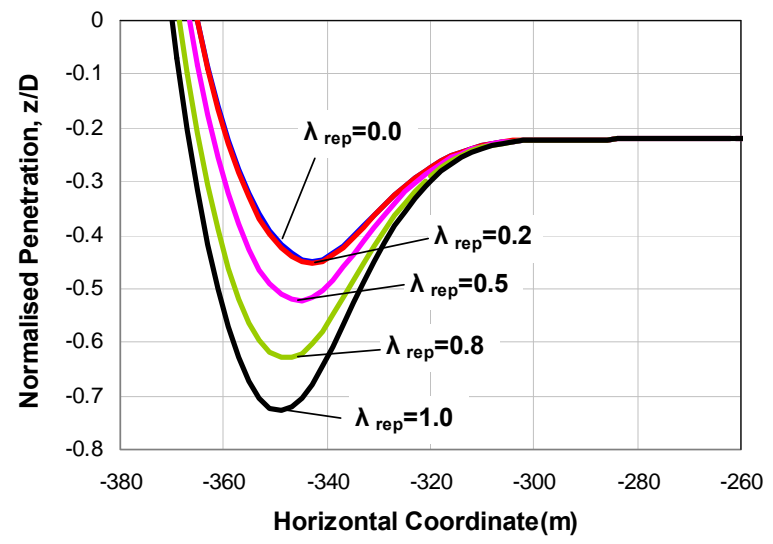

Figure B-12: SCR seabed profile with different values of repenetration offset parameter

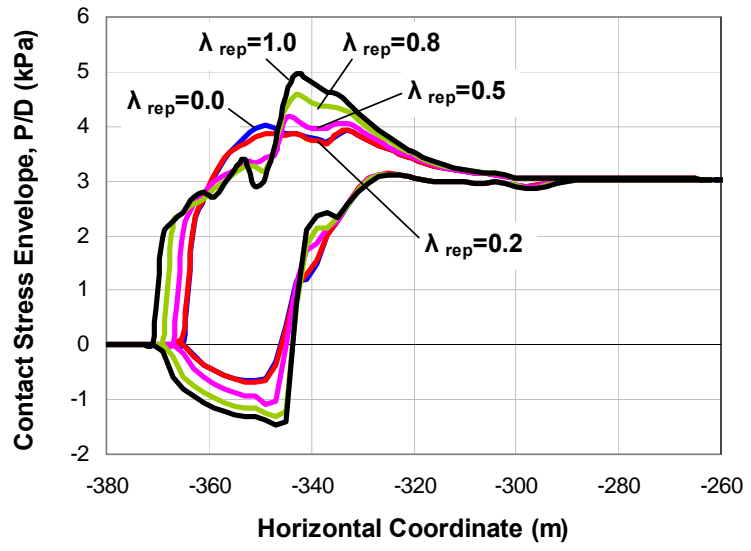

Figure B-13: Seabed contact stress envelope with different values of repenetration offset parameter

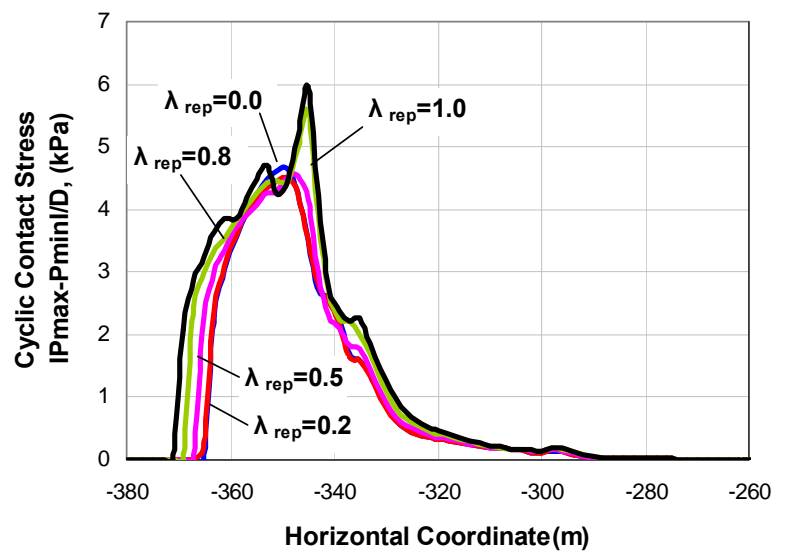

Figure B-14: Cyclic contact stress with different values of repenetration offset parameter 


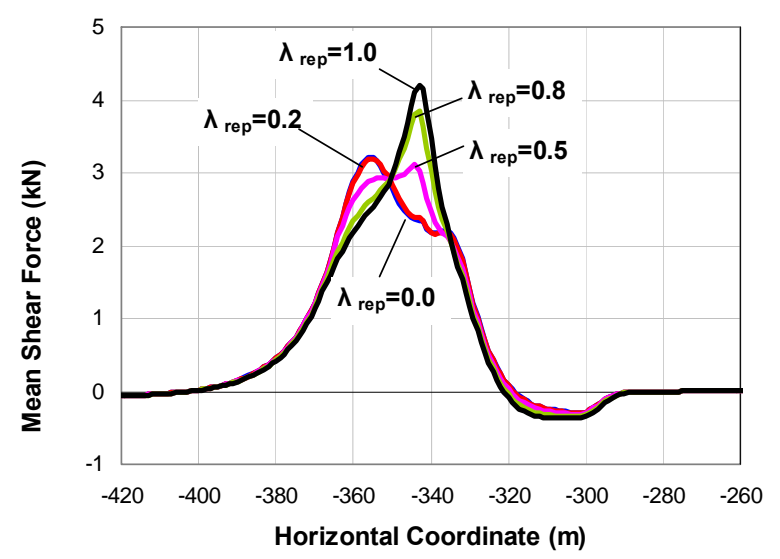

Figure B-15 Mean shear force distribution with different values of repenetration offset parameter

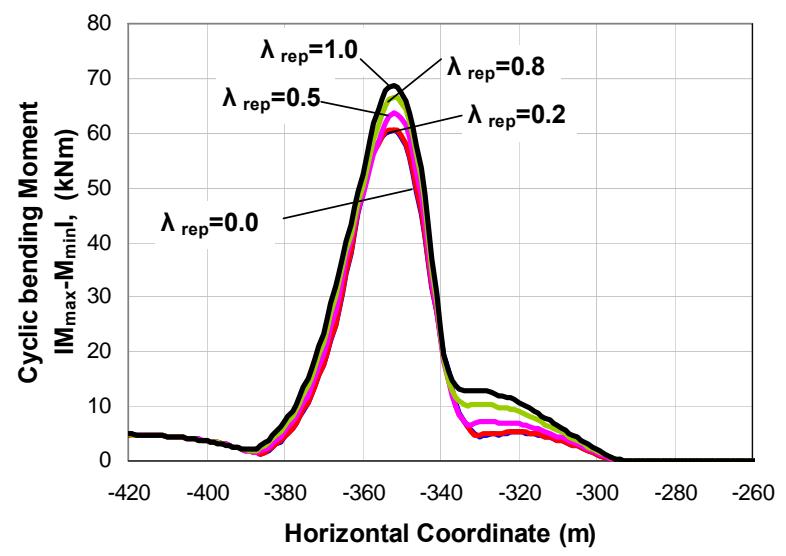

Figure B-16 Cyclic bending moment with different values of repenetration offset parameter 
B.6.2 Suction decay Parameter, $\lambda_{\text {suc }}$

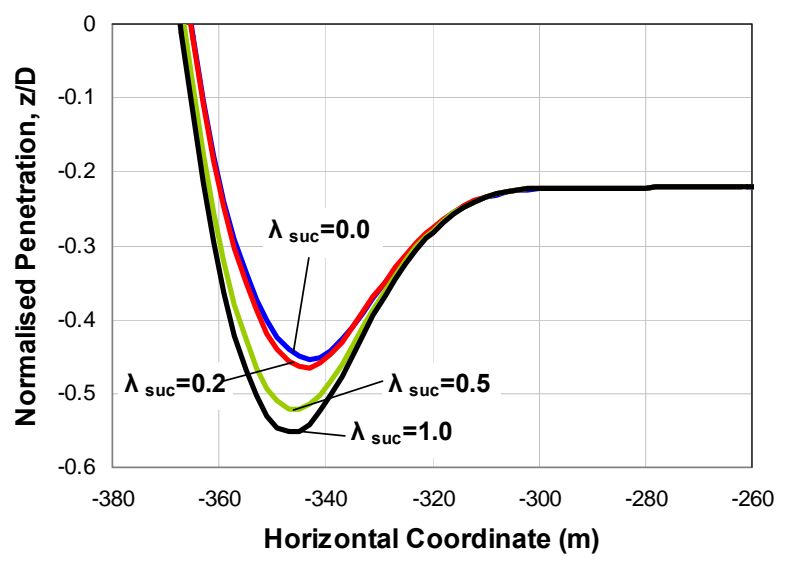

Figure B-17: SCR seabed profile with different values of suction decay parameter

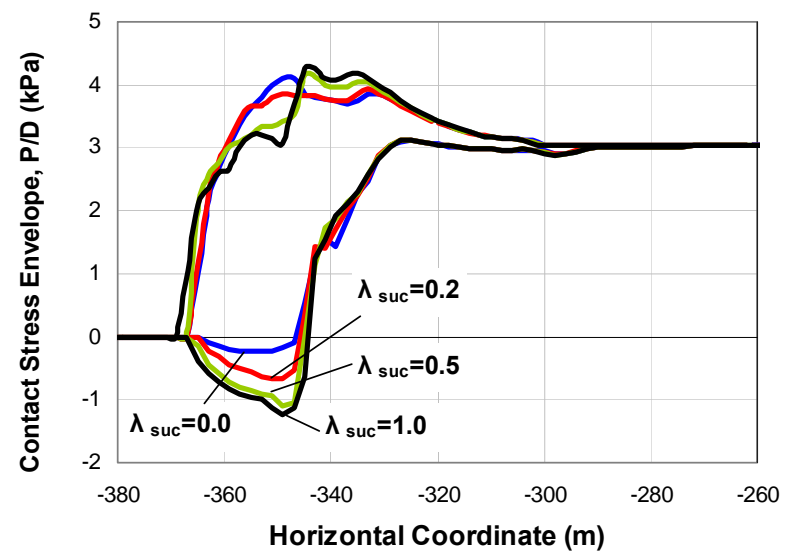

Figure B-18: seabed contact stress envelope with different values of suction decay parameter

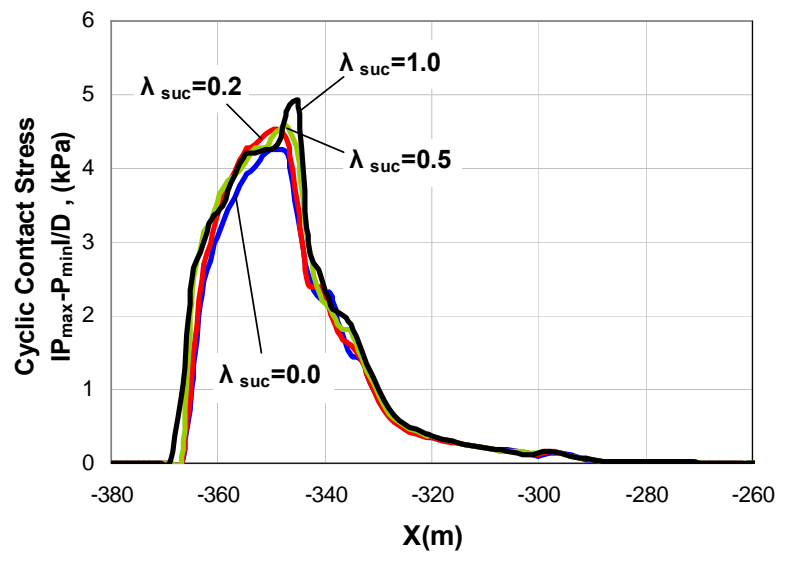

Figure B-19: Cyclic contact stress with different values of suction decay parameter 


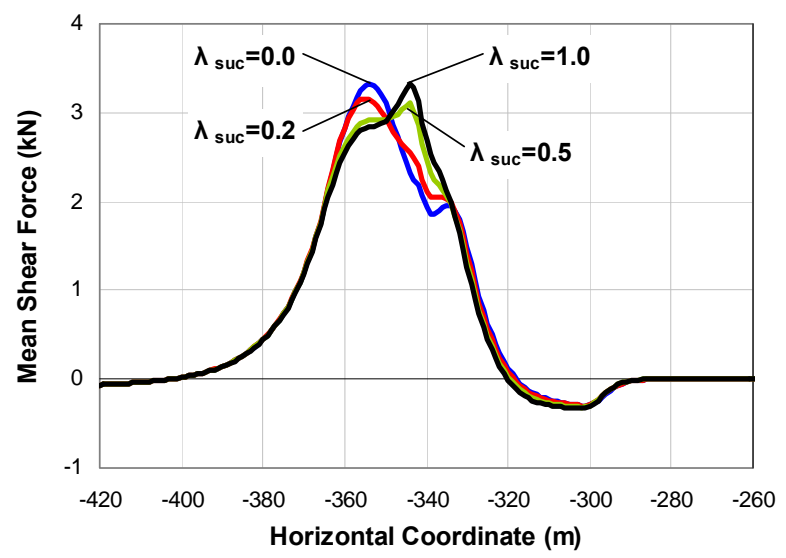

Figure B-20: Mean shear force distribution with different values of suction decay parameter

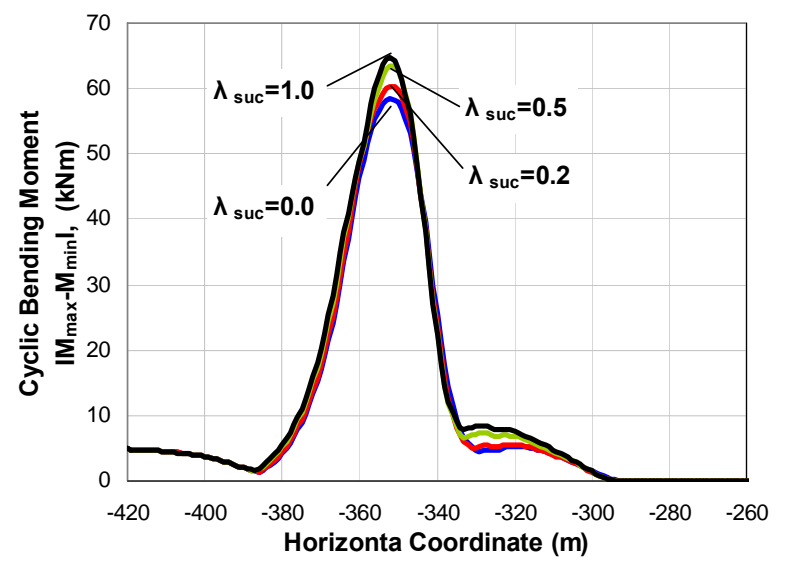

Figure B-21: Cyclic bending moment with different values of suction decay parameter 


\section{B.6.3 Ultimate Suction Parameter, $\mathbf{f}_{\text {suc }}$}

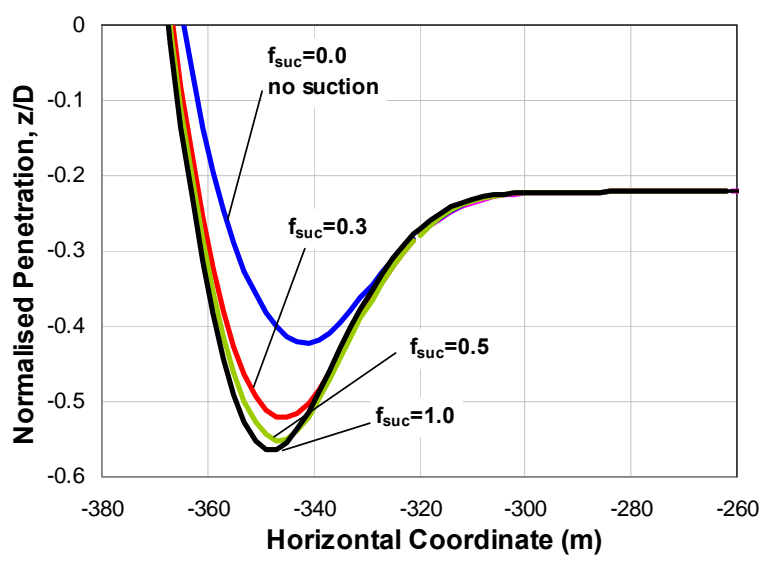

Figure B-22: SCR seabed profile with different values of ultimate suction parameter

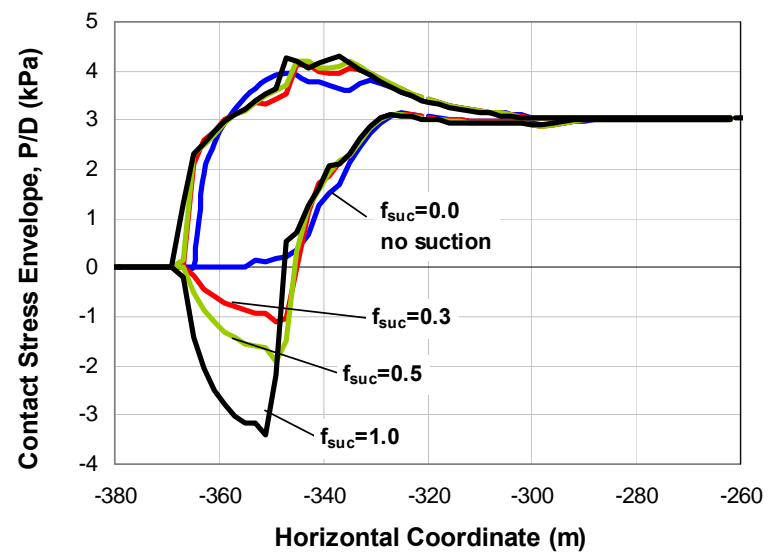

Figure B-23: Seabed contact stress envelope with different values of ultimate suction parameter

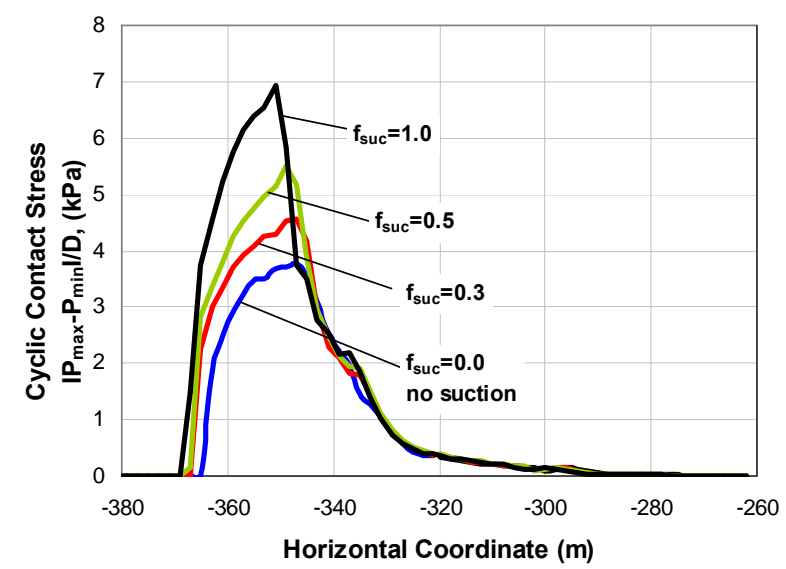

Figure B-24: Cyclic contact stress with different values of ultimate suction parameter 


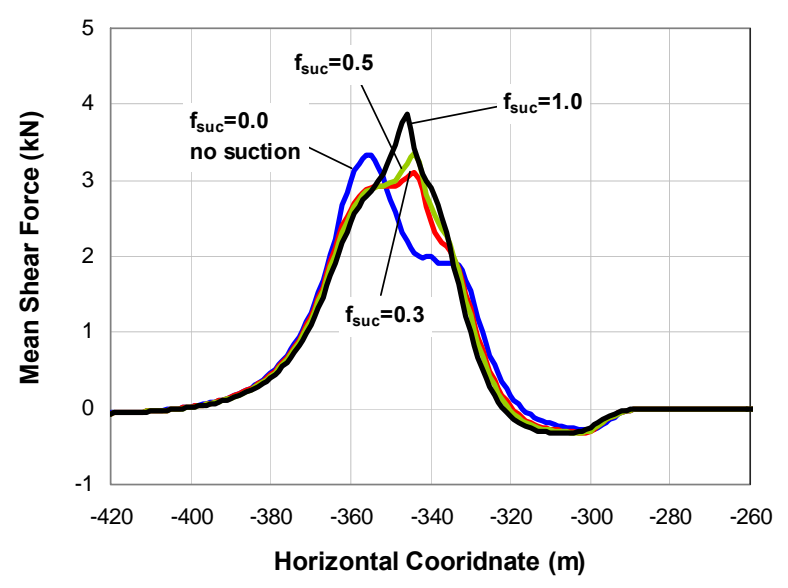

Figure B-25: Mean shear force distribution with different values of ultimate suction parameter

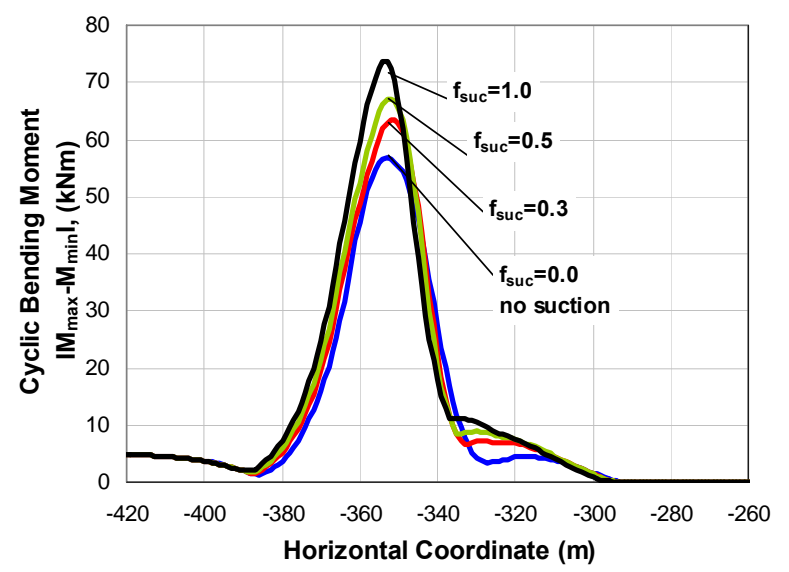

Figure B-26: Cyclic bending moment with different values of ultimate suction parameter 


\section{B.6.4 Surface Soil Undrained Shear Strength, $s_{u m}$ (values in kPa)}

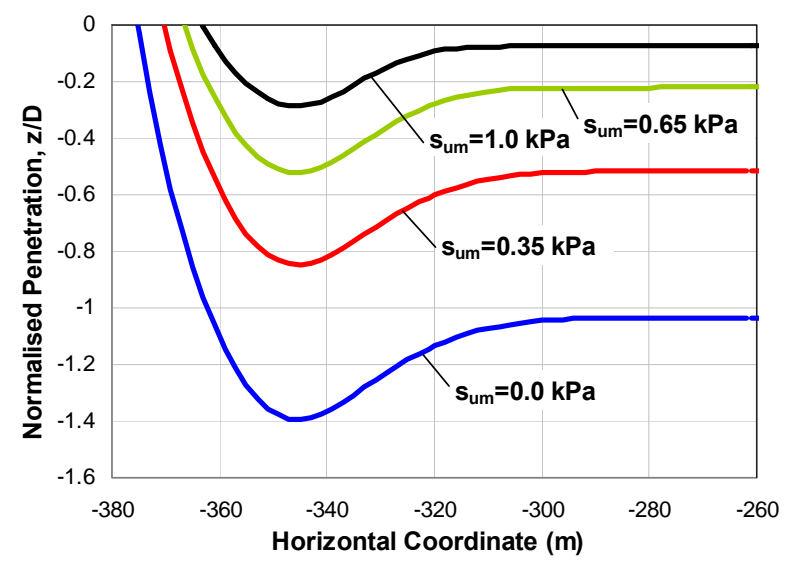

Figure B-27: SCR seabed profile with different values of surface soil shear strength

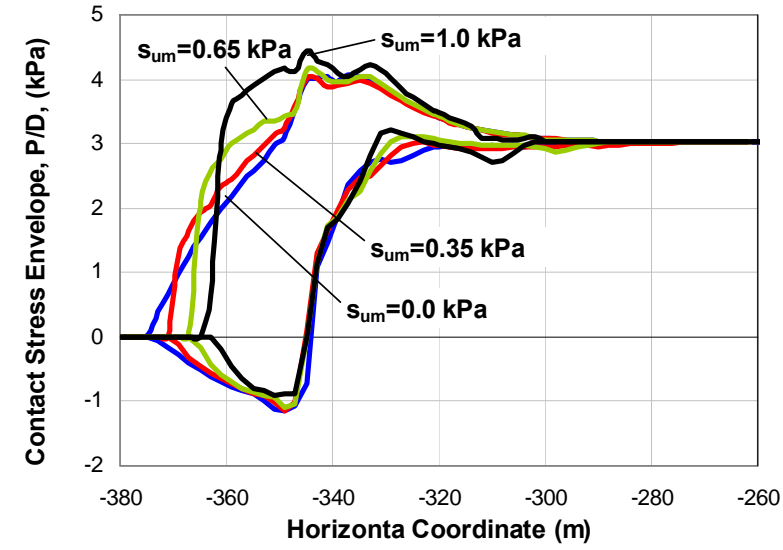

Figure B-28: Seabed contact stress envelope with different values of surface soil shear strength

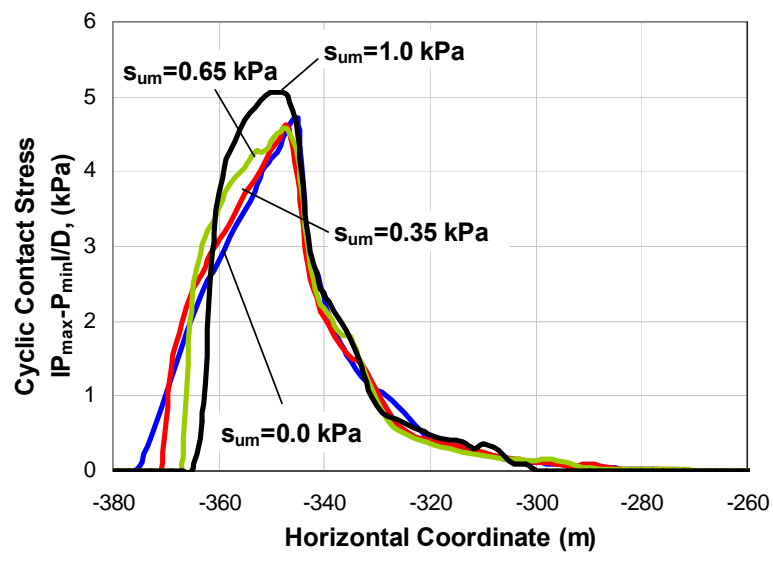

Figure B-29: Cyclic contact stress with different values of surface soil shear strength 


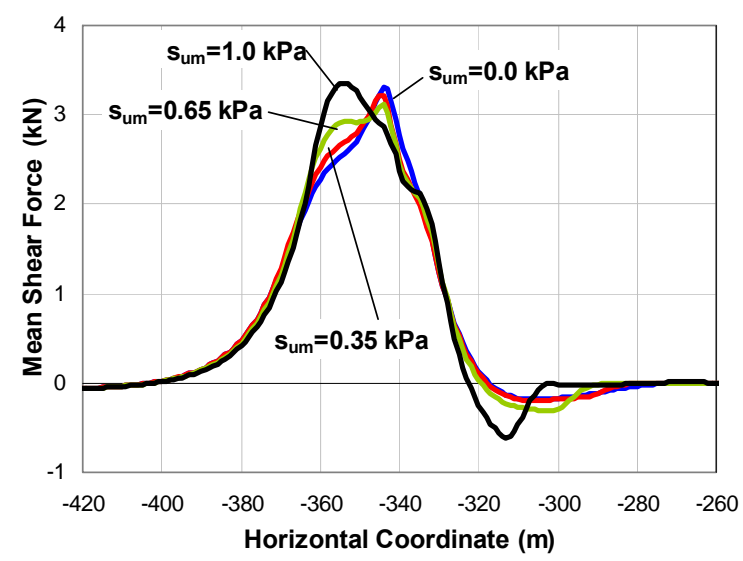

Figure B-30: Mean shear force distribution with different values of surface soil shear strength

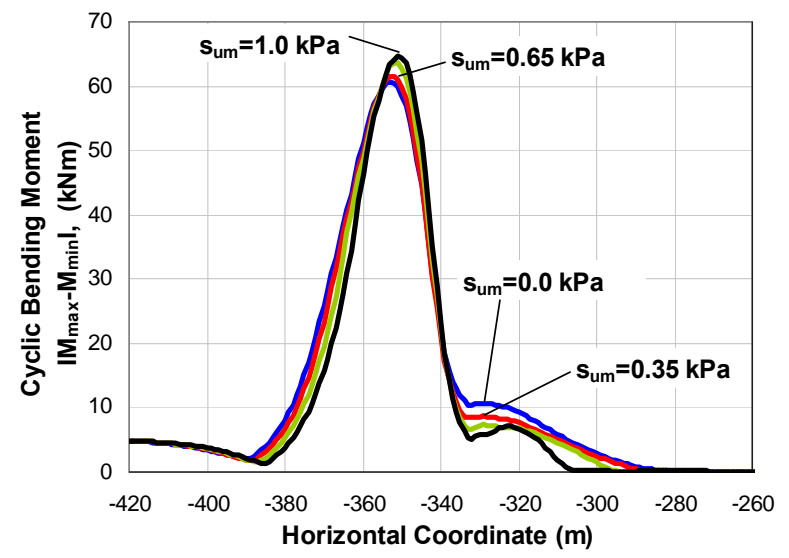

Figure B-31: Cyclic bending moment with different values of surface soil shear strength 


\section{B.6.5 Gradient of Undrained Soil Shear Strength, $\rho$ (values in kPa/m)}

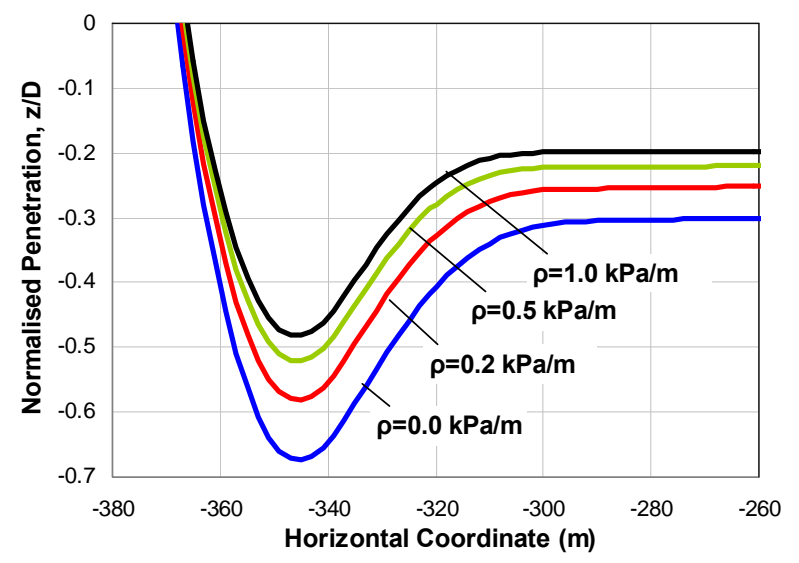

Figure B-32: SCR seabed profile with different values of soil shear strength gradient

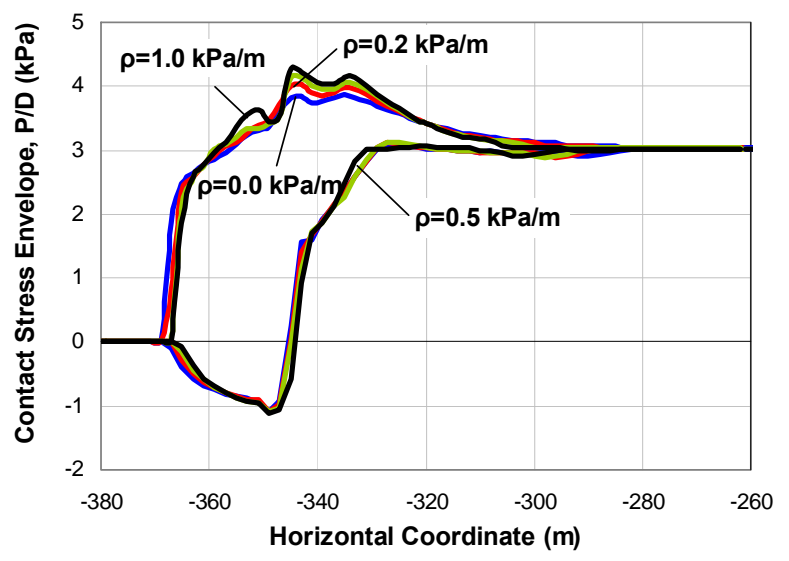

Figure B-33: Seabed contact stress envelope with different values of soil shear strength gradient

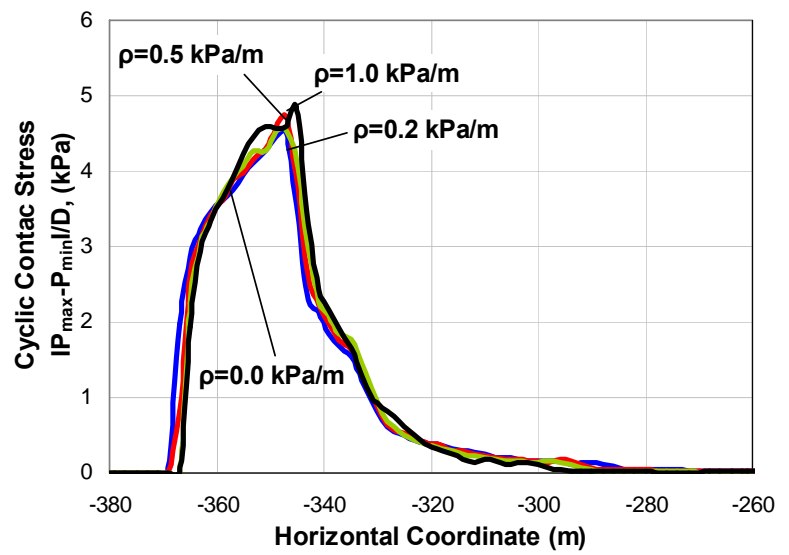

Figure B-34: Cyclic contact stress with different values of soil shear strength gradient 


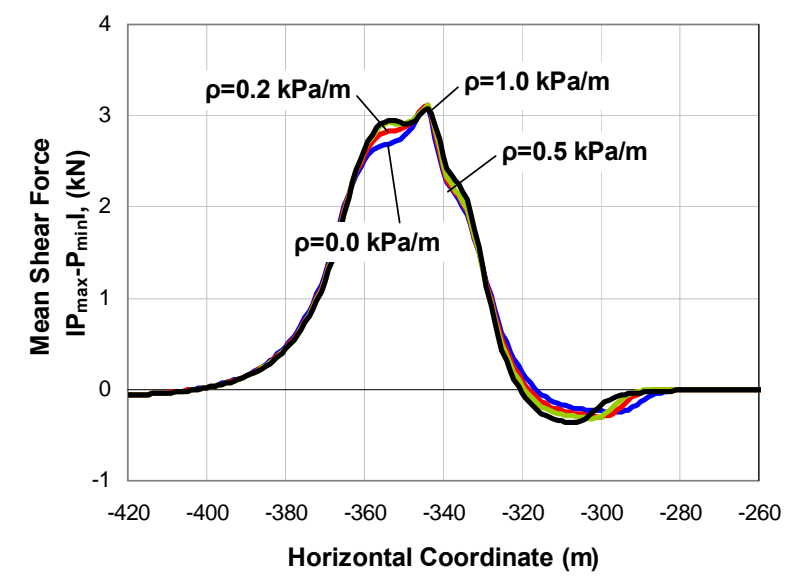

Figure B-35: Mean shear force distribution with different values of soil shear strength gradient

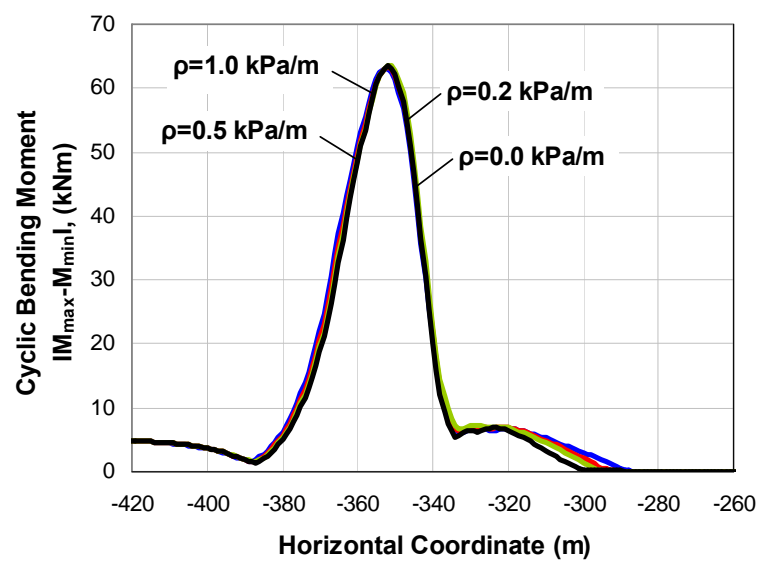

Figure B-36: Cyclic bending moment with different values of soil shear strength gradient 


\section{B.7 FATIGUE IN HYSTERETIC NON-LINEAR SEABED}

\section{B.7.1 Fatigue Damage Variation with Gradual Trench Development}

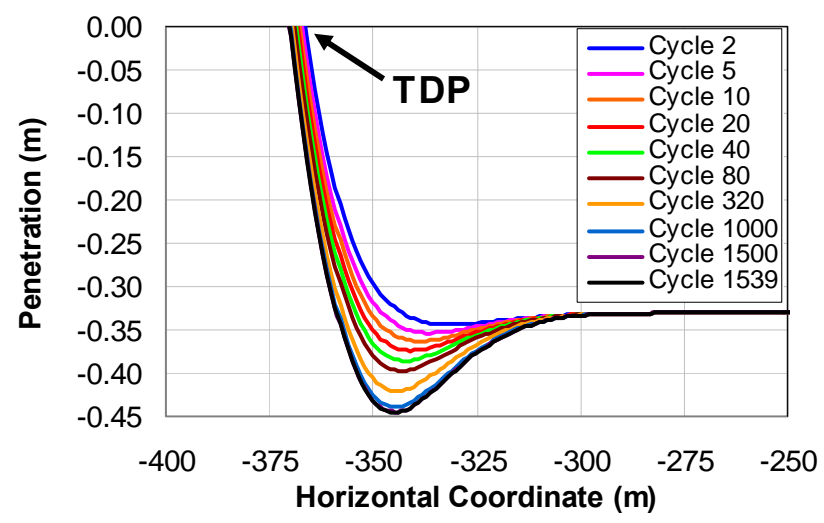

Figure B-37: Gradual trench development beneath the SCR with increasing the load cycles

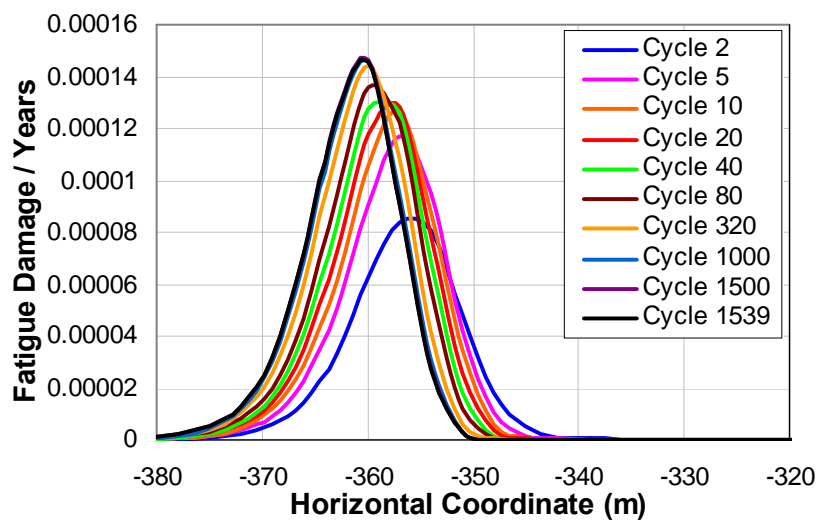

Figure B-38: Gradual variation of non-logarithmic fatigue damage in TDZ with increasing the load cycles

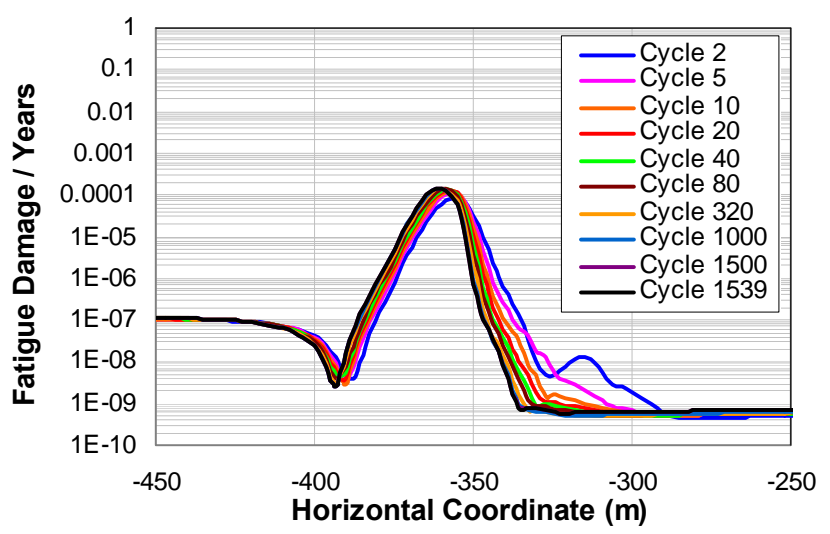

Figure B-39: Gradual variation of logarithmic fatigue damage in TDZ with increasing the load cycles 


\section{B.7.2 Influence of Suction Force Mobilisation on Fatigue Performance}

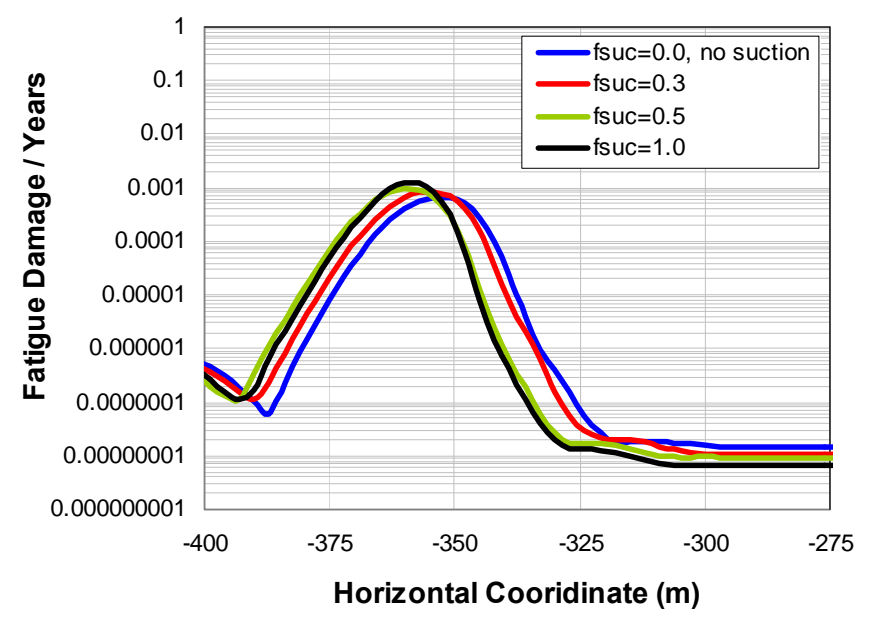

Figure B-40: Logarithmic fatigue damage distribution in $T D Z$ with various ultimate suction parameter values

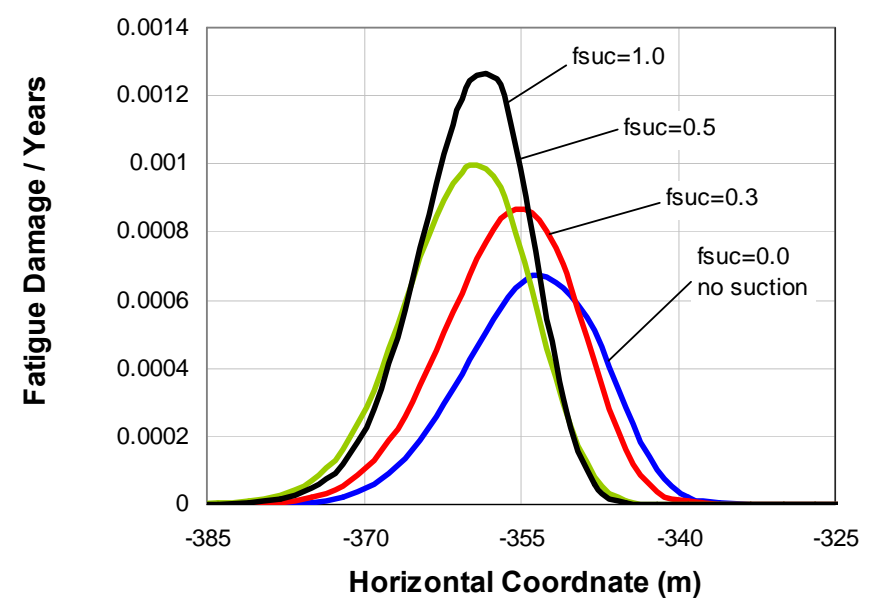

Figure B-41: Non-logarithmic fatigue damage in TDZ with various ultimate suction parameter values 


\section{B.7.3 Influence of Suction Decay Parameter on Fatigue Performance}

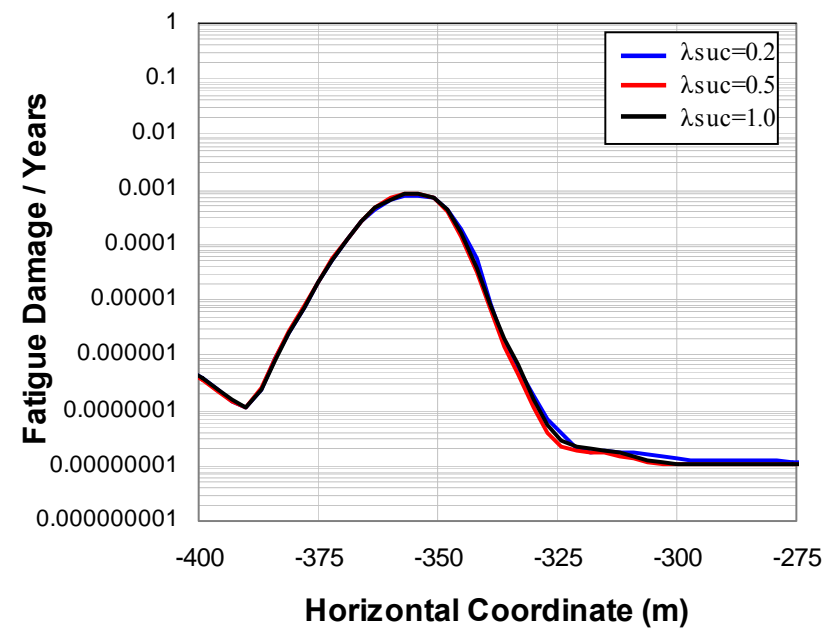

Figure B-42: : Fatigue damage distribution in TDZ with various suction decay parameter values 


\section{B.7.4 Influence of Seabed Soil Shear Strength and Gradient on Fatigue Performance}

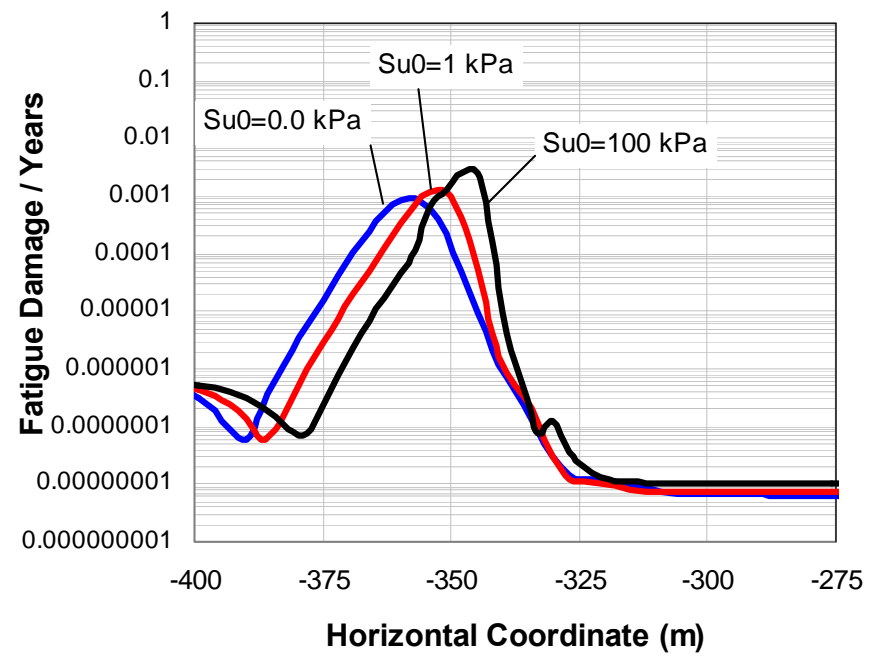

Figure B-43: Fatigue damage distribution in TDZ with various surface soil shear strength

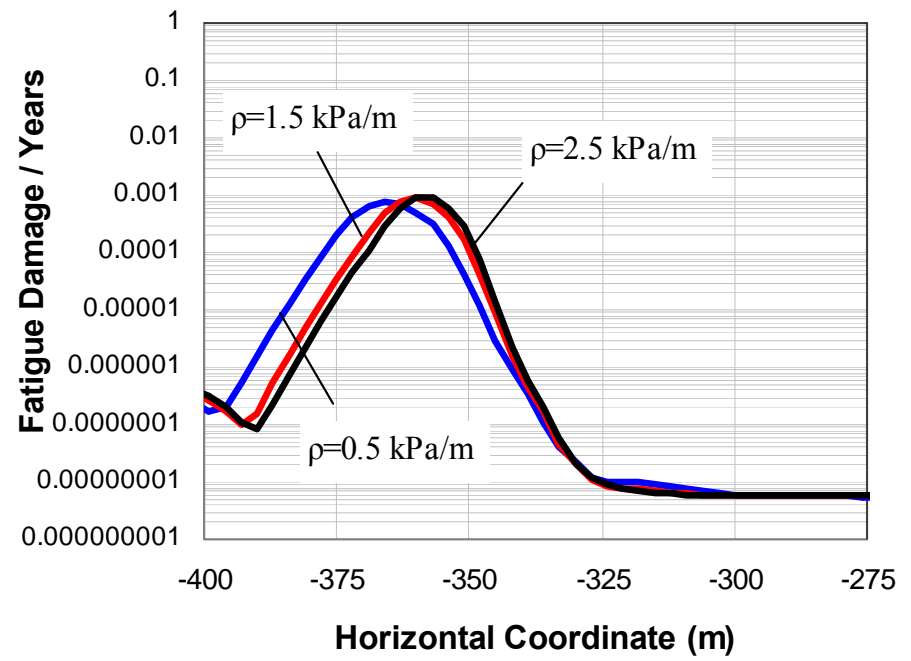

Figure B-44: Fatigue damage distribution in TDZ with various seabed soil shear strength gradient 


\section{B.8 INITIAL PRE-TRENCHING AND FATIGUE PERFORMANCE}

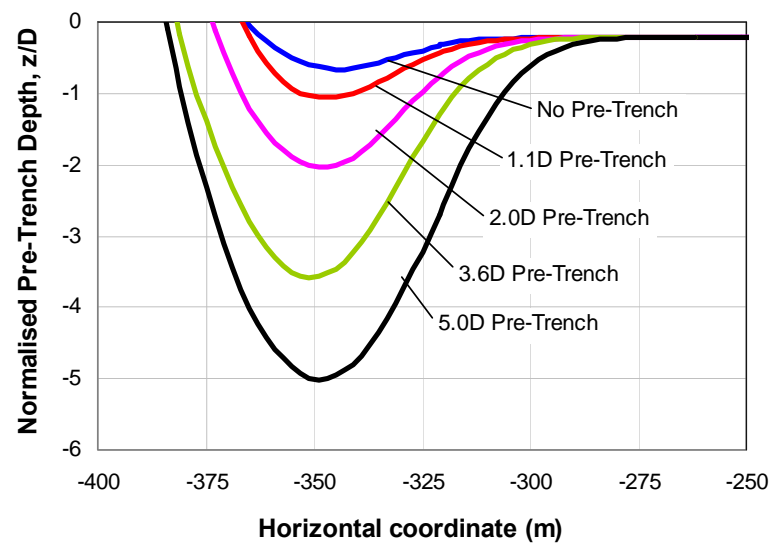

Figure B-45: Initial trench insertion with various depths

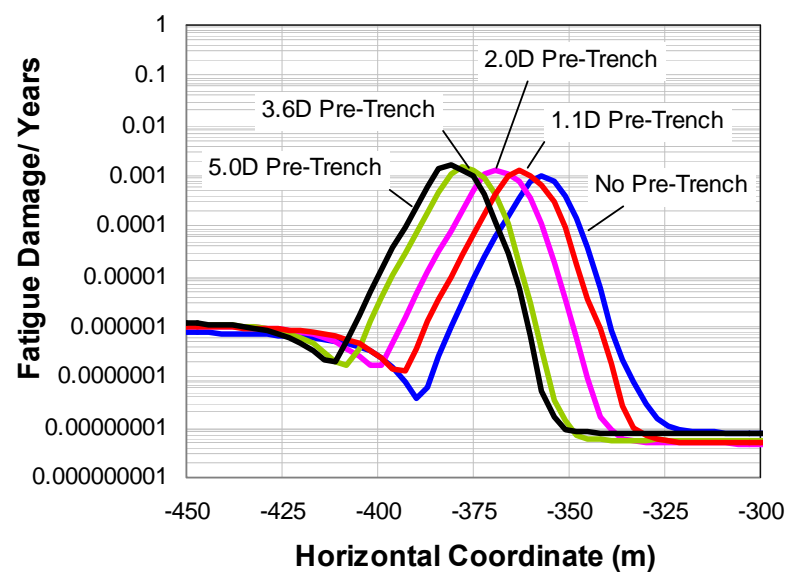

Figure B-46: Logarithmic fatigue damage by the initial trench insertion with various depths

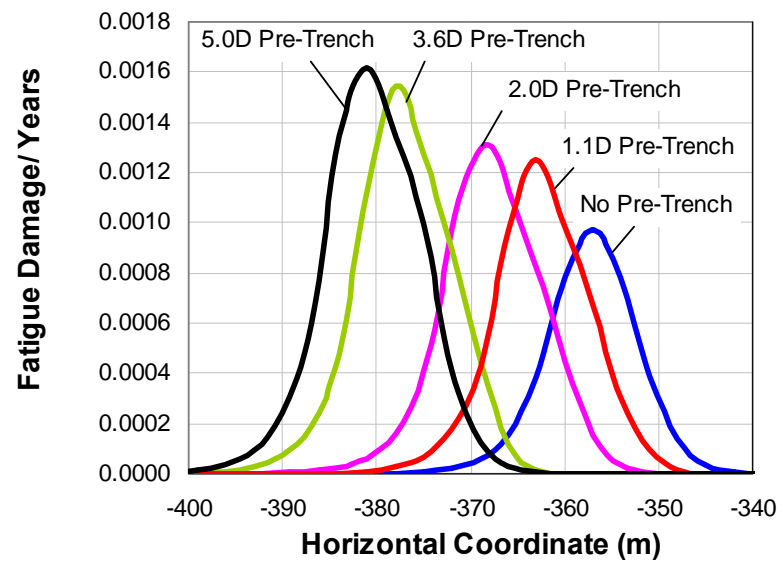

Figure B-47: Non-logarithmic fatigue damage by the initial trench insertion with various depths 


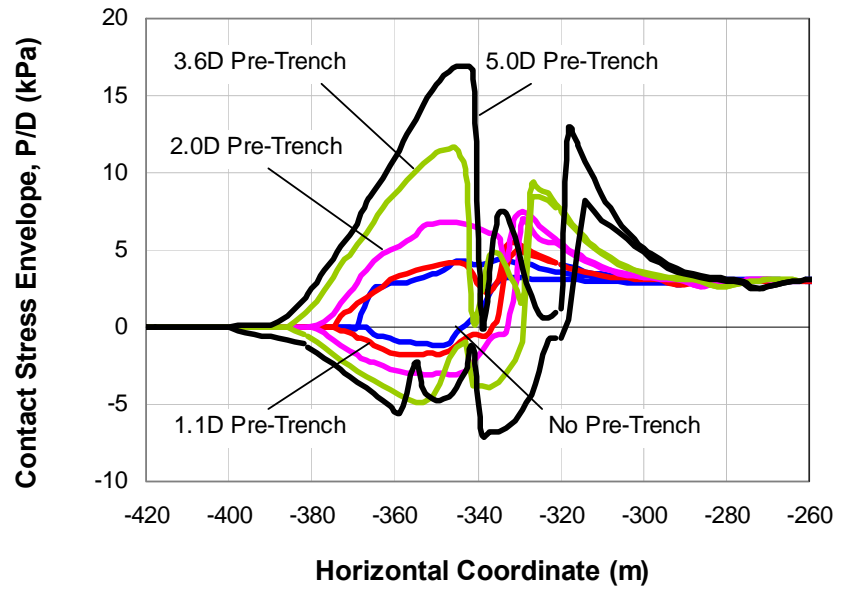

Figure B-48: Final contact stress envelope by the initial trench insertion with various depths

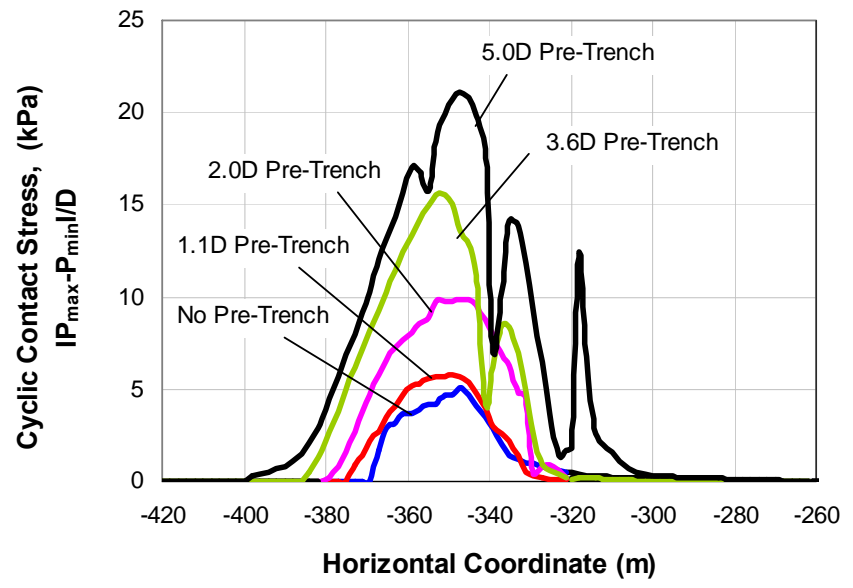

Figure B-49: Cyclic contact stress by the initial trench insertion with various depths

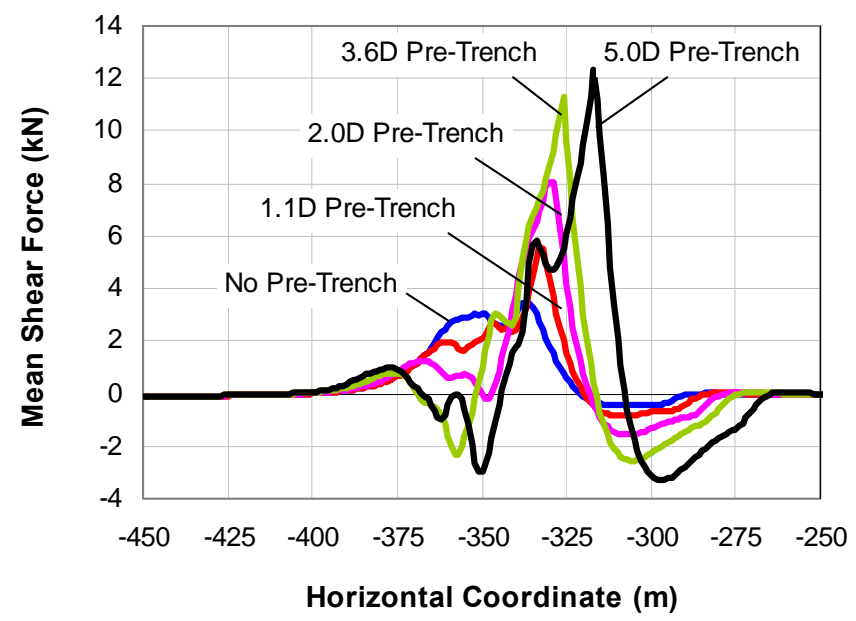

Figure B-50: Mean shear force distribution by the initial trench insertion with various depths 


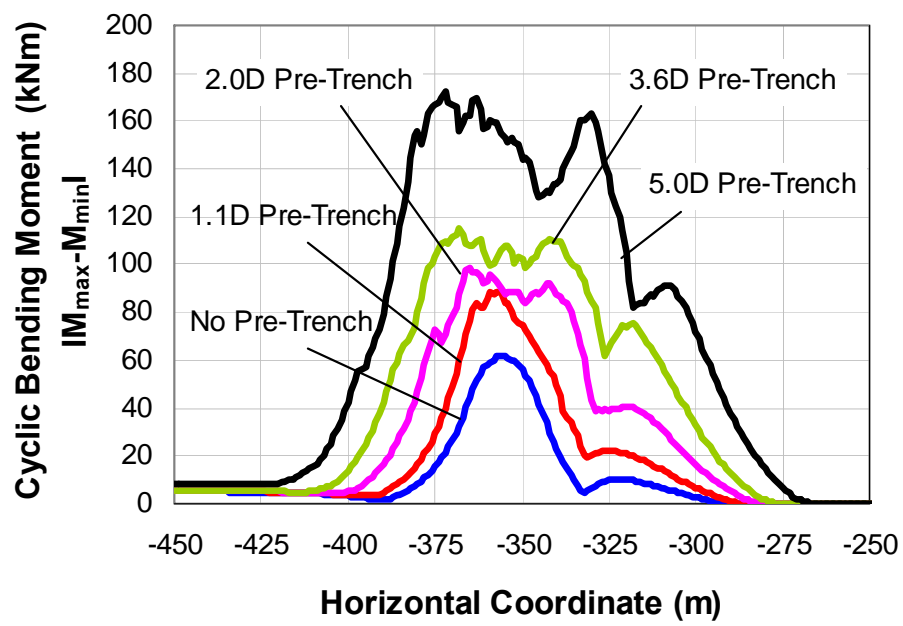

Figure B-51: Cyclic bending moment by the initial trench insertion with various depths 


\section{APPENDIX C.}

\section{PARAMETRIC STUDY OF NON-LINEAR HYSTERETIC SEABED MODEL FOR INDIVIDUAL NODES}

\section{C.1 INTRODUCTION}

The performance of the hysteretic non-linear seabed model was examined for different values of the various input parameters through a series of simulations. A sample of results are presented in the current appendix. The global setup of the steel centenary riser is the same as in Appendix B, repeated here for convenience (Figure C-1 and Table $\mathrm{C}-1$ to Table $\mathrm{C}-3$ ). The locations of key nodes for which results are presented are indicated in Figure C-2. Depending on the various input parameters, the node positions can vary slightly relative to the seabed and touchdown point, but will remain in the general area as indicated.

The model has been studied under a few vessel displacement cycles under quasi-static conditions. Specifically, the vessel has been subjected to 10 cycles of $\pm 5.13 \mathrm{~m}$ of horizontal motion, each of which leads to a TDP translation of approximately $\pm 3.8 \mathrm{~m}$. This appendix summarises the effect of different model parameters on the response at the indicated nodes.

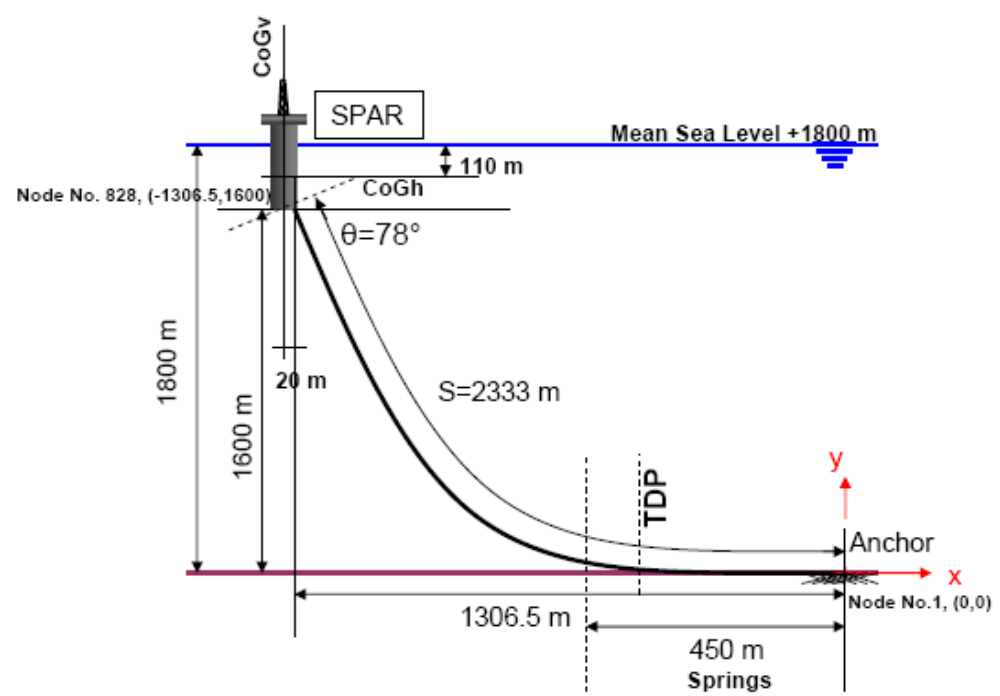

Figure C-1: The global geometry of SCR modelled by ABAQUS 


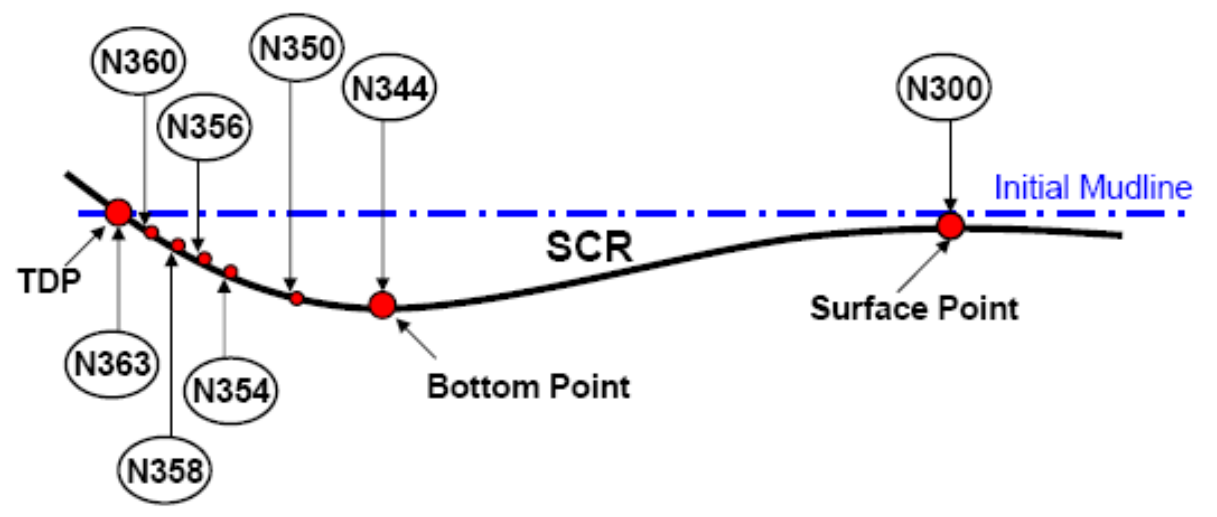

Figure C-2: Schematic position of the reported nodes (can vary with various model input parameters)

Table C-1: Default values of non-linear hysteretic model parameters

\begin{tabular}{|l|c|c|}
\hline \multicolumn{1}{|c|}{ Parameter } & Symbol & Value \\
\hline Mudline shear strength & $\mathrm{s}_{\mathrm{u} 0}$ & $0.65 \mathrm{kPa}$ \\
\hline Shear strength gradient & $\rho$ & $1.5 \mathrm{kPa} / \mathrm{m}$ \\
\hline Power law parameter & $\mathrm{a}$ & 6 \\
\hline Power law parameter & $\mathrm{b}$ & 0.25 \\
\hline Normalized maximum stiffness & $\mathrm{K}_{\max }$ & 200 \\
\hline Suction ratio & $\mathrm{f}_{\text {suc }}$ & 0.3 \\
\hline Suction decay parameter & $\lambda_{\text {suc }}$ & 0.5 \\
\hline Repenetration parameter & $\lambda_{\text {rep }}$ & 0.5 \\
\hline
\end{tabular}

Table C-2: key dimensions of SCR modelled by ABAQUS

\begin{tabular}{|l|c|}
\hline \multicolumn{1}{|c|}{ Dimension } & Value \\
\hline Water depth & $1800 \mathrm{~m}$ \\
\hline Height of attachment point & $1600 \mathrm{~m}$ \\
\hline SCR length & $2333 \mathrm{~m}$ \\
\hline Vessel distance from anchor end & $1306.5 \mathrm{~m}$ \\
\hline Lay angle & $78^{\circ}$ \\
\hline Attachment point offset from COG & $20 \mathrm{~m}$ \\
\hline Length of springs zone at seabed & $450 \mathrm{~m}$ \\
\hline Spacing of seabed springs & $1.0 \mathrm{~m}$ \\
\hline
\end{tabular}


Table C-3 : Riser pipe properties

\begin{tabular}{|l|c|}
\hline \multicolumn{1}{|c|}{ Dimension } & Value \\
\hline Outer Diameter, $\mathrm{D}_{\mathrm{o}}$ & $0.324 \mathrm{~m}\left(12^{3 / 4}\right.$ “ $)$ \\
\hline Wall Thickness, $\mathrm{t}$ & $0.0205 \mathrm{~m}$ \\
\hline Second Moment of Inertia, I & $2.26 \times 10^{-4} \mathrm{~m}^{4}$ \\
\hline Steel Density, $\rho_{\mathrm{s}}$ & $7850 \mathrm{~kg} / \mathrm{m}^{3}$ \\
\hline In service Submerged Weight, $\mathrm{m}_{\mathrm{s}}$ & $100 \mathrm{~kg} / \mathrm{m}$ \\
\hline Fatigue S-N Curve & $\begin{array}{l}\mathrm{DNV}(2008), \mathrm{E} \text { Class weld, } \overline{\mathrm{a}}=1.05 \times 10^{-12} \\
\mathrm{~m}=3.0, \mathrm{SCF}=1.13\end{array}$ \\
\hline
\end{tabular}




\section{C.2 NORMALISED REPENETRATION OFFSET}

$$
\lambda_{\text {rep }}=\mathbf{0 . 0}
$$
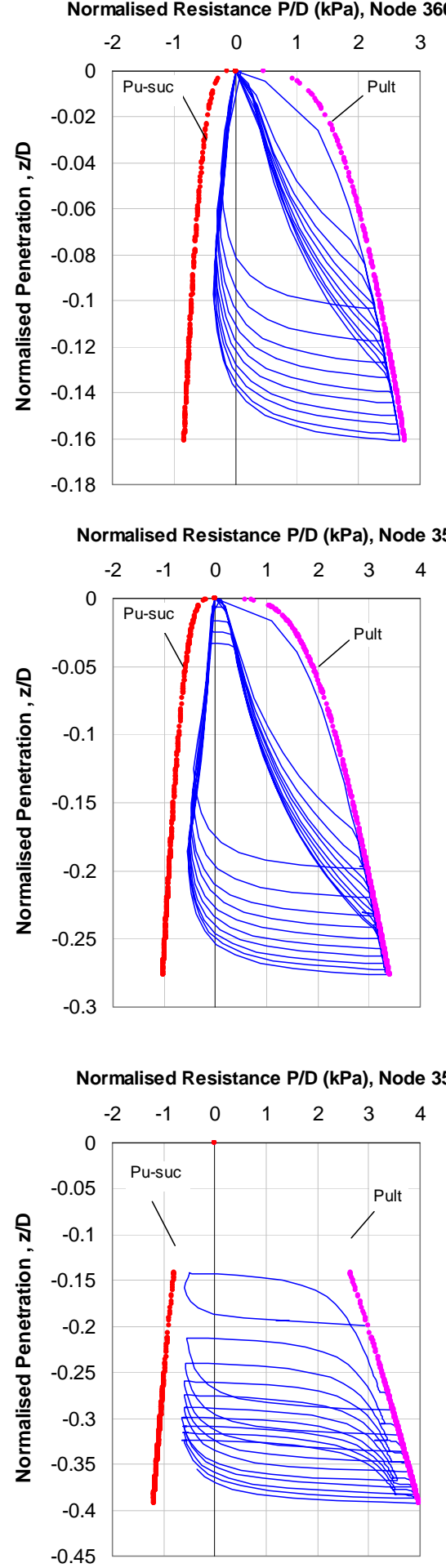
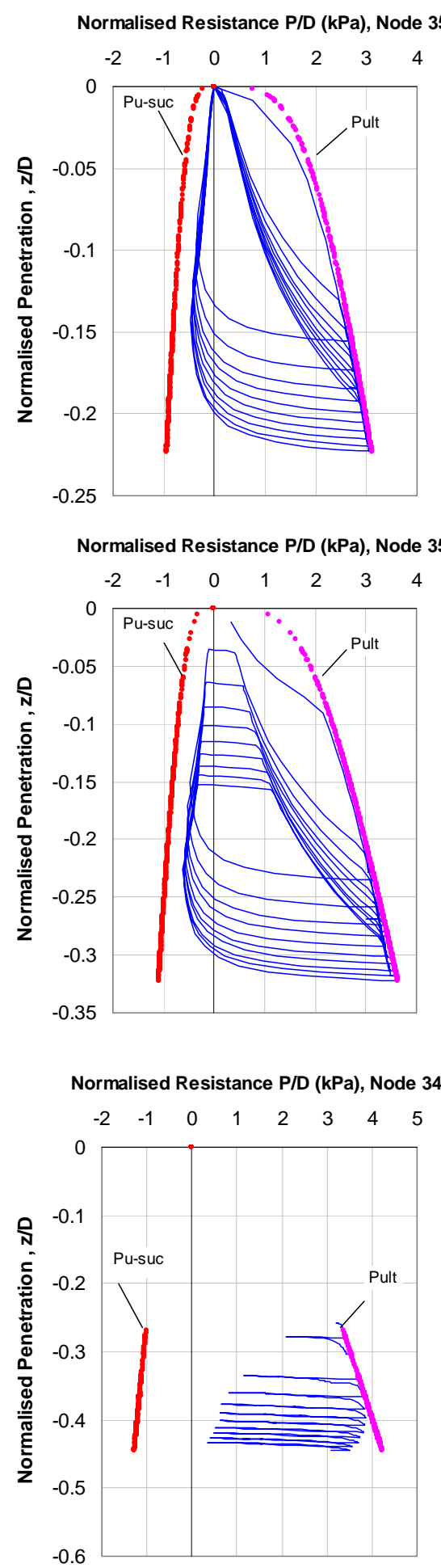


$$
\lambda_{\text {rep }}=0.2
$$
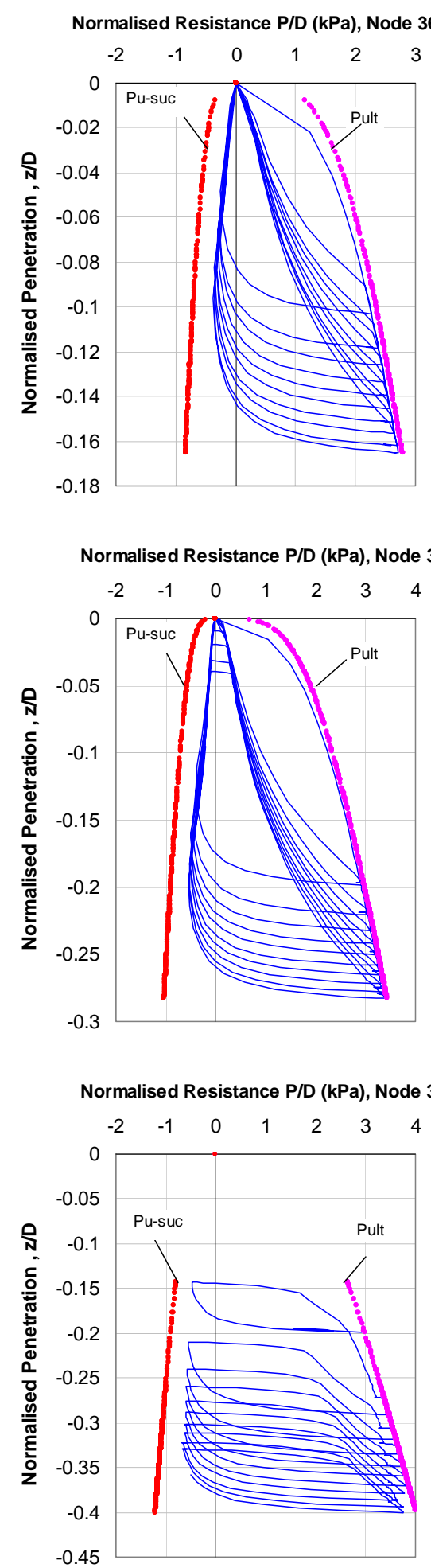

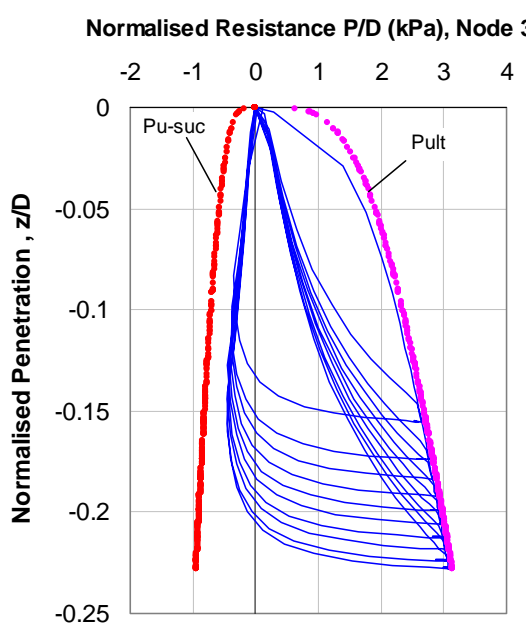

Normalised Resistance P/D (kPa), Node 354

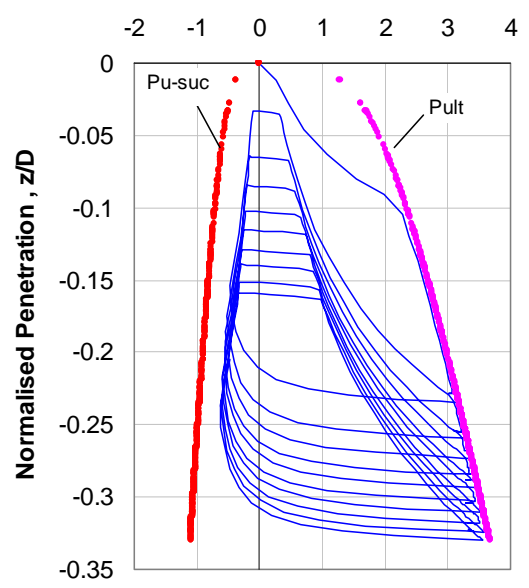

Normalised Resistance PID (kPa), Node 344

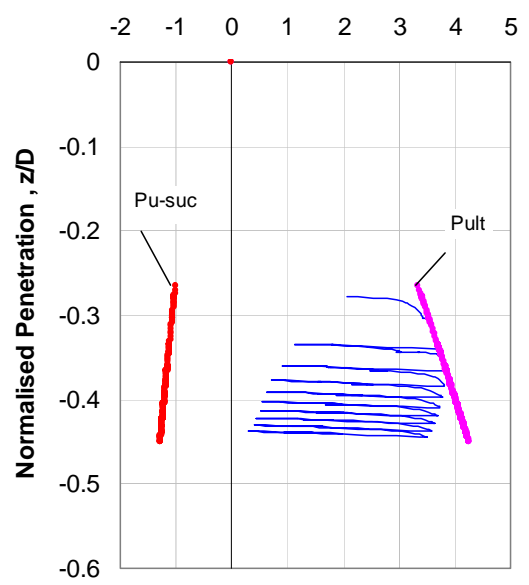




$$
\lambda_{\text {rep }}=0.5
$$
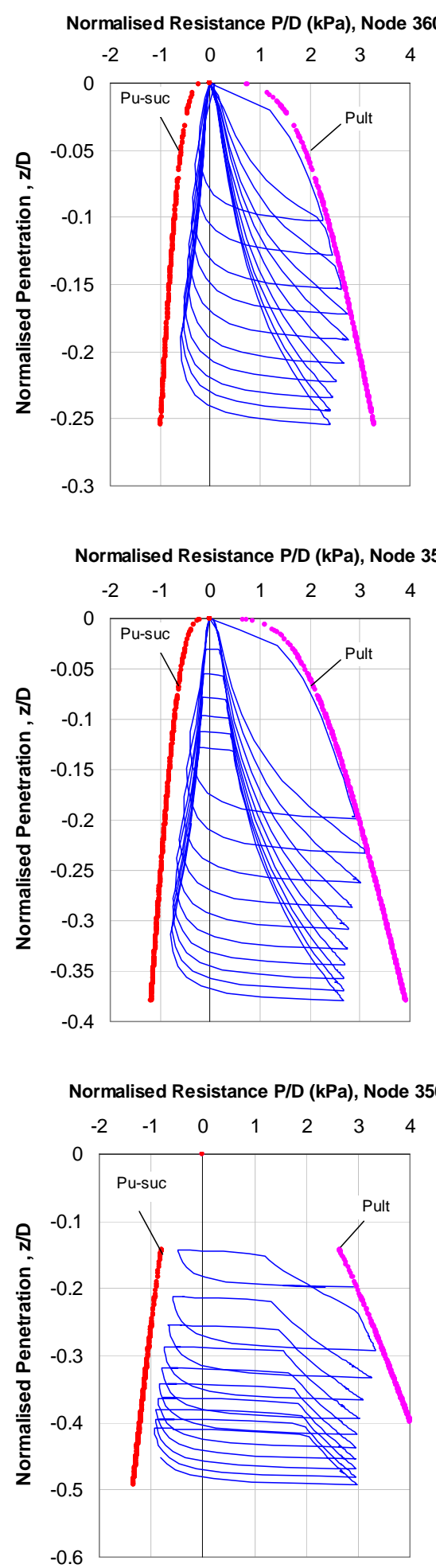
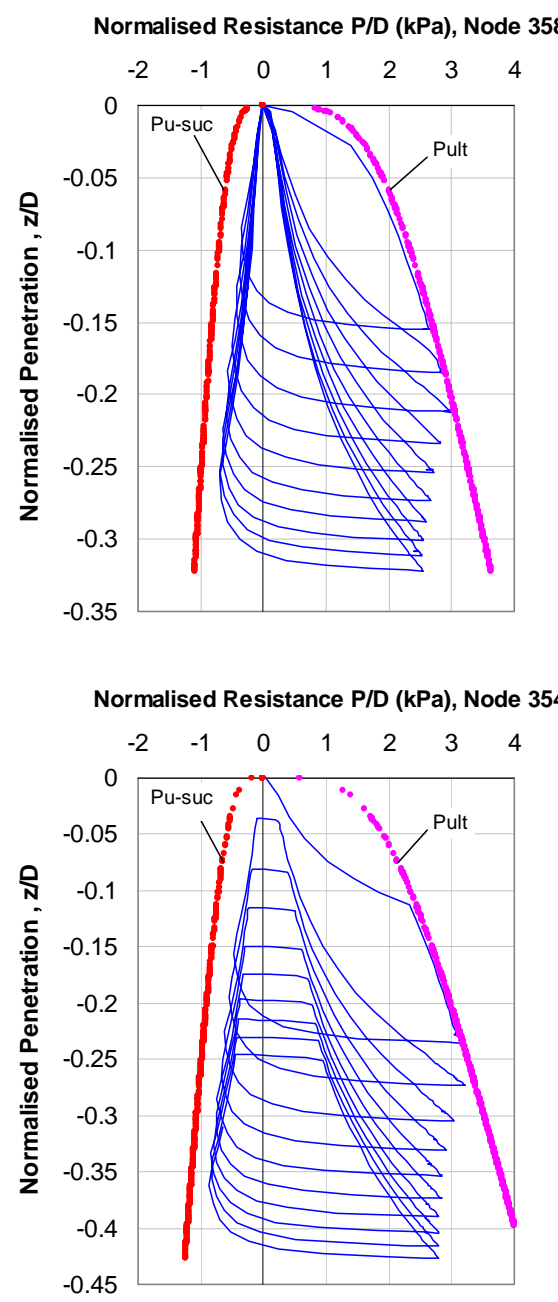

Normalised Resistance P/D (kPa), Node 344

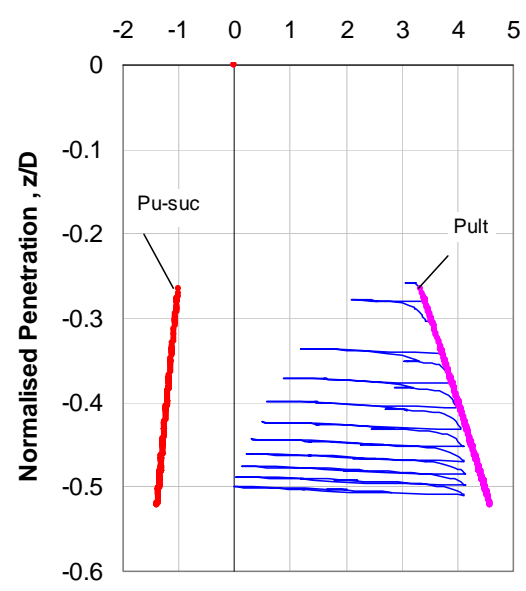




$$
\lambda_{\text {rep }}=0.8
$$

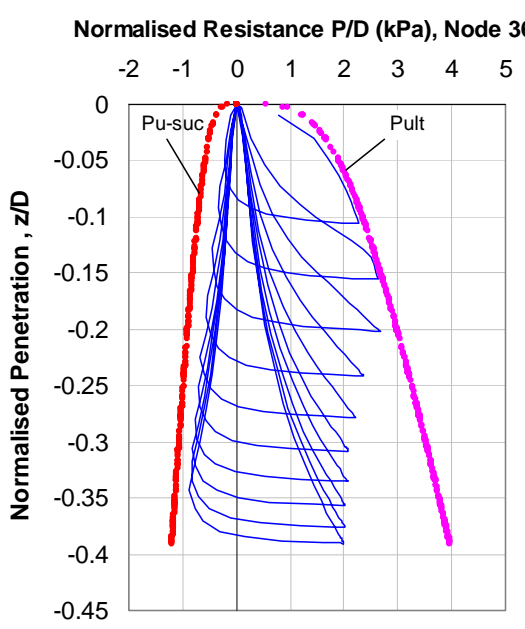

Normalised Resistance P/D (kPa), Node 356

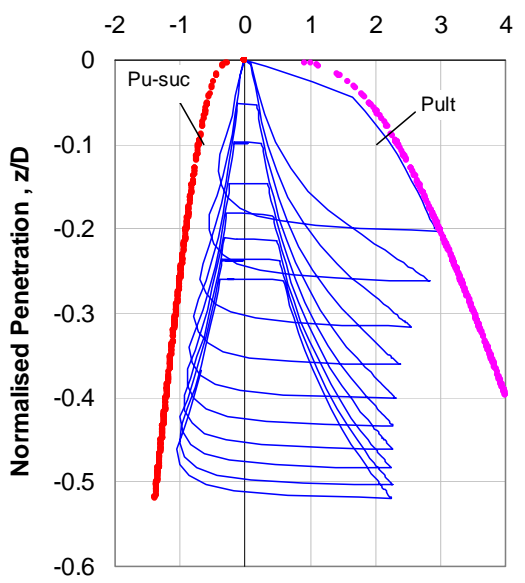

Normalised Resistance P/D (kPa), Node 350

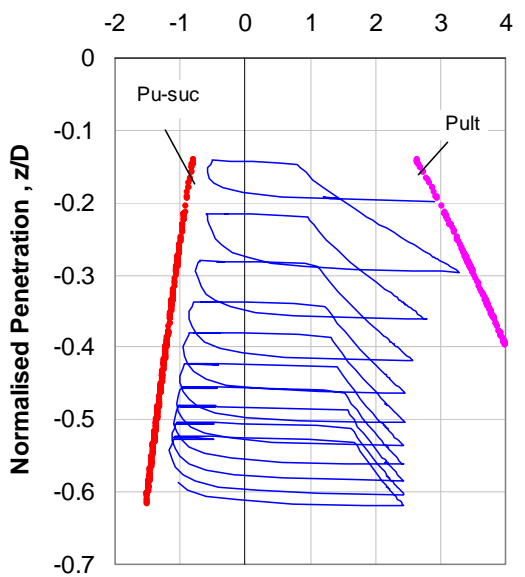

Normalised Resistance P/D (kPa), Node 358

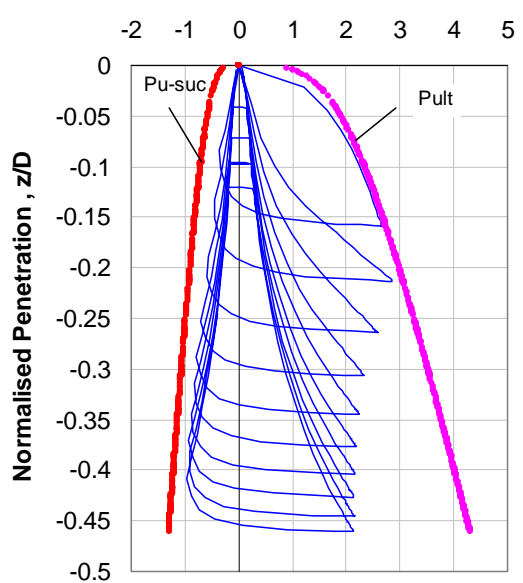

Normalised Resistance P/D (kPa), Node 354

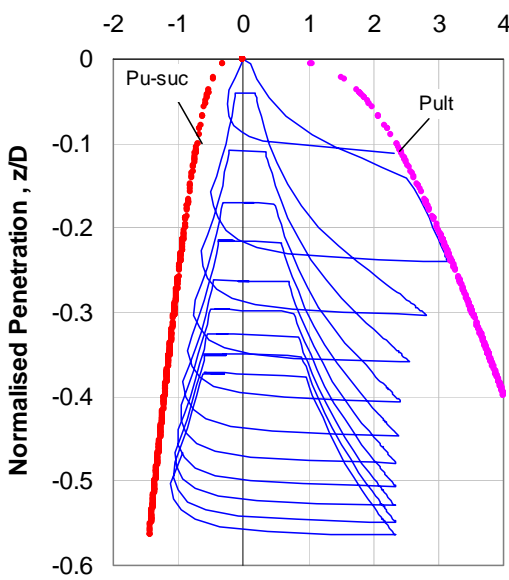

Normalised Resistance PID (kPa), Node 344

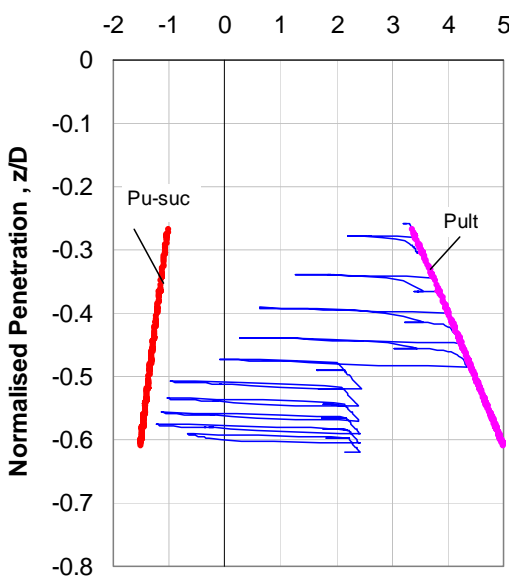




$$
\lambda_{\text {rep }}=\mathbf{1 . 0}
$$

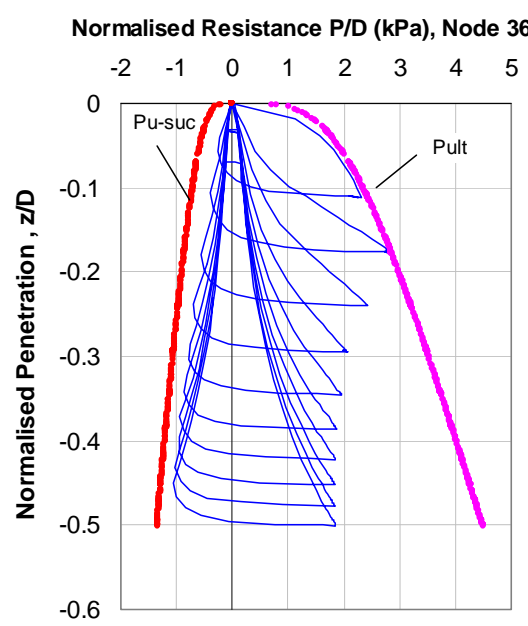

Normalised Resistance PID (kPa), Node 356

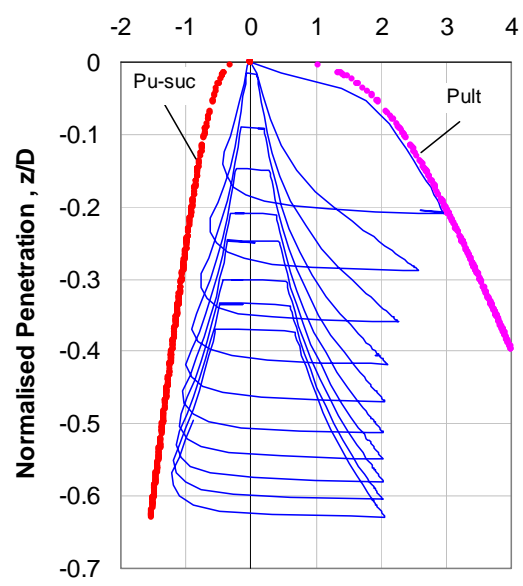

Normalised Resistance P/D (kPa), Node 350

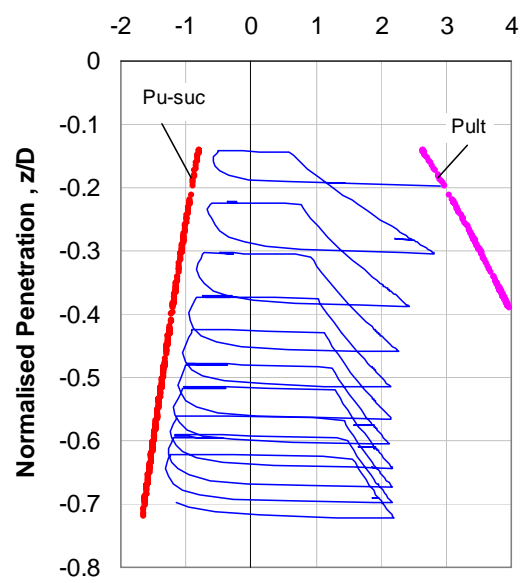

Normalised Resistance P/D (kPa), Node 358

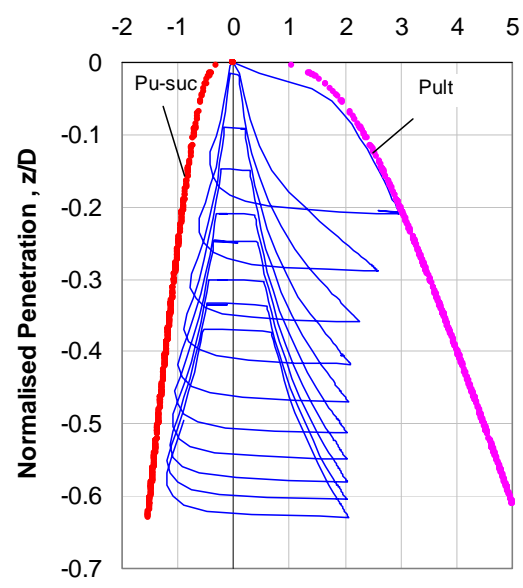

Normalised Resistance P/D (kPa), Node 354

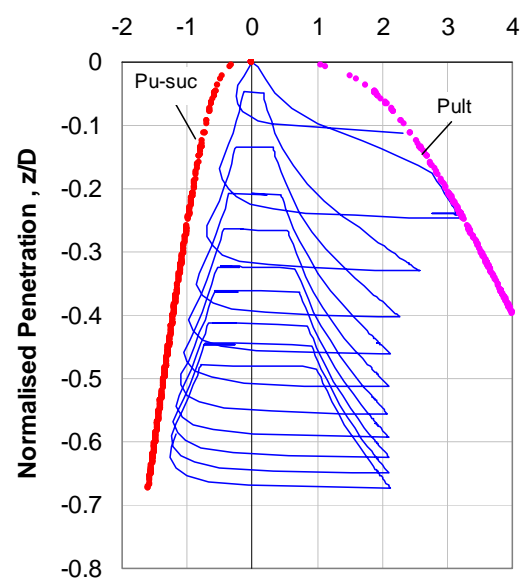

Normalised Resistance P/D (kPa), Node 344

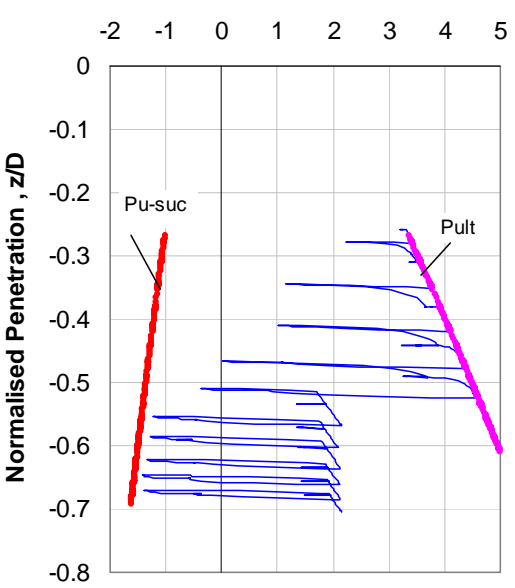




\section{C.3 NORMALISED SUCTION DECAY DISTANCE}

$$
\lambda_{\text {suc }}=0.2
$$

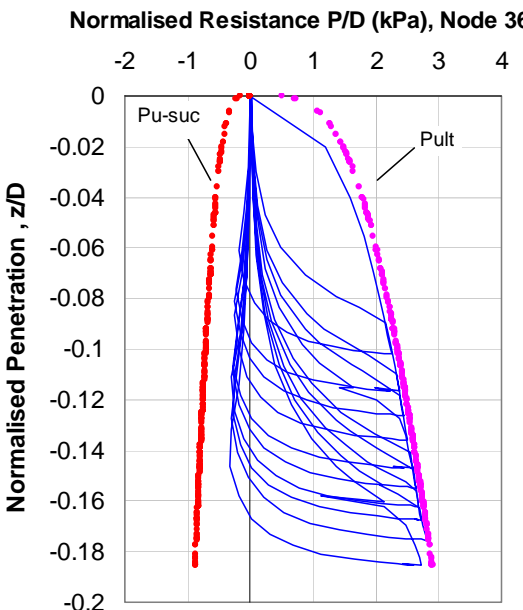

Normalised Resistance P/D (kPa), Node 356

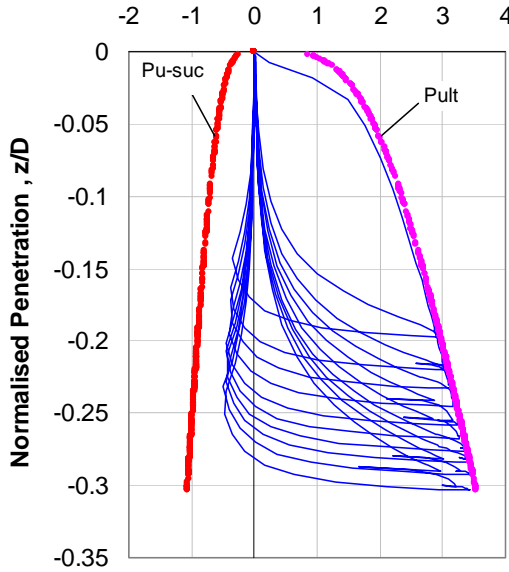

Normalised Resistance P/D (kPa), Node 350

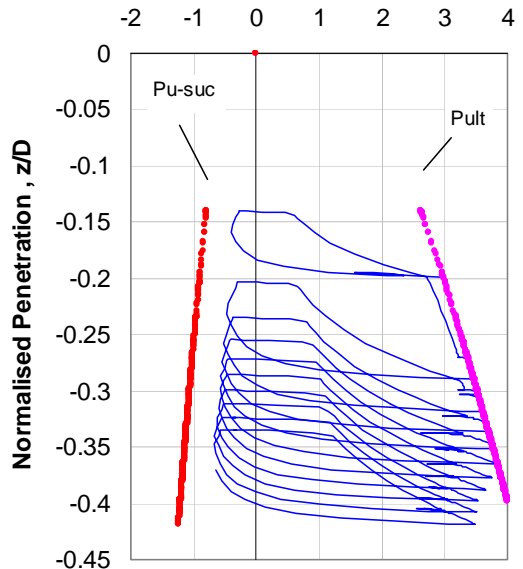

Normalised Resistance P/D (kPa), Node 358

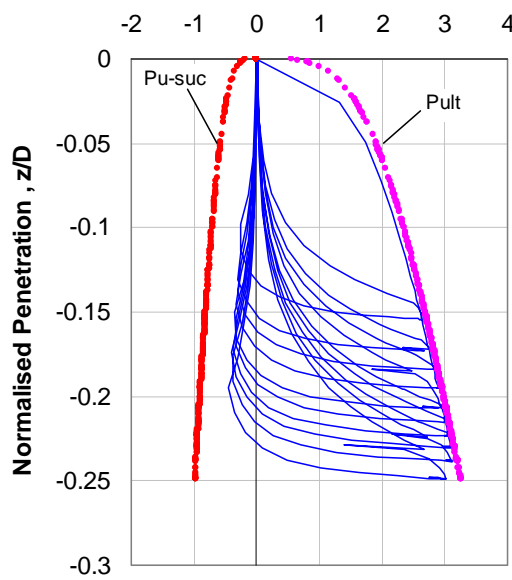

Normalised Resistance P/D (kPa), Node 354

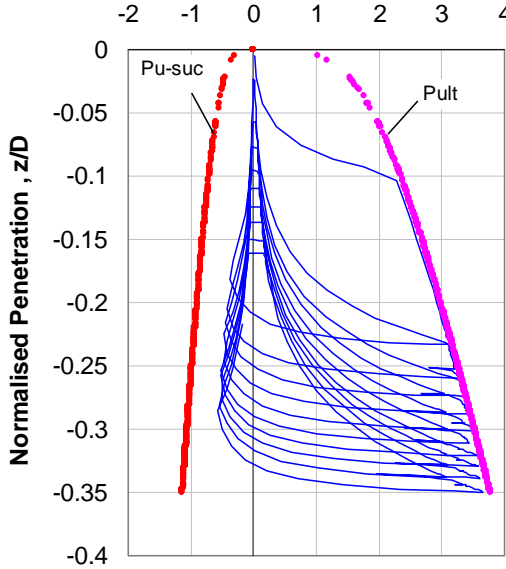

Normalised Resistance PID (kPa), Node 344 $\begin{array}{llllllll}-2 & -1 & 0 & 1 & 2 & 3 & 4 & 5\end{array}$

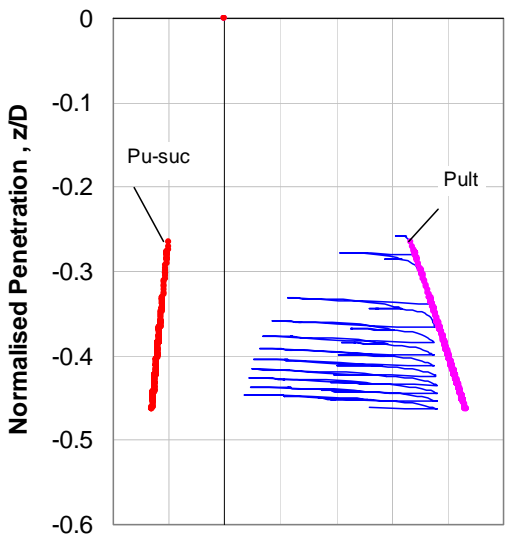




$$
\lambda_{\text {suc }}=0.5
$$
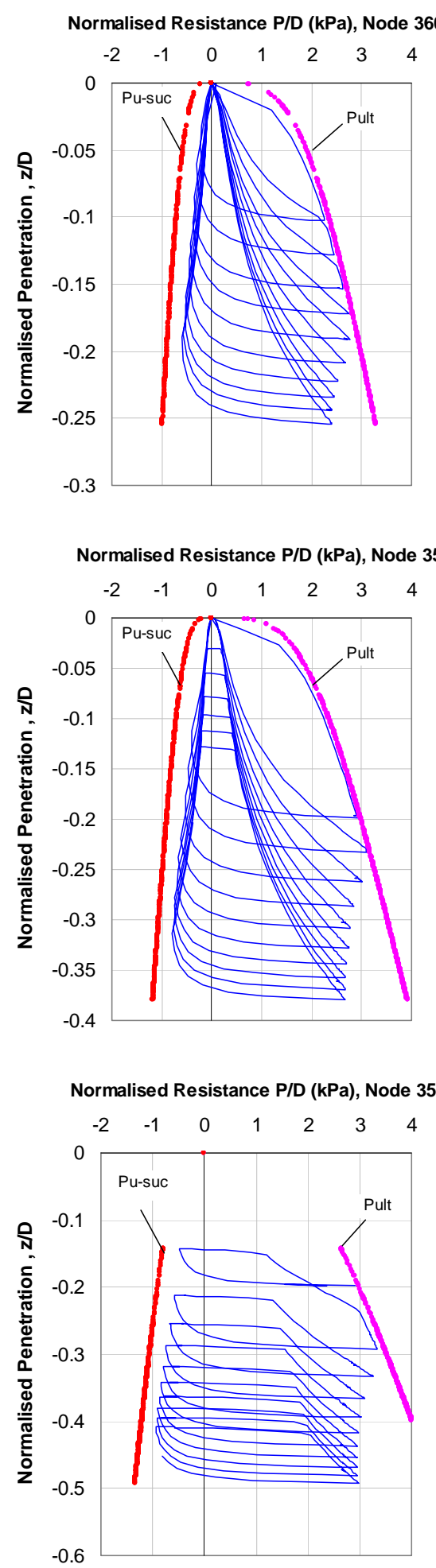
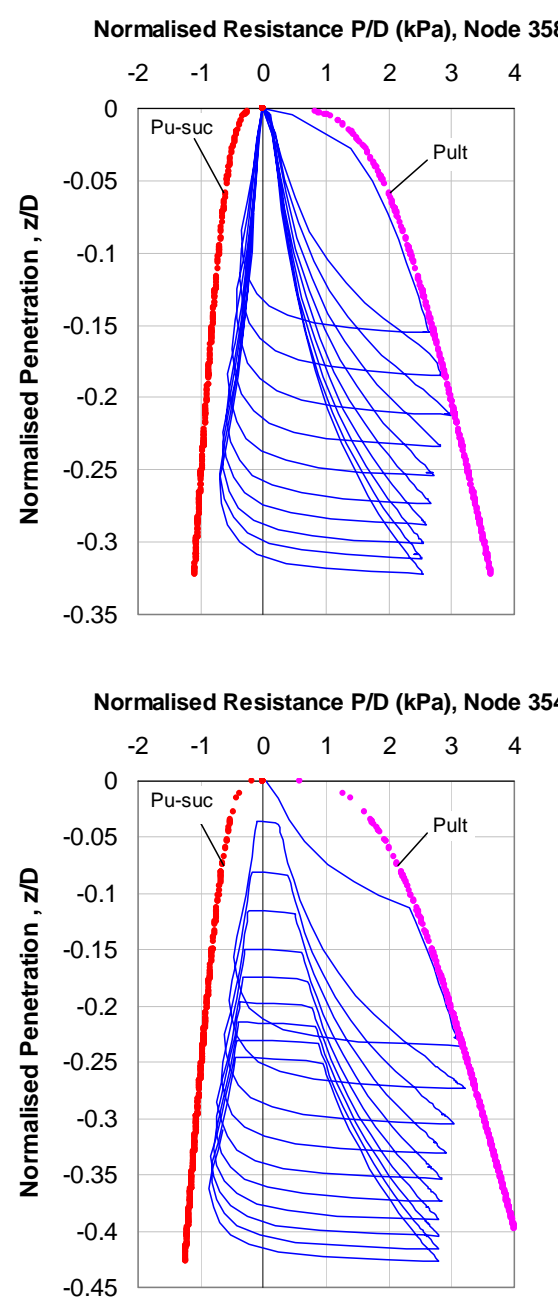

Normalised Resistance P/D (kPa), Node 344

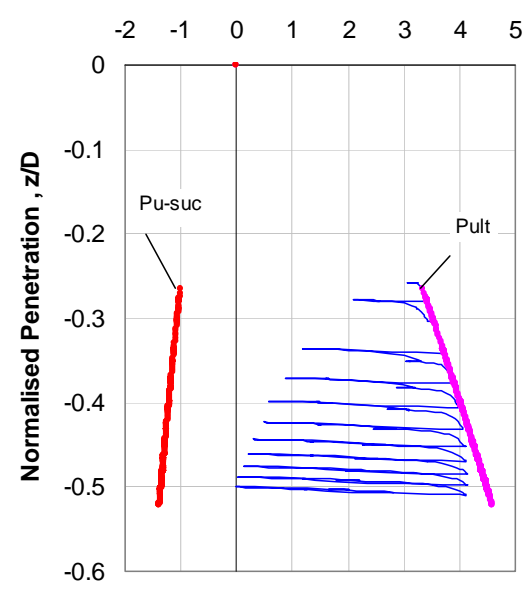




$$
\lambda_{\text {suc }}=\mathbf{1 . 0}
$$
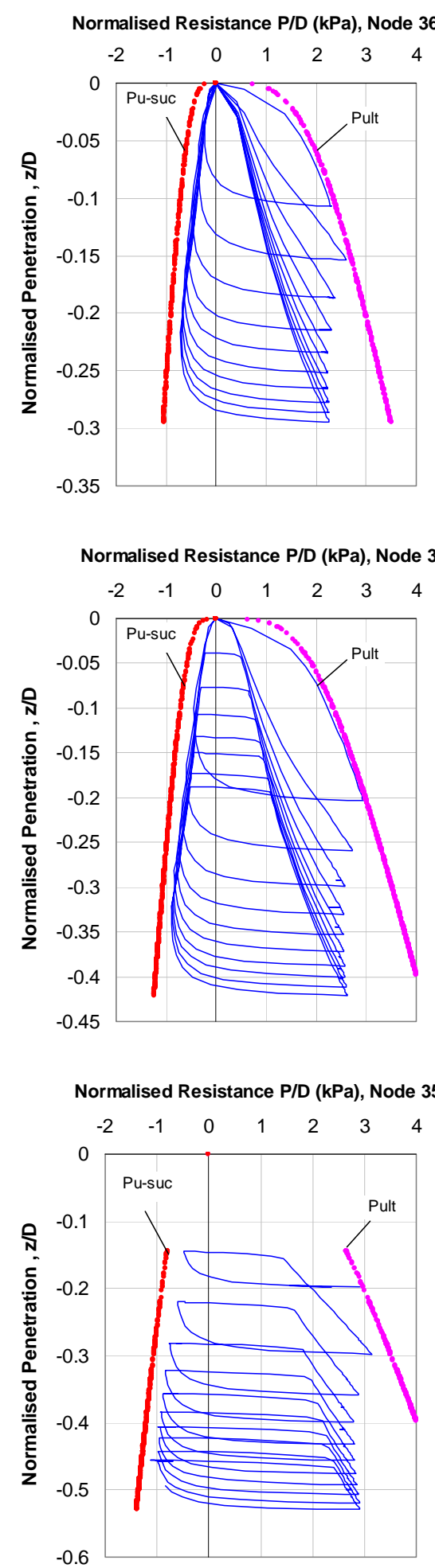

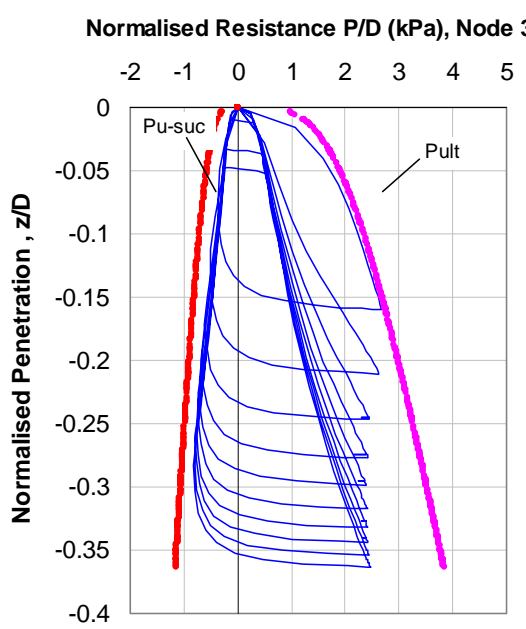

Normalised Resistance PID (kPa), Node 354

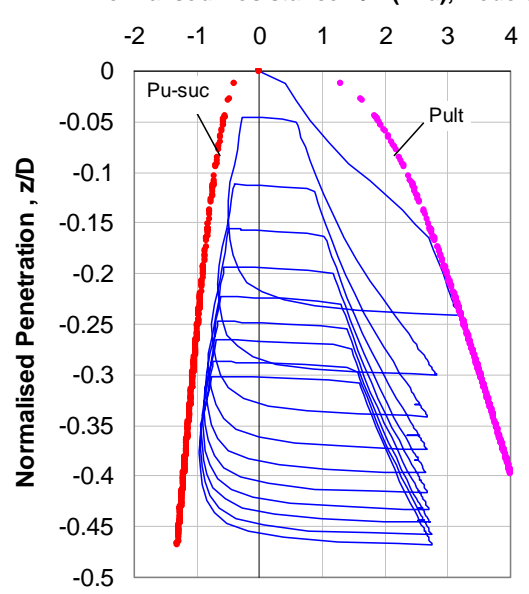

Normalised Resistance PID (kPa), Node 344

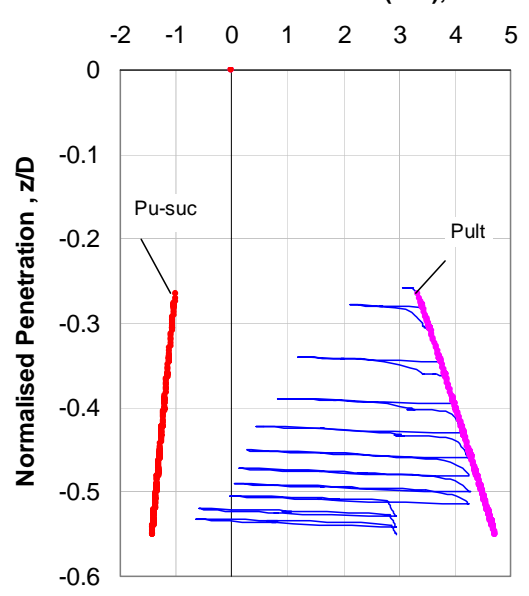




\section{C.4 SUCTION RESISTANCE RATIO}

$$
f_{\text {suc }}=\mathbf{0 . 3}
$$

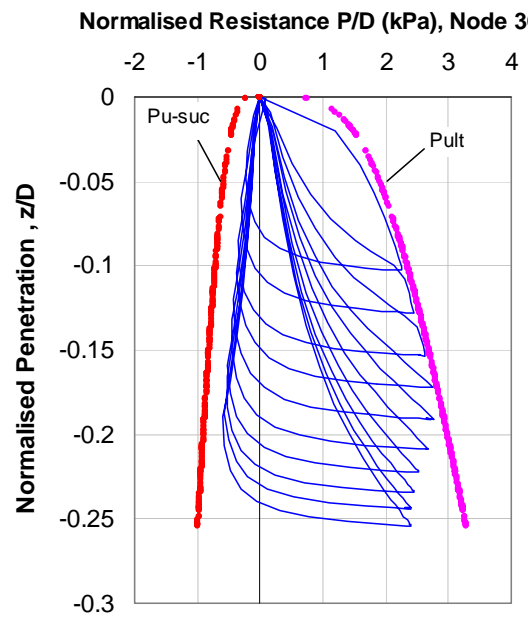

Normalised Resistance P/D (kPa), Node 356

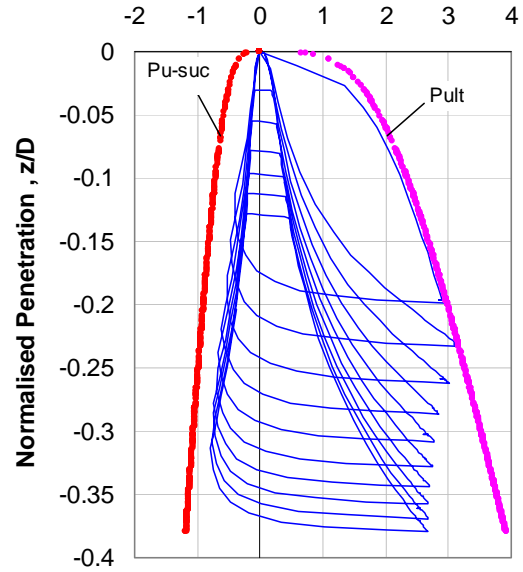

Normalised Resistance P/D (kPa), Node 350

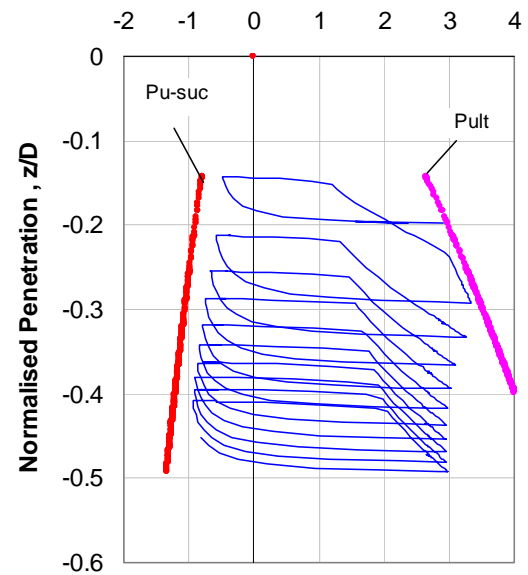

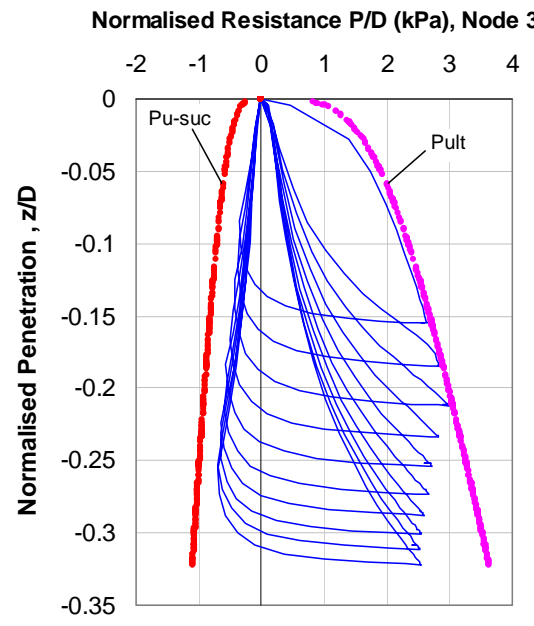

Normalised Resistance P/D (kPa), Node 354

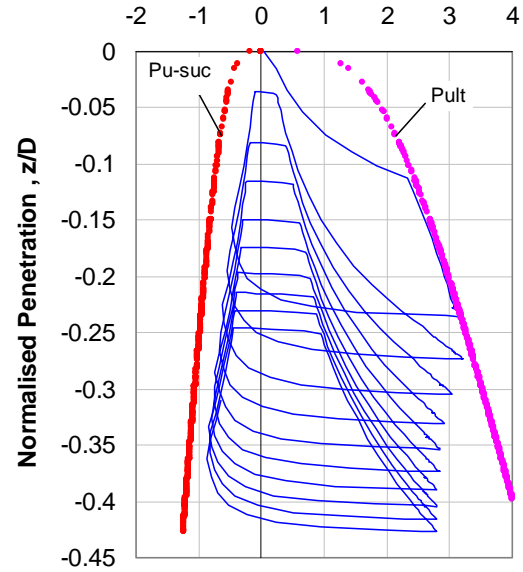

Normalised Resistance P/D (kPa), Node 344 $\begin{array}{llllllll}-2 & -1 & 0 & 1 & 2 & 3 & 4 & 5\end{array}$

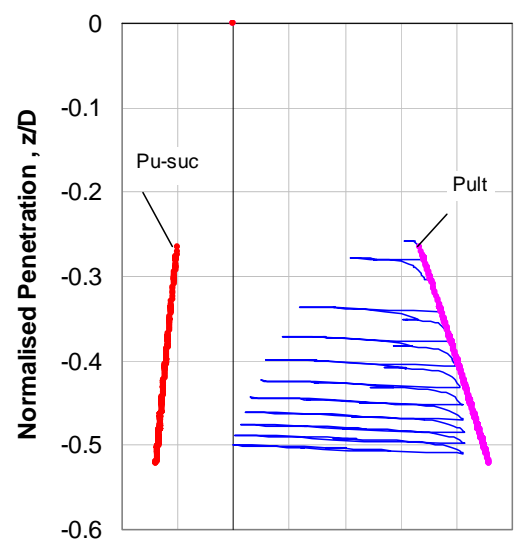




$$
\mathbf{f}_{\text {suc }}=0.5
$$

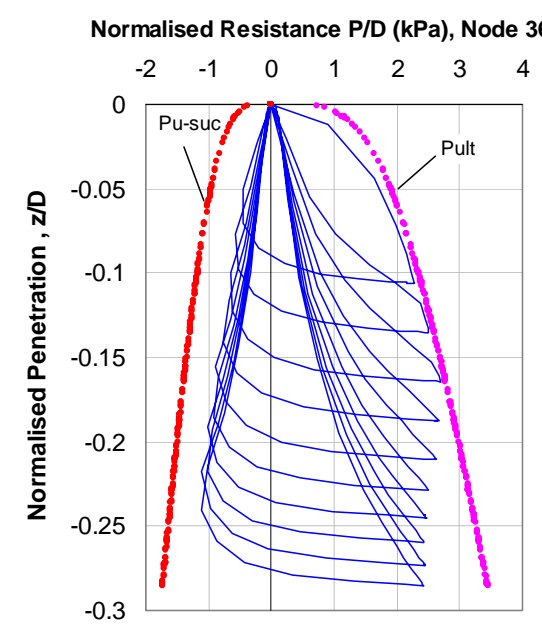

Normalised Resistance P/D (kPa), Node 356

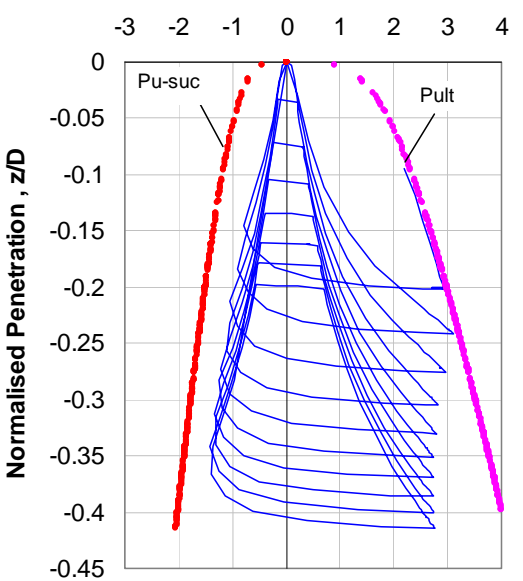

Normalised Resistance P/D (kPa), Node 350

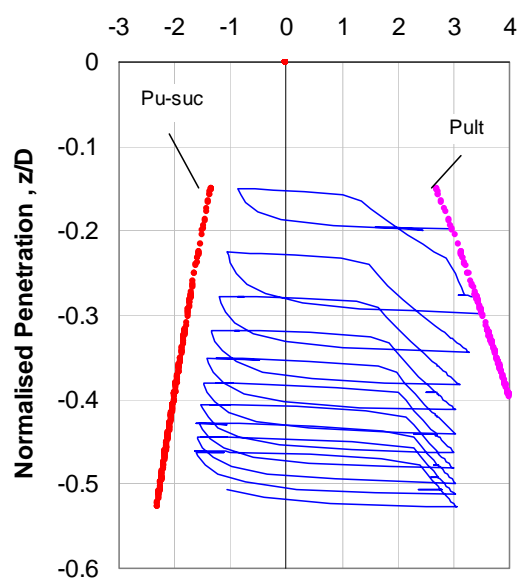

Normalised Resistance P/D (kPa), Node 358

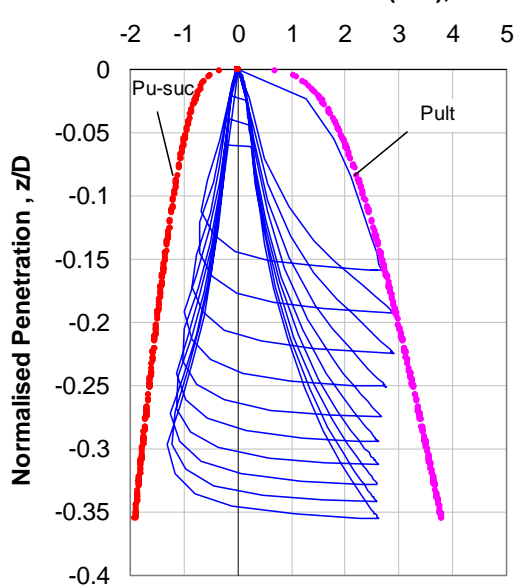

Normalised Resistance P/D (kPa), Node 354

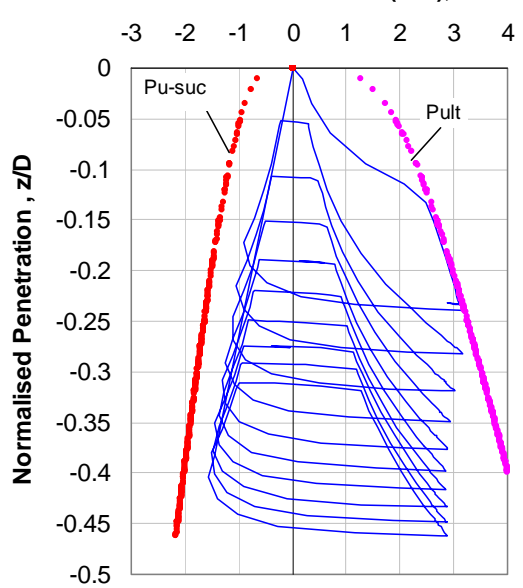

Normalised Resistance PID (kPa), Node 344

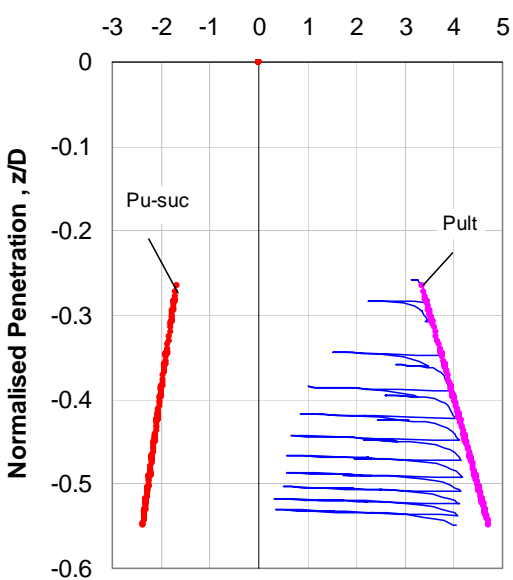




$$
\mathbf{f}_{\text {suc }}=\mathbf{1 . 0}
$$
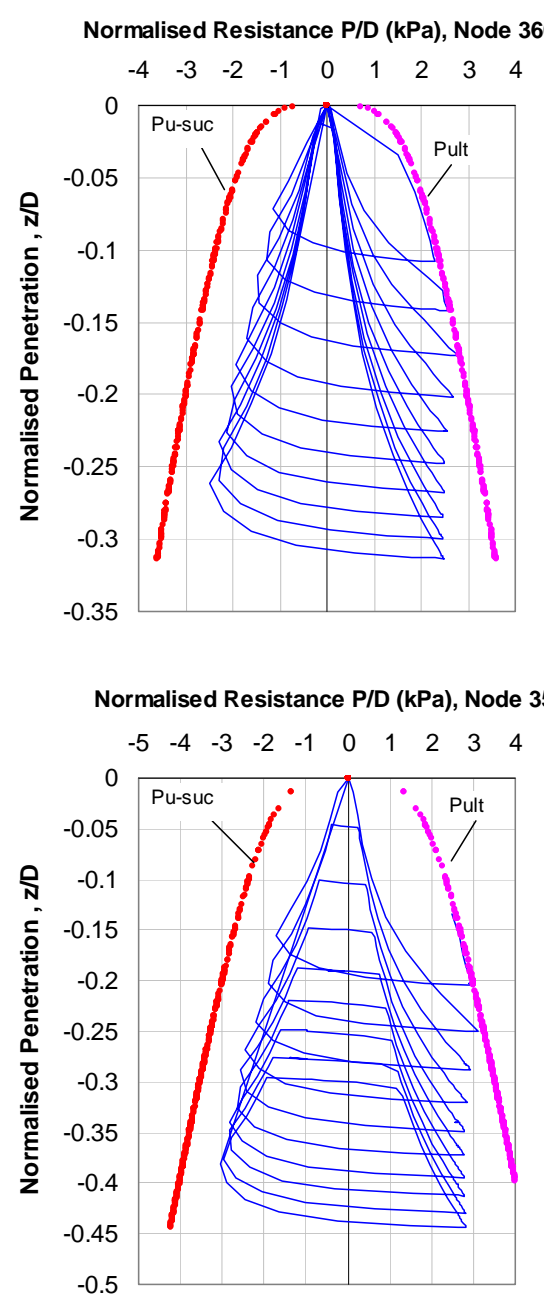

Normalised Resistance P/D (kPa), Node 350 $\begin{array}{lllllllllll}-6 & -5 & -4 & -3 & -2 & -1 & 0 & 1 & 2 & 3 & 4\end{array}$

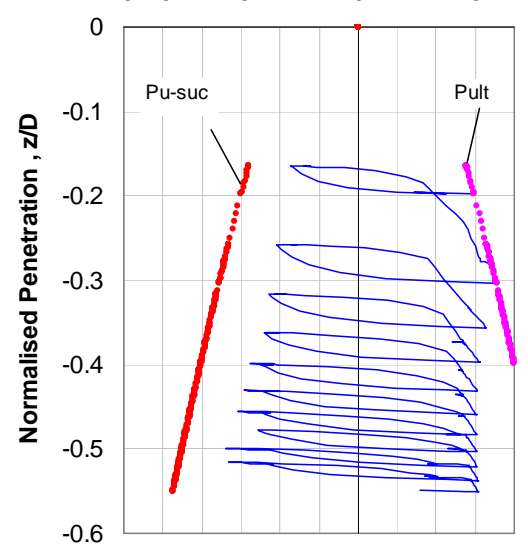

Normalised Resistance P/D (kPa), Node 358

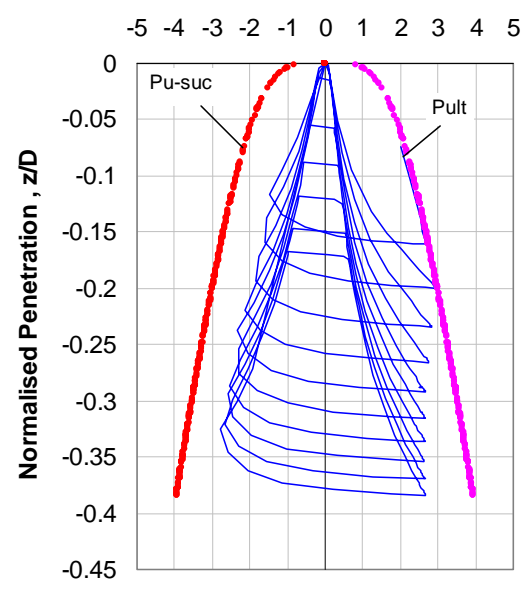

Normalised Resistance P/D (kPa), Node 354 $\begin{array}{llllllllll}-5 & -4 & -3 & -2 & -1 & 0 & 1 & 2 & 3 & 4\end{array}$

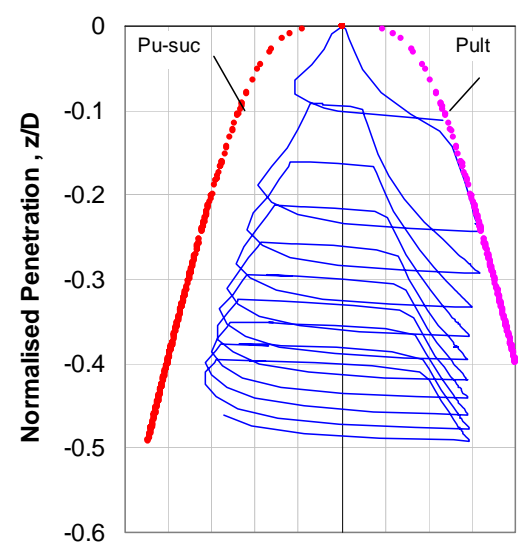

Normalised Resistance P/D (kPa), Node 344 $\begin{array}{lllllllllllll}-6 & -5 & -4 & -3 & -2 & -1 & 0 & 1 & 2 & 3 & 4 & 5\end{array}$

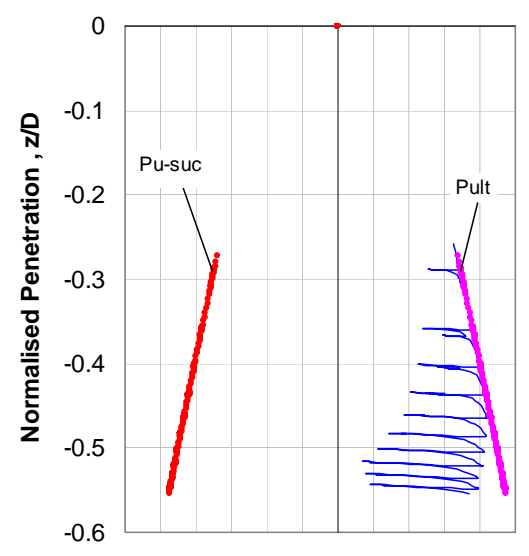




\section{C.5 SOIL UNDRAINED SHEAR STRENGTH AT MUDLINE}

$$
\mathrm{s}_{\mathrm{u} 0}=0.0 \mathrm{kPa}
$$

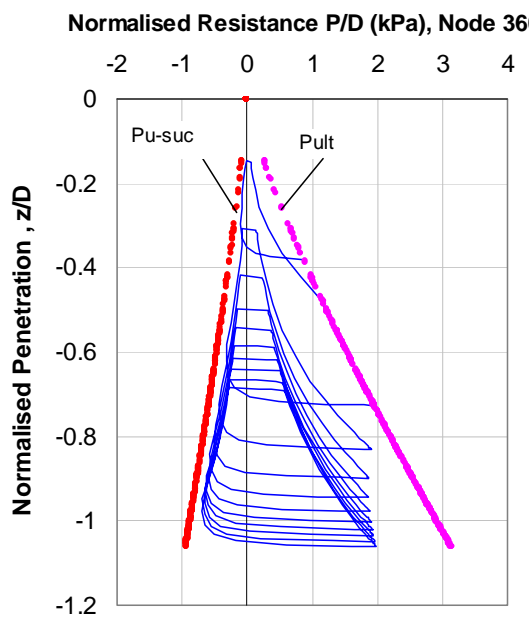

Normalised Resistance P/D (kPa), Node 356

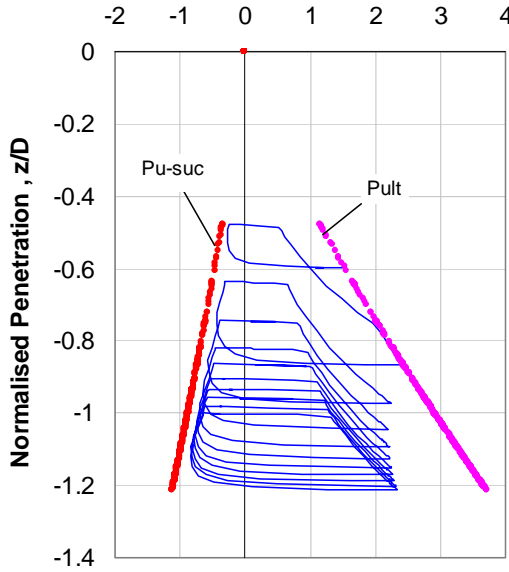

Normalised Resistance P/D (kPa), Node 350

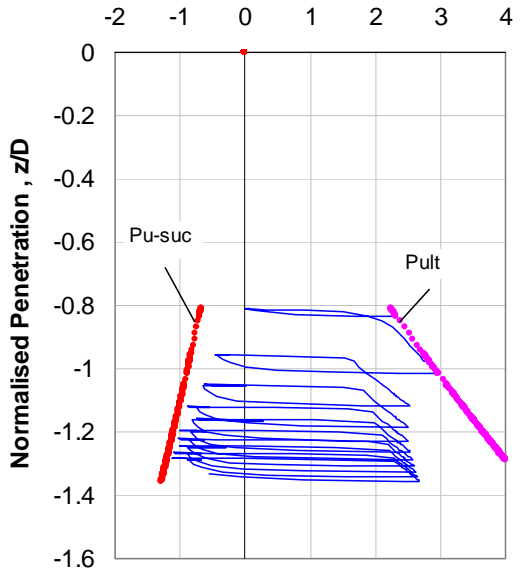

Normalised Resistance P/D (kPa), Node 358

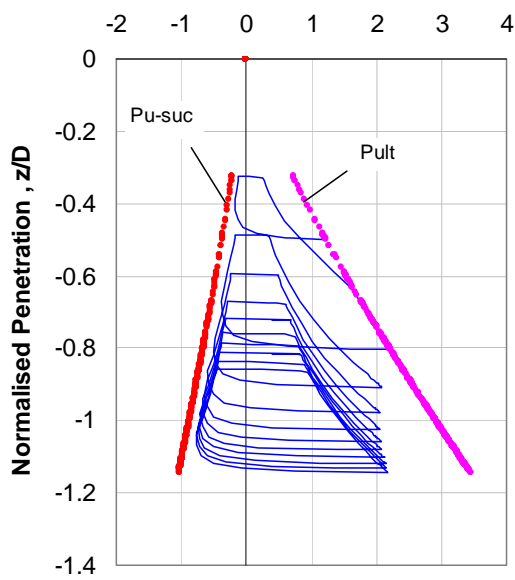

Normalised Resistance PID (kPa), Node 354

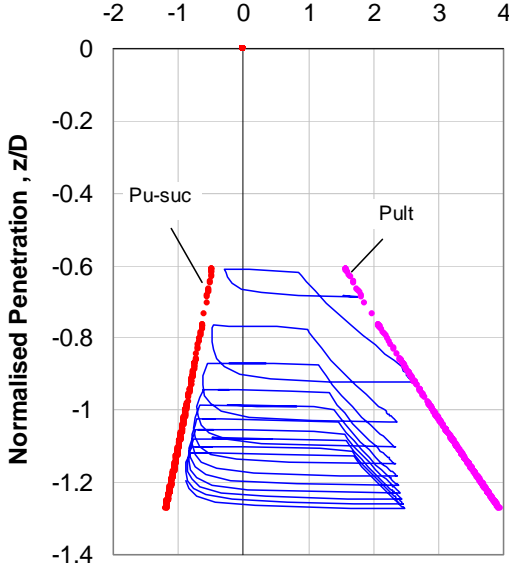

Normalised Resistance PID (kPa), Node 344

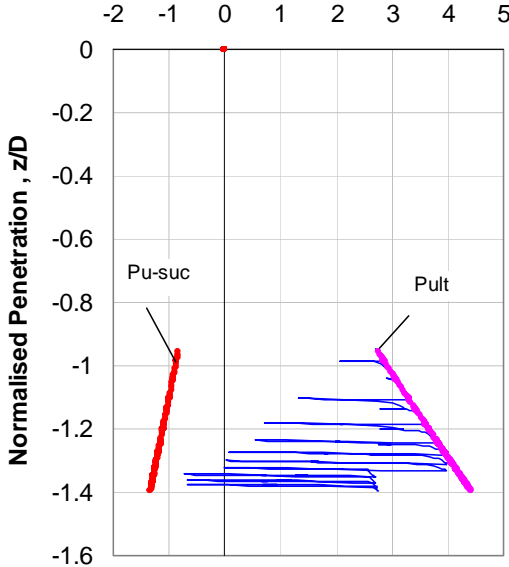




$$
\mathrm{s}_{\mathrm{u} 0}=0.35 \mathrm{kPa}
$$

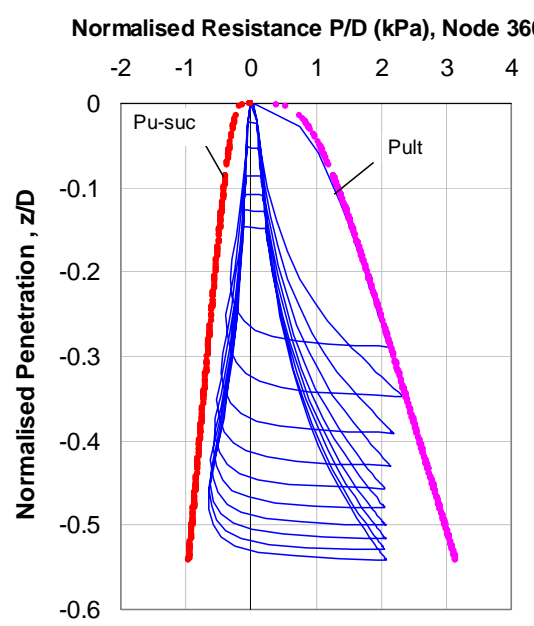

Normalised Resistance P/D (kPa), Node 356

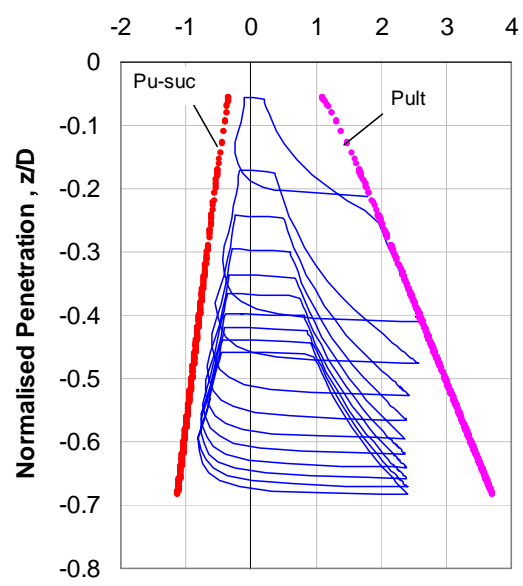

Normalised Resistance PID (kPa), Node 350

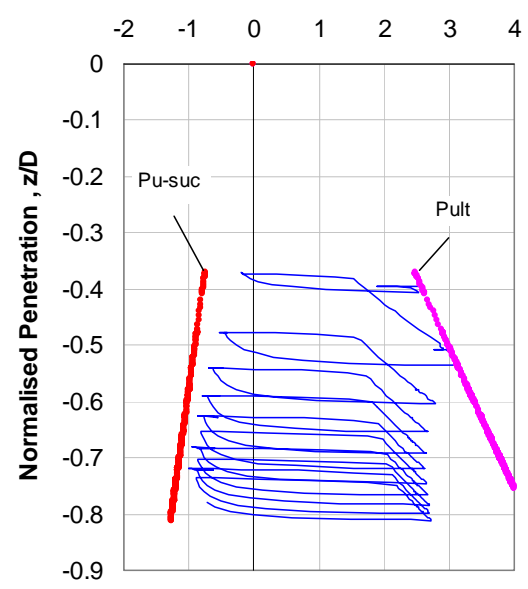

Normalised Resistance P/D (kPa), Node 358

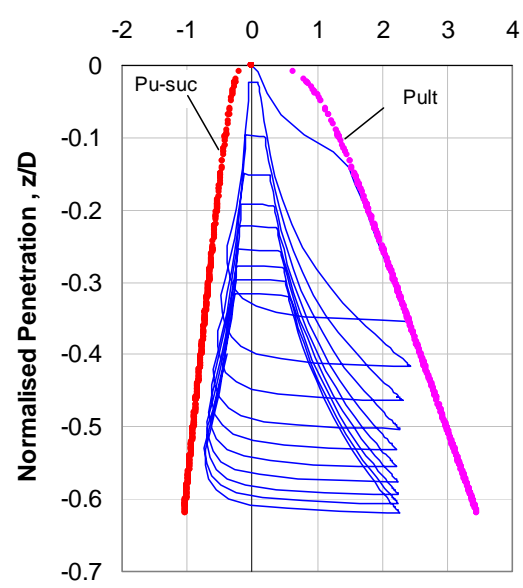

Normalised Resistance P/D (kPa), Node 354

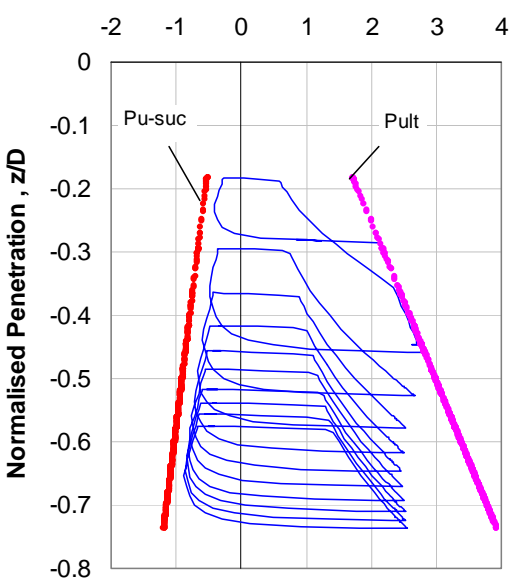

Normalised Resistance PID (kPa), Node 344

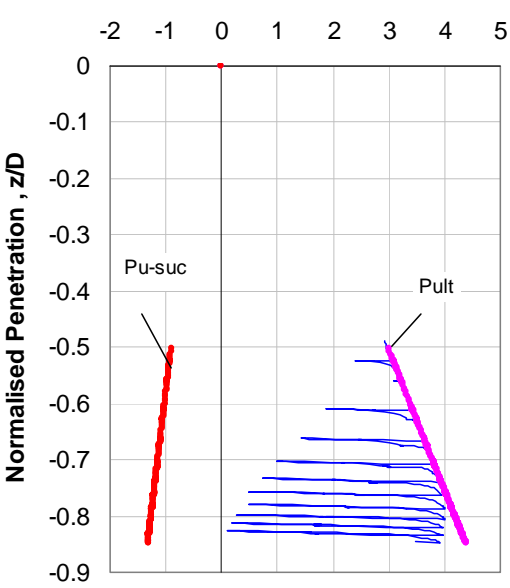




$$
S_{\mathrm{u} 0}=0.65 \mathrm{kPa}
$$

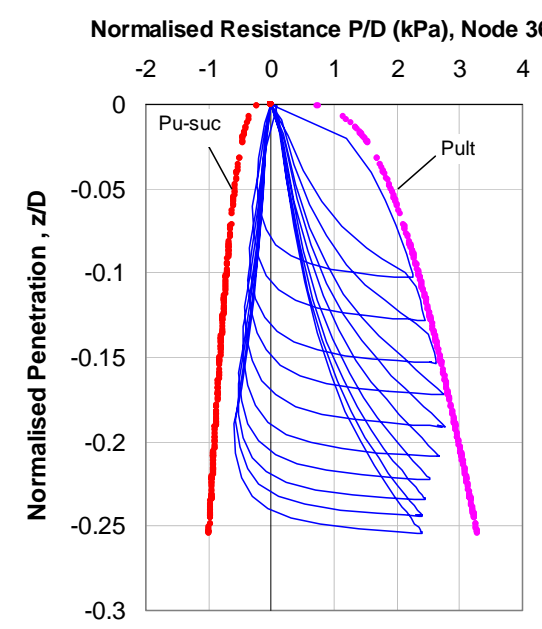

Normalised Resistance P/D (kPa), Node 356

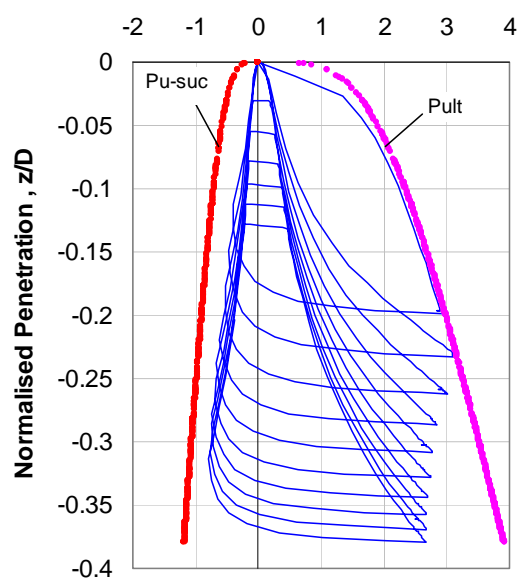

Normalised Resistance P/D (kPa), Node 350

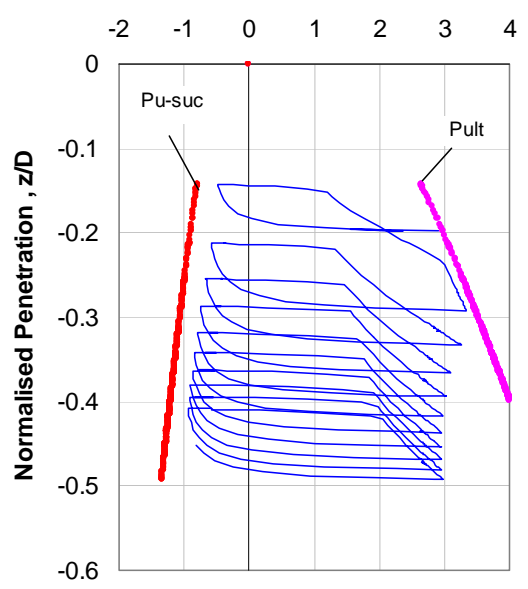

Normalised Resistance P/D (kPa), Node 358

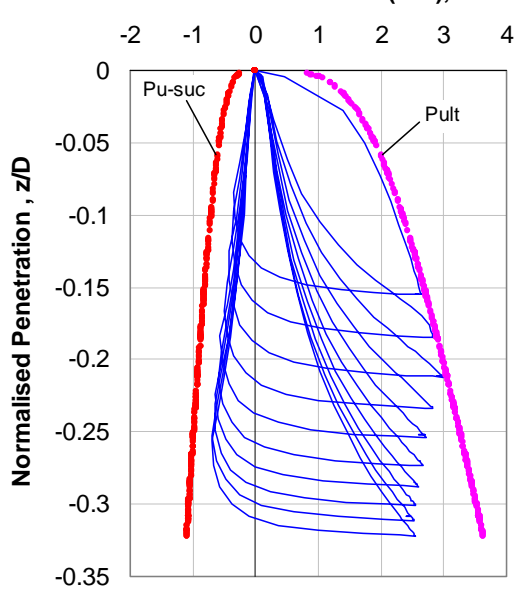

Normalised Resistance P/D (kPa), Node 354

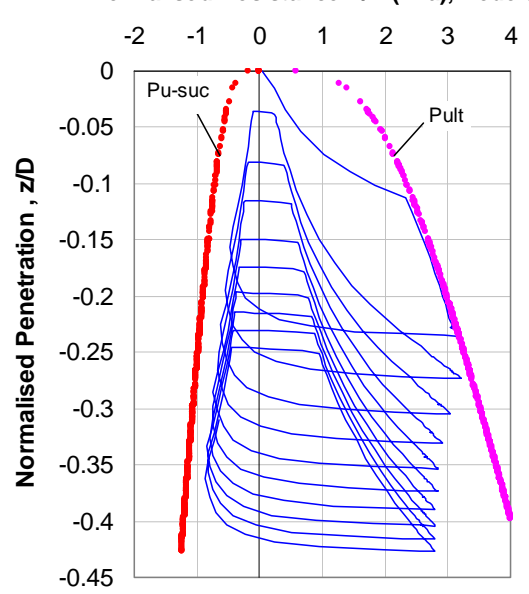

Normalised Resistance PID (kPa), Node 344

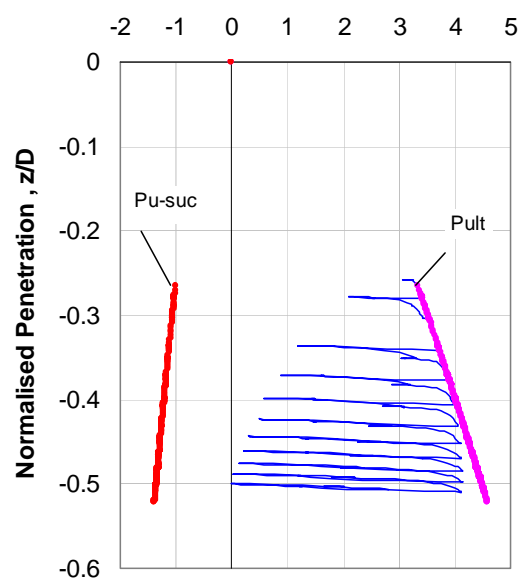




$$
\mathbf{S}_{\mathbf{u} 0}=1.0 \mathrm{kPa}
$$
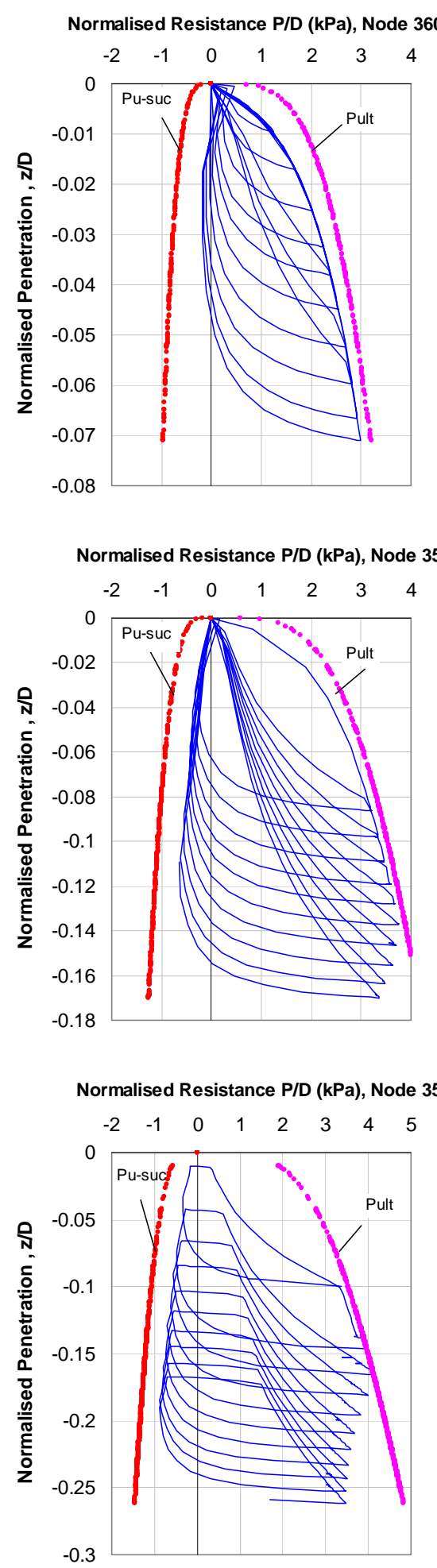
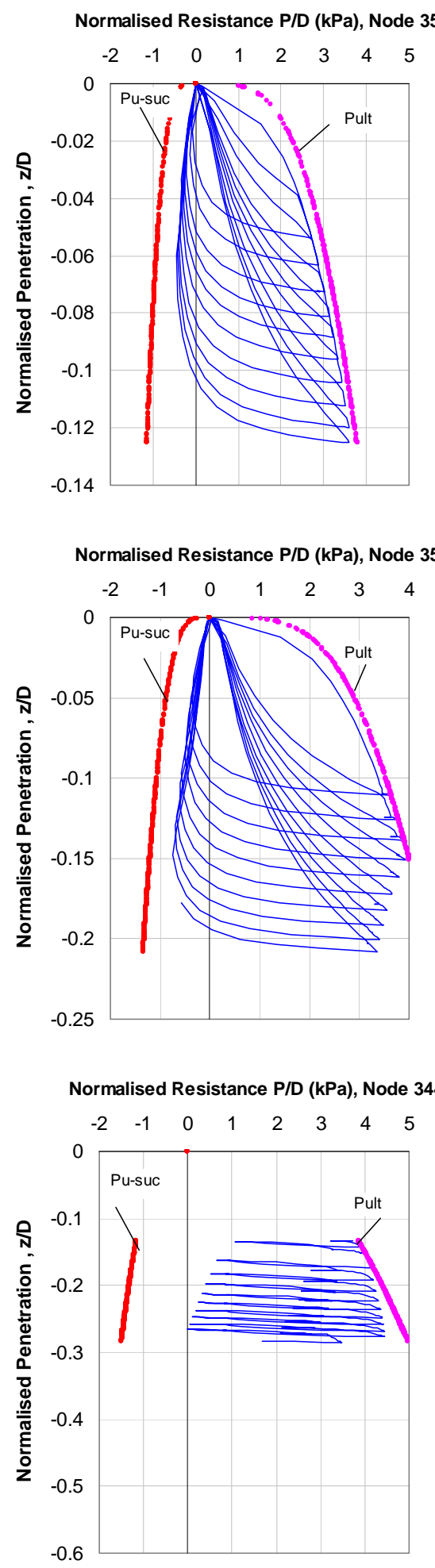


\section{C.6 SOIL UNDRAINED SHEAR STRENGTH GRADIENT}

$$
\rho=0.5 \mathrm{kPa} / \mathrm{m}
$$

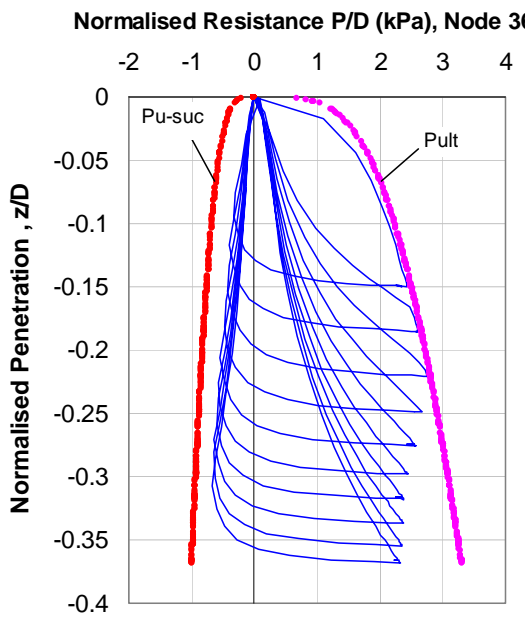

Normalised Resistance P/D (kPa), Node 356

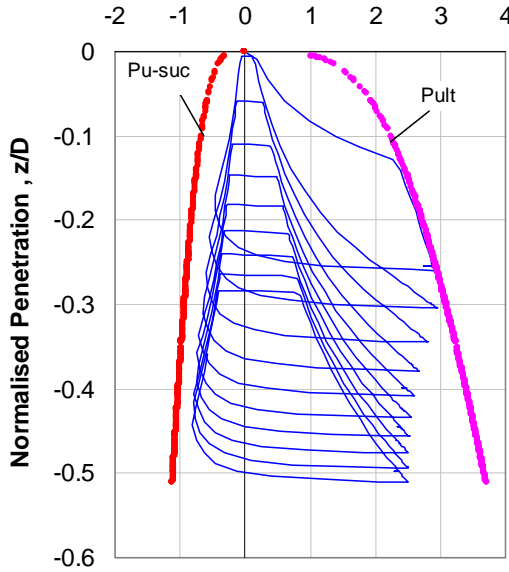

Normalised Resistance P/D (kPa), Node 350

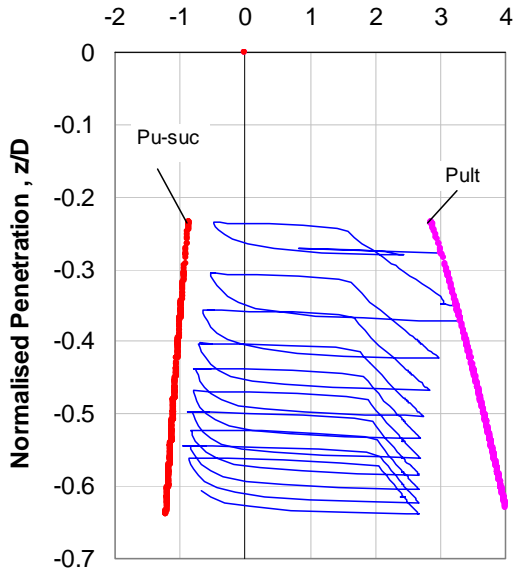

Normalised Resistance P/D (kPa), Node 358

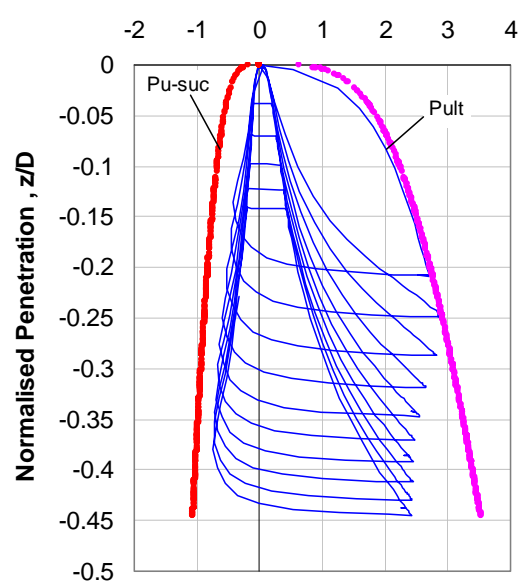

Normalised Resistance P/D (kPa), Node 354

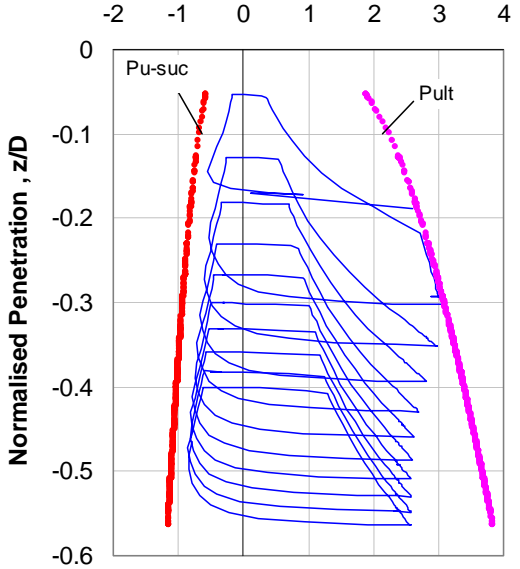

Normalised Resistance PID (kPa), Node 344

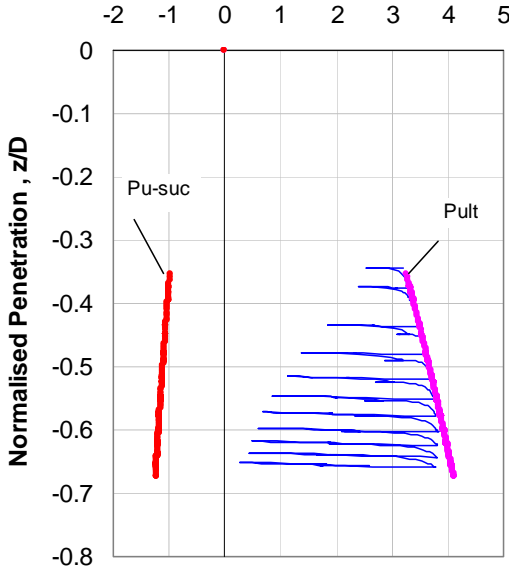




\section{$\rho=1.0 \mathrm{kPa} / \mathrm{m}$}
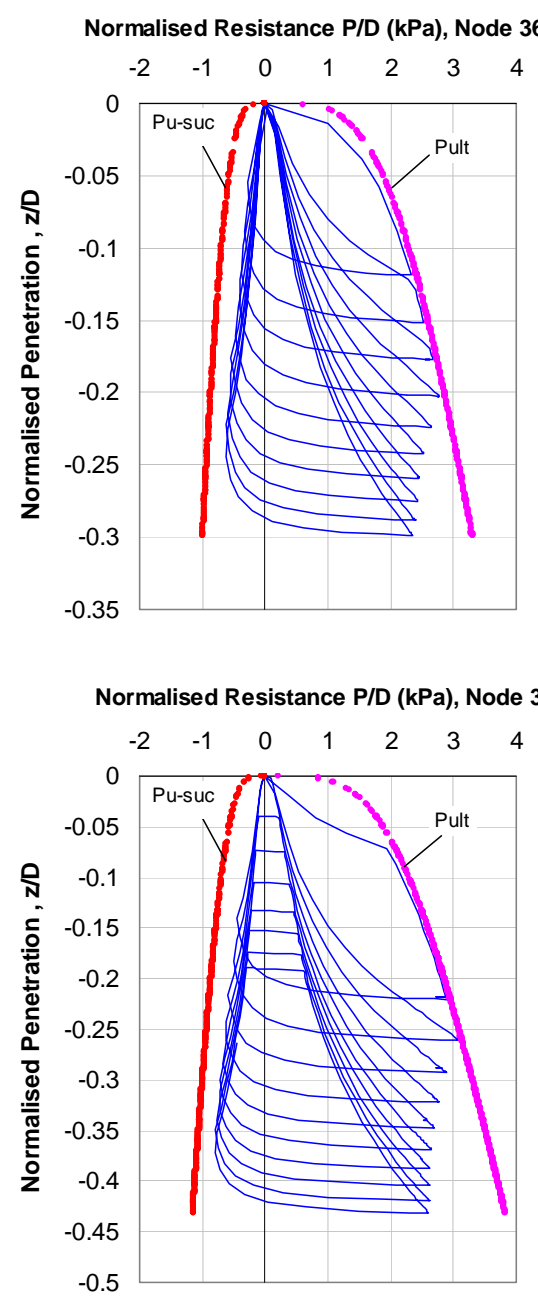

Normalised Resistance P/D (kPa), Node 350

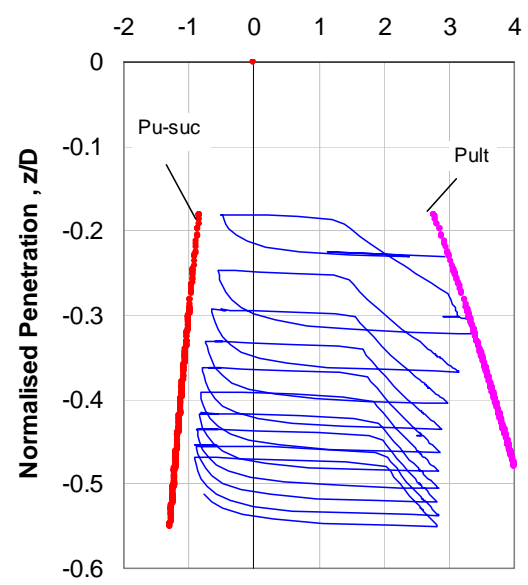

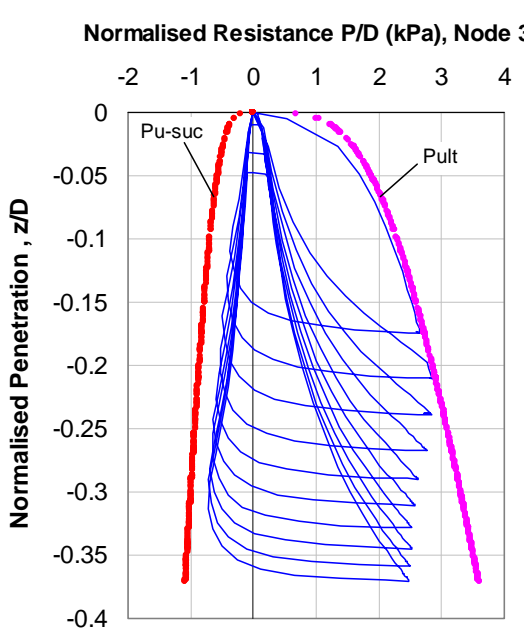

Normalised Resistance P/D (kPa), Node 354

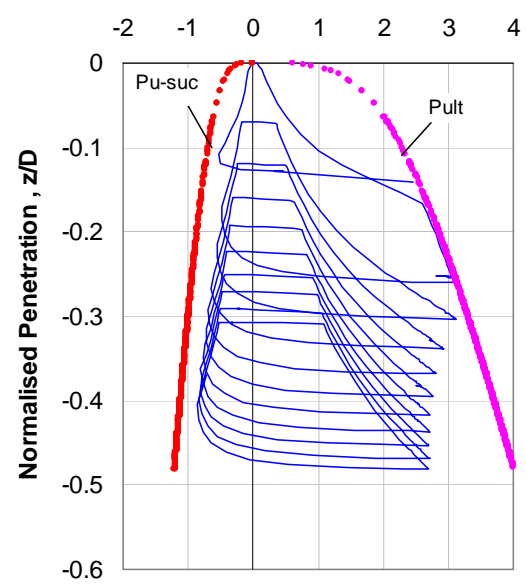

Normalised Resistance P/D (kPa), Node 344

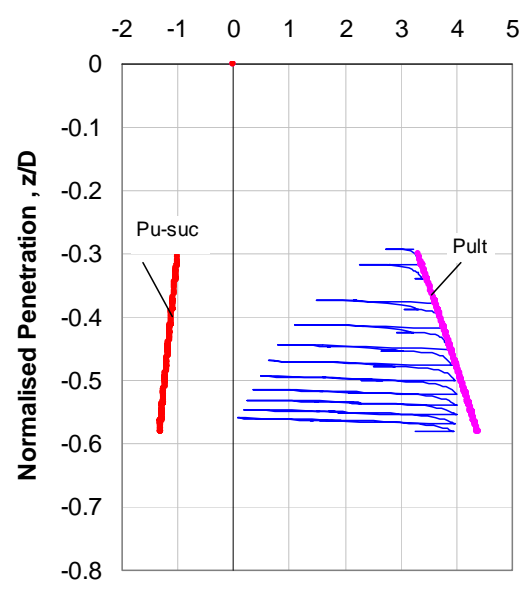




\section{$\rho=1.5 \mathrm{kPa} / \mathrm{m}$}

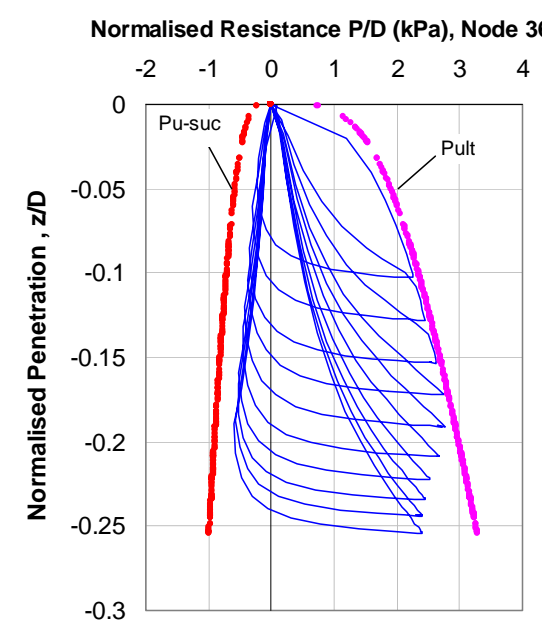

Normalised Resistance P/D (kPa), Node 356

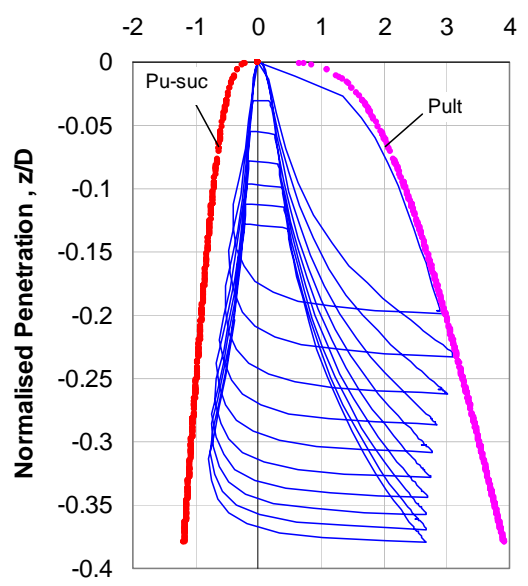

Normalised Resistance P/D (kPa), Node 350

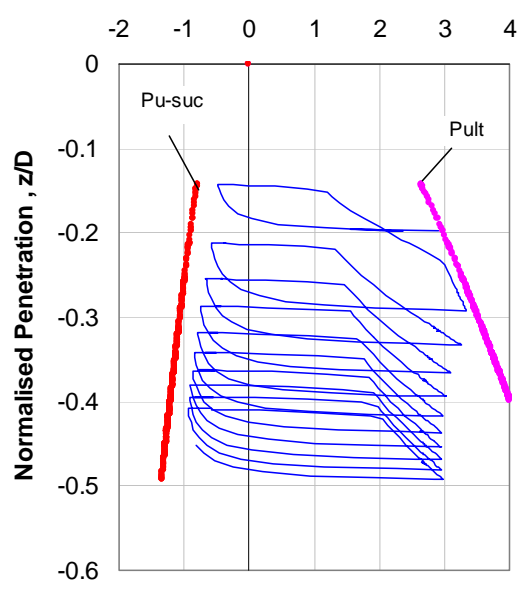

Normalised Resistance P/D (kPa), Node 358

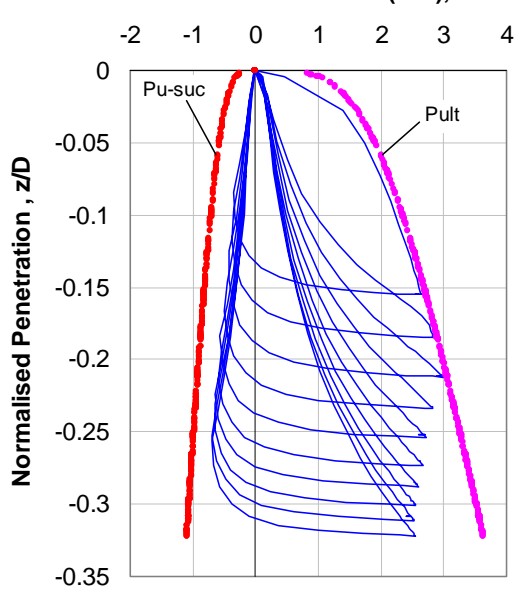

Normalised Resistance P/D (kPa), Node 354

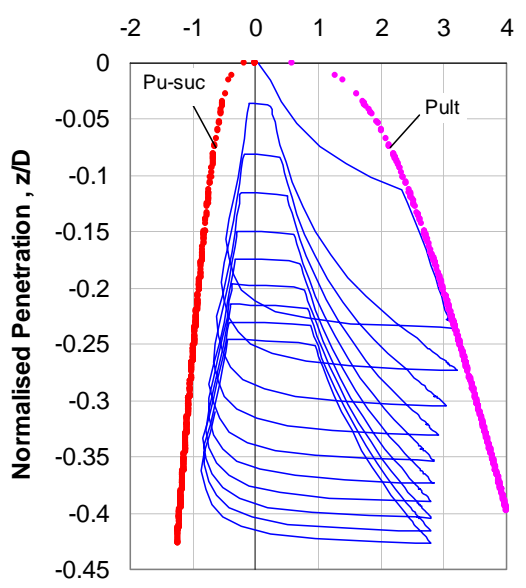

Normalised Resistance PID (kPa), Node 344

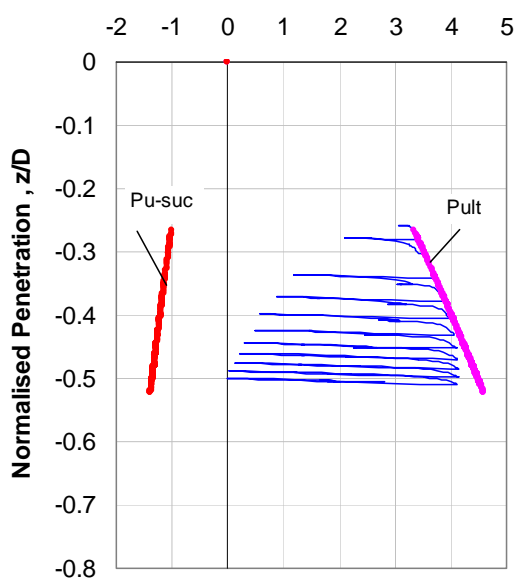




\section{$\rho=2.5 \mathrm{kPa} / \mathrm{m}$}
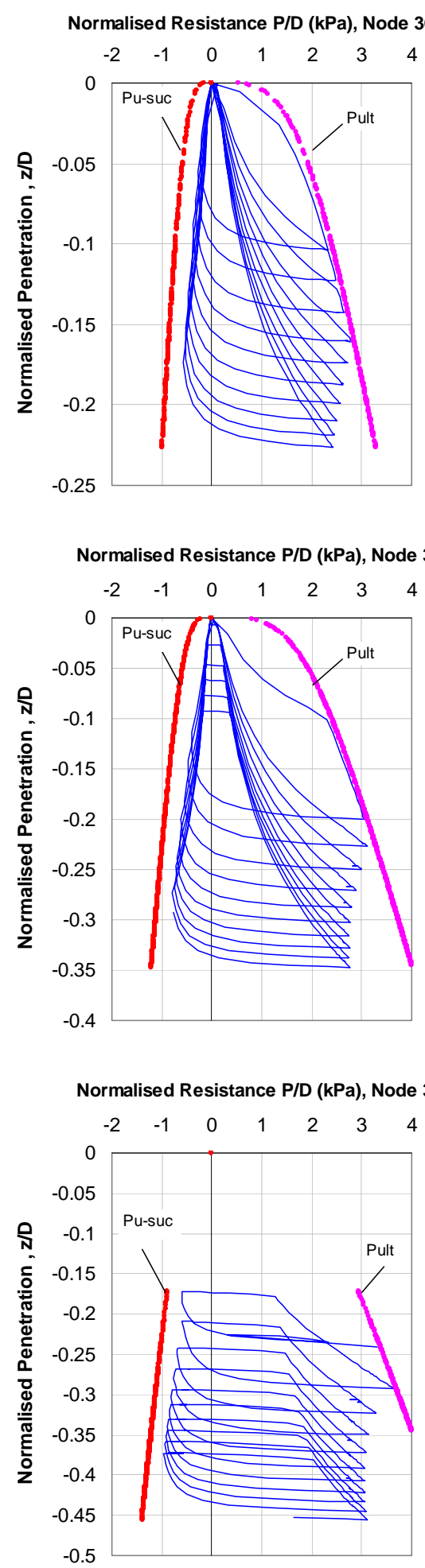
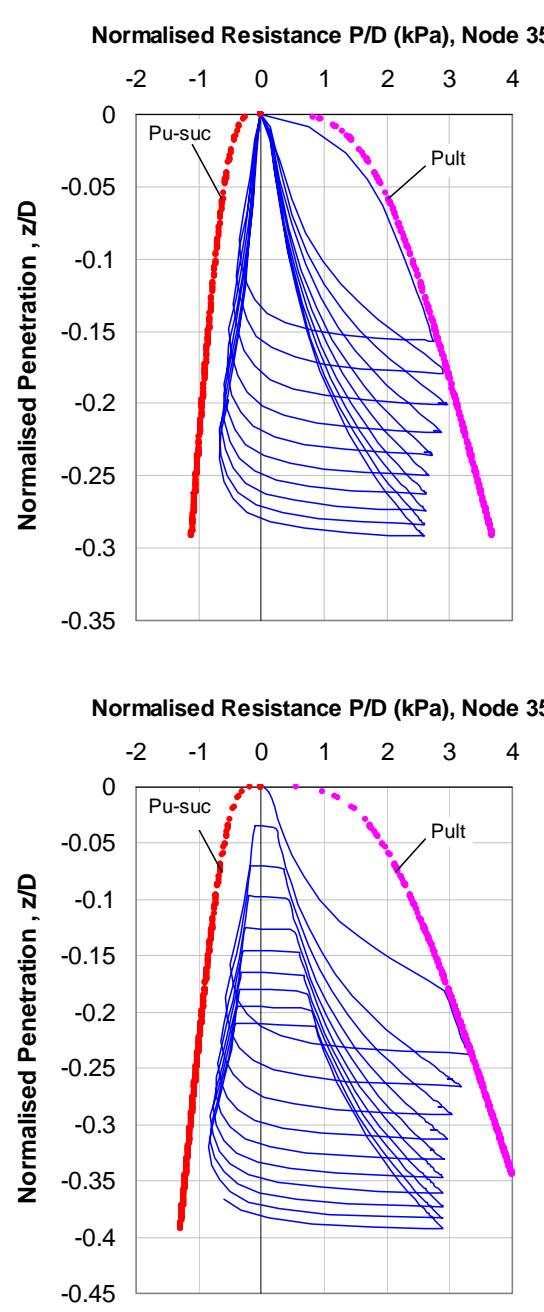

Normalised Resistance PID (kPa), Node 344

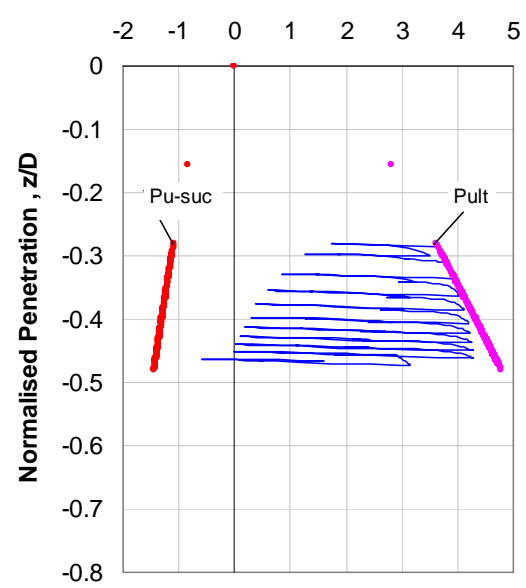


Parametric Study of Non-linear Hysteretic

Seabed Model

This page has been left blank deliberately
School of Civil and Resource Engineering The University of Western Australia 